
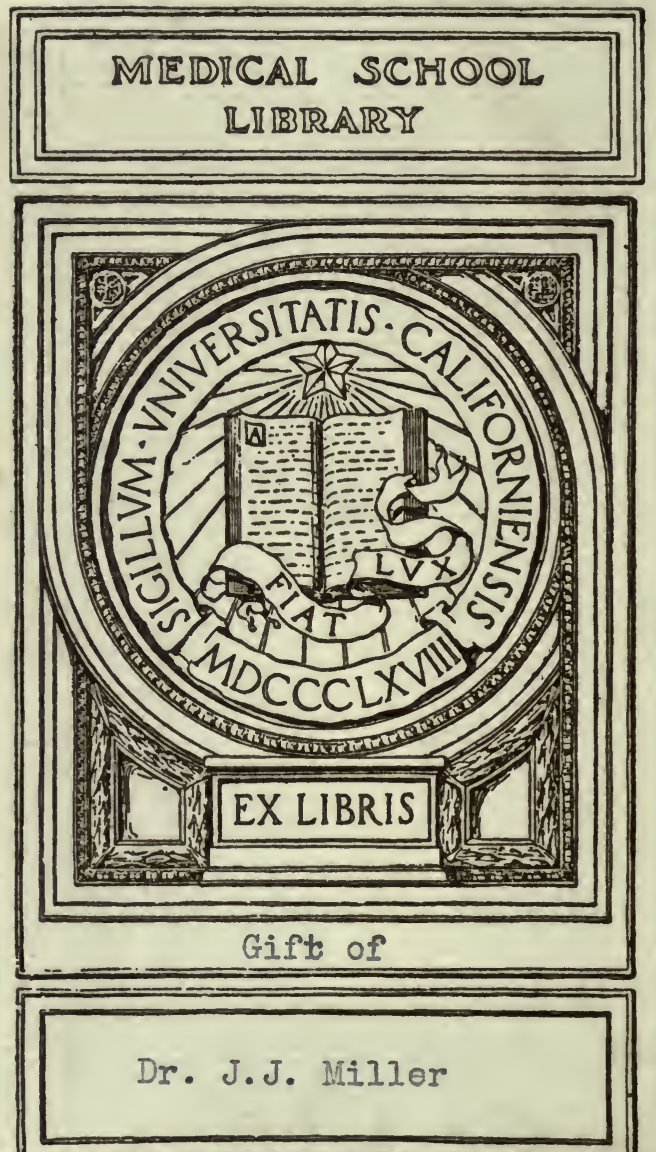

$\therefore$ 
Flounce brene Ratigll ' 24 intan fond 



\section{American \$cience ฐeries}

\section{Chemistry.}

By Ira Remsen, President of the Johns Hopkins University. Astronomy.

By Simon Newcomb and Edward S. Holden.

\section{Geology.}

By Thomas C. Chamberlin and Rollin D. Salisbury, Professors in the University of Chicago.

Physiography.

By Rollin D. Salisbury, Professor in the University of Chicago.

\section{General Biology.}

- By William T. Sedgwick, Professor in the Mass. Institute, and Edmund B. WrLson, Professor in Columbia University.

\section{Botany.}

By Charles E. Bessey, Professor in the University of Nebraska.

\section{Zoology.}

By A. S. PACKARD, Professor in Brown University.

The Human Body.

By H. Newell Martin.

\section{Psychology.}

By William James, Professor in Harvard University.

\section{Ethics.}

By John Dewey, Professor in Columbia University, and JAM ES H. Turts, Professor in the University of Chicago.

\section{Political Economy.}

By Francis A. Walker.

\section{Finance.}

By Henry C. Adams, Professor in the University of Michigan.'

For full descriptions of the Advanced, Briefer, and Elementary Courses published under each topic, see the publishers' Educational Catalog.

\section{HENRY HOLT AND COMPANY Publishers - NeW York}




\section{THE HUMAN BODY}

AN ACCOUNT OF

ITS STRUCTURE AND ACTIVITIES AND THE CONDITIONS OF ITS HEALTHY WORKING

BY

H. NEWELL MARTIN, D.Sc., M.A., M.D., F.R.S.

Late Professor of Biology in the Johns Hopkins University and of Physiology in the Medical Faculty of the same

TENTH EDITION, THOROUGHLY REVISED

BY

ERNEST G. MARTIN, Ph.D.

Professor of Physiology in Leland Stanford Junior University

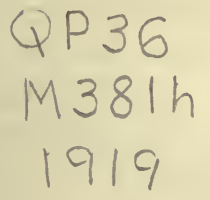

NEW YORK

HENRY HOLT AND COMPANY

1919 tc19/7 
Copyright, 1881, 1896.

BY

HENRY HOLT \& CO.

Copyright. 1909. 1910, 1917.

BY

HENRY HOLT AND COMPANY
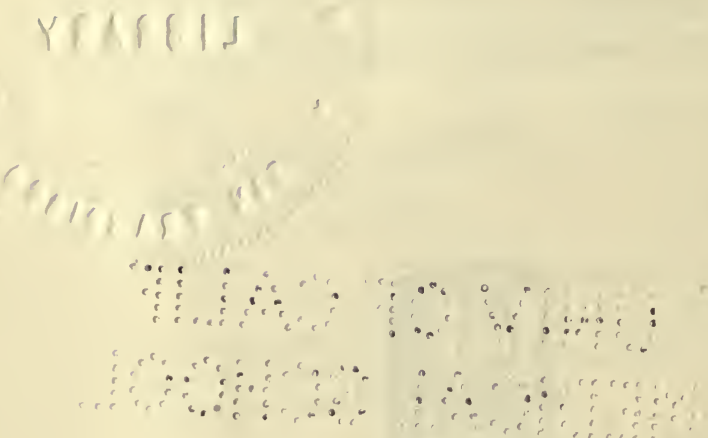


\section{PREFACE TO THE TENTH EDITION}

THE current tendency of physiological thought is clearly toward an increasing emphasis upon the unity of operation of the Human Body. Although the only feasible method of presentation of the subject continues to be by successive consideration of physiological systems one after another, physiologists recognize the desirability of keeping constantly before the mind of the student the fact that in reality the Body is more than an aggregate of systems; that it is an integer of parts so closely interdependent that the activity of any one calls forth related activities of the others. In the present revision the attempt has been made to keep this idea in the foreground. Increased emphasis is placed upon the manifestations of adaptation in the Body; cross references are freely used throughout. As a further assistance toward this end a changed method of treating the subject of chemical co-ordination is adopted. Instead of a chapter, or part of a chapter, devoted to internal secretions, the conception of chemical co-ordination is introduced, concurrently with that of nervous co-ordination, in an early chapter, and the various hormones are described in connection with the bodily processes they are known to modify.

Numerous minor changes have been made to bring the presentation abreast of present physiological knowledge. The following more or less extensive additions to the subject-matter of the former edition have also been included. A section on the physical chemistry of the Body is added to the first chapter. To this the paragraphs on filtration, osmosis, and dialysis are transferred from the chapter on blood in which they were formerly given. A brief discussion of crystalloids, colloids, solutions, and the significance of cell membranes is also included. The conception of the Body as a machine in its energy relationships, obeying the familiar laws of mechanics, is emphasized more strongly than hitherto. In connection with the discussion of muscle physiology a rather full account of the energy transformations in active muscle is given. The section on the nervous system has been modified 
in two respects. The description of the cerebellum and its functions is introduced before the account of the cerebrum, instead of after it as in the former presentation. The chapter on the autonomic system has been rewritten, with special emphasis on the "emergency" function of the system. The relation of the hormone adrenin to the autonomic system is discussed. In connection with the section on respiration the effect of muscular exercise on the respiratory function is given fuller consideration. In the chapter on foods the paragraphs dealing with dietary accessories are elaborated. The recent work on the relative food values of various proteins is described at some length. The conception of basal metabolism is introduced as a ground work for the discussion of general bodily metabolism. In the chapter on reproduction paragraphs dealing in an elementary way with Mendelian inheritance and the mechanism of sex-determination are included. In response to requests from several users of the book an appendix containing suggestions for laboratory experiments suitable for undergraduate classes is added. The basis of these is the laboratory course given for several years to undergraduates at Harvard and Radcliffe Colleges.

I beg to acknowledge the receipt of helpful suggestions from various colleagues. Among these I wish to mention especially Professor Chas. Wright Dodge, who very kindly furnished me a detailed list of comments based on his use of the former edition in his classes during a period of years.

A number of new cuts have been introduced. Some of these were drawn especially for this edition. Others were kindly furnished by the publishers of various text-books. I desire to make acknowledgment of this courtesy.

ERnest G. Martin.

Stanford University, Calif.

July, 1917. 


\section{PREFACE TO THE FIRST EDITION}

IN the following pages I have endeavored to give an account of the structure and activities of the Human Body, which, while intelligible. to the general reader, shall be accurate, and sufficiently minute in details to meet the requirements of students who are not making Human Anatomy and Physiology subjects of special advanced study. Wherever it seemed to me really profitable, hygienic topics have also been discussed, though at first glance they may seem less fully treated of than in many School or College Text-books of Physiology. Whoever will take the trouble, however, to examine critically what passes for Hygiene in the majority of such cases will, I think, find that, when correct, much of it is platitude or truism: since there is so much that is of importance and interest to be said it seems hardly worth while to occupy space with insisting on the commonplace or obvious.

It is hard to write a book, not designed for specialists, without running the risk of being accused of dogmatism, and some readers will, no doubt, be inclined to think that, in several instances, I have treated as established facts matters which are still open to discussion. General readers and students are, however, only bewildered by the production of an array of observations and arguments on each side of every question, and, in the majority of cases, the chief responsibility under which the author of a text-book lies is to select what seem to him the best supported views, and then to state them simply and concisely: how wise the choice of a side has been in each case can only be determined by the discoveries of the future.

Others will, I am inclined to think, raise the contrary objection that too many disputed matters have been discussed: this was deliberately done as the result of an experience in teaching Physiology which now extends over more than ten years. It would have been comparatively easy to slip over things still uncertain and subjects as yet uninvestigated, and to represent our knowledge of the workings of the animal body as neatly rounded off at all its contours and complete in all its details-totus, teres, et rotundus. 
But by so doing no adequate idea of the present state of physiological science would have been conveyed; in many directions it is much farther traveled and more completely known than in others; and, as ever, exactly the most interesting points are those which lie on the boundary between what we know and what we hope to know. In Gross Anatomy there are now but few points calling for a suspension of judgment; with respect to Microscopic Anatomy there are more; but a treatise on Physiology which would pass by, unmentioned, all things not known but sought, would convey an utterly unfaithful and untrue idea. Physiology has not finished its course. It is not cut and dried, and ready to be laid aside for reference like a specimen in an Herbarium, but is comparable rather to a living, growing plant, with some stout and useful branches well raised into the light, others but part grown, and many still represented by unfolded buds. To the teacher, moreover, no pupil is more discouraging than the one who thinks there is nothing to learn; and the boy who has "finished" Latin and "done" Geometry finds sometimes his counterpart in the lad who has "gone through" Physiology. For this unfortunate state of mind many Text-books are, I believe, much to blame: difficulties are too often ignored, or opening vistas of knowledge resolutely kept out of view: the forbidden regions may be, it is true, too rough for the young student to be guided through, or as yet pathless for the pioneers of thought; but the opportunity to arouse the receptive mental attitude apt to be produced by the recognition of the fact that much more still remains to be learned-to excite the exercise of the reasoning faculties upon disputed matters-and, in some of the better minds, to arouse the longing to assist in adding to knowledge, is an inestimable advantage, not to be lightly thrown aside through the desire to make an elegantly symmetrical book. While I trust, therefore, that this volume contains all the more important facts at present known about the working of our Bodies, I as earnestly hope that it makes plain that very much is yet to be discovered.

A work of the scope of the present volume is, of course, not the proper medium for the publication of novel facts; but, while the "Human Body," accordingly, professes to be merely a compilation, the introduction of constant references to authorities would have been out of place. I trust, however, that it will be found through- 
out imbued with the influence of my beloved master, Michael Foster; and on various hygienic topics I have to acknowledge a special indebtedness to the excellent series entitled Health Primers.

The majority of the anatomical illustrations are from Henle's Anatomie des Menschen, and a few from Arendt's Schulatlas, the publishers of each furnishing electrotypes. A considerable number, mainly histological, are from Quain's Anatomy, and a few figures are after Bernstein, Carpenter, Frey, Haeckel, Helmholtz, Huxley, McKendrick, and Wundt. About thirty, chiefly diagrammatic, were drawn specially for the work.

Quantities are throughout expressed first on the metric system, their approximate equivalents in American weights and.measures being added in brackets.

Baltimore, October, 1880.

H. Newell Martin. 



\section{CONTENTS}

\section{CHAPTER I}

THE GENERAL STRUCTURE AND COMPOSITION OF THE HUMAN BODY

Definitions. Tissues and organs. Histology. Zoological position of man. The vertebrate plan of structure. The mammalia. Microscopic structure of the Body. Chemical composition of the Body. Physico-

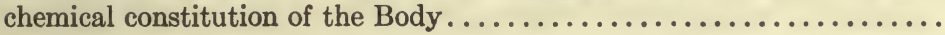

\section{CHAPTER II}

\section{THE FUNDAMENTAL PHYSIOLOGICAL ACTIONS}

The properties of the living Body. The Body as a machine. Cell growth. Details of cell structure. Mitotic cell division. Significance of the physiological properties. Adaptation. Co-ordination in the Body.

\section{CHAPTER III}

TISSUES, ORGANS, AND PHYSIOLOGICAL SYSTEMS

Development. The physiological division of labor. Classification of the tissues. The combination of tissues to form organs. Physiological systems. The relation of man to his environment. Adaptive systems. Maintenance systems. Chemical co-ordination. Animals compared with plants............................................

\section{CHAPTER IV}

THE SUPPORTING TISSUES

Connective tissue. Cartilage. Bone. Hormones of the supporting

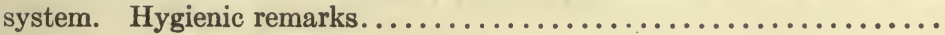

\section{CHAPTER V}

\section{THE SKELETON}

Exoskeleton and endoskeleton. The bony skeleton. Peculiarities of the human skeleton. Hygiene of the bony skeleton. Articulations. Joints.

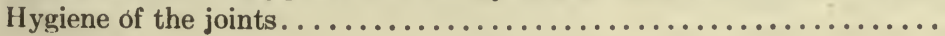




\section{CHAPTER VI}

THE STRUCTURE OF THE MOTOR ORGANS

PAGE

Motion in animals. The muscles. Histology of skeletal muscle. Structure of the smooth muscles. Cardiac muscular tissue. Ciliated cells. The physico-chemistry of skeletal muscle. The chemistry of muscular

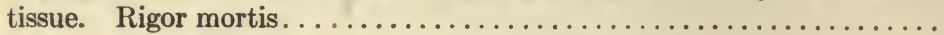

\section{CHAPTER VII}

\section{MUSCULAR ACTIVITY}

The study of isolated muscles. The necessity of stimulation. A simple muscular contraction. The influence of increasing stimulation strength. The influence of temperature. Heat rigor. The measure of muscular work. Influence of the form of the muscle on its working power. The beneficial effect of exercise. The nature of fatigue. The response to rapidly repeated stimuli. Tetanus. Voluntary muscular contraction. The electrical phenomena of muscle. The source of muscular energy. The chemistry of muscular contraction. The energy relationships of contracting muscle. Muscular efficiency. Energy units. The energy output of muscle. Significance of lactic acid in the contraction process. Summary of the contraction process. Oxidation in muscle. Hormone of skeletal muscle. Physiology of smooth muscle. Mechanism of contraction of smooth muscle. Physiology of cardiac muscle...............

\section{CHAPTER VIII}

THE USE OF MUSCLES IN THE BODY

Special physiology of the skeletal muscles. Levers in the Body. Loss to the muscles from the direction of their pull. The equilibrium of opposing muscles. Functional muscle groups. Posture. Locomotion. Prehension. Hygiene of the muscles. Varieties of exercise.......... 118

\section{CHAPTER IX}

\section{ANATOMY OF THE NERVOUS SYSTEM}

General statement. Nerve impulses. Neurons. Synapses. The myelin sheath. The central and peripheral nervous systems. Membranes of the central nervous system. Ventricles of the brain and central canal of the spinal cord. Cerebrospinal fluid. The spinal cord. The brain. The spinal nerves. Cranial nerves. White and gray matter. The sympathetic or autonomic system . . . . . . . . . . . . . . . . . . . . . 


\section{CHAPTER $\mathrm{X}$}

GENERAL PHYSIOLOGY OF THE NERVOUS SYSTEM. SPINAL AND CEREBELLAR REFLEXES

PAGE

Conduction within single neurons. Nature of the nerve impulse. Reflexes. Reflex ares. Irreversible conduction. Graded synaptic resistance. The orderly spreading of reflexes. Simple reflexes mediated by the spinal cord. Significance of the head senses in the control of reflexes. The sensory basis of locomotion. Structure and connections of the cerebellum. Functions of the cerebellum. Postural reflexes.................

\section{CHAPTER XI}

STRUCTURE, NERVE CONNECTIONS, AND FUNCTIONS OF THE CEREBRUM

The cerebrum in relation to muscular activity. A normal animal compared with a "reflex" one. The cerebrum dependent on the receptor system. Afferent paths of the cerebrum: Tracing nerve paths. Tracts of Body sense. Tracts of the head senses. General structure of the cerebrum. Structure of the cortex. Cortical localization. Cortical reflex paths. Cortical reflexes compared with spinal. Memory. Association. Volition. Inhibition. Will power. Habit formation. Language. Consciousness. Emotions. Cerebral functions compared in man and animals. Nourishment of the brain . . . . . . . . . . . . . . . . . . . . . . . . . .

\section{CHAPTER XII}

THE AUTONOMIC NERVOUS SYSTEM. NERVOUS FATIGUE. HORMONES OF THE NERVOUS SYSTEM

The brain stem (medulla and mid-brain). The autonomic or sympathetic system. The effect of nicotine. Reflex control of the autonomic system. Grand divisions of the autonomic system. This an emergency mechanism. The relation of the autonomic system to emotional states. Neuro-muscular fatigue. Adrenin. The thyroid. Emergency action of

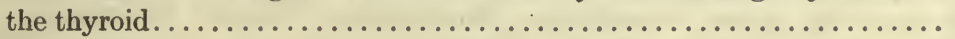

\section{CHAPTER XIII .}

THE RECEPTOR SYSTEM. INTERNAL AND CUTANEOUS SENSATIONS

The receptor system. The differences between sensations. Psychophysical law. Classification of receptors. Internal senses. Muscular sense. Hunger. Thirst. Cutaneous senses. Pain. Touch. Temperature sense. Peripheral reference of sensations. Perceptions. Illusions. . 204 


\section{CHAPTER XIV}

THE EAR. HEARING AND EQUILIBRATION. TASTE AND SMELL

Functions of the ear. Sounds. Sympathetic resonance. The external ear. Functions of the tympanic membrane. The middle ear. Auditory. ossicles. Internal ear. Bony labyrinth. Membraneous labyrinth. Organ of Corti. Function of the cochlea. Auditory perceptions. Nerveendings in semicircular canals and vestibule. Equilibrium sense. Smell.

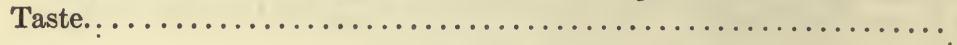

\section{CHAPTER XV}

\section{THE EYE AS AN OPTICAL INSTRUMENT}

The essential structure of an eye. Appendages of the eye. Lachrymal apparatus. Muscles of eye. Anatomy of eyeball. Optic nerves, chiasma, and tracts. Retina. Refracting media of the eye. Ciliary muscle. Properties of light. Refraction. Wide range of clear vision in the resting eye. Accommodation. Defects of the eye. Hygiene of the eyes...........

\section{CHAPTER XVI}

\section{THE EYE AS A SENSORY APPARATUS}

The excitation of the visual apparatus. Intensity of visual sensations. Function of the rods. Visual purple. Duration of luminous sensations. Localizing power of retina. Color vision. Function of the cones. Distribution of color sense over the retina. Color blindness. After images. Contrasts. Theories of color vision. Visual perceptions. Vision with two

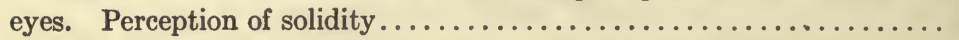

\section{CHAPTER XVII}

\section{THE STRUCTURE AND FUNCTIONS OF BLOOD AND LYMPH}

The external medium. The internal medium. Blood. Lymph. Renewal of lymph. Lymphatic vessels. Lacteals. Composition of blood. Red corpuscles. Hemoglobin. Origin and fate of red corpuscles. Spleen. Function of the spleen. Leucocytes. Blood plates. Plasma. Quantity of blood. Blood of other animals. Histology and chemistry of lymph. . 290

\section{CHAPTER XVIII}

THE HORMONE-CARRYING AND DISEASE-RESISTING FUNCTIONS OF THE BLOOD. BLOOD CLOTTING

Hormones. Infection. Resistance to infection. Recovery from infection. Opsonins, immune bodies, and agglutinins. Antitoxin. Immunity. Carriers. The use of antitoxin in disease. Protective inoculation. 
Anaphylaxis. Coagulation of blood. Cause of coagulation. Use of coagulation. Source of blood-fibrin. Thrombin. Antithrombin. Thromboplastic substance. Methods of hastening or retarding coagulation.

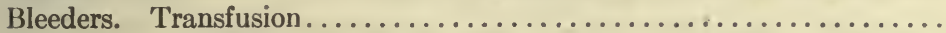

\section{CHAPTER XIX}

THE ANATOMY OF THE HEART AND BLOOD VESSELS

General statement. Position of heart. Membranes of heart. Cavities of heart. Anatomy of heart. Valves of heart. Arterial system. Capillaries. Veins. Pulmonary circulation. Course of blood. Portal circulation. Arterial and venous blood. Structure of vessels..............

\section{CHAPTER XX}

THE ACTION OF THE HEART. THE REGULATION OF THE HEART-BEAT

Beat of the heart. Cardiac impulse. Events of a cardiac cycle. Use of papillary muscles. Sounds of heart. Action of heart-valves. Effects of valvular insufficiency. Function of auricles. Work done by heart. Relation of nerve and muscle elements within heart. Physiological peculiarities of heart. Passage of beat over heart. Neurogenic and myogenic theories of beat. Nature of automatic rhythmicity. Extrinsic nerves of heart. Inhibitory and augmentor centers...................

\section{CHAPTER XXI}

THE CIRCULATION OF THE BLOOD. BLOOD PRESSURE AND BLOOD VELOCITY. THE PULSE

Circulation seen in frog's web. Resistance to blood-flow. Conversion of intermittent into continuous flow. Arterial pressure. Weber's schema. The pulse. Blood-pressure in man. Rate of the blood-flow. Secondary factors affecting the circulation. Aspiration of the thorax. Proofs of the

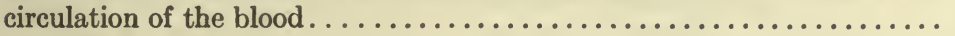

\section{CHAPTER XXII}

THE VASOMOTOR MECHANCISM. SLEEP. THE LYMPHATIC SYSTEM

Distribution of blood among various parts of Body. Nerves of bloodvessels. Vasoconstrictor nerves. Vasoconstrictor center. Control of vasoconstrictor center. Depressor nerve. Taking cold. Vasodilator nerves. Vasodilator center. Relation of vasomotor tone to cerebral activity. Sleep. Adrenin. The lymphatics. Structure of lymph vessels. Lymph-nodes. Tonsils and adenoids. Movements of lymph. Lympha-

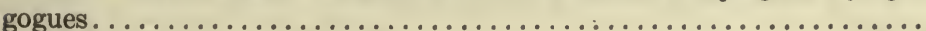




\section{CHAPTER XXIII}

RESPIRATION. THE MECHANISM OF BREATHING. THE REGULATION OF BREATHING

Definitions. Respiratory organs. Air-passages and lungs. Trachea and bronchi. Structure of lungs. Pleura. Respiratory movements. Anatomy of thorax. Changes in size of thorax. Forced respiration. Respiratory sounds. Capacity of lungs. Aspiration of thorax. Respiratory center. Excitation of respiratory center. Sensitiveness of respiratory center. Eupnea, hyperpnea, dyspnea, apnea. Holding the breath. Asphyxia. Artificial respiration. Modified respiratory movements.......

\section{CHAPTER XXIV}

\section{RESPIRATION. THE GASEOUS INTERCHANGES}

Nature of the problems. Changes produced in air by being breathed. Ventilation. Changes undergone by blood in lungs. Blood gases. Laws governing absorption of gases by liquid. Absorption of oxygen by blood. Oxygen interchanges in blood. Carbon dioxid in blood. Hormone action of carbon dioxid. Tissue respiration. Respiratory changes in muscular

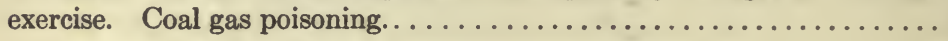

\section{CHAPTER XXV}

FOODS: THEIR CLASSIFICATION

What constitutes food. Function of food. Classes of foods. Occurrence of nutrients in food. Inorganic essential accessories. Organic essential accessories. Vitamines. Occurrence of occasional accessories in food. Nutrients. Mixed foods. Flesh. Eggs. Milk. Vegetable foods. Composition of foods. Alcohol. Tea, coffee, cocoa. Food poisoning.......

\section{CHAPTER XXVI}

\section{THE ALIMENTARY CANAL AND ITS APPENDAGES}

General arrangement. Subdivisions of the canal. Mouth. Teeth. Tongue. Salivary glands. Pharynx. Esophagus. Stomach. Small intestine. Large intestine. Nerves of intestines. Liver. Pancreas. Blood-

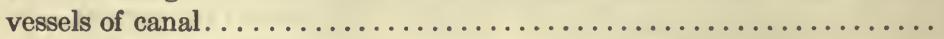

\section{CHAPTER XXVIII \\ THE CHEMISTRY OF DIGESTION}

Object of digestion. Nature of the digestive process. Digestion products. Saliva. Gastric juice. Pancreatic juice. Bile. Succus entericus. Summary of digestive process. Bacterial digestion. Prevention of self-

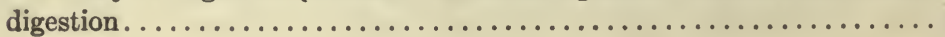




\section{CHAPTER XXVIII}

MOVEMENTS OF THE ALIMENTARY CANAL

PAGE

Mastication. Hygiene of mouth. Deglutition. Movements of stomach. Control of pyloric sphincter. Importance of stomach. Movements of small intestine. Extrinsic control of stomach and intestinal movements. Movements of large intestine. Importance of roughage. . . . . . . . .

\section{CHAPTER XXIX}

\section{THE DIGESTIVE SECRETIONS AND THEIR CONTROL}

Organs of secretion. Forms of glands. Secretory process. Nervous control of secretory process. Hormone control of gland activity. Control of salivary secretion. Control of gastric secretion. Nature of chemical stimulus to gastric secretion. Control of pancreatic secretion. Control of bile flow. Control of succus entericus. Digestive history of a meal.

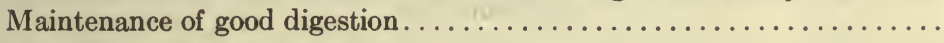

\section{CHAPTER XXX}

\section{THE ABSORPTION AND USE OF FOODS}

General statement. Absorption from stomach. Absorption in small intestine. Nature of absorptive process. Channels of absorption. Absorption and storage of carbohydrates. Glycogen in muscles. Relation of kidney to concentration of sugar in blood. Alimentary glycosuria. Emotional glycosuria. Diabetes mellitus. Glycosuria from increased permeability of kidney cells. Absorption of proteins. Absorption of fats. Absorption from large intestine. Food requirement of Body. Protein requirement of Body. Maintenance proteins and growth proteins. Fuel protein. Liberation of energy in Body. Basal metabolism. Metabolism of muscular work. Relative food values of proteins, carbohydrates, and fats. Specific dynamic action of proteins. Nutritive value of albuminoids. Special metabolism of fats. Principles of dietetics. Maintenance of constant weight. Water equilibrium. Nitrogen equilibrium. Carbon equilibrium. Influence of thyroid hormone on metabolism. Treatment for

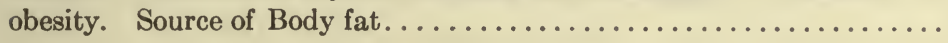

\section{CHAPTER XXXI}

\section{EXCRETION AND THE EXCRETORY ORGANS}

Exogenous and endogenous excreta. Channels of excretion. Liver as an excretory organ. General arrangement of urinary organs. Structure of kidney. Blood-flow through kidney. Urine. Secretory action of different parts of tubule. Relation of blood-flow to secretion of urine. Skin. Hairs. Nails. Glands of skin. Skin secretions. Factors in sweat secretion. Sebaceous secretion. Bathing 


\section{CHAPTER XXXII}

THE PRODUCTION AND REGULATION OF THE HEAT OF THE BODY

Cold- and warm-blooded animals. Temperature of Body. Sources of animal heat. Maintenance of uniform temperature. Local temperature. Fever. Clothing................................. 539

\section{CHAPTER XXXIII}

\section{VOICE AND SPEECH}

Voice. Larynx. Vocal cords. Muscles of larynx. Vowels. Consonants. .............................. 540

\section{CHAPTER XXXIV}

\section{REPRODUCTION}

Reproduction in general. Germ cells compared with tissue cells. Sexual reproduction. Maturation of the germ-cells. Accessory reproductive organs. Male reproductive organs. Seminal fluid. Female reproductive organs. Mammalian ovum. Ovulation. Menstruation. Fertilization. Heredity. Sex determination. Impregnation. Pregnancy. Intrauterine nutrition of embryo. Parturition. Lactation. Puberty. Hormones of reproductive system. Stages of life. Death..............

\section{APPENDIX}

Suggestions for laboratory work $\ldots \ldots \ldots \ldots \ldots \ldots \ldots \ldots \ldots, 587$

Index...........................................6 631 


\title{
THE HUMAN BODY
}

\author{
CHAPTER I
}

\section{THE GENERAL STRUCTURE AND COMPOSITION OF THE HUMAN BODY}

Definitions. The living Human Body may be considered from either of two aspects. Its structure may be especially examined, and the forms, connections and mode of growth of its parts be studied, as also the resemblances or differences in such respects which appear when it is compared with other animal bodies. Or the living Body may be more especially studied as an organism presenting definite properties and performing certain actions; and then its parts will be investigated with a view to discovering what duty, if any, each fulfils. The former group of studies constitutes the science of Anatomy, and in so far as it deals with the Human Body alone, of Human Anatomy; while the latter, the science concerned with the uses-or in technical language the functions-of each part is known as Physiology. Closely connected with physiology is the science of Hygiene, which is concerned with the conditions which are favorable to the healthy action of the various parts of the Body; while the activities and structure of the diseased body form the subject-matter of the science of Pathology.

Tissues and Organs. Histology. Examined merely from the outside our Bodies present a considerable complexity of structure. We easily recognize distinct parts as head, neck, trunk and limbs; and in these again smaller constituent parts, as eyes, nose, ears, mouth; arm, forearm, hand; thigh, leg and foot. We can, with such an external examination, go even farther and recognize different materials as entering into the formation of the larger parts. Skin, hair, nails and teeth are obviously different substances; simple examination by pressure proves that internally there are harder and softer solid parts; while the blood that flows from a cut finger shows that liquid constituents also exist in the Body. The 
conception of complexity which may be thus arrived at from external observation of the living, is greatly extended by dissection of the dead Body, which makes manifest that it consists of a great number of diverse parts or organs, which in turn are built up of a limited number of materials; the same material of ten entering into the composition of many different organs. These primary building materials are known as the tissues, and that branch of anatomy which deals with the characters of the tissues and their arrangement in various organs is known as Histology; or, since it is mainly carried on with the aid of the microscope, as Microscopic Anatomy. If, with the poet, we compare the Body to a house, we may go on to liken the tissues to the bricks, stone, mortar, wood, iron, glass and so on, used in building; and then walls and floors, stairs and windows, formed by the combination of these, would answer to anatomical organs.

Zoölogical Position of Man. External examination of the Human Body shows also that it presents certain resemblances to the bodies of many other animals: head and neck, trunk and limbs, and various minor parts entering into them, are not at all peculiar to it. Closer study and the investigation of internal structure demonstrates further that these resemblances are in many cases not superficial only, but that our Bodies may be regarded as built upon a plan common to them and the bodies of many other creatures: and it soon becomes further apparent that this resemblance is greater between the Human Body and the bodies of ordinary fourfooted beasts, than between it and the bodies of birds, reptiles or fishes. Hence, from a zoölogical point of view, man's Body marks him out as belonging to the group of Mammalia (see Zoölogy), which includes all animals in which the female suckles the young; and among mammals the anatomical resemblances are closer and the differences less between man and certain apes than between man and the other mammals; so that zoölogists still, with Linnæus, include man with the monkeys and apes in one subdivision of the Mammalia, known as the Primates. That civilized man is mentally far superior to any other animal is no valid objection to such a classification, for zoölogical groups are defined by anatomical and not by physiological characters; and mental traits, since we know that their manifestation depends upon the structural integrity of certain organs, are especially phenomena of 
function and therefore not available for purposes of zoölogical arrangement.

As man walks erect with head upward, while the great majority of Mammals go on all fours with the head forward and the back upward, and various apes adopt intermediate positions, confusion is apt to arise in considering corresponding parts in man and other animals unless a precise meaning be given to such terms as "anterior" and "posterior." Anatomists, therefore, give those words definite arbitrary significations. The head end is always anterior whatever the natural position of the animal, and the opposite end posterior; the belly side is spoken of as ventral, and the opposite side as dorsal; right and left of course present no difficulty: the terms cephalic and caudal as equivalent, respectively, to anterior and posterior, are sometimes used. Moreover, that end of a limb nearer the trunk is spoken of as proximal with reference to the other or distal end. The words upper and lower may be conveniently used for the relative position of parts in the natural standing position of the animal.

The Vertebrate Plan of Structure. Neglecting such merely apparent differences as arise from the differences of normal posture above pointed out, we find that man's own zoölogical class, the Mammals, differs very widely in its broad structural plan from the groups including sea-anemones, insects or oysters, but agrees in many points with the groups of fishes, amphibians, reptiles and birds. These four are therefore placed with man and all other Mammals in one great division of the animal kingdom known as the Vertebrata. The main anatomical character of all vertebrate animals is the presence in the trunk of the body of two cavities, a dorsal and a ventral, separated by a solid partition; in the adults of nearly all vertebrate animals, a hard axis, the vertebral column (backbone or spine), develops in this partition and forms a central support for the rest of the Body (Fig. 2, ee). The dorsal cavity is continued through the neck, when there is one, into the head, and there widens out. Within it are inclosed the chief organs of the nervous system: The bony axis is also continued through the neck and extends into the head in a modified form. The ventral eavity, on the other hand, is confined to the trunk. It contains the main organs connected with the blood-flow together with those of digestion and respiration. 
Upon the ventral side of the head is the mouth-opening leading into a tube, the alimentary canal, $f$ (Fig. 2), which passes back through the neck and trunk and opens again on the outside at the posterior part of the latter. In its passage through the trunk-region this canal lies in the ventral cavity.

The Mammalia. In many vertebrate animals the ventral cavity is not subdivided, but in the Mammalia it is; a membranous transverse partition, the diaphragm (Fig. 1, d), separating it into an anterior chest or thoracic cavity, and a posterior, or abdominal cavity. The alimentary canal and whatever else passes from one of these cavities to the other must therefore perforate the diaphragm.

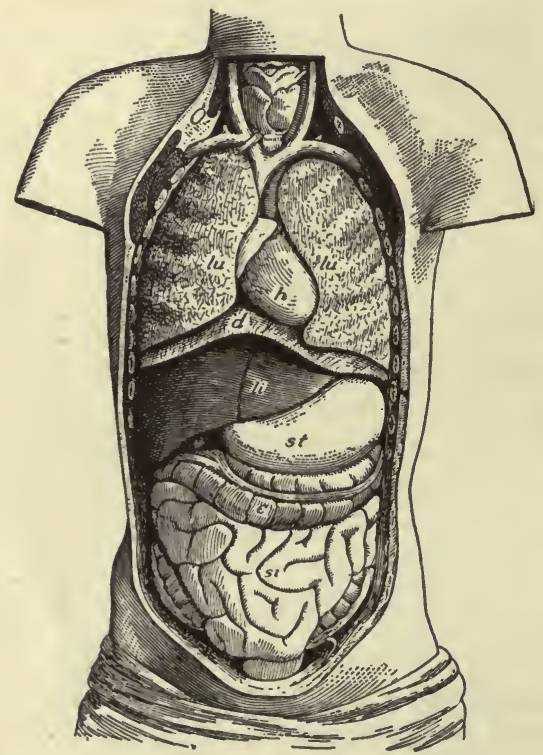

Fic. 1.-Diagram of the Body opened from the front to show the contents of the ventral cavity. $d$, diaphragm; $h$, heart; $l u$, lungs; st, stomach; $l i$, liver'si, small intestines; $c$, large intestine.

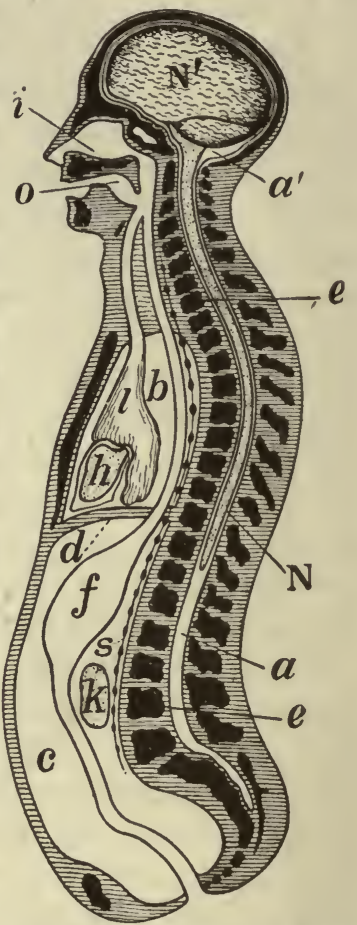

Frg. 2.-Diagrammatic longitudinal section of the Body. a, the neural tube, with its upper enlargement in the skull-cavity at $a^{\prime} ; N$, the spinal cord; $N^{\prime}$, the brain; $e e$, vertebræ forming the solid partition between the dorsal and ventral cavities; $b$, the pleural,.and $c$, the abdominal division of the ventral cavity, separated from one another by the diaphragm, $d ; i$, the nasal, and $o$, the mouth chamber, opening behind into the pharynx, from which one tube leads to the lungs, $l$, and another to the stomach, $f ; h$, the heart; $k$, a kidney; $s$, the sympathetic nervous chain. From the stomach, $f$, the intestinal tube leads through the abdominal cavity to the posterior opening of the alimentary canal. 
In the chest, besides part of the alimentary canal, lie important organs, the heart, $h$, and lungs, lu (Fig. 1); the heart being on the ventral side of the alimentary canal. The abdominal cavity is mainly occupied by the alimentary canal and organs connected with it and concerned in the digestion of food, as the stomach, $s t$, the liver, li, the pancreas, and the small and large intestines, si and $c$. Among the other more prominent organs in it are the kidneys and the spleen.

In the dorsal or neural cavity lie the brain and spinal cord, the former occupying its anterior enlargement in the head. Brain and spinal cord together form the cerebrospinal nervous center commonly called the central nervous system; in addition to this there are found in the ventral cavity a number of small nerve-centers united to each other and to the cerebrospinal center by connecting cords, and with their off-shoots forming the sympathetic nervous system.

The walls of the three main cavities are lined by smooth, moist serous membranes. That lining the dorsal cavity is the arachnoid; that lining the chest the pleura; that lining the abdomen the peritoneum; the abdominal cavity is in consequence often called the peritoneal cavity. Externally the walls of these cavities are covered by the skin, which consists of two layers: an outer horny layer called the epidermis, which is constantly being shed on the surface and renewed from below; and a deeper layer, called the dermis and containing blood, which the epidermis does not. Between the skin and the lining serous membranes are bones, muscles (the lean of meat), and a great number of other structures which we shall have to consider hereafter. All cavities inside the Body, as the alimentary canal and the air-passages, which open directly or indirectly on the surface are lined by soft and moist prolongations of the skin known as mucous membranes. In these two layers are found as in the skin, but the superficial bloodless one is called epithelium and the deeper vascular one corium.

Diagrammatically we may represent the Human Body in longitudinal section as in Fig. 2, where $a a^{\prime}$ is the dorsal or neural cavity, and $b$ and $c$, respectively, the thoracic and abdominal subdivisions of the ventral cavity; $d$ represents the diaphragm separating them; $e e$ is the vertebral column with its modified prolongation into the head beneath the anterior enlargement of the dorsal 
cavity; $f$ is the alimentary canal opening in front through the nose, $i$, and mouth, $o ; h$ is the heart, $l$ a lung, $s$ the sympathetic nervous system, and $k$ a kidney.

A transverse section through the chest is represented by the diagram Fig. 3, where $x$ is the neural canal containing the spinal

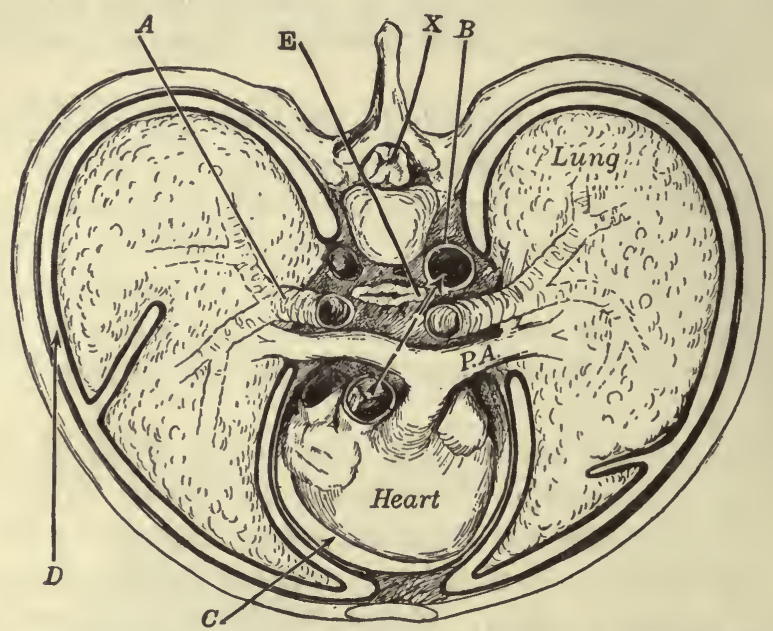

FIG. 3.-Cross-section of thorax. $A$, bronchus, entering the lung; $B$, the aorta cut at its origin and again at the descending part of its arch; $C$, the pericardial space; $D$, the pleural cavity; $E$, the alimentary canal; $P A$, the pulmonary artery; $\bar{X}$, the neural canal.

cord. In the thoracic cavity are seen the heart, the lungs, part of the alimentary canal, $E$; bronchial tubes, $A$, leading to the lungs; and blood vessels, $B$ and $P A$, communicating with the heart; the heavy line on each side covering the inside of the chest-wall and the outside of the lung represents the pleura.

Sections through corresponding parts of any other Mammal would agree in all essential points with those represented in Figs. 2 and 3.

The Limbs. The limbs present no such arrangement of cavities on each side of a bony axis as is seen in the trunk. They have an axis formed at different parts of one or more bones (as seen at $U$ and $R$ in Fig. 4, which represents a cross-section of the forearm near the elbow-joint), but around this are closely-packed soft parts, chiefly muscles, and the whole is enveloped in skin. The only cavities in the limbs are branching tubes which are filled with 
liquids during life, either blood or a watery-looking fluid known as lymph. These tubes, the blood and lymph-vessels respectively, are not, however, characteristic of the limbs, for they are present in abundance in the dorsal and ventral cavities and in their walls.

\section{Microscopic Structure of the Body.} For the detailed study of objects too small to be examined with the unaided eye the compound microscope is employed. Important optical conditions for the successful use of this instrument are adequate illumination and sharpness of focus. To secure these in the study of tissues the materials are cut in very thin slices and observed by transmitted light. Viewed thus tissues in their natural state are so nearly transparent that

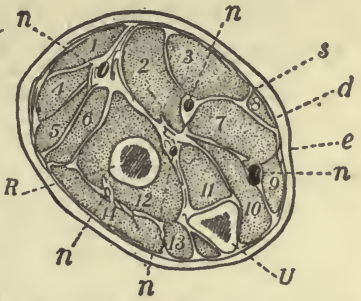

Frg. 4.-A section across the forearm a short distance below the elbow-joint. $R$ and $U$, its two supporting bones, the radius and ulna; $e$, the epidermis, and $d$, the dermis of the skin; the latter is continuous below with bands of connective tissue, $s$, which penetrate between and invest the muscles, which are indicated by numbers; $n$, $n$, nerves and vessels. relatively little of their detailed structure can be made out. The practice of histologists, therefore, is first to subject the tissues to the action of preservatives, and then to stain them with suitable dyes. By applying the principle that the different structures of

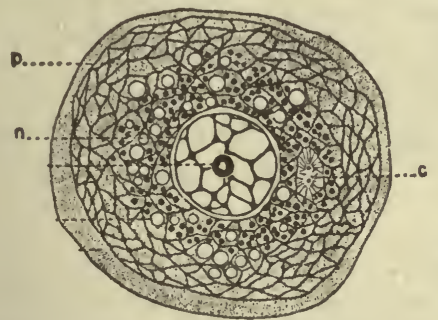
FIg. 5.-Diagram of a cell (Schäfer). ponents are unaffected by this $p$, protoplasm; $n$, nucleus; $c$, centrosome. dye but can be stained with another. This method of differential staining enables the various features of tissues to be made clearly visible.

Cells. Examination of the different tissues with the aid of the microscope reveals that they are made up of minute structures, the cells. These vary in form and size in different tissues. They are all constructed on a common plan, although in the more highly organized tissues, such as nerves and muscles, this plan is 
so modified to meet the special demands of these tissues as not to be easily recognized. The typical cell (Fig. 5) consists of a mass of living substance, known as protoplasm, of a semi-liquid, gelatinous consistency, about 0.01 millimeter $\left(\frac{1}{2500}\right.$ inch) in diameter. The protoplasm is usually not perfectly uniform throughout, but shows granules or fine, transparent net works through its substance. Imbedded in the protoplasm is a small structure of distinctly different appearance from the rest of the cell. This is the nucleus. It presents highly characteristic features which will be studied in later paragraphs (p. 23). The mass of cell protoplasm outside the nucleus is called cytoplasm. In general cytoplasm seems to be, as stated above, a relatively simple granular or vesicular mass. As figure 5 indicates, however, there are typically certain definite structures associated with the cytoplasm. The significance of these will be considered later (p. 24). In the highly organized tissues, muscle and nerve, the cytoplasm presents, in addition, complexities of structure suited to the special functions of these tissues.

Tissues. The individual cells are grouped into masses which are larger or smaller according to the region in which they occur. Obviously only by such a grouping can so large and complex a structure as the body be built of microscopic units. Any cell mass in which the cells are of one type is called a tissue. Thus we speak of muscle tissue or gland tissue according as the cells which make up the tissue in question are muscle cells or gland cells. Many kinds of tissue are widely distributed through the body, others occur only in special parts. The various tissues will be studied in detail in later chapters.

Chemical Composition of the Body. In addition to the study of the Body as composed of tissues and organs which are optically recognizable, we may consider it as composed of a number of different chemical substances. This branch of knowledge, which is still very incomplete, really presents two classes of problems. On the one hand, we may limit ourselves to the examination of the chemical substances which exist in or may be derived from the dead Body, or, if such a thing were possible, from the living Body entirely at rest; such a study is essentially one of structure and may be called Chemical Anatomy. But as long as the Body is alive it is the seat of constant chemical transformations in its 
material, and these are inseparably connected with its functions, the great majority of which are in the long run dependent upon chemical changes. From this point of view, then, the chemical study of the Body presents physiological problems, and might be called Chemical Physiology. At present it is customary to include under the term Biological Chemistry the study of the chemical structure of living matter and of the chemical changes occurring in it. At this point we may confine ourselves to the more important substances derived from or known to exist in the Body leaving questions concerning the chemical changes taking place within it for consideration along with those functions which are performed in connection with them.

Elements Composing the Body. Of the elements known to chemists only seventeen have been found to take part in the formation of the Human Body. These are carbon, hydrogen, nitrogen, oxygen, sulphur, phosphorus, chlorin, fluorin, iodin, silicon, sodium, potassium, lithium, calcium, magnesium, iron, and manganese. Copper and lead have sometimes been found in small quantities, but are probably accidental and occasional.

Uncombined Elements. Only a very small number of the above elements exist in the Body uncombined. Oxygen is found in small quantity dissolved in the blood; but even there most of it is in a state of loose chemical combination. It is also found in the cavities of the lungs and alimentary canal, being derived from the inspired air or swallowed with food and saliva; but while contained in these spaces it can hardly be said to form a part of the Body. Nitrogen also exists uncombined in the lungs ard alimentary canal, and in small quantity in solution in the blood. Free hydrogen has also been found in the alimentary canal, being there evolved by the fermentation of certain foods.

Chemical Compounds. The number of these which may be obtained from the Body is very great; but with regard to very many of them we do not know that the form in which we extract them is really that in which the elements they contain were united while in the living Body; since the methods of chemical analysis are such as always break down the more complex forms of living matter and leave us only its débris for examination. We know in fact, tolerably accurately, what compounds enter the Body as food and what finally leave it as waste; but the intermediate con- 
ditions of the elements contained in these compounds during their sojourn inside the Body we know very little about; more especially their state of combination during that part of their stay when they do not exist dissolved in the bodily liquids, but form part of a more or less compact living tissue.

For present purposes the chemical compounds existing in or derived from the Body may be classified as organic and inorganic, and the former be subdivided into those which contain nitrogen and those which do not.

Inorganic Constituents. Of the simpler substances entering into the structure of the Body the following are the most important:

Water; in all the tissues in greater or less proportion and forming about two-thirds of the weight of the whole Body. A man weighing 75 kilos (165 lbs.), if completely dried would therefore lose about 50 kilos (110 lbs.) from the evaporation of water. Of the constituents of the Body the enamel of the teeth contains least water (about 2 per cent), and the saliva most (about 99.5 per cent); between these extremes are all intermediate steps-bones containing about 22 per cent, muscles 75 , blood 79 .

Common salt-Sodium chlorid- $(\mathrm{NaCl})$; found in all the tissues and liquids, and in many cases playing an important part in keeping other substances in solution in water.

Potassium chlorid ( $\mathrm{KCl})$; in the blood, muscles, nerves and most liquids.

Calcium phosphate $\left[\mathrm{Ca}_{3}\left(\mathrm{PO}_{4}\right)_{2}\right]$; in the bones and teeth in large quantity. In less proportion in all the other tissues.

Besides the above, ammonium chlorid, sodium and potassium phosphates, magnesium phosphate, sodium sulphate, potassium sulphate, and calcium fluoride have been obtained from the Body.

Uncombined hydrochloric acid $(\mathrm{HCl})$ is found in the gastric juice.

Nitrogenous Organic Compounds. These fall into several main groups: proteins *-subdivided into simple proteins, conjugated proteins, and derived proteins-nitrogenous extractives, and pigments. The interesting substances known as enzyms probably form likewise a group under this head.

Simple Proteins. Under this head are grouped those proteins whose molecules contain only protein material; in contradistinc-

* The classification of proteins here given is that recommended by the joint committee on protein nomenclature of the American Physiological Society and the American Society of Biological Chemists, 1907. 
tion to the conjugated proteins whose molecules contain protein in combination with a non-protein substance.

Each of them contains carbon, hydrogen, oxygen, and nitrogen; most of them contain sulphur also, and a few phosphorus in addition. These elements are united into very complex molecules, and although different members of the group of simple proteins differ from one another in minor points they all agree in their broad features. The common body proteins have a similar percentage composition, falling within the limits given in the following table:

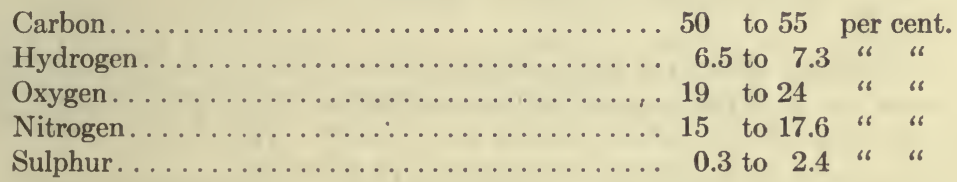

In addition a small quantity of ash is usually left when a protein is burned, showing that some inorganic salts are held in combination with it.

Recent chemical investigation has shown that the protein molecule is a complex, made up of a number of simpler molecules joined together. When a protein is boiled with a dilute acid its molecules are decomposed, and the resulting solution is found when examined to contain a mixture of the substances whose individual molecules were formerly parts of the complex protein molecules. Eighteen such substances have been obtained from decomposed proteins; they all contain nitrogen, and they all belong chemically to the group of amino acids. Some proteins contain all of them; others only a few. The characteristics of different proteins are supposed to depend on which of these amino acids are present in the molecules and also on their arrangement or grouping therein.

There are a number of chemical tests that may be used in detecting the presence of proteins; but only a few of them apply to the entire group. Of these the so-called biuret reaction is the most easily and most commonly used. It consists in making the protein solution strongly alkaline with caustic soda or potash and adding a small amount of very dilute solution of copper sulphate. A distinct purple color is evidence of the presence of protein. The common proteins of the body may also be recognized by the following char. acters: 
1. Boiled, either in the solid state or in solution, with strong nitric acid they give a yellow liquid which becomes orange on neutralization with ammonia. This is the xanthoproteic test.

2. Boiled with a solution containing subnitrate and pernitrate of mercury they give a pink precipitate, or, if in very small quantity, a pink colored solution. This is known as Millon's test.

3. If a solution containing a protein be strongly acidulated with acetic acid and be boiled with the addition of an equal bulk of a saturated watery solution of sodium sulphate, the protein will be precipitated.

The simple proteins which are found in the bodies of man and the lower animals fall into several groups as follows:

1. Albumins. Several proteins of this group are found in the Body; serum albumin, one of the proteins of the blood, myogen, a muscle protein, and cell albumin, found in the cellular tissues, are examples. Egg albumin (white of egg) is perhaps the most familiar example of an albumin.

The albumins are characterized by being coagulated by heat (illustrated by boiled white of egg); in this respect they are similar to the proteins of the next group, from which they differ by being soluble in pure water.

2. Globulins. These proteins, as indicated above, do not differ greatly from albumins. Like them they are coagulated by heat, but unlike them, are not soluble in pure water. If a small amount of an inorganic salt is added to the water they will go into solution. Two blood proteins, serum globulin or paraglobulin, and fibrinogen belong to this group; also myosin, one of the muscle proteins, and cell globulin, found in cellular tissues.

3. Albuminoids. In chemical structure these simple proteins are closely similar to those already described. They are found, however, exclusively in the supporting and protective tissues of the body, bone, connective tissue, epidermis, and hair, and evidently have some important structural difference as compared with the proteins of the cellular tissues since the Body cannot make use of them in building up its cell proteins in the way it uses other protein foods.

4. Protamins. These are the simplest proteins known. They have thus far been found only in the spermatozoa of fishes. Their molecules consist of a relatively small number of amino acid groupings and contain no sulphur.

5. Histons are intermediate in complexity between protamins and proteins of the albumin class. The one of chief importance in the body is globin, which is combined with a pigment to form hemoglobin, the red coloring matter of the blood.

Conjugated Proteins. In addition to the simple proteins described above there are present in the Body certain groups of compounds consisting of proteins combined with non-protein substances. The most important of these are: 
Nucleo proteins, consisting of protein combined with nucleic acid. These are of great interest physiologically since they form the chief constituents of cell nuclei, to which structures are assigned the function of exercising special control over the activities of living cells.

Glycoproteins, consisting of protein combined with a carbohydrate (see p. 15). Mucin, the substance which gives the secretions of the mouth, nose, and throat their peculiar viscous character, is an example of this group.

Phosphoproteins, consisting of protein combined with a phosphoruscontaining substance. The casein of milk, which forms the curd, is the most familiar member of this group.

Hemoglobins, compounds of protein with a pigment. These are of great physiological importance on account of the property, common to all of them, of acting as transporters of oxygen. The type member of the group, the hemoglobin of Mammalian blood, is of interest chemically on account of the great size of its molecules, which are estimated to contain not less than 2,300 atoms each and to have molecular weight exceeding 16,000 .

Derived Proteins. The members of this group are derived, as their name indicates, from the simple proteins. In the process of protein digestion, by which the protein portions of the food are made available for the needs of the Body by being split into simpler substances, the first steps in the digestive process give rise to compounds which differ from the simple proteins by a slight degree only. These are the derived proteins. The members of the group which occur most commonly in the Body are the proteoses and peptones. These are present in the stomach during protein digestion. They are characterized by greater solubility than simple proteins possess.

Nitrogenous Extractives. Under this head are grouped various nitrogen-containing substances most of which represent materials that have done their work in the Body and are about to be gotten rid of. Nitrogen is present in the living tissues of the Body chiefly as a part of their proteins. The vital activities of the tissues involve the breaking down of these complex proteins into simpler substances. Part of their carbon combines with oxygen and passes out through the lungs as carbon dioxid; their hydrogen is similarly in large part combined with oxygen and passed out as water; while their nitrogen, with some carbon and hydrogen and oxygen, is passed out in the form of crystalline extractives.

Urea is the most important substance of this class; fully nine-tenths of all the nitrogen that is eliminated from the Body is in this form. It is a diamide 
of carbonic acid, having the formula $\mathrm{CO}<\frac{\mathrm{NH}_{2}}{\mathrm{NH}_{2}}$; the relationship of urea to carbonic acid is clear when the formula for the latter is written thus: $\mathrm{CO}<\mathrm{OH}$. Fully 30 grams of urea are eliminated daily from the body of an adult man.

Creatinine $\left(\mathrm{C}_{4} \mathrm{H}_{7} \mathrm{~N}_{3} \mathrm{O}\right)$ is an interesting member of the group of extractives because the amount of it that is eliminated from the body daily is very constant, regardless of changes in amount of food or exercise taken, and seems to depend closely upon the amount of muscle tissue present in the Body; persons of great muscular development have a larger daily creatinine output than those of smaller build.

Creatine $\left(\mathrm{C}_{4} \mathrm{H}_{9} \mathrm{~N}_{3} \mathrm{O}_{2}\right)$ is closely related chemically to creatinine, but appears to play a very different part in the Body. Creatinine is undoubtedly a waste product of protein decomposition, being merely an incidental product of the vital processes which go on within the organism. Creatine, on the other hand, seems to be an essential constituent of living protoplasm, although just what purpose it serves is not clear. About one per cent of the solid substance of muscle is creatine.

The purin bodies, of which uric acid $\left(\mathrm{C}_{5} \mathrm{H}_{4} \mathrm{~N}_{4} \mathrm{O}_{3}\right)$ is the most familiar example, are derived chiefly, if not wholly, from the decomposition of nucleoproteins and are therefore interesting as being the end products of the vital activities of the cell nuclei.

Pigments. The most important of these that occur in the Body are:

Hemochromogen, an iron containing pigment which in combination with the histon globin forms hemoglobin, the red coloring matter of the blood. When hemochromogen is in the presence of oxygen it combines with it to form hematin.

Bilirubin and biliverdin are the bile pigments and give to bile its color. Bilirubin is yellow and biliverdin is green. The former usually predominates in the bile of man and the carnivora, making such bile yellow; the latter is the dominant color in the bile of herbivorous animals, which is green. They are closely related chemically and are derived from the decomposition of hemoglobin.

Urobilin is formed in the intestine as the result of the putrefaction there of the bile pigments. It is absorbed thence into the blood and excreted by the kidneys, and imparts to the urine its characteristic yellow color.

Enzyms are a group of substances which seem to be allied in chemical composition to the true proteins, but it is so difficult to be sure of the purity of any specimen that their composition is still in doubt. The enzyms have the power, even when present in very small quantity, of bringing about extensive changes in other sub- 
stances, and they are not themselves necessarily used up or destroyed in the process. Many enzyms of great physiological importance exist in the digestive fluids and play a part in fitting food for absorption from the alimentary canal. For example, pepsin found in the gastric juice converts, under suitable conditions, such complex proteins as albumins into simpler peptones; ptyalin, found in the saliva, converts starch into sugar. We shall have occasion later to study a number of enzyms more in detail in connection with their physiological uses. A characteristic property of all enzyms is their susceptibility to heat; a temperature of $60^{\circ} \mathrm{C}$. suffices to destroy them completely.

Non-Nitrogenous Organic Compounds. These may be conveniently grouped as hydrocarbons or fatty bodies; carbohydrates or amyloids; and certain non-nitrogenous acids.

Fats. The fats all contain carbon, hydrogen, and oxygen, the oxygen being present in small proportion as compared with the hydrogen. Three fats occur in the Body in large quantities, viz.: palmatin $\left(\mathrm{C}_{51} \mathrm{H}_{98} \mathrm{O}_{6}\right)$, stearin $\left(\mathrm{C}_{51} \mathrm{H}_{110} \mathrm{O}_{6}\right)$, and olein $\left(\mathrm{C}_{57} \mathrm{H}_{104} \mathrm{O}_{6}\right)$. The two former when pure are solid at the temperature of the Body, but in it are mixed with olein (which is liquid) in such proportions as to be kept fluid. The total quantity of fat in the Body is subject to great variations, but its average quantity in a man weighing 75 kilograms (165 pounds) is about 2.75 kilograms (6 pounds).

Each of these fats when heated with a caustic alkali, in the presence of water, breaks up into a fatty acid (stearic, palmitic, or oleic as the case may be), and glycerin. The fatty acid unites with the alkali present to form a soap.

Carbohydrates. These may be defined as substances composed of carbon, hydrogen, and oxygen, having the number of carbon atoms in each molecule usually six or some multiple thereof, and having the hydrogen and oxygen present in the same proportion as in water. The three chief groups are the sugars, starches, and cellulose.

Dextrose or grape sugar $\left(\mathrm{C}_{6} \mathrm{H}_{12} \mathrm{O}_{6}\right)$ is the most important representative of the sugars found in the Body. A large part of the food supply is received from the digestive tract into the blood in this form. It occurs constantly in small concentration in the blood and tissues.

Lactose, the sugar of milk, occurs in considerable quantity in milk. 
Glycogen or animal starch $\left(\mathrm{C}_{6} \mathrm{H}_{10} \mathrm{O}_{5}\right)$ is the anhydride of grape sugar. This is the form in which the excess of sugar is stored in the body to be drawn upon at need. Dextrose is readily converted into it, and it in turn is easily changed back into sugar. In many respects it resembles common vegetable starch. It is present in the muscles of the Body and in the liver, the latter organ alone containing about as much as all the muscles put together.

Cellulose, the woody fiber of plants, is not found in the Human Body, although a chemically identical substance, tunicin, is found in the bodies of tunicates.

Organic Non-Nitrogenous Acids. Of these the most important is carbon dioxid $\left(\mathrm{CO}_{2}\right)$, which is the form in which by far the greater part of the carbon taken into the Body ultimately leaves it. United with calcium it is found in the bones and teeth in large proportion.

Formic, acetic, and butyric acids are also found in the Body; stearic, palmitic, and oleic have been above mentioned as obtainable from fats. Lactic acid, $\mathrm{C}_{3} \mathrm{H}_{6} \mathrm{O}_{3}$, is often present in the digestive tract, and when milk turns sour is formed from lactose. Virtually the same substance, (sarcolactic acid), is formed in muscles when they work or die.

Glycerin phosphoric acid $\left(\mathrm{C}_{3} \mathrm{H}_{9} \mathrm{PO}_{6}\right)$ is obtained on the decomposition of lecithin, a complex nitrogenous fat found in nervous tissue and to some extent in all living cells.

Physico-Chemical Constitution of the Body. The functioning of the living body is the sum total of the functioning of the individual cells. The activity of any cell, in turn, is determined by the interactions of its constituent molecules. In the living tissues we have a great number of different molecules interacting in ways which depend, in part, on the chemical nature of the molecules, and in part, also, upon the manner in which the molecules are grouped and interrelated physically. The study of the manner in which molecules are related to each other under such conditions as obtain in the Body is a part of the science of Physical Chemistry and the structure of the Body from this standpoint is its Physico-chemical structure.

Liquid Environment. Of prime importance from the physicochemical standpoint is the fact that the active tissues of the body consist largely of water. Molecules in solution in a liquid move about freely, enter and leave chemical combinations readily, and in general display the degree of flexibility essential for the carry- 
ing out of complex chemical processes such as go on in the Body. Although the water of the Body does not itself take active part in the life processes, these processes could not go on in its absence.

Crystalloids. Substances which form crystals when solutions containing them are evaporated are classed as crystalloids. Examples are common salt and cane sugar. From the standpoint of physical chemistry as applied to the Body the important fact concerning crystalloids is not their crystal-forming ability, but the relatively small size of their molecules, which gives them a correspondingly high degree of freedom of motion in solution and facility in entering and leaving chemical combinations. All living tissues contain crystalloids as part of their substance. The amounts are relatively small, although the materials are as necessary to living protoplasm as are those that make up the greater part of its mass.

Colloids. These are substances which do not form crystals when solutions of them are evaporated, but appear as gelatinous or gummy masses. Examples are white of egg, and ordinary table gelatin. Colloids are composed, in general, of much larger molecules than are crystalloids. They have correspondingly less facility of chemical action. Proteins are colloidal in structure; hence living protoplasm, which is chiefly protein in its constitution, is colloidal.

Cell Membranes. To preserve definite structure in the watery, gelatinous protoplasm individual cells are enclosed in cell membranes. These should not be confused with the woody envelopes in which many plant cells are enclosed. Reference here is to the delicate sheaths which surround all cells, both plant and animal, and which serve to keep the semi-liquid contents of the cells from running together into a formless mass. The chemical nature of the membranes is not certainly known, although there is reason to believe that they consist of protoplasm which differs from that of the cells at large chiefly in its greater density. The significance of cell membranes in Physiology lies in the fact that all interchanges between living protoplasm and its surroundings must take place through them. Any nourishment any cell receives must pass through the cell membrane before it reaches active protoplasm. Similarly, all materials which cells discharge have to be expelled 
through the membrane. We shall learn later low much the membranes affect the cells which they enclose through the influence they have on the passage of materials into and out of the cells.

Intercellular Spaces and Intercellular Fluids. In the grouping of cells into tissues (p. 8) we find, even in those that are most compact, minute spaces among the cells. There are points of union between cell and cell, holding the tissue together, but these involve only relatively small portions of the total cell surfaces. Every cell has a large part of its surface fronting on intercellular spaces. These spaces are filled with watery fluid called lymph, which bathes the individual cells, and, in fact, forms their sole environment. The nourishment of the cells reaches them by way of the lymph; the discharges of waste materials from the cells are into the lymph. The interchanges between the cells and lymph are therefore the fundamental interchanges of the Body. They are subject, in part, at least, to certain definite laws given below.

Filtration, Osmosis, and Dialysis. At every step in the complex process of supplying the living cells with nourishment and removing from them their harmful waste products the membranes, described above, stand in the way of the substances involved and must be traversed by them. There are membranes between the protoplasm of the cells and the lymph which surrounds them. The digested food must pass through the membranous lining of the digestive tract before it can enter the blood; the oxygen of the air must pass through a membrane in the lungs on its way to the same medium. The juices which are secreted or excreted have to be forced through membranes in passing out from the organs from which they come. The movements of liquids through the membranes of the Body take place for the most part in accordance with certain physical principles which may conveniently be stated at this point.

Filtration. If a membranous bag such as an ox bladder be filled with a liquid and pressure be applied to the liquid in the bag a point may be reached where the liquid is squeezed through the membrane and appears in drops on its outer surface. This is an example of filtration. When a liquid is filtered in this way any solid particles which may have been suspended in it are left behind, but any substances which may be dissolved in it pass through as part of the liquid. Thus a salt solution which contained some 
particles of sand might be filtered and the sand removed, but the solution would have just as much salt dissolved in it after filtration as before.

Osmosis. If we should take such a membranous bag as described above filled with salt solution and dip it into a vessel of pure water, so that the surfaces within and without the bag are at the same level, it would be seen after a while that the level of liquid within the bag had risen while that in the vessel outside had correspondingly fallen. That is, there would have been an actual movement of water into the bag with sufficient force to overcome the pressure due to gravity resulting from the change of water level on the two sides of the membrane. Whenever two solutions of different concentrations are separated by a membrane which is permeable to water there will be a flow of water through the membrane in the direction of the greater concentration. This phenomenon is known as osmosis. The force which drives the water is called osmotic pressure and is said to be exerted by any solution of higher concentration toward any of lower concentration.

Dralysis. A membrane which is permeable to water but not to any particles which may be dissolved in it is known as a semipermeable membrane; one which allows dissolved substances as well as water to pass is a permeable membrane. When two solutions of different concentration are separated by a membrane of this latter class we have in addition to the movement of water under the driving force of osmotic pressure a movement of dissolved particles through the membrane. This is a special case of the general phenomenon of diffusion. Diffusion may be defined as the tendency of substances in solution to distribute themselves evenly throughout the solvent. Where this distribution necessitates the passage of particles through permeable membranes the phenomenon is called dialysis. The effect of both osmosis and dialysis is to equalize the concentrations of the solutions on the two sides of the membrane, but it must be remembered that they are entirely distinct phenomena. To illustrate: suppose we have on the two sides of a permeable membrane solutions respectively of sugar and salt of the same concentration, that is, having the same number of particles in solution; there would then be no flow of water in either direction since the osmotic pressure of both solutions is the same, but since neither the sugar nor the 
salt is evenly distributed throughout the solvent there will be dialysis of both substances until an even distribution is obtained.

Again, both osmosis and dialysis bring about changes in the concentration of the solutions affected by them whereas filtration does not. In considering the influence of the membranes of the Body upon its liquid contents these facts must be borne in mind.

Summary. We may summarize the physico-chemical structure of living tissues by picturing them as made up of a large percentage of colloids and a small percentage of crystalloids dissolved in a relatively considerable amount of water, and subdivided into units each of which is enclosed in a membrane. Among the units (cells) are spaces filled with a watery fluid (lymph). Interchanges between cell substance and lymph take place through the cell membranes in the main in accordance with the laws of filtration, osmosis, and dialysis. 


\section{CHAPTER II}

\section{THE FUNDAMENTAL PHYSIOLOGICAL ACTIONS}

The Properties of the Living Body. Just as the structure of the Body is the sum total of the structures of its individual cells, so the properties, or functions, of the Body are the sum total of the functions of the constituent tissues. With most of the properties of our Bodies we are familiar in a general way. The ability to perceive sensations and to make motions; the beating of the heart, the movements of breathing, the seeretion of saliva and of sweat, the maintenance of bodily warmth; all these we recognize as bodily functions. The power of performing them must reside with the constituent cells. We observe, however, that in our Bodies not all these properties are shared equally by all the cells. The cells that perceive sensations are not the same as perform active movements; those that secrete saliva have not the power of secreting sweat. A Body in which a large degree of specialization prevails we speak of as highly organized. In the simpler animals there is less specialization and we find individual cells doing more than one kind of work. In the simplest animals of all, the one-celled animals, all the properties possessed are necessarily combined in a single cell. We can observe, then, in the rudimentary form, to be sure, in the one-celled animals, all the fundamental properties of living protoplasm. Chief among these are: (1) assimilation, the power to take in food materials and to make them over into body substance; a typical one-celled animal, the ameba, can be observed under the microscope engulfing and dissolving in its own protoplasm minute food particles; (2) active motion, seen in all save a very few animal forms from the lowest to the highest. Motion in animals is brought about by forcible contractions of the moving parts; the property of motion is therefore more accurately described as contractility; (3) sensation, excitability, or irritability, the property of being affected by changes in the environment; (4) coordination, the power of causing all the parts of the organism to act in harmony; (5) reproduction, the property of separating off a 
portion of the protoplasm which may become eventually an independent organism, like the parent; (6) growth, the property, based on assimilation, of increasing in size by the building up of the body protoplasm faster than it is broken down.

The Body as a Machine. Dissimilation. All living things, from the simplest one-celled organism to man himself, obey the mechanical law of the conservation of energy. By this we mean that whenever energy is manifested, as in motion, heat production, or any other form of activity, an equivalent amount disappears from some antecedent source. The source of bodily energy is chemical, in animals being derived, ultimately, from the food. All cell activities involve the expenditure of energy. All cells, therefore, require to be fed. Energy is obtained in animal cells through the breakdown of the complex chemical substances of which food is composed into simpler ones. This breaking-down process is described as dissimilation, the opposite of the building-up process, assimilation. In living cells the two processes, assimilation and dissimilation, go on side by side, and under ordinary conditions exactly balance, so there is neither gain nor loss of cell substance. Since dissimilation is the process by which the Body obtains energy for its various activities we shall have occasion to study its manifestations in detail as the different phases of bodily function are considered.

Cell Growth. All cells during their early life possess the power of growth, or in terms of their chemical activities are able to assimilate faster than they dissimilate. The materials that are to be assimilated have to enter the cell through its surface membrane, and obviously, if there is no shortage of food, the larger the surface the more can enter. The processes of dissimilation, going on inside the cell are, on the other hand, relatively independent of the surface, being determined rather by the amount or mass of protoplasm making up the cell. Now a little consideration will make clear to us that the smaller an object is the larger is its relative surface. This we can demonstrate by placing two bricks together to make one block. The dimensions of the block will be $4^{\prime \prime} \times 4^{\prime \prime}$ $\times 8^{\prime \prime}$, and the surface area 160 sq. inches. If now we separate the two bricks each will have exactly half the mass of the former block but more than half the surface, the total surface area of a single brick being 112 sq. inches. Thinking now of cells instead of bricks 
we can see that if in the larger one the amount of material that can enter through the surface is just sufficient to balance the dissimilation, in the smaller one, with only half the mass to carry on dissimilation processes, the relatively larger surface permits the entrance of more than enough material for a balance, and there is an excess which can be built into the substance of the cell, the process which constitutes growth.

Cell Division. When the relation of surface to mass in the growing cell reaches the point where there is no excess of assimilation over dissimilation, growth necessarily stops. Since this relationship appears while both mass and surface are very small single cells are always quite or nearly microscopic. To build up such large structures as are present in the body cell multiplication takes place. Whenever a cell which has reached the limit of its growth divides, the greater relative reduction of mass as compared with surface gives opportunity for excess assimilation to occur once more and growth is resumed.

Details of Cell Structure and Nuclear Structure. The peculiar function of the cell nucleus appears to be the control of the processes of assimilation. Assimilation involves the transformation into cell protoplasm of the food substances which enter the cell; furthermore, the differences which distinguish one sort of cell from another sort, and in consequence, one sort of animal or plant from another, are at bottom differences in the character of the protoplasm; the nucleus is, therefore, the determining factor in the establishment of definite species, and much study has been given it in the hope of obtaining insight into the conditions upon which specific cell differences depend.

Microscopically the most striking feature of the nucleus as observed in suitably fixed and stained preparations is an irregular network of substance which has greater affinity for dyes than the other constituents of the cell and therefore stains much more deeply. Because of this affinity for coloring reagents the substance has been named chromatin. It occurs in the nucleus in the form of excessively minute granules, strung like beads upon a thread of different material, called linin, the whole twisted into an irregular network (Fig. 6).

During the process of cell division the chromatin network passes through a remarkable sequence of events, of such a character as to 
indicate most convincingly that the chromatin is vitally concerned in the determination of the nature of the cell.

Outside the nucleus, and imbedded in the cytoplasm is a structure, the attraction sphere, which takes active part in the process of cell division (Fig. 6).

Mitotic Cell Division. Since the chromatin is looked upon as determining the character of the cell, we will expect to find that in the process of cell division, whereby tissues are built up out of a

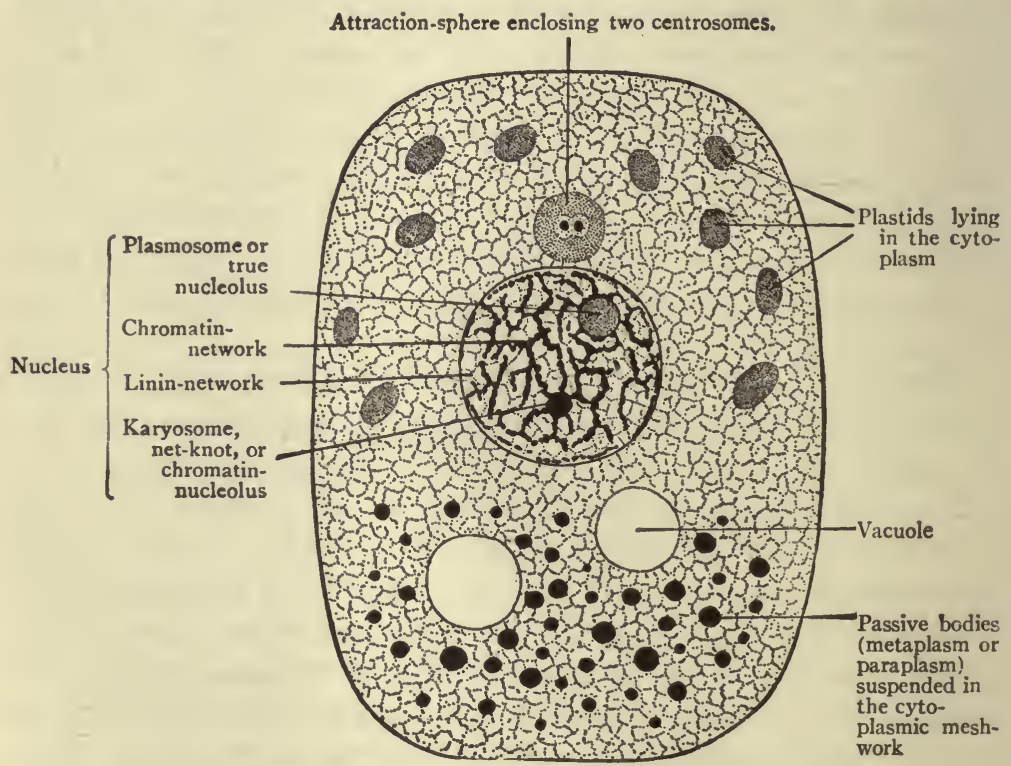

FIG. 6.-Minute structure of a cell (Wilson).

few antecedent cells, care will be taken to divide the chromatin of the dividing, or mother cell equally between the daughter cells, so as to insure that the latter shall be alike. Similarly, in the earlier stages of development, when the different tissues of the body are being formed from cells that are all alike, means must be afforded for dividing the chromatin of the mother cell unequally among the daughter cells, to make the desired differentiation possible. The processes by which these ends are secured are known as mitosis or mitotic cell division.

The first step in mitotic division is the splitting of the attraction sphere (Fig. 6) into two halves, called the centrosomes, which travel 
to opposite sides of the nucleus, but remain connected by a spindle of fine, colorless fibers, the achromatic spindle. This spindle, in passing from one centrosome to the other penetrates the nucleus and comes into close relationship with the chromatic network. This latter structure, meanwhile, has arranged itself into a continuous filament which presently breaks into segments, called the chromosomes (Fig. 7, 3). An interesting fact is that the number of chromosomes into which the chromatin filament divides is the same for all the cells of any given species. In the cells of the guinea pig, for example, the number is sixteen. The number of chromosomes, while characteristic, is probably not vitally significant, since the cells of the onion have the same number as those of the guinea pig.

Each individual chromosome becomes attached to a fiber of the spindle. Often the chromosomes take the form of a $\mathrm{V}$, in which case attachment is at the apex. The chromosome next splits lengthwise, each granule dividing into equal halves (Fig. 7, 4). By a shortening of the spindle fibers one subdivision of each chromosome is drawn to one of the centrosomes and the other subdivision to the other centrosome. The chromosomes then reunite to form a continuous filament which, in turn, shapes itself into the characteristic chromatin network of the resting cell nucleus. The cell protoplasm divides, meanwhile, and the process is complete. On the theory that the chromatin granules are the determiners of the cell characteristics, this method of division insures that the daughter cells shall resemble each other and the mother cell very closely. As supporting this theory of the function of the chromatin is the interesting observation that during the early stages of development, while tissue differentiation is in progress, those cells that are destined to become the progenitors of special tissues lose portions of their chromatin, by causing them to dissolve in the cell protoplasm and disappear. The deduction is that these specialized tissues are not going to need all the characteristics of undifferentiated protoplasm and so disburden themselves as early as possible of those determiners for which they have no further use.

Significance of the Physiological Properties. Adaptation. If we take the liberty of personifying Nature to the extent of ascribing purposes to her, we can say that the purpose of Nature with 
reference to the various species of living beings is to maintain them upon earth from generation to generation. The life of the individual is important only as it serves toward the perpetuation of the
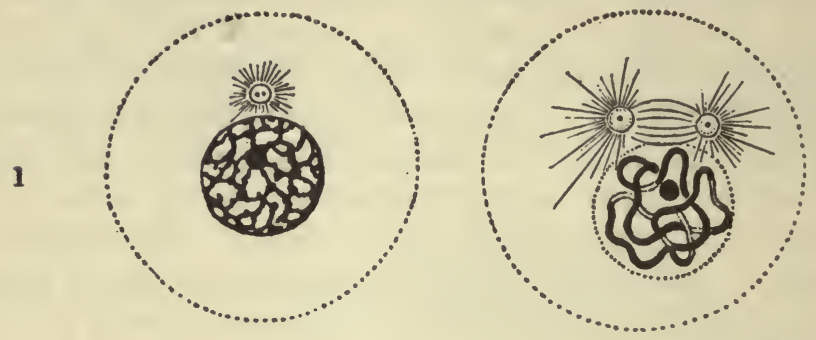

2

3
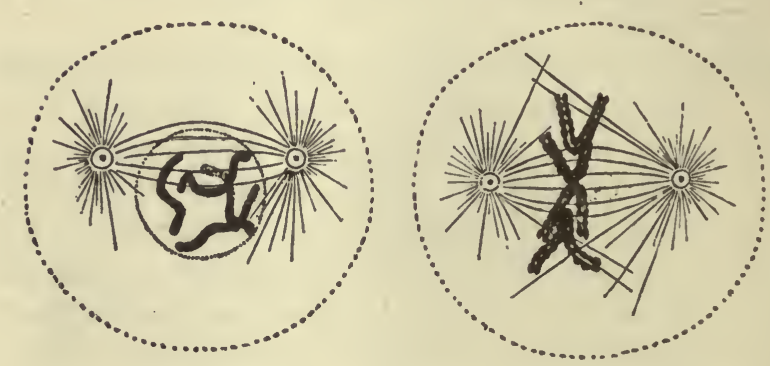

4
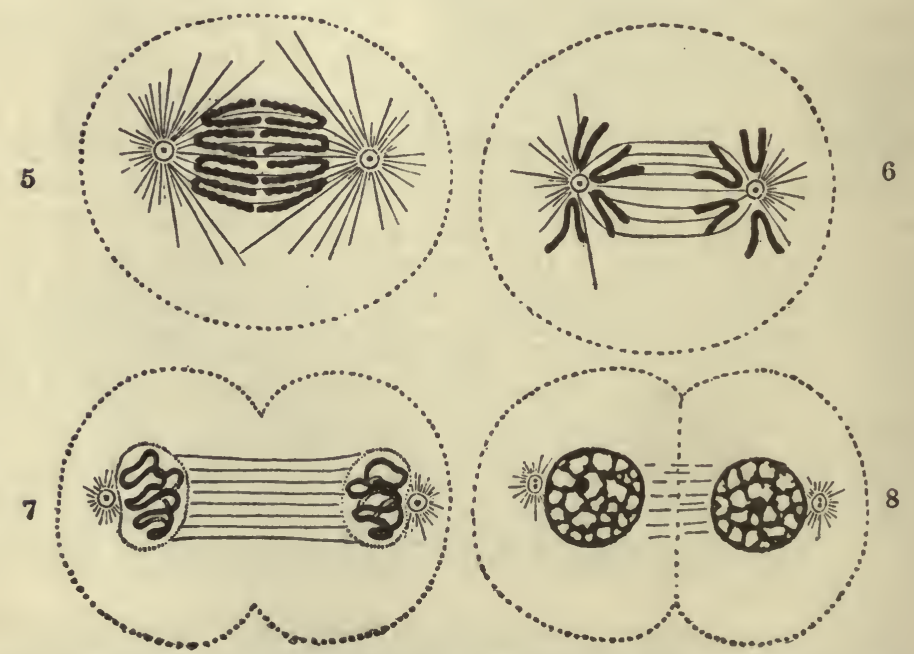

Fig. 7.-Diagram showing the changes which occur in the centrosomes and nucleus of a cell in the process of mitotic division. (Schäfer.) The nucleus is supposed to have four chromosomes. 
race. So long as the good of the individual does not run counter to that of the race the individual is conserved, but as soon as the good of the individual is opposed to that of the race the individual is sacrificed. Thus in a state of nature the old and feeble suffer destruction because their usefulness to the race is over, and they are consuming food which may be required by the young and vigorous. The elaborate humanitarian measures by which civilized men attempt to prevent the destruction of the old, the feeble, and the sick may seem, at first thought, absolutely opposed to the purposes of nature, and so, perhaps, from a purely physical standpoint they are, but when we recall that the really worth while part of Man's life is intellectual and spiritual rather than physical, and consider the influence of humanitarianism upon this part, we realize that humanitarianism represents, after all, one phase of the highest development of the purpose of Nature with respect to Man.

The Physiological Properties of organisms are the means by which they are enabled to carry out the purpose of Nature with regard to themselves. These properties are peculiarly fitted to enable organisms to maintain themselves and their race upon earth. Consider, for example, the usefulness of the functions of movement, sensation, and co-ordination. The power of motion is of great advantage to an animal, but only in connection with sensation and co-ordination. The chief usefulness of motion to the organism is the securing of food and the avoidance of harm. Neither of these ends is served by aimless motion. To secure food or to avoid harm the organism must have knowledge of its environment. This is gained through the operation of the property of sensation. The mere possession of knowledge is of no avail unless the movements can be directed in accordance with it. For this guidance the property of co-ordination serves.

Only through the successful co-operation of these three physiological properties is the organism able to adapt itself to its environment and so to live. Continued survival requires the continuous co-operation of these functions. The definition sometimes heard of life as continuous adaptation emphasizes this truth.

The function of assimilation and those phases of dissimilation not immediately concerned with the properties of motion, sensation, and co-ordination are, nevertheless, essential to the life of 
the organism, and upon them in great degree the perpetuation of the race depends. Their relationships are less familiar than those of the immediately adaptive functions, but as we study them, in due course, their fundamental importance will become clear.

Co-ordination in the Body. A very little study of our most common activities shows that in us the function of co-ordination is developed to a very high degree. In the act of walking, for example, sensations of sight direct the movements of the muscles of the legs. To cause the leg muscles to work adaptively in obedience to the sensations entering the eyes a special phase of co-ordination, namely, conduction of messages from one point to another, enters prominently. This form of conduction is accomplished through the operation of the nervous system, and the kind of co-ordination of which it is a part is called nervous co-ordination. There is another sort of co-ordination which is very important, and of which we shall have much to say, but which is in many respects less familiar in its workings. In the growth process, for instance, co-ordination enters constantly. In most individuals the two legs are the same length, so are the two arms; the ears are about the same size; the eyes are the same color. These things do not happen by accident, but because they are controlled by a definite co-ordinating mechanism. This type of co-ordination differs from that effected through the nervous system chiefly in that it is concerned with processes which go on more slowly. The body carries on this type of co-ordination by means of chemical substances, known as hormones, which are manufactured in certain tissues of the body, specially differentiated for that purpose, and conveyed to the tissues upon which they exert their influence through the blood stream. This method of control is called chemical co-ordination and shares with nervous co-ordination the task of causing the different parts of the exceedingly complex body machine to operate harmoniously.

Emphasis is placed upon the property of co-ordination thus early in our consideration of the Body because a true appreciation of Physiology requires not only an understanding of the working of the various tissues, but even -more a grasp of the manner in which they co-operate to secure that continuous adaptation of the organism to the environment upon which life depends. 


\section{CHAPTER III}

\section{TISSUES, ORGANS, AND PHYSIOLOGICAL SYSTEMS}

Development. Every Human Body commences its individual existence as a single nucleated cell. This cell, known as the ovum, divides or segments and gives rise to a mass consisting of a number

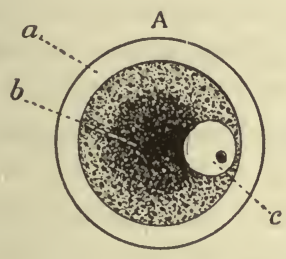

D

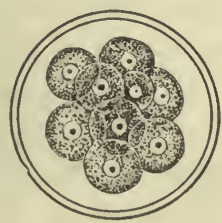

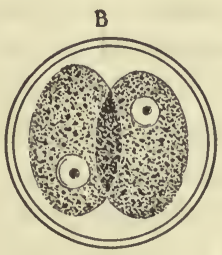

E

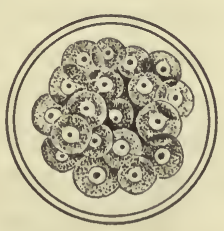

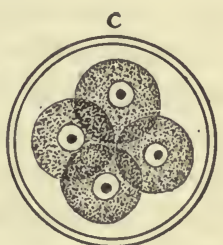

$\mathbf{f}$

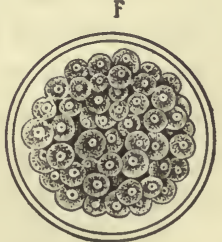

Fig. 8.- $A$, an ovum; $B$ to $E$, successive stages in its segmentation until the morula, $F$, is produced; $a$, cell-sac; $b$, cell contents; $c$, nucleus.

of similar units and called the mulberry mass or the morula. At this stage, long before birth, there are no distinguishable tissues entering into the structure of the Body, nor are any organs recognizable.

For a short time the morula increases in size by the growth and division of its cells, but very soon new processes occur which ultimately give rise to the complex adult body with its many tissues and organs. Groups of cells ceasing to grow and multiply like their parents begin to grow in ways peculiar to themselves, and so come to differ both from the original cells of the morula and from the cells of other groups, and this unlikeness becoming more and more marked, a varied whole is finally built up from one originally alike in all its parts. Peculiar growth of this kind, form- 
ing a complex from a simple whole, is called development; and the process itself in this case is known as the differentiation of the tissues, since by it they are, so to speak, separated or specialized from the general mass of mother-cells forming the morula.

As the differences in the form and structure of the constituent cells of the morula become marked, differences in property arise, and it becomes obvious that the whole cell-aggregate is not destined to give rise to a collection of independent living things, but to form a single human being, in whom each part, while maintaining its own life, shall have duties to perform for the good of the whole. In other words, a single compound individual is to be built up by the union and co-operation of a number of simple ones represented by the various cells, each of which thenceforth, while primarily looking after its own interests and having its own peculiar faculties, has at the same time its activities subordinated to the good of the entire community.

The Physiological Division of Labor. As the differentiation of tissues proceeds the fundamental physiological properties, originally exhibited in equal degree by all the cells, become distributed among the various tissues. Thus we find certain tissues adapted to execute movements and in these the property of active motion is developed to an especial degree. Other tissues, on the other hand, show little or no active motion but exhibit a marked degree of conductivity. The higher we look in the animal scale the more marked becomes this division of physiological duties among the tissues. In man it attains its highest development.

Classification of the Tissues.-As we might separate the inhabitants of the United States into groups, such as lawyers, doctors, clergymen, merchants, farmers, and so forth, so we may classify the tissues by selecting the most distinctive properties of each of those entering into the construction of the adult Body and arranging them into physiological groups; those of each group being characterized by some one prominent employment. No such classification, however, can be more than approximately accurate, since the same tissue has often more than one wellmarked physiological property. The following arrangement, however, is practically convenient.

1. Undifferentiated Tissues. These are composed of cells which have developed along no. one special line, but retain very 
much the form and properties of the cells forming the very young Body before different tissues were recognizable in it. The lymphcorpuscles and the colorless corpuscles of the blood belong to this class.

2. Supporting Tissues. Including cartilage (gristle), bone, and connective tissue. Of the latter there are several subsidiary varieties, the two more important being white fibrous connective tissue, composed mainly of colorless inextensible fibers, and yellow fibrous tissue, composed mainly of yellow elastic fibers. All the supporting tissues are used in the Body for mechanical purposes; the bones and cartilages form the hard framework by which softer tissues are supported and protected; and the connective tissues unite the various bones and cartilages, form investing membranes around different organs, and in the form of fine networks penetrate their substance and support their constituent cells. The functions of these tissues being for the most part passively to resist strain or pressure, none of them has any very marked physiological property; they are not, for example, excitable or contractile, and their mass is chiefly made up of an intercellular substance which has been formed by the actively living cells sparsely scattered through them, as, for instance, in cartilage (Fig. 11), where the cells are seen imbedded in cavities in a matrix which they have formed around them; and this matrix by its firmness and elasticity forms the functionally important part of the tissue.

3. Nutritive Tissues. These form a large group, the members of which fall into three main divisions, viz.:

Assimilative tissues, concerned in receiving and preparing food materials, and including-(a) Secretory tissues, composed of cells which make the digestive liquids poured into the alimentary canal and used to bring about chemical or other changes in the food. (b) Receptive tissues, represented by cells which line parts of the alimentary canal and take up the digested food.

Eliminative or excretory tissues, represented by cells in the kidneys, skin, and elsewhere, whose main business it is to get rid of the waste products of the various parts of the Body.

Respiratory tissues. These are concerned in the gaseous interchanges between the Body and the surrounding air. They are constituted by the cells lining the lungs and by the colored cor* puscles of the blood. 
As regards the nutritive tissues it requires especially to be borne in mind that although such a classification as is here given is useful, as helping to show the method pursued in the domestic economy of the Body, it is only imperfect and largely artificial. Every cell of the Body is in itself assimilative, respiratory, and excretory, and the tissues in this class are only those concerned in the first and last interchanges of material between it and the external world. They provide or get rid of substances for the whole Body, leaving the feeding and respiration and excretion of its individual tissues to be ultimately looked after by themselves, just as even the mandarin described by Robinson Crusoe who found his dignity promoted by having servants to put the food into his mouth, had finally to swallow and digest it for himself. Many secretory cells, too, have no concern with the digestion of food, as for example those which form the various hormones (p. 28).

4. Storage Tissues. The Body does not live from hand to mouth: it has always in health a supply of food-materials accumulated in it beyond its immediate needs. This lies in part in the individual cells themselves, but apart from this reserve there are certain cells, which store up considerable quantities of material and constitute what we will call the storage tissues. These are especially represented by the liver-cells and fat-cells, which contain in health a reserve fund for the rest of the Body.

5. Excitable, or Irritable Tissues. These include those tissues which are especially susceptible to changes in their surroundings and are therefore useful in giving to the Body information of what is going on around it. Any change in the environment which serves to arouse response in an excitable tissue constitutes a stimulus.

6. Conductive Tissues. While most, if not all, of the cells of the Body retain the property of conductivity in some degree, the nervous tissues exhibit it in very high degree. They serve therefore to bring into communication the various parts of the Body. As an incident in the conveying of messages from one part of the Body to another to fulfil the requirements of nervous co-ordination certain nervous structures have the power of modifying the messages which pass through them.

7. МотоR Tissues. These have the contractility of the original protoplasmic masses highly developed. The most important 
are the ciliated cells and muscular tissue. The former line certain surfaces of the Body, and possess on their free surfaces fine threads which are in constant movement. One finds such cells, for example (Fig. 46), lining the inside of the windpipe, where their threads or cilia serve, by their motion, to sweep any fluid formed there towards the throat, where it can be coughed up and got rid of. Muscular tissue occurs in three main varieties. One kind is found in the muscles attached to the bones, and is that used in the ordinary voluntary movements of the Body. It is composed of fibers which present cross-stripes when viewed under the microscope (Fig. 42), and is hence known as striped or striated muscular tissue. Because the muscles which are made of this sort of tissue are attached to bones they are often called skeletal muscles. A second kind of muscular tissue is found in the walls of the alimentary canal, the arteries, and some other hollow organs, and consists of elongated cells (Fig. 44) which present no cross-striation. It is known as smooth or unstriated muscular tissue. The third sort occurs only in the heart. It consists of branched cells presenting some indications of cross-striation (Fig. 45) and is called cardiac muscular tissue.

The cells enumerated under the heading of "undifferentiated tissues" might also be included among the motor tissues, since they are capable of changing their form.

8. Protective Tissues. These consist of certain cells lining

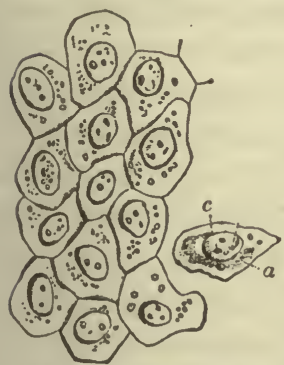

FIG. 9.-Flat epithelium cells from the surface of the peritoneum. $a$, cell-body; $c$, nucleus. cavities inside the body and called epithelial cells, and cells covering the whole exterior of the Body and forming epidermis, hairs, and nails. The enamel which covers the teeth belongs also to this group.

The class of protective tissues is, however, even more artificial than that of the nutritive tissues, and cannot be defined by positive characters. Many epithelial cells are secretory, excretory or receptive; and cilated cells have already been included among the motor tissues. The protective tissues may be best defined as including cells which cover free surfaces, and whose functions are mainly mechanical or physical. In their simplest form epithelial cells are flat scales, as, for example, those repre- 
sented in Fig. 9 from the lining membrane of the abdominal cavity.

9. The Reproductive Tissues. These are concerned in the production of new individuals, and in the Human Body are of two kinds, located in different sexes. The conjunction of the products of each sex is necessary for the origination of offspring, since the female product, egg-cell or ovum, which directly develops into the new human being, remains dormant until it has been fertilized, and fertilization consists essentially in the fusion of its nucleus with the nucleus of a cell produced by the male.

The Combination of Tissues to Form Organs. The various tissues above enumerated form the building materials of the Body; anatomy is primarily concerned with their structure, and physiology with their properties. If this, however, were the whole matter, the problems of anatomy and physiology would be much simpler than they actually are. The knowledge about the living Body obtained by studying only the forms and functions of the individual tissues would be comparable to that attained about a great factory by studying separately the boilers, pistons, levers, wheels, etc., found in it, and leaving out of account altogether the way in which these are combined to form various machines; for in the Body the various tissues are for the most part associated to form organs, each organ answering to a complex machine like a steam-engine with its numerous constituent parts. And just as in different machines a cogged wheel may perform very different duties, dependent upon the way in which it is connected with other parts, so in the Body any one tissue, although its essential properties are everywhere the same, may by its activity subserve very various uses according to the manner in which it is combined with others. For example: A nerve-fiber uniting the eye with one part of the brain will, by means of its conductivity, when its end in the eye is excited by the irritable tissue attached to it on which light acts, cause changes in the sensory nerve-cells connected with its other end and so arouse a sight sensation; but an exactly similar nerve-fiber running from the brain to the muscles will, also by virtue of its conductivity, when its ending in the brain is excited by a change in a nerve-cell connected with it, stir up the muscle to contract under the control of the will. The different results depend on the different parts connected with the ends of the nerve- 
fibers in each case, and not on differences in the properties of the nerve-fibers themselves.

It becomes necessary then to study the arrangement and uses of the tissues as combined to form various organs, and this is frequently far more difficult than to make out the structure and properties of the individual tissues. An ordinary muscle, such as one sees in the lean of meat, is a very complex organ, containing not only contractile muscular tissue, but supporting and uniting connective tissue and conductive nerve-fibers, and in addition a complex commissariat arrangement, composed in its turn of several tissues, concerned in the food-supply and waste-removal of the whole muscle. The anatomical study of a muscle has to take into account the arrangement of all these parts within it, and also its connections with other organs of the Body. The physiology of any muscle must take into account the actions of all these parts working together and not merely the functions of the muscular fibers themselves, and has also to make out under what conditions the muscle is excited to activity by changes in other organs, and what changes in these it brings about when it works.

Physiological Systems. Even the study of organs added to that of the separate tissues does not exhaust the matter. In a factory we frequently find machines arranged so that two or more shall work together for the performance of some one work: a steamengine and a loom may, for example, be connected and used together to weave carpets. Similarly in the Body several organs are often arranged to work together so as to attain some one end by their united actions. Such combinations are known as physiological apparatuses or systems. The circulatory system, for example, consists of various organs (each in turn composed of several tissues) known as heart, arteries, capillaries, and veins. The heart forms a force-pump by which the blood is kept flowing through the whole mechanism, and the rest, known together as the bloodvessels, distribute the blood to the various organs and regulate the supply according to their needs. Again, in the visual apparatus we find the co-operation of $(a)$ a set of optical instruments which bring the light proceeding from external objects to a focus upon (b) the retina, which contains highly irritable parts; these, changed by the light, stimulate $(c)$ the optic nerve, which is conductive and transmits a disturbance which arouses in turn $(d)$ sensory parts in 
the brain. In the production of ordinary sight sensations all these parts are concerned and work together as a visual apparatus. So, too, we find a respiratory system consisting primarily of two hollow organs, the lungs, which lie in the chest and communicate by the windpipe with the back of the throat, from which air enters them. But to complete the respiratory apparatus are many other organs, bones, muscles, nerves, and nerve-centers, which work together to renew the air in the lungs from time to time; and the act of breathing is the final result of the activity of the whole apparatus.

The Relation of Man to His Environment. From infancy the human organism is confronted with the task of maintaining itself alive. To this end all the bodily functions bend themselves. The maintenance of life in man, as in all animals, presents two distinct problems: first, to obtain the necessary food; and second, to cope successfully with the innumerable perils with which the organism is continually confronted. Failure in either of these endeavors means failure in maintaining life itself.

The labor of obtaining food and the struggle to escape harm take place in the midst of a world filled with creatures engaged in the same labor and the same struggle. Indeed it is the very prevalence of living beings that makes the securing of food labor, and the avoidance of harm a struggle. All the living beings that belong to the animal kingdom are in a more or less continuous state of activity. Each individual, therefore, finds himself surrounded by a continually shifting world of other beings. Nor is inanimate Nature stationary; winds and rains, heat and cold, come and go. To such a constantly changing environment the organism must adapt itself.

In the complex of systems which together make up the Body it is possible to distinguish between those whose immediate function is to maintain the necessary adaptation of the organism to its environment and those which function only indirectly to that end by keeping the Body itself in good working order and each part well supplied with the energy yielding materials without which activity is impossible. In making such a distinction, however, it must be borne in mind that all the bodily functions work together for the good of the whole Body so that no hard and fast line can be drawn between the two classes of systems. It will be convenient 
to consider first the systems which are particularly concerned in adapting the Body to its environment.

Adaptive Systems. The Motor System. In all members of the animal kingdom with the exception of certain parasites adaptation is secured mainly through movement. Both for obtaining food and for escaping danger movements either of the whole Body or of parts of it are constantly being resorted to.

In all higher animals the motor mechanism is made up of skeletal muscles, which by their action upon the movable bones of the jointed skeleton bring about the various bodily movements.

There are many types of movement in animals which are not concerned immediately with adaptation to the environment. The movements of breathing, for example, the beat of the heart, and the activities of the stomach. These have to do with maintenance. As emphasized in the last paragraph, however, the classification of systems as adaptive or maintenance is for convenience, and with reference to their most conspicuous functions, and is not to be taken as excluding the systems in one group from having important activities in the other.

The Supporting System. In all but the very simplest animal forms movements are made effective by the action of the muscles upon certain of the supporting tissues. These tissues play, thercfore, a very real, although passive part in adaptation. By including the supporting system among the adaptive systems of the Body we emphasize the importance of the supporting structures in making muscular action effective, although here again we must bear in mind that they are also intimately associated with other systems whose chief function is maintenance.

The Receptor System. It is obvious that the Body cannot execute movements adapting it to its surroundings unless it knows what its surroundings are. A blind man, be he never so agile, cannot escape the onward rush of the approaching car while he is ignorant of its coming. He will starve in the midst of abundant food if he does not know where it is to be found.

The Body obtains knowledge of its environment by means of a set of structures known as the sense-organs. In these the property of irritability is developed to a high degree, and so long as they all function properly not much that is important for the organism to know about need escape its knowledge. 
The Conductive System. Organs for making movements and organs for receiving impressions from the surroundings are not of themselves adequate to the maintenance of adaptation. It is necessary that the information gained by the sense-organs be transmitted to the muscles so that their movements may correspond to the requirements of the situation. This function is performed by the nervous system. The conduction of stimuli from sense-organs to muscles is not, however, a simple matter. Impressions are continually coming into the Body by way of a number of different channels. Movements must be made not in obedience to any one of these impressions by itself but for the advantage of the whole Body as indicated by all of them taken together. To this end a certain part of the nervous system is adapted for receiving all sorts of incoming stimuli and before passing them on to the motororgans combining and modifying them to produce the best results.

While for purposes of convenience, the conductive system is classed as one of the adaptive mechanisms, we need to bear in mind that nervous co-ordination, for which this system is the agency, although concerned primarily with direct adaptation, has also much to do with the control of those activities which are primarily concerned with maintenance and only indirectly adaptive.

Maintenance Systems. The systems which are not immediately concerned in the adaptation of the Body to its environment but which serve rather to keep it in proper condition for activity may next be considered.

Activity in the Body involves the manifestation of energy, and in its energy relations the Body is on exactly the same plane as any machine; it is without power to manufacture energy, and must receive whatever energy it obtains from without. The ultimate source of the Body's energy is chemical, being received in the complex substances which serve as food. This energy is made available for the use of the Body chiefly through the process of oxidation. Every living cell in the Body must share in this process, for the energy manifestations of the Body as a whole are simply the sum-total of those of its component cells.

The systems which are concerned with the maintenance of activity have, then, the task of furnishing to each cell of the Body oxidizable substance and oxygen; they must provide for making good the wear and tear of the cells themselves; and they must 
remove the waste materials which are formed in connection with the chemical activities of the cells and which would interfere with their proper working if allowed to accumulate.

The Circulatory System consists of the heart and blood-vessels. It serves to distribute to all the parts of the Body supplies of oxidizable material, of repair material, and of oxygen, and to remove therefrom the accumulated waste products. These functions are accomplished through the agency of a circulating medium, the blood.

The Respiratory System consists of the lungs, the bronchial tubes, and the trachea, together with the respiratory muscles. Its function is to bring the outside air to a region where the circulating medium can take up abundant supplies of oxygen, and where it can get rid of those waste products which are in gaseous form.

The Digestive System consists of the alimentary canal and certain associated glands (salivary, liver, pancreas). It serves to bring the various materials that are taken as food into the forms best adapted for use as repair materials or as oxidizable substance; when it has so prepared them it turns them over to the circulating medium for distribution.

The Excretory System consists of the kidney and bladder with their connecting tubes, the liver, and the skin. It serves to withdraw from the circulating medium and to eliminate from the body those waste products which are in liquid form.

Chemical Co-ordination is secured, as previously stated, by specific hormones which govern those bodily activities that are either not readily susceptible to nervous control or in which the best results are secured by supplementing nervous control with chemical. There are special organs, or parts of organs, which manufacture hormones. These are often called ductless glands, since they pour their secretions into the blood stream and not by ducts to the surface. They might be grouped together as a system, although nothing would be gained by so doing. Chemical co-ordination plays a part in nearly all forms of bodily activity, and the different hormones will be studied in connection with the activities over which they exert influence.

Through these systems provision is made for the activities of the individual cells. These activities are many and complex. They 
include oxidative processes, processes involving waste and repair, and doubtlessly many others of which we know nothing. The study of these cell activities is comprehended under the head of Metabolism.

The chemical activities which go on in the cells of the Body give rise to much heat. Some cells generate more heat than others. One of the functions of the circulating medium is to distribute this heat uniformly over the Body. There is constant loss of heat from the surface of the Body. In warm-blooded animals, which have a nearly constant body temperature, the maintenance of balance between heat production and heat loss in the face of constantly varying outside temperatures is a function of great importance. It is studied under the head of Heat Production and Heat Regulation.

Not immediately concerned with the well-being of the Body itself, but devoted to the well-being of the race as a whole through perpetuating the species is the Reproductive System.

Before we turn from this discussion of the various systems into which, for convenience, we have grouped the various Bodily structures, we may well emphasize again the unity of operation of the Body, so that we shall not fall into the habit of thinking of the different systems as separate mechanisms, operating independently of one another. This unity of operation is well illustrated in one of our commonest every-day experiences, namely, vigorous muscular exercise. Whenever we use our muscles briskly definite activities of the various systems we have classed as maintenance systems occur. Thus the heart is thrown into rapid beating; the skin is flushed; the breathing is quickened; the sweat glands are active; if the exercise is prolonged and not too fatiguing, there is likely to be a sharpening of appetite, leading to a greater consumption of food and so to increased digestive activity. All these manifestations accompany muscular exercise, as a matter of course. We shall see later how they are all part of the provision whereby the Body is able to use its muscles effectively. This is but one of many illustrations that might be cited to show the interdependence of the various systems. True insight into Human Physiology requires that this interdependence be thoroughly realized.

Animals Compared with Plants. We divide the world of living things into two kingdoms; the plant kingdom and the animal 
kingdom. The familiar members of the first kingdom seem to us to differ in nearly every important respect from the best known members of the second; an oak tree and a horse are superficially wholly dissimilar. Yet both plants and animals consist of living cells, and the protoplasm of which plant cells are composed is often indistinguishable from that found in animal cells. When we attempt to analyze the difference between plants and animals we find that it cannot be referred to difference in the protoplasm. The simplest plants and the simplest animals show the fundamental properties of protoplasm developed in about equal degrees. In fact it is by no means easy always to state positively whether a given one-celled organism should be considered a plant or an animal.

As we go up the scale to the region of higher organization, however, we find no difficulty in deciding whether a living form is plant or animal. The fundamental difference between the higher plants and animals, and the one which involves, as a natural sequence, the superficial differences which are so striking, is a difference in the manner of obtaining nourishment. The higher (green) plants are able to use the energy which falls upon them in the form of sunlight to build up from simple substances present in the air, and in the water to which their roots penetrate, the complex materials of which protoplasm is composed and which, through the processes of dissimilation, provide for the carrying on of the necessary activities of living cells. The higher animals, on the other hand, are nourished by means of complex materials which contain within themselves the energy required for the bodily activities. The ultimate source of animal energy, to be sure, is the same as of plant, for the complex materials consumed by animals are derived, directly or indirectly, from plants.

The simple chemical materials needed by plants are very widely distributed, and the sunlight falls, of course, on all parts of the earth. In any location, therefore, that is sufficiently suited to plant life to allow the plant to get a start, the chances of being able to continue to live are as good, on the whole, as they would be anywhere. Hence plants do net need to move about. The giant sequoias of the Pacific Slope have lived for centuries upon the spots where they became established as seedlings.

Animals, on the other hand, require materials that are not every. 
where present but must be sought in particular places. The successful search for this sort of food involves active motion subject to guidance in accordance with the environment. In a word, adaptation; and the presence of mechanisms for adaptation is the most striking feature of the higher animals, just as the presence of a mechanism for utilizing the energy of sunlight is the conspicuous feature of higher plants. 


\section{CHAPTER IV}

\section{THE SUPPORTING TISSUES}

Connective Tissue. This is the most widely distributed of the supporting tissues. It envelopes and pervades all the soft parts of the Body. The various constituents of individual organs are held together by it, and the organs themselves, are supported in their places by the same tissue. Beneath the skin and attaching it rather loosely to the underlying structures is a layer of connective tissue known as the fascia. So completely is the entire Body pervaded by connective tissue that if a solvent could be found which would dissolve away all the tissues of the Body except this one there would still remain in perfect outline not only the whole Body but also each organ down to minutest detail.

This connective tissue framework is commonly called areolar tissue. It is composed, in the main, of tough, inelastic strands; these are arranged, however, in most parts of the Body to form a. rather loose network, so that in removing the skin from an animal or in separating one muscle from another in making a dissection a blunt instrument readily tears the strands of areolar tissue apart.

The meshes of areolar tissue are everywhere filled with a fluid, lymph. Thus the various living tissues of the Body, all of which are surrounded by areolar tissue, are nourished.

There are in the body connective tissue structures in which the individual strands, instead of forming a loose network, are in parallel bundles, forming the toughest and strongest of cords and bands. These are the tendons, by which muscles are attached to bones, and the ligaments which hold the different bones of the skeleton together.

The functional part of connective tissue consists of two sorts of fibers. In most places the white fibers constitute the bulk of the tissue. These are flexible, inelastic strands composed of an albuminoid substance, collagen. The second sort of fibers are the elastic fibers. These are intermingled with the white fibers to some extent in nearly all regions where connective tissue occurs. In 
certain structures, where a high degree of elasticity is required, elastic fibers make up the entire connective tissue content. Examples of this sort are the walls of the large arteries and the ligaments connecting the vertebræ. In quadrupeds these fibers form the great ligament which helps to sustain the head (see p. 69). Elastic tissue is yellow in color, and consists chemically of an albuininoid, elastin, which in some important respects differs from the albuminoid of the white fibers.

Connective tissue fibers are not living structures. They owe their origin to certain living cells, the so-ealled connective tissue cells, which lie irregularly interspersed wherever connective tissue fibers occur (Fig. 10). In areolar tissue many of the eells have given up their function of forming fibers and have devoted themselves instead to storing within their substance masses of fat. Adipose tissue consists of cells of this sort, and occurs in regions where areolar tissue is most abundant, as just under the skin or in masses about certain internal organs.
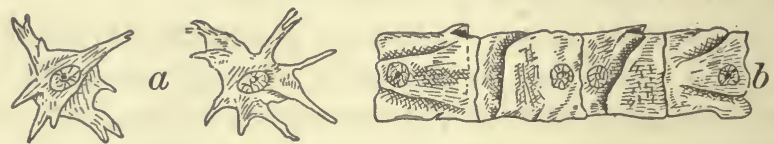

Fig. 10.-Connective tissue cells: $a$, from areolar tissue; $b$, from tendon.

Temporary and Permanent Cartilages. In early life a great many parts of the supporting framework of the Body, which afterwards become bone, consist of cartilage. Such for example is the case with all the vertebræ, and with the bones of the limbs. In these cartilages subsequently the process known as ossification takes place, by which a great portion of the original cartilaginous model is removed and replaced by true osseous tissue. Often, however, some of the primitive cartilage is left throughout the whole of life at the ends of the bones in joints where it forms the articular cartilages; and in various other places still larger masses remain, such as the costal cartilages, those in the external ears forming their framework, others finishing the skeleton of the nose which is only incompletely bony, and many in internal parts of the Body, as the cartilage of "Adam's apple," which can be felt in the front of the neck, and a number of rings around the windpipe serving to keep it open. These persistent masses are known 
as the permanent, the others as the temporary cartilages. In old age many so-called permanent cartilages become calcified-that is, hardened and made unyielding by deposits of lime-salts in themwithout assuming the histological character of bone, and this calcification of the permanent cartilages is one chief cause of the want of pliability and suppleness of the frame in advanced life.

Hyaline Cartilage. In its purest form cartilage is flexible and elastic, of a pale bluish-white color when alive and seen in large masses, and cuts readily with a knife. In thin pieces it is quite transparent. Everywhere except in the joints it is invested by a tough adherent membrane, the perichondrium.

When a thin slice of hyaline cartilage is examined with a microscope it is found (Fig. 11) to consist of granular nucleated cells, often collected into groups of two, four, or more, scattered through a homogeneous or faintly granular ground-substance or matrix. This matrix is composed of albuminoid substances, and owes its origin to the cells embedded within it. At the time the cartilage was in process of formation these cells laid down the matrix substance in concentric layers about themselves; thus they cut themselves off from each other and from communication with the outside. The substance of the matrix is sufficiently permeable, however, for a certain interchange of food materials between the cartilage cells and the blood, so that the cells

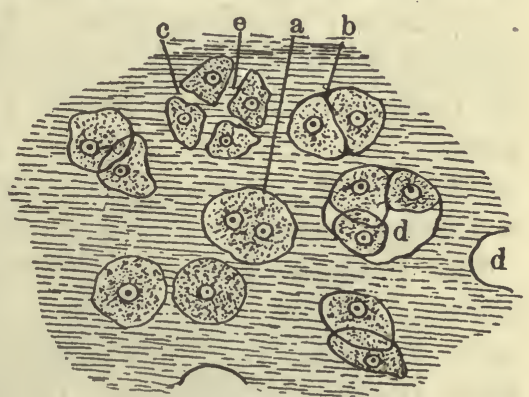

FIG. 11.-A thin slice of cartilage, magnified, to show the cells embedded in the homogeneous matrix. $a$, a cell in which the nucleus has divided; $b$, a cell in which division is just complete; $c, e$, a group of four cells resulting from further division of a pair like $b$; the new cells have formed some matrix between them, separating them from another; $d, d$, cavities in the matrix from which cells have dropped out during the preparation of the specimen. are able to remain alive, although their life is naturally an inactive one.

All temporary cartilages are of the hyaline type as are also the costal and articular permanent cartilages and the cartilage of the nose and of the windpipe.

Elastic Cartilage is a tissue whose cartilaginous matrix is interwoven with fibers of elastic connective tissue. The result of this 
interweaving is to give the cartilage a yellow color and a high degree of elasticity. Cartilage of this sort is found in the external ear, the epiglottis, and in certain parts of the larynx.

Fibrocartilage is really a dense fibrous connective tissue within whose spaces a certain amount of matrix material has been deposited. It makes up the intervertebral disks, pads which are interposed between the bones of the vertebral column, and is found also in certain joints, notably the knee-joints and the articulations of the lower jaw.

Bone. The bones which make up the skeleton vary greatly in shape and size, ranging from the long cylindrical bones of the arm and leg to the flat skull bones, and the tiny irregularly shaped ossicles of the middle ear. They all, however, have a similar microscopic structure and similar chemical composition.

The bones may be classified according to their origin as membrane bones or cartilage bones. To the first group belong the flat bones of the skull and the bones of the face (see p. 60). They do not replace cartilage but develop upon a foundation of connective tissue. The so-called cartilage bones replace the temporary cartilages and make up the whole of the bony skeleton, except the membrane bones mentioned above.

The Process of Bone Formation is complicated, and can be described only very briefly here. At the beginning of the development of a membrane bone the strands of connective tissue upon which the bone is to be built become covered with peculiar small cells which are bone-producing cells or osteoblasts. These osteoblasts deposit upon the strands whereon they rest albuminoid material which constitutes the organic matrix of bone. There is thus produced a rather open network of bone matrix. By the deposition. within the matrix of lime-salts it takes on the character of true bone. The original connective tissue is thus replaced by a network of bony spicules.

The surfaces of this bony mass now become covered with a stout connective tissue membrane, the periosteum, whose inner surface is beset with osteoblasts. These deposit upon the underlying mass a layer of compact bone. Thus the fully formed membrane bone consists of outer surfaces of compact bone inclosing a mass of spongy bone.

The replacement of temporary cartilage by bone proceeds from 
certain points in the cartilage known as centers of ossification. The cartilage itself becomes surrounded by a periosteum like that which incloses membrane bones. At the center of ossification the osteoblast layer of the periosteum begins to force its way into the cartilage, absorbing much of the latter and leaving only a coarse network, which is presently converted by the osteoblasts into true bone. Meanwhile the periosteum has deposited on the surface of the cartilage a layer of compact bone so that in time the cartilage bone presents a structure not unlike that of membrane bones, a spongy interior inclosed in a layer of compact bone. As the cartilaginous network is being ossified, many osteoblasts are imprisoned within the bony substance. The spaces which they occupy and the tiny canals which radiate therefrom into the bone substances are among the most characteristic appearances of bone viewed under the microscope (Fig. 12).

The growth in thickness of bone is accomplished by the addition of layer after layer of compact bone underneath the periosteum.

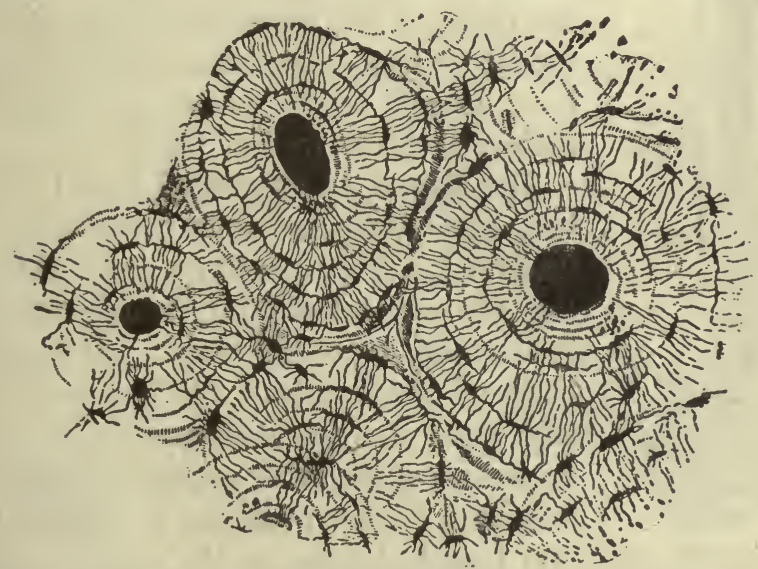

Fig. 12.-Cross-section of compact bone from the shaft of the humerus. (Sharpey, from Bailey's Text Book of Histology.)

During this process blood-vessels of the periosteum often become embedded within the bony mass. When this occurs the osteoblasts which accompany the blood-vessel surround it with concentric layers of bone. In this manner are formed the so-called Haversian Systems, each of which consists of the space through 
which the blood-vessel passes with its surrounding rings of bony material.

The increase in length of the long bones is brought about by plates of cartilage which persist between the shaft of the bone and

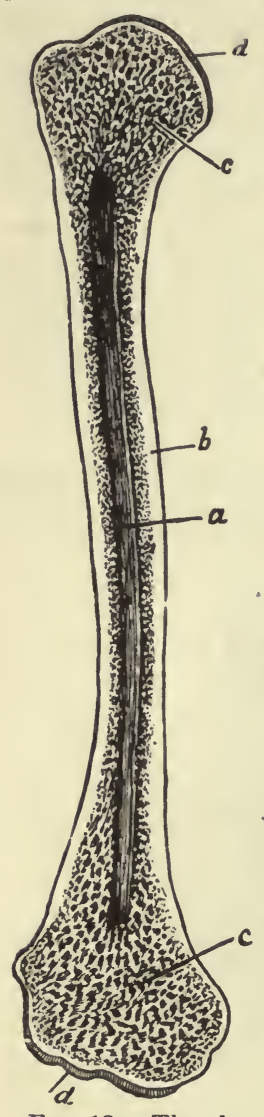

Fig. 13.-The humerus bisected lengthwise. $a$, marrow-cavity; $b$, hard bone; $c$, spongy bone; $d$, articular cartilage. its extremities. There is a continual growth of bone into these cartilages from both sides, but they grow in thickness with equal rapidity until the adult length of the bone is reached when their growth stops and they are gradually replaced by bone.

At the same time that the bone is growing by additions to its outer surfaces a continuous absorption of its inner portions is going on. This absorption is carried on by large, multinuclear cells known as osteoclasts. It serves the purpose of preventing the bone from becoming so heavy as to be unmanageable, without sacrificing unduly its strength. As the result of this absorption many adult bones, especially long ones, contain little or no spongy bone except at their ends, the shaft being hollow as shown in Fig. 13.

The Repair of Fractured Bone. When, as happens with unfortunate frequency, a bone is fractured, a sequence of processes is set in motion at the point of injury which results finally in the mending of the break. As an inevitable incident of the injury which caused the fracture there is marked laceration of the periosteum and of the other adjacent tissues. These lacerated tissues pour out a mixture of blood and lymph, known as the exudate, which permeates the region of injury. This exudate gradually stiffens until it affords considerable support to the injured bone. Osteoblasts from the inner surface of the periosteum and from the fractured ends of the bone penetrate the exudate. These osteoblasts, little by little, replace the exudate with spongy bone, which holds the injured parts even more firmly in place, and which in turn is grad- 
ually replaced by hard bone like that which was present before the injury. The spongy temporary bone is absorbed by the osteoclasts described above.

To secure proper knitting of the fracture two things are of great importance; the first of these is the reduction of the fracture, whereby the parts are brought as nearly as possible into their former positions; the second is immobilization of the part by means of splints, bandages, or casts to hold the broken ends in place during the formation of the new bony material.

Chemistry of Bone. Bone is composed of inorganic and organic portions intimately combined, so that the smallest distinguishable portion contains both. The inorganic matters form about twothirds of the total weight of a dried bone, and may be removed by soaking the bone in dilute hydrochloric acid. The organic portion left after this treatment constitutes a flexible mass, retaining the form of the original bone; it. consists chiefly of an albuminoid, ossein, which by long boiling, especially under pressure at a higher temperature than that at which water boils when exposed freely to the air, is converted into gelatin, which dissolves in the hot water. Much of the gelatin of commerce is prepared in this manner by boiling the bones of slaughtered animals, and even wellpicked bones may be used to form a good thick soup if boiled under pressure in a Papin's digestor; much nutritious matter being, in the common modes of domestic cooking, thrown away in the bones.

The inorganic salts of bone may be obtained free from organic matter by calcining a bone in a clear fire, which burns away the organic matter. The residue forms a white very brittle mass, retaining perfectly the shape and structural details of the original bone. It consists mainly of normal calcium phosphate, or boneearth $\left[\mathrm{Ca}_{3}\left(\mathrm{PO}_{4}\right)_{2}\right]$; but there is also present a considerable proportion of calcium carbonate $\left(\mathrm{CaCO}_{3}\right)$ and smaller quantities of other salts.

Hormones of the Supporting System. We learned in a previous chapter (p. 28) that co-ordination of many bodily processes, and notably of the growth process, is secured chemically by means of substances known as hormones. The size of the Body depends on the size of the bones which make up the skeleton. For that reason any clue to the mechanism which governs the growth of the bones is of great interest. Some years ago the discovery was made 
that a peculiar disease, acromegaly, in which there is abnormal enlargement of some of the bones, notably of the face and extremities, is associated with overgrowth of a mass of tissue at the base of the brain. This tissue mass, the pituitary body (Fig. 60), is formed in part by an outgrowth downward from the brain, and in part by an outgrowth upward from the roof of the mouth. The latter portion, known separately as the hypophysis, appears to be particularly concerned with bony growth. The theory of this control which best explains the known facts is that a hormone is secreted and poured out into the blood which by its presence stimulates growth of the bones. The more abundant the hormone the more vigorous is the growth. The enlargement of the hypophysis which occurs in acromegaly would account for the occurrence of a more abundant secretion of the hormone. A similar enlargement appears to characterize the condition of gigantism, which gives rise to the giants exhibited in side shows. Although there is no positive proof, it seems reasonable to suppose that the opposite condition, dwarfishness, is a result of a deficiency of the pituitary hormone.

We need to bear in mind in discussing hormone action that when an effect is attributed to a hormone we have not offered a complete explanation of it, but only moved the explanation a step farther along. We can say that a man's height is determined by the activity of his pituitary body, but we are still in the dark as to the factors that regulate the latter. Particularly are we ignorant of the manner in which secretion of the hormone is modified when the man has "gotten his growth."

Another body, the thyroid, located at the front of the neck, is believed to secrete a hormone which regulates the development of connective tissue. This function of the thyroid is, however, subordinate to its major function, which is concerned with the nervous system. Detailed discussion of the thyroid is deferred, therefore, to that connection,

Hygienic Remarks. Since in the new-born infant many parts which will ultimately become bone consist only of cartilage, the young child requires food which shall contain a large proportion of the lime-salts which are used in building up bone. Nature provides this in the milk, which is rich in such salts (see Chap. XXXIV), and no other food can thoroughly replace it. Long after infancy 
milk should form a large part of a child's diet. Many children though given food abundant in quantity are really starved, since their food does not contain in sufficient amount the mineral salts requisite for their healthy development.

At birth even those bones of a child which are most ossified are often not continuous masses of osseous tissue. In the large bone of the arm, the humerus, for example, the shaft of the bone is well ossified and so is each end, but between the shafts and each of the articular extremities there still remains a cartilaginous layer, and at those points the bone increases in length, new cartilage being formed and replaced by bone. The bone increases in thickness by new osseous tissue formed beneath the periosteum. The same thing is true of the bones of the leg. On account of the largely cartilaginous and imperfectly knit state of its bones, it is cruel to encourage a young child to walk beyond its strength, and may lead to "bow-legs" or other permanent distortions. Nevertheless here as elsewhere in the animal body, moderate exercise promotes the growth of the tissues concerned, and it is nearly as bad to wheel a child about forever in a baby-carriage as to force it to overexertion.

The best rule is to let a healthy child use its limbs when it feels inclined, but not by praise or blame to incite it to efforts which are beyond its age, and so sacrifice its healthy growth to the vanity of parent or nurse.

The final knitting together of the bony articular ends with the shaft of many bones takes place only comparatively late in life, and the age at which it occurs varies much in different bones. Generally speaking, a layer of cartilage remains between the shaft and the ends of the bone, until the latter has attained its full adult length. To take a few examples: the lower articular extremity of the humerus only becomes continuous with the shaft by bony tissue in the sixteenth or seventeenth year of life. The upper articular extremity only joins the shaft by bony continuity in the twentieth year. The upper end of the femur (p. 66) joins the shaft by bone from the seventeenth to the nineteenth year, and the lower end during the twentieth. In the tibia (p. 66) the upper extremity and the shaft unite in the twenty-first year, and the lower end and the shaft in the eighteenth or nineteenth: while in the fibula (p. 66) the upper end joins the shaft in the twenty- 
fourth year, and the lower end in the twenty-first. The separate vertebræ of the sacrum (p. 59) are only united to form one bone in the twenty-fifth year of life; and the ilium, ischium, and pubis unite to form the os innominatum about the same period. Up to about twenty-five then the skeleton is not firmly "knit," and is incapable, without risk of injury, of bearing strains which it might afterwards meet with impunity. To let lads of sixteen or seventeen row and take other exercise in plenty is one thing, and a good one; but to allow them to undergo the severe and prolonged strain of training for and rowing a long race is quite another, and not devoid of risk. 


\section{CHAPTER V}

\section{THE SKELETON}

Exoskeleton and Endoskeleton. The skeleton of an animal includes all its hard protecting or supporting parts, and is met with in two main forms. One is an exoskeleton developed in connection with either the superficial or deeper layer of the skin, and represented by the shell of a clam, the scales of fishes, the horny plates of a turtle, the bony plates of an armadillo, and the feathers of birds. In man the exoskeleton is but slightly developed, but it is represented by the hairs, nails, and teeth; for although the latter lie within the mouth, the study of development shows that they are developed from an offshoot of the skin which grows in and lines the mouth long before birth. Hard parts formed from structures deeper than the skin constitute the endoskeleton, which in man is highly developed and consists of a great many bones and cartilages or gristles, the bones forming the mass of the hard framework of the Body, while the cartilages finish it off at various parts. This framework is what is commonly meant by the skeleton; it primarily supports all the softer parts and is also arranged so as to surround cavities in which delicate organs, as the brain, heart, or spinal cord, may lie with safety. The gross skeleton thus formed is completed and supplemented by another made of the connective tissues, which not only, in the shape of tough bands or ligaments, tie the bones and cartilages together, but also in various forms pervade the whole Body as a sort of subsidiary skeleton running through all the soft organs and forming networks of fibers around their other constituents; they make, as it were, a microscopic skeleton for the individual modified cells of which the Body is solargely composed, and also form partitions between the muscles, cases for such organs as the liver and kidneys, and sheaths around the blood-vessels. The bony and cartilaginous framework with its ligaments might be called the skeleton of the organs of the Body, and this finer supporting meshwork the skeleton of the tissues.

The Bony Skeleton (Fig. 14). If the hard framework of the 


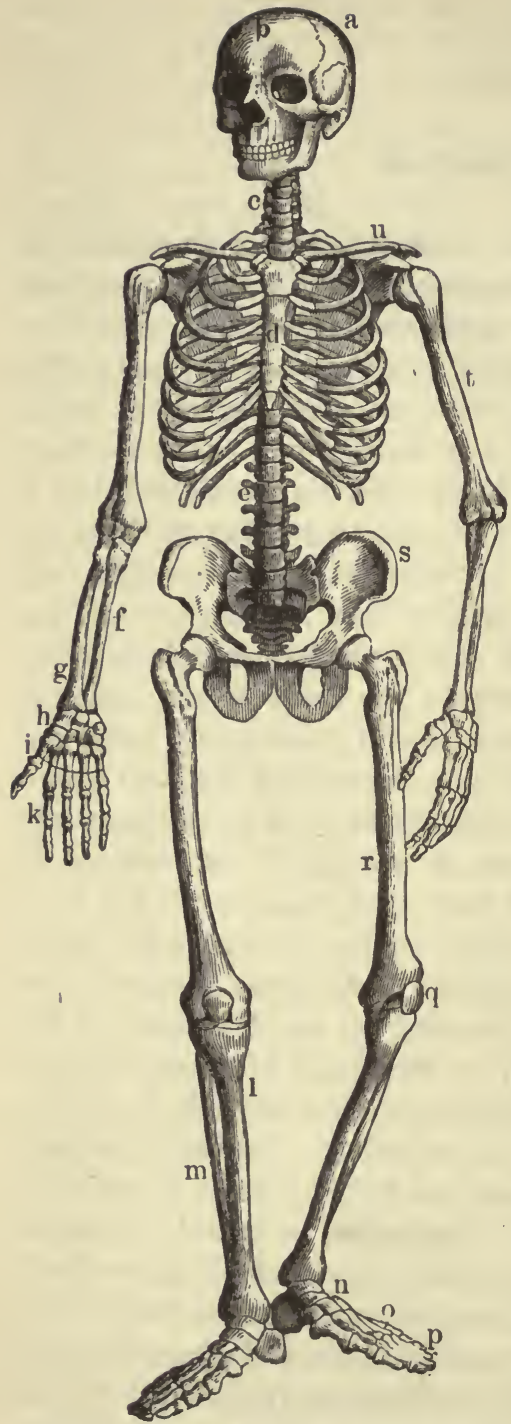

FIG. 14.-The bony and cartilaginous skeleton.

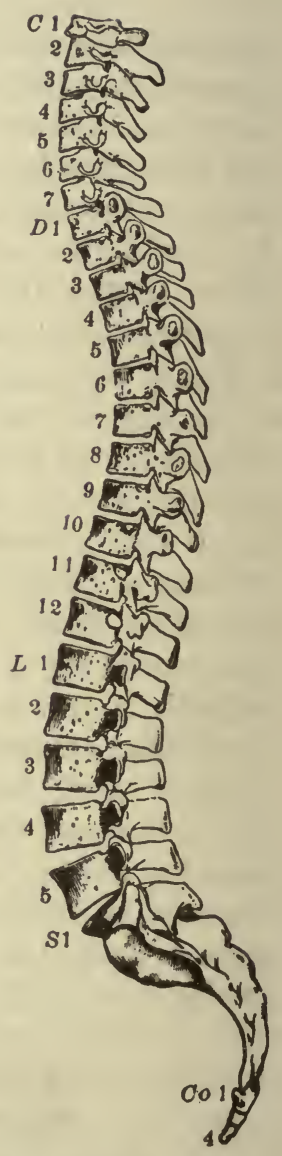

Frg. 15.-Side view of the spinal column. $C$ 1-7, cervical; $D$ 1-12, dorsal; $L 1-5$, lumbar; $S 1$, sacrum; Co 1-4, coccygeal. 
Body were joined together like the joists and beams of a house, the whole mass would be rigid; its parts could not move with relation to one another, and we should be unable to raise a hand to the mouth or put one foot before another. To allow of mobility the bony skeleton is made of many separate pieces which are joined together, the points of union being called articulations, and at many places the bones entering into an articulation are movably hinged together, forming what are known as joints. 'The total number of bones in the Body is more than two hundred in the adult; and the number in children is still greater, for various bones which are distinct in the child (and remain distinct throughout life in many lower animals) grow together so as to form one bone in the full-grown man. The adult bony skeleton may be described as consisting of an axial skeleton, found in the head, neck, and trunk; and an appendicular skeleton, consisting of the bones in the limbs and in the arches ( $u$ and $s$, Fig. 14) by which these are carried and attached to the trunk.

Axial Skeleton. The axial skeleton is made up of the vertebral column or spine, a side view of which is given in Fig. 15; the skull, Fig. 25; the sternum, Fig. 28; and the ribs, Fig. 29.

The vertebral column is the great supporting center for the whole skeleton and consists of 33 bones grouped as follows from above downward: 7 cervical, 12 dorsal or thoracic, 5 lumbar, 5 sacral, in the adult united into a single bone, the sacrum, and 4 coccygeal, or rudimentary tail bones.

The vertebral column occupies the mid-dorsal line of the trunk. On top of it is borne the skull (22 bones) made up of two parts; a great box above, composed of 8 bones, which incloses the brain and is called the cranium; and a group of 14 bones on the ventral side of this which form the skeleton of the face. Attached by ligaments to the underside of the cranium is the hyoid bone, to which the root of the tongue is fixed. There are 12 pairs of ribs, attached dorsally to the 12 thoracic vertebræ, one pair to each vertebra. The sternum, which occupies the mid-ventral line of the thorax and constitutes the anterior attachment for the ribs is made up of two bones, the manubrium and the body, and a cartilage, the ensiform cartilage.

Details of the Vertebral Column. The vertebral column is in a man of average height about twenty-eight inches long. Viewed 
from the side (Fig. 15) it presents four curvatures; one with the convexity forwards in the cervical region is followed, in the thoracic, by a curve with its concavity towards the chest. In the lumbar region the curve has again its convexity turned ventrally, while in the sacral and coccygeal regions the reverse is the case. These curvatures give the whole column a good deal of springiness such as would be absent were it a straight rod.

All the vertebræ are built upon the same plan, although with modifications in various parts of the column. Each consists: 1, of a stout bony body or centrum (Fig. 16, C), in shape a cylinder flattened at both ends; 2 , a bony arch, the neural arch (Fig. 16, A), attached to the dorsal side of the centrum and inclosing the neural ring (Fig. 16, Fv). The neural rings of all the vertebræ make up together a long bony tube, the neural canal, which contains the spinal cord. Between the bodies of adjoining vertebræ, except in the sacrum and coccyx, are thick pads of elastic cartilage. These permit bending movements which, while quite limited at each joint may be very considerable in the column as a whole. They also serve to take up a great deal of shock, preventing injury to the body when one sits down hard or comes down on his heels in walking or jumping. During the hours when one is on his feet these intervertebral pads are packed down by the weight of the body, and especially by the hammering effect of the movements of walking, running, etc., so that a man may be fro:n a half to threequarters of an inch shorter at night than he is in the morning. Strong ligaments fasten adjoining vertebræ together; there are also muscles passing from vertebræ to vertebræ, which by their contractions assist in bending the body. These muscles are arranged in antagonistic groups; that is, they are so placed that whenever the vertebral column is bent through the contraction of one group the muscles of the antagonistic group are put on the stretch. The neural arch of each vertebra bears a dorsal spinous process (Fig. 16, Ps), and a pair of lateral transverse processes (Fig. 16, Pt). These serve various purposes; the intervertebral muscles are attached to them; they also bear articular surfaces (Pas and Pai, Figs. 16 and 17) which sliding upon corresponding surfaces of adjoining vertebræ serve to limit the movements at each joint, and also help to prevent dislocation of the vertebral column. The spinous processes may be felt in the middle of the 
back. The neural arches are notched (Fig. 17, Is and $\mathrm{Fi}$ ), adjoining notches forming rounded openings through which the spinal nerves pass on their way out from the spinal cord.

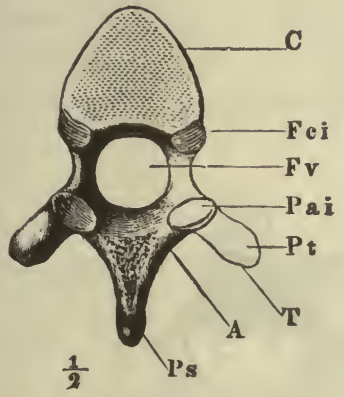

FIG. 16.

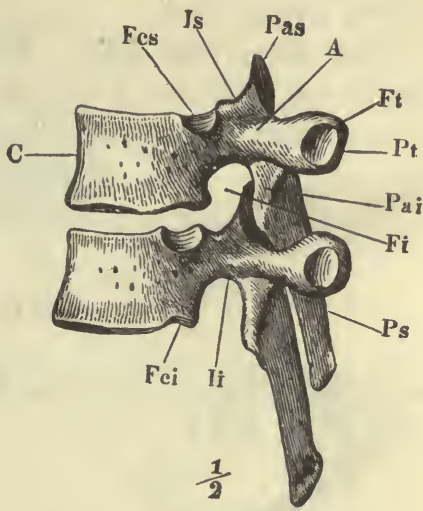

Fig. 17.

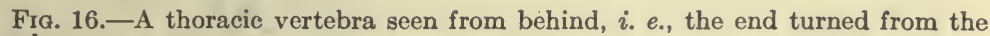
head.

Fig. 17. - Two thoracic vertebræ viewed from the left side, and in their natural relative positions. $C$, the body; $A$, neural arch; $P s$, spinous process; $P a s$, anterior articular process; $P a i$, posterior articular process; $P t$, transverse process; $F t$, facet for articulation with the tubercle of a rib; Fcs, $F c i$, articular surfaces on the centrum for articulation with a rib.

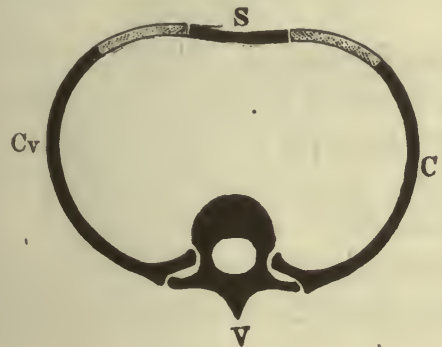

FIg. 18.

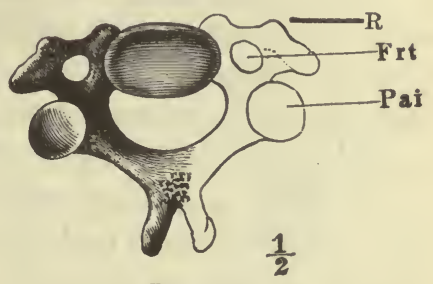

FIg. 19.

Fig. 18.-Diagrammatic representation of a segment of the axial skeleton $V$, a vertebra; $C, C v$, ribs articulating above with the body and transverse process of the vertebra; $S$, the breast-bone. 'The lighter-shaded part between $S$ and $C$ is the costal cartilage.

Fig. 19. - A cervical vertebra. Frt, vertebral foramen; Pai, anterior articular process; $R$, rudimentary rib.

The Cervical Vertebræ (Fig. 19), have rather small bodies and large neural arches; in some of them the spinous process is bifid. They move more freely upon each other than do the vertebræ lower down. A rudimentary rib ( $R$, Fig. 19) becomes united 
early in life to the ventral surface of each transverse process; the foramina (Fig. 19, Frt) thus formed give passage to an important artery which ultimately passes into the cranial cavity to carry blood to the brain.

The Atlas and Axis. The first and second cervical vertebræ differ considerably from the rest. The first, or atlas (Fig. 20), which carries the head, has a very small body, $A a$, and a large neural ring. This ring is subdivided by a cord, the transverse ligament, $L$, into a dorsal moiety in which the spinal cord lies and

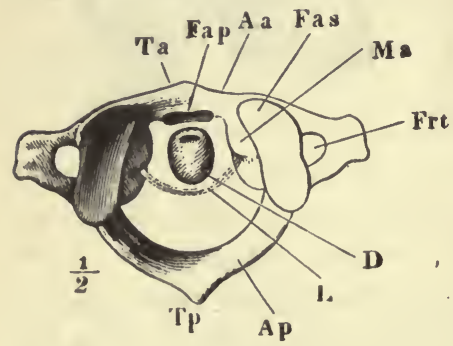

Fig 20.

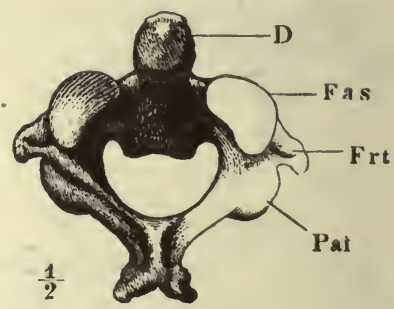

Fig 21.

Fig. 20.-The atlas. Fig. 21.-The axis. Aa, body of atlas; $D$, odontoid process; Fas, facet on front of atlas with which the skull articulates; arid in Fig. 21 anterior articular surface of axis; $L$, transverse ligament; Frt, vertebral foramen; $A p$, neural arch; $T p$, spinous process.

a ventral into which the bony process $D$ projects. This is the odontoid process, and arises from the front of the axis or second cervical vertebra (Fig. 21). Around this peg the atlas rotates when the head is turned from side to side, carrying the skull (which articulates with the large hollow surfaces $F a s$ ) with it.

The odontoid process really represents a large piece of the body of the atlas which in early life separates from its own vertebra and becomes united to the axis.

The Thoracic Vertebræ have larger bodies and longer processes than do the cervical vertebræ. They are specially modified for carrying the ribs. Each rib is attached at two points (Fig. 18). The head of the rib fits into an articulation at the junction of two vertebræ, a part of the articular surface being on the centrum of one and a part on the other (Fig. 17, Fcs and Fci). The second attachment is between a point on the neck of the rib and an articular surface at the end of the transverse process of the posterior of the two vertebræ which the rib touches (Fig. 17, $F t$ ). 
The Lumbar Vertebræ (Fig. 22) are the largest of all the movable vertebre and have no ribs attached to them. Their spines are short and stout and lie in a more horizontal plane than those of

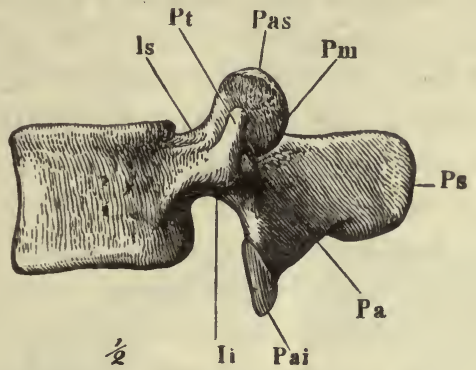

Fig. 22.-A lumbar vertebra, seen from the left side. $P s$, spinous process: $P a s$, anterior articular process; $P a i$, posterior articular process; $P t$, transverse process.

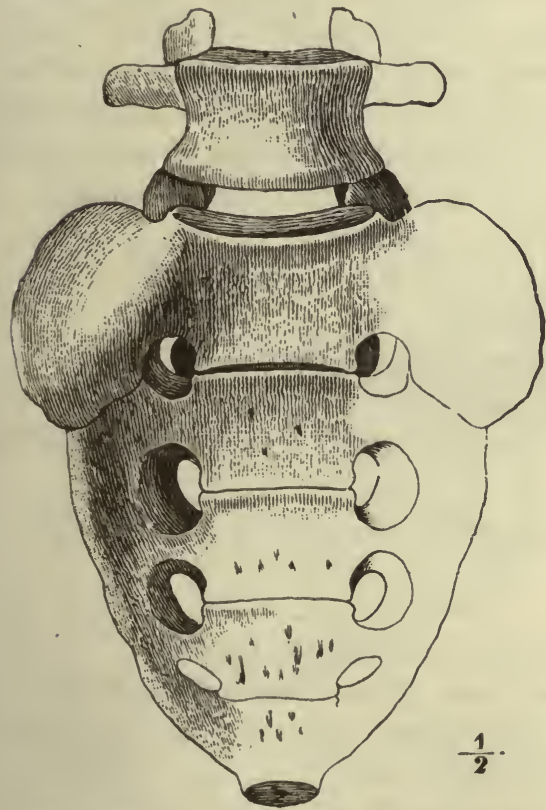

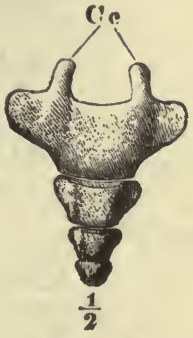

FIG. 24.

The coccyx.

FIG. 23.-The last lumbar vertebra and the sacrum seen from the ventral side. the vertebræ in front. The articular and transverse processes are also short and stout.

The Sacrum, which is represented along with the last lumbar 
vertebra in Fig. 23, consists in the adult of a single bone; but crossridges on its ventral surface indicate the limits of the five separate vertebræ of which it is composed in childhood. It is somewhat triangular in form, its base being directed upwards and articulating with the under surface of the body of the fifth lumbar vertebra. On its sides are large surfaces to which the arch bearing the lower limbs is attached (see Fig. 14). Its ventral surface is concave and smooth and presents four pairs of anterior sacral foramina, which communicate with the neural canal. Its dorsal surface, convex and roughened, has four similar pairs of posterior sacral foramina.

The $\operatorname{coccyx}$ (Fig. 24) calls for no special description. The four bones which grow together, or ankylose, to form it, represent only the bodies of vertebræ, and even those incompletely.

Details of the Skull. An account of the bones which make up the skull can conveniently be given in tabular form. Examination of the table will show that all the bones are either single or paired. Single bones are all median, paired bones occupy corresponding positions on each side of the mid-line. Figs. 25 and 26 will enable the reader to gain a fairly good notion of the form and relations of individual bones; for greater detail works on anatomy should be consulted. Cranium:

1 Frontal, forehead (Fig. 25, $F$ ).

2 Parietal, crown (Fig. 25, $P r$ ).

1 Occipital, base of skull (Fig. 25, 0 ).

2 Temporal, ear region (Fig. 25, T).

1 Sphenoid, base of cranium and back of orbit (Fig. 25, S.)

1 Ethmoid, between cavities of cranium and nose (Fig. $25, E)$.

Face:

1 Inferior maxilla, lower jaw (Fig. 25, $M d$ ).

2 Maxillæ, upper jaw, front of hard palate (Fig. 25, $M x$ ).

2 Palatine, back of hard palate, front of posterior nares

(Fig. 26, $P t$ ).

2 Nasal, bridge of nose (Fig. 25, $N$ ).

1 Vomer, partition between nostrils (Fig. 26, V).

2 Inferior turbinate, inside nostrils (not shown in Fig.).

2 Malar, cheeks (Fig. 25, Z).

2 Lachrymal, inside wall of orbit (Fig. 25, L). 
All these bones except the inferior maxilla are immovably joined together in the adult by irregular, saw-tooth like articulations. The inferior maxilla articulates with the temporal bones in such a way as to permit not only rotation about the points of articulation but also a certain amount of sliding from side to side and from back to front, thus making the grinding movements of chewing.

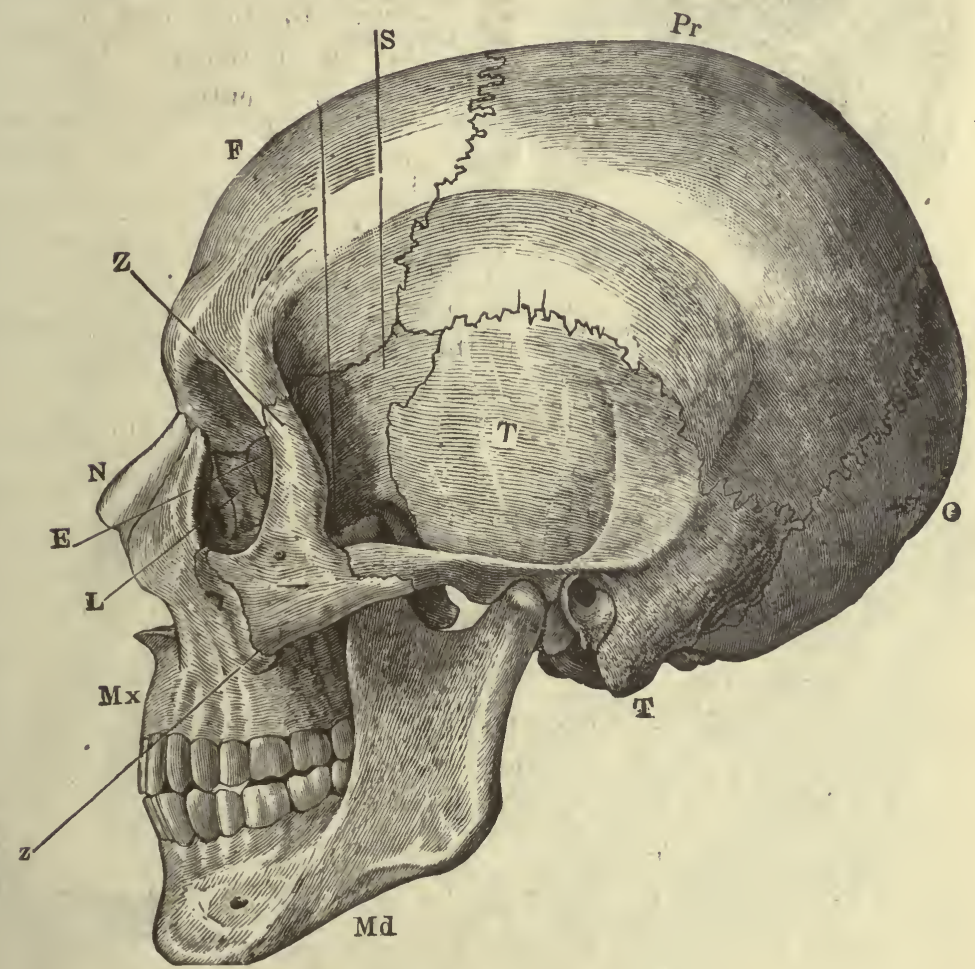

Fig. 25.-A side view of the skull. $O$, occipital bone; $T$, temporal; $\operatorname{Pr}$, parietal; $F$, frontal; $S$, sphenoid; $Z$, malar; $M x$, maxilla; $N$, nasal; $E$, ethmoid; $L$, lachrymal; $M d$, inferior maxilla.

There are several features of the skull which call for special comment. The foramen magnum (Fig. 26) is a large opening into the cranial cavity through the occipital bone; through it the spinal cord passes on its way to the brain. On each side of the 
foramen magnum is an occipital condyle (Fig. 26, oc). These are the points at which the skull rests upon the atlas. The orbits

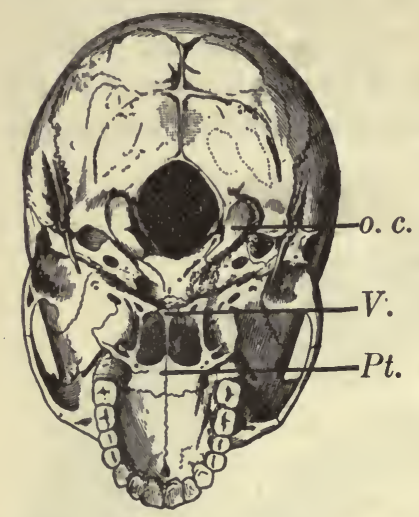

Fig. 26.-The base of the skull. The lower jaw has been removed. At the lower part of the figure is the hard palate forming the roof of the mouth and surrounded by the upper set of teeth. Above this are the paired openings of the posterior nares, and a short way above the middle of the figure is the large median foramen magnum, with the bony convexities (or occipital condyles) o. c., which articulate with the atlas, on its sides; $V$, the vonier; $P t$, the palatines.

of the throat, just above or eye. sockets are outlined in front by the frontal, malars, and maxillæ. The space behind the orbit, between the malar and temporal bones, is occupied by a large muscle which closes the jaw. The shape of the face depends very largely upon the malar bones. The anterior nares, or openings of the nostrils are bounded by the maxillæ and nasals. The posterior nares, by. which the nose communicates with the throat cavity, lie behind the palate bones (Fig. 23). Enlargements of the temporal bones contain the auditory apparatus.

The Hyoid. Besides the cranial and facial bones there is, as already pointed out, one other, the hyoid (Fig. 27), which really belongs to the skull, although it lies in the neck. It can be felt in the front "Adam's apple." The hyoid bone is U-shaped, with its convexity turned ventrally, and consists of a body and two pairs of processes called cornua. The smaller cornua (Fig. 27, 3) are attached to the base of the skull by long ligaments. The bone serves as an attachment for the base of the tongue. The hyoid is of much interest from the standpoint of comparative anatomy because in the very young Human Body it is a part of a structure which corresponds to the gill mechanism

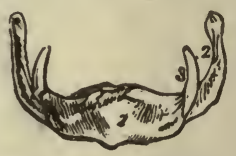

Fig. 27.-The hyoid bone. 1, body; 2 , great cornua; 3 , small cornua. of fish, tadpoles, and similar aquatic animals, consisting of several gill arches with gill clefts between them. In the human embryo the gill clefts close before birth, and all the gill arches disappear except those which persist as the hyoid. It is difficult to explain the development and subsequent disappearance of this structur: 
in the embryo except upon the theory which is part of the doctrine of evolution that each individual epitomizes in his own develop-

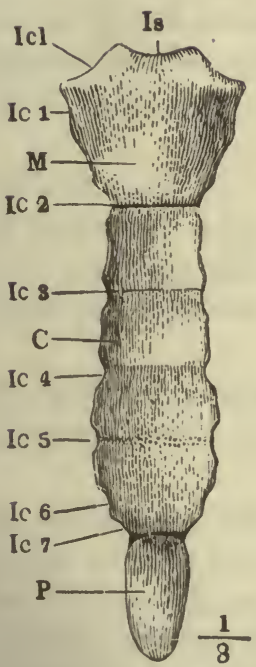

FIG. 28.

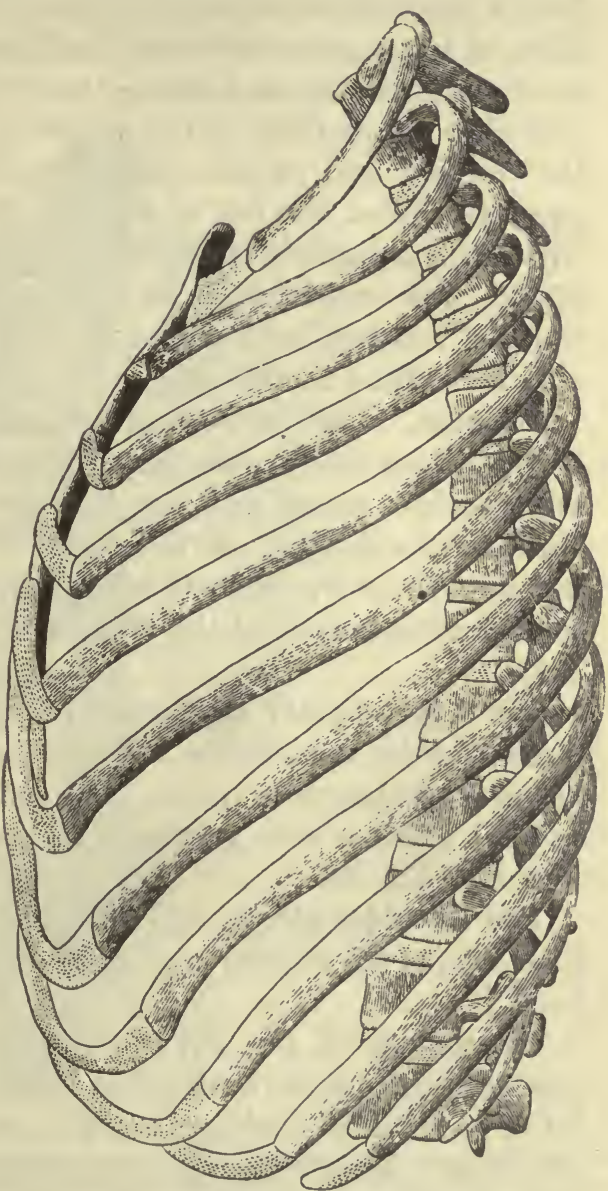

Fig. 29.

Fif, 28. - The sternum seen on its ventral aspect. $M$, manubrium; $C$, body; $P$, ensiform cartilage; Icl, notch for the collar-bone; Ic 1-7, notche's for the ribcartilages.

Fitr. 29.-The ribs of the left side, with the dorsal and two lumbar vertebræ, the rib-cartilages and the sternum.

mental history the evolutionary history of the race to which he belongs.

The Ribs (Fig. 29). There are twelve pairs of ribs, each being 
a slender curved bone attached dorsally to the body and transverse process of a vertebra in the manner already mentioned, and continued ventrally by a costal cartilage (Fig. 18). In the case of the anterior seven pairs, the costal cartilages are attached directly to the sides of the breast-bone; the next three cartilages are each attached to the cartilage of the preceding rib, while the cartilages of the eleventh and twelfth ribs are quite unattached ventrally, so these are called the free or floating ribs. The convexity of each curved rib is turned outwards so as to give roundness to the sides of the chest and increase its cavity, and each slopes downwards from its vertebral attachment, so that its sternal end is considerably lower than its dorsal.

Sternum. The sternum or breast-bone (Fig. 28 and Fig. 14) is wider from side to side than dorsoventrally. It consists in the adult of three pieces, and seen from the ventral side has somewhat the form of a dagger. At the upper end are notches for the articulations of the collar-bones (Fig. 28, Icl), and along each side notches for the articulations of the anterior costal cartilages (Fig. 28, Ic, 1-7).

The Appendicular Skeleton. This consists of the shouldergirdle and the bones of the fore limbs, and the pelvic girdle and the bones of the posterior limbs. The two supporting girdles in their natural position with reference to the trunk skeleton are represented in Fig. 30.

The Shoulder-girdle, or Pectoral Arch. This is made up, on each side, of the scapula or shoulder-blade, and the clavicle or collarbone.

The scapula ( $S$, Fig. 30) is a flattish triangular bone which can readily be felt on the back of the thorax. It is not directly articulated to the axial skeleton, but lies embedded in the muscles and other parts outside the ribs on each side of the vertebral column. From its dorsal side arises a crest to which the outer end of the collar-bone is fixed, and on its outer edge is a shallow cup into - which the top of the arm-bone fits: this hollow is known as the glenoid fossa.

The collar-bone ( $C$, Fig. 30$)$ is cylindrical and attached at its inner end to the sternum as shown in the figure. fitting into the notch represented at $I c l$ in Fig. 28.

The Pelvic Girdle (Fig. 30). This consists of a large bone, the 
os innominatum, Oc, on each side, which is firmly fixed dorsally to the sacrum and meets its fellow in the middle ventral line. In the child each os innominatum consists of three bones, viz., the ilium, the ischium, and pubis. Where these three bones meet and finally ankylose there is a deep socket, the acetabulum, into which the

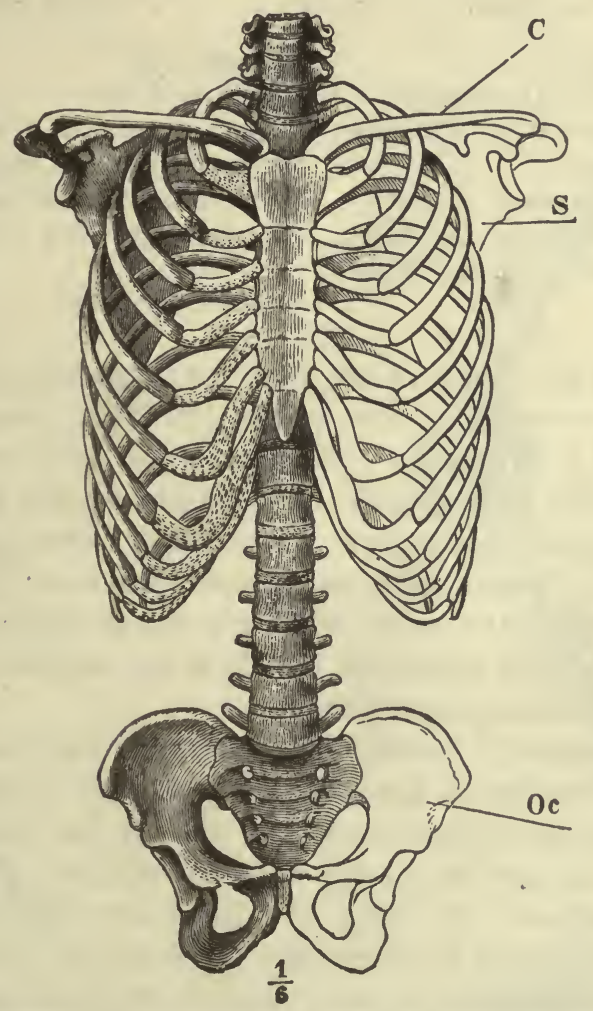

FIG. 30.-The skeleton of the trunk and the limb arches seen from the front. $C$, clavicle; $S$, scapula; $O c$, innominate bone attached to the side of the sacrum dorsally and meeting its fellow at the pubic symphysis in the ventral median line.

head of the thigh-bone fits (see Fig. 14). Between the pubic and ischial bones is the largest foramen in the whole skeleton, known as the doorlike or thyroid foramen. The pubic bone lies above and the ischial below it. The ilium forms the upper expanded portion of the os innominatum to which the line drawn from Oc in Fig. 30 points. 
Fore and Hind Limbs. Each of these contains thirty bones, and their arrangement is very similar. This is clearly seen in the figures (31 and 32), and is also brought out in the following table in which the bones of the extremities are enumerated.

\section{Fore Limb}

$a$. Humerus, upper arm.

b. Ulna, large bone of forearm.

c. Radius, smaller bone of forearm.

d. 8 carpals, wrist.

e. 5 metacarpals, hand.

f. 14 phalanges, fingers and thumb. ( 2 in thumb, 3 in each finger). $g$.

\section{Hind Limb}

Femur, thigh.

Tibia, shin bone.

Fibula, small bone of calf.

7 tarsals, heel and upper instep.

5 metatarsals, lower instep.

14 phalanges, toes ( 2 in great toe, 3 in others).

Patella, knee-cap.

In general the bones of the hind limb are larger and stronger than the corresponding ones of the fore limb; the femur is the longest bone in the body. The phalanges, however, are smaller in the foot than in the hand. The tarsals are one less in number than the carpals because one of the tarsal bones, the astragalus (Fig. 35, Ta), is composed of two bones which have united into one. A structure of the arm corresponding to the patella is the olecranon process of the ulna which can be felt at the back of the elbow; in early life this is a separate bone.

The differences in structure between fore and hind limb correspond to differences of function; the fore limb being a prehensile organ is capable of great freedom of motion; the hind limb, which is a supporting and locomotor organ, is adapted rather to maintain the weight of the body and to execute the movements of walking and running to advantage. The special adaptation of the arm to its purpose is seen particularly in three things: 1 , the comparatively flexible attachment of the pectoral girdle to the axial skeleton (Fig. 33), an attachment composed wholly of muscle and ligament except where the inner ends of the clavicles articulate with the sternum; 2 , the rotation of the radius over the ulna, an arrangement which increases very greatly the flexibility of the hand; 3 , the articulation of the thumb, which is of such a sort as to allow it to be opposed to any of the fingers, thus enabling the hand to manipulate small objects without difficulty. The leg, on the other hand, is characterized by much greater firmness, which 
is obtained at the expense of flexibility. The pelvic arch (Figs. 30 and 34) is not only heavy and strong, but is very firmly fixed to the axial skeleton, the sacrum and os innominatum becoming in mature life practically one bone. The socket into which the head

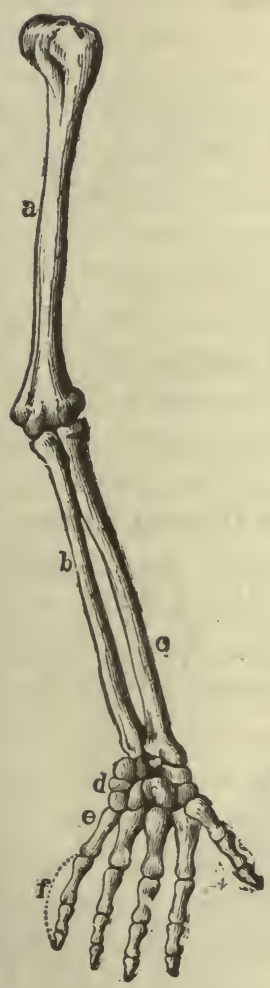

Fig. 31.

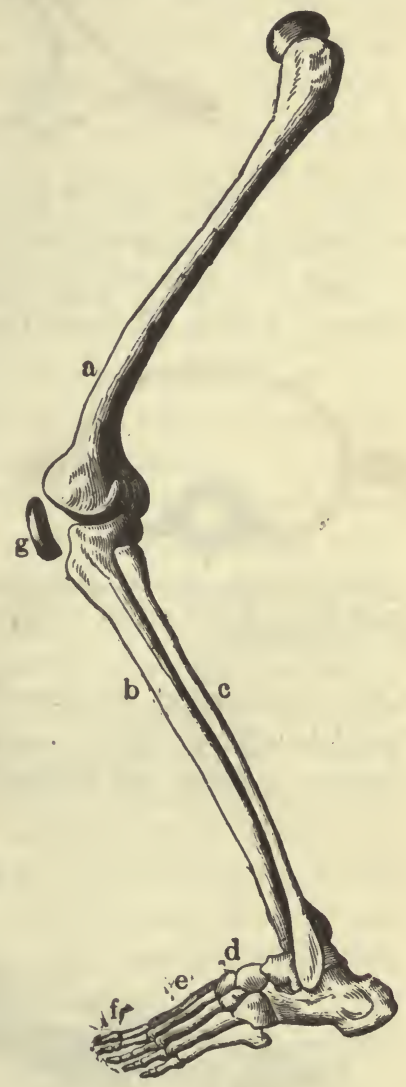

FIG. 32.

Fia. 31. -The bones of the arm. $a$, humerus; $b$, ulna; $c$, radius; $d$, the carpus; $e$, the fifth metacarpal; $f$, the three phalanges of the fifth digit (little finger).

Frg. 32.-Bones ol the leg. $a$, femur; $b$, tibia; $c$, fibula; $d$, tarsal bones; $e$, metatarsal bones; $f$, phalanges; $g$, patella.

of the femur fits is much deeper than that which receives the head of the humerus, rendering the leg much less liable to dislocation than the arm, but at the same time restricting its movements much more. The foot also in becoming adapted to form a 
support for the body has sacrificed its prehensile structure almost altogether; the toes are less flexible than the fingers and the great

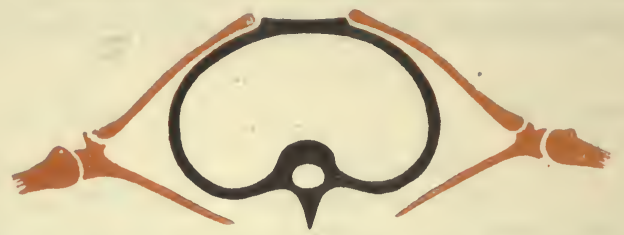

Fig. 33.-Diagram showing the relation of the pectoral arch to the axial skeleton. toe cannot be opposed to the others. A special modification of the foot for its particular function is seen in the arching of the instep. As Fig. 35 shows the bones of the foot form a springy arch,

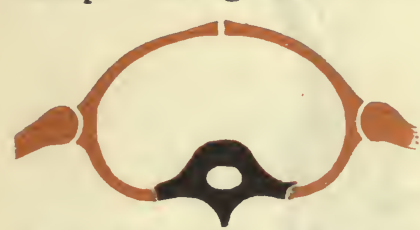

Fig. 34.-Diagram showing the attachment of the pelvic arch to the axial skeleton. the points of contact with the ground being at the extremity of the heel bone (os calcis, $C a$ of figure), and the distal ends of the metatarsals. The bones of the leg are mounted upon the crown of the arch ( $T a$ of figure).

Peculiarities of the Human Skeleton. These are largely connected with the division of labor between the fore and hind limbs referred to above, which is carried farther in man than in any other creature. Even the highest apes frequently use their fore limbs

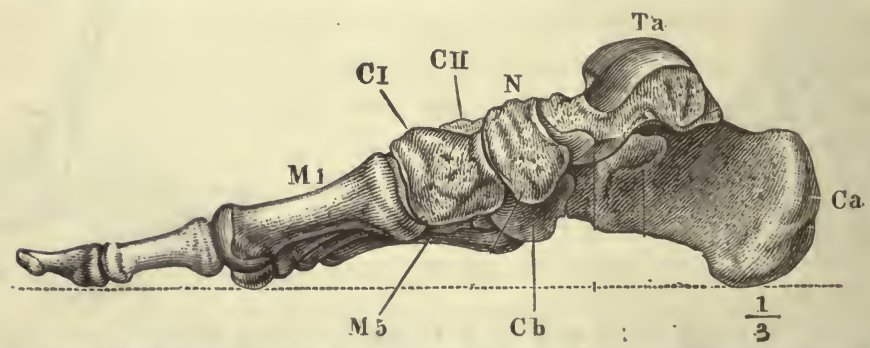

Fig. 35.-The bones of the foot. Ca, calcaneum, or os calcis; Ta, articular surface for tibia on the astragalus; $N$, scaphoid bone; $C I, C I I$, first and second cuneiform bones; $C b$, cuboid bone; $M 1$, metatarsal bone of great toe.

in locomotion and their hind limbs in prehension, and we find accordingly that anatomically they present less differentiation of hand and foot. The other more important characteristics of the human skeleton are correlated for the most part with the mainte- 
nance of the erect posture, which is more complete and habitual in man than in the animals most closely allied to him anatomically. These peculiarities, however, only appear fully in the adult. In the infant the head is proportionately larger, which gives the center of gravity of the Body a comparatively very high position and renders the maintenance of the erect posture difficult and insecure. The curves of the vertebral column are nearly absent, and the posterior limbs are relatively very short. In all these points the infant approaches more closely than the adult to the ape. The subsequent great relative length of the posterior limbs, which grow disproportionately fast in childhood as compared with the anterior, makes progression on them more rapid by giving a longer stride and at the same time makes it almost impossible to go on "all fours" except by crawling on the hands and knees. In other Primates this disproportion between the anterior and posterior limbs does not occur to nearly the same extent.

In man the skull is nearly balanced on the top of the vertebral column, the occipital condyles which articulate with the atlas being about its middle (Fig. 25), so that but little effort is needed to keep the head erect. In four-footed beasts, on the contrary, the skull is carried on the front end of the horizontal vertebral column and needs special ligaments to sustain it. For instance, in the ox and sheep there is a great elastic cord running from the cervical vertebræ to the back of the skull and helping to hold up the head. Even in the highest apes the skull does not balance on the top of the spinal column; the face part is much heavier than the back, while in man the face parts are relatively smaller and the cranium larger, so that the two nearly equipoise. To keep the head erect and look things straight in the face, "like a man," is for the apes far more fatiguing, and so they cannot long maintain that position.

The human spinal column, gradually widening from the neck to the sacrum, is well fitted to sustain the weight of the head, upper limbs, etc., carried by it; and its curvatures, which are peculiarly human, give it considerable elasticity combined with strength. The pelvis, to the sides of which the lower limbs are attached, is proportionately very broad in man, so that the balance can be more readily maintained during lateral bending of the trunk. The arched instep and broad sole of the human foot are also very characteristic. The majority of four-footed beasts, as horses. 
walk on the tips of their toes and fingers; and those animals, as bears and apes, which like man place the tarsus also on the ground, or in technical language are plantigrade, have a much less marked arch there. The vaulted human tarsus, composed of a number of small bones, each of which can glide a little over its neighbors, but none of which can move much, is admirably calculated to break any jar which might be transmitted to the spinal column by the contact of the sole with the ground at each step. A well-arched instep is therefore rightly considered a beauty; it makes progression easier, and by its springiness gives elasticity to the step. In London flat-footed candidates for appointment as policemen are rejected, as they cannot stand the fatigue of walking the daily "beat."

Hygiene of the Bony Skeleton. In early life the bones are less rigid, from the fact that the earthy matters then present in them bear a less proportion to the softer organic parts. Hence the bones of an aged person are more brittle and easily broken than those of a child. The bones of a young child are in fact tolerably flexible and may be distorted by any continued strain; therefore children should never be kept sitting for hours, in school or elsewhere, on a bench which is so high that the feet are not supported. If this be insisted upon (for no child will continue it voluntarily) the thighbones will almost certainly be bent over the edge of the seat by the weight of the legs and feet, and a permanent distortion may be produced. For the same reason it is important that a child be made to sit straight while writing, to avoid the risk of producing a lateral curvature of the spinal column. The facility with which the bones may be molded by prolonged pressure in early life is well seen in the distortion of the feet of the Chinese ladies of the old regime, produced by keeping them in tight shoes; and in the extraordinary forms which some races of man produce in their skulls, by tying boards on the heads of the children.

Throughout the whole of life, moreover, the bones remain among the most easily modified parts of the Body; although judging from the fact that dead bones are the most permanent parts of fossil animals we might be inclined to think otherwise. The living bone, however, is constantly undergoing changes under the influence of the protoplasmic cells embedded in it, and in the living Body is constantly being absorbed and reconstructed. The experience of 
physicians shows that any continued pressure, such as that of a tumor, will cause the absorption and disappearance of bone almost quicker than that of any other tissue; and the same is true of any other continued pressure. Moreover, during life the bones are eminently plastic; under abnormal pressures they are found to assume abnormal shapes quickly, being absorbed and disappearing at points where the pressure is most powerful, and increasing at other points; tight lacing may in this way produce a permanent distortion of the ribs.

When a bone is fractured a surgeon should be called in as soon as possible, for once inflammation has set in and the parts have become swollen it is much more difficult to place the broken ends of the bone together in their proper position than before this has occurred. Once the bones are replaced they must be held in position by splints or bandages, or the muscles attached to them will soon displace them again. With rest, in young and healthy persons complete union will commonly occur in three or four weeks; but in old persons the process of healing is slower and is apt to be imperfect.

Articulations. The bones of the skeleton are joined together in very various ways; sometimes so as to admit of no movement at all between them; in other cases so as to permit only a limited range or variety of movement; and elsewhere so as to allow of very free movement in many directions. All kinds of unions between bones are called articulations.

Of articulations permitting no movements, those which unite the majority of the cranial bones afford a good example. Except the lower jaw, and certain tiny bones inside the temporal bone belonging to the organ of hearing, all the skull-bones are immovably. joined together. This union in most cases occurs by means of toothed edges which fit into one another and form jagged lines of union known as sutures. Some of these can be well seen in Fig. 25 between the frontal and parietal bones (coronal suture) and between the parietal and occipital bones (lambdoidal suture); while another lies along the middle line in the top of the crown between the two parietal bones, and is known as the sagittal suture. In newborn children where the sagittal meets the coronal and lambdoidal sutures there are large spaces not yet covered in by the neighboring bones, which subsequently extend over them. These openings 
are known as fontanelles. At them a pulsation ean often be felt synchronous with each beat of the heart, which, driving more blood into the brain, distends it and causes it to push out the skin where bone is absent. Another good example of an articulation admitting of no movement is that between the rough surfaces on the sides of the sacrum and the innominate bones.

We find good examples of the second class of articulationsthose admitting of a slight amount of movement-in the vertebral column. Between every pair of vertebræ from the second cervical to the sacrum is an elastic pad, the intervertebral disk, which adheres by its surfaces to the bodies of the vertebræ between which it lies, and only permits so much movement between them as can be brought about by its own compression or stretching. When the back-bone is curved to the right, for instance, each of the intervertebral disks is compressed on its right side and stretched a little on its left, and this combination of movements, each individually but slight, gives considerable flexibility to the spinal column as a whole.

Joints. Articulations permitting of movement by the gliding of one bone over another are known as joints, and all have the same fundamental structure, although the amount of movement permitted in different joints is very different.

Joint Motions. The wide variety of motions possible to the body group themselves within a small number of classes: flexion, the bending of a joint as at elbow or knee; extension, the straightening of a joint, the opposite of flexion; abduction, the movement of a part away from the axis of the body, as in moving the arm out to the side at right angles, or the thumb and fore finger in spreading the hand; adduction, the opposite of abduction; rotation, the rolling movement seen when the hand is turned from the palm up to the palm down position, or when one ankle is placed on the opposite knee. There are a few movements, such as the sliding of the jaw from side to side in chewing, that do not fall in any of these classes, but the great majority of joint motions belong either to one of these groups or are combinations of two or more. Thus flexion and abduction of the hip can occur together, or extension and rotation.

Hip-joint. We may take this as a good example of a true joint permitting a great amount and variety of movement. On the 
os innominatum is the cavity of the acetabulum (Fig. 36), which is lined inside by a thin layer of articular cartilage which has an estremely smooth surface. The bony cup is also deepened a little by a cartilaginous rim. The proximal end of the femur consists of a nearly spherical smooth head, borne on a somewhat narrower neck, and fitting into the acetabulum. This head also is covered with articular cartilage; and it rolls in the acetabulum like a ball in a socket. To keep the bones together and limit the amount of move-

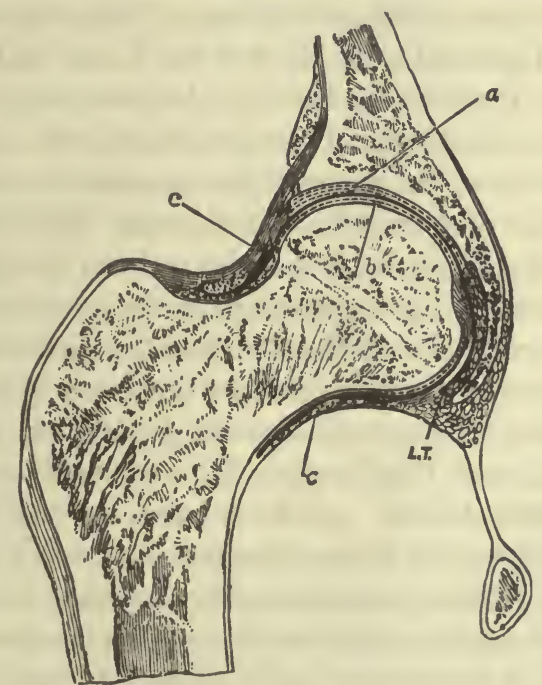

Fig. 36.-Section through the hip-joint. $a$ and $b$, articular cartilages; $c$, capsular ligament.

ment, ligaments pass from one to the other. These are composed of white fibrous connective tissue (Chap. IV) and are extremely pliable, but quite inextensible and very strong and tough. One is the capsular ligament, which forms a sort of loose bag all around the joint, and another is the round ligament, which passes from the acetabulum to the head of the femur. Should the latter rotate above a certain extent in its socket, the round ligament and one side of the capsular ligament are put on the stretch, and any further movement which might dislocate the femur (that is, remove the head from its socket) is checked. Covering the inside of the capsular ligament and the outside of the round ligament is a layer of flat cells, which are continued in a modified form over the ar- 
ticular cartilages and form the synovial membrane. This, which thus forms the lining of the joint, is always moistened in health by a small quantity of glairy synovial fluid, something like the white of a raw egg in consistency, and playing the part of the oil with which the contiguous moving surfaces of a machine are moistened; it makes all run smoothly with very little friction.

In the natural state of the parts, the head of the femur and the bottom and sides of the acetabulum lie in close contact, the two synovial membranes rubbing together. This contact is not maintained by the ligaments, which are too loose and serve only to check excessive movement, but by the numerous stout muscles which pass from the thigh to the trunk and bind the two firmly together. Moreover, the atmospheric pressure exerted on the surface of the Body and transmitted through the soft parts to the outside of the air-tight joint helps also to keep the parts in contact. If all the muscles and ligaments around the joint be cut away, it is still found in the dead Body that the head of the femur will be kept in its socket by this pressure, and so firmly as to bear the weight of the whole limb without dislocation, just as the pressure of the air will enable a boy's "sucker" to lift a tolerably heavy stone.

Ball-and-socket Joints. Such a joint as that at the hip is called a ball-and-socket joint and allows of more free movement than any other. Through movements occurring in it the thigh can be flexed, or bent so that the knee approaches the chest; or extended, that is, moved in the opposite direction. It can be abducted, so that the knee moves outwards; and adducted, or moved back towards the other knee again. The limb can also by movements at the hip-joint be made to describe a cone of which the base is at the foot and the apex at the hip. Finally, rotation can occur in the joint, so that with knee and foot joints held rigid the toes can be turned in or out, to a certain extent, by a rolling around of the femur in its socket.

At the junction of the humerus with the scapula is another balland-socket joint permitting all the above movements to even a greater extent. This greater range of motion at the shoulder-joint depends mainly on the shallowness of the glenoid cavity as compared with the acetabulum, and upon the absence of any ligament answering to the round ligament of the hip-joint. Another ball-and-socket joint exists between the carpus and the metacarpal 
bone of the thumb; and others with the same variety, but a much less range, of movement between each of the remaining metacarpal bones and the proximal phalanx of the finger which articulates with it.

Hinge-joints. Another form of synovial joint is known as a hinge-joint. In it the articulating bony surfaces are of such shape as to permit of movement, to and fro, in one plane only, like a door on its hinges. The joints between the phalanges of the fingers are good examples of hinge-joints. If no movement be allowed where the finger joins the palm of the hand it will be found that each can be bent and straightened at its own two joints, but not moved in any other way. The knee is also a hinge-joint, as is the articulation between the lower jaw and the base of the skull which allows us to open and close our mouths. The latter is, however, not a perfect hinge-joint, since it permits of a small amount of lateral movement such as occurs in chewing, and also of a gliding movement by which the lower jaw can be thrust forward so as to protrude the chin and bring the lower row of teeth outside the upper.

Pivot-joints. In this form one bone rotates around another which remains stationary. We have a good example of it between the first and second cervical vertebræ. The first cervical vertebra or atlas (Fig. 20) has a very small body and a very large arch, and its neural canal is subdivided by a transverse ligament ( $L$, Fig. 20 ) into a dorsal and a ventral portion; in the former the spinal cord lies. The second vertebra or axis (Fig. 21) has arising from its body the stout bony peg, $D$, called the odontoid process. This projects into the ventral portion of the space surrounded by the atlas, and, kept in place there by the transverse ligament, forms a pivot around which the atlas, carrying the skull with it, rotates when we turn the head from side to side. The joints on each side between the atlas and the skull are hinge-joints and permit only the movements of nodding and raising the head. When the head is leaned over to one side, the cervical part of the spinal column is bent.

Another kind of pivot-joint is seen in the forearm. If the limb be held straight out, with the palm up and the elbow resting on the table, so that the shoulder-joint be kept steady while the hand is rotated until its back is turned upwards, it will be found that the radius has partly rolled round the ulna. When the palm is up- 
wards and the thumb outwards, the lower end of the radius can be felt on the outer side of the forearm just above the wrist, and if this be done while the hand is turning over, it will be easily discerned that during the movement this end of the radius, earrying the hand with it, travels around the lower end of the ulna so as to get to its inner side. The relative position of the bones when the palm is upwards is shown at $\mathrm{A}$ in Fig. 37, and when the palm is

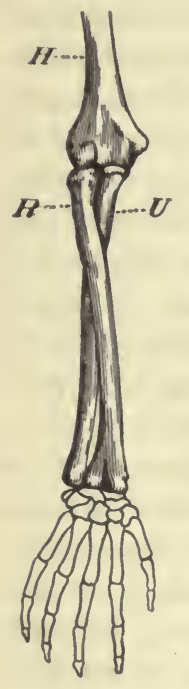

$\mathbf{B}$

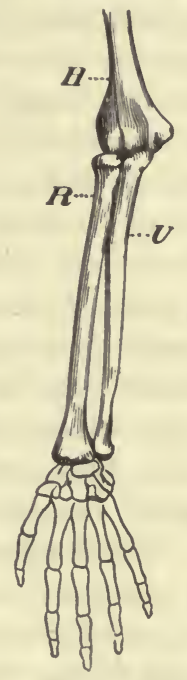

A down at $\mathrm{B}$. The former position is known as supination; the latter as pronation. The elbow end of the humerus (Fig. 37) bears a large articular surface: on the inner two-thirds of this, the ulna fits, and the ridges and grooves of both bones interlocking form a hinge-joint, allowing only of bending or straightening the forearm on the arm. The radius fits on the rounded outer third, and forms there a ball-and-socket joint at which the movement takes place when the hand is turned from the supine to the prone position; the ulna forming a fixed bar around which the lower end of the radius is moved.

FIG. 37.-A, arm in supination; $\mathrm{B}$, arm in pronation. $H$, humerus; $R$, radius; $U$, ulna.

Gliding Joints. These permit as a rule but little movement: examples are found between the closely packed bones of the tarsus and carpus (Figs. 31 and 32), which slide a little over one another when subjected to pressure.

Hygiene of the Joints. When a bone is displaced or dislocated the ligaments around the joint are more or less torn and other soft parts injured. This soon leads to inflammation and swelling which make not only the recognition of the injury but, afterdiagnosis, the replacement of the bone, or the reduction of the dislocation, difficult. Moreover, the muscles attached to it constantly pull on the displaced bone and drag it still farther out of place; so that it is of great importance that a dislocation be reduced as soon as possible: In most cases this can only be attempted with safety by one who knows the form of the bones, and possesses sufficient 
anatomical knowledge to recognize the direction of the displacement. No injury to a joint should be neglected. Inflammation once started there is often difficult to check and runs on, in a chronic way, until the synovial surfaces are destroyed, and the two bones perhaps grow together, rendering the joint permanently stiff.

Immediate and complete rest has been commonly supposed to be the only proper treatment for sprained joints, but it has been shown recently that massage, properly applied by one expert in its use, has a remarkably beneficial effect upon sprains. Injuries of this sort so severe that under the rest treatment they would require weeks for recovery yield so completely in a few days to massage treatment that the injured individual can participate in athletic contests. It should be borne in mind that massage to be effective must be applied by an expert in its use. 


\section{CHAPTER VI}

\section{THE STRUCTURE OF THE MOTOR ORGANS}

Motion in Animals. Motion is produced in animals by various sorts of motor tissues (p. 32), but in all the underlying mechanical principle is the same, namely, the forcible contraction of some element or elements. Various means of making these contractions effective exist in nature. The most familiar is that already cited (p. 37) of causing the contractile structure to pull across a movable joint. In some situations, the human stomach, for example, a hollow organ is completely surrounded by contractile tissues, whose contractions diminish, and whose relaxations permit increase of the capacity of the organ. Still another form of motion is that of the cilia previously mentioned (p. 33).

The Muscles. These are the main motor organs; their general appearance is well known to every one in the lean of butcher's meat. The majority of them being fixed to the skeleton can, by alterations in their form, bring about changes in the form and position of nearly all parts of the Body: With the skeleton and joints, they constitute preëminently the organs of motion and locomotion, and are governed by the nervous system which regulates their activity. In fact skeleton, muscles, and nervous system are correlated parts: the degree of usefulness of any one of them largely depends upon the more or less complete development of the others. Man's highly endowed senses and his powers of reflection and reason would be of little use to him, were his muscles less fitted to carry out the dictates of his will or his joints less numerous or mobile. All the muscles are under the control of the nervous system, but all are not governed by it with the coöperation of will or consciousness; some move without our having any direct knowledge of the fact. This is especially the case with certain muscles which are not fixed to the skeleton but surround cavities or tubes in the Body, as the blood-vessels and the alimentary canal, and by their movements control the passage of substances through them. The 
former group, or skeletal muscles, are also from their microscopic characters known as striped muscles, while the latter, or visceral muscles, are called unstriped or smooth muscles. The skeletal muscles being generally more or less subject to the control of the will (as for example those moving the limbs) are frequently spoken of as voluntary, and the visceral muscles, which change their form independently of the will, as involuntary. The heart muscle forms a sort of intermediate link; it is not directly attached to the skeleton, but forms a hollow bag which drives on the blood contained in it and that quite involuntarily; but in its microscopic structure it resembles somewhat the skeletal voluntary muscles. The muscles of respiration are striped skeletal muscles and, as we all know, are to a certain extent subject to the will; any one can draw a deep breath when he chooses. But in ordinary quiet breathing we are quite unconscious of their working, and even when attention is turned to them the power of control is limited; no one can voluntarily hold his breath long enough to suffocate himself. As we shall see hereafter, moreover, any one or all of the striped muscles of the Body may be thrown into activity independently of or even against the will, as, to cite no other instances, is seen in the "fidgets" of nervousness and the irrepressible trembling of extreme terror; so that the names voluntary and involuntary are not good ones, but so far as we use them they indicate no more than the general fact that the skeletal muscles are as a group responsive to the will while the smooth muscles are not.

The Skeletal Muscles. In its simplest form a skeletal muscle consists of a red soft central part, the belly, which tapers at each end and there passes into one or more dense white cords which consist almost entirely of white fibrous connective tissue. These terminal cords are called the tendons of the muscle and serve to attach it to parts of the skeleton. In Fig. 38 is shown the biceps muscle of the arm, which lies in front of the humerus. Its fleshy belly is seen to divide above and end there in two tendons, one of which, $B l$, is fixed to the scapula, while the other, $B b$, joins the tendon of a neighboring muscle (the coraco-brachial, $C b$ ), and is also fixed above to the shoulder-blade. Near the elbow-joint the muscle is continued into a single tendon, $B^{\prime}$, which is fixed to the radius, but gives an offshoot, $B^{\prime \prime}$, to the connective-tissue membranes lying around the elbow-joint. 


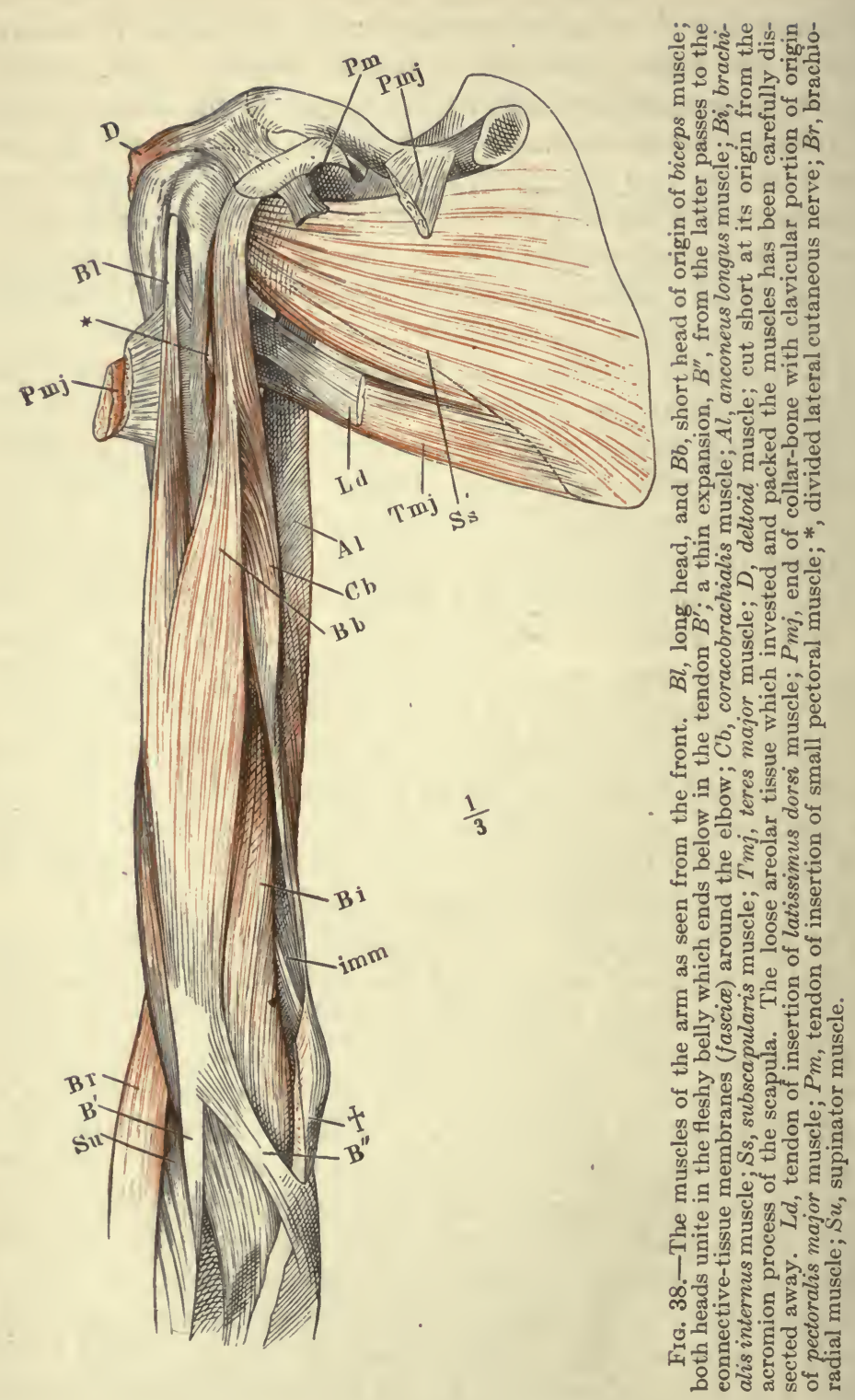


The belly of every muscle possesses the power of shortening forcibly under certain conditions. In so doing it pulls upon the tendons, which being composed of inextensible white fibrous tissue transmit the movement to the hard parts to which they are attached, just as a pull at one end of a rope may be made to act upon distant objects to which the other end is tied. The tendons are merely passive cords and are sometimes very long, as for instance in the case of the muscles of the fingers, the bellies of many of which lie away in the forearm.

If the tendons at each end of a muscle were fixed to the same bone the muscle would clearly be able to produce no movement, unless by bending or breaking the bone; the probable result in such a case would be the tearing of the muscle by its own efforts. In the Body, however, the two ends of a muscle are always attached

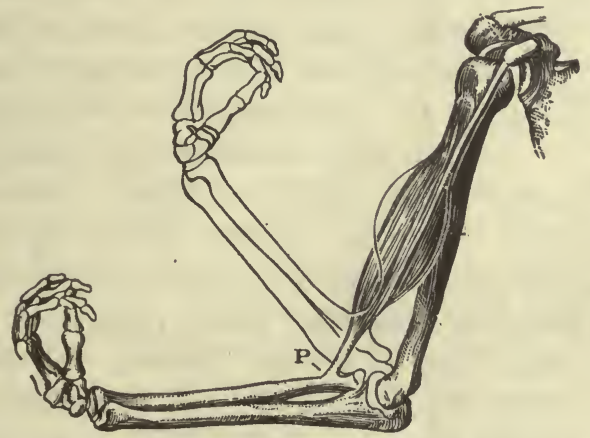

Fig. 39. - The biceps muscle and the arm-bones, to illustrate how, under ordinary circumstances, the elbow-joint is flexed when the muscle contracts.

to different parts, usually two bones, between which more or less movement is permitted, and so when the muscle pulls it alters the relative positions of the parts to which its tendons are fixed. In the great majority of cases a true joint lies between the bones on which the muscle can pull, and when the latter contracts it produces movement at the joint. Many muscles even pass over two joints and can produce movement at either, as the biceps of the arm which, fixed at one end to the scapula and at the other to the radius, can move the bones at either the shoulder or elbow-joint. Where a muscle passes over an articulation it is nearly always reduced to a narrow tendon; otherwise the bulky bellies lying around 
the joints would make them extremely clumsy and limit their mobility.

Origin and Insertion of Muscles. Almost invariably that part of the skeleton to which one end of a muscle is fixed is more easily moved than the part on which it pulls by its other tendon. The

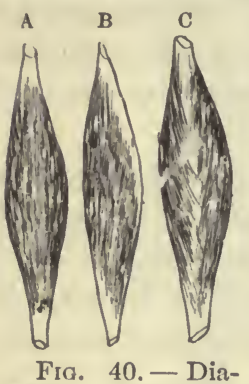

grams illustrating typical muscles with a central belly and two terminal tendons. $b$, a penniform muscle; $c$, a bi-
penniform muscle. less movable attachment of a muscle is called its origin, the more movable its insertion. Taking for example the biceps of the arm, we find that when the belly of the muscle contracts and pulls on its upper and lower tendons, it commonly moves only the forearm, bending the elbow-joint as shown in Fig. 39. The shoulder is so much more firm that it serves as a fixed point, and so that end is the origin of the muscle, and the forearm attachment, $P$, the insertion. It is clear, however, that this distinction in the mobility of the points of fixation of the muscle is only relative, for, by changing the conditions, the insertion may become the stationary and the origin the moved point; as for instance in going up a rope "hand over hand." In that case the radial end of the muscle is fixed and the shoulder is moved through space by its contraction.

Different Forms of Muscles. Many muscles of the Body have the simple typical form of a belly tapering to a single tendon at each end as $A$, Fig. 40, but others divide at one end and are called two-headed or biceps muscles; while some are even three-headed or triceps muscles. On the other hand, some muscles have no tendon at all at one end, the belly running quite up to the point of attachment; and some have no tendon at either end. In many muscles a tendon runs along one side and the fibers of the belly are attached obliquely to it: such muscles $(B$, Fig. 40) are called penniform or featherlike; or a tendon runs obliquely down the middle of the muscle and has the fibers of the belly fixed obliquely on each side of it ( $C$, Fig. 40), forming a

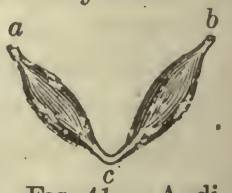

Frg. 41.-A digastrie muscle. bipenniform muscle: or even two tendons may run down the belly and so form a tripenniform muscle. In a few cases a tendon is found in the middle of the belly as well as at each end of it; such muscles are called digastric. A muscle of this form (Fig. 41) 
is found in connection with the lower jaw, It arises by a tendon attached to the base of the skull; from there its first belly runs downwards and forwards to the neck by the side of the hyoid bone, where it ends in a tendon which passes through a loop serving as a pulley. This is succeeded by a second belly directed upwards towards the chin, where it ends in a tendon inserted into the lower jaw. Running along the front of the abdomen from the pelvis to the chest is a long muscle on each side of the middle line called the rectus abdominis: it is polygatric, consisting of four bellies separated by short tendons. Many izuscles moreover are not rounded but form wide flat masses, as for example the muscle $S s$ seen on the ventral side of the shoulder-blade in Fig. 38.

Gross Structure of a Muscle. However the form of the skeletal muscles and the arrangement of their tendons may vary, the essential structure of all is the same. Each consists of a proper striated muscular tissue, which is its essential part, but which is supported by connective tissue, nourished by blood-vessels, and has its activity governed by nerves so that a great variety of things go to form the complete organ.

A loose sheath of areolar connective tissue, called the perimysium, envelops each muscle, and from this partitions run in and subdivide the belly into bundles or fasciculi which run from tendon to tendon, or for the whole length of the muscle when it has no tendons. The coarseness or firmness of butcher's meat depends upon the size of these primary fasciculi, which differs in different muscles of the same animal. These larger fasciculi are subdivided by finer connective tissue membranes into smaller ones, each of which consists of a certain number of microscopic muscular fibers bound together by very fine connective tissue and enveloped. in a close network of blood-vessels. Where a muscle tapers the fibers in the fasciculi become less numerous, and when a tendon is formed disappear altogether, leaving little but the connective tissue.

Histology of Skeletal Muscle. Each muscle-fiber is developed from a single cell and so constitutes a single histological element. In the adult form, however, a muscle-fiber differs from an ordinary cell in that it contains several nuclei. Muscle-fibers vary greatly in size; ranging in length from 1 up to $35 \mathrm{~mm}$. ( $\frac{1}{25}$ in. to $1 \frac{1}{3}$ in.), and in diameter from 0.034 to $0.055 \mathrm{~mm}$. ( $\left(\frac{1}{5} \sigma\right.$ to $\frac{1}{4} \frac{1}{5} \mathrm{in}$.). Each 
fiber consists of a certain amount of muscle substance, the muscle plasma, inclosed in a transparent connective tissue sheath, the sarcolemma. This latter structure serves not only to hold the semifluid muscle plasma in place, but also to transit the pull of the contracting fiber to the point of attachment of the muscle. The most striking characteristic of a fiber's appearance is the series of alternating light and dim transverse bands of nearly equal width with which it is marked, and from which its designation as striated muscle is derived (Fig. 42). Under the high power of the microscope the muscle plasma is seen to be made up of a number
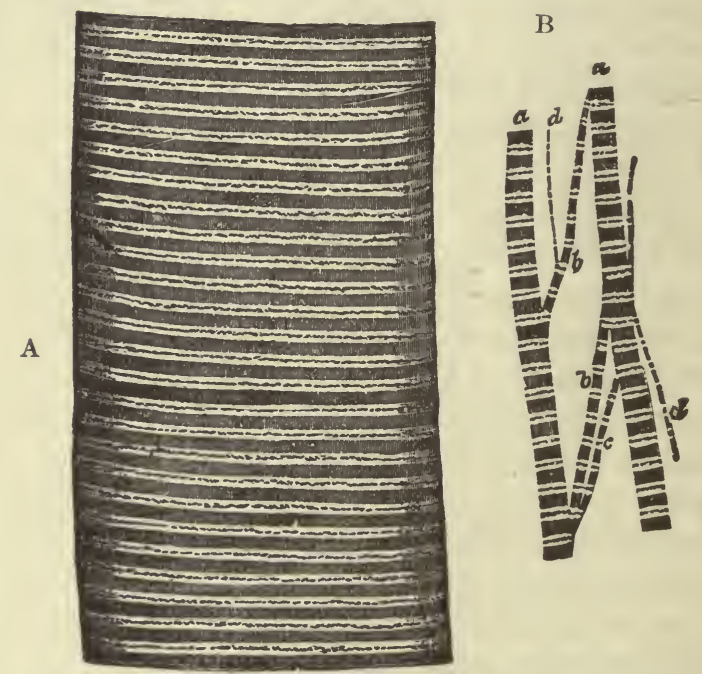

Frg. 42.-A, Portion of a Human muscle fiber. $B$, Separated bundles of sarcostyles, $d$, single sarcostyle. $\times 800$. (Sharpey.)

of longitudinal fibrils, the sarcostyles, surrounded by a homogeneous medium, the sarcoplasm.

Not all histologists are agreed as to the details of structure of the sarcostyles; they are so small that only the highest powers of the microscope can be used in studying them; they occur in ordinary muscle surrounded always by sarcoplasm and in company with many others. These circumstances combine to present to the eye of the observer a more or less distorted picture. It is no wonder, therefore, that differences of opinion as to the real structure of the sarcostyles have arisen. 
Certain insects' muscles happen to be so constituted that the sarcostyles can be separated one from another and isolated ones gotten under the field of the microscope for study. When examined thus singly and free from surrounding media which distort the view, these sarcostyles are seen to be tiny cylinders divided at regular intervals by transverse partitions, made, apparently, of delicate membrane. Many biologists think it likely that the sarcostyles of ordinary skeletal muscle have really this same structure; that the position of the transverse membranes is indicated by faint dark lines in the middle of the light bands and that the appearance of light and dim bands of nearly equal width is an optical

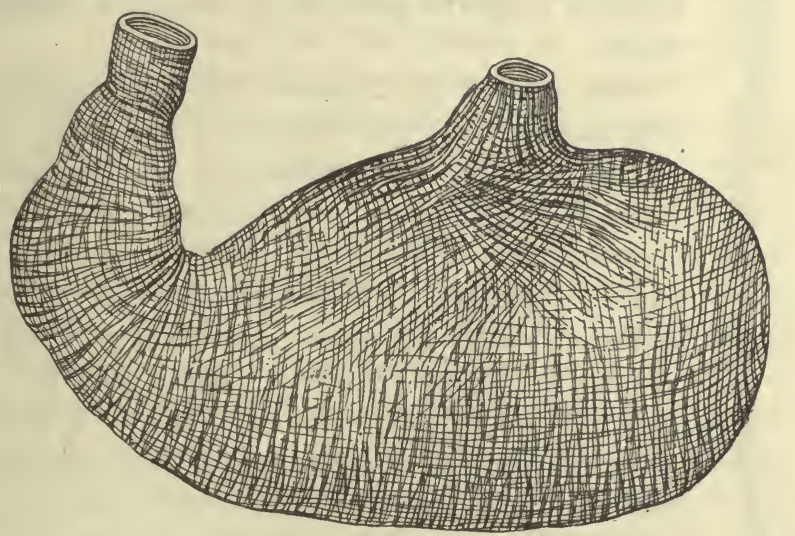

FIG. 43.-The muscular coat of the stomach.

illusion due to the unfavorable conditions of observation. Since the fiber as a whole contains many sarcostyles and since the cross striations are regular throughout the entire fiber it follows that all the sarcostyles of any fiber must have their partitions at corresponding levels. The sarcostyles are probably kept in place by an interfibrillar network of some sort.

The blood-vessels and nerve-fibers supplied to the skeletal muscles are numerous. - The larger blood-vessels run in the coarser partitions of the connective tissue lying between the fasciculi and give off fine branches which form a network between the individual fibers but never penetrate the sarcolemma.

Connected with each muscle-fiber is a nerve-fiber. The central core of the nerve-fiber ends in an oval expansion (end plate) which 
contains many nuclei and lies close under the sarcolemma, its deeper side being in immediate contact and possibly continuous with the striated contents. These nerve-fibers are motor or con-

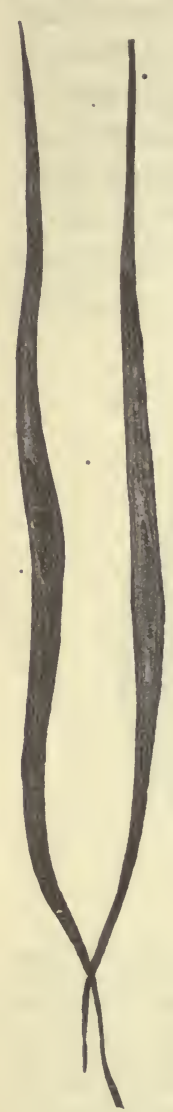

FIG. 44.

Isolated smooth muscle cells from human small intestine. cerned in exciting a contraction of the muscle-fiber. Other nerve-fibers are connected with very peculiar bodies found scattered throughout the muscle, but especially numerous near the tendons. They are usually of a size just visible to the unaided eye and from their form have been named muscle-spindles. They are doubtless sensory in function. Somewhat similar bodies (Golgi's tendon-organs) are found in the tendons and are also richly supplied with nervefibers.

Structure of the Smooth Muscles. Of these the muscular coat of the stomach (Fig. 43) is a good example. They have no definite tendons, but form expanded membranes surrounding cavities, so that they have no definite origin or insertion. Like the skeletal muscles they consist of proper contractile elements, with accessory connective tissue, bloodvessels, and nerves. Their fibers, however, have a very different microscopic structure. They present a slightly marked longitudinal but.no cross striation and are made up of elongated cells (Fig. 44), bound together by a small quantity of cementing material. The cells vary considerably in size, but on the average are about $\frac{1}{24} \mathrm{~mm}$. ( $\frac{1}{60} \mathrm{in}$.) in length. Each is flattened in one plane, tapers off at each end, and possesses a very thin enveloping membrane; in its interior lies an elongated nucleus. These cells have the power of shortening in the direction of their long axes, and so of diminishing the capacity of the cavities in the walls of which they lie.

Cardiac Muscular Tissue. This consists of nu-cleated branched cells which unite to form a network, in the interstices of which blood-capillaries and nerve-fibers run. The cells present transverse striations, but not so distinct as those of the skeletal muscles, and are said to have no sarcolemma (Fig. 45).

Ciliated Cells. As the growing Body develops from its prim- 
itive simplicity we find that the cells lining some of the tubes and cavities in its interior undergo a very remarkable change, by which each cell differentiates itself into a nutritive and a highly motile

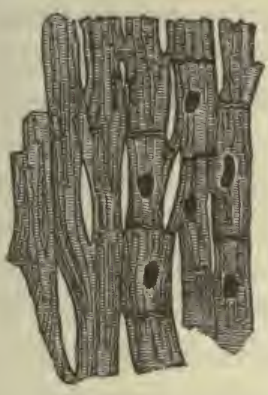

Frg. 45. - Cardiac muscular tissue, magnified about 400 diameters. The cell-boundindicated only in the right-hand portion of the figure.

portion. Such cells are found for example lining the windpipe, and are represented in Fig. 46. Each has a conical form, the base of the cone being turned to the cavity of the air-tube, and contains an oval nucleus. On the broader free end are a number (about thirty on the average) of extremely fine processes called cilia. During life these are in constant rapid movement, lashing to and fro in the liquid which moistens the interior of the passage; and as the cells are very closely packed, a bit of the inner surface of the windpipe, examined with a microscope, looks like a field of wheat or barley when the wind blows over it. Each cilium strikes with more force in one direction than in the opposite, and as this direction of more powerful stroke is the same for all the cilia on any one surface, the resultant effect is that the liquid in which they move is driven one way. In the case of the windpipe for example it is driven up towards the throat, and the tenacious liquid or mucus which is thus swept along is finally coughed or "hawked" up and got rid of, instead of accumulating in the deeper air-passages away down in the chest.

These cells afford an extremely interesting example of the division of physiological employments. Each proceeds from a cell which was primitively equally motile and nutritive in all its parts. But in the fully developed state the nutritive duties have been especially assumed by the conical cell-body, while the contractile properties have been condensed, so to speak, in that modified portion of the primitive proto-
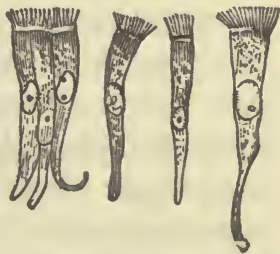

Fig. 46.-Ciliated cells.

plasmic mass which forms the cilia. These, being supplied with elaborated food by the rest of the cell, are raised above the vulgar cares of life and have the opportunity to devote their whole attention to the performance of automatic movements; which are accordingly far more rapid and precise than those executed 
by the whole cell before any division of labor had occurred in it.

That the movements depend upon the structure and composition of the cells themselves, and not upon influences reaching them from the nervous or other tissues, is proved by the fact that they continue for a long time in isolated cells, removed and placed in a liquid, as blood-serum, which does not alter their physical constitution. In cold-blooded animals, as turtles, whose constituent tissues frequently retain their individual vitality long after that bond of union has been destroyed which constitutes the life of the whole animal as distinct from the lives of its different tissues, the ciliated cells in the windpipe have been found still at work three weeks after the general death of the animal.

The Physico-Chemistry of Skeletal Muscle. The activity of a muscle is the sum of the activities of its individual fibers. To understand the operation of the muscle engine, therefore, we must analyze the activity of the muscle fiber. Some help toward this may be gained by studying the structure of the fiber from the physico-chemical standpoint. Throughout this study the fundamental fact of muscular activity should be kept before the mind, namely, that the muscle is a device for executing a forcible shortening at the expense of energy derived from the food (p. 22).

From the account of the anatomical structure of the musclefiber, given previously (p. 85), we have learned to think of the fiber as consisting of a large number of tiny, longitudinal cylinders, the sarcostyles, inclosed in, and attached by their ends to, a sheath, the sarcolemma, and surrounded by a fluid, the sarcoplasm, which occupies all the space within the sarcolemma not taken by the sarcostyles. Physico-chemical studies indicate that the sarcostyles are colloidal in nature. In fact there is reason to think that the colloids of which they are composed may be quite dense, containing less water than do most of the colloidal tissues of the body. The sarcoplasm, on the other hand, is thought of as a very watery fluid, with salts and other relatively simple substances dissolved in it, but containing little if any colloid. When we reach the consideration of the precise manner in which the forcible shortening of the muscle comes about we shall find that the densely colloidal consistency of the sarcostyles and the watery nature of the sarcoplasm are of great significance. 
The Chemistry of Muscular Tissue. When we subject a mass of muscle to chemical analysis we, of course, kill the fibers, if they were not dead when the analysis was started. The difference between life and death is undoubtedly at bottom a chemical difference, so that we cannot hope by the ordinary methods of analysis as applied to dead tissues to learn the exact chemistry of the living muscles. On the other hand, the constituents we find in dead muscle were derived from those of the living muscle, and are undoubtedly nearly related to them, so that knowledge of the chemistry of dead muscle cannot fail to give us a degree of insight into the nature of the living muscle.

To understand clearly the facts brought out by an analysis of muscle we need to bear in mind that a muscle, as stated previously, is an engine, whose property is to convert chemical energy into mechanical. Our analysis will demonstrate the presence of some substances which are part of the machinery; of others which make up the fuel from which the energy for operation is derived; of still others which are nothing more than the waste products from previous activity. It is as though a locomotive in full career suddenly fell into so deep a chasm as to reduce it with its tender to a mass of indistinguishable fragments. Chemical examination of the mass would bring to light various materials, such as steel, brass, and nickel, which were part of the engine; coal, which was the fuel; and ashes, representing the waste products. In similar fashion we may pick out from a chemical study of muscle some constituents which probably represent the machinery; others which form the fuel; and still others which are waste substances.

The most abundant single constituent of muscle is water, which forms 75 per cent of its mass. Dissolved in the water are small quantities of a number of simple inorganic salts, chiefly phosphates and chlorides of potassium, sodium, and magnesium. Since experiment has shown that these salts, as well as the water in which they are dissolved, are essential to the life and operation of the muscle we may look upon them as part of the machinery. Similarly the most abundant solid constituents of muscle, the proteins, are to be included as portions of the mechanism.

At least three proteins have been obtained from mammalian striped muscle, myogen, an albumin, myosin, a globulin, both coagulable by heat, and a protein which is insoluble in pure water 
or dilute saline solution and which appears to form a framework within the fiber. This latter is called the muscle stroma and constitutes 9 per cent of the weight of striated muscle. Muscle tissue contains three or four times as much myogen as myosin. Both of these proteins possess the property of passing over into insoluble forms known respectively as myogen fibrin and myosin fibrin.

Heart muscle contains relátively much less myogen and myosin and much more stroma than does ordinary striated muscle, its stroma constituting 56 per cent of its weight. Smooth muscle contains an even larger proportion of stroma, 72 per cent. In striated muscle the proteins appear to be confined largely to the colloidal sarcostyles, except in so far as the sarcolemma is protein in constitution.

Another constituent of muscle which is apparently part of the contractile machinery is the relatively simple nitrogenous compound creatine (p. 14). Recent studies of the part played by this substance in tissue indicate that living protoplasm always contains it, and suggest that it may be an essential part of the chemical complex upon which life depends.

The known fuel substances found in muscle are two, dextrose, which is found in very small amount, and glycogen, which forms about 1 per cent of the weight of the muscle. The muscle is probably able to use other substances as fuel, fats, for example, and perhaps proteins themselves on occasion, but chemical analysis does not enable us to distinguish these from similar substances which belong to the mechanism.

Urea and other nitrogenous extractives, and sodium carbonate are found in muscle also. These are to be classed as waste products, formed during the operation of the machine. An interesting and very significant fact is that a muscle analyzed immediately after a period of vigorous activity is found to contain lactic acid, or the compound of this acid with sodium, sodium lacrate, whereas muscles that have been resting do not contain lactates. This fact, as we shall see, has important bearing on the question of the nature of muscle contraction. We shall recur to it in connection with the analysis of the process of contraction.

Beef Tea. From the facts about proteins, stated above, it is clear that when a muscle is boiled in water its myogen and myosin 
are coagulated and left behind in the meat; even if cooking be commenced by soaking in cold water the myogen still remains, as it is as insoluble in cold water as in hot. Beef tea as ordinarily made, then, contains little but the flavoring matters and salts of the meat, traces of some albumins and some gelatin, the latter derived from the connective tissues of the muscle. The flavoring matters and salts make it deceptively taste as if it were a strong solution of the whole meat, and the gelatin causes it to "set" on cooling, so the cook feels quite sure she has got out "all the strength of the meat," whereas the beef tea so prepared contains but little of the most nutritious protein portions, which in an insipid shrunken form are left when the liquid is strained off. Various proposals have been made with the object of avoiding this and getting a really nutritive beef tea; as for example chopping the raw meat fine and soaking it in strong brine for some hours to dissolve out the myogen; or extracting it with dilute acids which dissolves the myogen and myosin and at the same time render it non-coagulable by heat when subsequently boiled. Such methods, however, make unpalatable compounds which invalids will not take. Beef tea is a slight stimulant, and often extremely useful in preparing the stomach for other food, but its direct value as a food is slight, and it cannot be relied upon to keep up a patient's strength for any length of time. There can be no doubt that thousands of sick persons have in the past been starved to death on it. Liebig's extract of meat is essentially a very strong beef tea; containing much of the flavoring substances of the meat, nearly all its salts and the crystalline nitrogenous bodies, such as creatine, which exist in muscle, but hardly any of its really nutritive parts, as was pointed out by Liebig himself. From its stimulating effects it is often useful to persons in feeble health, but other food should be given with it. It may also be used on account of its flavor to add to the "stock" of soup and for similar purposes; but the erroneousness of the common belief that it is a highly nutritious food cannot be too strongly insisted upon. Under the name of liquid extracts of meat other substances have been prepared by subjecting meat to chemical processes in which it undergoes changes similar to those experienced in digestion: the myosin is thus rendered soluble in water and uncoagulable by heat, and such extracts if properly prepared are nutritious and can often be absorbed when meat in the 
solid form cannot be digested: they may thus help the stomach over a crisis, but are not, even the best of them, to be depended on as anything but temporary substitutes for other food; or in some cases as useful additions to it.

Rigor Mortis. During life and for a certain time after general death the muscles are soft, translucent, extensible and elastic, and neutral or feebly alkaline in reaction; after a period which in warmblooded animals is brief (varying from a few minutes to three or four hours) they gradually become harder, more opaque, less extensible and less elastic, and distinctly acid in reaction. The result of these changes is the well-known cadaveric rigidity or rigor mortis. It was formerly very generally believed that the cause of rigor is the change of soluble myogen and the myosin to insoluble myogen fibrin and myosin fibrin. Quite recently, however, some physiologists have called attention to the strong probability that death stiffening may be due to the considerable production of lactic acid which is known to accompany the death process. In support of their view may be cited the well-known tendency of animals or men killed suddenly in the midst of violent exertion to stiffen very quickly. Men killed in battle often retain the postures in which death overtook them. Hard muscular work involves a large production of lactic acid, a condition favorable according to the view quoted, to a prompt onset of rigor. 


\section{CHAPTER VII}

\section{MUSCULAR ACTIVITY}

The Study of Isolated Muscles. There are some simple facts of muscle activity that one can learn by observing his own muscles; for example, when the arm is bent at the elbow the muscle that produces the movement, the biceps, can be seen under the skin to shorten and thicken, and if felt will be found hard when contracted, as compared with its soft flabbiness when relaxed. This knowledge is the possession of every school boy.

More detailed knowledge can be gained by the direct examination of living muscles, dissected away from their bodily attachments. To isolate the muscles of the higher animals thus is obviously impractical, but fortunately the "cold-blooded" animals, notably frogs and turtles, are peculiarly suited for such studies as this. If a frog is quickly killed, as by destroying the brain with a sharp instrument, the large muscle of the calf of the leg, the gastrocnemius, can be dissected out, and if properly cared for will remain alive for several hours, during which its activity can be studied. The chief precaution to be observed is to prevent such evaporation of water from the muscle as would disturb the physico-chemical equilibrium and injure the tissue. This loss of water is prevented by repeated moistening, but here again a precaution must be observed since the application of pure water to the surface of the muscle would be followed by a flow of water into the tissue under the driving force of osmotic pressure (p. 19). This would bring about a disturbance of equilibrium in the direction of too great dilution as harmful to the tissue as the evaporation it is designed to prevent. For moistening the muscle a liquid of the same osmotic pressure as the tissue fluids must be employed. A solution of common salt with a concentration of 0.7 per cent satisfies this condition and is the fluid commonly used for keeping living tissues moist.

The Necessity of Stimulation. An important fact about skeletal muscle, one indeed which has much to do with the adaptive 
character of its action, is that it remains in a condition of inactivity except when in receipt of definite stimulation. A moment's thought will show the importance of this property. The usefulness of a muscle depends quite as much upon its ability to be inactive when not wanted as upon its property of contracting when contraction is desired. The difficulties frequently experienced by sufferers from chorea (St. Vitus Dance) illustrate this point sufficiently.

For the study of activity in isolated muscles some form of artificial stimulation must be employed. By far the most satisfactory is an electric shock such as may be generated by a small induction coil. By carrying fine wires from the terminals of the coil to opposite ends of the muscle the latter becomes a part of the circuit. Thus when the coil discharges a spark the muscle receives it and is stimulated. Besides being peculiarly effective as stimuli induction shocks have the advantage of being easily modified in intensity, and, when not excessive, of having no injurious effect on the tissue.

A Simple Muscular Contraction. When a single electric shock is sent through a muscle, it rapidly shortens and then rapidly lengthens again. The whole series of phenomena from the moment of stimulation until the muscle regains its resting form is known as a simple muscular contraction or a "twitch": it occupies in frog's muscle about one-tenth of a second. So brief a movement as this cannot be followed in its details by direct observation, but it is possible to record it and study its phases at leisure. This may be done by firmly fixing the upper tendon of an isolated muscle, $M$, Fig. 47 , and attaching the other end at $d$ to a lever, $l$, which can move about the fulcrum $f$ : the end of the long arm of the lever bears a point, $p$, which scratches on a smooth smoked surface, $S$. Suppose the surface to be placed so that the writing point of the lever is at $a$; if the muscle now contracts it will raise the point of the lever, and a line $a c$ will be drawn on the smoked surface, its vertical height, $\mathrm{cm}$, being dependent, first, on the extent of the shortening of the muscle, and second, on the proportion between the long and short arms of the lever: the longer $f p$ is as compared with $f d$, the more will the actual shortening of the muscle be magnified. With the lever shown in the figure this magnification would be about ten times, so that one-tenth of $\mathrm{cm}$ would be 
the extent of the shortening of the muscle. Suppose, next, the smoked surface to be moved to such position that the writing point of the lever touches it at $i$, and, the muscle being left at rest the surface to be moved evenly from left to right; the horizontal line io would then be traced, its length depending on the distance through which $S$ moved during the time the lever was marking on it: and it is clear that if $S$ move uniformly, and we know its rate of movement, we can very readily calculate from the length of $i o$ how long $S$ was moving while that line was being traced: for example, if we know the rate of movement to be ten centimeters per

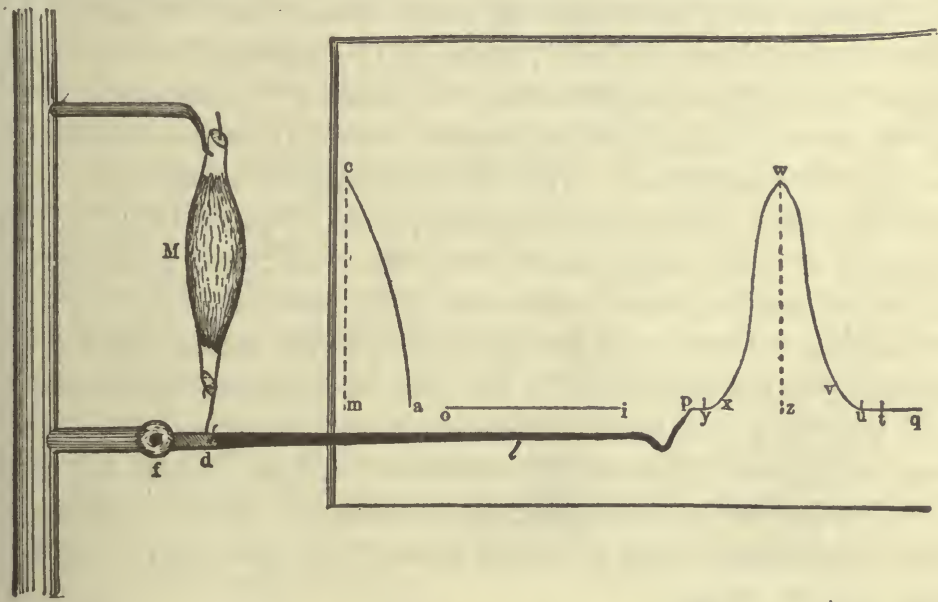

Fig. 47.-Diagram to illustrate the method of obtaining a graphic record of a muscular contractión.

second, and on measurement find io to be one centimeter long, the time during which the surface was moving must have been $\frac{1}{10}$ of a second; and each tenth of $i o$ correspond to $\frac{1}{100}$ of a second.

If we set the recording surface in motion and while the lever point is tracing a horizontal line cause the muscle to contract, the point will be raised as long as the muscle is contracted, and the line drawn by it will be due to a combination of two simultaneous movements - a horizontal, due to the motion of $S$, a nearly vertical, due to the shortening of the muscle; the resulting line is a curve known as the curve of a simple muscular contraction. Let the surface $S$ be placed so that the writing point is at $q$ and then be set in uniform motion from left to right at the same rate as before 
(ten centimeters per second). When the point is opposite $t$, stimulate the muscle by an electric shock; the result, until the muscle has fully lengthened again, will be the curve tuvwxy, from which many things may be learned. In the first place we see that the muscle does not commence to contract at the very instant of stimulation, but at an appreciably later time, and during the interval the lever draws the horizontal line $t u$; this period, occupied by preparatory changes within the muscle, is known as the latent period. Then the muscle begins to shorten and the lever to rise, until the summit of the contraction is reached at $w$. The muscle then, but only gradually passes back to the resting state, tracing the line wxy. The curve shows three distinct phases in the contraction: the latent period; the period of shortening; the period of relaxation. Knowing the rate of horizontal movement, we can measure off the time occupied by each phase. The horizontal distance from $t$ to $u$ represents the time taken by the latent period; from $u$ to $z$, the time occupied in shortening; from $z$ to $y$, the time taken in elongation; in a fresh frog's muscle these times are respectively $\frac{1}{100}, \frac{4}{100}, \frac{5}{100}$ of a second. In the muscles of warm-blooded animals they are all shorter, but the difficulties in the way of accurate experiment are very great. If we know the relative lengths of the arms of the lever we can of course readily calculate from the height, $w z$, of the curve the extent of shortening of the muscle. With a single electrical stimulation this is never more than one-fourth the total length of the muscle.

In Fig. 47 the accessory apparatus used in practice to indicate on the moving surface the exact instant of stimulation and to measure the rate at which $S$ moves have been omitted.

The Influence on Contraction Height of Increasing Stimulation Strength. If an isolated muscle is stimulated at regular intervals, as once in two seconds, by induction shocks made stronger each time, there will be at first, if the shocks to begin with are weak enough, no visible response. Presently, as stronger shocks are used, barely perceptible twitches are given. These become higher and higher as the stimuli are increased until a point is reached beyond which no further increase in height appears, no matter how much the intensity of the shock may be increased. These highest contractions which the muscle is capable of giving as the result of any single stimulus, however strong, are called 
maximal contractions. The gradation of response by grading the intensity of stimulation is obviously the means employed in our own bodies to produce graded movements. We realize without difficulty that a powerful contraction requires a great effort (strong stimulation), while a gentle contraction is produced with very little effort (weak stimulation).

Every skeletal muscle, even the smallest, is made up of a great number of fibers. This must be borne in mind when we attempt to explain the production of graded responses, for when the muscle contracts feebly it may be that all the fibers are contracting, each one feebly, or it may be that a few fibers scattered through the muscle are contracting powerfully while the others are inactive, If the first of these suppositions is correct we must look upon all the fibers as equally sensitive to stimulation, so that all respond feebly to weak stimuli and powerfully to strong ones. If the second view is the true one we must picture the various fibers as differing in sensitiveness over a wide range. Some are aroused by very feeble stimuli, others require stronger ones, and others still stronger ones; but whether aroused by a weak stimulus or a strong one the response of the individual fibers is powerful. According to this view the muscle fibers could be compared to the cartridge in a rifle and the stimulus to the pull of the trigger. In some rifles the trigger is harder to pull than in others, but the explosion of the cartridge is as violent in the rifle with the hair trigger as in the stiffer one.

The first of these theories, the one that makes the gradation of response a gradation within the fibers, is the older and the one that has formerly been generally accepted. The second view, according to which the gradation of response depends on the number of fibers involved, has been urged only recently, and while many known facts are in accord with it, it cannot be said to be conclusively proven.

The Influence of Temperature on Contraction. If an isolated muscle is cooled down and then stimulated, the contraction will occur much more slowly than at ordinary temperatures. On the other hand, a muscle that is warmed contracts and relaxes more rapidly than one that is at room temperature. This variation in the speed of contraction with change of temperature is in accord with a general chemical law which states that chemical processes 
are more rapid at higher temperatures than at lower; a law which applies to muscular contraction because the mechanical act of shortening is based on a preceding chemical process. In nature the influence of temperature on muscular contraction is seen only in the lower (cold-blooded) animals, whose bodies are at substantially the temperature of the surroundings and which, as can easily be observed, are sluggish in cold weather and active in warm. In man and the higher (warm-blooded) animals, and in birds, which are also warm-blooded, the body temperature is high and relatively constant, and the muscles are not subjected, therefore, to such temperature variations as occur in lower forms. One advantage of the warm-blooded state is that it insures for the muscles a favorable temperature for effective operation in cold weather as well as in warm.

Heat Rigor. If an isolated muscle is heated above $40-45^{\circ} \mathrm{C}$. $\left(104-113^{\circ} \mathrm{F}\right.$.) it is killed by the heat and undergoes a marked contraction known as heat rigor. Heat rigor like the death stiffening (rigor mortis, p. 92) is accompanied by, and probably caused by, a great production of lactic acid within the muscle.

The Measure of Muscular Work. The work done by a muscle in a given contraction, when it lifts a weight vertically against gravity, is measured by the weight moved, multiplied by the distance through which it is moved. When a muscle contracts carrying no load it does very little work, lifting only its own weight; when loaded with one gram and lifting it five millimeters it does five gram-millimeters of work, just as an engineer would say an engine had done so many kilogrammeters or foot-pounds. If loaded with ten grams and lifting it six millimeters it would do sixty gram-millimeters of work. Even after the weight becomes so great that it is lifted through a less distance, the work done by the muscle goes on increasing, for the heavier weight lifted more than compensates for the less distance through which it is raised. For example, if the above muscle were loaded with fifty grams it would maybe lift that weight only 1.5 millimeters, but it would then do 75 gram-millimeters of work, which is more than when it lifted ten grams six millimeters. A load is, however, at last reached with which the muscle does less work, the lift becoming very little indeed, until at last the weight becomes so great that the muscle cannot lift it at all and so does no work when stimulated. Starting 
then from the time when the muscle carried no load and did no work, we pass with increasing weights, through phases in which it does more and more work, until with one particular load it does the greatest amount possible to it with that stimulus: after that, with increasing loads less work is done, until finally a load is reached with which the muscle again does no work. What is true of one muscle is of course true of all, and what is true of work done against gravity is true of all muscular work, so that there is one precise load with which a beast of burden or a man can do the greatest possible amount of work in a day. With a lighter or heavier load the distance through which it can be moved will be more or less, but the actual work done always less. In the living Body, however, the working of the muscles depends so much on other things, as the due action of the circulatory and respiratory systems and the nervous energy or "grit" (upon which the stimulation of the muscles depends) of the individual man or beast, that the greatest amount of work obtainable is not a simple mechanical problem as it is with the excised muscle.

\section{Influence of the Form of the Muscle on its Working Power.} The amount of work that any muscle can do depends of course largely upon its physiological state; a healthy well-nourished muscle can do more than a diseased or starved one; but allowing for such variations the work which can be done by a muscle varies with its form. The thicker the muscle, that is the greater the number of fibers present in a section made across the long axes of the fasciculi, the greater the load that can be lifted or the other resistance that can be overcome. On the other hand, the extent. through which a muscle can move a weight increases with the length of its fasciculi. A muscle a foot in length can contract more than a muscle six inches long, and so would move a bone through a greater distance, provided the resistance were not too great for its strength. But if the shorter muscle had double the thickness, then it could lift twice the weight that the longer muscle could. IVe find in the Body muscles constructed on both plans; some to have a great range of movement, others to overcome great resistance, besides numerous intermediate forms which cannot be called either long and slender or short and thick; many short muscles for example are not specially thick, but are short merely because the parts on which they act lie near. to- 
gether. It must be borne in mind, too, that many apparently long muscles are really short stout ones-those namely in which a tendon runs down the side or middle of the muscle, and has the fibers inserted obliquely into it. The muscle (gastrocnemius) in the calf of the leg, for instance (Fig. $40, B$ ), is really a short stout muscle, for its working length depends on the length of its fasciculi and these are short and oblique, while its true cross-section is that at right angles to the fasciculi and is considerable. The force with which a muscle can shorten is very great. A frog's muscle of 1 square centimeter ( $0.39 \mathrm{inch})$ in section can just lift 2,800 grams (98.5 ounces), and a human muscle of the same area more than twice as much.

The Beneficial Effect of Exercise. An interesting fact about skeletal muscle, that is in the experience of every athlete, and can also be demonstrated upon an isolated muscle, is that the response to stimulation after a period of inaction is less vigorous than the response to precisely the same amount of stimulation after the muscle has been exercised for a while. This fact explains the necessity under which base ball pitchers and other athletes labor of "warming up" before they can use their muscles effectively. In the case of an isolated muscle stimulated at regular intervals the effect is seen in a well-marked increase in the height of contraction during the first dozen or so of the series. This increase from contraction to contraction is often very regular, suggesting a flight of stairs. For this reason it is often spoken of as the "staircase phenomenon."

Fatigue of Muscle - Contracture. If an isolated muscle is subjected to a series of stimulations at fairly frequent intervals - one a second or oftener - the period of the "staircase" described above, is usually followed by a period in which the relaxation of the muscle is definitely slowed. So far as can be seen the muscle contracts as rapidly and forcibly as ever, but the relaxations are drawn out. The effect of this in a regular series of stimulations is that the muscle fails to relax completely from one stimulation by the time the next one is sent in, so that it continues in a state of partial contraction during the intervals between stimulations. This condition is called contracture. It consists essentially of a slowing down of the relaxation rate, and is the first indication of the condition of impairment that we call fatigue. Aside from its signifi-

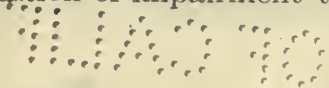


cance as a phase of fatigue, contracture is important as proving that the relaxation of muscle is not a mere passive falling back from the contracted state, for if relaxation were just that the rate of the falling back should be the same at one time as at another. Since the relaxation rate varies, becoming slower at the beginning of fatigue, there must be a definite relaxation process and we are bound, in our analysis of the muscle machine to take the relaxation process into account as well as the contraction process itself.

If the recurrent stimulation of the isolated muscle is continued beyond the phase of contracture there soon develops the phase which accords more fully with our ordinary conception of fatigue. The muscle contracts more and more feebly until finally it refuses to respond at all to stimulation. After this stage is reached a rest of a few minutes often suffices to restore the muscle to a condition in which it will show a considerable degree of activity, although usually not so much as it exhibited when fresh.

The Nature of Fatigue. We can understand why muscles become fatigued if we recall the fact that the muscle is a chemical engine. The energy for contraction is furnished by chemical transformations within the muscle whereby substances containing large amounts of energy enter combinations of less energy value, and liberate the surplus energy for the use of the muscle. These resulting compounds, of low energy value, are waste products. Their relation to the muscle is that of the ashes to the furnace. Unless they are gotlen rid of they interfere with further activity. This hampering of a chemical process by its own products is a wellknown principle of chemistry. To avoid the effect the products should be removed as fast as they are formed. In the body the agency for removing waste products from the muscles is the bloodstream flowing through them. Under ordinary circumstances this is sufficient, so that muscular fatigue is not commonly felt. In isolated muscles, however, there is no stream of blood. The only way in which waste products can be gotten rid of is by the slow process of dialysis from the fibers into the surrounding lymph. Fatigue comes on rather quickly, therefore, in isolated muscles that are stimulated repeatedly.

The reader should be cautioned at this point against attempting to apply the description of fatigue just given to all his own experiences of weariness. The fatigue of isolated muscles is muscular 
fatigue, and its study is exceedingly instructive in aiding us to analyze the workings of the muscle machine, but it is not the type of fatigue that human beings most commonly feel. Our muscles are, to be sure, so much like the muscles of the frog that they would, if isolated and stimulated, show similar fatigue, but as used in the body they are usually saved from experiencing serious fatigue by the fact that the swiftly flowing blood tends to sweep out the "fatigue products" and prevent their accumulation, and also because in the nervous system, by which the muscles are stimulated to activity, there are regions which become fatigued while the muscles are yet in good condition. Only under exceptional circumstances, as in athletic contests, or severe manual labor, when the nervous system is driven far beyond its usual activity and the blood is unable to remove the waste products as fast as they are formed, do we experience genuine muscular fatigue.

The Response to Rapidly Repeated Stimuli. Tetanus. Since a simple muscular contraction occupies one-tenth of a second, it is obvious that a second stimulus following the first within that interval will find the muscle in a state of partial or complete contraction, depending on the exact length of the interval. In such a case the muscle executes in response to the second stimulus a second contraction, which is fused with the first. Similarly a third can be elicited, which will be fused with the second, and so on. A series of such fused contractions is known as a physiological tetanus. If the interval between stimuli does not exceed $\frac{1}{10}-\frac{1}{12}$ of a second in fresh frog's muscle the fusion is complete. That is, there are no signs of relaxation between stimuli, and the contraction is perfectly steady: An interesting fact about physiological tetanus, or tetanic contraction as it is often called, is that the extent of the contraction may be markedly greater than in a simple contraction, even though the latter may have been maximal. Evidently the so-called maximal simple contraction is not maximal in the sense that it represents the mechanical limit of the muscle's power to shorten, but only in that it is the utmost the muscle can do in response to a single stimulus. In two respects, then, the tetanic contraction differs from the simple twitch: in being more prolonged, and in being higher.

Voluntary Muscular Contraction. In view of the superiority of the tetanus over the simple twitch it is interesting to note that 
all voluntary muscular movements, even the briefest, are tetanic in character. They owe this special character to a peculiarity of the nervous system, through which the muscles are excited to contract. When nerve-cells discharge their impulses into muscles the discharge is never a single one but always a series in rapid succession. The result in the muscle is, of course, a tetanus.

The Electrical Phenomena of Muscle. When a living muscle is carefully exposed and suitable electrodes connected with a sensitive galvanometer or electrometer are applied to its surface the entire surface is found to be isoelectric, $i$. e., having a uniform electric potential. If, however, an injury such as cutting or burning is inflicted upon any part of the muscle the injured surface is found to possess a different potential from the surrounding uninjured surfaces. This difference of potential is shown by movements of the indicator of the galvanometer or electrometer. These movements are usually in such a direction as to indicate that the injured region has a lower potential than uninjured parts of the same tissue. This difference of potential existing between injured and uninjured living tissue is often referred to as the current of injury, although no current actually flows unless the two regions are connected by an electrical conductor. No current of injury can be obtained by connecting living tissue with dead tissue. Only while the injured tissue is in act of dying does it exhibit the altered potential which may give rise to an injury current.

The explanation of the change of electric potential accompanying an injury to living tissue is found in the fact that the death process which follows injury involves extensive chemical changes in the tissue. This disturbance in chemical relationship brings about corresponding disturbance in the electric equilibrium which finds expression in an altered electric potential in the part where the chemical activity is going on.

Just as the chemical changes which follow injury to the tissue give rise to the change of electrical potential which we call the current of injury, so the chemical changes which accompany normal activity in the tissue give rise to electrical changes which are designated currents of action. Action currents cannot easily be demonstrated in an ordinary contracting muscle because the whole muscle goes into contraction at once and so the electriz 
potential of its entire surface rises and falls uniformly. In the heart we have a muscle, however, which does not contract all at once, the contraction sweeping over it from base to apex. The action currents of the heart, therefore, can be demonstrated without difficulty if the apparatus used for detecting them is able to respond quickly enough to recurrent changes of potential in opposite directions. Delicate galvanometers have been devised which answer admirably for the purpose. Another interesting method of demonstrating the action currents of the heart is by causing them to act as stimuli for an irritable tissue. If in a recently killed frog the sciatic nerve is dissected out as far as the knee and cut away from its connection with the spinal cord, being left in connection with the leg below, and if this nerve is laid on the exposed beating heart of the same frog or some other recently killed animal, often the muscles of the lower leg and foot which are connected with the nerve will contract at each beat of the heart. The nerve where it lies on the heart serves as a conductor for the action currents as they are generated in the heart, and the action currents in turn stimulate the nerve during their flow through it.

The Source of Muscular Energy. In the physical sense a muscle is a machine. By this we mean that whatever energy it gives out must have been supplied to it previously from the outside. The work which a muscle does in contracting is at the expense of its available store of energy. We know that the energy exhibited by a steam-engine is derived from the combustion or oxidation of the fuel under the boiler. We know also that the energy exhibited by a contracting muscle is derived from the oxidation of fuel substances within it. The physical accompaniments of oxidation are not the same in the two cases; the fuel under the boiler burns with flame and at a high temperature; the fuel substance within the muscle burns without flame and at a temperature only slightly higher than that of the body. The energy yield, however, for corresponding amounts of fuel is as great in one case as in the other.

The fuel substances used in the Body are ehiefly dextrose (grape sugar), or the closely related substance glycogen, and fats. That the third group of energy yielding foods, the proteins, are not ordinarily used as fuel for the muscles was proven by a very inter- 
esting experiment which stands as one of the classical experiments in Physiology. The proof rests upon the fact that the decomposition of proteins gives rise to compounds which contain nitrogen and which are discharged from the Body, except for a negligible residue, by way of the kidneys. If the urine is collected and its nitrogen content determined, the amount of protein decomposition that has occurred in the Body can be calculated (see table, p. 11). Two German Physiologists, Fick and Wislicenus, determined their average daily loss of nitrogen over a period of several days of relative inactivity and then engaged in a day of exceptionally vigorous muscular exercise. The form of activity chosen was mountain climbing; the mountain ascended was the Faulhorn in the Alps, 1,956 meters (6,000 feet) high. In spite of the very great increase in the amount of muscular energy manifested, and the consequent great increase in the amount of fuel consumed, the total loss of nitrogen from the Body was virtually the same as on the previous days of inactivity. This experiment, which has been repeated and confirmed many times, shows that proteins are not ordinarily used by the muscles as fuel. Therefore the other energy yielding foods must serve. (See also Chap. XXX).

While fats are excellent fuel foods, and are, in all likelihood, used by the muscles when brought to them by the blood, there is abundant evidence that the muscles can get along without fats provided they have enough fuel in the form of sugar. This evidence-is found in the experience of grazing animals which may never after weaning have a particle of fat in their food and which, nevertheless, are able to use their muscles to the very best advantage. When dextrose or fats are burned the products of the oxidation are carbon dioxid and water. The reaction in the case of dextrose is represented by the equation $\mathrm{C}_{6} \mathrm{H}_{12} \mathrm{O}_{6}+6 \mathrm{O}_{2}=6 \mathrm{CO}_{2}+6 \mathrm{H}_{2} \mathrm{O}$. That one or the other of the fuel substances mentioned above is oxidized during muscular activity is shown by an increase in the amount of carbon dioxid produced in the Body and breathed out from the lungs. By a comparatively simple device the amount of carbon dioxid breathed out per minute can be determined, and there is invariably a pronounced increase during and immediately following muscular exercise. If the exercise is sharp the increase may be 7 or 8 fold. There is, of course, an equivalent increase in water production, but the water so produced merely adds itself to the 
abundant water already present in the Body and cannot be identified as can the gaseous carbon dioxid.

The Chemistry of Muscular Contraction. In addition to the fact just stated, that there is oxidation of fuel substances, with production of carbon dioxid and water, about the only definite chemical process we know to be associated with muscular activity is the production of lactic acid (p. 16). Lactic acid is chemically closely related to dextrose in that one molecule of dextrose can be split into two molecules of lactic acid with no residue. The relationship is expressed by the equation $\mathrm{C}_{6} \mathrm{H}_{12} \mathrm{O}_{6}=2 \mathrm{C}_{3} \mathrm{H}_{6} \mathrm{O}_{3}$, the latter symbol being that of lactic acid. For a long time the lactic acid that appears in active muscles was supposed to be merely a stage in the oxidation of dextrose, and its invariable appearance was taken to mean that dextrose is the only fuel that muscles are able to use. This conception involves the view that before muscles can use fats as fuel the fats must be converted into dextrose. Chemically the conversion of fat into sugar is exceedingly difficult, and it seemed a very remarkable thing that a chemical transformation so hard to bring about in the laboratory should occur constantly in the Body. As a result of this difficulty a very celebrated controversy has arisen in Physiology over the question of whether or not fats are converted into sugar in the Body before being utilized.

Of recent years evidence has been accumulating that the lactic acid which appears in active muscles is not a mere incidental stage in the transformation of dextrose, but an essential feature of the contraction process. In fact the view held at present by many physiologists is that lactic acid is associated in intimate fashion with the mechanical act of shortening, but is not involved at all in the chemical processes by which the energy for the contraction is obtained. In other words, lactic acid is not a fuel substance, and the fact that it can be obtained from sugar by a simple splitting of the molecule does not prove that it is so derived in the muscle.

The importance of this newer conception is that it releases us from the necessity of supposing sugar to be the only possible fuel for muscles. The question of whether or not fats are transformed into sugar in the Body, instead of being absolutely fundamental, becomes an interesting problem in Biological Chemistry.

The Energy Relationships of Contracting Muscle. Muscular Efficiency. Since the muscle is an engine for the conversion of 
one kind of energy (chemical) into another kind (mechanical) its energy relationships can be studied in the same manner as in other sorts of engines. We are familiar with various classes of these. A steam power plant and an automobile fall in the same group with muscles in that they are devices for transforming chemical energy (oxidation of fuel) into mechanical. A hydro-electric installation converts the mechanical energy of the water-fall into electrical energy. An ordinary dry cell is an engine for the conversion of chemical energy into electrical, and a motor for the conversion of electrical energy into mechanical. All these engines have in common the feature of relative inefficiency. When we speak of the efficiency of an engine we mean the ratio of the amount of useful energy it gives out to the total amount it uses up. No engine is 100 per cent efficient; in none can all the energy put in be recovered in available form. There is always a fraction of the total energy which manifests itself in the form of heat. The hot flue gases from the furnace, the hot bearings on the locomotive, the hot water in the cooling system of the automobile; all these signify energy which from the standpoint of the machine is wasted. Muscles share with other engines this feature of inefficiency. As used in the Body the muscles are only about 20 per cent efficient. That is, in order to do a given amount of muscular work we must burn in our bodies enough fuel to give a total energy output five times as great. The balance, of 80 per cent, takes the form of heat, and explains why we find ourselves so warm after vigorous exercise. Isolated. muscles under favorable circumstances may show an efficiency of nearly 50 per cent.

Energy Units. In order to be able to discuss energy relationships intelligently we need to have some means of designating definite amounts. The form of energy into which all other forms tend to convert themselves is, as we have seen, heat. A convenient energy unit, then, is the heat unit. The amount of heat required to raise the temperature of 1 gram $\left(\frac{1}{28} \mathrm{oz}\right.$.) of water 1 degree centigrade (strictly from zero to $1^{\circ}$ ) is taken as the unit. This is known as the calorie. For convenience when large amounts of heat are involved a second unit just one thousand times as great is also used. This is called the kilocalorie or simply the large Calorie, distinguished from the small calorie by the use of the capital initial. Although the calorie is strictly a heat unit it serves 
as an expression for any form of energy. If we speak of any engine as able to furnish a certain number of calories we mean that if all the energy were to appear as heat that many calories would be liberated. As a matter of fact much of the energy may actually take other forms, as it does in the case of the contracting muscle.

When the energy is manifested as mechanical work it is measured in terms of the work done. Since the simplest form of work is probably the raising of weights against the resistance of gravity the units of work are based on weight and height. Thus the foot-pound is the amount of energy involved in raising a weight of one pound to a height of one foot. A calorie is approximately equivalent to 3 foot-pounds. In the metric system the unit of mechanical energy is the gram-centimeter. In round numbers 41,000 gram-centimeters represent the same amount of energy as 1 calorie.

The Energy Output of Muscle. Studies of the mechanical energy developed by selected groups of muscles in the Body can be made directly. An excellent method is by means of a stationary bicycle. The wheel can be made to revolve against a measured resistance, and thus the work done can be readily determined. As already stated, muscles in the Body work very inefficiently. This is in part due to the less favorable conditions of energy liberation in the physiological tetanus as compared with the simple twitch, but more because of the mechanical disadvantages at which muscles work. They pull at the short arms of levers, and the direction of their pull is usually oblique (Chap. VIII). The energy output of an isolated muscle is also easy to determine, although to be certain that the utmost possible has been obtained is not so simple. The muscle operates by forcible shortening. That means that the muscle pulls upon the weight to which it is attached. If the tension developed is greater than the weight the latter is lifted and work is done. If the tension is insufficient to raise the weight there is no manifestation of mechanical energy. All the energy liberated takes the form of heat. It has been found that in a given muscle under given conditions the tension developed is fairly constant and directly proportional to the total energy manifested in the contraction; whereas the actual mechanical work done depends, as we have already seen (p. 98) on various factors, such as the relation of the load to the absolute strength of the muscle. If 
the most favorable load is selected about half the energy of the contraction may, as we learned above (p. 107), appear as mechanical work.

In the study of the muscle as an engine knowledge of the energy relationships is of the greatest moment. We know that muscular activity is based on chemical transformations, and to explain the actions of the muscle we must know what these chemical transformations are. If we assume any particular transformation to be one from which the muscle derives its energy we must be able to show that that transformation yields the amount of energy which the muscle actually manifests. If it does not do so the assumption which selected it as the source of the muscle's power is obviously erroneous.

There are two facts of the chemistry of muscular activity which are fully demonstrated and which have already been stated. These are the oxidation of sugar or fat as fuel, and the production of lactic acid as an essential feature of the contraction process. Reference has already been made to the view formerly held that the lactic acid represents merely a stage in the oxidation of sugar, and to the replacement of that view by the present one. The evidence upon which is based this new recognition of the part played by lactic acid rests in large part upon consideration of the energy relationships.

When a skeletal muscle is exposed to the vapor of chloroform it passes into a condition of pronounced rigor. The strong contraction of rigor is the result of the production of lactic acid within the muscle. It differs chemically from ordinary contractions of the living muscle in that the production of acid goes on without concurrent oxidation of fuel, and in the fact that the lactic acid produced is not immediately removed. Mechanically, as already pointed out, the rigor differs from ordinary contraction in its persistence. The rigor contraction may last for hours, whereas the ordinary contraction ends with the cessation of stimulation. If the muscle that is to be subjected to the action of the chloroform is so firmly fixed at both ends that no actual shortening can occur all the energy of the rigor contraction appears in the form of heat and can be measured as such. Because there is no concurrent oxidation all the energy thus manifested must be derived directly from the chemical transformation which gives rise to the lactic 
acid. The amount of energy thus manifested has been found to equal about 1.3 calories for each gram of muscle. By chemical analysis the amount of lactic acid produced can be measured. It does not exceed 0.004 gram for each gram of muscle. The production of 0.004 gram lactic acid has given rise, then, to 1.3 calories of energy. The conversion of this amount of sugar to lactic acid, however, yields only 0.43 calories, or only one-third the amount of energy actually liberated. Evidently the transformation which actually occurs, the result of which is the production of $0.004 \mathrm{gm}$. lactic acid and 1.3 calories of energy for each gram of muscle, is some other than the direct conversion of sugar into acid.

These facts, which hold for the energy manifested during the contraction of rigor, are true also for the ordinary contractions of muscle except in so far as the latter are attended by oxidation processes, which liberate additional energy and complicate the determinations. If, however, oxidation be prevented, as can be done by surrounding the muscle with an atmosphere of pure nitrogen, substantially the same relationship between lactic acid production and energy manifestation appears as in rigor. A curious and important feature of the energy liberation of an ordinary contraction is that the oxidation that takes place in connection with the contraction accompanies, not the contraction phase proper, but the relaxation phase. For this reason it has been called the "recovery oxidation." This oxidation, as stated previously, is the ultimate source of the energy shown by the contracting muscle. How are we to connect this chemical process, occurring after the contraction is well under way, or even after it is over, with the energy shown during the contraction itself? The analogy that has been suggested, and that corresponds with the facts so far as we know them, is that of the pile driver. ' In this machine the energy of the burning fuel under the boiler is used in raising the weight to the top of the derrick. When the trip is opcrated the weight falls and by the energy of its impact does the work for which the machine is designed. In our analogy the production . of lactic acid corrresponds to the fall of the weight. The stimulus which excites the muscle is represented by the operation of the trip which releases the weight. The process, which, in the muscle, is analogous to the raising of the weight, is pictured as the formation of a substance which is decomposed into lactic acid under the 
influence of the stimulus and which in connection with this decomposition yields the amount of energy manifested by the contraction. The substance so pictured has not been demonstrated chemically. For lack of a definite name it has been called the "lactic acid precursor" to indicate its position as the energy-yielding antecedent substance to lactic acid. Since this substance must contain more energy than sugar, or it would not by its decomposition into lactic acid yield enough energy, it cannot be one of the fuel substances, but must be built up within the muscle at the expense of energy furnished by the fuel. If so built up it is not necessary that the oxidation of fuel occur in immediate connection with the contraction process, since all the oxidation has to do is to provide energy by which a supply of precursor is kept on hand. The muscle is then ready to respond whenever stimulation occurs.

To complete the picture we must account for the mechanical act of relaxation. Since contraction depends on the production of lactic acid in the muscle relaxation necessarily involves its disappearance. That the removal of lactic acid is a definite process is shown by the persistent contraction of rigor, which is due to the loss in the dead muscle of the means for getting rid of the acid. The immediate discharge of lactic acid in living muscles from the contractile elements is in all probability a simple outward diffusion from sarcostyles into sarcoplasm. Under ordinary. conditions the relaxation is too rapid to suggest a more complicated action. Unless the lactic acid is removed from the sarcoplasm, however, equilibrium will soon be reached between it and the sarcostyles and further diffusion will be impossible. There are at least three ways in which lactic acid might be removed. The simplest one, chemically, is by reacting with the alkaline salt of the muscle, sodium carbonate, to form neutral sodium lactate. A second possible method is by oxidation with the formation of carbon dioxid and water and with the liberation of much energy that would be available for the building up of the precursor. A third method, suggested by the analogy of the pile driver above, is the rebuilding of the acid itself into the precursor from which it was originally derived. The energy of the burning fucl might as well be devoted to the reconversion of lactic acid into precursor as to the building up of the precursor from some other substance than lactic acid, 
and if so devoted would have the additional advantage of caring for the removal of the acid. That either the second or third of these possibilities represents the method ordinarily operating in the muscle seems certain. Either of them would satisfy the known energy relationships in most respects. There is, however, one fact that seems to favor the replacement theory as against the oxidation theory. This is that if all the lactic acid produced in a simple contraction were oxidized in connection with the relaxation the total energy liberation would be several times that which actually occurs. This fact seems to identify the oxidation that does occur with the production of enough energy for the manufacture of the precursor, rather than with the removal of the lactic acid, and strengthens the view that the acid is removed by being rebuilt into precursor.

Under conditions of extreme muscular activity the blood is not able to deliver oxygen to the tissues fast enough to enable the oxidations by which lactic acid is removed to keep up with the production of the acid. There is, therefore, under these circumstances, an excess of lactic acid which must be removed if normal activity is to continue. In this situation the method of removal suggested first above comes into play, namely, the neutralization of the acid by sodium carbonate. The sodium lactate thus formed escapes from the muscles into the blood, is carried with the blood stream to the kidneys, and there eliminated. The proof of this lagging behind of the oxidations when the exercise is very vigorous is furnished by the appearance of considerable sodium lactate in the urine of persons who have recently undergone violent exertion. As compared with the removal of acid by oxidation this is evidently a wasteful process. The acid which escapes in combination with sodium is no longer available for reconstruction into precursor, and some other of the constituents of muscle must be used in its stead.

Significance of Lactic Acid in the Contraction Process. The relationship of lactic acid to the liberation of energy in muscle has been discussed in detail. There remains for consideration the manner in which the presence of the acid in the muscle brings about the mechanical act of contraction.

The most satisfactory explanation of this mechanism thus far suggested is based upon a property possessed by colloids of show- 
ing when acidified a greater affinity for water than they show when their reaction is neutral. The cylindrical colloidal sarcostyles which make up the actual contractile elements of muscle are surrounded by watery sarcoplasm (p. 88). If these sarcostyles are suddenly acidified we can picture a rush of water into them from the surrounding sarcoplasm, which would cause them to swell.

Studies of the contraction process in the individual sarcostyles of insects' wing muscles (p. 85) show that during contraction they present a beaded appearance. This beading could be brought about by a swelling of the segments of which the sarcostyles are composed, provided the membranes between the segments remain undistended (Fig. 48). That such a swelling might cause a forcible shortening can be proven with the aid of a suitable model. A similar swelling of the sarcostyles appears to occur during the contraction of skeletal muscle. If microscopic cross-sections of relaxed and contracted skeletal muscle are compared the sarcostyles of the relaxed specimen are seen to be smaller and further apart than are those of the contracted one. This suggests a transfer of sarcoplasm from the spaces between the sarcostyles

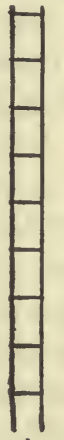

A

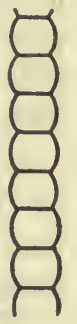

B

Fig. 48.-Diagrams of sarcostyles of insects' wing muscles. $A$, relaxed; $B$, contracted. into the sarcostyles themselves. When we recall the minute size of the elements and the correspondingly short distances through which any given particles of fluid would have to pass we can understand without difficulty how the contraction is able to occur in so small a fraction of a second.

Summary of the Contraction Process. The mechanism of contraction of skeletal muscle will be more readily grasped. as a whole, perhaps, if summarized briefly. In the resting muscle there has been some oxidation of fuel which has furnished energy for the building up of a substance of high energy value, the "lactic acid precursor." Stimulation of the muscle causes decomposition of some of this precursor into lactic acid with the liberation of energy. This energy is made available for the act of contraction through the property the colloidal sarcostyles have of absorbing water when acidified. The forcible absorption of water from the sur- 
rounding sarcoplasm brings about a swelling of the sarcostyles, which, by virtue of their peculiar segmental structure, results in turn in a forcible contraction. The shortening of the whole muscle is nothing more than the sum total of the contractions of the individual sarcostyles. Relaxation is brought about through the removal of the lactic acid, immediately by diffusion, but ultimately by being rebuilt into precursor at the expense of energy obtained through further oxidation of fuel.

Oxidation in Muscle. Reference has already been made (p. 104) to the fact that in the muscle oxidation occurs at about the temperature of the body, instead of at the high temperature characteristic of ordinary combustion. This is true of all oxidations in living cells. It is accomplished through the presence of special substances known as oxidases, belonging to the class of enzyms (p. 14). These have the property of bringing the oxygen and the fuel into such intimate relationship that they will combine chemically without first having to be heated to a high temperature.

Hormone of Skeletal Muscle. A curious fact about muscle is that the oxidation of sugar within it is subject to the control of a hormone. Why this control should exist is not clear, but that it does exist is proven beyond doubt. The hormone is secreted by certain masses of cells which are embedded in one of the digestive glands, the pancreas (p. 460). The importance of the hormone is shown by the dire results that follow its absence. A well-known, and unfortunately rather common disease, diabetes, is caused by the failure of these cell masses in the pancreas to manufacture their hormone in normal amounts. The muscles thereupon lose in greater or less degree the power to utilize sugar as fuel, and suffer, in consequence, more or less serious impairment of function. The unused sugar accumulates in the blood and is discharged through the kidneys, giving rise to the most conspicuous symptom of the disease, sugar in the urine. This condition is discussed in greater detail in a later chapter (p. 496).

Physiology of Smooth Muscle. Smooth muscle differs strikingly from skeletal muscle, not only, as stated previously, in structure, but also in mode of action. Aside from the fact that both sorts of muscle produce their effects by contraction they have almost no features in common. Weight for weight smooth muscle is much less powerful than skeletal muscle. Its movements are 
also much slower. Smooth muscle constitutes the operating machinery of the maintenance. systems (except the respiratory system), and it is as nicely adjusted to the special requirements of these systems as is skeletal muscle to the needs of external adaptation. One striking peculiarity of smooth muscle tissue is illustrated by the bladder. This organ sometimes contains a large amount of urine, at other times there is little or none in it. When the bladder is empty it is shrunken to a fraction of its size when full. The muscular walls are distended or contracted as the organ is full or empty. These pronounced changes appear to be effected, in part at least, by rearrangement of the cells which make up the muscle coats. When the organ is distended there is a smaller number of layers of cells than when it is contracted. Just how this rearrangement is brought about is not known.

Another feature in which smooth muscle differs strikingly from skeletal is in the tendency it often shows to carry on spontaneous contractions. Skeletal muscle, as previously emphasized, contracts only when subjected to definite stimulation. Smooth muscle, on the other hand, often undergoes periods of rhythmic contraction and relaxation when no obvious sources of stimulation are present.

Still another peculiarity of smooth muscle is its ability to remain indefinitely in the contracted state without fatigue. This property is seen in the muscular coats of the small arteries, many of which are never relaxed. They may be more strongly contracted at some times than at others but in health they are always in some degree of contraction.

There are in the body a number of sphincters, circular bands of smooth muscle which guard the openings of various organs such as the stomach, large intestine, and bladder. These are strongly contracted the greater part of the time, relaxation being for them only an occasional occurrence. They maintain their condition of strong contraction without fatigue and apparently without much expenditure of energy, offering in this regard a sharp contrast to skeletal muscle.

To excite skeletal muscle sharp stimuli, like the electric shock, are most efficient. Smooth muscle, on the other hand, responds best to slower, more prolonged excitants. A pull or pinch, which will ordinarily fail to cause contraction in such a muscle as the 
gastrocnemius, arouses a strip of stomach muscle to pronounced activity.

Mechanism of Contraction of Smooth Muscle. The structure of smooth muscle is, as shown formerly, much less complex than of skeletal muscle. No elaborate system of sarcostyles, sarcoplasm, and sarcolemma exists. The spindle-shaped cells with their environment of lymph are the contractile elements. In fact when we attempt to compare smooth muscle with skeletal we find that the smooth muscle-cell corresponds better to the sarcostyle than to the fiber, although the fiber is the cell unit. The lymph which bathes the cell of smooth muscle functions toward it as does the sarcoplasm toward the sarcostyle. Contraction of smooth muscle depends, then, on interaction of muscle-cell with lymph, as in skeletal muscle on interaction of sarcostyle with sarcoplasm. This latter interaction is of such a sort that to keep the sarcostyles in a state of contraction a continuous expenditure of energy is necessary, and fatigue is bound ultimately to occur. The expenditure of energy in smooth muscle, on the other hand, appears to take place only during an actual change in length, whether the change is a shortening or a lengthening; the maintenance of a given length after it is once attained seems not to require further energy liberation. This difference accounts for the ability of smooth muscle to continue in contraction without fatigue.

The suggestion made above, that energy expenditure occurs during change in length in smooth muscle, even though the change be a relaxation, is in harmony with the interesting fact that most, smooth muscle tissues are supplied with two sets of nerves. Stimulation by way of one set induces contraction; by way of the other, relaxation. This is in marked contrast with the situation in skeletal muscle, where the only function of stimulation is to arouse contraction; relaxation following spontaneously upon the release from excitation.

Heat rigor in smooth muscle shows an interesting difference from the same phenomenon in skeletal muscle. In the latter tissue the result of heating above the death point is a pronounced contraction. When smooth muscle is thus heated, instead of contracting it undergoes marked relaxation. We know that when skeletal muscle is heated there is production of lactic acid within it, and that this lactic acid brings about the shortening 
If the same treatment causes lactic acid to be produced in smooth muscle, we are obliged to conclude that the presence of this acid may cause relaxation in this tissue instead of contraction. This, again, is in harmony with the general idea that in smooth muscle any change in length may require the liberation of energy.

Physiology of Cardiac Muscle. In some features of its activity heart muscle resembles skeletal muscle; in others it is more like smooth muscle. The contraction of heart muscle is rapid and vigorous, in this respect corresponding to skeletal muscle. The liberation of energy in heart muscle is associated with contraction rather than with change in length as such. Here again the resemblance is with skeletal muscle rather than with smooth.

Cardiac muscle is like smooth muscle in that it has the power of executing spontaneous contractions. The heart receives, also, the double innervation referred to above as characteristic of smooth muscle.

In a number of important regards the heart differs from either of the other types of muscle. For convenience discussion of these special features is deferred to the section in which the heart is studied as an organ of the circulation (Chap. XX). 


\section{CHAPTER VIII}

\section{THE USE OF MUSCLES IN THE BODY}

The Special Physiology of the Skeletal Muscles. Having now considered separately the structure and properties in general of the skeleton, the joints, and the muscles, we may go on to consider how they all work together in the Body. Although the properties of muscular tissue are everywhere the same, the uses of different muscles are very varied, by reason of the different parts with which they are connected. Some are muscles of respiration, others of deglutition; many are known as flexors because they bend joints, others as extensors because they straighten them. The exact use of any particular muscle, acting alone or in concert with others, is known as its special physiology, as distinguished from its general physiology, or properties as a muscle without reference to its use as a muscle in a particular place. The functions of those muscles forming parts of the physiological mechanisms concerned in breathing and swallowing will be studied hereafter; for the present we may consider the muscles which cooperate in maintaining postures of the Body; in producing movements of its larger parts with reference to one another; and in producing locomotion or movement of the whole Body in space.

In nearly all cases the striated muscles carry out their functions with the co-operation of the skeleton, since nearly all are fixed to bones at each end, and when they contract primarily move these, and only secondarily the soft parts attached to them. To this general rule there are, however, exceptions. The muscle for example which lifts the upper eyelid and opens the eye arises from bone at the back of the orbit, but is inserted, not into bone: but into the eyelid directly; and similarly other muscles arising at the back of the orbit are directly fixed to the eyeball in front and serve to rotate it on the pad of fat on which it lies. Many facial muscles again have no direct attachment whatever to bones, 
as for example the muscle (orbicularis oris) which surrounds the mouth-opening, and by its contraction narrows it and purses out the lips; or the orbicularis palpebrarum which similarly surrounds the eyes and when it contracts closes them.

Levers in the Body. When the muscles serve to move bones the latter are in nearly all cases to be regarded as levers whose fulcra lie at the joint where the movement takes place. Examples of all the three forms of levers recognized in mechanics are found in the Human Body.

Levers of the First Order. Examples of the first form of lever (fulcrum between power and weight, Fig. 49), are not numerous in' the Human Body. One is afforded in the nodding movements of

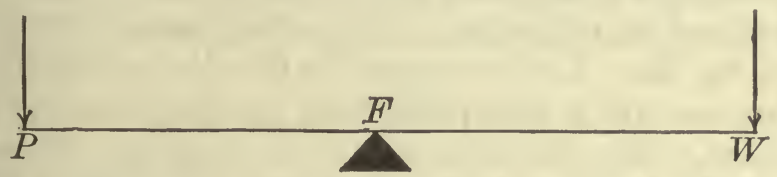

FIg. 49.-A lever of the first order. $F$, fulcrum; $P$, power; $W$, resistance or weight.

the head, the fulcrum being the articulations between the skull and the atlas. When the chin is elevated the power is applied to the skull, behind the fulcrum, by small muscles passing from the vertebral column to the occiput; the resistance is the excess in the weight of the part of the head in front of the fulcrum over that behind it, and is not great. To depress the chin as in nodding does not necessarily call for any muscular effort, as the head will fall forward of itself if the muscles keeping it erect cease to work, as those of us who have fallen asleep during a dull discourse on a hot day have learnt. If the chin however be depressed forcibly, as in the athletic feat of suspending one's self by the chin, the muscles passing from the chest to the skull in front of the occipital condyles are called into play. Another example of the employment of the first form of lever in the Body is afforded by the curtsey with which formerly one lady saluted another. In curtseying the trunk is bent forward at the hip-joints, which form the fulcrum; the weight is that of the trunk acting as if all concentrated at its center of gravity, which lies a little above the sacrum and behind the hip-joints; and the power is afforded by muscles passing from the thighs to the front of the pelvis. 
Levers of the Second Order. As an example of the employment of such levers (weight between power and fulcrum, Fig. 50) in the Body, we may take the act of standing on the toes. Here the foot represents the lever, the fulcrum is at the contact of its fore part with the ground; the weight is that of the Body acting

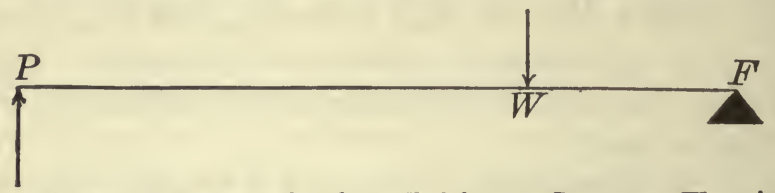

FIG. 50.-A lever of the second order. $F$, fulcrum; $P$, power; $W$, weight. The arrows indicate the direction in which the forces act.

down through the ankle-joints at Ta, Fig. 35; and the power is the great muscle of the calf acting by its tendon inserted into the heelbone ( $\mathrm{Ca}$, Fig. 35). Another example is afforded by holding up the thigh when one foot is kept raised from the ground, as in hopping on the other. Here the fulcrum is at the hip-joint, the power is applied at the knee-cap by a great muscle (quadriceps femoris) which is inserted there and arises from the pelvis; and the weight is that of the whole lower limb acting at its center of gravity, which lies somewhere in the thigh between the hip and knee-joints, that is, between the fulcrum and the point of application of the power.

Levers of the Thi-d Order. These are the levers most commonly used on the Human Body (power between weight and fulcrum, Fig. 51). For example, when the arm is bent at the elbow the fulcrum is the elbow-joint, the power is applied at the insertion of the biceps muscle (Fig. 39) into the radius and of

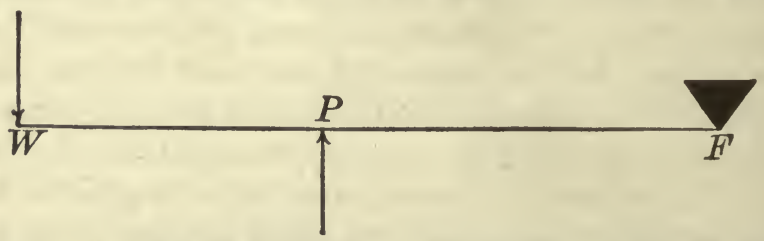

Fra. 51.-A lever of the third order. $F$, fulcrum; $P$, power; $W$, weight.

another muscle (not represented in the figure, the brachialis anticus,) into the ulna, and the weight is that of the forearm and hand, with whatever may be contained in the latter, acting at the center of gravity of the whole somewhere on the distal side 
of the point of application of the power. In the Body the powerarm is usually very short so as to gain speed and range of movement, the muscles being powerful enough to do their work in spite of the mechanical disadvantage at which they are placed. The limbs are thus made much more shapely than would be the case were the power applied near or beyond the weight.

It is of course only rarely that simple movements as those described above take place. In the great majority of those executed several or many muscles co-operate.

The Loss to the Muscles from the Direction of their Pull. It is worthy of note that, owing to the oblique direction in which the muscles are commonly inserted into the bones, much of their force is lost so far as producing movement is concerned. Suppose the log of wood in the diagram (Fig. 52) to be raised by pull-

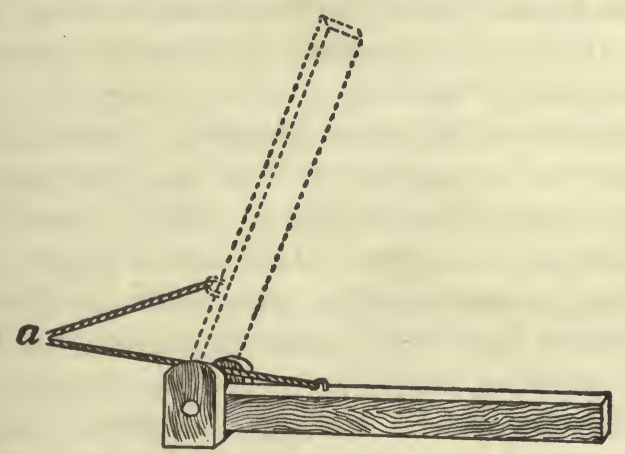

Frg. 52.-Diagram illustrating the disadvantage of an oblique pull.

ing on the rope in the direction $a$; it is clear at first that the rope will act at a great disadvantage; most of the pull transmitted by it will be exerted against the pivot on which the log hinges, and only a small fraction be available for elevating the latter. But the more the log is lifted, as for example into the position indicated by the dotted lines, the more useful will be the direction of the pull, and the more of it will be spent on the log and the less lost unavailingly in merely increasing the pressure at the hinge. If we now consider the action of the biceps (Fig. 39) in flexing the elbow-joint, we see similarly that the straighter the joint is, the more of the pull of the muscle is wasted. Beginning with the arm straight, it works at a great disadvantage, but as the forearm is 
raised the conditions become more and more favorable to the muscle. Those who have practiced the gymnastic feat of raising one's self by bending the elbows when hanging by the hands from a horizontal bar know practically that if the elbow-joints are quite straight it is very hard to start; and that, on the other hand, if they are kept a little flexed at the beginning the effort needed is much less; the reason being of course the more advantageous direction of traction by the biceps in the latter case.

Experiment proves that the power with which a muscle can contract is greatest at the commencement of its shortening, the very time at which, we have just seen, it works at most mechanical disadvantage; in proportion as its force becomes less the conditions become more favorable to it. There is, however, it is clear, nearly always a considerable loss of power in the working of the skeletal muscles, strength being sacrificed for variety, ease, rapidity, extent, and elegance of movement.

The Equilibrium of Opposing Muscles. The muscles are highly elastic bodies, and on account of their elasticity exert, even while not actively contracting, a definite elastic tension upon their points of insertion. We have, therefore, at every joint, constant opposing pulls of the resting muscles. The efficient manner in which the elastic tensions of opposing muscles balance each other is often very striking, particularly when one considers the marked differences in size and in mechanical advantage apt to exist in upposing muscles. Take, for example, the muscles which move the fore arm. The flexor muscle (biceps) is a larger and stronger muscle than the extensor (triceps). It has, moreover, a better leverage. Yet so far as elastic tension is concerned the two muscles balance perfectly. One appreciates best the significance of this equilibrium when a case is seen in which it has been lost. The disease known as infantile paralysis injures or destroys the nervous connections of the skeletal muscles, and often affects one group while leaving the opposing group unimpaired. The normal tension is lost in the affected muscles but persists in the unaffected ones. Unless special precautions are taken to prevent the occurrence, the muscles which still exert elastic tension will gradually shorten, pulling the joint into the position into which it is drawn by the normal contractions of these muscles, and holding it there 
permanently, and so forcibly that extreme measures must often be adopted to return the part to its normal resting situation. The importance of this constant tension during life is probably in the instant readiness it gives the muscles. There is never any slack to be taken up; motion of the joint is simultaneous with the contraction of the muscle.

Functional Muscle Groups. In attempting to analyze the exceedingly numerous and diverse muscular movements of which we are capable we have to bear in mind that in the state of nature for which our bodies were primarily adapted men's movements were directed to fewer and simpler ends. Our fundamental activities fall into a small number of groups, and we shall see that our more complex movements are but modifications of the fundamental ones.

According to this principle we can classify our muscular acts as follows: posture; locomotion; prehension (grasping); mastication (and swallowing); vision; voice production (including breathing). Of these groups posture, locomotion, and prehension are treated in following paragraphs. A separate chapter is devoted to voice production (Chap. XXXIII). The others are treated in detail in connection with the discussion of the particular bodily functions with which they are associated. Some points of general interest concerning them may not be out of place here. Among the facial muscles are found groups devoted to mastication; to vision; to voice production; and to prehension. The masticatory and visual muscles do not show in man any very striking differences from their functioning in other mammals. Prehension, which in man and the higher monkeys is taken over so largely by the front limbs, manifests itself among the facial muscles in the grasping power of the very flexible lips, which are in most of the lower mammals important grasping organs, but in man confined chiefly to the safe guidance of food into the mouth.

The tongue is a muscular mass composed of several distinct museles which are interwoven in such a manner that by their interaction they can draw, thrust, or twist the organ into the numerous positions and shapes it is capable of taking. The larger tongue muscles have a bony attachment at one end, either in the hyoid bone (p. 62) or the lower jaw. Their insertions are within the fleshy mass itself. There are besides these large muscles a number 
of smaller ones which are embedded wholly within the tongue. These have no bony attachments at either end.

In addition to its masticatory function (p. 469) the tongue is an essential part of the speech apparatus. Its importance is shown not only by the loss of the power of articulate speech from paralysis or removal of the tongue, but also by the fact that only those lower forms which have tongues at once fleshy and flexible (parrots, tongue-cut crows) can learn to talk. The ape's tongue is very similar to that of man. The fact that the parrot can learn to talk while the ape cannot, or will not, raises the interesting question in connection with the lower animals as to how much speech depends on tongue-structure and how much on intelligence or willingness to imitate sounds. The solution of this problem is a matter for the student of animal behavior rather than for the physiologist.

Postures. The term posture is applied to those positions of equilibrium of the Body which can be maintained for some time, such as standing, sitting, or lying, compared with leaping, running, or falling. In all postures the condition of stability is that the vertical line drawn through the center of gravity of the Body shall fall within the basis of support afforded by objects with which it is in contact; and the security of the posture is proportionate to the extent of this base, for the wider it is the less is the risk of the perpendicular through the center of gravity falling outside of it on slight displacement.

The Erect Posture. This is pre-eminently characteristic of man, his whole skeleton being modified with reference to it. Nevertheless the power of maintaining it is only slowly learnt in the first years after birth, and for a long while it is unsafe. And though finally we learn to stand erect without conscious attention, the maintenance of that posture always requires the co-operation of many muscles, co-ordinated by the nervous system. The influence of the latter is shown by the fall which follows a severe blow on the head, which may nevertheless have fractured no bone nor injured any muscle: the concussion of the brain, as we say, "stuns" the man, and until its effects have passed off he cannot stand upright. In standing with the arms straight by the sides and the feet together the center of gravity of the whole adult Body lies in the articulation between the sacrum and 
the last lumbar vertebra, and the perpendicular drawn from it will reach the ground between the two feet, within the basis of support afforded by them. With the feet close together, however, the posture is not very stable, and in standing we commonly make it more so by slightly separating them so as to increase the base. The more one foot is in front of the other the more swaying back and forward will be compatible with safety; and the greater the lateral distance separating them the greater will be the lateral sway which is possible without falling. Consequently we see that a man about to make great movements with the upper part of his Body, as in fencing or boxing, or a soldier preparing for the bayonet exercise, always commences by thrusting one foot forward obliquely, so as to increase his basis of support in both directions.

The ease with which we can stand is largely dependent upon the way in which the head is almost balanced on the top of the vertebral column, so that but little muscular effort is needed to keep it upright. In the same way the trunk is almost balanced on the hip-joints, but not quite, its center of gravity falling rather behind them; so that just as some muscular effort is needed to keep the head from falling forwards, some is needed to keep the trunk from toppling backwards at the hips. In a similar manner other muscles are called into play at other joints: as between the vertebral column and the pelvis, and at the knees and ankles; and thus a certain rigidity, due to

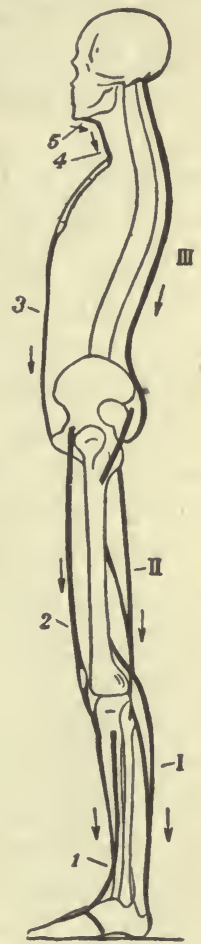

Fig. 53.-Diagram illustrating the muscles (drawn in thick black lines) which pass before and behind the joints and by their balanced activity keep the joints rigid and the body erect. muscular effort, extends all along the erect Body: which, on account of the flexibility of its joints, could not otherwise be balanced on its feet, as a statue can. Beginning (Fig. 53) at the ankle-joint, we find it kept stiff in standing by the combined and balanced contraction of the muscles passing from the heel to the thigh, and from the dorsum of the foot to the shin- 
bone (tibia). Others passing before and behind the knee-joint keep it from yielding; and so at the hip-joints: the others again, lying in the walls of the abdomen and along the vertebral column, keep the latter rigid and erect on the pelvis; and finally the skull is kept in position by muscles passing from the sternum and vertebral column to it, in front of and behind the occipital condyles.

Posture the Task of the Extensor Muscles. In the maintenance of posture the muscles which bear the strain are in general the extensors, since posture requires that the joints shall be kept from bending. So far as the flexors co-operate they do so by preventing overextension, a part which calls for relatively little exertion. The degree of pull manifested by the extensor muscles in the maintenance of posture is slight but steady. In the course of a day we may become aware of postural fatigue, showing that muscular activity has been present, although we may not have been conscious of definite volition. This mild degree of sustained contraction, which differs strikingly from the ordinary rapid and extensive contractions of skeletal muscle, is known as tonus. In the maintenance of posture it is chiefly extensor tonus. In what respects tonus is comparable with ordinary contraction and in what respects different, is not yet known.

Locomotion includes all the motions of the whole Body in space, dependent on its own muscular efforts: such as walking, running, leaping and swimming.

Walking. In walking the Body never entirely quits the ground, the heel of the advanced foot touching the ground in each step before the toe of the rear foot leaves it. The advanced limb supports the Body, and the foot in the rear at the commencement of each step propels it.

Suppose a man standing with his heels together to commence to walk, stepping out with the left foot: the whole Body is at first inclined forwards, the movement taking place mainly at the ankle-joints. By this means the center of gravity would be thrown in front of the base formed by the feet and a fall on the face result, were not simultaneously the left foot slightly raised by bending the knee and then swung forwards, the toes just clear of the ground, and, in good walking the sole nearly parallel to it. When the step is completed the left knee is straightened and the 
sole placed on the ground, the heel touching it first, and the base of support being thus widened from before back, a fall is prevented. Meanwhile the right leg is kept straight, but inclines forwards above with the trunk. when the latter advances, and as this occurs the sole gradually leaves the ground, commencing with the heel. When the step of the left leg is completed the great toe of the right alone is in contact with the support. With this a push is given which sends the trunk on over the left leg, which is now kept rigid, except at the ankle-joint; and the right knee being bent that limb swings forwards, its foot just clearing the ground as the left did before. The Body is meanwhile supported on the left foot alone, but when the right completes its step the knee of that leg is straightened and the foot thus placed, heel first, on the ground. Meanwhile the left foot has been gradually leaving the ground, and its toes only are at that moment upon it: from these a push is given, as before, with the right foot, and the knee being bent so as to raise the foot, the left leg swings forwards at the hip-joint to make a fresh step.

During each step the whole Body sways up and down and also from side to side. It is highest at the moment when the advancing trunk is vertically over the foot supporting it, and then sinks until the moment when the advancing foot touches the ground, when it is lowest. From this moment it rises as it swings forward on this foot, until it is vertically over it, and then sinks again until the other touches the ground; and so on. At the same time, as its weight is alternately transferred from the right to the left foot and vice versa, there is a slight lateral sway, commonly more marked in women than in men, and which when excessive produces an ugly "waddling" gait.

The length of each step is primarily dependent on the length of the legs; but can be controlled within wide limits by speciat muscular effort. In easy walking little muscular work is employed to carry the rear leg forwards after it has given its push. When its foot is raised from the ground it swings on, like a pendulum; but in fast walking the muscles, passing in front of the hipjoint, from the pelvis to the limb, by their contraction forcibly carry the leg forwards. The easiest step, that in which there is most economy of labor, is that in which the limb is let swing freely, and since a short pendulum swings faster than a longer, 
the natural step of short-legged people is quicker than that of long-legged ones.

In fast walking the advanced or supporting leg also aids in propulsion; the muscles passing in front of the ankle-joint contracting so as to pull the Body forwards over that foot and aid the push from the rear foot. Hence the fatigue and pain in front of the shin which is felt in prolonged, very fast walking. From the fact that each foot reaches the ground heel first, but leaves it toe last, the length of each stride is increased by the length of the foot.

Running. In this mode of progression there is a moment in each step when both feet are off the ground, the Body being unsupported in the air. The toes alone come in contact with the ground at each step, and the knee-joint is not straight when the foot reaches the ground. When the rear foot is to leave the support, the knee is suddenly straightened, and at the same time the ankle-joint is extended so as to push the toes forcibly on the ground and give the whole Body a powerful push forwards and upwards. Immediately after this the knee is greatly flexed and the foot raised from the ground, and this occurs before the toes of the forward foot reach the latter. The swinging leg in each step is violently pulled forwards and not suffered to swing naturally, as in walking. By this the rapidity of the succession of steps is increased, and at the same time the stride is made greater by the sort of one-legged leap that occurs through the jerk given by the straightening of the knee of the rear leg just before it leaves the ground.

Leaping. In this mode of progression the Body is raised completely from the ground for a considerable period. In a powerful leap the ankles, knees, and hip-joints are all flexed as a preparatory measure, so that the Body assumes a crouching attitude. The heels, next, are raised from the ground and the Body balanced on the toes. The center of gravity of the Body is then thrown forwards, and simultaneously the flexed joints are straightened, and by the resistance of the ground, the Body receives a propulsion forwards; much in the same way as a ball rebounds from a wall. The arms are at the same time thrown forwards. In leaping backwards, the Body and arms are inclined in that direction; and in jumping vertically there is no leaning either way and the arms are kept by the sides. 
Prehension. In man, and to a less extent in monkeys, the fore limbs are differentiated from organs of locomotion into prehensile structures. To a large degree the effectiveness of the fore limb as an organ of prehension depends on peculiarities of its bony framework. 'These have been described in detail in a previous chapter (p. 66). The chief special features, which it may not be amiss to recall, are three; the attachment of the shoulder girdle to the trunk by muscles rather than by a firm bony articulation; the rotation of the radius over the ulna; the opposibility of the thumb to each of the fingers. These skeletal features, which afford a groundwork for great flexibility of action, are made effective by the arrangement of the arm muscles. Although detailed description of this arrangement is outside the scope of this work, some general statements may properly be presented.

The muscles of the arm fall into three groups, shoulder muscles, muscles of the upper arm, muscles of the fore arm and hand. The muscles of the shoulder are arranged so that the arm can be raised or lowered; drawn forward or backward. Those at the back of the shoulder include within their mass the shoulder blade (scapula) in such a manner that in ordinary upward movements of the arm the rotation is about the shoulder joint, but in extensive upward movements, as in raising the arms above the head, the shoulder blade itself is pulled out of its ordinary horizontal position into a nearly vertical one.

The muscles of the upper arm are simple flexors and extensors since the elbow-joint is a hinge-joint, permitting no variety of movements. The muscles of the fore arm and hand have a great variety of movements to provide for', and are accordingly numerous and complicated. Only the arrangements for securing flexion and extension will be mentioned here. The front of the fore arm is made up of a number of muscles, of which most are flexors of the wrist or of the fingers. Similarly the back of the fore arm contains the extensors of wrist and of fingers. The tendons of the latter muscles form the conspicuous cords at the back of the hand. These flexors and extensors interact in an interesting fashion. Thus the flexors of the finger aid in flexion of the wrist. If flexion of the fingers without accompanying flexion of the wrist is desired the latter must be prevented by simultaneous contraction of the wrist extensors. Similarly the extensors of the fingers 
act also to extend the wrist. To secure extension of che fingers while the wrist is flexed the latter position must be maintained by activity of the wrist flexor to overcome the tendency of the finger extensors to extend the wrist.

Hygiene of the Muscles. The healthy working of the muscles needs of course a healthy state of the Body generally, so that they shall be supplied with proper materials for growth and repair, and have their wastes rapidly and efficiently removed. In other words, good food and pure air are necessary for a vigorous muscular system, a fact which trainers recognize in insisting upon a strict dietary, and in supervising generally the mode of life of those who are to engage in athletic contests. The muscles should also not be exposed to any considerable continued pressure, since this interferes with the flow of blood and lymph through them.

As far as the muscles themselves are directly concerned, exercise is the necessary condition of their best development. The muscles are so compactly built that the movement of blood and lymph through them is less free than in other tissues. During the act of contraction, however, the circulation both of blood and of lymph is augmented by the pressure of the muscle upon its own contents. For their proper nourishment most of the muscles are largely dependent upon this self-massage. A muscle which is permanently unused suffers serious impairment of nutrition, and usually degenerates and is absorbed, little finally being left but the connective tissue of the organ and a few muscle-fibers filled with oil-drops. This is well seen in cases of paralysis dependent on injury to the nerves. In such cases the muscles may themselves be perfectly healthy at first, but lying unused for weeks they become altered, and finally, when the nervous injury has been healed, the muscles may be found incapable of functional activity. The physician therefore is often careful to avoid this by exercising the paralyzed muscles daily by means of electrical shocks sent through the part; passive exercise, as by proper massage, is frequently of great use in such cases. The same fact is illustrated by the feeble and wasted condition of the muscles of a limb which has been kept for some time in splints. After the latter have been removed it is only slowly, by judicious and persistent exercise, that the long-idle muscles regain their former size and power. 
The great muscles of the "brawny" arm of the blacksmith or wrestler illustrate the reverse fact, the growth of the muscles by exercise. We may note, incidentally, that in this growth from exercise there is no increase in the number of muscle-fibers. The greater size is due to growth of the individual fibers. Exercise, to be effective, must be judicious; repeated frequently to the point of exhaustion it does harm; the period of repair is not sufficient to counteract the injurious effects of fatigue, and the muscles thus waste under too violent exercise as with too little. Rest should alternate with work, and that regularly, if benefit is to be obtained. Moreover, violent exercise should never be suddenly undertaken by one unused to it, not only lest the muscles suffer, but because muscular effort greatly increases the work of the heart. No general rule can be laid down as to the amount of exercise to be taken; for a healthy man in business the minimum would perhaps be represented by a daily walk of five miles.

Varieties of Exercise. In walking and running the muscles chiefly employed are those of the lower limbs and trunk. This is in part true of rowing, which when good is performed much more by the legs than the arms; especially when sliding seats are used. Hence any of these exercises alone is apt to leave the muscles of the chest and arms imperfectly exercised. Indeed, no one exercise employs equally or proportionately all the muscles: therefore gymnasia in which various feats of agility are practiced, so as to call different parts into play, have very great utility. It should be borne in mind, however, that the legs especially need strength; while the upper limbs, in which delicacy of movement, as a rule, is more desirable than power, do not require so much exercise; and the fact that gymnastic exercises are commonly carried on indoors is a great drawback to their value. When the weather permits, out-of-door exercise is far better than that carried on in even the best ventilated and lighted gymnasium. For those who are so fortunate as to possess a garden there is no better exercise, at suitable seasons, than an hour's daily digging in it; since this calls into play nearly all the muscles of the Body; while of games, lawn-tennis is perhaps the best from a hygienic view that has ever been invented, since it not only demands great muscular agility in every part of the Body, but trains the hand to work with the eye in a way that walking, running, row- 
ing and similar pursuits do not. For the same reasons baseball, cricket, and boxing are excellent.

Exercise in Infancy and Childhood. Young children have not only to strengthen their muscles by exercise, but also to learn to use them. Watch an infant trying to convey something to its mouth, and you will see how little control it has over its muscles. On the other hand, the healthy infant is never at rest when awake; it constantly throws its limbs around, grasps at all objects within its reach, coils itself about, and so gradually learns to exercise its powers. It is a good plan to leave every healthy child more than a few months old several times daily on a large bed, or even on a rug or carpeted floor, with as little covering as is safe, and that as loose as possible, and let it wriggle about as it pleases. In this way it will not only enjoy itself thoroughly, but gain strength and a knowledge of how to use its limbs. To keep a healthy child swathed all day in tight and heavy clothes is cruelty.

When a little. later the infant commences to crawl it is safe to permit it to as much as it wishes, but unwise to tempt it to do so when disinclined: the bones and muscles are still feeble and may be injured by too much work. The same is true of learning to walk.

From four or five to twelve years of age almost any form of exercise should be permitted, or even encouraged. During this time, however, the epiphyses of many bones are not firmly united to their shafts, and so anything tending to throw too great a strain on the joints should be avoided. After that up to commencing manhood or maidenhood any kind of outdoor exercise for healthy persons is good, and girls are all the better for being allowed to join in their brothers' sports. Half of the debility and general ill-health of so many of our women is the consequence of deficient exercise during early life.

Exercise in Youth should be regulated largely by sex; not that women are to be shut up and made pale, delicate, and unfit to share the duties or participate fully in the pleasures of life; but the other calls on the strength of the young woman render vigorous muscular work often unadvisable, especially under conditions where it is apt to be followed by a chill.

A healthy boy or young man may do nearly anything; but until twenty-two or twenty-three very prolonged effort is un- 
advisable. The frame is still not firmly knit or as capable of endurance as it will subsequently become.

Girls should be allowed to ride or play outdoor games in moderation, and in any case should not be cribbed in tight stays or tight boots. A flannel dress and proper lawn tennis shoes are as necessary for the healthy and safe enjoyment of an afternoon at that game by a girl as they are for her brother in the baseball field. Rowing is excellent for girls if there be any one to teach them to do it properly with the legs and back, and not with the arms only, as women are so apt to row. Properly practiced it strengthens the back and improves the carriage.

Exercise in Adult Life. Up to forty a man may carry on safely the exercises of youth, but after that sudden efforts should be avoided. A lad of twenty-one or so may, if trained, safely run a quarter-mile race, but to a man of forty-five it would be dangerous, for with the rigidity of the cartilages and blood-vessels which begins to show itself about that time comes a diminished power of meeting a sudden violent demand. On the other hand, the man of thirty would more safely than the lad of nineteen or twenty undertake one of the long-distance walking matches such as used to be in vogue; the prolonged eifort would be less dangerous to him, though a six-days' match, with its attendant loss of sleep, cannot fail to be more or less dangerous to any one. Probably for one engaged in active business a walk of two or three miles to it in the morning and back again in the afternoon is . the best and most available exercise. The habit which Americans have everywhere acquired, of never walking when they can take a street car, is certainly detrimental to the general health; though the extremes of heat and cold to which we are subject often render it unavoidable.

For women during middle life the same rules apply: there should be some regular but not violent daily exercise.

In Old Age the needful amount of exercise is less, and it is still more important to avoid sudden or violent effort.

Exercise for Invalids. This should be regulated under medical advice. For feeble persons gymnastic exercises are especially valuable, since from their variety they permit of selection according to the condition of the individual; and their amount can be cunveniently controlled. 
Training. If any person attempt some unusual exercise he soon finds that he loses breath, gets perhaps a "stitch in the side," and feels his heart 'beating with unwonted violence. If he persevere he will probably faint - or vomit, as is frequently seen in the case of imperfectly trained men at the end of a hard boat-race. These phenomena are avoided by careful gradual preparation known as "training." The immediate cause of them lies in disturbances of the circulatory and respiratory organs, on which excessive work is thrown. 


\section{CHAPTER IX}

\section{ANATOMY OF THE NERVOUS SYSTEM}

General Statement. In Chapter III the special function of the nervous system was outlined, and was shown to involve the transmission of stimuli from the sensory regions of the Body to the active tissues (muscles and glands), and in the course of such transmission to make whatever modifications are necessary to the production of the best,results. The sensory regions of the Body are numerous; there are likewise many muscles. Successful adaptation of the individual to his surroundings may call at one time or another for the passage of stimuli from any sensory region to any muscle, or for the combination of stimuli from several sensory regions to form stimuli to go to any group of muscles. A somewhat analogous situation occurs in the telephone systems which are such important features of modern life. In these communication may be desired between any pair of instruments in the system. To make this possible all the telephones in any one system are led into a central exchange where provision is made for connecting any instrument with any other. Flexibility of communication between sensory and motor regions in the Body is secured in somewhat similar fashion. All nerves from sensory regions are led into a central "exchange" from which start all nerves to the motor organs.

Nerve Impulses. Since it is impossible to describe the nervous system without frequent reference to the messages which nerves carry it is desirable before proceeding farther to state that it has become the custom to call these messages nerve impulses. When we speak of a nerve impulse we have in mind the process by which the message is transmitted along the nerve. The situation corresponds to that in a telephone wire. When the latter is transmitting a message the words spoken into the transmitter are not carried along, but an electrical disturbance which they set up. So the nerve does not transmit the exact stimulus which acts upon it, but a nerve impulse which the stimulus arouses. 
Neurons. The nervous system as a whole is made up of struclures called neurons, each of which seems to be a single nervecell.

A typical neuron consists of a cell-body containing a nucleus and from whose surface project many rather short branching processes called dendrites, and a single long process having few if any branches and known as the axon (Fig. 55A). Neurons which convey impulses to muscles (motor neurons) have this structure (Fig.

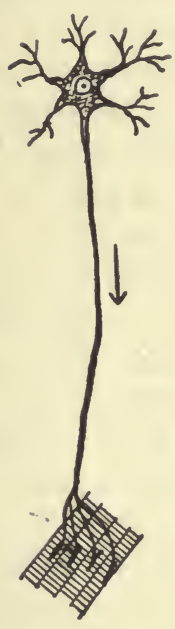

$A$

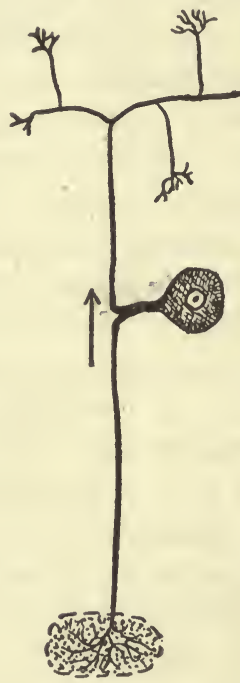

$B$

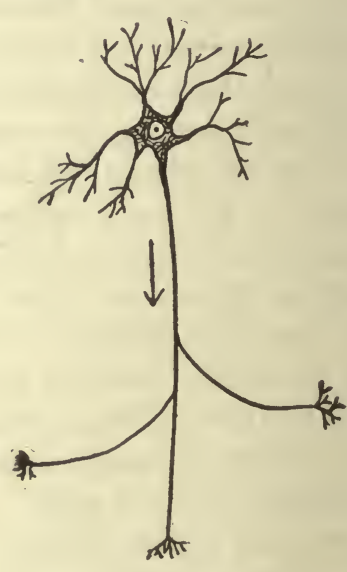

$C$

Fig. 54.-Types of neurons. $A$, motor; $B$, sensory; $C$, association.

$54 A$ ). The only branching of a motor neuron is at its very end, where it is distributed to the muscle fibers of which it has control. The number of muscle fibers thus innervated by one motor neuron varies in different muscles, ranging from a half dozen to fifty or more.

The neurons which convey impulses from sensory regions to the center (sensory neurons) have a structure which appears at first view, to be altogether different from that of the typical neuron just described. They have cell-bodies with nuclei but instead of a single axon and numerous much-branched dendrites the cell-body gives rise to two long axon-like processes, one connecting with the 
receptor and the other having a number of branches. The bipolar character of these neurons, moreover, is concealed in many through the union of the two processes for a short distance from the cellbody, giving an appearance as though the latter were on a side branch of a long axon (Fig. 54B). The underlying similarity of these to the type neuron appears if we consider that the dendrites of the typical neuron are replaced in the sensory neuron by the axon-like process which connects with the receptor.

A third sort of neurons occurring in the Body resembles the first or motor type in the possession of cell-body and many branching dendrites. Instead of long, slightly branched axons, however, neurons of this sort have short and very much-branched ones. These neurons occur interposed in the pathway of impulses from sensory to motor neurons and are often called association neurons (Fig. 54C): they are not, however, the only sort of association neurons; many neurons which belong physiologically to the group of association neurons in that they form communicating paths between sensory and motor neurons are anatomically of the type to which all motor neurons belong.

If we adopt the usual view that each single neuron represents one nerve-cell, neurons are the largest cells known. Although axons are so small in cross-section as to be microscopic they may have a length of three feet or more, as in the nerve trunks which extend down the legs to the feet.

The nervous system as a whole is made up of neurons of these three types. The sensory neurons, as stated above, lead from the receptors to the center; motor neurons lead from the center to the active tissues; and association neurons form the connecting links wherever such are necessary. All sensory neurons communicate with other neurons at their central terminations. Since the central axons are branched (see above) each sensory neuron has a number of such connections. All motor neurons have likewise connections with other neurons at their central ends. Association neurons connect with other neurons at both ends, as they must if they are to serve as links in a chain whose ends are sensory and motor neurons.

Synapses. Communication between neuron and neuron is always according to a certain scheme. The axons of all except motor neurons end in masses of fine branches known as end arbori- 
zations. These are in contact with the branching dendrites of some other neuron. The surfaces of contact between the end arborization of one neuron and the dendrites of another constitute what is called a synapse. In order for a nerve impulse to pass from one neuron to another it must cross this synapse.

The Myelin Sheath. All true nerve tissue has a characteristic gray color. This statement applies equally to cell-bodies, dendrites, and axons. Most, but not all, of the long axons of the body are inclosed within sheaths composed chiefly of a substance, myelin, which has a characteristic glossy white color. The myelin sheath where present does not inclose the axon throughout its entire length; near the cell-body and again near its termination the axon is not inclosed. Surrounding the myelin sheath, or, where it is absent, the axon itself, is a delicate membrane, the neurilemma. The myelin sheath is made up of short segments which are separated one from another by the nodes of Ranvier.

The myelin sheath is not composed of living cells and so does not contain nuclei. The neurilemma, however, is a living membrane; scattered along it at intervals are nuclei. The function of the myelin sheath is not known. Perhaps the most satisfactory suggestion that has been offered is that it serves as an insulator to keep the nerve impulse within its own axon and prevent its escape to adjacent ones.

Axons which are inclosed in myelin sheaths are spoken of as medullated or myelinated nerve-fibers.

It is the presence of myelin sheaths that gives to certain parts of the nervous system their characteristic white appearance. All "white matter" is made up of medullated axons. "Gray matter," on the other hand, is made of cell-bodies and dendrites, together with some non-medullated axons.

The Central and Peripheral Nervous Systems. In a preceding paragraph was pointed out the analogy between the nervous system and a telephone system. That part of the nervous system corresponding to the telephone "exchange," to which sensory neurons lead and from which motor neurons spring is called the central nervous system. It consists of the brain and spinal cord. (The analogy between the central nervous system and a telephone exchange should not be pushed too far, for the central nervous 
system has numerous functions in addition to the simple one of making connections between sensory and motor neurons. These special functions have to do with the modification of the impulses passing through it for the best advantage of the organism as a whole.)

Springing from the central nervous system and corresponding to the cables bearing wires to individual telephones are fortythree pairs of nerve-trunks. Twelve pairs arise from the brain and are called cranial nerves; the remaining thirty-one pairs arise from the spinal cord and are called spinal nerves. Each nerve-trunk contains a large number of axons, and in most nerve-trunks the axons of both motor and sensory neurons are present. These forty-three pairs of nerve-trunks with their ramifications to all parts of the Body constitute the peripheral nervous system (Fig. 55).

There are in the Body a set of neurons which though part of the peripheral nervous system are specially adapted for a certain function and are therefore usually considered independently. These constitute the sympathetic or autonomic system.

The Central Nervous System and its Membranes. Lying inside the skull is the brain and in the neural canal of the vertebral column the spinal cord, the two being continuous through the foramen magnum of the occipital bone. The central nervous system is bilaterally symmetrical throughout except for slight differences on the surfaces of parts of the brain, which are often found in the higher races of mankind. Both brain and spinal cord are very soft and easily crushed; nervous tissue as well as the connective tissue and a peculiar supporting tissue (neuroglia) which pervades it being delicate; accordingly both organs are placed in nearly completely closed bony cavities and are also enveloped by membranes which give them support. These membranes are three in number. Externally is the dura mater, very tough and strong and composed of white fibrous and elastic connective tissues. In the cranium the dura mater adheres by its outer surface to the inside of the skull chamber, serving as the periosteum of its bones; this is not the case in the vertebral column, where the dura mater forms a loose sheath around the spinal cord and is only attached here and there to the surrounding bones, which have a separate periosteum of their own. The innermost membrane lies in immediate contact with the proper nervous parts. 


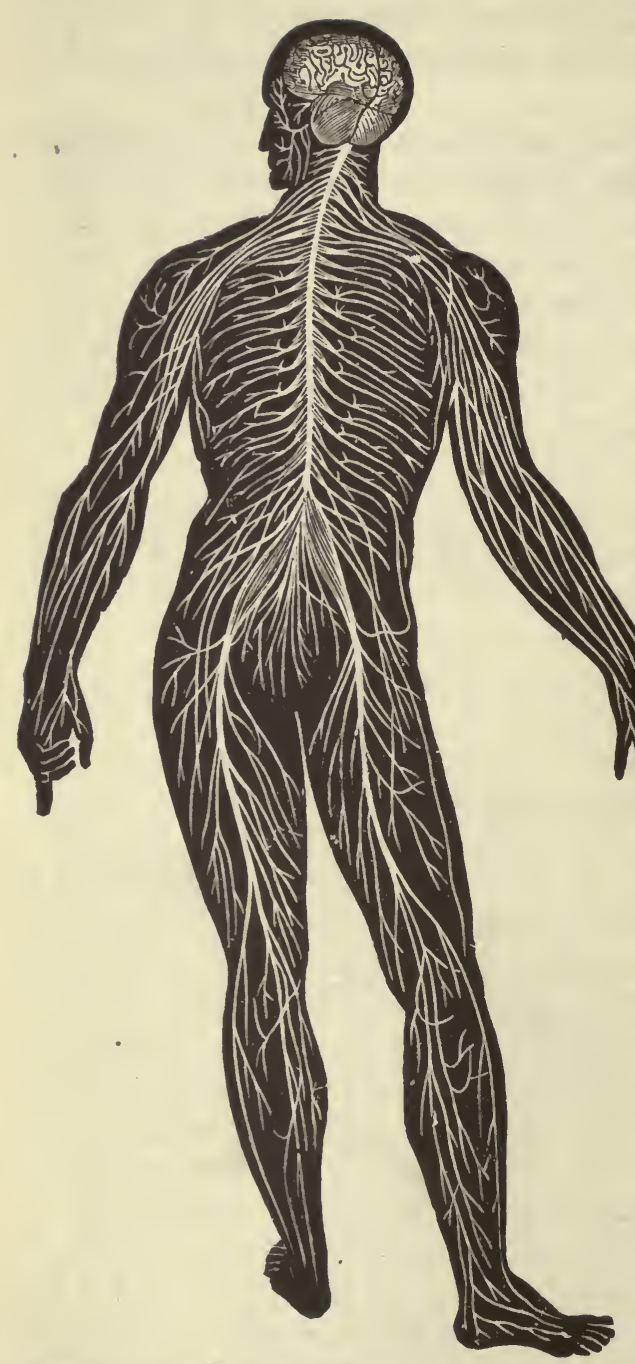

Fu. 55.-Diagram illustrating the general arrangement of the nervous system.
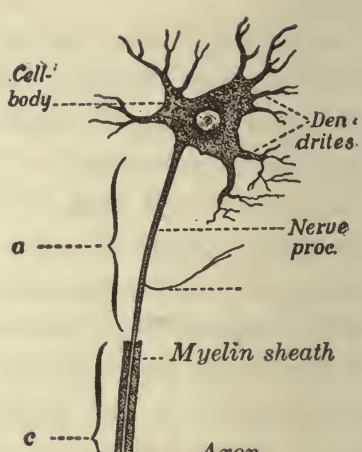
This is the pia mater, also made up of white fibrous tissue interwoven with elastic fibers, but less closely than in the dura mater, so as to form a less dense and tough membrane. The pia mater contains many blood-vessels which break up in it into small branches before entering the nervous mass beneath. Covering the outside of the pia mater is a layer of flat closely fitting cells; a similar layer lines the inside of the dura mater, and these two layers make up the third membrane, called the arachnoid. In the space between the two layers of the arachnoid is contained a small quantity of watery cerebrospinal liquid. The surface of the brain is folded and the pia mater follows closely these folds; the arachnoid often stretches across them: in the spaces thus left between it and the pia mater is contained some of the cerebrospinal liquid.

Ventricles of the Brain and Central Canal of the Spinal Cord. The central nervous system begins its embryonic development as a groove in the layer of cells which forms the upper surface of the embryo. This groove deepens, and finally cuts itself off from the cell layer of which it was at first a part. The edges grow together transforming the groove into a tube. The cell layer heals over, leaving the neural tube beneath it. The hollow in the tube persists throughout life. In the adult spinal cord it is represented by the tiny central canal (Fig. 65). As the front end of the neural tube develops into the complex brain the hollow in this region

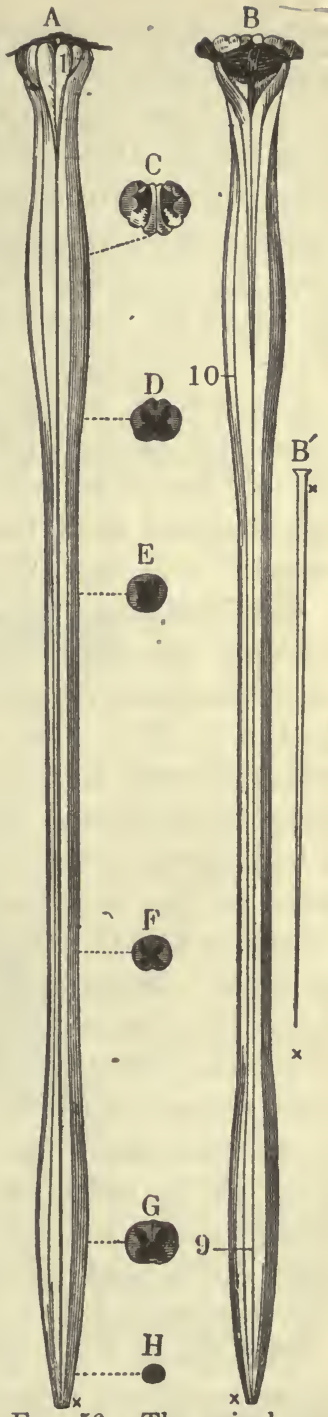

FIG. 56.-The spinal cord and medulla oblongata. A, from the ventral, and $B$, from the dorsal aspect; $C$ to $H$, crosssections at different levels. takes the form of a series of chambers of extremely irregular shape, communicating with each other and with the central canal of the 
spinal cord by narrow channels. The chambers are four in number, and are known as the ventricles of the brain. There is one in each cerebral hemisphere (p. 145). These are called the iateral ventricles. Numerically they rank as first and second. The lateral ventricles open into a narrow chamber in the base of the cerebrum in the mid line, known as the third ventricle. This in turn communicates by a narrow channel (the aqueduct of Sylvius) with the cavity of the brain stem (p. 146) which is called the fourth ventricle.

The cavity of the fourth ventricle communicates with the arachnoid space (p. 141) by three small openings in its roof, one in the mid line and one at each lateral border. By these openings the cerebrospinal fluid which occupies the arachnoid space is continuous with that which fills the ventricles and central canal.

Cerebrospinal Fluid. This fluid, which occupies-all the spaces within and around the central nervous system, is in general similar to the medium, lymph, by which the other tissues of the body are bathed (p. 18). It represents some chemical differences, however, which become accentuated in certain diseases. In the lumbar region there is room between the processes of the vertebræ so that a hypodermic needle can be thrust into the arachnoid space and some of the cerebrospinal fluid withdrawn. The operation is simple and by the application of cocaine to the skin made virtually painless. Chemical examination of the fluid so obtained is often helpful in diagnosing obscure complaints. Under certain diseased conditions there is a great accumulation of cerebrospinal fluid. The pressure of this upon the delicate nervous structures is likely to do them harm, and "lumbar puncture" is often resorted to to draw off the accumulated fluid and relieve the pressure. Sometimes in young children the accumulation of fluid distends the head far beyond its normal size, giving the condition known as "hydrocephalus."

The Spinal Cord (Fig. 56) is nearly cylindrical in form, being however a little wider from side to side than dorsiventrally, and tapering off at its posterior end. Its average diameter is about 19 millimeters ( $\frac{8}{4}$ inch) and its length 0.43 meter (17 inches). It weighs 42.5 grams ( $1 \frac{1}{2}$ ounces). There is no marked limit between the spinal cord and the brain, the one passing gradually into the other (Fig. 62), but the cord is arbitrarily said to commence 
opposite the outer margin of the foramen magnum of the occipital bone: from there it extends to the articulation between the first and second lumbar vertebræ, where it narrows off to a slender nonnervous filament, the filum terminale (cut off and represented separately at $B^{\prime}$ in Fig. 56), which runs back to the end of the neural canal behind the sacrum. In its course the cord presents two expansions, an upper, 10, the cervical enlargement, reaching from the third cervical to the first dorsal vertebræ, and a lower or lumbar enlargement, 9, opposite the last dorsal vertebra.

Running along the middle line on both the ventral and the dorsal aspects of the cord is a groove, and a cross-section shows

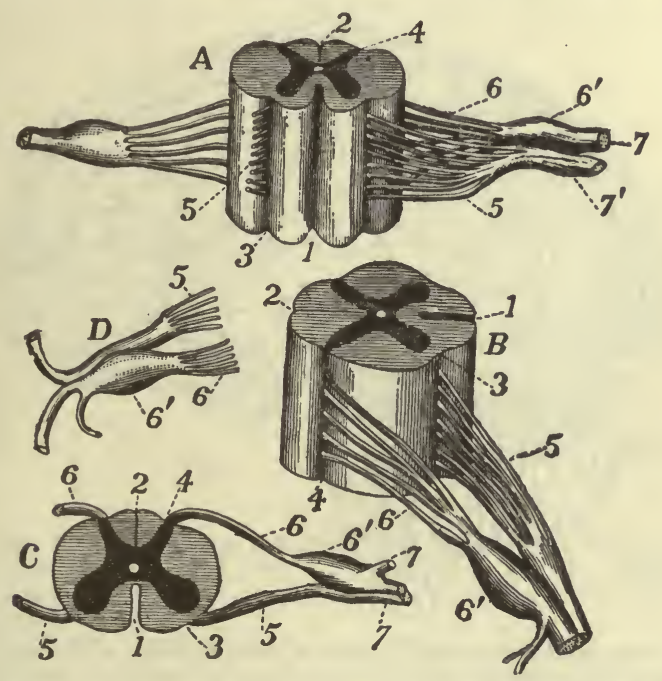

FIG. 57.-The spinal cord and nerve-roots. $A$, a small portion of the cord seen from the ventral side; $B$, the same seen laterally; $C$, a eross-section of the cord; $D$, the two roots of a spinal nerve; 1 , ventral fissure; 2 , dorsal fissure; 3 , surface groove along the line of attachment of the ventral nerve-roots; 4 , line of origin of the dorsal roots; 5 , ventral root filaments of spinal nerve; 6 , dorsal root filaments; $6^{\prime}$, ganglion of the dorsal root; $7,7^{\prime}$, the first two divisions of the nerve-trunk after its formation by the union of the two roots. The grooves are much exaggerated.

that these grooves are the surface indications of fissures which extend deeply into the cord ( $C$, Fig. 57$)$ and nearly divide it into right and left halves.

The ventral fissure (1, Fig. 57) is wider and shallower than the dorsal, 2, which indeed is hardly a true fissure, being completely filled up by an ingrowth of pia mater. The transverse section, 
$C$, shows also that the substance of the cord is not alike throughout, but that its white superficial layers envelop a central gray substance arranged somewhat in the form of a capital H. Each half of the gray matter is crescent-shaped, and the crescents are turned back to back and united across the middle line by the gray commissure. The tips of each crescent are called its horns or cornua, and the ventral horn on each side is thicker and larger than the dorsal. In the cervical and lumbar enlargements the proportion of white to gray matter is greater than elsewhere; and as the cord approaches the medulla oblongata its central gray mass becomes irregular in form and begins to break up into smaller

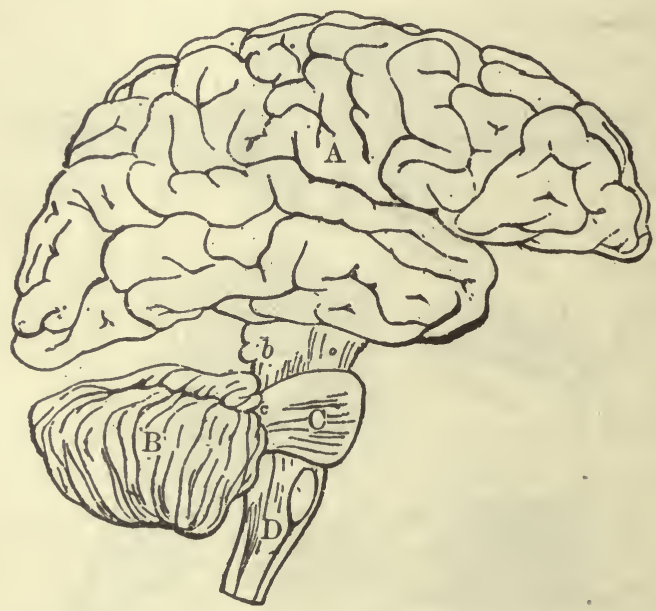

FrG. 58.-Diagram illustrating the general relationships of the parts of the brain. $A$, fore-brain; $b$, midbrain; $B$, cerebellum; $C$, pons Varolii; $D$, medulla oblongata; $B, C$, and $D$ together constitute the hind-brain.

portions. If lines be drawn on the transverse section of the cord from the tip of each horn of the gray matter to the nearest point of the surface, the white substance in each half will be divided into three portions: one between the ventral fissure and the ventral cornu, and called the ventral white column; one between the dorsal fissure and the dorsal cornu, and called the dorsal white column; while the remaining one lying in the hollow of the crescent and between the two horns is the lateral column: the ventral and lateral columns of the same side are frequently named the ventrolateral column. A certain amount of white substance crosses the middle 
line at the bottom of the ventral fissure; this forms the ventral white commissure. There is no dorsal white commissure, the bottom of the dorsal fissure being the only portion of the cord where the gray substance is uncovered by white. Running along the middle of the gray commissure, for the whole length of the cord, is the central canal, previously described. It is a tiny channel, just visible to the unaided eye.

The Brain (Fig. 58) is far larger than the spinal cord and more complex in structure. It weighs on the average about 1,415 grams (50 ounces) in the adult male, and about 155 grams ( 5.5 ounces) less in the female. In its simpler forms the vertebrate brain con-

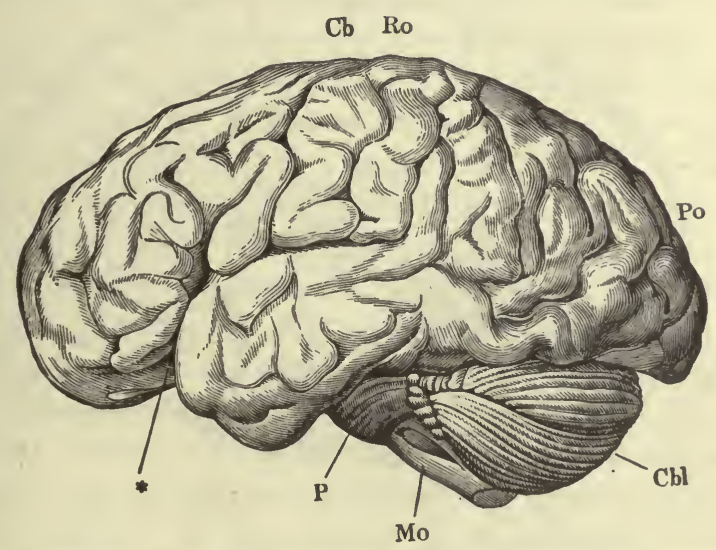

Fig. 59.-The brain from the left side. $C b$, the cerebral hemispheres forming the main bulk of the fore-brain; $C b l$, the cerebellum; $M o$, the medulla oblongata; $P$, the pons Varolii; *, the fissure of Sylvius; Ro, the fissure of Rolando; $P o$, the Parieto-occipital fissure.

sists of three masses, each with subsidiary parts, following one another in series from before back, and known as the fore-brain, midbrain, and hind-brain respectively. In man the fore-brain, $A$, weighing about 1,245 grams (44 ounces), is much larger than all the rest put together and laps over them behind. It consists mainly of two large convoluted masses, separated from one another by a deep median fissure, and known as the cerebral hemispheres. The immense proportionate size of these is very characteristic of the human brain. Beneath each cerebral hemisphere is an olfactory lobe, inconspicuous in man but in many animals larger than the cerebral hemispheres. Buried in the fore-brain 
on each side are two large gray masses, the corpora striata and optic thalami. The midbrain forms a connecting. isthmus between the two other divisions and presents on its dorsal side four hemispherical eminences, the corpora quadrigemina or collicuti. On its ventral side it exhibits two semicylindrical pillars (seen under the nerve $I V$ in Fig. 62), known as the crura cerebri. The hindbrain consists of three main parts: on its dorsal side is the cerebellum, $B$ (Fig. 58), consisting of a right, a left, and a median lobe; on the ventral side is the pons Varolii, $C$ (Fig. 58), and behind

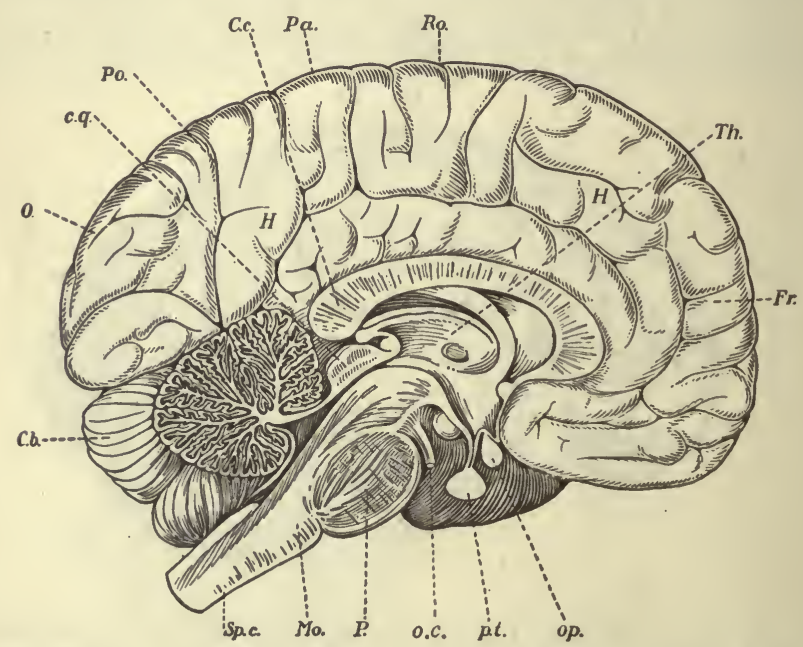

FIG. 60.-Diagram of the left half of a vertical median section of the brain, (Sobotta-McMurrich, Atlas and Text-book of Human Anatomy). H, H, convoluted inner surface of left cerebral hemisphere; $C c$, corpus callosun; $T h$, optic thalamus; c.q., corpora quadrigemina; $C b$, cerebellum; Sp.c, spinal cord; $M o$. medulla oblongata; $P$, pons Varolii; $o c$, oculo-motor nerve; $p t$, pituitary body; $o p$, optic nerve; $R o$, fissure of Rolando; $\mathrm{Po}$, parieto-occipital fissure; $\mathrm{Fr}$, frontal lobe; $P a$, parietal lobe; $O$, occipital lobe.

that the medulla oblongata, $D$ (Fig. 58), which is continuous with the spinal cord. The medulla and midbrain together make up the brain stem.

In nature, the main divisions of the brain are not separated so much as has been represented in the diagram for the sake of clearness, but lie close together, as represented in Fig. 59, only some folds of the membranes extending between them; and the midbrain is entirely covered in on its dorsal aspect. Nearly everywhere the surface of the brain is folded, the folds, known as gyri 
or convolutions being deeper and more numerous in the brain of man than in that of the animals nearest allied to him; and in the human species more marked in the higher than in the lower races. It should however be added that some species of animals which are not markedly intelligent have much convoluted cerebral hemispheres.

The brain, like the spinal cord, consists of gray and white nervous matter, but somewhat differently arranged, for while the brain, like the cord, contains gray matter in its interior, a great part of its surface is also covered with it. By the external convolutions of the cerebellum and the cerebral hemispheres the surface over which this gray substance is spread is very much increased (see Fig. 59).

The Spinal Nerves. Thirty-one pairs of spinal nerve-trunks enter the neural canal of the vertebral column through the intervertebral foramina (p. 57). Each divides in the foramen into a dorsal and ventral portion known respectively as the dorsal and ventral roots of the nerve ( 6 and 5 , Fig. 57), and these again subdivide into finer branches which are attached to the sides of the cord, the dorsal root at the point where the dorsal and lateral white columns meet, and the ventral root at the junction of the lateral and ventral columns. Although the nerve-trunks contain both sensory and motor neurons these are completely separated in the roots; the dorsal root contains only sensory neurons; the ventral only motor. At the lines on which the roots are attached there are superficial furrows on the surface of the cord. On each dorsal root is a spinal ganglion (6', Fig. 57), placed just before it joins the ventral root to make up the common nerve-trunk. This spinal ganglion contains the cell-bodies of the bipolar sensory neurons. Immediately after its formation by the mixture of fibers from both roots, the trunk divides ( $D$, Fig. 57), into a dorsal primary, a ventral primary, and a communicating branch. The branches of the first set go for the most part to the skin and muscles on the back; from the second the nerves for the sides and ventral region of the neck and trunk and for the limbs arise; the communicating branches form part of the sympathetic system.

The various spinal nerves are named from the portions of the vertebral column through the intervertebral openings of which they pass out; and as a general rule each nerve is named from the vertebra in front of it. For example, the nerve passing out be- 
tween the fifth and sixth thoracic vertebræ is the "fifth thoracic" nerve, and that between the last thoracic and first lumbar vertebræ, the "twelfth thoracic." In the cervical region, however, this rule is not adhered to. The nerve passing out between the occipitai bone and the atlas is called the "first cervical" nerve, that between the atlas and axis the second, and so on; that between seventh cervical and first thoracic vertebræ being the "eighth cervical" nerve. The thirty-one pairs of spinal nerves are then thus distributed: 8 cervical, 12 thoracic, 5 lumbar, 5 sacral, and 1 coccygeal; the latter passing out between the sacrum and coccyx. Since the spinal cord ends opposite the upper lumbar vertebræ while the sacral and coccygeal nerves pass out from the neural canal much farther back, it is clear that the roots of those nerves, on their way to unite in the foramina of exit and form nerve-trunks, must run obliquely backwards in the spinal canal for a considerable distance. One finds in fact the neural canal in the lumbar and sacral regions, behind the point where the spinal cord has tapered off to form the filum terminale, occupied chiefly by a great bunch of nerve-roots forming the so-called "horse's tail" or cauda equina.

Plexuses. Very frequently several neighboring nerve-trunks send off communicating branches to one another, each branch carrying fibers from one trunk to the other. Such networks are called plexuses (Fig. 61), and through the interchanges taking place in them it often happens that the distal branches of a nervetrunk contain fibers which it does not possess as it leaves the central nervous system.

Distribution of the Spinal Nerves. It would be out of place here to go into detail as to the exact portions of the Body supplied by each spinal nerve, but the following general statements may be made. The ventral primary branches of the first four cervical nerves form on each side the cervical plexus (Fig. 61) from which branches are supplied to the muscles and skin of the neck: also to the outer ear and the back part of the scalp. The ventral primary branches of the remaining cervical nerves and the first dorsal form the brachial plexus, from which the upper limb is supplied. The roots of the trunks which form this plexus arise from the cervical enlargement of the spinal cord.

From the fourth and fifth cervical nerves on each side, small 
branches arise and unite to make the phrenic nerve (4', Fig. 61) which runs down through the chest and ends in the diaphragm.

The ventral primary branches of the thoracic nerves, except part of the first which enters the brachial plexus, form no plexus,

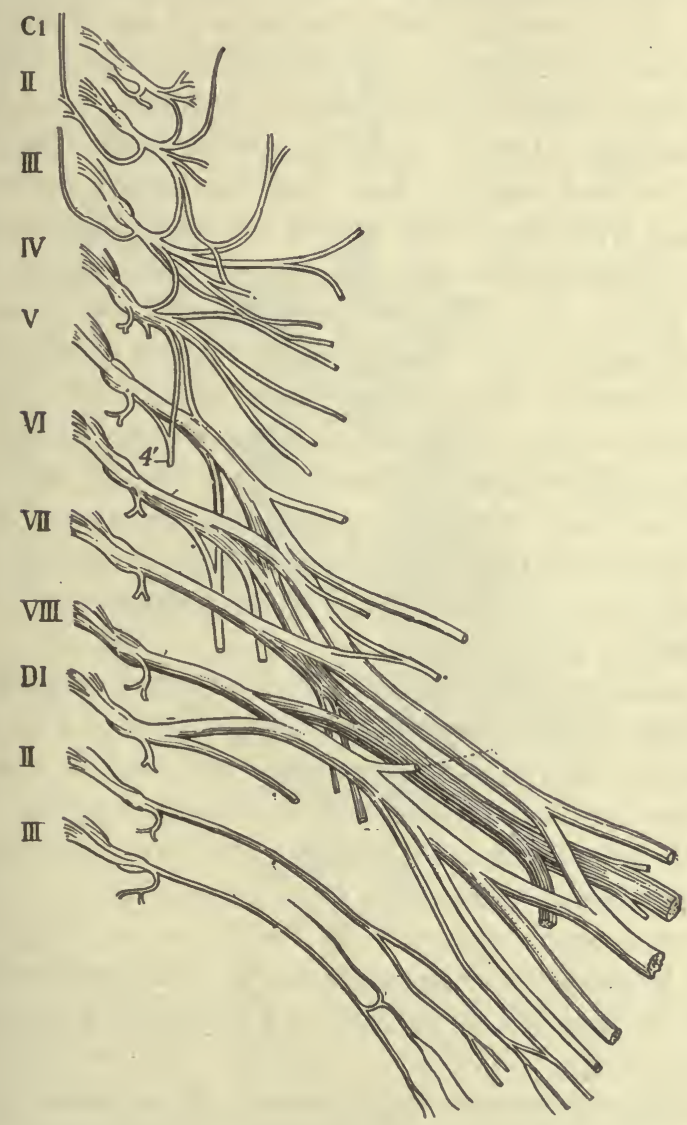

Fig. 61. -The cervical and brachial plexuses of the left side of the Body.

but each runs along the posterior border of a rib and supplies branches to the chest-walls, and the lower ones to those of the abdomen also.

The ventral primary branches of the four anterior lumbar nerves are united by branches to form the lumbar plexus. It sup- 
plies the lower part of the trunk, the buttocks, the front of the thigh, and inner side of the leg.

The sacral plexus is formed by the anterior primary branches of the fifth lumbar and the first four sacral nerves, which unite in one great cord and so form the sciatic nerve, which is the largest in the Body and, running down the back of the thigh, ends in branches for the lower limb. The roots of the trunks which form the sacral plexus arise from the lumbar enlargement of the cord.

Cranial Nerves. Twelve pairs of nerves leave the skull by apertures in its base, and are known as the cranial nerves. Most of them spring from the under side of the brain, and so they are best studied in connection with the base of that organ, which is represented in Fig. 62. The first pair, or olfactory nerves, spring from the under sides of the olfactory lobes, $I$, and pass out through the roof of the nose. They are the nerves of smell. The second pair, or optic nerves, $I I$, spring from the optic thalami and corpora quadrigemina, and, under the name of optic tracts, run down to the base of the brain, where they appear passing around the crura cerebri, as represented in the figure. In the middle line the two optic tracts unite to form the optic chiasma, from which an optic nerve proceeds to each eyeball.

All the remaining cranial nerves arise from the hind-brain. The third pair (motores oculi) arise from the front of the pons Varolii, and are distributed to most of the muscles which move the eyeball and also to that which lifts the upper eyelid.

The fourth pair of nerves (pathetici) $I V$, arise from behind the crura cerebri. From there, each curls around a crus cerebri (the cylindrical mass seen beneath it in the figure, running from the pons Varolii to enter the under surface of the cerebral hemispheres) and appears on the base of the brain. Each goes to one muscle of the eyeball.

The fifth pair of nerves (trigeminales), $V$, resemble the spinal nerves in having two roots; one of these is much larger than the other and possesses a ganglion (the Gasserian or semilunar ganglion) like the dorsal root of a spinal nerve. Beyond the ganglion the two roots form a common trunk which divides into three main branches. Of these, the ophthalmic is the smallest and is mainly distributed to the muscles and skin over the forehead and upper eyelid; but also gives branches to the mucous membrane 
lining the nose, and to the integument over it. The second division (superior maxillary nerve) of the trigeminal gives branches to the skin over the temple, to the cheek between the eyebrow and the angle of the mouth, and to the upper teeth; as well as to the mucous membrane of the nose, pharynx, soft palate and roof

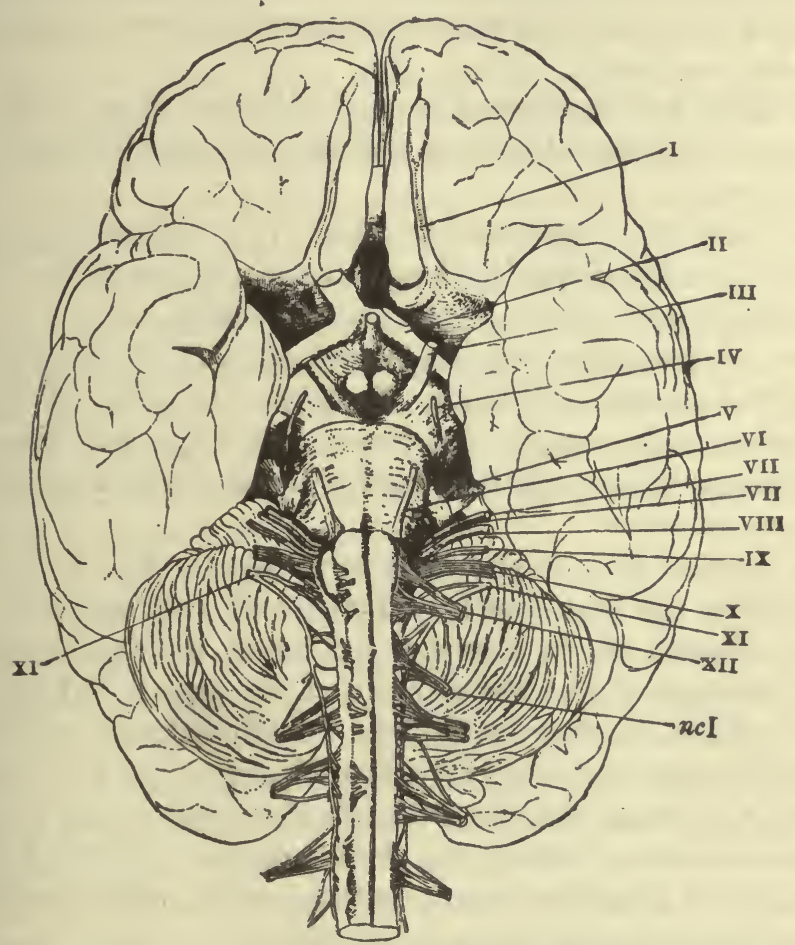

Fig. 62.-The base of the brain. The cerebral hemispheres are seen overlapping all the rest. I, olfactory lobes; $I I$, optic tract passing to the optic chiasma from which the optic nerves proceed; $I I I$, the third nerve or motor oculi; $I V$, the fourth nerve or patheticus; $V$, the fifth nerve or trigeminalis; $V I$, the sixth nerve or $a b$ ducens; VII, the seventh or facial nerve; VIII, the auditory nerve; $I X$, the ninth or glossopharyngeal; $X$, the tenth or pheumogastric or vagus; $X I$, the spinal accessory; $X I I$, the hypoglossal; $n c I$, the first cervical spinal nerve.

of the mouth. The third division (inferior maxillary) is the largest branch of the trigeminal; it receives some fibers from the larger root and all of the smaller. It is distributed to the side of the head and the external ear, the lower lip and lower part of the face, the inucous membrane of the mouth and the anterior two-thirds 
of the tongue, the lower teeth, the salivary glands, and the inuscles which move the lower jaw in mastication.

The sixth pair of cranial nerves VI, or abducentes arises from the posterior margin of the pons Varolii, and each is distributed to one muscle of the eyeball.

The seventh pair (facial nerves), VII, appear also at the posterior margin of the pons. They are distributed to most of the muscles of the face and scalp.

The eighth pair (auditory nerves), VIII, arise close to the facial. They are the nerves of hearing and are distributed entirely to the internal ear.

The ninth pair (glossopharyngeals), IX, arising close to the auditories, are distributed to the mucous membrane of the pharynx, the posterior part of the tongue, and the middle ear.

The tenth pair (pneumogastric nerves or vagi), $X$, arise from the sides of the medulla oblongata. Each gives branches to the pharynx, gullet, and stomach, the larynx, windpipe, and lungs, and to the heart. The vagus runs farther through the Body than any other cranial nerve.

The eleventh pair (spinal accessory nerves), XI, do not arise mainly from the brain but by a number of roots attached to the lateral columns of the cervical portion of the spinal cord, between the ventral and dorsal roots of the proper cervical spinal nerves. Each, however, runs into the skull cavity alongside of the spinal cord and, getting a few filaments from the medulla oblongata, passes out along with the glossopharyngeal and pneumogastric nerves. Outside the skull it divides into two branches, one of which joins the pneumogastric trunk, while the other is distributed to muscles about the shoulder.

The twelfth pair of cranial nerves (hypoglossa), XII, arise from the sides of the medulla oblongata; they are distributed mainly to the muscles of the tongue and hyoid bone.

It must be remembered that the cranial nerves, like the spinal nerves, are really bundles containing hundreds of axons having various destinations. Just as in the spinal nerve plexuses bundles of axons cross over from one nerve-trunk to another, so in many of the cranial nerves, especially the fifth and seventh, there are branchings from one nerve to another, making it difficult to tell in many cases from what part of the brain the nerves to a special 
part have come; for example, it was believed for a long time that the axons mediating the sense of taste enter the brain as part of the trigeminal nerve. It is now practically certain that they enter instead by way of the facial and glossopharyngeal.

White and Gray Matter. In preceding paragraphs the occurrence of white and gray matter in the central nervous system has been mentioned. In the paragraph on myelin sheaths (p. 138) the difference between them was described. It may be worth while, for emphasis, to state again this difference before discussing more specifically their distribution in the nervous system. White matter consists of medullated axons, and is concerned functionally, therefore, with the conduction of impulses from point to. point. Gray matter consists of cell-bodies, dendrites, and parts of axons, and in it and it alone are the synapses found over which impulses pass across from one neuron to another. Gray matter, therefore, is concerned with the distribution of nerve impulses among the neurons. In it also, as we shall see, take place the modifications which nerve impulses undergo during their passage through the central nervous system.

Most of the gray matter of the Body is found in three special regions. These are: (1) the gray columns of the spinal cord; (2) a layer about $2 \mathrm{~mm}$. ( $\frac{8}{32} \mathrm{in}$.) thick over the entire outer surface of the cerebral hemispheres, including the mesial surface of each, and (3) a similar layer over the surface of the cerebellum. In addition to these chief gray regions there are a number of small masses of gray matter distributed in various parts of the Body. Some of these are embedded in the brain; others are outside the central nervous system altogether. Those within the central nervous system are known as nucle ${ }^{*}$, those outside it as ganglia.

The gray nuclei are found in the following regions: (1) The base of the cerebrum; these are known as the basal nuclei and include the optic thalami, the caudate, and the lenticular nuclei; (2) the base of the cerebellum; here are several pairs of nuclei, including the dentate nuclei; (3) the midbrain; here are several small nuclei, the superior and inferior colliculi (corpora quadrigemina), the external and internal geniculate bodies, and the red

* It must be understood that the term nucleus as applied to a mass of gray nervous matter has an entirely different significance than when applied to a part of a single cell. 
nucleus; (4) the medulla; all the gray matter of the medulla is contained within its nuclei. They constitute the so-called deeporigins of those cranial nerves which arise in the medulla.

All nerve-ganglia in the Body, using the term ganglia in the restricted sense suggested above, fall into two groups: (1) Those which contain the cell-bodies of sensory neurons; in this group belong all dorsal root-ganglia of spinal nerves (see p. 143), likewise the ganglia which are found on some of the cranial nerves; (2) the so-called sympathetic ganglia which are described in the next paragraph.

The Sympathetic or Autonomic System. The ganglia which form the main centers of the sympathetic nervous system lie in two rows (s, Fig. 2), one on either side of the bodies of the vertebræ. Each ganglion is united by a nerve-trunk with the one in front of it, and so two great chains are formed reaching from the base of the skull to the coccyx. In the trunk region these chains lie in the ventral cavity. The ganglia on these chains are forty-nine in number, viz., twenty-four pairs, and a single one in front of the coccyx in which both chains terminate. They are named from the regions of the vertebral column near which they lie; there being three cervical, twelve thoracic, four lumbar, and five sacral pairs.

Each sympathetic ganglion is united by communicating branches with the neighboring spinal nerves, and near the skull with various cranial nerves also; while from the ganglia and their uniting cords arise numerous trunks, many of which, in the thoracic and abdominal cavities, form plexuses, from which in turn nerves are given off to the viscera. These plexuses frequently possess numerous ganglia of their own; two of the most important are the cardiac plexus which lies on the dorsal side of the heart, and the solar plexus which lies in the abdominal cavity and supplies nerves to the stomach, liver, kidneys, and intestines. Many of the sympathetic nerves finally end in the walls of the blood-vessels of various organs. To the naked eye they are commonly grayer in color than the cerebro-spinal nerves. 


\section{CHAPTER $\mathrm{X}$}

\section{GENERAL PHYSIOLOGY OF THE NERVOUS SYSTEM. SPINAL AND CEREBELLAR REFLEXES}

Conduction within Single Neurons. Since the nervous system, whose function as a whole is the conduction of impulses from sensory regions to motor ones, is made up of individual neurons, the study of its physiology can best be begun by considering the phenomenon of conduction as exhibited in single neurons, passing later to conduction as it involves more neurons than one.

The passage of an impulse along a nerve is attended by no visible alteration of the nerve itself; it is impossible to tell by looking at a nerve whether it is carrying impulses or not. For this reason nerve impulses can only be studied indirectly. If as the result of stimulating a motor nerve at some point along its course the muscle in which it terminates is thrown into contraction the obvious conclusion is that nerve impulses are passing from the point of stimulation to the muscle. When the prick of a finger gives rise within the brain to a conscious sensation of pain we know that a nerve impulse must have passed between the finger and the brain, although we would be unable to detect any sign of its passage if the nerve were visible throughout its length. In addition to these methods of studying nerve impulses, in which the passage of the impulse is made known through its effect on some other part of the Body, we have a method which depends upon the fact that activity of nerve, like activity of muscle or any other living tissue, is accompanied by changes of electrical potential which may give rise to action currents. Every time an impulse passes along a nerve it is accompanied by this electrical alteration. Sensitive electrometers applied to nerves will indicate the passage of impulses under their points of contact.

By the use of these methods of studying nerve impulses we have learned many things about them, although much more remains unknown. 
How Nerve Impulses Are Aroused. We know that nerve impulses may be started in various ways. A sharp blow on a living nerve starts impulses traveling along it; a good example of this is the effect of striking the "funny" bone. Nerves may be stimulated by heat or by cold, by chemical agents or by an electric spark. Whatever the nature of the stimulus the nerve impulse which it arouses is, so far as we can tell, the same in all cases.

Speed of Nerve Impulses. The nerve impulse travels from the point of stimulation over the neuron at a regular and rather slow rate which probably varies somewhat in different animals and in different nerves of the same animal. In frogs' nerves at ordinary temperatures the rate approximates 30 meters ( $97 \mathrm{ft}$.) per second. In human nerves the rate is probably two or three times as high.

Spread of Impulses in Both Directions. Through observations of the action currents of nerves it has been shown that the impulse spreads from the point of stimulation in both directions along the neuron, finally traversing all parts of it. This fact could never have been demonstrated if the existence of the action currents (p. 103) were unknown because our only other method of detecting the presence of nerve impulses depends upon the production of effects in the organs to which the neurons lead; and in the body each neuron has such connection only at one end; a nerve impulse imparted to a motor nerve will cause contraction in its connected muscle but produces no effect whatever at its other end.

Fatigue. It has been proven beyond question that the passage of impulses over nerve-fibers does not fatigue them to an appreciable degree. In this respect the nerve is comparable to a telephone wire; in each case the message is transmitted without impairing the ability of the structure to transmit other messages.

We learned in connection with our study of muscular fatigue (p. 101) to look upon fatigue as the result of the accumulation of waste substances. Its absence from active nerve-fibers indicates one of two things. Either the transmission of nerve impulses does not involve the production of fatigue substances or the fiber is able to get rid of such as are produced so quickly that they cannot affect its working. Exceedingly delicate tests which have recently been devised indicate that in nerve trunks there is a small production of carbon dioxid. This gas is known to be a product of oxida- 
tion and its occurrence is taken to mean that chemical processes do go on in nerve-fibers. They must be of very small magnitude, however, for their products are so quickly dissipated as not to hinder the functioning of the nerve at all.

While indefatigability is thus seen to be a property of axons, we shall learn presently that other nervous structures are highly susceptible to it. Nervous fatigue is a common phenomenon, but it is localized in the region of the synapses and not in the axons.

Nature of the Nerve Impulse. Although we know these things about nerve impulses, we do not know what the nerve impulse itself really is. There have been many interesting and ingenious theories of its nature proposed. Some of these attempt to describe it as a purely physical process, the transmission of a physical stress from particle to particle along the nerve; others would consider it as a chemical process, too delicate and transitory to be detected. All theories of its nature agree that the change transmitted along the nerve is not a continuous flow, like an electric current along a wire, but is an exceedingly brief impulse or series of impulses. The name given to the nervous discharge implies this character. During continuous excitation of a nerve, as in prolonged voluntary contraction of a muscle, the individual impulses follow each other in rapid succession. The exact rate is not known, but is believed to be in the general neighborhood of 50-100 a second. Quite recently evidence has accumulated which indicates that individual nerve impulses are on the whole of nearly equdl intensity. This is important in view of the familiar fact that nervous activities in general may show widely different intensities. The prevailing explanation accounts for this on the ground that nervous activities depend, not on single impulses but on streams of impulses which latter may vary even though the individual component impulses are equal. In accordance with this view we have to suppose that a weak stimulus gives rise to one sort of stream of impulses, a stronger stimulus to a different sort, and so on.

Conduction Involving More Than One Neuron. Reflexes. In the actual passage of nerve impulses through the Body more neurons than one are always involved. Let us examine a simple case of conduction by which the Body adapts itself to its surroundings. Accidentally my finger comes in contact with a hot surface. Quite involuntarily I jerk my hand away. The chain of events is 
as follows: the skin of the hand is stimulated by the heat; the sensory neurons in the nerve supplying that part of the hand convey the nerve impulses thus aroused to the central nervous system; here the impulses are conveyed to the motor neurons leading to the muscle which retracts the arm; upon the arrival of the impulses within the muscle the latter is stimulated to contract. The whole process is entirely mechanical; none of the structures involved has any knowledge that the hand is in danger of being severely burnt, or that retraction of the arm will save it. It is an example of an adaptive mechanism. Such a chain of events as the one described constitutes a simple reflex and typifies the fundamental basis of all nervous activity within the organism. Our

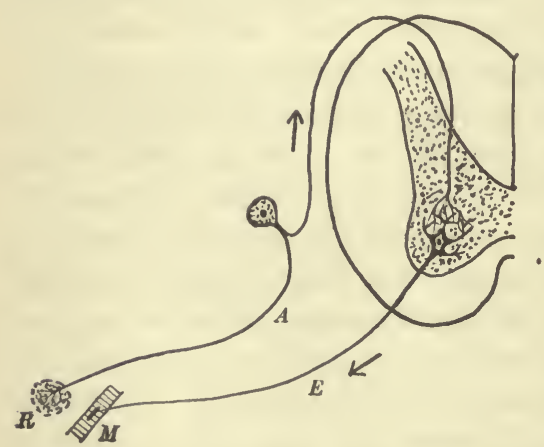

FIG. 63.-Diagram of the simple reflex arc. $R$, receptor; $A$, afferent (sensory) neuron; $E$, efferent (motor) neuron; $M$, muscle.

make up the reflex arc. The simplest imaginable reflex arc must include at least two neurons, the sensory neuron which brings the impulses from the receptor to the center, and the motor neuron which conveys the impulses from the center to the motor organ. Undoubtedly most reflex ares in the Body include, in addition, one or more association neurons interposed between the sensory and the motor neuron.

Anatomical Arrangement. The anatomical relationships of the various neurons which make up the reflex arc are indicated in figure 63. For simplicity the spinal cord is taken as the part of the central nervous system pictured. The receptor communicates with the cell-body of the sensory neuron by means of the axon-like 
process previously described (p. 136). The cell-body of the sensory neuron always lies outside the central nervous system in a dorsal root ganglion; its axon extends thence by way of the dorsal root of the spinal nerve into the spinal cord (or brain) and enters the dorsal column of white matter (p. 144).

Within the central nervous system is located the cell-body of the motor neuron which forms the outgoing part of the path. This will be found in the ventral horn of gray matter of the spinal cord. The simple reflex arc is formed by one of the branches of the sensory axon which penetrates the gray matter and whose end arborization makes synaptic connection with the dendrites of the motor neuron.

Reflex Arcs Not Rigidly Fixed Paths. Although a given sensory stimulus usually arouses the same sort of reflex response every time it is applied, this does not mean that the reflex path followed in such a case is the only one into which that sensory neuron leads. Very different reflex responses may originate in the same receptor. A good illustration of this is furnished by certain reflexes through the eye. If I see that a small floating particle threatens my eye I am apt to wink; if a flying insect approaches I am more likely to turn my head to one side; if the threatening object is a swiftly thrown baseball I will probably bring the hands before the face, or perhaps dodge to one side. All these actions are performed mechanically and are therefore true simple reflexes. The originating sensory impulses travel in each case over the same sensory neurons, those of the optic nerves. It is evident, then, that impulses coming in over the sensory neurons of the optic nerve do not have to pass over to any particular motor neuron, such as the one which leads to the muscle of winking, but may follow any one of various courses, finally terminating in muscles far distant from the eye. In fact, and this is one of the most important things to remember about the nervous system, there is such an extraordinary richness of connection among the various neurons within the central nervous system that any sensory neuron may be brought into communication with any motor neuron.

This richness of connection is afforded anatomically through two rather simple arrangements. In the first place the axons of sensory neurons after entering the central nervous system continue 
along it for some distance, giving off branches, called collaterals, at various levels. In the spinal cord the dorsal white columns contain these axons. They extend toward the brain, but each gives off a branch which extends a short distance down the cord in the opposite direction. Each collateral terminates in an end arborization which communicates in turn with the dendrites of another neuron, either motor or association. Thus each sensory neuron, besides its connection with one or more motor neurons, has connection with various association neurons located in different parts of the central nervous system. The association neurons likewise are richly branched, each branch terminating in a synaptic con-

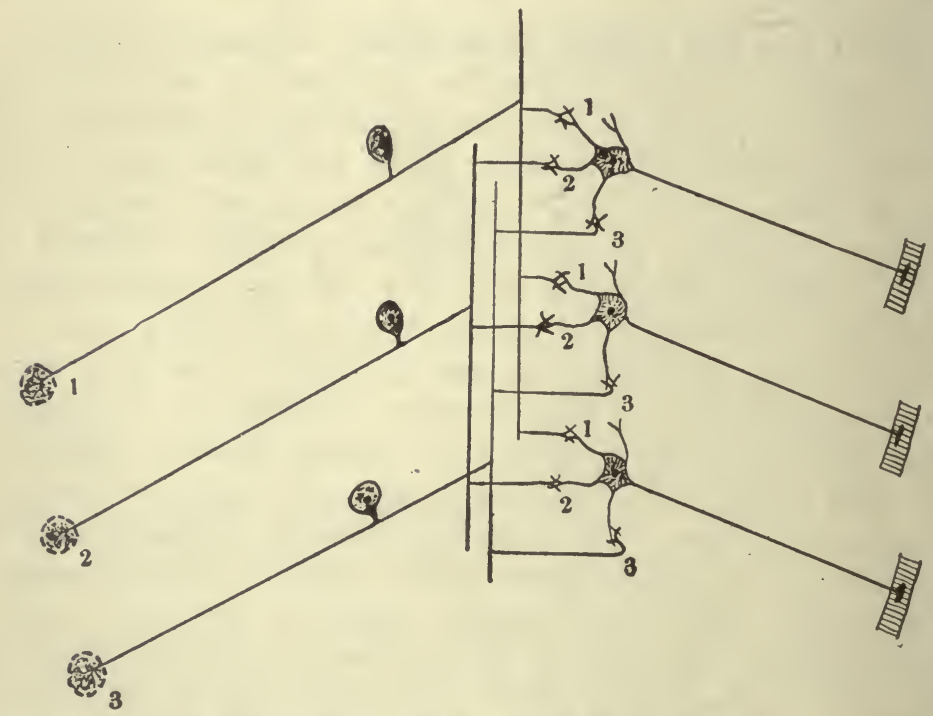

FIG. 64.-Diagram to illustrate how a single sensory neuron may communicate with several motor neurons, and a single motor neuron with several sensory neurons.

nection with another neuron, and this in turn may be an association neuron, or may be a motor neuron. In the second place the dendrites of all association and motor neurons doubtless have synaptic connection with end arborizations of numerous neurons, sensory or association as the case may be. Thus a sensory neuron has a wide choice of paths over which to send its impulses; and a motor neuron may recerve impuises from a great variety of sources (Fig. 64). 
Irreversible Conduction. In all this maze of connections and interconnections within the central nervous system, how is it that the impulses coming in at a sensory neuron always come out finally at a motor neuron instead of becoming switched sometimes to another sensory neuron? The orderly progress of impulses is insured by a very simple arrangement, namely, that impulses can pass freely across a synapse from end arborization to dendrites but can never pass in the reverse direction, from dendrites to end arborization. When a sensory neuron delivers its impulses to an association neuron the impulses doubtless spread to all parts of the latter. They can leave it, however, only by way of its end arborizations, and these communicate only with the dendrites of motor neurons or of other association neurons. The final outcome is bound to be a motor neuron since all association neurons lead ultimately to them. Sensory neurons never receive impulses from other neurons because they have no dendrites within the central nervous system by which impulses might be received. The portion of a sensory neuron which corresponds to the dendrites of a motor neuron is the long axon-like process communicating with the receptor.

Graded Synaptic Resistance. Another question which naturally arises when one considers the innumerable courses which an impulse may take within the central nervous system is what determines the course it actually does take? Why, for instance, when my eye is threatened do I wink instead of opening my mouth, or why do I sometimes. wink and sometimes dodge? A complete answer to this question cannot be made in the present state of our knowledge, but we have a fairly good general idea of the way in which nerve impulses are probably guided. A sensory neuron has several collaterals, each with its synaptic connection with another neuron. If we suppose these synapses are not all alike, but that certain ones transmit the sort of stream of impulses generated by feeble stimuli more readily than do the others, such a stream spreading over the sensory neuron will pass most easily to that connecting neuron whose synapse offers least resistance to its passage. Thus we may imagine a stream of impulses spreading from neuron to neuron following always the path of least resistance until it finally terminates in a muscle which it arouses to activity. In the central nervous system the various paths of least resistance are 
so blocked out as to lead to adaptive motions; a prick on the finger causes retraction of the hurt hand; irritation in the nose causes the convulsive movements of the respiratory muscles which constitute a sneeze; in each case the motions are calculated to get rid of the source of irritation.

That adaptive reflexes are due to paths of least resistance blocked out from an infinite number of possible paths is strikingly illustrated by the effects of strychnine poisoning. This drug acts on the central nervous system in such a way as to abolish differences of synaptic resistance. When one suffering from the drug receives a stimulus by way of any sensory nerve the impulses, instead of following the usual path, spread over the whole central nervous system; all the muscles are stimulated simultaneously and the well-known strychnine convulsion results.

The Orderly Spreading of Reflexes. The conception of graded synaptic resistances explains also in a very satisfactory way the phenomenon of the orderly spreading of reflexes. A feeble stimulus produces reflex movement in those muscles only which are immediately concerned in the adaptive response; stronger stimuli involve more muscles, but only such as by their movement make the response more effective. For example, if a frog's hind leg is touched gently it will be drawn away. from the source of irritation; a stronger stimulus is likely to cause contractions of such additional muscles as are required for jumping away from the point of danger. If we assume that the reflex paths to the first set of muscles have such low resistances as to allow feeble impulse streams to pass them, and that stronger impulse streams can overcome enough additional resistance to enter the paths of higher resistance leading to the jumping muscles, while the paths to muscles not concerned in any way in an adaptive response have too high resistance to be passed at all, we can account for reflex actions of very great complexity.

Simple Reflexes Mediated by the Spinal Cord. The simple reflexes described in the preceding paragraphs are all of a sort that can be carried on through the lowest part of the central nervous system, the spinal cord. A frog whose brain has been destroyed and which is therefore wholly devoid of feeling and consciousness can still perform highly complicated reflex acts; he will retract a foot which is pinched; he will wipe off a bit of acid-soaked paper 
from his flank, and if unable to reach it with one foot will bring the other into service. All his acts, however, are purely mechanical, and are determined by the spread of impulses over reflex paths of less or greater complexity.

The grading of the synaptic resistances in the spinal cord of any animal, including man, is established with the development of the cord itself. The organism is born with paths of least resistance from the different receptors to adaptive muscles laid down. These paths are as much part of the hereditary equipment of the individual as is the spinal cord itself. Spinal cord reflexes apparently do not require to be developed by training. They seem to be performed as perfectly the first time as at any later time. An excellent example of this class of reflexes in man is the sneezing reflex, and we all know that the new-born infant does not have to learn how to sneeze. He can do it from birth. Moreover, and this is important as regards spinal reflexes as a class, if the stimulus is strong enough he cannot help doing it. Although, as we shall learn, we have a certain degree of voluntary control over some spinal reflexes, their essentially automatic character should be emphasized.

In general the higher we look in the animal scale the less varied and extensive are the spinal reflexes. A large proportion of all the activities of such animals as fish and frogs are in this class, while in man they are confined to a few relatively simple acts, such as coughing, sneezing, winking, and simple withdrawal of an extremity from a source of irritation.

Significance of the Head Senses in the Control of Reflexes. We have noted how, in the lower animals, highly complicated acts are performed automatically through the operation of the "spinal cord" reflexes. When we study such activities in an animal whose brain has been destroyed we note that on the sensory side they are based exclusively on the body senses, touch, temperature, pain, etc. (p. 172). The destruction of the brain has cut off all possibility of any action on the part of the head senses, sight, hearing, taste, and smell (p. 173). One result of this dependence on the body senses of spinal cord reflexes is that they are, as a class, immediately protective. The adaptive response consists of the withdrawal from or removal of a direct source of irritation. The chief significance of the head senses is in their property of giving information of what is 
happening at a distance. The bodily adjustments based on them are therefore long range adjustments rather than immediate ones. Other adaptations than the simple one of withdrawing from a source of injury are possible. Notably reflexes concerned with the quest for food, a quest based in most animals largely on the senses of smell and sight, are added to the purely protective reflexes. Associated with these long range adjustments is a great group of movements which constitute our most frequent and, in general, most important muscular acts, movements of locomotion. These make up a separate class of reflexes and will be studied by themselves next in order.

The Sensory Basis of Locomotion. Locomotion takes various forms in individual animals and in different classes of animals. Walking, running, leaping, swimming, flying, these are all fundamental locomotor acts. With them must be classed also the more artificial forms of locomotion of civilized man, as bicycle riding or aviation. All these have certain primary features in common. They all require the accurately co-ordinated use of a number of muscles, and all of them involve the maintenance of equilibrium. More, in fact, than the simple maintenance of balance is involved. In every sustained locomotion there is constant restoration of an equilibrium that is continually disturbed. Of great importance for the guidance of co-ordinated muscular movement is a sense whose receptors are embedded within the muscles themselves and distributed about the joints to which the contracting muscles impart movement. This is the muscle and joint sense, or briefly, muscle sense. Less well known than some of our other senses it is, as we shall learn (Chap. XIII), of equal rank with the others, and in connection with our muscular movements more important than most. Every bodily movement results in stimulation of the receptors of muscle sense. Any locomotor act is accompanied by a great stream of impulses from these receptors which serve not only to guide but to maintain the activity. There are definite organs of equilibrium, the semicircular canals and vestibule of the ear (Chap. XIV), by which the equilibrium sense is mediated. These two senses constitute the essential sensory basis for the locomotor reflexes. They are reinforced and modified by some of the other senses, notably touch and sight. Since the locomotor reflexes require the co-operation of several senses they are more 
complicated on the sensory side than the most elaborate spinal cord reflexes, the latter being based on stimulation of single groups of receptors. On the motor side, also they are more complicated. The degree of muscular co-ordination involved is greater than in any spinal cord reflex. For the translation of the complex stream of sensory impulses into an equally complicated stream of motor impulses a more elaborate arrangement of interconnecting neurons is required than the spinal cord affords. For this purpose a special portion of the brain, the cerebellum, is set apart, and our next concern is with the structure and connections of this organ, and its functioning in the mediation of reflexes of locomotion.

Structure and Connections of the Cerebellum. This organ, as shown in Figs. 58-60 is a distinct portion of the brain, lying underneath the posterior part of the cerebrum, and behind and above the midbrain and medulla. It consists of a thin layer of . gray matter superposed upon white matter, and having embedded within the white matter at its base gray masses, the nuclei of the cerebellum. The thin outer gray layer, known as the cortex, is the region in which the incoming streams of sensory impulses are converted into outgoing streams of co-ordinated motor impulses. The cerebellum communicates with the brain stem, as, for convenience, the midbrain and medulla together are often called, by three pairs of stalks or peduncles. These consist of bundles of axons. The upper and lower stalks (Fig. 66) lead directly into the brain stem. The middle peduncles form the backward extension of the pons varolii (Fig. 58).

The senses which are concerned with locomotor reflexes all have connection, either directly or by means of association neurons, with the brain stem, and thence, by neurons whose axons extend through the peduncles, with the cerebellum itself. The detailed anatomy of these paths will be presented later in connection with the study of the cerebrum (p. 173).

The outgoing paths from the cerebellum, the paths over which pass the co-ordinated streams of impulses which carry on the acts of locomotion, consist of chains of association neurons. These begin in the cerebellar cortex and pass thence over the peduncles to the brain stem. Here communication is made with others which pass down the spinal cord to final terminations in the ventral horn 
of gray matter in immediate synaptic connection with the cell bodies of the motor neurons.

We can trace reflex arcs for locomotor reflexes as for the simpler spinal reflexes. In both cases the paths begin with sensory neurons and terminate with motor neurons. Many more association neurons are always involved in locomotor reflexes than in spinal, and they always include the cerebellum in their course. As indicated above, however (p. 164), not one, but several senses co-operate in locomotor reflexes, and many muscles are concerned in their performance, so that no single reflex arc suffices to carry them on, but several paths into the cerebellum and a number out of it must be thought of as involved.

Functions of the Cerebellum. In a previous paragraph the general function of the cerebellum was stated, namely, to translate the streams of impulses from the receptors of muscle sense, equilibrium, touch, and sight into co-ordinated motor impulses by which are carried on the important reflexes of locomotion. We need to bear in mind, in this connection, that our muscles will not work spontaneously. We can cause them to contract by an act of the will (p. 184) or they can be operated reflexly by means of stimuli conducted to them from receptors. We know from our own experience that our common locomotor acts, such as walking, are not volitional in the sense that every muscular movement is voluntary. We can see, also, that in such an act as walking there are abundant sources of sensory stimulation. The pressure of the feet upon the ground, the muscular movements themselves, the disturbances of equilibrium, the appearance of the footing, all give rise to streams of sensory impulses which, if properly co-ordinated can be made to operate complicated muscular movements. This co-ordination is the function of the cerebellum.

All the reflexes which the cerebellum mediates are reflexes of skeletal muscles. They are all such as the higher parts of the brain through the property of volition are competent to carry on. If their performance depended on the higher brain regions, however, these would have little time left for other, and more important activities. We may view the cerebellum, therefore, as an organ which by taking up complicated but not highly intellectual tasks leaves the higher parts of the brain free for higher forms of activity.

An important difference between cerebellar and spinal reflexes 
is that while the latter are instinctive, born in us, the former are not. Fvery one has to learn to stand, walk, run, and so on; at first all are difficult, but after a time become easy and are performed unconsciously. In standing or walking very many muscles are concerned, and if the mind had all the time to look directly after them we could do nothing else at the same time; we have forgotten how we learnt to walk, but in acquiring a new mode of progression in later years, as skating, we find that at first it needs all our attention, but when once learnt we have only to start the series of movements and they are almost unconsciously carried on for us. At first we had to learn to contract certain muscle groups when we got particular sensations, either tactile, from the soles, or muscular, from the general position of the limbs, or visual, or equilibrium sensations from the semicircular canals. But the oftener a given group of sensations has been followed by a given muscular contraction the more close becomes the association of the two; the path of connection between the incoming and outgoing fibers becomes easier the more it is traveled, and at last the sensory impulses arouse the proper movement without volitional interference at all, and while hardly exciting any consciousness; we can then walk or skate without thinking about it. The will, which had at first to excite the proper motor neurons in accordance with the felt directing sensations, now has no more trouble in the matter; the sensory impulses stimulate the proper motor centers in an unconscious and unheeded way. Injury or disease of the cerebellum produces great disturbances of locomotion and insecurity in maintaining various postures, as well as marked loss of endurance. The functions normally performed by it are transferred to other parts of the brain, and these, which are less fitted for the task, do it less well and with more fatigue.

Postural Reflexes. In a previous chapter (p. 126) the dependence of posture on extensor tonus was described. This tonus is maintained reflexly, and to the extent that it involves equilibrium, as in the erect posture in man, is to be looked upon as belonging to the class of cerebellar reflexes. The most striking fact about postural tonus, perhaps, is the completeness with which it disappears in the presence of any active movement which would conflict with it. This is a striking example of the adaptive character of the nervous system. So long as one is maintaining any 
posture quietly, the tonus is present and suffices to keep the Body in position, but let any active movement be started, either volitionally or reflexly, and the opposition to that movement which would be offered by a persistence of the tonus is removed by its complete cessation. This fact has been proven conclusively. For details the reader is referred to larger works on the nervous system. 


\section{CHAPTER XI}

\section{STRUCTURE, NERVE CONNECTIONS, AND FUNCTIONS OF THE CEREBRUM}

The Cerebrum in Relation to Muscular Activity. In the preceding chapter two classes of reflexes, spinal and cerebellar, have been described. A fact it is important we should grasp, is that a large part of all the activities of all animals belong to one or the other of these classes. Indeed as we go down the animal scale and examine such animals as fish, frogs, and turtles, it is a matter of some difficulty to prove that any of their acts involve higher nervous manifestations. In man and the higher animals, however, we recognize many activities which cannot be assigned to either category. Among these are all acts which we describe as volitional. For the performance of these the cerebrum is essential. We will get an idea of the significance of the cerebrum in relation to muscular activity, by noting the way in which it may modify such activity.

A Normal Animal Compared with a "Reflex" One. Let us imagine that we have side by side before us two living animals of the same species, one normal in every respect, the other in the "reflex" condition; that is, having had the cerebrum destroyed but the remainder of the nervous system uninjured. Disregarding for the present the phenomenon of consciousness and looking at both animals simply as pieces of machinery three striking differences between them are manifest: 1 . The "reflex" animal always responds to adequate stimulation by a predictable response; the intact animal sometimes responds and sometimes does not. 2. The "reflex" animal does not move except when stimulated, while the intact animal often moves without any apparent reason. 3. The amount of response given by the "reflex" animal bears some relation to the intensity of the exciting stimulus, whereas in the normal animal an apparently feeble stimulus may arouse a vigorous and long-continued response. An example of 
this last is the running of a dog to its master upon hearing his whistle. The stimulus may be a very faint one, the motions which it arouses are exceedingly vigorous and complicated.

All these differences depend at bottom upon a single fundamental difference between the two animals which is this: in the "reflex" animal the immediate stimulus dominates the situation completely; in the intact animal the immediate stimulus is only one factor of many which together determine what the response shall be. The superior practical efficiency of the intact animal as an adaptive organism depends upon this power, resident in the cerebrum, of modifying immediate stimuli in accordance with the demands of less obvious considerations. To illustrate: a hungry man perceiving food would inevitably respond to the double stimulus of hunger and the sight of food by taking the food and eating it if he acted upon a purely reflex basis; his actual response to these stimuli will depend, however, upon whether they are in harmony with or opposed to certain more remote factors, such as the question whether the food is of a sort that will agree with him, or whether he is likely to need it more urgently at some future time than at present.

Before entering upon a fuller discussion of the functions of the cerebrum, its structure and its connections with lower nervecenters must be described.

The Cerebrum Dependent on the Receptor System. If the cerebrum is to introduce remote considerations as factors in determining the nature of reflex responses it must have within it the knowledge upon which these remote considerations are based. That the cerebrum has little original endowment of knowledge is evident from study of infants, who during the first months are perfect examples of "reflex" organisms. The equipment which the cerebrum finally obtains must be gotten bit by bit by experience or the teaching of others. Since the receptor system is the organism's only means of acquiring information, the cerebrum must be in communication with this system if it is to learn anything whatsoever.

Afferent Paths of the Cerebrum. We have learned in previous paragraphs that all sensory neurons lead directly into the central nervous system and there have numerous synaptic connections with association neurons. These connections are all, however, 
with the possible exception of those of the sense of smell, made in gray matter of the spinal cord, the medulla, or the midbrain. In order for impulses coming in over these sensory neurons to reach the cerebrum there must be communication by association neurons between the terminations of the sensory neurons and the cerebrum. As a matter of fact such connections are richly supplied. Some of the most conspicuous tracts of white matter in the central nervous system consist of the myelinated axons of association neurons which form connecting links between sensory neurons and the cerebrum. Since the cerebrum is the crown of the entire nervous system it is used as a landmark in describing other nervous structures. Thus nerve paths which convey impulses toward the cerebrum are called afferent paths; those carrying impulses away from it are efferent paths. According to this classification all sensory neurons are afferent and all motor ones efferent, while association neurons are either afferent or efferent according as they carry impulses toward the cerebrum or away from it.

Tracing Nerve Paths. Wallerian Degeneration. One of the very satisfactory achievements of biologists has been the resolution of the apparently inextricable tangle of gray and white matter of the central nervous system into a system of fairly definite nerve tracts whose origins, courses, and terminations are known. Our present knowledge is the result of various methods of study. Perhaps the most fruitful has rested upon recognition of three facts: first, that white matter always consists of myelinated axons; second, that axons always are outgrowths of cell-bodies which are to be looked for in gray matter; and third, the fact discovered by the English physiologist, Waller, in 1852, that axons cut off from connection with their cell-bodies undergo degeneration in a few days. Because of this latter fact if a cut be made anywhere in the central nervous system of an animal, and the animal be killed a few days later and its spinal cord and brain examined microscopically, the direction and extent of degeneration reveal the relation of the severed axons to the rest of the nervous system. If the degeneration is all toward the head the severed tract must be an afferent one with cell-bodies somewhere below the cut. Backward degeneration would signify an efferent tract with its origin somewhere forward of the point of injury. Wallerian degeneration is 
not difficult to follow because it is fatty and the drops of fat in the degenerated region can be plainly revealed by the application of osmic acid, which turns them black.

Successive Myelination. Another valuable method of tracing nerve tracts was discovered by Flechsig, who found that during the embryological development of the animal the axons of individual tracts all become myelinated together, while different tracts receive their myelin sheaths at different periods of development. Thus by examining a large series of embryos in all stages the various tracts can be picked out.

Paths of the Various Senses. For convenience in describing the paths by which information is conveyed from the various receptors to the cerebrum, the receptors will be classified as body sense receptors and head sense receptors. The group of body senses includes all those senses such as touch, pain, muscle sense, etc., whose receptors are for the most part in parts of the Body other than the head, and which therefore communicate with the central nervous system by way of spinal nerves. The head senses, sight, hearing, taste, and smell, are those from which stimuli are carried over cranial nerves to the medulla or midbrain, or in the case of the sense of smell directly into the cerebrum.

Tracts of Body Sense. Sensory neurons of body sense enter the spinal cord all along its length. Afferent paths within the cord begin, therefore, at its extreme end. These are to be looked for, as previously stated, in the columns of white matter which make up the greater part of the substance of the cord. Two distinct regions of white matter in each half of the cord have been shown to consist chiefly of afferent neurons leading toward the cerebrum. These are: first, the dorsal columns, each of which consists of two rather well-marked bundles of axons, the so-called fasciculus gracilis (Column of Goll) next the dorsal fissure, 'and the fasciculus cuneatus (Column of Burdach) next to the dorsal horn of gray matter; second, the ventrolateral tracts which lie next to the ventral horns of gray matter, surrounding them on the sides and below (Fig. 65). It is thought that the dorsal columns consist chiefly if not wholly of the axons of sensory neurons which, entering the cord by the dorsal roots of spinal nerves, extend forward within the dorsal columns, giving off collaterals into the gray matter at various levels. Only a part of the sensory axons which 
enter the dorsal columns continue along them as far as the medulla; the others after extending a short distance plunge into the gray matter and terminate in synaptic connection with association neurons. The ventrolateral afferent columns consist chiefly of association neurons which communicate, presumably, with those sensory neurons which do not themselves extend all the way to the medulla; these columns serve, therefore, to afford cerebral communication to those sensory neurons which terminate within the gray matter of the cord.

None of the afferent axons coming up the cord by the tracts just described extend further than the medulla; they all terminate there in masses of gray matter known as the gracile and cuneate nuclei; here they form synaptic connections with a new set of association neurons which continue the path toward the cerebrum. These tracts, which from their ribbon-like appearance have been named the fillets, cross the mid-line at a point in the medulla known as the sensory decussation; so that sensory stimuli from the right half of the Body are carried to the left cerebral hemisphere, and those from the left half of the Body to the right hemisphere.

In the lateral margins of the spinal cord are tracts known as the direct cerebellar tract and Gower's tract (Fig. 65), which consist of the axons of association neurons that pass up to the brain stem and directly through it by way of the peduncles to the cerebellum. The cell-bodies of these axons are in the gray matter of the cord and have synaptic connection with branches of sensory neurons, particularly neurons of muscle sense. These tracts are believed to constitute the chief channels by which muscle sense exerts its influence on the cerebellum in the mediation of locomotor reflexes.

Tracts of the Head Senses. The senses of sight and hearing are the head senses whose central connections are best known. The central connections of the sense of smell are imperfectly known; those of taste practically not at all. Axons conveying visual impulses enter the midbrain by way of the optic nerves and optic tracts and terminate for the most part in nuclei of the midbrain, the external geniculates and superior colliculi; some of them appear to terminate in basal nuclei of the cerebrum, the optic thalami. In all these nuclei synaptic connection is made with new neurons which carry the impulses into the cerebrum. 
Auditory impulses enter the medulla by way of the auditory nerves. The axons of the nerves themselves terminate in nuclei of the medulla, the auditory nuclei; new neurons continue the path thence across the mid-line of the medulla and forward into the midbrain terminating in the internal geniculate nuclei and the inferior colliculi. From these nuclei a third set of neurons continue the path to the cerebrum.

General Structure of the Cerebrum. This organ consists, as previously stated, of an outer surface of gray matter, two milli-

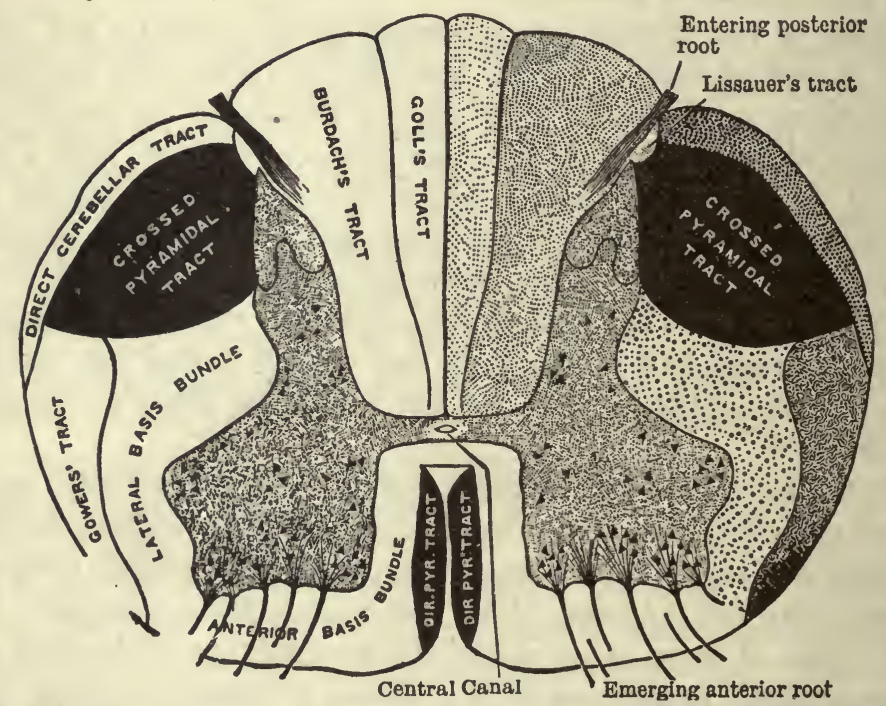

Frg. 65.-Diagrammatic transverse section of the spinal cord showing the conduction paths. (Cunningham.)

meters thick, overlying a mass of white matter; the whole held together by neuroglia and connective tissue, and mounted upon the midbrain as upon a stalk. Because of the convoluted surface of the cerebrum the total amount of superficial gray matter is much greater than it would be if the cerebrum were smooth. This layer of gray matter is the region wherein occur those special activities which set the cerebrum above the rest of the nervous system. It is called the cortex cerebri, or for convenience simply the cortex.

Structure of the Cortex. The cortex cerebri consists for the most part of neurons with small cell-bodies having much branched 
processes, signifying rich synaptic connections. Many of these neurons appear to be confined altogether within the cortex; others give off myelinated axons into the underlying white matter. Interspersed with these small cell-bodies are others which are much larger, which are pyramidal in shape, and which always give off a large axon into the white matter. From their shape and size these are known as large pyramidal cells. In a certain region of the cortex, known as the motor area, the pyramidal cells are relatively gigantic, being just at the limit of naked eye visibility.

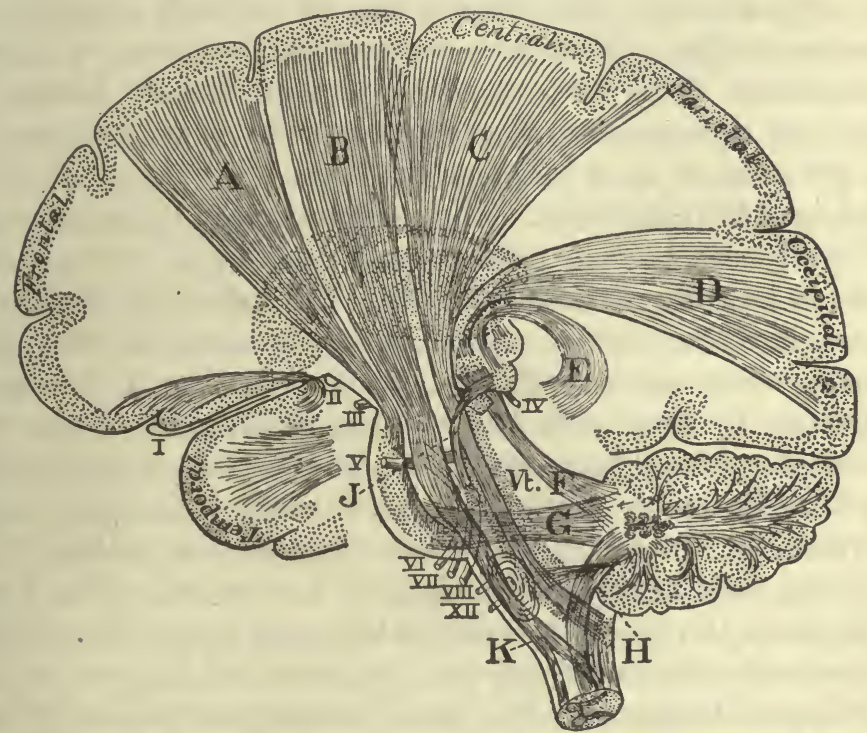

Frg. 66.-Diagram of the projection fibers of the cerebrum (from Starr). $B$, motor (pyramidal) tract; $C$, body-sense tract; $D$, visual tract; $E$, auditory tract; $F, G$ and $H$, upper, middle and lower peduncles of cerebellum; $K$, decussation of pyramids. Numerals refer to cranial nerves.

The White Matter of the Cerebrum. This consists of myelinated axons classified according to their course and distribution into three groups. The so-called projection fibers (Fig. 66) are the axons by which the cortex is brought into connection with the other parts of the nervous system. These include afferent projection fibers, which are the continuations within the cerebrum of the various sensory paths described in previous paragraphs (see p. 172), and efferent projection fibers, which convey impulses from the cortex to the rest of the Body. 
At the base of the cerebrum, where it rests upon the midbrain, all the projection fibers, both afferent and efferent, are crowded together into a restricted space between two of the basal nuclei. This region is known as the internal capsule. As the fibers emerge thence into the roomy cerebrum they spread apart on their way to the different parts of the cortex forming the corona radiala.

The second group of cerebral axons are the association fibers. These pass between one part of the cortex and another within the same hemisphere, enabling impulses to travel freely among the cortical cells. The third group of cerebral axons are the commissural fibers which pass between cortical areas in opposite hemispheres; these serve to unify the anatomically double cerebrum into a single physiological organ; the corpus callosum $(C c$, Fig. 60) is made up of commissural fibers.

Lobes of the Cerebrum. The convolutions of the cerebrum are sufficiently constant in number and position to serve as landmarks in locating particular regions. The individual convolutions, or gyri, have been given specific names, as have also the fissures, or sulci, which separate them. For our purposes it is necessary to mention by name only those fissures which mark off the grand divisions, or lobes, of the cerebrum. The division of the cerebrum into lobes is purely arbitrary, and is made for greater ease in describing it. In general the lobes correspond in position to the overlying skull bones for which they are named. The fissures which mark the boundaries of the lobes are indicated in Fig. 59. They are the fissure of Sylvius, the fissure of Rolando, and the Parieto-occipital fissure. The frontal lobe is that part of the cerebrum above the fissure of Sylvius and in front of the fissure of Rolando; the parietal lobe is between the fissure of Rolando and the parieto-occipital fissure; the occipital lobe is the wedge-shaped portion behind the parieto-occipital fissure; the temporal lobe is below the fissure of Sylvius; it is the only one of the lobes which is sharply set off as a distinct region.

Cortical Localization. A problem of much interest in connection with the study of cerebral functions is whether there is division of labor among the various parts of the cortex. Do certain groups of cells perform certain special functions, or are all cortical activities shared in by all the cells? This is not the place for a history of the solution of this problem. Suffice it to say that we 
now have positive proof of a high degree of specialization of function in the cortex.

Sensory Areas. In previous paragraphs the paths of the various senses were traced as far as their entrance into the cerebrum by way of the internal capsule. We must now continue the paths to their cortical terminations. The body sense fibers pass to that part of the parietal lobe just behind the fissure of Rolando; the region where they terminate is the body serise area. The visual tracts end in the occipital lobes in the visual areas. The auditory tracts terminate in the temporal lobes in a region just below and within the fissure of Sylvius; this region constitutes the auditory area. Although the paths of smell and taste are imperfectly known, their cortical terminations have been fairly well established. The olfactory area is supposed to be in the temporal lobe, and possibly at its very tip. The area for taste, gustatory area, is thought to be also in the temporal lobe, probably adjacent to the area for smell. Since the nerve-paths of the various senses lead directly to these areas, and since destruction of any one of them, by accident or disease, results in loss of the particular sense whose area is involved, we must conclude that the sensory areas are the receiving stations of the cerebrum. All afferent projection fibers entering the cerebrum terminate in one or another of the sensory areas. Within these areas they have synaptic connection with the association neurons of the region.

The Motor Area and the Pyramidal Tracts. In each hemisphere a region of the frontal lobe just in front of the fissure of Rolando contains numerous giant pyramidal cells whose axons extend into the white matter and are grouped together in the internal capsule as a conspicuous nerve tract, called the pyramidal tract. It extends through the midbrain to the medulla and appears upon the ventral surface of the latter as a well-marked anatomical feature. About midway of the medulla the pyramidal tracts cross the mid-line in the decussation of the pyramids ( $K$. fig. 66 ). This decussation is not complete; part of the fibers of each pyramidal tract continue along the same side of the medulla to the spinal cord and down the latter in the ventral column, forming the direct pyramidal tract. That part of each pyramidal tract which crosses over at the "decussation" proceeds along the spinal cord in the lateral column as the crossed pyramidal tract (Fig. 65). 
It appears that most of the fibers of the direct pyramidal tracts cross the mid-line in the spinal cord before reaching their terminations; so that the pyramidal tracts are finally crossed tracts. All the pyramidal axons have synaptic connection with the cells of motor neurons in the ventral horns of gray matter of the cord.

The pyramidal axons are branched at their tips, so that each communicates with several motor neurons. On page 136 we saw that each motor neuron connects with a number of muscle-fibers. It follows that a considerable group of muscle-fibers is under the control of each pyramidal axon. When we recall the large numbers of fibers which go to make up even our smallest muscles, we see that this arrangement, which cuts down the number of nervous elements required to operate the muscular system, does not at all impair the delicacy and efficiency of our muscular movements.

Since the pyramidal axons arise from cell-bodies within the cortex it is evident that the pyramidal tracts must be efferent paths. The intimate way in which the pyramidal fibers connect with the cell-bodies of motor neurons indicates that they form the paths by which the cerebrum exercises control over bodily movements. The anatomical evidence for that view has been corroborated and strengthened by physiological evidence. The German physiologists, Fritsch and Hitzig, showed that in dogs electrical excitation of those areas of the brain from which spring the pyramidal tracts is followed by movements of the muscles of the Body. They showed also that these are the only areas from which such movements can be elicited.

Upon the basis of all this evidence we are justified in looking upon the regions immediately in front of the Rolandic fissures as motor areas. These areas have been much studied physiologically in recent years. The brains of the higher apes have been preferred in these studies to those of lower animals because of their greater similarity to the human brain.

There have been a few observations upon the brains of human beings in cases where the surgical treatment of certain diseases has involved removal of portions of the skull overlying the Rolandic areas.

These recent studies have shown that there is a considerable localization within the motor areas themselves; stimulation of one point causes movements of the hand, of another the foot, of still 
another the head. They have shown incidentally, also, that the cerebral cortex is not painfully sensitive to direct stimulation. The men whose brains were excited electrically in the observations cited above were conscious throughout the procedure and reported no sensations of pain or discomfort at any stage.

Cortical Reflex Paths. The various sensory areas with their afferent nerve-paths afford means whereby impulses may enter the cerebrum from the different receptors; the motor areas, one in each hemisphere, with their efferent paths, provide for the passage of impulses from the cerebrum to the motor organs of the Body; the abundant equipment of association fibers within the cerebrum makes possible the passage of impulses across from sensory areas to motor areas. We can picture, then, reflex ares involving the cerebrum. Such arcs are necessarily complex, involving many more neurons than do the simple spinal cord reflex arcs already described. In a previous paragraph (p. 158) we saw that the simplest reflex arc through the cord involves at least two neurons, one sensory, and one motor. If we trace a reflex are involving the cortex from a receptor in the skin of the right hand, for example, to a retractor muscle of the right arm, we find in it at least five neurons and possibly many more. The five which are. necessarily included are: 1 , the sensory neuron which we suppose extends all the way from the receptor into the cord and up the dorsal column to a termination in the cuneate or gracile nucleus; 2 , a neuron of the fillet tract, having its cell-body in the cuneate or gracile nucleus, and its axon extending through the medulla and midbrain and the white matter of the cerebrum, crossing the midline in the "sensory decussation" of the fillet, and terminating in synaptic connection with a neuron of the body sense area in the left cerebral hemisphere; 3 , the neuron just mentioned, having its cell-body in the body sense area and an axon which passes by way of the cerebral white matter to the motor area; 4 , a pyramidal neuron of the motor area whose dendrites receive the impulse from the body sense neuron (3), and whose axon forms part of the pyramidal tract, crossing back to the right side of the Body in the decussation of the pyramids, and terminating in synaptic connection with the cell-body of a motor neuron in the ventral horn of gray matter of the cord; 5 , the motor neuron which forms the last link in the reflex chain, conveying the impulse from the 
pyramidal neuron to the muscle. It is doubtful whether any cortical reflex ares are actually composed of as few neurons as five; probably the simplest ones contain several additional association neurons within the cerebrum.

Cortical Reflexes Compared with Spinal Reflexes. As an example of a simple spinal reflex was cited the involuntary withdrawal of the hand from accidental contact with a hot body. To illustrate a simple cortical reflex suppose that my finger rests upon the terminals of an apparatus for generating electric shocks; I am told that when I feel the shock I must withdraw my hand. The shock may be so feeble as to be barely perceptible. Under such circumstances the withdrawal must be voluntary and the response, therefore, must involve the cerebrum. The chief objective difference between voluntary withdrawal of the hand in response to feeble stimulation, and its involuntary retraction in response to strongly painful stimulation is that the former reaction requires a noticeably longer time than does the latter. The only simple reflex whose time has been satisfactorily measured in man is the winking reflex; this requires about 0.06 second for its completion. The quickest cortical reflexes take about 0.15 second. This difference in time is much greater than can be accounted for by supposing the cortical reflex to involve a greater length of nerve-fibers, and therefore must be due to the fact that the cortical reflex involves a greater number of neurons and consequently more synapses to be crossed.

An additional difference which we recognize subjectively between spinal and cortical reflexes is that while the former are involuntary and unconscious, the latter are voluntary responses to stimuli consciously perceived. This difference will be discussed. more fully in a later paragraph, when the meaning of the terms "voluntary" and "consciously" shall have been considered.

Memory. We have seen that the primary function of the cerebrum is to introduce remote considerations as determining factors in the responses of the organisms. We have seen also that in order to do this the cerebrum must have an equipment of knowledge, which can be gained only through the receptor channels of the Body. The information which reaches the brain, to be of service, must be retained there until needed, and must be held in such a way as to be available when required. 
The neurons of the nervous system generally act, in the main, as conductors pure and simple. When they are stimulated nerve impulses are aroused; these spread over them and escape by those synapses whose resistance is not too high; thus other neurons are involved and so the impulses advance to a motor termination.

The cortical neurons of the cerebrum owe their dominant position in the nervous system chiefly to a peculiar ability which they possess of "holding up" impulses which come to them, retaining them indefinitely, and giving them out again in the future, if necessary, over and over. This storing of impulses constitutes memory. The "reflex" animal, because he is deprived of this property, must always respond immediately to adequate stimulation; the intact animal may respond immediately or may retain the stimulus as a memory to modify his future activities. Since the intact animal has within his cerebrum a store of impulses "held in leash," he may at any time become active through the liberation of some of them, without immediate external stimulation.

Association Areas. The different sensory areas and the motor areas occupy only a small part of the whole cerebral cortex. Most of the frontal lobes and large areas of the parietal and temporal lobes are not involved in the immediate reception of impulses, nor in their transmission to the Body. These areas are as richly supplied with interconnecting neurons as any part of the cortex. They are assumed, without very positive proof, to be the seat of a function we know the cerebrum to possess, that of association.

The Nature and Mechanism of Association. At birth the brain of the infant may be compared to a clean page. It bears no impressions of any sort. Such activities as the infant shows are purely reflex. In course of time sense impressions begin to come into the sensory areas of the cortex. These register themselves more or less definitely as memories, and presently the child is in possession of a considerable store of memories of various sorts. He may know the sound of his mother's voice or may recognize her face. As yet, however, there is no connection between these independent impressions. When in the child's mind that voice is associated with that face, so that he knows them as parts of a single whole, he has performed an act of association. From. this time throughout his life his memory is not alone of the simple sound of the voice or the appearance of the face but of the mother 
whom he has learned to know by these associated impressions.

Acts of association are supposed to be earried on within the association areas of the cortex. We may picture the process in the example cited above somewhat as follows: The impression of the voice is stored in the auditory area; that of the appearance of the face is in the visual area; both these sensory areas have rich communications with neurons of the association areas. By some means impulses from the sensory cells where these impressions are stored meet in a cell of an association area. That cell builds from these single related sense impressions, a composite, which is stored in turn as a memory. As additional related information is gained the composite, or concept, is enlarged.

The union of related impressions into concepts does not necessarily involve loss or impairment of the fundamental impressions themselves; the child in whose mind is a definite concept of his mother retains also clear memories of her voice and her face. The paths of communication between the cells where are stored the primary sense impressions and those where the resulting concepts are formed seem to remain always very easy of passage. The sound of the mother's voice calls up the entire concept of the mother with great clearness, even though years may have elapsed since it was heard.

Since concepts are stored as memories they may serve in their turn as bases for more complex associations; these again by becoming memories may contribute to the associative process, and so the complex structure of the mind is built up, resting at bottom always upon primary sense impressions.

The act of association is essentially one of combining related memories; the formed associations become memories in their turn. For these reasons the term associative memory is used as more truly describing the nature of associative processes than the older expression "the association of ideas."

The use of a memory in forming one association does not interfere with its use in the formation of others. This ability of the cerebrum to use memories over and over again is a very valuable property since it enables us to make the utmost of all our knowledge.

Development of the Cortex. The increase in intellectual power which accompanies the growth of the child is not the re- 
sult of any increase in the number of nerve-cells, for the child is born with his full number. It is, however, based upon their continuous development; this development consisting chiefly of greater and greater branching with correspondingly richer synaptic connections. At birth scarcely any cortical cells are sufficiently developed to be functional. The sensory areas first become so. The association areas reach their highest point of development at about the thirty-fifth year. At this age the anatomical progress of the brain comes to an end; all possible paths of association have been laid down. This does not mean, however, that all possible associations have been formed. These continue to be formed so long as the brain continues active. It is probably true, however, that with advancing years there is a diminution in the freedom of associative activity; the brain no longer accomplishes daring feats of thought, such as constitute creative genius, but plods along in the ruts established by its earlier activities. This fact explains why conservative tendencies usually become more pronounced as age advances.

The Functions of Associative Memory. It is because the cerebrum is able to form associative memories that the organism can adjust its responses with due regard to remote as well as to immediate considerations. Incoming stimuli, which in a "reflex" animal would produce a definite response of a certain kind, are in an intact animal balanced against such related associative memories as the animal possesses; if these indicate that the natural reflex response is the proper one to make, the animal responds as does the "reflex" one; if, however, they indicate a different line of action as more advantageous, the animal substitutes for the natural reflex response a different one, suited to the situation.

Associative memory also forms the basis for the execution of complex movements from feeble, immediate stimuli, or in their absence; the young puppy responds to his master's whistle only by a pricking of the ears; in the older dog the sound of the whistle arouses a chain of associative memories and under their impelling force he executes the complex movements which carry him to his master's feet.

In order that associative memory may influence bodily activities it must have access to the efferent nerve-paths of the cerebrum. This access it has through rich connections from the 
association areas to the motor areas. It must have also the power to stimulate the efferent nerves. This power it exercises through the function of volition.

Volition. Although all voluntary acts result from nerve impulses which have come from the motor areas of the cerebrum by way of the pyramidal tracts, we cannot suppose that they originate in the cells of the motor cortex. There is no evidence that these or any cortical cells are able to originate any activities whatever. All voluntary acts, as a matter of fact, are based upon associative memory; the immediate stimulus to the performance of the voluntary act comes, not from the motor areas, but from that part of the association areas where the exciting memory is stored. All memories, as we have seen, are at bottom stored sensory impressions. What happens, then, when we perform voluntary acts is that we cause to pass on to the motor areas stimuli which originally entered the nervous system by way of the receptors, and which have since been combined in various ways, and the resulting associations stored as memories. Voluntary acts are, therefore, the completion of reflexes.

The Usefulness of Associative Memory Depends on its Orderliness. It is perfectly obvious that associations to be of value must be formed from related impressions or related concepts. We know that our brains normally form associations in this orderly way. How the brain is guided in its selection of material for making associations so as to include what is relevant and exclude the rest is quite beyond our knowledge or even imagination. That in the highly complex associative processes which we call thinking there may be a conscious selection or rejection of memories we know from our own experience.

It is true, of course, that the brain; being an imperfect instrument, often makes mistakes and forms associations that instead of being useful give rise to harmful activities. The resulting disaster, through the additional knowledge it affords, may enable the brain to form correct associations next time. Thus we profit by our mistakes.

The Interaction of Associative Memories. Inhibition. The human brain acquires in the course of years such a wealth of associative memories, based upon so many phases of experience, that the determination of the conduct to be employed in any particular 
situation becomes often a matter of much difficulty. One set of memories point toward one course and another set toward quite the opposite course. When this happens it is necessary to call in more and more remote considerations until the balance tips unmistakably in one way or the other. When even this procedure fails to be decisive, or when the mind wishes to avoid the labor of deciding by this method recourse is often had to a selective external stimulus. Deciding a course of action by the flip of a coin is a case in point.

Associative memories also come into conflict when immediate considerations point toward one course and remote considerations toward a different one. Associative memories are classified by placing those of remote bearing higher than those of immediate bearing. Highest of all, because most remote, are abstract conceptions of right and wrong; conceptions of altruism, care for mankind, are higher than conceptions of family love; these in turn rank above purely personal considerations. Personal considerations which have regard to the future are higher than those dealing only with the immediate present. The progress of civilization is largely measured by the degree to which remote considerations outweigh immediate ones in determining conduct.

Because the cerebrum rests upon an underlying reflex mechanism the tendency of the organism is always toward immediate response to sensory stimulation; the hungry man tends to take the first food that comes to hand; the cold man tends to seek the nearest available shelter. The action of associative memory, when higher considerations dictate a different course, is to prevent or inhibit the carrying out of the immediate response. Inhibition is, then, one of the important functions of associative memory. The man who deliberately does what he knows to be wrong, acts as he does because his conceptions of right are not powerful enough to inhibit the response to the lower stimulus. The importance of inculcating the highest principles of right living by training and example, during the receptive period of the brain's development; is therefore clearly manifest.

Will Power. In some persons there is an inborn tendency to respond to immediate stimulation, even though associative memory shows that such response is not for the best. Such persons we describe as weak-willed. Those in whom the dictates of associative 
memory are supreme we call strong-willed. The weak-willed person yields to temptations which are powerless to move the one whose will power is great. In this definition of will power we have set associative memory against immediate stimulation, and usually the conflict is between these. Sometimes, however, the struggle comes between different immediate stimuli. In the weak-willed man the more insistent ones are likely to control, rather than the more important. If he is beseeched by various friends to accompany them different ways the most vociferous is usually the one to carry him off. The strong-willed man, on the other hand, makes his decision on other grounds. On the other hand, various associative memories may be in conflict, and here again, whether obedience will be to the most elamorous or the most important depends on the strength of the will.

Cerebral Control of Spinal and Cerebellar Reflexes. The, exercise of the inhibitory function of associative memory as just described involves an ability on the part of the cerebrum to modify the reflexes of the lower parts of the nervous system. There is abundant evidence that such ability actually exists. In the case of spinal reflexes it may be supposed to act through the discharge of impulses from the motor area which in some manner increase synaptic resistances in the course of the reflexes sufficiently to block them. A feature of the inhibition of spinal reflexes which points to this as the means of bringing it about is that in the case of sharp sensory stimulation the inhibition must be established before the stimulus is received. If one unexpectedly touches a hot object he automatically and inevitably jerks his hand away, but if he knew the object was hot, and nevertheless found it necessary to grasp it he could, through an act of volition, block his reflex path so effectively that the tendency to draw the hand away would be completely overcome.

Cerebellar reflexes are also subject to cerebral control. There is evidence that part of the pyramidal tract from the motor area terminates in the brain stem in relationship with paths leading into and out of the eerebellum. Apparently thus voluntary control of locomotion is exercised. That we have such voluntary control is evident. We can start, stop, or modify our locomotor acts at will, although as we have previously seen, the performance of the reflexes as distinet from their guidance, is automatic. 
Habit Formation. Just as a single sensory impression repeated orer and over becomes more firmly fired in memory than does ane received only once, there seeming to be some sort of impression upos the remembering nerve-cell which becomes deeper at each repetition of the stimulus; so every intersetion of associative memories in deternining a course of conduct leaves a track upon the cells involved, which is deepened by repetition of the same series of memories leading to the same conduct. One of the strong teadencies of the brain is to arrange its associative memories thus in groups leading to certain definite responses. It is this tendency which lies at the basis of habit formations."

Habits which are formed in this way, by repeated following of the same line of thought to the same actions, take on much of the character of simple reflexes. A stimulus which arouses the chaim of associative mecorories results in immediate canving out of the habitcal action. A definite act of inhibition on the part of other associative memories is necessary to prevent the response. All of us here meny babits of this sort and they are of the greatest value in our daily lives because on account of them many things that we have to do are more easily done than they would be if the abole mental process upon which the acts depend bad to be gooe through with at each repetition.

The teodener to babit formation can be used tery effectively in training the child to right actions. It can be used as well in training to right tboughte, since thoughts are associative proccoses, and these terod to follow the lines laid down by habit.

Language. Of all the powers of the human mind its power to use language has had as wanch to do with the progress of the race as any other property it presesses. Language, from the standpriet of escebral function, is simply a special sort of ascociation; the association of artitrarily selected sounds or written symbinds with objects and concepte. Let us, for illustration, consider again the case of -tbe child forming the conoept motber. Coindilent with the association of ber voice, appearance, and otber persical attribetes into a definite concept is the repeated auditory

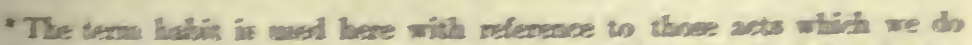

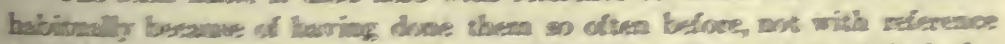

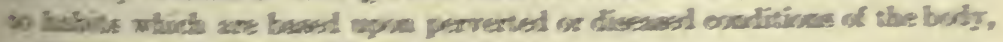

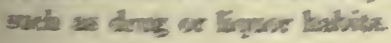


stimulus of the word mother, heard when she is present or when she is indicated in some way. In course of time this particular succession of syllables is included as part of the concept. Several years later the group of written symbols making up the word mother is included in the same concept. Thus larguage, spoken and written, becomes indissolubly included in our whole mental equipment.

It is a curious fact that in man the use of language seems to be not optional, but a necessary factor in his mental development. Two lines of evidence favor this view. The first is the common experience of all of us that we are incapable of thought except in terms of words; coupled with the observation that no race of men exists or is known to have existed without some form of language. The second, and more striking, fact is that certain regions in the association areas of the cerebrum are specially devoted to language associations. Four such regions are known, having been revealed by the physiological effects of their impairment through accident or disease. Two of these areas have to do with spoken, and two with written, language. One of the two areas for each form of language is sensory and the other motor. An interesting thing about these language association areas is that they seem to be confined to one of the cerebral hemispheres; in right-handed people the left hemisphere contains them, and in left-handed people they occur in the right hemisphere. It has been observed, moreover, that the development of right or lefthandedness in infants is coincident with their learning to use language. Just what the relationship between the two properties may be is not clear.

Impairment of a sensory language area results in word-deafness or word-blindness; the sounds are heard, or the words are seen, but they are without meaning because the power to associate language with concepts is affected. When motor language areas are injured the power of expression is lost. The commonest of all these abnormalities is the loss of power to use spoken language, a condition known as motor aphasia. The sufferer from this condition knows what he wants to say but is unable to recall the words by which to express his ideas. Embarrassment often gives rise to a momentary inhibition of the motor aphasia region, resulting in the same inability to recall the needful words. 
Since all our mental processes are dependent on language, impairment of the language areas would be expected to lower the whole mental power. This appears to be the case in most sufferers from this condition.

There is no evidence that any species of animals except the human species possesses the power to use language. This difference sets man sharply apart from the animals most nearly approaching him in intelligence.

Consciousness. This is a phenomenon that we all recognize as existing in ourselves and as accompanying most if not all of our cerebral activities. That it is present when the cortical cells are actively functioning and absent when they are inactive is indicated by the fact that any treatment, such as anæsthesia, which depresses nerve-cells, tends to abolish consciousness. It is a phenomenon whose nature is wholly unknown, and for whose existence, even, there is no objective evidence. We cannot prove that any lower animal has the same sort of consciousness that we have. We can only suppose from the general similarity of their cerebral processes to ours that this particular phenomenon is also in them as in us. As we go down the animal scale where mental processes become simpler and simpler does it follow that consciousness becomes dimmer and dimmer? We ordinarily assume this to be true, but without any positive evidence upon which to base the assumption.

Emotions. Another set of phenomena accompanying cerebral activity, but known chiefly by subjective experience, are the emotions. We know that certain sensory stimuli give us pleasure, others arouse disgust. Love and hate, sorrow and joy, are mental states which are associated with certain sense impressions immediate or remembered. Emotion, like consciousness, does not lend itself to objective study, and therefore does not come within the realm of physiology beyond simple recognition of the existcnce of the phenomenon. It is true that emotional states are usually accompanied by reactions of other parts of the body, the blush which accompanies embarrassment being an example, but this allows us only to judge whether an emotion is present, and tells us nothing about its actual nature.

Cerebral Functions Compared in Man and Animals. In the higher animals as well as in man associative memory is the rep- 
resentative cerebral activity. So far as we can judge it represents in animals the climax of intellectual achievement. No animal has ever been seen to perform any act, not purely reflex, as are all "instinctive" actions, which associative memory cannot account for. The activities of man are for that matter based upon associative memory almost as fully as are those of animals. The important-intellectual difference between man and animals is the possession by man of the faculty of reason, which is denied to animals. The power to reason is itself, however, based upon associative memory. It may be roughly explained as the association of concepts whose relationship is not obvious. An animal, according to this idea, cannot reason because he cannot form associations except of concepts that manifestly belong together. The man reasons by perceiving relationships in apparently unrelated facts or ideas.

We must admit, however, that the most complicated acts of associative memory that have been observed in animals simulate so closely mental processes which in man are ordinarily thought of as reason, that no hard and fast limit of the latter can be set. We would scarcely venture to establish any point as marking the utmost intellectual achievement in animals, although we would not hesitate to say that in comparison with the possibilities of the human brain the extremest mental process of the most intelligent animal dwindles to insignificance.

The powers of using language and of reasoning are the only cerebral functions possessed by man and not by animals of which we have positive objective proof. They are therefore the only ones of which physiology can take account at the present time. Physiology does not thereby deny, however, the existence of many activities in the human brain which are without counterpart in the brains of lower animals. While associative memory accounts completely for all non-instinctive actions of the lower animals, the history of the human race and the experience of individuals contain much that baffles explanation in terms of associative memory or of reason. The factors which lead the race always onward and upward to greater and greater heights of spiritual achievement are beyond the power of present-day physiology to analyze or even discuss.

Nourishment of the Brain. The cells of the cerebral cortex 
are very dependent upon their blood supply. A slight diminution in the rate of blood flow through the brain may depress the coltical cells to such an extent that consciousness is lost. The problem of retaining consciousness is, then, the problem of keeping the cerebral circulation up to the proper level. How this is accomplished during our waking hours, and how its falling off affords opportunity for needed intervals of sleep will be discussed in connection with the circulation of the blood (Chap. XXII). 


\section{CHAPTER XII}

THE AUTONOMIC NERVOUS SYSTEM. NERVOUS FATIGUE. HORMONES OF THE NERVOUS SYSTEM

The Brain Stem (Medulla and Midbrain). If our attention had been called to the matter when the courses of the various afferent and efferent pathways of the cerebrum and cerebellum were being described, we should have noted that the brain stem forms a great highway through which pass virtually all impulses on their way to or from the higher brain structures. Moreover, most of the nerve tracts leading through the brain stem do not pass directly through, but suffer interruption in one or the other of the many nuclei which occur therein. Wherever a nerve tract is interrupted by a nucleus the axons leading into the nucleus terminate in synaptic connection with new neurons by which the tract is continued. There is always the possibility, where such connections are being formed, of a certain amount of diversion from the main channel into side channels. The medulla and midbrain, then, are strategically located for concentrating into small areas influences from all the receptors of the Body. This region has also its own efferent pathways. It affords, therefore, an additional field for the establishment of reflex arcs, but, as we shall see, of a somewhat less specialized sort than are afforded by the cerebrum and cerebellum.

There are a number of so-called "vital processes" going on in the Body. These are activities whose continuance is essential to the maintenance of life, and which must, therefore, go on quite independently of the will; they are of a sort, however, to require modification in accordance with the demands of the Body. Examples of such activities are the beating of the heart, breathing, the secretion of sweat.

Many of these so-called "vital" activities are really as purely reflex as any of the ordinary reflex acts of the Body, and those that are truly automatic are subject to constant reflex influence. 
Their immediate control is vested in certain centers located in the medulla. This location for the centers insures that they shall never be wholly free from sensory stimulation, for no matter how quiet the surroundings of the Body may be the processes going on within it give rise to sensory stimuli, and, as we have seen, whatever impulses are aroused are sure to pass through the brain stem. Detailed consideration of the various centers is not necessary here as each will be treated in connection with the vital process with which it is related.

The Autonomic or Sympathetic System. This system is treated as a distinct portion of the nervous system because to a rather special physiological function it adds peculiar anatomical relationships. In spite of its anatomical and physiological peculiarities, however, it forms an integral part of the whole nervous system, and interacts with other parts as completely as though nothing distinguished it from them. Its old name has no present significance, having been given to it in the erroneous belief that its function is to bring remote organs into sympathy with each other. The name autonomic, by which it is at present known, signifies a mechanism not under voluntary control, and in thus emphasizing an important feature of the system, constitutes a more satisfactory designation. The special physiological function of the autonomic system may be stated in a sentence: it forms the efferent connection between the central nervous system and all the smooth muscles and glands of the Body, and the heart.

It will be recalled that the skeletal muscles have motor connection with the central nervous system by means of motor neurons, structures whose cell-bodies lie in the ventral horns of gray matter and whose axons extend directly to the muscles. The autonomic system differs from the motor system to skeletal muscles in that each pathway from the central nervous system to a smooth muscle or to a gland is made up of a succession of two neurons. The first neuron has its cell-body in the ventral horn of gray matter; its axon passes out by way of the ventral root of the spinal nerve and the communicating branch (see p. 147) to one of the sympathetic ganglia where it forms synaptic connection with the second neuron of the chain. This neuron sends its axon back over the communicating branch to the spinal nerve along which it passes to its destination in a smooth muscle or a gland. 
Because of their positions with regard to sympathetic ganglia the first and second neurons are known respectively as pre-ganglionic and post-ganglionic neurons. The latter present the anatomical peculiarity of being for the most part devoid of myelin sheaths; nerve-trunks made up of post-ganglionic fibers can therefore be distinguished from other nerve-trunks by their gray color.

The structures innervated by the autonomic system perform their functions by acting to a considerable extent in groups together; not individually as do skeletal muscles. To enable them to be stimulated in groups single autonomic pathways commonly involve numerous end structures. This is accomplished by rich branching of the pre-ganglionic fibers, enabling each to have synaptic connection with a number of post-ganglionic neurons, and so to influence simultaneously numerous end organs.

The Effect of Nicotine. Much of our knowledge of the autonomic system has resulted from the discovery that application of the drug nicotine to sympathetic ganglia prevents the passage of impulses over whatever synapses may be contained therein. By the use of this drug, therefore, the point of contact of pre-ganglionic with post-ganglionic fibers in the pathway to any particular organ can be determined. To illustrate how its use brings out these points of contact we may take the autonomic innervation of the eye. The size of the pupil is regulated by opposing autonomic fibers; one set tending to constrict it, the other to dilate it. By the use of nicotine it has been shown that the contact of pre-ganglionic with post-ganglionic fibers in the constrictor pathway is in the ciliary ganglion, which is in the orbit; while for the dilator pathway the connection between pre-ganglionic and post-ganglionic fibers is in one of the sympathetic ganglia of the neck.

Reflex Control of the Autonomic System. The autonomic system, as we have seen, forms only the last step in the conducting pathway by which influences are brought to bear on the structures it innervates. Like the motor system for the skeletal muscles it conveys only those impulses which are imparted to it from without. It is, in other words, the efferent portion of a reflex mechanism.

The so-called "vital" processes of the Body are, with the exception of respiration, largely carried on through the agency of smooth muscles and glands. The autonomic system is, therefore, 
the system through which these processes have their nervous control. In the paragraph dealing with the brain stem the existence therein of reflex "centers" for the various "vital" processes was inentioned. On the afferent side these centers are subject to all sensory stimulations which affect the Body. On the efferent side they act through the autonomic system.

This reflex mechanism is not subject to voluntary control except for the single case of the muscle of accommodation of the eye, the ciliary muscle. This muscle is innervated through the autonomic system, but can be voluntarily controlled as completely as any muscle in the Body. When we say that the autonomic system is not under voluntary control we are simply stating in other words that the motor area of the cerebrum is not able to establish connection through the pyramidal tracts with the neurons of this system. Since this sytem is outside the control of the motor area all reflexes which affect it must be immediate ones. Only present stimuli can arouse it to activity. When we bear in mind that the proper functioning of the Body requires its vital activities to be adjusted to its immediate circumstances and not to its circumstances of a week or a year ago, the necessity that autonomic reflexes be immediate is manifest.

Grand Divisions of the Autonomic System. Not all parts of the central nervous system give rise to autonomic pre-ganglionic neurons. A group originates in the brain stem. Its fibers are distributed through cranial nerves. These are called cranial autonomics. The vagus nerve (p. 152) consists largely of cranial autonomic fibers distributed to various organs of the trunk. A second group arises in the thoracic and lumbar regions of the spinal cord. These are distributed through the sympathetic system of the old classification. They are known as thoracico-lumbar autonomics. The third group of fibers arises in the sacral portion of the cord. These are distributed to the pelvic region and constitute the sacral autonomics.

In a previous paragraph (p. 116) attention was called to the peculiar feature of smooth muscle, shared by heart muscle, of requiring double innervation. These tissues must have stimulation to augment their activity and other stimulation to inhibit it. Both sorts of innervation are furnished through the autonomic system. An important feature of the system as a whole is that the opposing 
described in the last paragraph. If we note, however, that the conditions that arouse these reactions are such as call for a rallying of the Body for flight or struggle we begin to see wherein the importance of this mechanism lies. In general its effect on the Body is a diversion of resources from the maintenance organs to those of cxternal adaptation, the latter making up the mechanism on which the Body must depend for salvation in time of stress. The dilation of the pupil may be supposed to enhance the sensitiveness of vision. The dryness of the mouth signifies that energy ordinarily einployed in producing saliva is now set free for use elsewhere. The erection of the hair, of no importance in man, is in many of the lower animals an important part of the scheme of defense. The pallor of the face is the result of the diversion of blood from the skin, whence it can be spared, to the muscles and brain. where it is greatly needed. The acceleration of the heart results in a quickened circulation of blood through the regions of heightened activity. The inhibition of the digestive organs is another example, like the cessation of salivary secretion, of the suspension of functions not immediately essential, in order that all the Bodily energy shall be available for the emergency. Numerous other reactions of the thoracico-lumbar mechanism also contribute to the general plan of defense. These we shall examine in due course. Here we need only note that all of them tend toward increasing the efficiency of the skeletal muscles and the central nervous system, which together make up the emergency mechanism.

The Relation of the Autonomic System to Emotional States. In a previous paragraph (p. 189) the fact was noted that emotion in general is accompanied by activity of the autonomic system. We have just examined the basis for this relationship in those emotions that are associated with the immediate need of self-preservation. An interesting fact, and one of great practical importance, is that the emotion of worry or anxiety, which is responsible for much of the discomfort of life, has significance as a means of preparing before hand for a time of trouble. We may describe it as an anticipatory emotion. We bring about in our Bodies through worry the characteristic reactions of the thoracico-lumbar autonomic system. Unfortunately, these reactions, useful indeed when the actual stress is at hand, are inimical to the carrying on of the ordinary bodily processes, so that their occurrence in advance of 
the emergency does no particular good, and when, as usually happens, the worry proves to have been needless, real harm.

Emotion of satisfaction and contentment appear to manifest themselves chiefly through the cranial autonomics. The sacral autonomics control the activities of the generative organs. Their emotional associations are for the most part those concerned with reproduction.

Neuro Muscular Fatigue. In a previous chapter (p. 101) muscular fatigue was discussed, and the fact pointed out that under ordinary circumstances the muscles are protected from fatigue by precurring nervous fatigue. Two general locations are recognized in the nervous system for the occurrence of fatigue. The first of these is in the synapses. The delicate junctions between neuron and neuron are believed to be highly susceptible to fatigue. In terms of the prevailing theory we would say that the accumulation of waste products at the synapses increases their resistance to the passage of nervous impulses, and that the resulting hindrance to nervous action constitutes fatigue. The second place of fatigue is at the junctions between motor nerves and the fibers of skeletal muscle. These junctions consist of minute flat plates pressed against the muscle-fibers and in which the nerve-fibers terminate. They are known as motor end plates. There is ample proof that as the result of continued excitation of a muscle the neuro muscular junctions show a falling off in the ease with which impulses pass through them to muscle-fibers. Synaptic fatigue and end plate fatigue occur in such minute structures that we would be apt to expect recovery to be rather rapid. As a matter of fact quick recovery seems often to occur. It is a common experience to obtain marked relief from fatigue by the briefest sort of a nap. Nevertheless, we are bound to recognize that although the feelings of fatigue may be quickly dissipated actual restoration of the fatigued structures requires time. An ordinary night's rest is none too long for recovery from nervous fatigue. In fact there is definite evidence that under many conditions a single night's sleep does not suffice for complete restoration. The almost universal and very valuable habit of abstaining from ordinary duties one day in seven has its physiological significance in the necessity of allowing at intervals a longer period of restoration than the usual nightly ones, in order that any fatigue which failed to be overcome in ordinary course 
might be gotten rid of therein. That this longer rest period be spent in sleep is by no means always desirable. When we recall that the synapses which experience fatigue primarily are the ones that are being used we realize that the essential for rest is often diversion rather than sleep. During the rest periods one's mental activities should be along as different lines as possible from those of his ordinary workaday life. Thus his fatigued synapses can be resting while others are busy. This same fact emphasizes the importance of diversity of interests. Where one's thoughts cling in certain ruts mental fatigue is apt to be more pronounced than where various lines can be followed. In those whose occupation requires prolonged concentration it is particularly advantageous to have widely different interests to turn to during the intervals of relaxation.

Hormones of the Nervous System. Adrenin. This hormone is interesting chemically because it was the first hormone to be obtained pure, and is even yet by far the best known of the numerous hormones produced in the Body. Various names have been applied to it (suprarenin, epinephrin, adrenalin). The name given in the paragraph heading is coming into general use at present.

The Suprarenal Capsules or Adrenals are a pair of small organs, weighing together about 12 grams $\left(\frac{1}{2} \mathrm{oz}\right.$.) placed one on the top of each kidney. They have, however, no intimate connection with the kidneys, and in many animals are placed at some distance from them. Each consists of a denser less colored external cortex, and a central deep yellow-brown softer medulla. The cortex is subdivided into chambers by connective tissue, and the chambers are filled by closely packed, polygonal nucleated cells. Similar cells are found in the medulla, which is, moreover, closely connected with the sympathetic system and is richly supplied with nerves.

It was noticed some seventy-five years ago by a physician named Addison that certain obscure diseased conditions characterized by great debility and by the appearance of bronzed patches on the skin, and leading to death, were found on post-mortem examination to be accompanied by disease of the adrenals. The disease has since been named Addison's disease. When the suprarenal capsules are completely removed from animals a similar fatal 
diseased condition results, death taking place in warm-blooded animals within two or three days, and being preceded by muscular weakness, dilation of the arteries, mental feebleness and general prostration.

These symptoms show that the hormone is essential to life, although they do not afford any very positive evidence as to the manner of its working. Careful studies have shown that adrenin is present in the blood under ordinary circumstances in almost inconceivably minute amounts. A striking feature of this, and of hormones in general, is their remarkable potency as chemical stimulants. Our detailed knowledge of the functioning of adrenin has been gained chiefly by observing the results of its introduction into the blood in larger than normal amounts. The Body responds to these enlarged doses by a considerable number of very definite reactions which, when first observed, seemed to be quite unrelated, but are now recognized as combining to bring about a particular bodily condition, and one which, as we shall see, is sometimes of great importance to the organism. Not all these effects of adrenin can be described in this place, some will have to be deferred to later chapters; but enough can be presented to make clear the significance of its action.

One of the properties of adrenin is to stimulate chemically the terminations of the thoracico-lumbar autonomic system. It is thus able to bring about the same bodily reactions as are called forth through thoracico-lumbar autonomic activity. Dilation of the pupil, acceleration of the heart, constriction of the blood-vessels, with consequent heightened blood pressure, all are brought about by the injection of adrenin into the Body. These manifestations, as we saw above, are part of what we have described as the emergency reaction of the Body, and the ability of adrenin to bring them about reveals its function as the emergency hormone. The emergency reaction is so vital in time of stress that the Body does not depend wholly on the nervous system to evoke it. The action of the thoracico-lumbar autonomics is reinforced by the chemical stimulation of adrenin. This adrenin action depends, as we have seen, on the presence in the blood of larger than normal amounts of the hormone. The adrenal bodies are under the control of nerves which form part of the thoracico-lumbar system. Whenever, in a time of excitement, there is an outrush of impulses over this sys- 
tem, the adrenals are stimulated to great activity, and pour out their product into the blood stream. Thus at the time when increased adrenin is advantageous to the organism it is provided. The persistence of the bodily effects of strong emotion after the emotion itself has subsided may be explained by the continued presence of adrenin in the blood.

The reinforcement of the thoracico-lumbar autonomic mechanism is only one phase of the emergency function of adrenin. Another, and very interesting, feature of its action is in connection with the fatigue of the neuro-muscular junctions described in an earlier paragraph (p. 198). We saw there that the effect of fatigue on these junctions is to make the passage of impulses over them difficult. Recently the important discovery has been made that adrenin has the property of counteracting this fatigue, and thus making the muscles more accessible to nervous impulses. The value of this property in time of emergency is obvious. It explains a familiar fact that was unexplained before, namely, the "strength of desperation." Why a man in a tight place should suddenly experience an access of strength we now know is because in connection with the powerful emotions engendered by his situation there is an outpouring of impulses over his thoracico-lumbar autonomic system. His adrenal bodies are stimulated thereby to abundant production of adrenin; the adrenin is carried by his blood to all his. muscles, and there makes the access of nerve impulses to the muscles more ready. The gain is not in actual muscular strength, but in ability to use to the full the strength already present.

The Thyroid. This organ lies in the neck on the sides of the windpipe and consists usually of a right and a left lobe united by a narrow isthmus across the front of the air-tube. It is about thirty grams (one ounce) in weight; in the disease known as goiter it is greatly enlarged and its structure altered. The thyroid is dark red in color and very vascular, richly supplied with nerves, and is subdivided by connective tissue into cavities or alveoli, the largest of which are just visible to the unaided eye. Each alveolus is lined by a single layer of cuboidal cells, and filled by a glairy fluid known as the thyroid colloid.

From the gland can be obtained, in addition to the usual organic compounds, a peculiar substance containing a large percentage of iodine, and known as iodothyrin. This compound was 
thought, when first discovered, to be the hormone of the gland, but fuller study showed that iodothyrin as such is not the hormone although it probably has to do in some way with it. Although the chemistry of the hormone is not perfectly known its physiology can be studied indirectly by observing the effect of changes in the amount present in the Body. These changes may be brought about experimentally or may occur as the result of disease.

Studies thus made show that the hormone of the thyroid gland has a great deal to do with the proper carrying on of those chemical activities of living cells which constitute their "vital" processes and which are grouped together under the term metabolism. The nervous system is peculiarly dependent upon this hormone for its proper development and for the proper carrying on of its metabolic activities. This fact appears strikingly in cases in which the hormone is deficient in amount. In adults a condition known as myxedema is the result of such deficiency; its chief manifestation is distressing mental deterioration. Sometimes children are born in whom the thyroid gland fails to develop properly; they grow into dwarfish, misshapen idiots. To such a condition the name cretinism is applied. The sufferers are called cretins. Thanks to the discovery that by simple feeding of thyroid material the hormone can be supplied in ample quantity, sufferers from myxedema and -cretinism are now restored to perfectly normal condition; although it is said that for the treatment to be wholly successful for cretins it must be begun quite early in life.

There is a disease known as exophthalmic goiter (Grave's disease), named from the protrusion of the eyes which is a prominent symptom. This disease is due to an increase in the amount of the thyroid hormone. The effects on the Body are just the opposite of those seen in myxedema. There is heightened nervous activity, often proceeding so far beyond the normal as to constitute mental instability. One of the triumphs of modern surgery is the establishment of a method whereby enough of the thyroid can be removed to reduce the hormone to normal amount, and so cure the complaint. In this connection only the effects of the hormone on the nervous system are discussed. In a later chapter (p. 513) its influence on general metabolism is considered.

Emergency Action of the Thyroid. An interesting fact of recent discovery is that during the outpouring of autonomic in- 
fluences in time of stress the thyroid shows augmented secretory activity. The organ is innervated by the thoracico-lumbar system, and so may be excited directly. In addition to this means of arousing it, the thyroid may be stimulated to activity chemically by means of adrenin. Whenevier the blood is charged with this latter hormone the thyroid is thrown into activity. So far as we are able to judge from present knowledge the importance of this emergency action of the thyroid is in the general speeding up of the chemical activities of the Body; and possibly also in heightening nervous irritability, although that the latter effect can be brought about so promptly as would be necessary in an emergency mechanism has not been demonstrated. 


\section{CHAPTER XIII}

\section{THE RECEPTOR SYSTEM. INTERNAL AND CUTANEOUS SENSATIONS}

The Receptor System constitutes the Body's means of gaining information of its surroundings and of such internal conditions as it needs to know about. Since the surroundings may play upon the Body in many different ways and through the operation of many forms of energy, receptors are provided which respond to all sorts of stimuli. Inasmuch as proper adaptation requires that different sorts of stimuli affect the Body differently particular receptors are specialized to respond most readily to particular kinds of stimulation.

An interesting thing about the responses of the different receptors is that while their adaptation to special forms of stimulation does not exclude the possibility of their being aroused by other sorts of stimuli than the normal ones, when so aroused the effect in consciousness is as though the normal stimulus had been applied. Pressure on the eyes gives rise to sensations of light; electrical stimulation of the tongue may cause sensations of taste. This fact has led physiologists to take the view that the quality of any sensation depends on the region of the cerebrum to which it comes, and that it is quite independent of the structure of the receptor or the manner of its stimulation. If this is true it, accords well with another conception which most physiologists find very attractive, that the nerve impulse, whatever it may be, is the same sort of process wherever it occurs. It is, of course, evident that this idea, the so-called "doctrine of specific nerve energies," cannot be true if the quality of sensation depends in any manner upon the nature of the receptor or the way in which it is stimulated. It must be confessed that many known facts about the senses, that of sight particularly, cannot at present be explained upon any basis which excludes differences in the receptor as determining factors of the quality of sensation.

The Differences between Sensations. We distinguish among 
our sensations kinds which are absolutely distinct for our consciousness, and not comparable mentally. We can never get confused between a sight, a sound, and a touch, nor between pain and hunger; nor can we compare them with one another: each is sui generis. The fundamental difference which thus separates one sensation from another is its modality. Sensations of the same modality may differ; but they shade imperceptibly into one another, and are comparable between themselves in two ways. First, as regards quality: while a high and a low pitched note are both auditory sensations, they are nevertheless different and yet intelligibly comparable; and so are blue, purple, and red objects. In the second place, sensations of the same modality are distinguishable and comparable as to amount or intensity: we readily recognize and compare a loud and a weak sound of the same pitch; a bright and feeble light of the same color; an acute and a slight pain of the same general character. Our sensations thus differ in the three aspects of modality, quality within the same modality, and intensity. Certain sensations also differ in what is known as the "local signs," a difference by which we tell a touch on one part of the skin from a similar touch on another; or an object exciting one part of the eye from an object like it, but in a different location in space and exciting another part of the visual surface.

As regards modality, we commonly distinguish five senses, those of sight, sound, touch, taste, and smell; to these at least six others must be added to make the list approximately complete. These additional senses are temperature, pain, hunger, thirst, muscle sense, and equilibrium sense. The last five of this list were formerly set apart as common sensations, but there seems to be no good reason for viewing them in any different light from the others.

The Psychophysical Law. Although our sensations are, in modality or kind, independent of the force exciting them, they are not so in degree or intensity, at least within certain limits. We cannot measure the amount of a sensation and express it in footpounds or calories, but we can get a sort of unit by determining how small a difference in sensation can be perceived. This smallest perceptible difference varies in the different senses and for different amounts of stimulation in the same sense. Its variation in any single sense follows, however, a certain law. The increase of stimulus necessary to produce the smallest perceptible change in a sensation 
is proportional to the strength of the stimulus already acting; for example, the heavier a pressure already acting on the skin the more must it be increased or diminished in order that the increase or diminution may be felt. Examples of this, which is known as "Weber's" or "Fechner's psychophysical law" will be hereafter pointed out, and are readily observable in daily life; we have, for example, a luminous sensation of certain intensity when a lighted candle is brought into a dark room; this sensation is not doubled when a second candle is brought in; and is hardly affected at all by a third. The law is only true, however (and then but approximately), for sensations of medium intensity; it is applicable, for example, to light sensations of all degrees between those aroused by the light of a candle and ordinary clear daylight: but it is not true for luminosities so feeble as only to be seen at all with difficulty, or so bright as to be dazzling.

Besides their variations in intensity, dependent on variations in the strength of the stimulus, our sensations also vary with the irritability of the sensory apparatus itself; which is not constant from time to time or from person to person. In the above statements the condition of the sense-organ and its nervous connections is presumed to remain the same throughout.

Classification of Receptors. It is possible to group the senseorgans in several different ways according to the properties upon which the classification is based. If we group them according to the forms of energy to which they respond they fall into four classes: 1, the senses aroused by mechanical stimulation, touch, pain, hunger, muscle sense, equilibrium, and hearing; 2, those aroused by chemical stimulation, taste, smell, and probably the sensation of thirst; 3 , the temperature sense, aroused by thermal stimuli; 4 , the sense of sight, aroused by stimuli of light.

Another classification, and a more convenient one to follow in describing the receptors, is based upon their position in the Body. This classification gives us two main groups: 1, the internal senses, whose receptors lie within the Body; here belong muscle sense, equilibrium, pain, hunger, and thirst; 2 , the external senses, whose receptors are on the surface of the Body and which therefore obtain information of the outside world. These senses fall again into two subgroups; the first includes the contact senses which are stimulated only by things in immediate contact with the Body; the second in- 
cludes the projecting senses which tell us of the surroundings not immediately touching us.

The group of contact senses includes the eutaneous senses, touch, temperature, and pain, the latter being both external and internal, and the sense of taste. The group of projecting senses includes hearing, smell, and sight.

It is not desirable to follow this classification exactly in the discussion of the various senses, but it represents in the main the order of their consideration.

Not Included in this Classification are a group of feelings which in consciousness have features in common with the senses, although from the standpoint of physiology they seem not to fall in the same eategory. Examples are fatiguc, nausea, and the general state of ill-feeling ealled malaise. While these are well-marked sensations there is reason to doubt whether they are mediated by definite receptors as are the senses. They are more probably induced by general bodily states in some manner not now understood.

The Internal Senses. Of these only muscle sense, hunger, and thirst will be considered here. The sense of pain is treated more satisfactorily in connection with the cutaneous senses. The equilibrium sense requires an account of the structure of the ear and will be given in connection with the sense of hearing. The functions of these senses are to inform the Body of its own condition. They are recognized in consciousness as bodily states, being in this respect very different from the external senses, which we interpret altogether in terms of the sources from which the stimuli arise. The difference in consciousness between internal and external senses may be illustrated by supposing that a knife is held in the hand. The sensations we have are referred in our consciousness to the knife. It is hard, cold, etc. Let the knife now cut through the skin. The stimulus arises from the knife as much as before, but it is to the hand and not to the knife that we refer the feeling of pain.

The Muscular Sense. From the muscles arise sensations of great importance, although they do not often become so obtrusive in consciousness as to arouse separate attention. They are due to the excitation of sensory nerves ending within the muscles themselves, or in the tendons or joints with which the muscles are connected. 
We have at any moment a fairly accurate knowledge of the position of various parts of our Bodies, even when we do not see them; and we can also judge fairly accurately the extent of a movement made with the eyes shut. The afferent nerve impulses concerned in the development of such judgments may be various; different parts of the skin are pressed or creased; different joints are subjected to pressure; different tendons are put on the stretch and different muscles are in different states of contraction, and it is by no means easy to determine the part played in each case by the sensory nerves of the different organs. Moreover, when we push against an object, or lift it, we are able to form a judyment as to the amount of effort exerted; but here again pressure on skin and joints and tension of tendons come in. Although under normal circumstances the skin sensations are undoubtedly of importance, they are not necessary: persons with cutaneous paralysis can, apart from sight, judge truly the position of a limb and the extent of movement made by it; and in many movements change in joint pressure must be very little if any. We have then to look to muscles and tendons themselves for an important part of the sensations, and in both muscles and tendons there are organs in connection with nerve-fibers which are certainly sensory in nature: moreover, muscle sensory nerves appear to be excited by mere passive change of form in the muscle; with the eyes closed each of us can tell how much another person has lifted one of our arms.

The sensations by which we judge the extent of a muscular movement enable us to determine very minute differences of contraction; the ocular determination of the distance of an object not too far off to have its absolute distance determined with considerable accuracy, depends almost entirely upon judgments based upon very small changes in the degree of contraction of the internal and external straight (recti) muscles, converging or diverging the eyeballs. A singer, too, must be able to judge with great minuteness the degree of contraction of the small muscles of the larynx necessary to produce a certain tension of the vocal cords. It may be well to point out that we do not refer a muscular sensation to any given muscle or muscles; it is merely associated with a certain movement or position, and a person who knows nothing about his ocular muscles can judge distance through sensations derived from them, quite as well as any anatomist. This fact is of course cor- 
related with the fact that in voluntary movement we do not make a conscious effort to contract any particular muscles: the higher nerve-centers are merely concerned with the initiation of a given movement of a given extent, and all the details are carried out by lower co-ordinating centers. In ordinary daily life in fact we have no interest whatever in a muscular contraction per se; all we are concerned with is the result, and consciousness has never had need to trouble itself, if it could, with associating a particular feeling or a particular movement with any individual muscle.

Muscular feelings are, as already pointed out, frequently and closely combined not only with visual but also with tactile, in providing sensations on which to base judgments: in the dark, when an object is of such size and form that it cannot be felt all over by any one region of the skin, we deduce its shape and extent by combining the tactile feelings it gives rise to, with the muscular feelings accompanying the movements of the hands over it. Even when the eyes are used the sensations attained through them mainly serve as short-cuts which we have learned by experience to interpret, as telling us what tactile and muscular feelings the object seen would give us if felt; and, in regard to distant points, although we have learnt to apply arbitrarily selected standards of measurement, it is probable that distance, in relation to perception, is primarily a judgment as to how much muscular effort would be needed to come into contact with the thing looked at.

When we wish to estimate the weight of an object we always, when possible, lift it, and so combine muscular with tactile, sensations. By this means we can form much better judgments. While with touch alone just perceptibly different pressures have the ratio $1: 3$, with the muscular sense added differences of $\frac{1}{17}$ can be perceived.

Hunger. In discussing this sense we must first draw a distinction between true hunger and appetite. The latter is a feeling that food would be acceptable, with usually a degree of pleasurable anticipation included. It is often heightened by the odor and taste of food. There is reason to doubt whether appetite should be called a sense in the strict meaning of that term. It might, perhaps, be better classed with fatigue, nausea, and the other feelings mentioned in a former paragraph as not representing the results of definite receptor stimulation. True hunger, on the other hand, is a 
definite sense aroused in a specific manner. In consciousness it takes the form of sensations arising from the stomach, which, when pronounced, are of a character sufficiently disagreeable to justify their description as "pangs of hunger." During a period of hunger the feeling is not continuous, but comes and goes, usually at fairly regular intervals. By means of interesting experiments, in which records were obtained of the movements of the stomach, the fact was demonstrated that spasms of hunger are the result of vigorous contractions of the muscular walls of the organ. Apparently these contractions stimulate, mechanically, receptors embedded in the stomach walls. Most of the facts about hunger are readily explicable in accordance with this idea of its nature when we recall that the stomach, whose contractions evoke the sensations, is governed by the autonomic system, which, in turn, is subject to emotional as well as to reflex influences. The well-known capriciousness of hunger can thus be accounted for. If the need for food were the necessary incitement to hunger we should expect the greatest hunger to be after the longest fast, but the experience of a great many people is that their least hunger before any meal is before breakfast, which is the meal at the end of the longest interval. Moreover, those who have endured long fasts testify that hunger disappears completely after a period of two or three days, particularly if not much exercise is taken.

The function of hunger is to insure the taking of food. This is an act essential to life, but in the lower animals, and in children, is not recognized as such through the operation of associative memory, and, therefore, is not to be depended on to be performed volitionally. It is essentially a reflex act and hunger is the sensory basis for the reflex. As we shall learn in a later chapter, an important feature of proper eating is the maintenance of regular habits in regard to it. Hunger serves as a powerful aid to regularity, for it tends to come on at about the time we are in the habit of eating. Many people suffer rather severely if obliged to wait through the period of a usual meal, although the interval measured in hours may be no longer than others to which they are accustomed and which cause no discomfort.

Thirst. This sense, in its ordinary form, arises from dryness of the throat. Apparently there are receptors in that region which are stimulated by deficiency of moisture. The throat is moistened by 
the saliva which is swallowed at frequent intervals, and the sense of thirst thus kept in abeyance. There is a constant loss of water from the Body by means of the various channels of excretion, lungs, sweat glands, etc. When the resultant diminution in the water content of the tissues reaches a certain point the swallowing of saliva no longer prevents stimulation of the thirst receptors, and liquid from outside the Body must be taken if the thirst is to be relieved. The liquid need not necessarily be swallowed. Injections directly into the veins are effective in abolishing thirst sensations.

If, as the result of prolonged deprivation, the water content of the Body is seriously diminished, ordinary thirst gives way to much more pronounced and finally very painful sensations. From these there is no relief with the passage of time as there is in case of hunger. The distress becomes more and more marked leading ultimately, it is said, to mental breakdown. Thirst is believed to be the only sense of which the Body may not be deprived through accident or disease.

The Cutaneous Senses. These occur over the entire Body, not uniformly distributed but scattered in fine dots over the surface. This punctiform arrangement can be demonstrated by exploring the skin with fine needles. Such a procedure shows that the different cutaneous senses occur in distinct spots which do not overlap, but which in most parts of the Body are so intermingled as to leave no area of any size devoid of any one of the senses. Sensory spots are much more numerous and more closely packed together in such regions as the hands and face which are liable to come in contact with foreign bodies, than they are in the better protected surfaces of the trunk and limbs. Four sorts of cutaneous sense spots are recognized: those of pain, touch, warmth, and cold. Pain spots are more numerous than any of the others; touch spots rank next in number, it being estimated that on the trunk and limbs there are a half million of them; cold spots are only half as numerous as touch spots; warmth spots are fewest of all, their number being estimated at thirty thousand for the entire Body.

Pain. When the skin is powerfully stimulated by heat, cold or pressure, or is inflamed, we get a sensation which we call pain. This is something quite different from the unpleasantness caused by a dazzling light or a musical discord or a disagreeable odor or taste. We recognize these as being still sight or sound or smell. 
or taste sensations. Pain, however, is always recognized as a distinet sensation having its own modality. Its function seems to be wholly one of warning; only when something is amiss do we feel it. Since danger results from strong stimulation but not from feeble stimulation pain receptors are less irritable than other sorts; it is estimated that the sense of touch is one thousand times as delicate as the sense of pain. Harm may result from excessive stimulation of any sort. Pain receptors, therefore, are irritable to all forms of energy except that of light.

Because pain results from any sort of stimulation, but only when excessive, it was formerly thought to be not a distinct sense but the result of overstimulation of the other senses. On this theory it would be hard to account for the fact that skin pain is so very different in modality from a touch or temperature feeling, and to understand why it gives rise in consciousness to conceptions concerning a condition of the Body and not of some external object: it is not extrinsically referred by the mind to a quality of anything but the painful part itself, as a dazzling light sensation or a fetid odor is. There is also experimental and pathological evidence that the paths taken in the spinal cord by nerve impulses causing pain are different from those leading to a consciousness of touch. If certain parts of the cord are cut in the thoracic region of a rabbit, gentle touches on the hind limb appear to be felt; the animal erects its ears or moves its head: but powerful stimulation of the sciatic nerve causes no signs of pain, while if the dorsal white columns be cut the animal still can feel stimuli applied to the hind limb and sufficient to cause pain under normal conditions, but it appears insensible to gentle pressure on the skin. In human beings very similar phenomena have been observed in cases of spinal cord disease: and in a certain stage of chloroform or ether narcosis the patient feels the surgeon's hand or his knife where it touches the skin, but he experiences no pain when deeper parts are cut. Such considerations seem to lead to the conclusion that the nerve-fibers and receptors concerned with painful sensations are quite distinct from those of the other senses. If that be so we must assume that there are "pain" fibers very widely distributed over the skin and through most other parts of the Body. In accident or disease these are stimulated powerfully enough to arouse perception and imperiously call attention to danger. 
The pain nerves of the skin do not seem to be provided with special end organs but to end nakedly among the cells of the epidermis. Such a mode of termination accords with the low irritability of the pain mechanism and with its absence of adaptation to particular forms of energy, since nerve-tissue proper exhibits these same qualities.

The interior of the Body, in certain regions at least, seems to be provided with special pain receptors. These are the Pacinian corpuscles (see Fig. 67). They are specially numerous in the mesentery, the connective tissue membrane which supports the abdominal viscera.

Pains can be localized, though only imperfectly, and the less perfectly the more severe they are. The exact place of a needle prick after removal of the needle (so that there is no guiding concomitant touch sensation) cannot be recognized as well as a pin touch on the same region of the skin, but still fairly well; while the acute pain caused by a small abscess (bone felon) under the periosteum of a finger bone is often felt all over the forearm; and a single diseased tooth may cause pain felt over the whole of that side of the face.

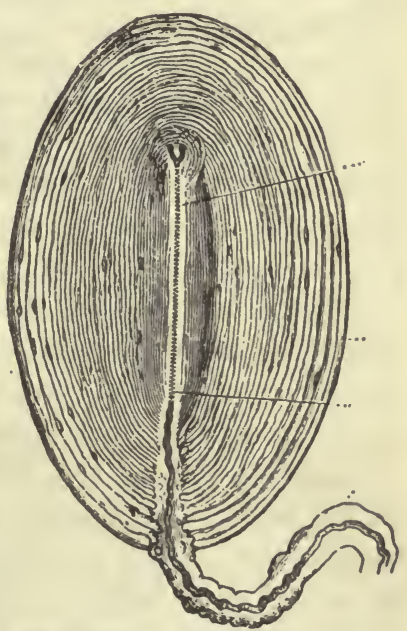

Frg. 67.-A Pacinian corpuscle, magnified.

Many internal pains instead of being felt as coming from the organ where they originate are referred to areas of the skin. So constant is this misreference that the physician is able to judge of the seat of many disturbances from the particular skin areas that exhibit tenderness. The explanation of this misreference of internal pain to the skin is not easy to make. It has been suggested that the nerve-paths over which internal pain reach the body sense-area of the cortex lie close to those of pains from certain skin areas; and that since painful skin stimulation is much more common than internal pains, the brain interprets all impulses reaching it over a restricted nerve-path as coming from the particular skin area whose nerve-path forms part of the whole nerve-path in question. 
Touch, or the Pressure Sense. Through touch proper we recognize pressure or traction exerted on the skin, and the force of the pressure, the softness or hardness, roughness or smoothness, of the body producing it; and the form of this, when not too large to be felt all aver. When to learn the form of an object we move the hand over it, muscular sensations are combined with proper tactile, and such a combination of the two sensations is frequent; moreover, we rarely touch anything without at the same time getting temperature sensations; therefore pure tactile feelings are rare.

From an evolution point of view, touch is probably the first distinctly differentiated sensation, and this primary position it still largely holds in our mental life; we mainly think of the things about us as objects which would give us certain tactile sensations if we were in contact with them. Though the eye tells us much quicker, and at a greater range, what are the shapes of objects and whether they are smooth, rough, and so on, our real conceptions of round and square and rough bodies are derived through touch, and we largely translate unconsciously the teachings of the eye into mental terms of the tactile sense.

The delicacy of the pressure sense varies on different parts of the skin; it is greatest on the forehead, temples, and back of the forearm, where a weight of 2 milligr. (0.03 grain) pressing on an area of 9 sq. millim. (0.0139 sq. inch) can be felt. On the front of the forearm 3 milligr. (0.036 grain) can be similarly felt, and on the front of the forefinger 5 to 15 milligr. (0.07-0.23 grain).

In order that the sense of touch may be excited neighboring skin areas must be differently pressed; when we lay the hand on a table this is secured by the inequalities of the skin, which prevent end organs, lying near together, from being equally compressed. When, however, the hand is immersed in a Tiquid, as mercury, which fits into all its inequalities and presses with practically the same weight on all neighboring immersed areas, the sense of pressure is only felt at a line along the surface, where the immersed and non-immersed parts of the skin meet.

It was in connection with the tactile sense that the facts on which the so-called psychophysical law (p. 205) is based, were first observed. The smallest perceptible difference of pressure recognizable when touch alone is used, is about $\frac{1}{3}, i$. e., we can just tell 
a weight of 20 grams (310 grains) from one of 30 (465 grains) or of 40 grams (620 grains) from one of 60 (930 grains); the change which can just be recognized being thus the same fraction of that already acting as a stimulus. The rate only holds good, however, for a certain mean range of pressure; it is not true for very small or very great pressures. The experimental difficulties in determining the question are considerable; muscular sensations inust be rigidly excluded; the time elapsing between laying the different weights on the skin must always be equal; the same region and area of the skin must be used; the weights must have the same temperature; and fatigue of the organs must be eliminated. Considerable individual variations are also observed, the least perceptible difference not being the same in all persons.

The Localizing Power of the Skin. When the eyes are closed and a point of the skin is touched we can with some accuracy indicate the region stimulated; although tactile feelings are in general characters alike, they differ in something (local sign) besides intensity by which we can distinguish them; some sensation quality must be present enabling us to tell from one another two precisely similar contacts of an external object when applied, say, to the tips of the fore and ring fingers respectively. The accuracy of the localizing power is not nearly so great as in the eye and varies widely in different skin regions; it may be measured by observing the least distance which must separate two objects (as the blunted points of a pair of compasses) in order that they may be felt as two. The following table illustrates some of the differences observed:

Tongue-tip..................... $1.1 \mathrm{~mm}$. (0.04 inch)

Palm side of last phalanx of finger......... $2.2 \mathrm{~mm}$. (0.08 inch)

Red part of lips. . . . . . . . . . (0.16 inch)

Tip of nose................... $6.6 \mathrm{~mm}$. (0.24 inch)

Back of second phalanx of finger.......... $11.0 \mathrm{~mm}$. (0.44 inch)

Heel. ........................ $22.0 \mathrm{~mm}$. (0.88 inch)

Back of hand . . . . . . (1.23 inches)

Forearm........................ 39.6 mm. (1.58 inches)

Sternum...................... $44.0 \mathrm{~mm}$. (1.76 inches)

Back of neck. . . . . . .

Middle of back. . . . . . . . .

The localizing power is a little more acute across the long axis, of a limb, and is better when the pressure is only strong enough 
just to cause a distinct tactile sensation, than when it is more powerful; it is also very readily and rapidly improvable by practice.

It might be thought that this localizing power depended directly on nerve distribution; that each touch nerve had connection with a special brain-center at one end (the excitation of which caused a sensation with a characteristic local sign), and at the other end was distributed over a certain skin area, and

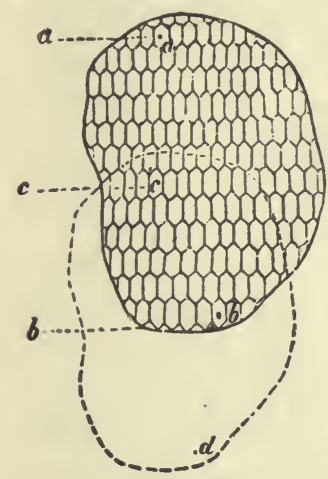

FIG. 68. that the larger this area the farther apart might two points be and still give rise to only one sensation. If this were so, however, the peripheral tactile areas (each being determined by the anatomical distribution of a nerve-fiber) must have definite unchangeable limits, which experiment shows that they do not possess. Suppose each of the small areas in Fig. 68 to represent a peripheral area of nerve distribution. If any two points in $c$ were touched we would according to the theory get but a single sensation; but if, while the compass points remained the same distance apart, or were even approximated, one were placed in $c$ and the other on a contiguous area, two fibers would be stimulated and we ought to get two sensations; but such is not the case; on the same skin region the points 1 must be always the same distance apart, no matter how they be shifted, in order to give rise to two just distinguishable sensations.

- It is probable that the nerve areas are much smaller than the tactile; and that several unstimulated must intervene between the excited, in order to produce sensations which shall be dis $/$ tinct. If we suppose twelve unexcited nerve areas must intervene, then, in Fig. 68, $a$ and $b$ will be just on the limits of a single tactile area; and no matter how the points are moved, so long as eleven, or fewer, unexcited areas come between, we would get a single tactile sensation; in this way we can explain the fact that tactile areas have no fixed boundaries in the skin, although the nerve distribution in any part must be constant. We also see why the back of a knife laid on the surface causes a continuous linear sensation, although it touches many distinct nerve areas; 
if we could discriminate the excitations of each of these from that. of its immediate neighbors we would get the sensation of a series of points touching us, one for each nerve region excited; but in the absence of intervening unexcited nerve areas the sensations are fused together.

The Temperature Sense. By this we mean our faculty of perceiving cold and warmth; and, with the help of these sensations, of perceiving temperature differences in external objects. Its organ is the whole skin, the mucous membrane of mouth and fauces, pharynx and upper part of gullet, and the entry of the nares. Direct heating or cooling of a sensory nerve may stimulate it and cause pain, but not a true temperature sensation; and the amount of heat or cold requisite is much greater than that necessary when a temperature-perceiving surface is acted upon; hence we must assume the presence of temperature receptors. As previously stated these are of two kinds, those that are stimulated by cold, and those that are stimulated by warmth.

In a comfortable room we feel at no part of the Body either heat or cold, although different parts of its surface are at different temperatures; the fingers and nose being cooler than the trunk which is covered by clothes, and this, in turn, cooler than the interior of the mouth. The temperature which a given region of the temperature organ has (as measured by a thermometer) when it feels neither hot nor cold is its temperature-sensation zero for that time, and is not associated with any one objective temperature; for not only, as we have just seen, does it vary in different parts of the organ, but also on the same part from time to time. Whenever a skin region passes with a certain rapidity to a temperature above its sensation zero we feel warmth; and vice versa: the sensation is more marked the greater the difference, and the more suddenly it is produced; touching a metallic body, which conducts heat rapidly to or from the skin, causes a more marked hot or cold sensation than touching a worse conductor, as a piece of wood, of the same temperature.

The change of temperature in the organ may be brought about by changes in the circulatory apparatus (more blood flowing through the skin warms it and less leads to its cooling), or by temperature changes in gases, liquids, or solids in contact with it. Sometimes we fail to distinguish clearly whether the cause is 
external or internal; a person coming in from a windy walk often feels a room uncomfortably warm which is not really so; the exercise has accelerated his circulation and tended to warm his skin, but the moving outer air has rapidly conducted off the extra heat; on entering the house the stationary air there does this less quickly, the skin becomes hotter, and the cause is supposed to be oppressive heat of the room. Hence, frequently, opening of windows and sitting in a draught, with its concomitant risks; whereas keeping quiet for five or ten minutes, until the circulation had returned to its normal rate, would attain the same end without danger.

The acuteness of the temperature sense is greatest at temperatures within a few degrees of $30^{\circ} \mathrm{C}$. $\left(86^{\circ} \mathrm{F}\right.$.); at these differences of less than $0.1^{\circ} \mathrm{C}$. can be discriminated. As a means of measuring absolute temperature, however, the skin is very unreliable, on account of the changeability of its sensation zero. We can localize temperature sensations much as tactile, but not so accurately.

The receptors for cold are near the surface of the skin; those for warmth are embedded deeply within it. While the latter respond only to temperatures above their own, the cold receptors are stimulated not only by temperatures below their own but also by temperatures above $45^{\circ} \mathrm{C}$. $\left(140^{\circ} \mathrm{F}\right.$.). It is for this reason that a sensation of cold is felt when one first steps into a hot bath; the receptors for cold being nearer the surface than those for warmth are stimulated an instant before them. It is said that the sensation of "hot" as distinguished from "warm" results from simultaneous stimulation of warmth and cold spots by temperatures above $45^{\circ} \mathrm{C}$.

The Peripheral Reference of our Sensations. Repeated mention has been made of the fact that we refer our external sensations to the outside world; this is only one case of a more general law, in accordance with which we do not ascribe our sensations, as regards their locality, to the brain, where the sensation is actually aroused, but to a peripheral part. With respect to the brain, other parts of the Body are external objects as much as the rest of the material universe, yet we locate the majority of our internal sensations at the places where the sensory nerves concerned are irritated, and not in the brain. Even if a nerve- 
trunk be stimulated in the middle of its course, we refer the resulting sensation to its outer endings. A blow on the inside of the elbow-joint, injuring the ulnar nerve, produces not only a local pain, but a sense of tingling ascribed to the fingers to which the ends of the fibers go. Persons with amputated limbs have feelings in their fingers and toes long after they have been lost, if the nerve-trunks in the stump be irritated. This persistent reference is commonly ascribed to the results of experience. The events of life have taught us that in the great majority of instances the sensory impulses which excite a given tactile sensation, for example, have acted upon the tip of a finger. The sensation goes when the finger is removed, and returns when it is replaced; and the eye confirms the contact of the external object with the finger-tip when we get the tactile sensation in question. We thus come firmly to associate a particular region of the skin with a given sensation, and whenever afterwards the nerve-fibers coming from the finger are stimulated, no matter where in their course, we ascribe the origin of the sensation to something acting on the finger-tip.

Perceptions. In every sensation we have to distinguish carefully between the pure sensation and certain judgments founded upon it; we have to distinguish between what we really feel and what we think we feel; and very often firmly believe we do feel when we do not.

The most important of these judgments is that which leads us to ascribe certain sensations, those aroused through organs of special sense, to external objects-that outer reference of our sensations which leads us to form ideas concerning the existence, form, position, and properties of external things. Such representations as these, founded on our senses, are called perceptions. Since these always imply some mental activity in addition to a mere feeling, their full discussion belongs to the domain of Psychology. Physiology, however, is concerned with them so far as it can determine the conditions of stimulation under which a given mental representation concerning a sensation is made. It is quite certain that we can feel nothing but states of ourselves, but, as already pointed out, we have no hesitation in saying we feel a hard or a cold, a rough or smooth body. When we look at a distant object we usually make no demur to saying that we 
perceive it. What we really feel is, however, the change produced by it in our eyes. There are no parts of our Bodies reaching to a tree or a house a mile off-and yet we seem to feel all the while that we are looking at the tree or the house and feeling them, and not merely experiencing modifications of our own eyes or brains. When reading we feel that what we really see is the book; and yet the existence of the book is a judgment founded on a state of our Body, which alone is what we truly feel.

We have the same experience in other cases, for example with regard to touch.

Hairs are quite insensible, but are embedded in the sensitive skin, which is excited when they are moved. But if the tip of a hair be touched by some external object we believe we feel the contact at its insensible end, and not in the sensitive skin at its root. So, the hard parts of the teeth are insensible; yet when we rub them together we refer the seat of the sensation aroused to the points where they touch one another, and not to the sensitive parts around the sockets where the sensory nerve impulse is really started.

Still more, we may refer tactile sensations, not merely to the distal ends of insensible bodies implanted in the skin, but to the far ends of things which are not parts of our Bodies at all; for instance, the distant end of a rod held between the finger and a table while the finger is moved a little from side to side. We then believe we feel touch or pressure in two places; one where the rod touches our finger, and the other where it comes in contact with the table. A blind man gropes his way along by feeling at the end of his stick.

This irresistible mental tendency to refer certain of our states of feeling to causes outside of our Bodies, whether in contact with them or separated from them by a certain space, is known as the phenomenon of the extrinsic reference of our sensations. It seems largely to depend on the fact that the sensations extrinsically referred can be modified by movements of our Bodies. Hunger, thirst, and toothache all remain the same whether we turn to the right or left, or move away from the place we are standing in. But a sound is altered. We may find that in a certain position of the head it is heard more by the right ear than the left; but on turning round the reverse is the case; and halfway round the 
loudness in each ear is the same. Hence we are led, by mental laws outside of the physiological domain, to suspect that its cause is not in our Body, but outside of it; and depends not on a condition of the Body but on something else.

Sensory Illusions. "I must believe my own eyes" and "we can't always believe our senses" are two expressions frequently heard, and each expressing a truth. No doubt a sensation in itself is an absolute incontrovertible fact: if I feel redness or hotness I do feel it, and that is an end of the matter: but if I go beyond the fact of my having a certain sensation and conclude from it as to properties of something else-if I form a judgment from my sensation-I may be totally wrong; and in so far be unable to believe my eyes or skin. Such judgments are almost inextricably woven up with many of our sensations, and so closely that we cannot readily separate the two; not even when we know that the judgment is erroneous.

For example, the moon when rising or setting appears bigger than when high in the heavens-we seem to feel directly that it arouses more sensation, and yet we know certainly that it does not. With a body of a given brightness the amount of change produced in the end organs of the eye will depend on the size of the image formed in the eye, provided the same part of its sensory surface is acted upon. Now the size of this image depends on the distance of the object; it is smaller the farther off it is and greater the nearer, and measurements show that the area of the sensitive surface affected by the image of the rising moon is no larger than that affected by it when overhead. Why then do we, even after we know this, see it bigger? The reason is that when the moon is near the horizon we imagine, unconsciously and irresistibly, that it is farther off; even astronomers who know perfectly well that it is not, cannot help forming this unconscious and erroneous judgment-and to them the moon appears in consequence larger when near the horizon, just as it does to less well-informed mortals. In fact we have a conception of the sky over which the moon seems to travel, not as a half sphere but as somewhat flattened, and hence when the moon is at the horizon we unconsciously judge that it is farther off than when overhead. But any body which excites the same extent of the sensitive surface of the eye at a great distance that another does at less, must be larger than 
the latter; and so we conclude that the moon at the horizon is larger than the moon in the zenith, and are ready to declare that we see il so.

Erroneous perceptions of this sort are known as sensory illusions; and we ought to be constantly on guard against them. 


\section{CHAPTER XIV}

THE EAR. HEARING AND EQUILIBRATION. TASTE AND SMELL

Functions of the Ear. The ear is not solely an organ of hearing. It includes, in addition, the highly important structures by which is mediated the sense of equilibrium. Hearing is, however, its familiar function, and we will consider it first in order. To be able to discuss intelligently the apparatus for hearing we must have in mind the fundamental facts about the agency by which the sense is aroused, namely, sound.

The Loudness, Pitch, and Timbre of Sounds. Sounds, as sensations, fall into two groups-notes and noises. Phyșically, sounds consist of vibrations, and these, under most circumstances, when they first reach our auditory organs, are alternating rarefactions and condensations of the air, or aërial waves. When the waves follow one another uniformly, or periodically, the resulting sensation (if any) is a note; when the vibrations are irregular it is a noise. In notes we recognize (1) loudness or intensity; (2) pitch; (3) quality or timbre, or, as it has been called, tone color; a note of a given loudness and pitch produced by a flute and by a violin has a different character or individuality in each case; this quality is its timbre. Before understanding the working of the auditory mechanism we must get some idea of the physical qualities in objective sound of which the subjective differences of auditory sensations are signs.

The loudness of a sound depends on the force of the aërial waves; the greater the intensity of the alternating condensations and rarefactions of these, the louder the sound. The pitch of a note depends on the length of the waves, that is, the distance from one point of greatest condensation to the next, or (what amounts to the same thing) on the number of waves reaching the ear in given time, say a second. The shorter the waves the more rapidly they follow one another, and the higher the pitch of the note. When audible 
vibrations bear the ratio $1: 2$ to one another, we hear the musical interval called an octave. The middle $C$ of the musical scale is due to 256 vibrations per second. Its octave has 512 vibrations.

Sound vibrations may be too rapid or too slow in succession to produce sonorous sensations. The highest-pitched audible note answers to about 38,000 vibrations in a second, but it differs in individuals; many persons cannot hear the cry of a bat nor the chirp of a cricket, which lie near this upper audible limit. On the other hand, sounds of vibrational rate about 40 per second are not well heard, and a little below this become inaudible. The highest note used in orchestras is the $d^{\mathrm{v}}$ of the fifth accented octave, produced by the piccolo flute, due to 4,752 vibrations in a second; and the lowest-pitched is the $E_{1}$, of the contra octave, produced by the double bass. Modern grand pianos and organs go dowis to $C$, in the contra octave (33 vibrations per second) or even $A^{\prime \prime}$, $\left(27 \frac{1}{2}\right)$, but the musical quality of such notes is imperfect; they produce rather a "hum" than a true tone sensation, and are only used along with notes of higher octaves to which they give a character of greater depth.

Timbre. Since the loudness of a tone depends on the vibrational amplitude of its physical antecedent, and its pitch on the vibrational rate, we have still to seek the cause of timbre; the quality by which we recognize the human voice, the violin, the piano, and the flute, even when all sound the same note and of the same loudness. Helmholtz showed that the quality of any tone is determined by the particular overtones or harmonic partials that are combined in it with the fundamental tone. Most vibrating bodies are able to vibrate both as a whole and in sections. Since the sections are smaller than the whole body their vibrations are more rapid than those of the body as a whole. The vibrating sections may be halves, thirds, fourths, or any other fraction of the whole body. Also one and the same body may be vibrating at once in halves, quarters, and several other smaller divisions. These vibrations in parts are the sources of overtones, the pitch of the tone being determined by its vibration as a whole, the socalled fundamental vibration.

The air waves set in motion by a body vibrating in such complex fashion must necessarily be themselves very complex. Since they are periodic, however, they produce audible notes, if rapid 
and intense enough. The actual form of air wave which proceeds from a body vibrating thus depends upon the particular components which make it, and it has been shown that any complex periodic vibration can be analyzed mathematically into its constituents, and these unerringly determined. The timbre of a tone depends, then, according to our former definition, upon the form of air wave which enters the ear. A tone composed of a fundamental and three overtones will come to the ear as a wave having quite a different form from one having in addition to the fundamental five partials.

Whereas we ordinarily hear compound tones merely as tones of certain quality, the trained ear is able to hear and pick out the overtones by which the quality is determined. It is evident, therefore, that the ear is able to analyze compound tones into their individual constituents.

Sympathetic Resonance. Imagine slight taps to be given to a pendulum; if these be repeated at such intervals of time as always to help the swing and never to retard it, the pendulum will soon be set in powerful movement. If the taps are irregular, or when regular come at such intervals as sometimes to promote and sometimes retard the movement, no great swing will be produced; but if they always push the pendulum in the way it is going at that instant, they need not come every swing in order to set up a powerful vibration; once in two, three, or four swings will do. A stretched string, such as that of a piano, is so far like a pendulum that it tends to vibrate at one rate and no other; if aërial waves hit it at exactly the right times they soon set it in sufficiently powerful vibrations to cause it to emit an audible note. By using such strings we can analyze compound tones and thus prove objectively that they are made up of partials. If the dampers of a piano be raised and a note be sung loudly to it, it will be found that several strings are set in vibration, such vibrations being called sympathetic. The human voice emits compound tones which can be mathematically analyzed into simple vibrations, and if the piano strings set in movement by it be examined, they will be found to be exactly those which answer to these vibrations and to no others. We thus get experimental grounds for believing that compound tones are really made up of a number of simple vibrations, and get an additional justification for the supposition that in 
the ear each note is analyzed into its components; and that the difference of sensation which we call timbre is due to the effect of the secondary partial tones thus perceived. If so, the ear must have in it an apparatus adapted for sympathetic resonance.

The External Ear. The auditory organ in man consists of three portions, known respectively as the external ear, the middle ear or tympanic cavity, and the internal ear or labyrinth; the latter contains the end organs of the auditory nerve. The external ear consists of the expansion seen on the exterior of the head, called the concha, $M$, Fig. 69, and a passage leading in from it, the $e x-$

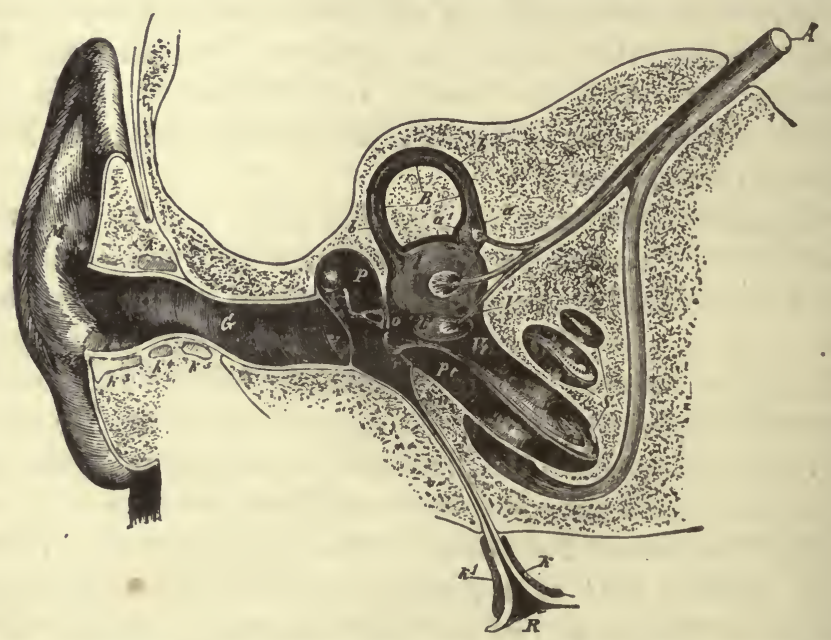

Fig. 69.-Semidiagrammatic section through the right ear (Czermak). $M$, concha; $G$, external auditory meatus; $T$, tympanic membrane; $P$, middle ear; $o$, oval foramen; $r$, round foramen; $R$, pharyngeal opening of Eustachian tube; $V$, vestibule; $B$, a semicircular canal; $S$, the cochlea; $V t$, scala vestibuli; $P t$, scala tympani; $A$, auditory nerve.

ternal auditory meatus, $G$. This passage is closed at its inner end by the tympanic or drum membrane, $T$. ' It is lined by skin, through which numerous small glands, secreting the wax of the ear, open.

The Functions of the Tympanic Membrane. If a stretched membrane, such as a drumhead, be struck, it will be thrown into periodic vibration and emit for a time a note of a determined pitch. The smaller the membrane and the tighter it is stretched the higher the pitch of its note; every stretched membrane thus has a rate of its own at which it tends to vibrate, just as a piano or violin string 
has. When a note is sounded in the air near such a membrane, the alternating waves of aërial condensation and rarefaction will move it; and if the waves succeed at the vibrational rate of the membrane the latter will be set in powerful sympathetic vibration; if they do not push the membrane at the proper times, their effects will neutralize one another: hence such membranes respond well to only one note. The tympanic membrane, however, responds equally well to a large number of notes; at the least for those due to aërial vibrations of rates from 60 to 4,000 per second, running over eight octaves and constituting those commonly used in music. This faculty depends on two things: (1) the membrane is comparatively loosely and not uniformly stretched; (2) it is loaded by the tympanic bones.

The drum-membrane is a shallow funnel with its sides convex towards the external auditory meatus; something like an umbrella turned inside out; in such a membrane the tension is not uniform but increases towards the center, and it has accordingly no proper note of its own. Further, whatever tendency such a membrane may have to vibrate rather at one rate than another, is almost completely removed by "damping" it, $i$. e., placing in contact with it something comparatively heavy and which has to be moved when the membrane vibrates. This is effected by the tympanic bones, fixed to the drum-membrane by the handle of the malleus. Another advantage is gained by the damping; once a stretched membrane is set vibrating it continues so doing for some time; but if loaded its movements cease almost as soon as the moving impulses. The dampers of a piano are for this purpose; and violin-players have to "damp" with the fingers the strings they have used when they wish the note to cease. The tympanic bones act as dampers.

The Middle Ear ( $P$, Fig. 69$)$ is an irregular cavity in the temporal bone, closed externally by the drum membrane. From its . inner side the Eustachian tube $(R)$ proceeds to the pharynx, and the mucous membrane of that cavity is continued up the tube to line the middle ear; the proper tympanic membrane composed . of connective tissue is therefore covered by mucous membrane on its inner, as it is by very thin skin on its outer, side. In the bony inner wall of the middle ear are two small apertures, the oval and round foramens, $o$ and $r$, which lead into the labyrinth. During life the round aperture is closed by the lining mucous membrane, 
and the oval in another way, to be described presently. The tympanic membrane, $T$, stretched across the outer side of the middle ear, forms a shallow funnel with its concavity outwards. It is pressed by the external air on its exterior, and by air entering the tympanic cavity through the Eustachian tube on its inner side. If the middle ear were closed the pressures on the inner and outer sides of the drum membrane would not be always equal when barometric pressure varied, and the membrane would be bulged in or out according as the external or internal pressure on it were the greater. This unequal pressure would interfere seriously with the freedom of vibration of the membrane and so impair hearing. On the other hand, were the Eustachian tube always open the sounds of our own voices would be loud and disconcerting, so it is usually closed; but every time we swallow it is opened, and thus the air-pressure in the cavity is kept equal to that in the external auditory meatus. By holding the nose, keeping the mouth shut, and forcibly expiring, air may be forced under pressure into the middle ear, and will be held in part imprisoned there until the next act of swallowing. On making a balloon ascent or going rapidly down a deep mine, the sudden and great change of aërial pressure outside frequently causes painful tension of the drum membrane, which may be greatly alleviated by frequent swallowing movements.

The great importance of the Eustachian tubes in hearing is illustrated by the deafness that results from their continued closure. This condition is frequently brought about in children by the growth of adenoids (see p. 383) in the back of the throat, which press upon and close the Eustachian tubes.

Essential as these tubes are for good hearing they constitute a frequent source of ear trouble. The congestion of the mucous membranes of the throat and nose in a "cold in the head" is apt to involve the Eustachian tubes and the lining of the middle ear. Sometimes an exudate from the congested membranes fills the middle ear completely, and by its pressure causes acute pain, as well as deafness. Unless relief is obtained the tympanic membrane may be ruptured. Earache resulting from a cold should therefore not be neglected. Less commonly but more seriously actual infection (p. 306) of the middle ear may occur, the infection invading the region by way of the Eustachian tubes. The mastoid process 
(the prominent bony mass just behind the ear) has many hollows in it which communicate with the cavity of the middle ear and may become infected from it. Infection of these hollows gives rise to the extremely grave condition, mastoiditis.

The Auditory Ossicles. Three small bones lie in the middle ear forming a chain from the drum membrane to the oval foramen. The external bone (Fig. 70) is the malleus or hammer; the middle one, the incus or anvil; and the internal, the stapes or stirrup. The malleus, $M$, has an upper enlargement or head, which carries on its inner side an articular surface for the incus; below the head is a constriction, the neck, and below this two processes complete the bone; one, the long or slender process, is embedded in a ligament which reaches from it to the front wall of the tympanic cavity; the other process, the handle, reaches down between the mucous membrane lining the inside of the drum membrane and the membrane proper, and is firmly attached to the latter near its center and keeps the membrane dragged in there so as to give it its peculiar concave form, as seen from the outside. The incus has a body and two processes, and is much like a molar tooth with two roots. On its body is an articular hollow to receive the head of the malleus; its short process $(\mathrm{Jb})$ is attached by ligament to the back wall of the tympanum; the long process $(J l)$ is directed inwards to the stapes; on the tip of this process is a little knob, which represents a bone (os orbiculare) distinct in early life. The stapes $(S)$ is extremely like a stirrup, and its base (the footpiece

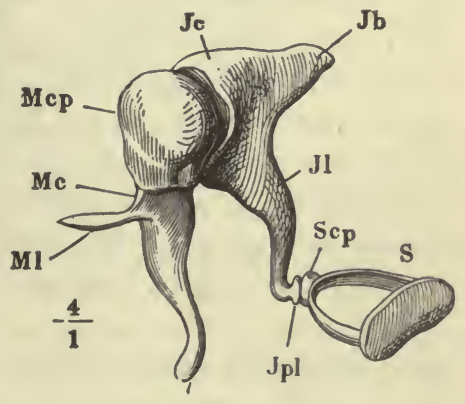

$\mathrm{Mm}$

Frg. 70.-The auditory ossicles of the right ear, seen from the front. $M$, malleus; $J$, incus; $S$, stapes; $M c p$, head of the malleus; $M c$, neck of ditto; $M l$, long process; $M m$, handle; $J c$, body; $J b$, short, and $J l$, long process of incus; $J p l$, os orbiculare; Scp, head of stapes. of the stirrup) fits into the oval foramen, to the margin of which its edge is united by a fibrous membrane, allowing of a little play in and out.

From the posterior side of the neck of the malleus a ligament passes to the back wall of the middle ear: this, with the ligament embedding the slender process and fixed to the front wall of the 
cavity, forms an anteroposterior axial ligament, on which the malleus can rotate slightly, so that the handle can be pushed in and the head out and vice versa. If a pin be driven through Fig. 70 just below the neck of the malleus and perpendicular to the paper it will very fairly represent this axis of rotation. Connected with the malleus is a tiny muscle, called the tensor tympani; it is inserted on the handle of the bone below the axis of rotation, and when it contracts pulls the handle in and tightens the drum membrane. Another muscle (the stapedius) is inserted into the outer end of the stapes, and when it contracts fixes the bone so as to limit its range of movement in and out of the fenestra ovalis.

Functions of the Auditory Ossicles. When the air in the external auditory meatus is condensed it pushes in the tympanic membrane which carries with it the handle of the malleus. This bone then slightly rotates on the axial ligament and, locking into the incus where the two bones articulate, causes the long process ( $J l$, Fig. 70) of the latter to move inwards. The incus thus pushes in the stapes; the reverse occurs when air in the auditory passage is rarefied. Aërial vibrations thus set the chain of bones swinging, and push in and pull out the base of the stapes, which sets up waves in the fluid of the labyrinth. This fluid being chiefly water, and practically incompressible, the end of the stapes could not work in and out at the oval foramen, were the labyrinth elsewhere completely surrounded by bone: but the membrane covering the round foramen bulges out when the base of the stapes is pushed in, and vice versa; and so allows of waves being set up in the labyrinthic fluid. These correspond in period and form to those in the auditory meatus; their amplitude is determined by the extent of the vibrations of the drum membrane.

The form of the tympanic membrane causes it to transmit to its center, where the malleus is attached, vibrations of its lateral parts in diminished amplitude but increased power; so that the tympanic bones are pushed only a little way but with considerable force. Its area, too, is about twenty times as great as that of the oval foramen, so that force collected on the large area is, by pushing the tympanic bones, all concentrated on the smaller. The ossicles also form a bent lever (Fig. 70) of which the fulcrum is at the axial ligament and the effective outer arm of this lever is about half as long again as the inner, and so the movements transmitted 
by the drum membrane to the handle of the malleus are communicated with diminished range, but increased power, to the base of the stapes.

Ordinarily sound-waves reach the labyrinth through the tympanum, but they may also be transmitted through the bones of the head; if the handle of a vibrating tuning-fork be placed on the top of the head, the sounds heard by the person experimented upon seem to have their origin inside his own cranium. Similarly when a vibrating body is held between the teeth, sound reaches the end organs of the auditory nerve through the skull-bones; and persons who are deaf from disease or injury of the tympanum can thus be made to hear, as with the audiphone. Of course if deafness be due
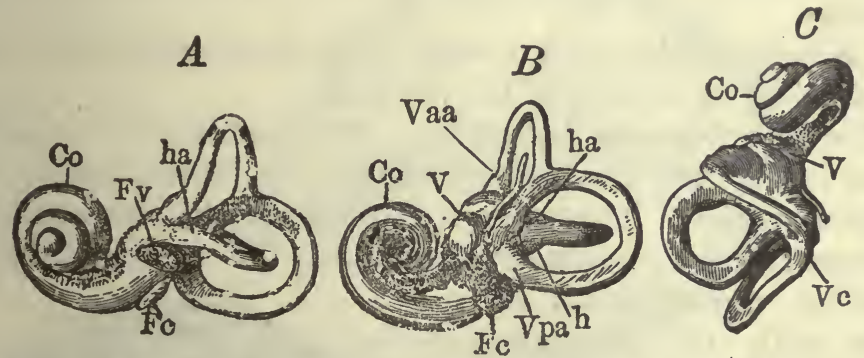

Fig. 71.-Casts of the bony labyrinth. $A$, left labyrinth seen from the outer side; $B$, right labyrinth from the inner side; $C$, left labyrinth from above; $F c$, round foramen; $F v$, oval foramen; $h$, horizontal semicircular canal; $h a$, its ampulla; $V a a$, ampulla of anterior vertical semicircular canal; $V p a$, ampulla of posterior vertical semicircular canal; $V c$, conjoined portion of the two vertical canals.

to disease of the proper nervous auditory apparatus no device can make the person hear.

The Internal Ear. The labyrinth consists primarily of chambers and tubes hollowed out in the temporal bone and inclosed by it on all sides, except for the oval and round foramina on its exterior, and certain apertures on its inner side by which bloodvessels and branches of the auditory nerve enter; during life all these are closed water-tight in one way or another. Lying in the bony labyrinth thus constituted, are membranous parts, of the same general form but smaller, so that between the two a space is left; this is filled with a watery fluid, called the perilymph; and the membranous internal ear is filled by a similar liquid, the endolymph.

The Bony Labyrinth. The bony labyrinth is described in three portions, the vestibule, the semicircular canals, and the cochlea; 
casts of its interior are represented from different aspects in Fig. 71. The vestibule is the central part and has on its exterior the oval foramen $(F v)$ into which the base of the stirrup-bone fits. Behind the vestibule are three bony semicircular canals, communicating with the back of the vestibule at each end, and dilated near one end to form an ampulla (Vpa, Vaa, and $h a$ ). The horizontal canal lies in the plane which its name implies, and has its ampulla at the front end. The two other canals lie vertically, the anterior at right angles, and the posterior parallel, to the median anteroposterior vertical plane of the head. Their ampullary ends are turned forwards and open close together into the vestibule; their posterior ends unite $(V c)$ and have a common vestibular opening.

The bony cochlea is a tube coiled on itself somewhat like a snail's shell, and lying in front of the vestibule.

The Membranous Labyrinth. The membranous vestibule, lying in the bony one, consists of two sacs communicating by a narrow aperture. The posterior is called the utriculus, and into it the membranous semicircular canals open. The anterior, called the sacculus, communicates by a tube with the membranous cochlea. The membranous semicircular canals much resemble the bony, and each has an ampulla; in most of their extent they are only united by a few irregular connective-tissue bands with the periosteum lining the bony canals; but in the ampulla one side of the membranous tube is closely adherent to its bony

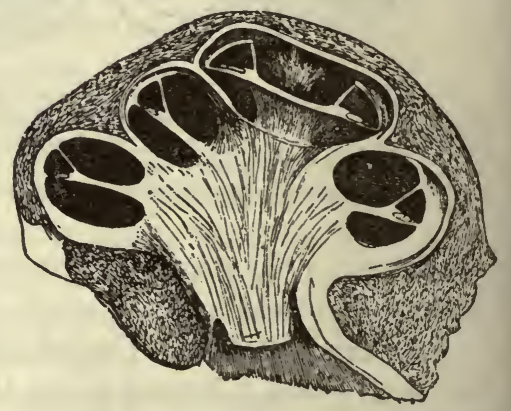

Fig. 72.-A section through the cochlea in the line of its axis.

protector; at this point nerves enter the former. The relations of the membranous to the bony cochlea are more complicated. A section through this part of the auditory apparatus (Fig. 72) shows that its osseous portion consists of a tube wound two and a half times (from left to right in the right ear and vice versa) around a central bony axis, the modiolus. From the axis a shelf, the lamina spiralis, projects and partially subdivides the tube, extending farthest across in its lower coils. Attached to the outer 
edge of this bony plate is the membranous cochlea (scala mediu), a tube triangular in cross-section and attached by its base to the outer side of the bony cochlear spiral. The spiral lamina and the membranous cochlea thus subdivide the cavity of the bony tube (Fig. 73) into an upper portion, the scala vestibuli, SV, and a lower, the scala tympani, ST. Between these lie the lamina spiralis (lso) and the membranous cochlea $(C C)$, the latter being bounded above by the membrane of Reissner $(R)$ and below by the basilar membrane $(b)$. The free edge of the lamina spiralis is thickened and covered with connective tissue which is hollowed out so as to form a spiral groove (the sulcus spiralis, ss) along the whole length of the membranous cochlea. The latter does not extend to the

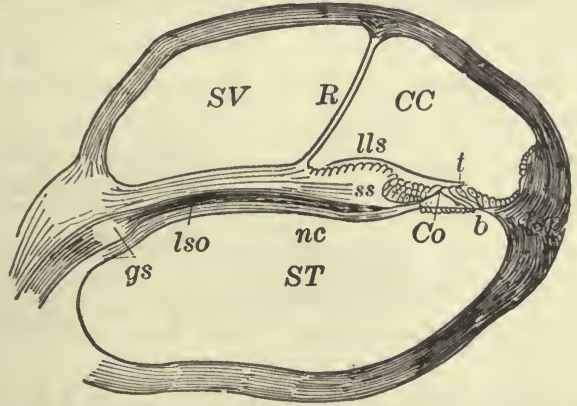

FIG. 73.-Section of one coil of the cochlea, magnified. $S V$, scala vestibuli; $R$, membrane of Reissner; $C C$, membranous cochlea (scala media); lls, limbus lamince spiralis; $t$, tectorial membrane; ST, scala tympani; lso, spiral lamina; $C o$, rods of Corti; $b$, basilar membrane.

tip of the bony cochlea; above its apex the scala vestibuli and scala tympani join; both are filled with perilymph, and the former communicates below with the perilymph cavity of the vestibule, while the scala tympani abuts below on the round foramen, which, as has already been pointed out, is closed by a membrane. The membranous cochlea contains certain solid structures seated on the basilar membrane and forming the organ of Corti; the rest of its cavity is filled with endolymph, which has free passage to that in the sacculus.

The Organ of Corti. This contains the end organs of the cochlear nerves. Lining the sulcus spiralis are cuboidal cells; on the inner margin of the basilar membrane the cells become columnar, and then are succeeded by a row which bear on their upper ends a 
set of short stiff hairs, and constitute the inner hair-cells, which are fixed below by a narrow apex to the basilar membrane; nerve-fibers enter them. To the inner hair-cells succeed the rods of Corti $(\mathrm{Co}$, Fig. 73), which are represented much magnified in Fig. 74. These rods are stiff and arranged side by side in two rows, leaned against one another by their upper ends so as to cover in a tunnel; they are known respectively as the inner and outer rods, the former being nearer the lamina spiralis. Each has a somewhat dilated base, firmly fixed to the basilar membrane; an expanded head where it meets its fellow (the inner rod presenting there a concavity into which the rounded head of the outer fits); and a slender shaft uniting the two, slightly curved like an italic $f$. The inner rods are more slender and more numerous than the outer, the numbers be-

A

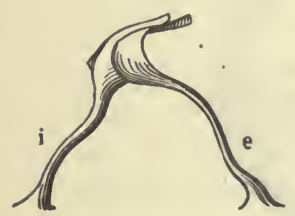

B

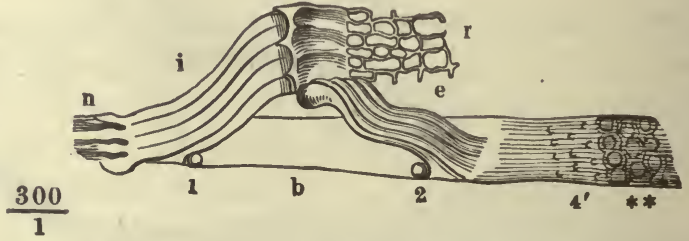

Fig. 74.-The rods of Corti. $A$, a pair of rods separated from the rest; $B$, a bit of the basilar membrane with several rods on it, showing how they cover in the tunnel of Cort $i$; $i$, inner, and $e$, outer rods; $b$, basilar membrane; $r$, reticular membrane.

ing about 6,000 and 4,500 respectively. Attached to the external sides of the head of the outer rods is the reticular membrane $(r$, Fig. 74), which is stiff and perforated by holes. External to the outer rods come four rows of outer hair-cells, connected like the inner row with nerve-fibers; their bristles project into the holes of the reticular membrane. Beyond the outer hair-cells is ordinary columnar epithelium, which passes gradually into cuboidal cells lining most of the membranous cochlea. The upper lip of the sulcus spiralis is uncovered by epithelium, and is known as the limbus lamino spiralis; from it projects the tectorial membrane ( $t$, Fig. 73) which extends over the rods of Corti and the hair-cells.

Function of the Cochlea. We have already seen reason to believe that in the ear there is an apparatus adapted for sympathetic resonance, by which we recognize different musical tone colors; the 
minute structure of the membranous cochlea is such as to lead us to look for it there. Of the various structures making up the membranous cochlea the basilar membrane seems to satisfy best the requirements of an apparatus for registering sounds by sympathetic resonance. It increases in breadth twelve times from the base of the cochlea to its tip (the less width of the lamina spiralis at the apex more than compensating for the less size of the bony tube there). Careful histological examination has shown that instead of being a true membrane it is really made up of a large number of transverse strands tightly stretched, and varying in length as the space between the lamina spiralis and the wall of the bony cochlea varies.

Probably each strand vibrates to simple tones of its own period, and excites the hair-cells which lie on it, and through them the nerve-fibers. Perhaps the rods of Corti, being stiff, and carrying the reticular membrane, rub that against the upper ends of the hair-cells which project into its apertures and so help in a subsidiary way, each pair of rods being especially moved when the band of basilar membrane carrying it is set in vibration. The tectorial membrane is probably a "damper"; it is soft and inelastic, and suppresses the vibrations as soon as the moving force ceases.

According to various estimates that have been made, from six thousand to eleven thousand different tones can be distinguished in the whole range of the ear. The basilar membrane is more than adequate to distinguish this number as it consists of twenty-four thousand strands. Fourteen thousand nerve-fibers communicate with the hair-cells of the organ of Corti.

We must suppose that compound tones entering the ear set the fluids of the cochlea into vibrations whose form depends upon the make-up of the tone producing them. These vibrations are analyzed by the basilar membrane, the particular strands having the vibration rates of the fundamental and the partials which are present being set into sympathetic vibration and stimulating the nerve-fibers with which they communicate.

Auditory Perceptions. Sounds, as a general rule, do not seem to us to originate within the auditory apparatus; we refer them to an external source, and to a certain extent can judge the distance and direction of this. As already mentioned, the extrinsic reference 
of sounds which reach the labyrinth through the general skullbones instead of through the tympanic chain is imperfect or absent. The recognition of the distance of a sounding body is possible only when the sound is well known, and then not very accurately; from its faintness or loudness we may make in some cases a pretty good guess. Judgments as to the direction of a sound are also liable to be grossly wrong, as most persons have experienced. However, when a sound is heard louder by the left than the right ear we can recognize that its source is on the left; when equally with both ears, that it is straight in front or behind; and so on.

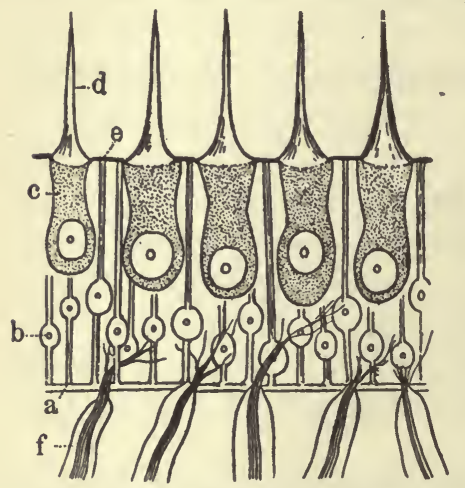

Fig. 75.-Diagram of epithelium in nervous region of ampulla of a semicircular canal.

The concha has perhaps something to do with enabling us to detect whether a sound originates before or behind the ear, since it collects, and turns with more intensity into the external auditory meatus, sound-waves coming from the front. By turning the head and noting the accompanying changes of sensation in each ear we can localize sounds better than if the head be kept motionless. The large movable concha of many animals, as a rabbit or a horse, which can be turned in several directions, is probably an important aid to them in detecting the position of the source of a sound. That the recognition of the direction of sounds is not a true sensation, but a judgment, founded on experience, is illustrated by the fact that we can estimate much more accurately the direction of the human voice, which we hear and heed most, than that of any other sound.

Nerve-Endings in the Semicircular Canals and the Vestibule. Myelinated fibers ( $f$, Fig. 75) from the vestibular branch of the auditory nerve are distributed along a line across the ampulla of each semicircular canal. They lose their myelin sheath close to the basement membrane, $a$, which the axons pierce. The axons branch among the epithelium cells, which at this place are several rows thick, but have not yet been traced into direct con- 
tinuity with any of them. The cells of the epithelium are of two varieties. The columnar cells or hair-cells, c, do not reach the basement membrane, are nucleated or slightly granular: from the free end of each projects a rigid hair process, $d$. The remaining cells, rod-cells, $b$, are in several rows: each has a slender inner process extending to the basement membrane and an outer which reaches to the bases of the columnar cells and appears there to end in a rigid membrane, $e$, which is perforated for the passage of the hairs. They probably are mere supporting structures.

In some parts of the utricle and saccule are regions of epithelium very similar to that above described, and also supplied with nervefibers. In connection with them are found minute calcareous particles,-otoliths or ear-stones.

The Equilibrium Sense. An important group of afferent impulses concerned with the maintenance of bodily equilibrium is derived through the semicircular canals and vestibule of the ear, which are supplied by the vestibular portion of the auditory nerve.

Experiment shows that cutting a semicircular canal is followed by violent movements of the head in the plane of the canal divided; the animal staggers, also, if made to walk; and, if a pigeon and thrown into the air, cannot fly. All its muscles can contract as before, but they are no longer so co-ordinated as to enable the animal to maintain or regain a position of equilibrium. It is like a creature suffering from giddiness; and similar phenomena follow, in man, electrical stimulation of the regions of the skull in which the semicircular canals lie.

If, moreover, a person lie perfectly quiet with closed eyes or a table which can be rotated, he is able to tell when the table is turned and in which direction, and often with considerable accuracy through what angle. If the rotation be continued for a time the feeling of it is lost, and then when the movement ceases there is a sense of rotation in the opposite direction. In such case neither tactile, muscular, nor visual sensations can help, and in the semicircular canals we seem to have a mechanism through which rotation of the head could give origin to afferent impulses, whether the head be passively moved with the rest of the Body or independently by its own muscles. Movements of endolymph 
in relation to the walls of the canals may act as stimuli by causing a swaying of the projecting hairs of the ampullæ (Fig. 75). Place a few small bits of cork in a tumbler of water, and rotate the tumbler; at first the water does not move with it; then it begins to go in the same direction, but more slowly; and, finally, moves at the same angular velocity as the tumbler. Then stop the tumbler, and the water will go on rotating for some time. Now if the head be turned or rotated in a horizontal plane similar phenomena will occur in the endolymph of the horizontal canal; if it be bent sidewise in the vertical plane, in the anterior vertical canal; and if nodded, in the posterior vertical; the hairs moving with the canal would meet the more stationary water and be pushed and so, possibly, excite the nerves at the deep ends of the cells which bear them, and generate afferent impulses which will cause the general nerve-centers of bodily equilibration to be differently acted upon in each case. Under ordinary circumstances the results of these impulses do not become prominent in consciousness as definite sensations; but they are probably always present. If one spins round for a time, the endolymph takes up the movement of the canals, as the water in the tumbler does that of the glass; on stopping, the liquid still goes on moving and stimulates the hairs which are now stationary; and we feel giddy, from the ears telling us we are rotating and the eyes that we are not; hence difficulty in standing erect or walking straight. A common trick illustrates this very well: make a person place his forehead on the handle of an umbrella, the other end of which is on the floor, and then walk three or four times round it, rise, and try to go out of a door; he will nearly always fail, being unable to combine his muscles properly on account of the conflicting afferent impulses. This and the feeling of rotation in the contrary direction when a previous rotation ceases become readily intelligible if we suppose feelings to be excited by relative movements of the endolymph and the canals inclosing it.

The sense of equilibrium as mediated by the semicircular canals. is a dynamic sense, one dealing with equilibrium of motion. That we have also a static sense of equilibrium, which tells us our position when at rest is well known. The swimmer immersed in water knows perfectly whether he is on his face or on his back; whether his head is up or down. This static equilibrium sense is 
thought to be mediated by structures of the vestibule, the utricle and saccule. These are hollow structures having stiff hairs projecting into their cavities and tiny stones caught among the hairs. The weight of the stones will affect the hairs among which it rests in one way when the head is erect, in quite another way when the head is horizontal. Thus the nerves may be stimulated differently for different positions of the head, fulfilling the conditions that the sense requires. In many invertebrate animals structures similar to the utricle and saccule represent their only organs resembling our ears in any way. Experiments upon these animals have shown that in them these structures are not hearing organs but organs of equilibrium.

Smell. The region of the nostril nearest its outer end possesses the sense of touch: the olfactory organ proper consists of the upper portions of the two nasal cavities, over which the endings of the olfactory nerves are spread and where the mucous membrane has a brownish-yellow color. This region (regio olfactoria) covers the upper and lower turbinate bones, which are expansions of the ethmoid on the outer wall of the nostril chamber,

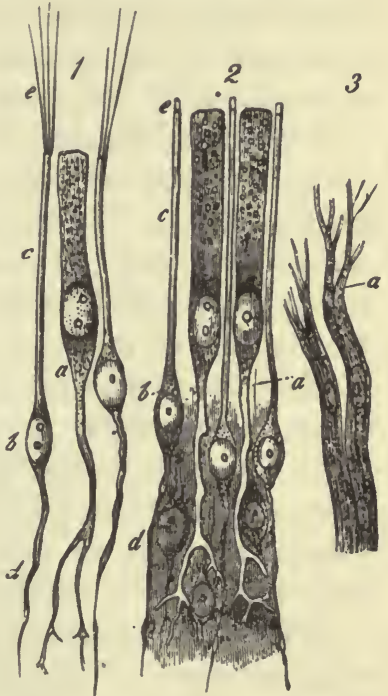

Fig. 76.-Cells from the olfactory epithelium. 1, from the frog; 2, from man; $a$, columnar cell, with its branched deep process; $b$, so-called olfactory cell; $c$, its narrow outer process; $d$, its slender central process; 3 , gray nerve-fibers of the olfactory nerve, seen dividing into fine peripheral branches at $a$. the opposite part of the partition between the nares, and the part of the roof of the nose separating it from the cranial cavity. The epithelium covering the mucous membrane contains three varieties of cells (2, Fig. 76). The cells of one set are much like ordinary columnar epithelium, but with long branched processes attached to their deeper ends; mixed with these are peculiar cells, each of which has a large nucleus surrounded by a little protoplasm; a slender external process reaching to the surface; and a very slender deep one. The latter cells have been supposed to be the proper olfactory end organs, and to be connected with the fibers of the ol- 
factory nerve, which enter the deeper strata of the epithelium and there divide. In Amphibia the corresponding cells have fine filaments on their free ends. The cells of the third kind are irregular in form and lie in several rows in the deeper parts of the epithelium. It may be that the cylindrical cells if not (as is possible) directly concerned in olfaction, have important functions in regard to the nourishment of the olfactory cells which they surround; they may supply them with needful material.

Odorous substances, the stimuli of the olfactory apparatus, are always gaseous and frequently act powerfully when present in very small amount. We cannot, however, classify them by the sensations they arouse, or arrange them in series; and smells are but minor sensory factors in our mental life, although very powerful associations of memory are often aroused by odors. We commonly refer them to external objects, since we find that the sensation is intensified by "sniffing" air into the nose, and ceases when the nostrils are closed. Their peripheral localization is, however, imperfect, for we confound many smells with tastes (see below); nor can we well judge of the direction of an odorous body through the olfactory sensations which it arouses.

Although the sense of smell in man is aroused by inconceivably small amounts of odoriferous substance, one part of mercaptan to thirty billion of air being detectible, it is much "less keen than the sense of smell in many animals, canines in particular. In such animals the sense of smell as a source of information seems to be of the first importance, approaching our eyes in rank.

A striking thing about the sense of smell is the ease with which it is fatigued. One may notice a bad odor upon entering a room, but in a few minutes ceases to perceive it because his olfactory apparatus has become fatigued. For this reason the sense of smell is wholly untrustworthy as a guide by which to regulate the ventilation of a room.

Taste. The organ of taste is the mucous membrane on the dorsum of the tongue * and, in some persons, of the soft palate and fauces. The nerves concerned are the glossopharyngeals, distributed over the hind part of the tongue, and the lingual branches of the inferior maxillary division of the trigeminals on its anterior two-thirds. It has been shown that the nerves of

*A description of the tongue will be found on page 446. 
taste which reach the tongue by way of the trigeminal nerve spring from the medulla as part of the sensory branch of the facial.

On the tongue most of the sensory nerves run to papillæ; the circumvallate have the richest supply, and on these are peculiar end organs (Fig. 77) known as taste-buds; they are oval and embedded in the epidermis covering the side of the papilla. Each consists, externally, of a number of flat, fusiform, nucleated cells and, internally, of six or eight so-called taste-cells. The latter are much like the olfactory cells of the nose, and are probably connected with nerve-fibers at their deeper ends. The capsule formed by the enveloping cells has a small opening on the surface; each taste-cell terminates in a very fine thread which there protrudes. Taste-buds are also found on some of the fungiform papillæ, and

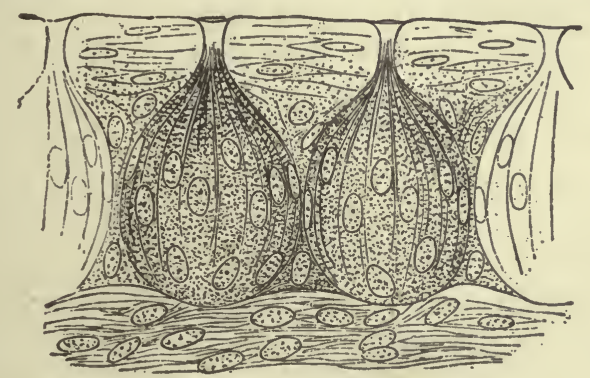

Frg. 77.-Taste-buds.

it is possible that simpler structures, not yet recognized, and consisting of single taste-cells are widely spread over the tongue, since the sense of taste exists where no taste-buds can be found. The filiform papillæ are probably tactile.

In order for substances to be tasted they must be in solution: wipe the tongue dry and put a crystal of sugar on it; no taste will be felt until exuding moisture has dissolved some of the crystal. Excluding the feelings aroused by acid substances, tastes proper may be divided into sweet, bitter, acid, and saline. Although contributing much to the pleasures of life, they are intellectually of small value; the perceptions we attain through them as to qualities of external objects being of little use, except as aiding in the selection of food, and for that purpose they are not safe guides at all times.

Many so-called tastes (flavors) are really smells; odoriferous 
particles of substances which are being eaten reach the olfactory region through the posterior nares and arouse sensations which, since they accompany the presence of objects in the mouth, we take for tastes. Such is the case, $e$. g., with most spices; when the nasal chambers are blocked or inflamed by a cold in the head, or closed by compressing the nose, the so-called taste of spices is not perceived when they are eaten; all that is felt, when cinnamon, $e . g$., is chewed under such circumstances is a certain pungency due to its stimulating nerves of touch in the tongue. This fact is sometimes taken advantage of in the practice of domestic medicine when a nauseous dose, as rhubarb, is to be given to a child.

As the tongue, in addition to taste functions, possesses tactile and temperature sensibility, its nerve apparatus must be complex; and there is even reason to believe that different nerve-fibers with presumably different end organs are concerned in the different true tastes. Most persons taste bitter things better with the back part of the tongue and sweet things with the tip, and in some persons the separation of function is quite complete. Chemical compounds are known which in such persons cause a pure sweet sensation if placed on the tongue tip and a pure bitter sensation if placed in the region of the circumvallate papillæ; these facts seem to show that the fibers concerned in bitter and sweet sensation are distinct. Again, if leaves of a certain plant (Gymnema sylvestre) be chewed, the capacity to taste sweet or bitter things is lost for some time, but salts and acids are tasted as well as usual; and most persons taste salines better at the sides of the tongue than elsewhere; so that the salt and acid sensations seem to have a different apparatus, not only from the sweet and bitter, but from one another. 


\section{CHAPTER XV}

\section{THE EYE AS AN OPTICAL INSTRUMENT}

The Essential Structure of an Eye. Every visual organ consists primarily of a nervous expansion, provided with end organs by means of which light is enabled to excite nervous impulses, and exposed to the access of objective light; such an expansion is called a retina. By itself, however, a retina would give no visual sensations referable to distinctly limited external objects; it would enable its possessor to tell light from darkness, more light from less light, and (at least in its highly developed forms) light of one color from light of another color; but that would be all. Were our eyes merely retinas we could only tell a printed page from a blank one by the fact that, being partly covered with black letters (which reflect less light), it would excite our visual organ less powerfully than the spotless white page would. In order that distinct objects and not merely degrees of luminosity may be seen, some arrangement is needed which shall bring all light entering the eye from one point of a luminous surface to a focus again on one point of the sensitive surface. If $A$ and $B$ (Fig. 78) be two red spots on a black surface, $K$, and $r r$ be a retina, then rays of light diverging from $A$ would fall equally on all parts of the retina and excite it all a little; so with rays starting from $B$. The sensation aroused, supposing the retina in connection with the rest of the nervous visual apparatus, would be one of a certain amount of red light reaching the eye; the red spots, as definite objects, would be indistinguishable. If, however, a convex glass lens $L$ (Fig. 79) be put in front of the retina, it will cause to converge again to a single point all the rays from $A$ falling upon it; so, too, with the rays from $B$ : and if the focal distance of the lens be properly adjusted these points of convergence will both lie on the retina, that for rays from $A$ at $a$, and that for rays from $B$ at $b$. The sensitive surface would then only be excited at two limited and separated points by the red light emanating from the spots; consequently only some of its end organs and nerve- 
fibers would be stimulated and the result would be the recognition of two separate red objects. In our eyes there are certain refract-

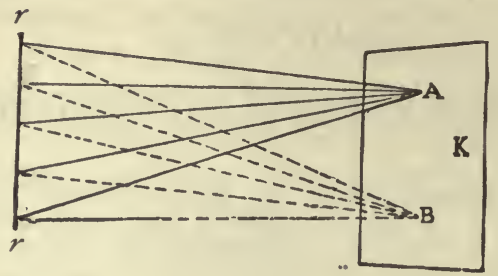

FIG. 78.-Diagram illustrating the indistinctness of vision with a retina alone. $K$, a surface on which are two spots, $A$ and $B ; r r$, the retina. The diverging lines represent rays of light spread uniformly over the retina from each spot.

ing media which lie in front of the retina and take the place of the lens $L$ in Fig. 79. That portion of physiology which treats of the

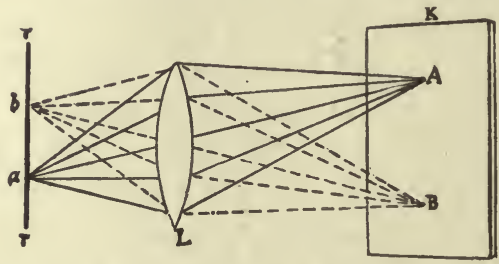

Fra. 79.-Illustrating the use of a lens in giving definite retinal images. $A, B, K$, $r r$, as in Fig. 78. $L$, a biconvex lens so placed that it brings to a focus on the points $a$ and $b$ of the retina, rays of light diverging from $A$ and $B$ respectively.

physical action of these media or, in other words, of the eye as an optical instrument, is known as the dioptrics of the eye.

The Appendages of the Eye. The eyeball itself consists of the retina and refracting media, together with supporting and nutritive structures and other accessory apparatuses, as, for example, some controlling the light-converging power of the media, and others regulating the size of the aperture (pupil) by which light enters. Outside the ball lie muscles which bring about its movements, and other parts serving to protect it.

Each orbit is a pyramidal cavity occupied by connective tissue, muscles, blood-vessels, and nerves, and in great part by fat, which forms a soft cushion on which the back of the eyeball lies and rolls during its movements. The contents of the orbit being for the most part incompressible, the eye cannot be drawn into its 
socket. It simply rotates there, as the head of the femur does in the acetabulum. When the orbital blood-vessels are gorged, however, the eyeball may protude (as in strangulation); and when these vessels empty it recedes somewhat, as is commonly seen after death. The front of the eye is exposed for the purpose of allowing light to reach it, but can be covered up by the eyelids, which are folds of integument, movable by muscles and strengthened by plates of fibrocartilage. At the edge of each eyelid the skin which covers its outside is turned in, and becomes continuous with a mucous membrane, the conjunctiva, which lines the inside of each lid, and also covers all the front of the eyeball as a closely adherent layer.

The upper eyelid is larger and more mobile than the lower, and when the eye is closed covers all its transparent part. It has a special muscle to raise it, the levator palpebra superioris. The eyes are closed by a flat circular muscle, the orbicularis palpebrarum which, lying on and around the lids, immediately beneath the skin, surrounds the aperture between them. At their outer and inner angles (canthi) the eyelids are united, and the apparent size of the eye depends upon the interval between the canthi, the eyeball itself being nearly of the same size in all persons. Near the inner canthus the line of the edge of each eyelid changes its direction and becomes more horizontal. At this point is found a small eminence, the lachrymal papilla, on each lid. For most of their extent the inner surfaces of the eyelids are in contact with the outside of the eyeball, but near their inner ends a red vertical fold of conjunctiva, the semilunar fold (plica semilunaris) intervenes. This is a representative of the third eyelid, or nictitating membrane, found largely developed in many animals, as birds, in which it can be drawn all over the exposed part of the eyeball. At the inner or nasal corner is a reddish elevation, the caruncula lachrymalis, caused by a collection of sebaceous glands * embedded in the semilunar fold. Opening along the edge of each eyelid are from twenty to thirty minute compound sebaceous glands, named the Meibomian follicles. Their secretion is sometimes abnormally abundant, and then appears as a yellowish matter along the edges of the eyelids, which often dries in the night and causes the lids to be glued together in the morning. The eyelashes are short

* For a description of the glands see p. 535. 
curved hairs, arranged in one or two rows along each lid where the skin joins the conjunctiva.

The Lachrymal Apparatus consists of the tear-gland in each orbit, the ducts which carry its secretion to the upper eyelid, and the canals by which the tears, unless when excessive, are carried off from the front of the eye without running down over the face. The lachrymal or tear-gland, about the size of an almond, lies in the upper and outer part of the orbit, near the front end. It is a compound racemose gland, (see Chap. XXIX) from which twelve or fourteen ducts run and open in a row at the outer corner of the upper eyelid. The secretion there poured out, is spread evenly over the exposed part of the eye by the movements of winking, and keeps it moist; finally the tear is drained off by two lachrymal canals, one of which opens by a small pore (punctum lachrymalis) on each lachrymal papilla. The aperture of the lower canal can be readily seen-by examining the corresponding papilla by the aid of a looking-glass. The canals run inwards and open into the lachrymal sac, which lies just outside the nose, in a hollow where the lachrymal and superior maxillary bones ( $L$ and $M x$, Fig. 25) meet. From the sac the nasal duct proceeds to open into the nose-chamber, below the inferior turbinate bone and within the nostril.

Tears are constantly being secreted, but ordinarily in such quantity as to be drained off into the nose, from which they flow into the pharynx and are swallowed. When the lachrymal ducts are stopped up, however, their continual presence makes itself unpleasantly felt, and may need the aid of a surgeon to clear the passage. In weeping the secretion is increased, and then not only more of it enters the nose, but some flows down the cheeks. The frequent swallowing movements of a crying child, sometimes spoken of as "gulping down his passion," are due to the need of swallowing the extra tears which reach the pharynx.

The Muscles of the Eye (Fig. 80). The eyeball is spheroidal in form and attached behind to the optic nerve, $n$, somewhat as a cherry might be to a thick stalk. On its exterior are inserted the tendons of six muscles, four straight and two oblique. The straight muscles lie, one (superior rectus), s, above, one (inferior rectus), not appearing in the figure, below, one (external rectus), $a$, outside, and one (internal rectus), $i$, inside the eyeball. Each 
arises behind from the bony margin of the foramen through which the optic nerve enters the orbit. In the figure, which represents the orbits opened from above, the superior rectus of the right side has been removed. The superior oblique or pulley (trochlear) muscle, $t$, arises behind near the straight muscles and forms anteriorly a tendon, $u$, which passes through a fibrocartilaginous ring, or pulley, placed at the notch in the frontal bone where it

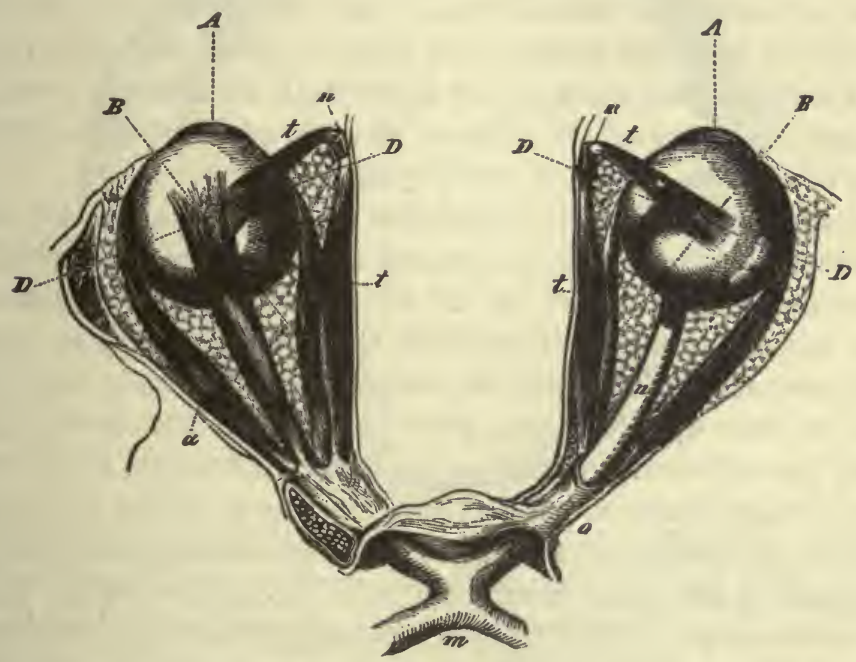

Fig. 80.-The eyeballs and their muscles as seen when the roof of the orbit has been removed and the fat in the cavity has been partly cleared away. On the right side the superior rectus muscle has been cut away. $a$, external rectus; $s$, superior rectus; $i$, internal rectus; $t$, superior oblique.

bounds superiorly the front end of the orbit. The tendon then turns back and is inserted into the eyeball between the upper and outer recti muscles. The inferior oblique muscle does not arise, like the rest, at the back of the orbit, but near its front at the inner side, close to the lachrymal sac. It passes thence outwards and backwards beneath the eyeball to be inserted into its outer and posterior part.

The inner, upper, and lower straight muscles, the inferior oblique, and the elevator of the upper lid are supplied by branches of the third cranial nerve. The sixth cranial nerve goes to the outer rectus; and the fourth to the superior oblique.

The eye may be moved from side to side; up or down; obliquely, 
that is, neither truly vertically nor horizontally, but partly both; or, finally, it may be rotated on its anteroposterior axis. The oblique movements are always accompanied by a slight amount of rotation. When the glance is turned to the left, the left external rectus and the right internal contract, and vice versa; when up, both superior recti; when down, both the inferior. 'The superior oblique muscle acting alone will roll the front of the eye downwards and outwards with a certain amount of rotation; the inferior oblique does the reverse. In oblique movements two of the recti are concerned, an upper or lower with an inner or outer; at the same time one of the oblique also always contracts. Movements of rotation rarely, if ever, occur alone.

The natural combined movements of the eyes by which both are directed simultaneously towards the same point depends on the accurate adjustment of all its nervo-muscular apparatus. When the coördination is deficient the person is said to squint. A left external squint would be caused by paralysis of the inner rectus of that eye, for then, after the eyeball had been turned out by the external rectus, it would not be brought back again to its median position. A left internal squint would be caused, similarly, by paralysis of the left external rectus; and probably by disease of the sixth cranial nerve or its brain-centers. Dropping of the upper eyelid (ptosis) indicates paralysis of its special elevator muscle and is often a serious symptom, pointing to disease of the brain-parts from which it is innervated.

The Globe of the Eye is on the whole spherical, but consists of segments of two spheres (see Fig. 81), a portion of a sphere of smaller radius forming its anterior transparent part and being set on to the front of its posterior segment, which is part of a larger sphere. From before back it measures about 22.5 millimeters $\left(\frac{9}{10}\right.$ inch), and from side to side about 25 millimeters (1 inch). Except when looking at near objects, the anteroposterior axes of the eyeballs are nearly parallel, though the optic nerves diverge considerably (Fig. 80 ); each nerve joins its eyeball, not at the center, but about $2.5 \mathrm{~mm}$. ( $\frac{1}{10}$ inch) on the nasal side of the posterior end of its anteroposterior axis. In general terms the eyeball may be described as consisting of three coats and three refracting media.

The outer coat, 1 and 3, Fig. 81 , consists of the sclerotic and the 
cornea, the latter being transparent and situated in front; the former is opaque and white and covers the back and sides of the globe and part of the front, where it is seen between the eyelids

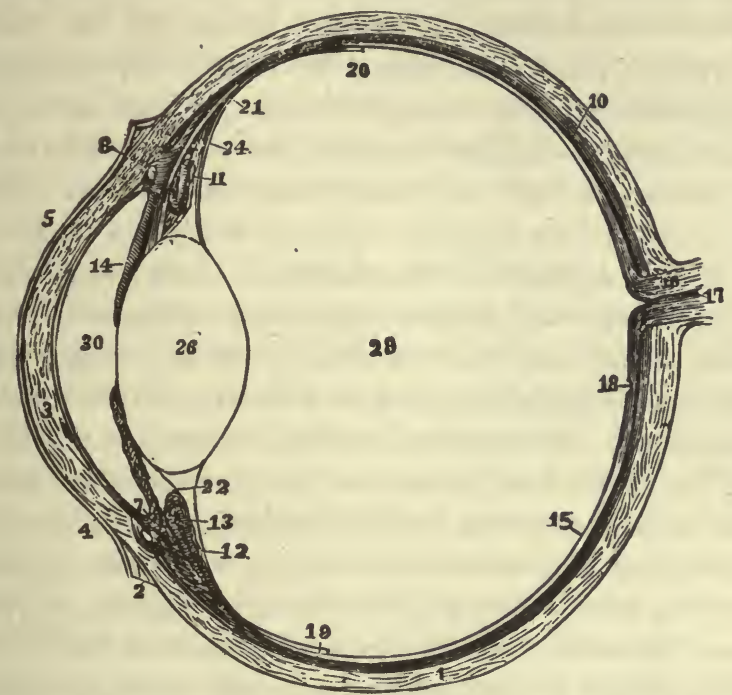

FIG. 81.-The left eyeball in horizontal section from before back. 1, sclerotic; 2 , junction of sclerotic and cornea; 3 , cornea; 4,5 , conjunctiva; 7 , ciliary muscle; 10 , choroid; 11,13 , ciliary processes; 14 , iris; 15 , retina; 16 , optic nerve; 17 , artery entering retina in optic nerve; 18 , fovea centralis; 19,20 , region where sensory part of retina ends; 22 , suspensory ligament; 24 , the anterior part of the hyaloid membrane; 26 , the lens; 29 , vitreous humor; 30 , aqueous humor.

as the white of the eye. Both are tough and strong, being composed of dense connective tissue.

The second coat consists of the choroid, 10, the ciliary processes, 11, 13, and the iris, 14 . The choroid is made up of bloodvessels supported by loose connective tissue containing numerous corpuscles, which in its inner layers are richly filled with darkbrown or black pigment granules. Towards the front of the eyeball, where it begins to diminish in diameter, the choroid is thrown into plaits, the ciliary processes, 11, 13. Beyond these it continues as the iris, which forms the colored part of the eye seen through the cornea; and in the center of the iris is a circular aperture, the pupil: so its second coat does not, like the outer one, completely envelop the eyeball. In the iris is a ring of plain muscular tissue encircling the aperture of the pupil: when its fibers contract they narrow the pupil. Radiating from this ring to the 
edges of the iris are muscle-fibers which by their contraction enlarge the pupil. Both sets of muscles are under the control of autonomic nerves. Those to the constrictor-fibers reach the eye by way of the third cranial nerve and belong to the cranial autonomic system; those innervating the dilator-fibers enter by way of the ophthalmic branch of the fifth nerve. These latter fibers belong to the thoracico-lumbar autonomic system and have a rather tortuous connection with the central nervous system. The pathway starts in the upper thoracic region of the spinal cord where the cell-body of the preganglionic neuron lies. The axon of this neuron passes out from the cord to the sympathetic chain and in this chain up the neck to the superior cervical ganglion at the base of the skull. Here the preganglionic neuron terminates in connection with a post-ganglionic. The axon of the latter passes to the fifth nerve and along this to its termination in the pupillo-dilator muscle.

The iris contains pigment which is yellow, or of lighter or darker brown, according to the color of the eye, and more or less abundant according as the eye is black, brown, or gray. In blue eyes the pigment is confined to the deeper layers, and modified in tint by light absorption in the anterior colorless strata through which the light passes.

The third coat of the eye, the retina, 15, is its essential portion, being the part in which the light produces those changes that give rise to impulses in the optic nerve. It is a still less complete envelope than the choroid, extending forwards only as far as the commencement of the ciliary processes, at least in its typical form. It is extremely soft and delicate; and, when fresh, transparent. Usually when an eye is opened the retina is colorless; but when the eye has been cut open in faint yellow light and the exposed retina quickly examined in white light it is seen to be purple. The coloring substance (visual purple) very rapidly bleaches when a dead eye is exposed to daylight. On the front or inner surface of the human retina two special areas can be distinguished in a fresh eye. One is the point of entry of the optic nerve, 16, the fibers of which, penetrating the sclerotic and choroid, spread out in the retina. At this place the retina is whiter than elsewhere and presents an elevation, the optic disk. The other peculiar region is the fovea centralis, 18, which lies nearly at the posterior end of the axis of the eyeball and therefore out- 
side the optic disk; in it the retina is thinner than elsewhere and so a pit is formed. This appears black, the thinned retina there allowing the choroid to be seen through it more clearly than elsewhere. In Fig. 82 is represented the right retina as seen from the front, the elliptical darker patch about the center indicating the fovea and the white circle on one side, the optic disk. The vessels of the retina arise from an artery (17, Fig. 81) which runs in with the optic nerve and from which branches diverge as shown in Fig. 82.

The Optic Nerves, Chiasma, and Tracts. The optic nerves converge to meet in the optic chiasma ( $m$, Fig. 80), from which the optic tracts pass to the region of the midbrain. They terminate mainly in the anterior corpora quadrigemina, (superior colliculi) (Chap. IX) and in the corpora geniculata. The behavior of the nerve-fibers in the chiasma is interesting in that part of them cross to the opposite side and part continue into the tract of the same side. The fibers which cross over in each optic nerve are those coming from the inner half of the retina, the right half of the left retina and the left half of the right retina. The effect of this arrangement is to include in the right optic tract, behind the chiasma, the nerve-fibers from the right halves of both retinas, and in the left optic tract those from the left halves of both retinas. Cutting the right optic nerve, therefore, causes total blindness of the right eye, but cutting the right optic tract blindness of the right half of each retina (hemianopia).

The half crossing of the optic nerve-fibers in man is correllated with the fact that his eyes are so placed that most of the field of vision is common to both. In mammals whose eyes are so laterally placed that at any given moment the objects seen by the two eyes are quite different, the crossing at the commissure is complete; this condition obtains also in birds with the exception of owls, whose eyes like those of man have their visual axes parallel; in owls the crossing is only partial. It should be noted that the fovea centralis, which is the center of distinct vision, has nerve connections from both eyes with both optic tracts. For this reason unilateral injury to the visual mechanism back of the chiasma interferes practically not at all with ordinary vision, and sufferers from hemianopia may be unaware of their infirmity until careful examination by a physician reveals it. 
The Microscopic Structure of the Retina. This, the sensitive portion of the eye, has the form of a thin membrane lining the entire back part of the cavity of the eyeball as far forward as the ciliary processes. Although only 0.15 millimeter (0.006 inch)

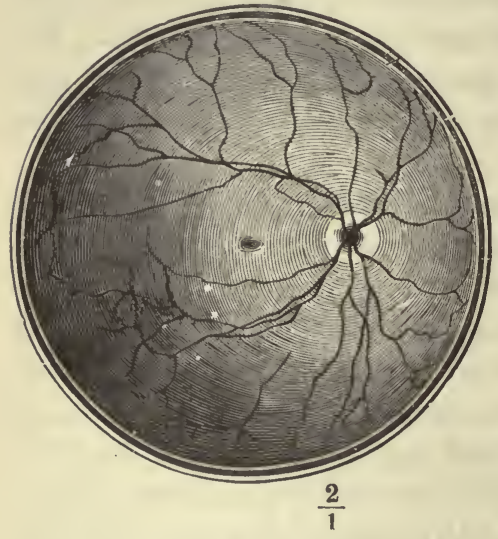

FIG. 82.-The right retina as it would be seen if the front part of the eyeball with the lens and vitreous humor were removed.

tween themselves and the source of light the nerve structures which connect them with the optic nerve, and the supporting tissues and blood-vessels of the retina. Fortunately all these structures are so transparent or so placed as not to interfere seriously with vision. In the fovea, where all clear sight is located, blood-vessels are absent and the other structures are much reduced.

The sensitive elements of the eye are called, from their shape, rods and cones. The rods consist of basal enlarged portions from which slender rod-like processes project toward the choroid coat. These processes contain a peculiar reddish substance (visual pur$p l e$ ), which has the property of bleaching out when exposed to light ( $R$, Fig. 83). The cones have somewhat thicker basal portions than the rods and much shorter processes containing no visual purple ( $C$, Fig. 83 ). Rods and cones make up layer number two of the ten retinal layer's. The first layer, which is between the rods and cones and the choroid coat, is a layer of pigment cells which send processes in among the rods, and seem to have something to do with forming the visual purple. 
The rods and cones appear to constitute the peripheral or dendritic portions of bipolar sensory neurons. They communicate with cell-bodies from which in turn pass typical, though very short, axons. The third retinal layer is composed of these cell-bodies with their axons. The axons of the rod and cone neurons come into synaptic connection with dendrites of a second

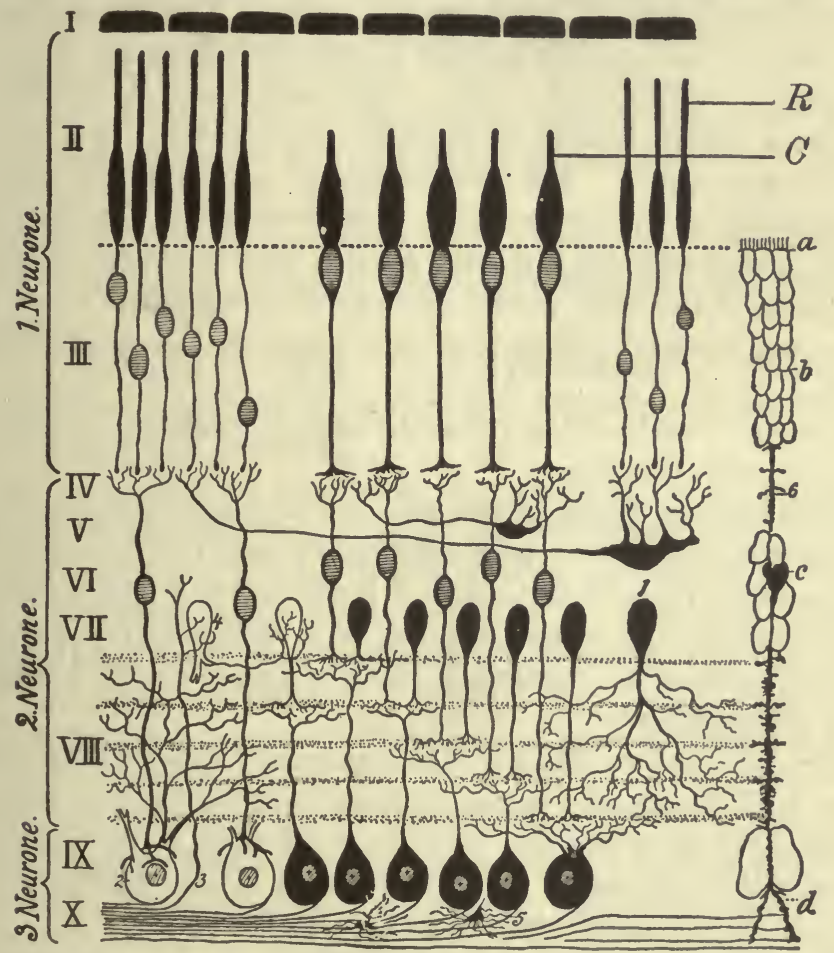

FIG. 83.-Diagram of the structure of the human retina (Greeff): $I$, pigment layer; $I I$, rod and cone layer; $R$, rods; $C$, cones; $I I I-I X$, int raretinal nerve-elements; $X$, axons which pass to optic nerve.

set of retinal neurons, the synapses making up the fourth retinal layer. The fifth, sixth, and seventh retinal layers contain the cell-bodies and short axons of these second retinal neurons; in the eighth layer these come into synaptic connection with the dendrites of the third set of retinal neurons. The large cellbodies of these neurons make up the ninth retinal layer, and their axons, converging from all parts of the retina upon the optic 
disk, constitute the tenth and front layer of the retina. These axons continue uninterrupted to terminations in the midbrain ganglia. The relations of the three sets of retinal neurons are shown in the diagram (Fig. 83).

Rods and cones are not uniformly distributed over the retina. The fovea, where distinct vision is centered, contains only cones. The peripheral portions of the retina contain a larger and larger proportion of rods as the margin is approached, until the outermost regions contain only rods. This difference of distribution indicates a differentiation of function between the two sorts of sensitive structures. "The probability of such differentiation is strengthened by the observation that each cone communicates through the intervening retinal neuron with a single and separate neuron of the optic nerve, whereas the connection of the rods is such that several of them may send impulses into a single optic neuron.

The blood-vessels of the retina lie almost entirely in the ninth and tenth retinal layers.

The Refracting Media of the Eye are, in succession from before back, the aqueous humor, the crystalline lens, and the vitreous humor.

The aqueous humor fills the space between the front of the lens, and the back of the cornea (30, Fig. 81). Chemically, it consists of water holding in solution a small amount of solid matters, mainly common salt.

The crystalline lens (26, Fig. 81 ) is colorless, transparent, and biconvex, with its anterior surface less curved than the posterior. It is surrounded by a capsule, and the inner edge of the iris lies in contact with it in front. In consistence it is soft, but its central layers are rather more dense than the outer.

The capsule is continuous at the margin of the lens with the suspensory ligament which in turn is attached all around to the ciliary processes. The suspensory ligament is stretched and its pull upon the capsule keeps the lens more flattened than it would be if free.

The vitreous humor (29, Fig. 81 ) is a soft jelly enveloped in a thin capsule, the hyaloid membrane. It consists mainly of water and contains some salts, a little albumin, and some mucin. It is divided up, by delicate membranes, into compartments in which its more liquid portions are imprisoned. 
The Ciliary Muscle. (7, Fig. 81.) Between the sclerotic and choroid coats, just where the former merges into the cornea, are small masses of smooth muscle-fibers which make up the ciliary muscle. These fibers are attached in front to the sclerotic coat and pass back a short distance to an insertion in the choroid coat just in front of the ciliary processes. The contraction of the ciliary muscle pulls the margin of the choroid coat forward and inward. The effect of this is to bring the ciliary processes nearer together and loosen the suspensory ligament, which is attached to them. The tension upon the capsule of the crystalline lens is thus diminished.

The ciliary muscle is interesting as being the only voluntary muscle in the Body which is innervated through the autonomic system.

The Properties of Light. Before proceeding to the study of the eye as an optical instrument, it is necessary to recall briefly certain properties of light.

Light is considered as a form of movement of the particles of an hypothetical medium, or ether, the vibrations being in planes at right angles to the line of propagation of the light. Starting from a luminous point light travels in all directions along the radii of a sphere of which the point is the center; the light propagated along one such radius is called a ray, and in each ray the ethereal particles vibrate from side to side in a plane perpendicular to the direction of the ray.

Any ray, all of whose particles are vibrating at the same rate, is a ray of monochromatic light. It has a pure spectral color. The wave length of a beam of monochromatic light is measured by the distance between any ethereal particle of the beam and the next one which is in precisely the same phase of vibration. Since the rate at which light travels is nearly fixed, the wave length must vary inversely as the vibration rate. Light of high vibration rate has short wave length and vice versa. The color of monochromatic light depends upon its wave length. Where lights of various wave lengths are mixed together in a beam a compound light results. To the eye such a beam gives a definite color sensation but not one of the pure spectral colors.

Refraction. When light passes obliquely from one transparent medium into another of different density it is bent from its 
course, or refracted. The amount of refraction depends upon the optical nature of the two media and also upon the angle at

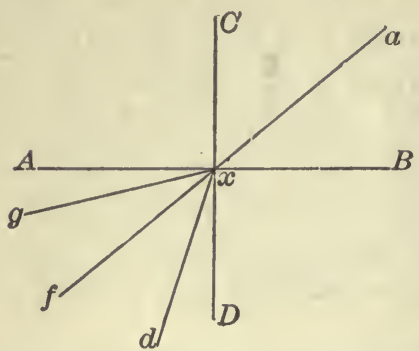

Fig. 84.-Diagram illustrating the refraction of light. $A B$, surface of separation between two transparent media; $C D$, the perpendicular to the surface at the point of incidence; $x$, $a x$, incident ray; $x d$, refracted ray, if the second medium be denser than the first; $x g$, refracted ray, if the second medium is less refractive than the first.

by the law of refraction which is: the ratio of the sine of the angle of incidence to that of the angle of refraction is always constant for the same two media and for light of the same wave length.

This ratio of sines is the index of refraction. It is usually ex-

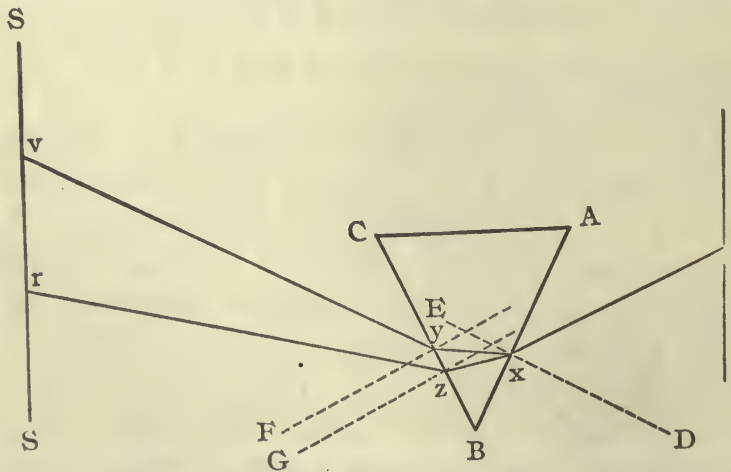

FIG. 85.-Diagram illustrating the dispersion of mixed light by a prism.

pressed for various refractive media with air as the second and less refractive one.

Dispersion of Mixed Light. The shorter the vibration periods of light-rays the more they are deviated by refraction. Hence 
mixed light when sent through a prism is spread out, and decomposed into its simple constituents. For let ax (Fig. 85) be a ray of mixed light composed of a set of short and a set of long ethereal waves. When it falls on the surface $A B$ of the prism, that portion which enters will be refracted towards the normal $E D$, but the short waves more than the longer. Hence the former will take the direction $x y$, and the latter the direction $x z$. On emerging from the prism both rays will again be refracted, but now from the normals $F y$ and $G z$, since the light is passing from a more to a less refracting medium. Again the ray $x y$, made up of shorter waves, will be most deviated, as in the direction $y v$, and the long waves less, in the direction $z r$. If a screen were put at $S S^{\prime}$, we would receive on it at separate points, $v$ and $r$, the two simple lights which were mixed together in the compound incident ray ax. Such a separation of light-rays is called dispersion.

Ordinary white light, such as that of the sun, is composed of ethereal vibrations of every rate, mixed together. When such light is sent through a prism it gives a continuous band of lightrays, known as the solar spectrum, reaching from the least refracted to the most refracted and shortest waves. The exceptions to this statement due to Frauenhofer's lines (see Physics) are unessential for our present purpose. Not all the rays of the solar spectrum are visible to the human eye. The least refracted ones, called the ultra red, and the most refracted ones, the ultra violet, do not stimulate the retina; they are determined by their physical and chemical effects. The visible spectrum includes in order of increasing refrangibility the seven spectral colors red, orange, yellow, green, blue, indigo, and violet. These merge insensibly into one another, showing the sun's light to be a mixture of all possible wave lengths, and not of certain selected ones.

Refraction of Light by Lenses. In the eye the refracting media have the form of lenses thicker in the center than towards the periphery; and we may here confine ourselves, therefore, to such convex lenses. If simple light from a point $A$, Fig. 79, fall on such a lens its rays, emerging on the other side, will take new directions after refraction and meet anew at a point, $a$, after which they again diverge. If a screen, $r r$, be held at $a$ it will therefore receive an image of the luminous point $A$. For every convex lens there is such a point behind it at which the rays 
from a given point in front of it meet: the point of meeting is called the conjugate focus of the point from which the rays start. If instead of a luminous point a luminous object be placed in front of the lens an image of the object will be formed at a certain

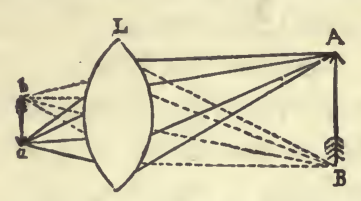

Fig. 86.-Diagram illustrating the formation of an image by a convex lens. distance behind it, for all rays proceeding from one point of the object will meet in the conjugate focus of that point behind. The image is inverted, as can be readily seen from Fig. 86 . All rays from the point $A$ of the object meet at the point $a$ of the image; those from $B$ at $b$, and those from intermediate points at intermediate positions. If the single lens were replaced by several combined so as to form an optical system the general result would be the same, provided the system were thicker in the center than at the periphery.

A moment's consideration of the diagram (Fig. 86) shows us that the nearer any luminous point is to the lens the further behind the lens its conjugate focus will be: The rays from near points are more divergent when they strike the lens than are those from far points, they are therefore not so much bent toward each other upon emerging, and their point of meeting is further back. There must be some point near the lens from which rays are so divergent that after emerging they do not meet at all, but continue to diverge or form a parallel beam. A plane so located with reference to a lens that rays from any point in it striking the lens emerge in a parallel beam is the principal focal plane of the lens. The thicker a lens the nearer to it is its principal focal plane.

The Ordinary Photographic Camera is an instrument which serves to illustrate the formation of images by converging systems of lenses. It consists of a box blackened inside and having on its front face a tube containing the lenses; the posterior wall is made of ground glass. If the front of the instrument be directed on exterior objects, inverted and diminished images of them will be formed on the ground glass; those images only are well defined, at any one time, which are at such a distance in front of the instrument that the conjugate foci of points on them fall exactly on the glass behind the lens: objects nearer or farther off give confused and indistinct images; but by altering the dis- 
tance between the lenses and the ground glass, in common language "focussing the instrument," either can be made distinct. For near objects the lenses must be farther from the surface on which the image is to be received, and for distant nearer.

The Refracting Media of the Eye Form a Convergent Optical System, made up of cornea, aqueous humor, lens, and vitreous humor. These four media are reduced to three practically, by the fact that the indices of refraction of the cornea and aqueous humor are the same, so that they act together as one converging lens. The surfaces at which refraction occurs are: (1) that between the air and the cornea; (2) that between the aqueous humor and the front of the lens; (3) that between the vitreous humor and the back of the lens. The refractive indices of those media are: the air, 1; the aqueous humor, 1.3379; the lens (average), 1.4545; the vitreous humor, 1.3379. From the laws of the refraction of light it therefore follows that (Fig. 87) the rays $C d$ will at the corneal surface be refracted towards the normals $N, N$, and take the course de. At the front of the lens they will again be refracted towards the normals to that surface and take the course ef; at the back of the lens, passing from a more refracting to a less refracting medium, they will be bent from the normals $N^{\prime \prime}$ and take the course $f g$. If the retina be there, these parallel rays will therefore be brought to a focus on it. In the resting condition of the natural eye this is what happens to parallel rays entering it: and, since distant objects send into the eye rays which are practically parallel, such objects are seen distinctly without any effort, because all rays emanating from a point of the object meet again in one point on the retina.

Wide Range of Clear Vision in the Resting Eye. While in the normal resting eye only parallel rays focus exactly on the retina, the fact is that it sees clearly all objects that are as far as 18-20 feet away. The rays of light from points on such objects are divergent when they strike the cornea, and their focus is therefore behind the retina. How then can the resting eye see such objects clearly? The explanation is found in the structure of the retina. The light perceiving elements, the rods and cones (Fig. 83), although extremely minute, are not mathematical points, but objects with measurable diameter. When light falls on one of them the effect is the same whether only a part of the element is illuminated or the 
whole, provided the light does not lap over into adjacent elements. Any beam of light entering the eye forms a cone with its apex at the focus. Near the focus a cross-section of the cone consists of a small circular area, which is larger the further away from the focus it is taken. Such an area is known as a dispersion circle. The convergent beams from the points of an object 18 feet away strike the retina before reaching their exact focus, but the dispersion circles formed by them are too small to stimulate more than one element; the effect is therefore the same as though an accurate focus had been reached, and objects at this distance are seen clearly.

Accommodation. Points on objects nearer than 18 feet send into the eye beams so diverging, and therefore focussing so far

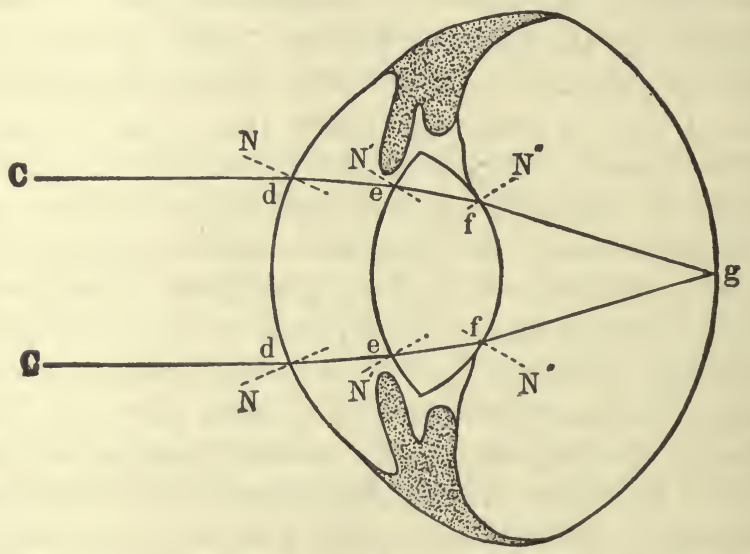
eye.

Frg. 87.-Diagram illustrating the surfaces at which light is refracted in the

behind the retina, that the dispersion circles formed at the retina are too large to stimulate only single elements. Near objects, therefore, would not be seen distinctly, did not some change occur in the eye; since we can see them quite plainly if we choose (unless they be very near indeed), there must exist some means by which the eye is focussed or accommodated for looking at objects at different near distances. That some change does occur one can, also, readily prove by observing that we cannot see distinctly, at the same moment, both near and distant objects. For example, standing behind a lace curtain, at a window, we can as we choose look at the threads of the lace or at the houses across the street; but when 
we look at the one we see the other only indistinctly; and if, after looking at the more distant object, we look at the nearer we experience a distinct sense of effort. It is clear, then, that something in the eye is different in the two cases. The resting eye, suited for seeing distinctly distant objects, might conceivably be accommodated for near vision in several ways. The refracting indices of its media might be increased; that of course does not happen; the physical properties of the media are the same in both cases: or the distance of the retina from the refracting surfaces might be increased, for example, by compression of the eyeball by the muscles around it; however, experiment shows that changes of accommoda-

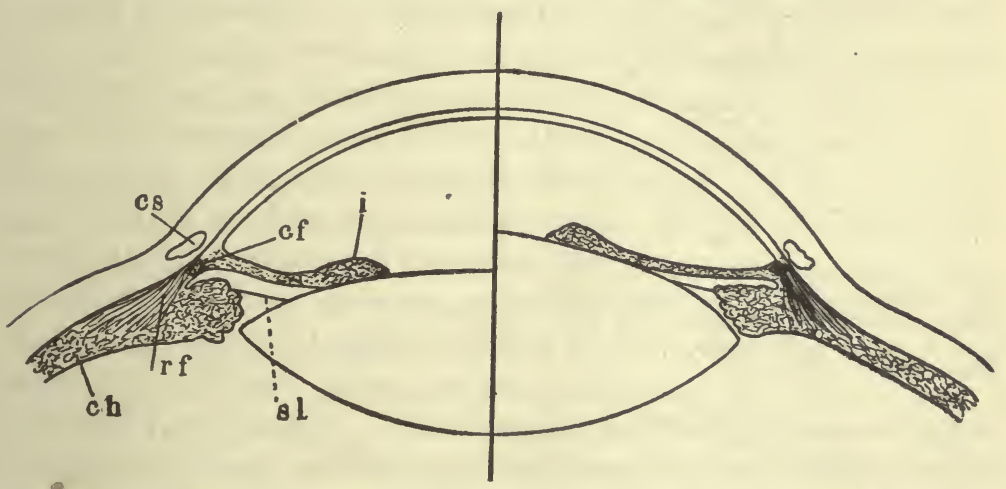

Fig. 88.-Diagram to illustrate the mechanism of accommodation; on the right half of the figure for a near, on the left for a distant, object; $r f$, ciliary muscle; $c h$, ciliary process of choroid; sl, suspensory ligament; $i$, iris.

tion can, by stimulating the third cranial nerve, be brought about in the fresh excised eyes of animals from which the muscles lying outside the eyeball have been removed, in which no such compression is possible; we are thus reduced to the third explanation, that the refracting surfaces, or some of them, become more curved, and so bring diverging rays sooner to a focus. Observation shows that this is what actually happens: the corneal surface remains unchanged when a near object is looked at after a distant one, but the lens becomes considerably more convex.

Accommodation is brought about by the ciliary muscle (Fig. 88). In the resting eye it is relaxed and the suspensory ligament of the lens is taut, and, pulling on its edge, drags it out laterally a little and flattens its surfaces, especially the anterior, since the ligament 
is attached a little in front of the edge. To see a nearer object the ciliary muscle is contracted, and according to the degree of its contraction slackens the suspensory ligament, and then the elastic lens, relieved from the lateral drag, bulges out a little in the center.

When the eye is focussed for seeing a near object the circular muscle of the iris contracts, narrowing the pupil, but this has nothing directly to do with the accommodation.

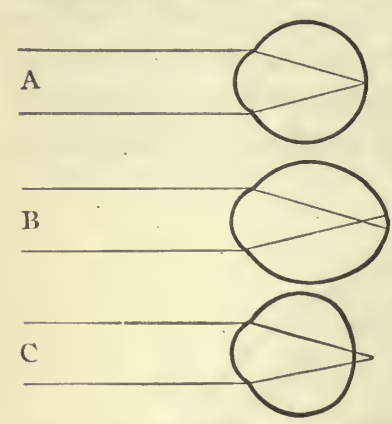

Fig. 89.-Diagram illustrating the path of parallel rays $(A)$, a myopic $(B)$, and a hypermetropic $(C)$ eye.

Short Sight and Long Sight. In the normal eye parallel rays meet on the retina when the ciliary muscle is completely relaxed ( $A$, Fig. 89 ). Such eyes are emmetropic. In other eyes the eyeball is too long from before back; in the resting state parallel rays meet in front of the retina $(B)$. Persons with such eyes, therefore, cannot see distant objects distinctly without the aid of diverging (concave) spectacles; they are shortsighted or myopic. Or the eyeball may be too short from before back; then, in the resting state, parallel rays are brought to a focus behind the retina $(C)$. To see even infinitely distant objects, such persons must therefore use their accommodating apparatus to increase the converging power of the lens; and when objects are near they cannot, with the greatest effort, bring the divergent rays proceeding from them to a focus soon enough. To get distinct retinal images of near objects they therefore need converging (convex) spectacles. Such eyes are called hypermetropic or in common language long-sighted.

Optical Defects of the Eye. The eye, though it answers admirably as a physiological instrument, is by no means perfect optically; not nearly so good, for example, as a good microscope objective. The main defects in it are due to:

1. Chromatic Aberration. As already pointed out, the rays at the violet end of the solar spectrum are more refrangible than those at the red end. Hence they are brought to a focus sooner. The light emanating from a point on a white object does not, therefore, all meet in one point on the retina; but the violet rays come to a focus first, then the indigo, and so on to the red, farthest back of 
all. If the eye is accommodated so as to bring to a focus on the retina parallel red rays, then violet rays from the same source will meet half a millimeter in front of it, and crossing and diverging there make a little violet circle of diffusion around the red point on the retina. In optical instruments this defect is remedied by combining together lenses made of different kinds of glass; such compound lenses are called achromatic.

The general result of chromatic aberration, as may be seen in a bad opera-glass, is to cause colored borders to appear around the edges of the images of objects. In the eye we usually do not notice such borders unless we especially look for them; but if, while a white surface is looked at, the edge of an opaque body be brought in front of the eye so as to cover half the pupil, colorations will be seen at its margin. If accommodation be inexact they appear also when the boundary between a white and a black surface is observed. The phenomena due to chromatic aberration are much more easily seen if light containing only red and violet rays be used instead of white light containing all the rays of intermediate refrangibility. Ordinary blue glass only lets through these two kinds of rays. If a bit of it be placed over a very small hole in an opaque shutter and sunlight be admitted through the hole, it will be found that with one accommodation (that for the red rays) a red point is seen with a violet border, and with another (that at which violet rays are brought to a focus on the retina) a violet point is seen with a red aureole.

2. Spherical Aberration. It is not quite correct to state that ordinary lenses bring to a focus in one point behind them rays proceeding from a point in front, even when these are all of the same refrangibility. Convex lenses whose surfaces are segments of spheres, as are those of the eye, bring to a focus sooner the rays which pass through their marginal than those passing through their central parts. If rays proceeding from a point and traversing the lateral part of a lens be brought to a focus at any point, then those passing through the center of the lens will not meet until a little beyond that point. If the retina receive the image formed by the peripheral rays the others will form around this a small luminous circle of light-such as would be formed by sections of the cones of converging rays in Fig. 78, taken a little in front of $r r$. This defect exists in all glass lenses, as it is found impossible in practice 
to grind them of the non-spherical curvatures necessary to avoid it. In our eyes its effect is to a large extent corrected in the following ways: $(a)$ The opaque iris cuts off many of the external and more strongly refracted rays, preventing them from reaching the retina. (b) The outer layers of the lens are less refracting than the central; hence the rays passing through its peripheral parts are less refracted than those passing nearer its axis.

3. Irregularitics in Curvature. The refracting surfaces of our eyes are not even truly spherical; this is especially the case with

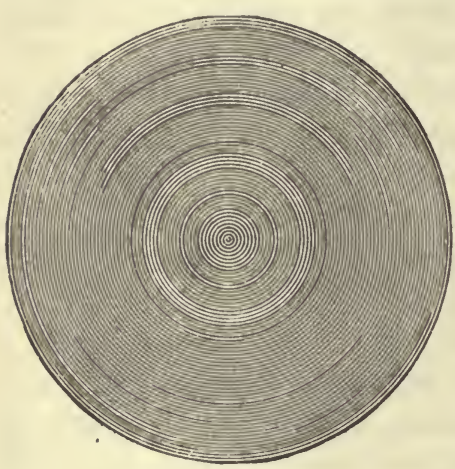

Fig. 90. the cornea, which is very rarely curved to the same extent in its vertical and horizontal diameters. Suppose the vertical meridian to be the most curved; then the rays proceeding from points along a vertical line will be brought to a focus sooner than those from points on a horizontal line. If the eyc is accommodated to see distinctly the vertical line, it will see indistinctly the horizontal and vice versa. Few people therefore see equally clearly at once two lines crossing one another at right angles. The phenomenon is most obvious, however, when a series of concentric circles (Fig. 90) is looked at: then when the lines appear sharp along some sectors, they are dim along the rest. This defect is known as astigmatism; it is corrected by the use of lenses which are curved only in one plane. The lens is so adjusted that its curvature combines with the less curvature of the eye to equal the greater.

4. Opaque Bodies in the Refracting Media. In diseased eyes the lens may be opaque (cataract) and need removal; or opacities from ulcers or wounds may exist on the cornea. But even in the best eye there are apt to be small opaque bodies in the vitreous humor causing muscoe volitantes; that is, the appearance of minute bodies floating in space outside the eye, but changing their position when the position of the eye changes, by which fact their origin in internal causes may be recognized. Many persons never see them until their attention is called to their sight by some weakness of it, 
and then they think they are new phenomena. Visual phenomena due to causes in the eye itself are called entoptic; the most interesting are those due to the retinal blood-vessels (Chap. XVI). Tears, or bits of the secretion of the Meibomian glands, on the front of the eyeball often cause distant luminous objects to look like ill-defined luminous bands or patches of various shape. The cause of such appearances is readily recognized, since they disappear or are changed after winking.

Hygienic Remarks. Since muscular effort is needed by the normal eye to see near objects, it is clear why the prolonged contemplation of such is more fatiguing than looking at more distant things. If the eye be hypermetropic still more is this apt to be the case, for then the ciliary muscle has no rest when the eye is used, and to read a book at a distance such that enough light is reflected from it into the eye in order to enable the letters to be seen at all, requires an extraordinary effort of accommodation. Such persons complain that they can read well enough for a time, but soon fail to be able to see distinctly. This kind of weak sight should always lead to examination of the eyes by an oculist, to see if glasses are needed; otherwise severe neuralgic pains about the eyes are apt to come on, and the overstrained organ may be permanently injured. Old persons are apt to have such eyes; but young children frequently also possess them, and if so should at once be provided with spectacles. Astigmatism is another fruitful source of eye strain. Although sharp focussing is impossible the eye constantly strives for it. This involves great activity of the muscles of accommodation, which suffer from the effort. The occurrence of headache at frequent intervals, particularly in connection with the use of the eyes, as in reading or sewing, is more often than not an indication of visual defects which proper glasses would overcome. Sufferers from such headaches should therefore have their eyes examined and if glasses are necessary should wear them.

Short-sighted eyes appear to be much more common now than formerly, especially in those given to literary pursuits. Myopia is rare among those who cannot read or who live mainly out of doors. It is not so apt to lead to permanent injury of the eye as is the opposite condition, but the effort to see distinctly objects a little distant is apt to produce headaches and other symptoms of nervous exhaustion. If the myopia become gradually worse the 
eyes should be rested for, several months. Short-sighted persons are apt to have, or acquire, peculiarities of appearance: their eyes. are often prominent, indicative of the abnormal length of the eyeball. They also get a habit of "screwing" up the eyelids, probably an indication of an effort to compress the eyeball from before back so that distant objects may be better seen. They often stoop, too, from the necessity of getting their eyes near objects they want to see. The acquirement of such habits may be usually prevented by the use of proper glasses. On the other hand, "it is said that myopia even induces peculiarities of character, and that myopes are usually unsuspicious and easily pleased; being unable to observe many little matters in the demeanor or expression of those with whom they converse, which, being noticed by those of quicker sight, might induce feelings of distrust or annoyance."

In old age the lens loses some of its elasticity and becomes more rigid. This leads to the long-sightedness of old people, known as presbyopia. The stiffer lens does not become as convex as it did in early life, when the ciliary muscle contracts and the suspensory ligament is relaxed. In order to adapt the eye to see near objects distinctly, therefore, convex glasses are required.

In all forms of defective vision too strong glasses will injure the eyes irreparably, increasing the defects they are intended to relieve. Skilled advice should therefore be invariably obtained in their selection, except perhaps in the long-sightedness of old age; when the sufferer may tolerably safely select for himself any glasses that allow him to read easily a book about 30 centimeters (12 inches) from the eye. As age advances stronger lenses must usually be obtained. 


\section{GHAPTER XVI}

\section{THE EYE AS A SENSORY APPARATUS}

The Excitation of the Visual Apparatus. The excitable visual apparatus for each eye consists of the retina, the optic nerve, and the brain-centers connected with the latter; however stimulated, if intact, it causes visual sensations. In the great majority of cases its excitant is objective light, and so we refer all stimulations of it to that cause, unless we have special reason to know the contrary. As already pointed out pressure on the eyeball causes a luminous sensation (phosphene), which suggests itself to us as dependent on a luminous body situated in space where such an object must be in order to excite the same part of the retina. Since all rays of light penetrating the eye, except in the line of its long axis, cross that axis, if we press the outer side of the eyeball we get a visual sensation referred to a luminous body on the nasal sicle; if we press below we see the luminous patch above, and so on.

Of course different rays entering the eye take different paths through it, but on general optical principles, which cannot here be detailed, we may trace all oblique rays through the organ by assuming that they meet and leave the optic axis at what are known as the nodal points of the system; these ( $k k^{\prime}$, Fig. 91) lie near together in the lens. If we want to find where rays of light from $A$ will meet the retina (the eye being properly accommodated for seeing an object at that distance) we draw a line from $A$ to $k$ (the first nodal point) and then another, parallel to the first, from $k^{\prime}$ (the second nodal point) to the retina. 'The nodal points of the eye lie so near together that for practical purposes we may treat them as one ( $k$, Fig. 92), placed near the back of the lens. By manifold experience we have learnt that a luminous body ( $A$, Fig. 92) which we see, always lies on the prolongation of the line joining the excited part of the retina, $a$, and the nodal point $k$. Hence any excitation of that part of the retina makes us think of a luminous body somewhere on the line $a A$, and, similarly, any 
excitation of $b$, of a body on the line $b B$ or its prolongation. It is only other conflicting experiences, as that with the eyes closed

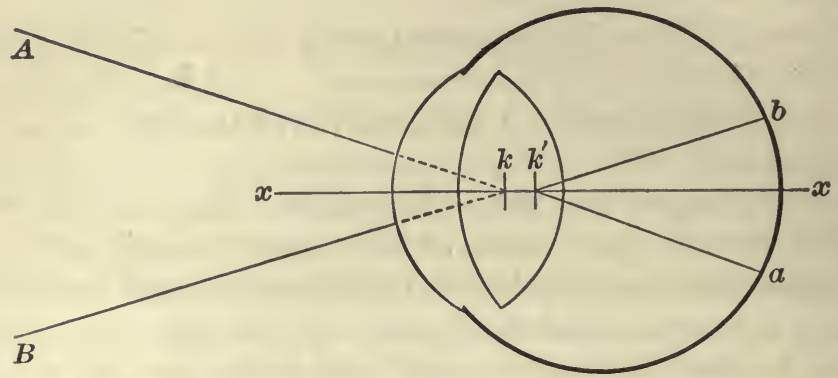

FIG. 91.-Diagram illustrating the points at which incident rays meet the retina. $x x$, optic axis; $k$, first nodal point; $k^{\prime}$, second nodal point; $b$, point where the image of $B$ would be formed, were the eye properly accommodated for it; $a$, the retinal point where the image of $\boldsymbol{A}$ would be formed.

external bodies do not excite visual sensations, and the constant connection of the pressure felt on the eyelid with the visual sen-

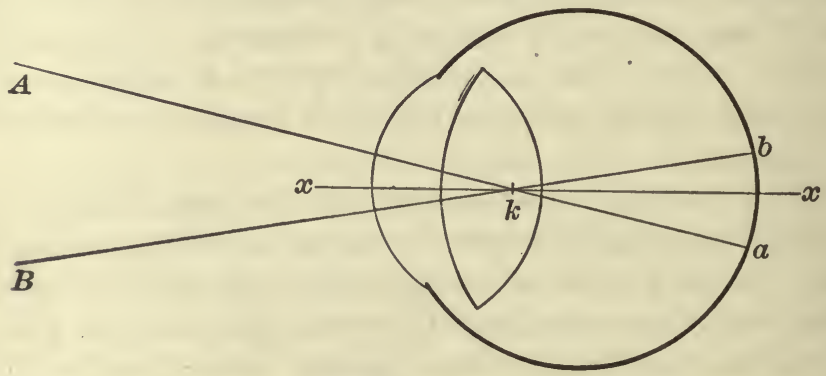

Fig. 92.-Diagrammatic section through the eyeball. $x x$, optic axis; $k$, nodal point.

sation, that enable us when we press the eyeball to conclude that, in spite of what we seem to see, the luminous sensation is not due to objective light from outside the eye.

The Excitation of the Visual Apparatus by Light. Light only excites the retina when it reaches its nerve end organs, the rods and cones. The proofs of this are several.

1. Light does not arouse visual sensations when it falls directly on the fibers of the optic nerve. Where this nerve enters there is a retinal part possessing only nerve-fibers, and this part is blind. Close the left eye and look steadily with the right at the cross in 
Fig. 93, holding the book vertically in front of the face, and moving it to and fro. It will be found that at about 25 centimeters

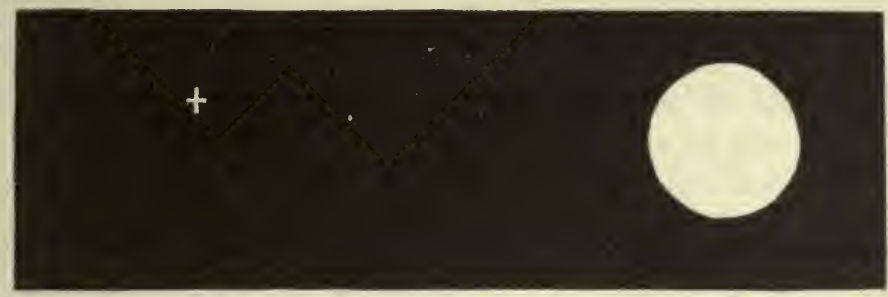

Fig. 93.

(10 inches) off the white circle disappears; but when the page is nearer or farther, it is seen. During the experiment the gaze must be kept fixed on the cross. There is thus in the field of vision a blind spot, and it is easy to show by measurement that it lies where the optic nerve enters.

When the right eye is fixed on the cross, it is so directed that rays from this fall on the fovea ( $y$, Fig. 94). The rays from the circle then cross the visual axis at the nodal point, $n$, and meet the retina at $o$. If the distance of the nodal point of the eye from the paper be $f$, and from the retina (which is $15 \mathrm{~mm}$.) be $F$, then the distance, on the paper, of the cross from the circle will be to the distance of $y$ from $o$ as $f$ is to $F$. Measurements made in this way show that the circle disappears when its image is thrown on the entry of the optic nerve, which lies to the nasal side of the fovea.

2. The above experiment having shown that light does not act directly on the optic nervefibers any more than it does on any other nervefibers, we have next to see in what part of the retina those changes do first occur which form the link between light and nervous impulses.

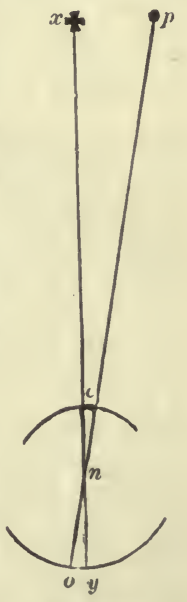

Fig. 94. They occur in the outer part of the retina, in the rods and cones. This is proved by what is called Purkinje's experiment. Take a candle into a dark room and look at a surface not covered with any special pattern, say a whitewashed wall or a plain 
window-shade. Hold the candle to the side of one eye and close to it, but so far back that no light enters the pupil from it; that is, so far back that the flame just cannot be seen, but so that a strong light is thrown on the white of the eye as far back as possible. Then move the candle a little to and fro. The surface looked at will appear luminous with reddish-yellow light, and on it will be seen dark branching lines which are the shadows of the retinal vessels. Now in order that these shadows may be seen the parts on which the light acts must be behind the vessels, that is, in the layers of the retina next the choroid since the bloodvessels lie in its front strata.

If the light be kept steady the vascular shadows soon disappear; in order to continue to see them the candle must be kept moving. The explanation of this fact may readily be made clear by fixing the eyes for ten or fifteen seconds on the dot of an " $i$ " somewhere about the middle of this page: at first the distinction between the slightly luminous black letters and the highly luminous white page is very obvious; in other words, the different sensations arising from the strongly and the feebly excited areas of the retina. But if the glance be not allowed to wander, very soon the letters become indistinct and at last disappear altogether; the whole page looks uniformly grayish. The reason of this is that the powerful stimulation of the retina by the light reflected from the white part of the page soon fatigues the part of the visual apparatus it acts upon; and as this fatigue progresses the stimulus produces less and less effect. The parts of the retina, on the other hand, which receive light only from the black letters are but little stimulated and retain much of their original $\mathrm{cx}$ citability, so that, at last, the feebler excitation acting upon these more irritable parts produces as much sensation as the stronger stimulus acting upon the fatigued parts; and the letters become indistinguishable. To see them continuously we must keep shifting the eyes so that the parts of the visual apparatus are alternately fatigued and rested, and the general irritability of the whole is kept about the same. So, in Purkinje's experiments if the position of the shadows remain the same, the shaded part of the retina soon becomes more irritable than the more excited unshaded parts, and its relative increase of irritability makes up for the less light falling on it, so that the shadows cease to be per- 
ceived. It is for this reason that we do not see the retinal vessels under ordinary circumstances. When light, as usual, eniters the eye from the front through the pupil the shadows always fall on the same parts of the retina, and these parts are thus kept sufficiently more excitable than the rest to make up for the less light reaching them through the vessels.

Further evidence that the rod and cone layer is the true receptor of the eye is furnished by the fact that the seat of most acute vision is the fovea centralis, where only this layer and the conefibers diverging from it are present. When we want to see anything distinctly we always turn our eyes so that its image shall fall on the fovere.

The Intensity of Visual Sensations. Light considered as a form of energy may vary in quantity; physiologically, also, we distinguish quantitative differences in light as degrees of brightness, but the connection between the intensity of the sensation excited and the quantity of energy represented by the stimulating light is not a direct one. In the first place, some rays excite our visual apparatus more powerfully than others: a given amount of energy in the form of yellow light, for example, causes more powerful visual sensations than the same quantity of energy in the form of violet light.

Furthermore, the sense of vision, like all the other senses, obeys the psychophysical law (Chap. XIII). That is, differences of sensation are proportional not to absolute but to relative changes in the amount of stimulating energy. If a room is lighted by one candle and another is brought in we perceive an increase of illumination, but if it is lighted by an arc light the bringing in of a single lighted candle makes no perceptible difference in the illumination. Another illustration of the application of the psychophysical law to the visual sense is found in the fact that the stars which are ordinarily invisible in the daytime, can be seen from the bottom of deep wells or from deep and narrow cañons. The explanation is that in open day the general illumination of the sky is so intense that the additional light of the stars is unperceived. To one in the bottom of a well, however, the general illumination is cut down so much as to bring the additional light from the stars within the limits of perception. The smallest difference in luminous intensity which we can perceive is about 
100 of the whole, for all the range of lights we use in carrying on our ordinary occupations. For strong lights the smallest perceptible fraction is considerably greater; finally we reach a limit where no increase in brightness is felt. For weak illumination the sensation is more nearly proportioned to the total differences of the objective light. Thus in a dark room an object reflecting all the little light that reaches it appears almost twice as bright as one reflecting only half; in a stronger light it would not so appear. Bright objects in general obscurity thus appear unnaturally bright when compared with things about them, and indeed often look self-luminous. A cat's eyes, for example, are said to "shine in the dark"; and painters to produce moonlight effects always make the bright parts of a picture relatively brighter, when compared with things about them, than would be the case if a sunny scene were to be represented; by a relatively excessive use of white pigment they produce the relatively great brightness of those things which are seen at all in the general obscurity of a moonlight landscape.

Function of the Rods. Inasmuch as the rods are absent from the foveæ, they cannot be concerned with ordinary conscious vision since clear vision, as we know from experience, is confined to these areas. It is easy to demonstrate by a simple experiment that the parts of the retina containing rods are more susceptible to feeble lights than is the fovea, which is devoid of them. The constellation of the Pleiades consists of seven stars; one of these is so faint, however, as to be invisible to most eyes when the constellation is looked at directly. If the gaze be turned to a point in the sky a degree or two to one side of the constellation, so as to throw its image off the fovea unto a rod-containing area, the seventh star becomes immediately visible.

This evidence indicates that the function of the rods is some how related to the reception of light stimuli of feeble intensity. The portions of the retina outside the fovea seem to function for the most part more reflexly than consciously; stimuli striking these portions of the retina bring about reflex movements of the eyes and head so that the source of stimulation throws its light upon the fover, and we derive conscious perceptions as to its nature. For such reflex activity a high degree of irritability is desirable. 
It is said that some animals, such as snakes, have no rods in their retinas, while the retinas of others of nocturnal habits, such as owls, consist exclusively of rods.

Visual Purple. If a perfèctly fresh retina be excised rapidly, its outer layers will be found of a rich purple color. In daylight this rapidly bleaches, but in the dark persists even when putrefaction has set in. In pure yellow light it also remains unbleached a long time, but in other lights disappears at different rates. If a rabbit's eye be fixed immovably and exposed so that an image of a window is focussed on the same part of its retina for some time, and then the eye be rapidly excised in the dark and placed in solution of potash alum, a colorless image of the window is found on the retina, surrounded by the visual purple of the rest which is, through the alum, fixed or rendered incapable of change by light. Photographs, or optograms, are thus obtained which differ from the photographer's only in the nature of the chemical substances and processes involved. Both depend on the production of a chemical reaction by light. If the eye be not rapidly excised and put in the alum after its exposure, the optogram will disappear; the vision purple being rapidly regenerated at the bleached part. This reproduction of it is due mainly to the cells of the pigmentary layer of the retina, which in living eyes exposed to light thrust long processes between the rods and cones. Portion of frogs' retinas raised from this, bleach more rapidly than those left in contact with it, but become soon purple again if let fall back upon the pigment-cells.

The visual purple, as stated previously, occurs only in the outer segments of the rods. Whatever function it has is probably connected, therefore, with their special property of reacting to feeble lights. The nature of its function is, further than this, unknown.

The Duration of Luminous Sensations. This is greater than that of the stimulus, a fact taken advantage of in making fireworks: an ascending rocket produces the sensation of a trail of light extending far behind the position of the bright part of the rocket itself at the moment, because the sensation aroused by it in a lower part of its course still persists. So, shooting stars appear to have luminous tails behind them. By rotating rapidly before the eye a disk with alternate white and black sectors we get for each point of the retina on which a part of its image falls, 
alternating stimulation (due to the passage of white sector) and rest (when a black sector is passing). If the rotation be rapid enough the sensation aroused is that of a uniform gray, such as would be produced if the white and black were mixed and spread evenly over the disk. In each revolution the eye gets as much light as if that were the case, and is unable to distinguish that this light is made up of separate portions reaching it at intervals: the stimulation due to each lasts until the next begins and so all are fused together. If, while looking at the flame, one turns out suddenly the gas in a room containing no other light, the image of the flame persists a short time after the flame itself is extinguished.

The Localizing Power of the Retina. As already pointed out a necessary condition of seeing definite objects, as distinguished from the power of recognizing differences of light and darkness, is that all light entering the eye from one point of an object shall be focussed on one point of the retina. This, however, would not be of any use had we not the faculty of distinguishing the stimulation of one part of the retina from that of another part. This

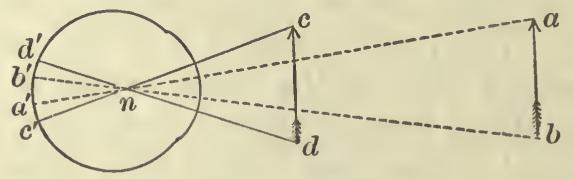

Fig. 95.

power the visual apparatus possesses in a very high degree; while with the skin we cannot distinguish from one, two points touching it less than $1 \mathrm{~mm}$. ( $\frac{1}{25}$ inch) apart, with our eyes we can distinguish two points whose retinal images are not more than $.004 \mathrm{~mm}$. (.00016 inch) apart. The distance between the retinal images of two points is determined by the "visual angle" under which they are seen; this angle is that included between lines drawn from them to the nodal point of the eye. If $a$ and $b$ (Fig. 95) are luminous points, the image of $a$ will be formed at $a^{\prime}$ on the prolongation of the line $a n$ joining $a$ with the node, $n$. Similarly the image of $b$ will be formed at $b^{\prime}$. If $a$ and $b$ still remaining the same distance apart, be moved nearer the eye to $c$ and $d$, then the visual angle under which they are seen will be greater and their retinal images will be farther apart, at $c^{\prime}$ and $d^{\prime}$. If $a$ and $b$ are 
the highest and lowest parts of an object, the distance between their retinal images will then depend, clearly, not only on the size of the object, but on its distance from the eye; to know the discriminating power of the retina' we must therefore measure the visual angle in each case. In the fovea centralis two objects seen under a visual angle of 50 to 70 seconds can be distinguished from one another; this gives for the distance between the retinal images that above mentioned, and corresponds pretty accurately to the diameter of a cone in that part of the retina. We may conclude, therefore, that when two images fall on the same cone or on two contiguous cones they are not discriminated; but that if one or more unstimulated cones intervene between the stimulated, the points may be perceived as distinct. The diameter of a rod or cone, in fact, marks the anatomical limit up to which we can by practice raise our acuteness of visual discrimination; and in the fovea which we constantly use all our lives in looking at things which we want to see distinctly, we have educated the visual apparatus up to about its highest power. Elsewhere on the retina our discriminating power is much less and diminishes as the distance from the fovea increases.

While we can tell the stimulation of an upper part of the retina from a lower, or a right region from a left, it must be borne in mind that we have no direct knowledge of which is upper or lower or right or left in the ocular image. All our visual sensations tell us is that they are aroused at different points, and nothing at all about the actual positions of these on the retina. There is no other eye behind the retina looking at it to see the inversion of the image formed on it. Suppose I am looking at a pane in a secondstory window of a distant house: its image will then fall on the fovea centralis; the line joining this with the pane is called the visual axis. The image of the roof will be formed on a part of the retina below the fovea, and that of the front door above it. I distinguish that the images of all these fall on different parts of the retina in certain relative positions, and have learnt, by the experience of all my life, that when the image of anything arouses the sensation due to excitation of part of the retina below the fovea the object is above my visual axis, and vice versa; similarly with right and left. Consequently I interpret the stimulation of lower retinal regions as meaning high objects, and of right retinal 
regions as meaning left objects, and never get confused by the inverted retinal image about which directly I know nothing. A new-born child, even supposing it could use its muscles perfectly, could not, except by mere chance, reach towards an object which it saw; it would grasp at random, not yet having learnt that to reach an object exciting a part of the retina above the fovea needed movement of the hand towards a position in space below the visual axis; but very soon it learns that things near its brow, that is $u p$, excite certain visual sensations, and objects below its eyes others, and similarly with regard to right and left; in time it learns to interpret retinal stimuli so as to localize accurately the direction, with reference to its eyes, of outer objects, and never thenceforth is puzzled by retinal inversion.

Color Vision. Sunlight reflected from snow gives us a sensation which we call white. The same light sent through a prism and reflected from a white surface excites in us no white sensation but a number of color sensations, gradating insensibly from red to violet, through orange, yellow, green, blue-green, blue, and indigo. The prism separates from one another light-rays of different periods of oscillation and each ray excites in us a colored visual sensation, while all mixed together, as in sunlight, they arouse the entirely different sensation of white. If the light fall on a piece of black velvet we get still another sensation, that of black; in this case the light-rays are so absorbed that but few are reflected to the eye and the visual apparatus is left at rest. Physically black represents nothing: it is a mere zero-the absence of ethereal vibrations; but, in consciousness, it is as definite a sensation as white, red, or any other color. We do not feel blackness or darkness except over the region of the possible visual field of our eyes. In a perfectly dark room we only feel the darkness in front of our eyes, and in the light there is no such sensation associated with the back of our heads or the palms of our hands, though through these we get no visual sensations. It is obvious, therefore, that the sensation of blackness is not due to the mere absence of luminous stimuli, but to the unexcited state of the retinas, which are aione capable of being excited by such stimuli when present. This fact is a very remarkable one, and is not paralleled in any other sense. Physically, complete stillness is to the ear what darkness is to the eye; but silence impresses itself on us as the ab- 
sence of sensation, while darkness causes a definite feeling of "blackness."

Our color sensations insensibly fade into one another; starting with black we can insensibly pass through lighter and lighter shades of gray to white: or beginning with green through darker and darker shades of it to black or through lighter and lighter to white: or beginning with red we can by imperceptible steps pass to orange, from that to yellow and so on to the end of the solar spectrum: and from the violet, through purple and carmine, we may get back again to red. Black and white appear to bo fundamental color sensations mixed up with all the rest: we never imagine a color but as light or dark, that is, as more or less near white or black; and it is found that as the light thrown on any given colored surface weakens, the shade becomes deeper until it passes into black; and if the illumination be increased, the color becomes "lighter" until it passes into white. Of all the colors of the spectrum yellow most easily passes into white with strong illumination. Black and white, with the grays which are mixtures of the two, thus seem to stand apart from all the rest as the fundamental visual sensations, and the others alone are in common parlance named "colors." It has even been suggested that the power of differentiating them in sensation has only lately been acquired by man, and a certain amount of evidence has been adduced from passages in the Iliad to prove that the Greeks in Homer's time confused together colors that are very different to most modern eyes; at any rate there seems to be no doubt that the color sense can be greatly improved by practice; women whose mode of dress causes them to pay more attention to the matter, have, as a general rule, a more acute color sense than men.

Leaving aside black, white, gray, and the various browns (which are only dark tints of other colors), we may enumerate our color sensations as red, orange, yellow, green, blue, violet, and purple; between each there are, however, numerous transition shades, as yellow-green, blue-green, etc., so that the number which shall have definite names given to them is to a large extent arbitrary. Of the above, all but purple are found in the spectrum given when sunlight is separated by a prism into its rays of different refrangibility; rays of a certain wave-length cause in us the feeling red; others yellow, and so on; for convenience we 
may speak of these as red, yellow, blue, etc., rays; all together, in about equal proportions, they arouse the sensation of white.

Peculiarities of Color Vision. A remarkable fact is that most color feelings can be aroused in several ways. White, for example, not only by the above general mixture, but red and bluegreen rays, or orange and blue, or yellow and violet, taken in pairs in certain proportions, and acting simultaneously or in very rapid succession on the same part of the retina, cause the sensation of white: such colors are called complementary to one another. The mixture may be made in several ways; as, for example, by causing the red and blue-green parts of the spectrum to overlap, or by painting red and blue-green sectors on a disk and rotating it rapidly; they cannot be made, however, by mixing pigments, since what happens in such cases is a very complex phenomenon. Painters, for example, are accustomed to produce green by mixing blue and yellow paints, and some may be inclined to ridicule the statement that yellow and blue when mixed give white. When, however, we mix the pigments we do not combine the sensations of the same name, which is the matter in question. Blue paint is blue because it absorbs all the rays of the sunlight except the blue and some of the green; yellow is yellow because it absorbs all but the yellow and some of the green, and when blue and yellow are mixed the blue absorbs all the distinctive part of the yellow and the yellow does the same for the blue; and so only the green is left over to reflect light to the eye, and the mixture has that color. Grass-green has no complementary color in the solar spectrum; but with purple, which is made by mixing red and blue, it gives white. Several other colors taken three together, give also the sensation of white. If then we call the light-rays which arouse in us the sensation red, $a$, those giving us the sensation orange $b$, yellow $c$, and so on, we find that we get the sensation white with $a, b, c, d, e, f$, and $g$ all together; or with $b$ and $e$, or with $c$ and $f$, or with $a, d$, and $e$; our sensation white has no determinate relation to ethereal oscillations of a given period, and the same is true for several other colors; yellow feeling, for example, may be excited by ethereal vibrations of one given wave-length (spectral yellow), or by a light containing only such waves as taken separately cause the sensations red and grass-green; in otner words, a physical light in which there are no waves of the 
"yellow" length may cause in us the sensation yellow, which is only one more instance of the general fact that our sensations, as such, give us no direct information as to the nature of external forces; they are but signs which we have to interpret.

Function of the Cones. These structures, since they are the only sensitive elements of the fovea centralis, must be the receptors for all ordinary conscious vision. Their special function is doubtless the perception of color. This perception is a part of all our conscious visual sensations. We never think of a luminous object as being merely light; but always as having some color.

Distribution of Color Sense over the Retina. By means of an apparatus called the perimeter it is possible to determine the boundaries of visual sensation in the retina. In using this apparatus the subject with head supported in one position looks fixedly at a point straight in front; the observer then brings small squares of paper from the side toward the front and the subject reports the instant the square of paper comes into his field of vision. The angle is marked on a specially prepared chart and the observation repeated along different radii. By this means the field of vision is mapped out. The visual field for any particular color can be determined similarly, the subject in this case being required to report as soon as he is certain what the color of the square of paper is. Such studies have brought out the interesting fact that ability to perceive the different colors is unequally distributed over the retina. The margins of the visual field are sensitive only to white and black, and to their mixtures of gray; the fields for blue and yellow cover the whole area except the margins; the fields for red and green sensation are the smallest of all, occupying only the central part of the field and covering about half its entire surface. According to most determinations the boundaries of the yellow and blue fields do not coincide exactly, nor those of green and red; but it is quite probable that they do coincide exactly in reality, and that experimental errors account for their apparent divergences.

It is clear from these observations that the cones in the central part of the visual field are sensitive to all colors; that those further out are sensitive to all except red and green; and that the marginal ones are insensitive to color as such, and distinguish only degrees of light and darkness. 
Color Blindness. This is a deficiency in color vision whereby certain colors fail to produce the characteristic color sensations that they do in normal eyes. The commonest sort of color blindness is so-called red-green blindness. In it neither red nor green has the same value as in normal eyes. Both colors seem to give the sensation of "neutral" tints, grays and browns. Two varieties of red-green blindness are recognized; the difference between them is, however, apparently one of perception of luminosity rather than of color. To the red-blind person a red object looks dim as well as of neutral tint; to the green-blind person a red object appears to be bright, although in color of neutral tint likewise.

Red-green blindness is the common form. It is usually congenital and occurs more frequently in males than in females. One male in twenty-five, on the average, is color blind, and less than one female in a hundred. It has been suggested that this difference is at bottom one of training; women have from time immemorial used brighter colors and more colors in their clothing than have men, and have therefore become more accustomed to making nice color discriminations.

A form of violet blindness has been described as occurring in rare pathological conditions. It can be brought on temporarily, it is said, by taking the drug santonin. This form of color blindness has not been thoroughly studied. Monochromatic blindness, in which the only sensation is of degrees of grayness, shading at one end into white, at the other into black, is also described. This is accompanied in most cases by blindness of the fovea, and is probably therefore the result of complete loss of cone function.

A full explanation of red-green blindness cannot be had, of course, until the mechanism of color vision is understood. From what was said about the distribution of color perception in the retina it is clear, however, that in all eyes there is an area of redgreen blindness between the area of complete color perception and the area of white-black vision. If we suppose the cones in the central area to be undifferentiated from those of this immediately surrounding zone we have a condition of red-green blindness involving the whole eye and corresponding to that of the color-blind person.

The detection of color blindness is often a matter of considerable importance, especially in sailors and railroad operators since the 
two colors most commonly confounded, red and green, are those used in maritime and railroad signals. Persons attach such different names to colors that a decision as to color blindness cannot be safely arrived at by simply showing a color and asking its name. The best plan is to take a heap of worsted of all tints, select one, say a red, and tell the man to put alongside it all those of the same color, whether of a lighter or a darker shade; if red blind he will select not only the reds but the greens, especially the paler tints, as well as the grays and browns. This test, which is almost universally used, was devised by the Swedish physiologist, Holmgren.

After-Images and Contrasts. These are well-marked visual phenomena, and have to be taken into account in attempting to explain the mechanism of color vision. After-images are visual sensations which remain after the withdrawal of the stimulus. They are best seen after looking at bright objects, or fixedly for several seconds at the same object. After-images are of two sorts, positive and negative. Positive after-images are always the same color as the object looked at; if one looks for an instant at an incandescent filament and then shuts his eyes he perceives a positive after image of the filament. This is due, probably, to the persistence of the chemical process in the retina after the light which causes it is withdrawn. Negative after-images, instead of being the color of the object looked at, are always of its complimentary color; if a red paper is looked at fixedly for several seconds and the eyes then turned to a white wall, a bluish green after-image is seen, instead of a red one. Negative after-images can also be seen by closing the eyes after looking fixedly at a bright object for some seconds.

Contrasts are effects produced by bringing side by side different colors; blue appears bluer when near yellow than when near other shades or the same shade of blue. Red and green heighten each other in a similar way. If a large black square and a large white square are placed side by side the black square looks blacker on the edge next the white than elsewhere, and the white looks whiter next the black than elsewhere.

Theories of Color Vision. A theory of color vision to be acceptable must explain first the fundamental facts of color perception, our ability to distinguish innumerable shades of color, 
and the fact that pairs or groups of fused colors give rise to sensations entirely unrelated to any of the constituent colors. The theory must account for the distribution of color perception over the retina and for the facts of color blindness; it must also explain after-images and contrasts. The fact that black, the absence of stimulation, has all the subjective qualities of a true sensation is also to be explained in some way. No theory yet proposed is satisfactory in accounting for all the known facts. Each one lays special emphasis on some group of visual phenomena and disregards such facts as cannot be harmonized with it. Three interesting theories will be briefly summarized for the sake of showing how such a problem is attacked. Each of them assumes that the excitation of the visual nerve endings depends upon the action of light upon certain photochemical substances in the cones.

The Young-Helmholtz Theory. This theory, proposed by Young in 1807 and elaborated by Helmholtz many years later, may be described rather as an attempt to apply the doctrine of specific nerve energies to color vision than as an attempt to explain the facts of color vision as we know them.

It is an interesting illustration of the extent to which this doctrine has come to physiologists to seem fundamental in forming conceptions of the nervous system that the theory of Young, manifestly impossible as it is, because of the numerous facts with which it cannot be harmonized, has received much more attention and consideration than other theories, agreeing with many of the facts as we know them, but not in accord with the doctrine of specific nerve energies.

The theory assumes all our color sensations to be based on three primary ones, red, green, and violet, each of which is aroused by the decomposition of its special photochemical substance, and each having distinct nervous connection with the visual area of the cerebrum. Since anatomical study shows that each cone has a single nerve-fiber leading from it we must either suppose that there are three sorts of cones, one red-perceiving, one greenperceiving, and one violet-perceiving, and that these are scattered in groups of three over the retina; or we must conclude that the nerve-fiber is not the unit of nervous conduction but that it is made up of smaller units in the same way that the nerve-trunk is made up of fibers. The originators of the theory held the first 
of these views; they assumed that any method of stimulating a red-perceiving cone would give rise to red sensations; if red- and green-perceiving cones were stimulated simultaneously the effect in consciousness would be very different from that of stimulating either one alone, the red cone and the green cone together, giving yellow; and if all three sorts were stimulated at once in equal amounts the effect would be a sensation of white. All our color perceptions are supposed to be based on proper combinations of stimuli acting on the groups of three cones. To explain some facts, such as that pure red light as it becomes brighter and brighter approaches and finally becomes white, the theory supposes that no light stimulates only one cone; all three of the group are stimulated by light of any color, and the effect in consciousness depends on which is more strongly stimulated.

It is easy to demonstrate that the color of a spot of light whose retinal image is of such a size as to fall within the boundaries of a single cone can be accurately distinguished. According to the theory white light should seem to be one or the other fundamental color under such circumstances, instead of looking white as it actually does.

When the theory was proposed it was thought that red-blindness and green-blindness were entirely distinct forms of color blindness. The theory fits that idea very well, since it supposes distinct red-perceiving and green-perceiving cones. Now that we know that both red and green blindness are really forms of redgreen blindness in which neither red nor green gives normal color sensations the theory does not agree at all with the facts in this regard. The theory also fails to explain the distribution of color vision over the retina or the fact that black is a true sensation. It explains very well on the basis of fatigue the negative afterimages that one sees when the eyes are turned to a white surface after looking at a colored body; for if one particular set of cones is fatigued by looking steadily at any color, white light coming upon the retina stimulates the unfatigued ones more powerfully than the fatigued ones, and instead of the sensation of white which follows equal stimulation of all cones, the complimentary color to that one which fatigued the cones in the first place is seen. The theory does not explain well the negative after-images seen with closed eyes, nor does it explain the phenomena of contrast. 
The Hering Theory. This theory frankly makes no attempt to accord with the doctrine of specific nerve energies, but seeks rather to explain on a rational basis those visual phenomena which the Young-Helmholtz theory explains poorly or not at all. It is based upon the observation that whereas we recognize certain colors as being combinations of two others, as bluish-green, or reddish-yellow, there are no colors which we recognize as combinations of complementary colors; greenish-red or yellowish-blue do not occur. The existence of these mutually exclusive colors suggested to the author of this theory that there might be two opposing processes going on in the retina, one a process of chemical breaking down or dissimilation; the other a process of building up, or assimilation.

He therefore postulated three photochemical substances, a white-black substance, a yellow-blue substance, and a red-green substance. He supposed that white light falling on the retina breaks down the white-black substance and gives rise to the sensation of white; whenever no white light is falling on the retina this substance is building itself up; this gives rise to the sensation of black. Similarly the sensation of red is the result of breaking down the red-green substance, and green of its assimilation. The white sensation resulting from stimulation of complementary colors is explained as due to neutralization of opposing effects. When red and green light come together into the retina the red-green substance is neither broken down nor built up. Both red and green light have a dissimilatory effect on the white-black substance as do rays of all colors, according to the theory. The only effect, therefore, of the complementary colors, is to produce a sensation of white. Contrast is explained as due to the maintenance of a sort of chemical balance in the retina whereby a breaking down of one of the elements in part of it is accompanied by building up of the same element in neighboring areas. So, if the yellow-blue substance is being broken down in part of the retina by yellow light, and built up in adjoining part by blue light, at the border between them each process is heightened by the near presence of the other.

The theory explains very well, also, the facts of negative afterimages, of color blindness, and of the distribution of color vision in the retina. The chicf criticism that has been offered against it, 
apart from its failure to accord with the doctrine of specific nerve energies, is that its assumption of similar nervous activities resulting from opposing chemical processes is unwarranted by any knowledge that we have of the relation between chemical processes and nervous activities in other parts of the body.

The Franklin Theory is based on the idea that the peculiar distribution of color vision over the retina is significant as suggesting that the more complex color perceptions are evolved from simpler ones. According to this theory the primary photochemical substance is a gray-perceiving substance; white and black representing the ends of the gray color series. This substance is in all the rods, and in the cones of the retinal margin where only gray perception occurs. In the cones of the yellow-blue field, the fundamental gray-perceiving photochemical substance is supposed to be dissociated into two different photochemical substances, one yellow-perceiving, the other blue-perceiving. Since these are products of the gray-perceiving substance when both are stimulated together the effect is the same as when the gray-perceiving substance itself is stimulated, namely, a shade of gray.

In the central cones of the retina a still further decomposition is assumed to have occurred, of the yellow-perceiving substance into red and green-perceiving substances. The central cones, then, contain three photochemical substances, a red-perceiving one, a green-perceiving one, and a blue-perceiving one. Since all are ultimately derived from the gray-perceiving substance their combined stimulation produces gray sensations; simultaneous stimulation of the red and green substances gives the same result as stimulation of their parent substance, that for perceiving yellow.

This theory puts the distribution of color vision in the retina and the phenomenon of color blindness, which it explains as due to failure of dissociation of the yellow-perceiving substance, upon a more rational basis than do either of the other theories described. In most other respects it offers little advantage over them.

While we must admit that at present a full understanding of color vision is beyond us we may properly look forward to its ultimate mastery, as physiology is able to penetrate more deeply the processes which underly it.

Visual Perceptions. The sensations which light excites in us we interpret as indications of the existence, form, and position of ex- 
ternal objects. The conceptions which we arrive at in this way are known as visual perceptions. The full treatment of perceptions belongs to the domain of Psychology, but Physiology is concerned with the conditions under which they are produced.

The Visual Perception of Distance. With one eye our perception of distance is very imperfect, as illustrated by the common trick of holding a ring suspended by a string in front of a person's face, and telling him to shut one eye and pass a rod from one side through the ring. If a penholder be held erect before one eye, while the other is closed, and an attempt be made to touch it with a finger moved across towards it, an error will nearly always be made. (If the finger be moved straight on towards the pen it will be touched because with one eye we can estimate direction accurately and have only to go on moving the finger in the proper direction till it meets the object.) In such cases we get the only clue from the amount of effort needed to "accommodate" the eye to see the object distinctly. When we use both eyes our perception of distance is much better; when we look at an object with two eyes the visual axes are converged on it, and the nearer the object the greater the convergence. We have a pretty accurate knowledge of the degree of muscular effort required to converge the eyes on all tolerably near points. When objects are farther off, their apparent size, and the modifications of their retinal images brought about by aërial perspective, come in to help. The relative distance of objects is easiest determined by moving the eyes; all stationary objects then appear displaced in the opposite direction (as for example when we look out of the window of a railway car) and those nearest most rapidly; from the different apparent rates of movement we can tell which are farther and nearer. We so inseparably and unconsciously bind up perceptions of distance with the sensations aroused by objects looked at, that we seem to see distance; it seems at first thought as definite a sensation as color. That it is not is shown by cases of persons born blind, who have had sight restored later in life by surgical operations. Such persons have at first no visual perceptions of distance: all objects seem spread out on a flat surface in contact with the eyes, and they only learn gradually to interpret their sensations so as to form judgments about distances, as the rest of us did unconsciously in childhood before we thought about such things. 
The Visual Perception of Size. The dimensions of the retinal image determine primarily the sensations on which conclusions as to size are based; and the larger the visual angle the larger the retinal image; since the visual angle depends on the distance of an object the correct perception of size depends largely upon a correct perception of distance; having formed a judgment, conscious or unconscious, as to that, we conclude as to size from the extent of the retinal region affected. Most people have been surprised now and then to find that what appeared a large bird in the clouds was only a small insect close to the eye; the large apparent size being due to the previous incorrect judgment as to the distance of the object. The presence of an object of tolerably well-known height, as a man, also assists in forming conceptions (by comparison) as to size; artists for this purpose frequently introduce human figures to assist in giving an idea of the size of other objects represented.

The Visual Perception of a Third Dimension of Space. This is very imperfect with one eye; still we can thus arrive at conclusions from the distribution of light and shade on an object, and so that amount of knowledge as to the relative distance of different points which is attainable monocularly; the different visual angles under which objects are seen also assist us in concluding that objects are farther and nearer, and so are not spread out on a plane before the eye, but occupy depth also. Painters depend mainly on devices of these kinds for representing solid bodies, and objects spread over the visual field in the third dimension of space.

Single Vision with Two Eyes. When we look at a flat object with both eyes we get a similar retinal image in each. Under ordinary circumstances we see, however, not two objects but one. In the habitual use of the eyes we move them so that the images of the object looked at fall on the two fover. A point to the left of this object forms its image on the inner (right) side of the left eye and the outer (right) side of the right. An object vertically above that looked at would form an image straight below the fovea of each eye; an object to the left and above, its image to the inner side and below in the left eye and to the outer side and below in the right eye; and so on. We have learned that similar simultaneous excitations of these corresponding points mean single objects, and so interpret our sensations. When the eyes do not work together, as in the muscular incoördination of one stage of 
intoxication, then they are not turned so that images of the same objects fall on corresponding retinal points, and the person sees double. When a squint comes on, as from paralysis of the external rectus of one eye, the sufferer at first sees louble for the same reason, but after a time he makes new associations of corresponding retinal points.

When a given object is looked at, lines drawn from it through the nodal points reach the fovea centralis in each eye. Lines so drawn at the same time from a more distant object diverge less and meet each retina on the inner side of its fovea; but as above pointed out the corresponding points for each retinal region on the inside of the left eye, are on the outside of the right, and vice versa. Hence the more distant object is seen double. So, also, is a nearer object, because the more diverging lines drawn from it through the nodal points lie outside of the fovea in each eye. Most people go through life unobservant of this fact; we only pay attention to what we are looking at, and nearly always this makes its images
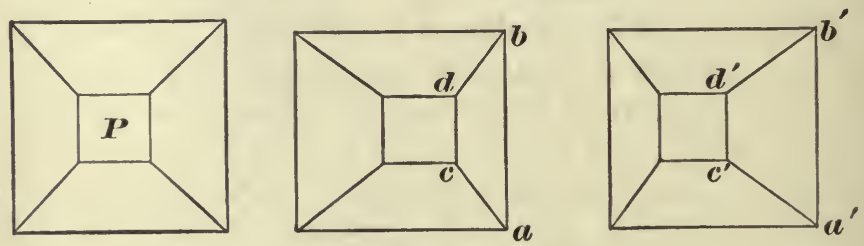

FIG. 96.

on the two fover. That the fact is as above stated may, however, be readily observed. Hold one finger a short way from the face and the other a little farther off; looking at one, observe the other without moving the eyes; it will be seen double. For every given position of the eyes there is a surface in space, all objects on which produce images on corresponding points of the two retinas: this surface is called the horopter for that position of the eyes: all objects in it are seen single; all others in the visual field, double.

The Perception of Solidity. When a solid object is looked at the two retinal images are different. If a truncated pyramid be held in front of one eye its image will be that represented at P, Fig. 96. If, however, it be held midway between the eyes, and looked at with both, then the left-eye image will be that in the middle of the figure, and the right-eye image that to the right. The small sur- 
face, $b d c a$, in one answers to the large surface, $b^{\prime} d^{\prime} c^{\prime} a^{\prime}$, in the other. This may be readily observed by holding a small cube in front of the nose and alternately looking at it with each eye. In such cases, then, the retinal images do not correspond, and yet we combine them in consciousness so as to see one solid object. This is known as stereoscopic vision, and the illusion of the common stereoscope depends on it. Two photographs are taken of the same object from two different points of view, one as it appears when seen from the left, and the other when seen from the right. These are then mounted for the stereoscope so that each is looked at by its proper eye, and the object appears in distinct relief, as if, instead of flat pictures, solid objects, occupying three dimensions of space, were looked at. 


\section{CHAPTER XVII}

\section{THE STRUCTURE AND FUNCTIONS OF BLOOD AND LYMPH}

Introductory. We turn at this point from study of the mechanism by which the Body adapts itself to its surroundings to a consideration of the structures and processes engaged in body maintenance. These have the task of providing the living tissues of the Body with supplies of energy-yielding material and of keeping them in good working order. Their dependence upon the environment is not so obvious perhaps as is that of the adaptive mechanism proper, although as a matter of fact, changes in the environment do influence the maintenance mechanisms, and often very promptly and strikingly. For example, variations in the surrounding temperature bring about adaptive responses in the mechanism for keeping the Body at the proper degree of warmth. We shall have constant occasion, therefore, to recall the facts brought out in preceding chapters.

The External Medium. During the whole of life interchanges of material go on between every living being and the external world; by these exchanges material particles that one time constitute parts of inanimate objects come at another to form part of a living being; and later on these same atoms, after having been a part of a living thing, are passed out from it in the form of lifeless compounds. As the foods and wastes of various organisms differ more or less, so are more or less different environments suited for their existence; and there is accordingly a relationship between the plants and animals living in any one place and the conditions of air, earth, and water prevailing there. Even such simple unicellular animals as the amœbæ live only in water or mud containing in solution certain gases, and in suspension solid food-particles; and they soon die if the water be changed either by essentially altering its gases or by taking out of it the solid food. So in yeast we find a unicellular plant which thrives 
and multiplies only in liquids of certain composition, and which in the absence of organic compounds of carbon in solution will not grow at all. Each of these simple living things, which corresponds to one only of the innumerable cells composing the full-grown Human Body, thus requires for the manifestation of its vital properties the presence of a surrounding medium suited to itself: the yeast would die, or at the best lie dormant, in a liquid containing only the solid organic particles on which the amoba lives; and the amœba would die in such solutions as those in which yeast thrives best.

The Internal Medium. A similar close relationship between the living being and its environment, and an interchange between the two like that which we find in the amoba and the yeast-cell, we find also in even the most complex living beings. When, however, an animal comes to be composed of many cells, some of which are placed far away from the surface of its body and from immediate contact with the environment, there arises a new need-a necessity for an internal medium or plasma which shall play the same part toward the individual cells as the surrounding air, water and food to the whole animal. This internal medium kept in movement and receiving at some regions of the bodily surfaces materials from the exterior, while losing substances to the exterior at the same or other surfaces, forms a sort of middleman between the individual tissues and the surrounding world, and stands in the same relationship to each of the cells of the Body as the water in which an amœba lives does to that animal, or beer-wort does to a yeast-cell. We find accordingly the Human Body pervaded by a liquid plasma, containing gases and foodmaterial in solution, the presence of which is necessary for the maintenance of the life of the tissues. Any great change in this medium will affect injuriously few or many of the groups of cells in the Body, or may even cause their death; just as altering the media in which they live will kill an amœba or a yeast-cell.

In a body so large and complex as that of man, moreover, the internal medium must do more than merely bring food to the individual cells and carry waste materials away from them. All the cells have to be kept at just the right degree of warmth, but some produce more heat than others; so part of its work is to maintain an even distribution of heat over the whole Body or 
when excess is generated to provide for its escape. Many bodily processes, particularly the slower ones, such as growth, are not of a nature to be conveniently controlled by the nervous system. Their control is vested in the hormones with which we are already familiar. These are conveyed by the internal medium to all parts of the Body, being thus sure of reaching the structures upon which their influence is to be exerted. Finally, the environment which is favorable for the life and growth of the body-cells is also favorable for the life and growth of foreign and harmful organisms. That the Body is subject to invasion by such organisms is only too well known, and but for the system of defense which the internal medium affords these invasions could not fail to be even more disastrous than they are.

We can summarize the functions of the internal medium as follows:

1. To convey to all the living cells their needed supplies of food material and oxygen.

2. To convey away from the body-cells the waste materials generated by their activities.

3. To distribute heat uniformly over the Body and provide for getting rid of the excess.

4. To convey from the regions where they are produced to those where they are used the special substances, hormones, which regulate many bodily processes.

5. To defend the Body against the inroads of disease-producing micro-organisms. .

The Blood. In the Human Body the internal medium is primarily furnished by the blood which, as every one knows, is a red liquid very widely distributed over the frame, since it flows from any part when the skin is cut through. There are in fact very few portions of the Body into which the blood is not carried. One of the exceptions is the epidermis or outer layer of the skin: if a cut be made through it only, leaving the deeper skin-layers intact, no blood will flow from the wound. Hairs and nails also contain no blood. In the interior of the Body the epithelial layers lining free surfaces, such as the inside of the alimentary canal, contain no blood, nor do the hard parts of the teeth, the cartilages, and the refracting media of the eye (see Chap. XV), but these interior parts are moistened with liquid of some kind, and unlike 
the epidermis are protected from rapid evaporation. All these bloodless parts together form a group of non-vascular tissues; they alone excepted, a wound of any part of the Body will cause bleeding.

In many of the lower animals there is no need that the liquid representing their blood should be renewed very rapidly in different parts. Their cells live slowly, and so require but little food and produce but little waste. In a sea-anemone, for example, there is no special arrangement to keep the blood moving; it is just pushed about from part to part by the general movements of the body of the animal. But in higher animals, especially warm-blooded ones, such an arrangement, or rather absence of arrangement, as this would not suffice. In them the constituent cells live very fast, making much waste and using much food, and altering the medium in their neighborhood very rapidly. Besides, we have seen that in complex animals certain cells are set apart to get food for the whole organism and certain others to remove its wastes, and there must be a sure and rapid interchange of material between the feeding and excreting tissues and all the others. This can only be brought about by a rapid movement of the blood in a definite course, and that is accomplished by shutting it up in a closed set of tubes, and placing somewhere a pump, which constantly takes in blood from one end of the system of tubes and forces it out again into the other. Sent by this pump, the heart, through all parts of the Body and back to the heart again, the blood gets food from the receptive cells, takes it to the working cells, carries off the waste of these latter to the excreting cells; and so the round goes on.

The Lymph. The blood, however, lies everywhere in closed tubes formed by the vascular system, and does not come into direct contact with any cells of the Body except those which float in it and those which line the interior of the blood-vessels. At one part of its course, however, the vessels through which it passes have extremely thin coats, and through the walls of these capillaries liquid transudes from the blood and bathes the various tissues. The transuded liquid is the lymph, and it is this which forms the immediate nutrient plasma of the tissues except the few which the blood moistens directly.

The Renewal of the Lymph. Osmotic phenomena (p. 18) play 
a great part in the nutritive processes of the Body. The lymph present in any organ gives up things to the cells there and gets things from them; and thus, although it may have originally been tolerably like the liquid part of the blood, it soon acquires a different chemical composition. Diffusion or dialysis then commences between the lymph outside and the blood inside the capillaries, and the latter gives up to the lymph new materials in place of those which it has lost and takes from it the waste products it has received from the tissues. When this blood, altered by exchanges with the lymph, gets again to the neighborhood of the receptive cells, having lost some food-materials it is poorer in these than the richly supplied lymph around those cells, and takes up a supply by dialysis from it. When it reaches the excretory organs it has previously picked up a quantity of waste matters and loses these by dialysis to the lymph there present, which is specially poor in such matters, since the excretory cells constantly deprive it of them. In consequence of the different wants and wastes of various cells, and of the same cells at different times, the lymph must vary considerably in composition in various organs of the Body, and the blood flowing through them will gain or lose different things in different places. But renewing during its circuit in one what it loses in another, its average composition is kept pretty constant, and, through interchange with it, the average composition of the lymph also.

The Lymphatic Vessels. The blood, on the whole, loses more liquid to the lymph through the capillary walls than it receives back the same way. This depends mainly on the fact that the pressure on the blood inside the vessels is greater than that on the lymph outside, and so a certain amount of filtration of liquid from within out occurs through the vascular wall in addition to the dialysis proper. The excess is collected from the various organs of the Body into a set of lymphatic vessels which carry it directly back into some of the larger blood-vessels near where these empty into the heart; in this way the liquid which is forced out of the blood stream in the capillaries gets back into it again.

The Lacteals. In the walls of the alimentary canal certain food-materials after passing through the receptive cells into the lymph are not transferred locally, like the rest, by dialysis into the blood, but are carried off bodily in the lymph-vessels and 
poured into the veins of a distant part of the Body. The lymphatic vessels concerned in this work, being frequently filled with a white liquid during digestion, are called the milky or lacteal vessels.

Summary. To sum up: the blood and lymph form the internal medium in which the tissues of the Body live; the lymph is primarily derived from the blood and forms the immediate plasma for the great majority of the living cells of the Body; and the excess of it is finally returned to the blood. The lymph moves but slowly, but is constantly renovated by the blood, which is kept in rapid movement, and which, besides containing a store of new food-matters for the lymph, carries off the wastes which the various cells have poured into the latter, and thus is also a sort of sewage stream into which the wastes of the whole Body are primarily collected.

Composition of the Blood. The average specific gravity of human blood is 1,055 . It has an alkaline reaction to litmus. About one-third its mass consists of moist corpuscles and the remainder of the liquid part or plasma. Exposed in a vacuum, 100 volumes of blood yield about 60 of gas consisting of a mixture of oxygen, carbon dioxid, and nitrogen.

Microscopic Characters of Blood. If a finger be pricked, and the drop of blood flowing out be spread on a glass slide, covered, protected from evaporation, and examined with a microscope magnifying about 400 diameters, it will be seen to consist of innumerable solid bodies floating in a liquid. The solid bodies are the blood-corpuscles, and the liquid is the blood-plasma.

The corpuscles are not all alike. While currents still exist in the freshly-spread drop of blood, the great majority of them are readily carried to and fro; but a certain number more commonly stick to the glass and remain in one place. The former are the red, the latter the pale or colorless blood-corpuscles. With proper precautions a third sort of corpuscles, the blood-plates, may also be seen.

Red Corpuscles. Form and Size. The red corpuscles as they float about frequently seem to vary in form, but by a little attention it can be made out that this appearance is due to their turning round as they float, and so presenting different aspects to view; just as a silver dollar presents a different outline according as it is looked at from the front or edgewise or in three-quarter profile. 
Sometimes the corpuscle (Fig. 97, B) appears circular; then it is seen in full face; sometimes linear $(C)$, and slightly narrowed in the middle; sometimes oval, as the dollar when halfway between a full and a side view. These appearances show that each red corpuscle is a circular disk, slightly hollowed in the middle (or biconcave) and about four times as wide as it is thick. The average transverse diameter is 0.008 millimeter ( $\frac{1}{3} \frac{1}{20}$ inch). Shortly after blood is drawn the corpuscles tend to arrange themselves in rows, or rouleaux, adhering to one another by their broader surfaces.

Color. Seen singly each red corpuscle is of a pale yellow color; it is only when collected in masses that they appear red. The

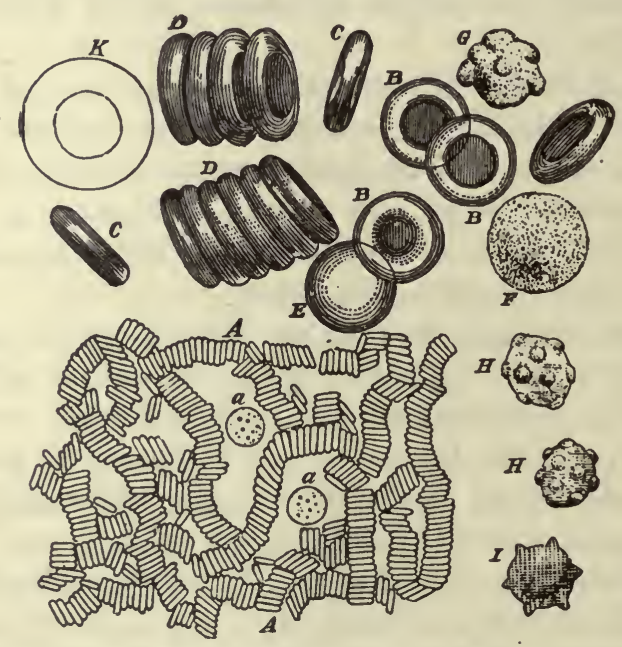

Fig. 97.-Blood-corpuscles. $A$, magnified about 400 diameters. The red corpuscles have arranged themselves in rouleaux; $a, a$, colorless corpuscles; $B$, red corpuseles more magnified and seen in foeus; $E$, a red corpuscle slightly out of focus. Near the right-hand top corner is a red corpuscle seen in three-quarter face, and at $C$ one is seen edgewise. $F, G, H, I$, white corpuscles highly magnified.

blood owes its red color to the great numbers of these bodies in it; if it is spread out in a very thin layer it, too, is yellow.

Structure. There is no satisfactory evidence that these corpuscles have any enveloping sac or cell-wall. All the methods used to bring one into view under the microscope are such as would coagulate the outer layers of the substance composing the corpuscle and so make an artificial envelope. So far as optical 
analysis goes, then, each corpuscle is homogeneous throughout. By other means we can, however, show that at least two materials enter into the structure of each red corpuscle. If the blood be diluted with several times its own bulk of water and examined with the microscope, it will be found that the formerly red corpuscles are now colorless and the plasma colored. The dilution has caused the coloring matter to pass out of the corpuscles and dissolve in the liquid. This coloring constituent of the corpuscle is hemoglobin, and the colorless residue which it leaves behind and which swells up into a sphere in the diluted plasma is the stroma. In the living corpuscle the two are intimately mingled throughout it, and so long as this is the case the blood is opaque; but when the coloring matter dissolves in the plasma, then the blood becomes transparent, or, as it is called, laky. The difference may be very well seen by comparing a thin layer of fresh blood diluted with ten times its volume of ten per cent salt solution with a similar layer of blood diluted with ten volumes of water. The watery mixture is a dark transparent red; the other, in which the coloring matter still lies in the corpuscles, is a brighter opaque red.

Red corpuscles do not possess nuclei; they are not, therefore, living cells in the ordinary sense. Whether they contain any living protoplasm cannot be told certainly. So far as we can judge their activities they are purely mechanical and do not require the participation of living substance.

Consistency. Each red corpuscle is a soft jelly-like mass which can be readily crushed out of shape. Unless the pressure be such as to rupture it, the corpuscle immediately reassumes its proper form when the external force is removed. The corpuscles are, then, highly elastic; they frequently can be seen much dragged out of shape inside the vessels when the circulation of the blood is watched in a living animal (Chap. XX), but immediately springing back to their normal form when they get a chance.

Composition. In the fresh moist state there are in 100 parts of red corpuscles 57 to 64 of water and 36 to 43 of solids. Of the solids nearly ninety per cent is hemoglobin, about one per cent inorganic salts, chiefly phosphates and chlorides of potassium, the residue the proteins and other materials of the stroma.

Number. There is considerable variation in the number of red corpuscles in any given volume of the blood. The average for the 
adult male is stated at 5,000,000 per cubic millimeter $\left({ }_{15,0} \frac{1}{100}\right.$ cubic inch); for the adult female the figure is half a million less. The method of determining this number is to draw from the ear or finger tip an accurately measured volume of blood; this is diluted to a known volume and the number of corpuscles in a known amount of this diluted blood is counted under the microscope. From this the total number in any volume of undiluted blood can easily be calculated.

It must be remembered that the liquid part of the blood is subject to changes of volume, either in the way of increase as liquid is received into it from the digestive tract, or decrease as liquid passes from it into the lymph; therefore a variation in the number of red corpuscles per cubic millimeter does not necessarily mean a corresponding variation in the total number in the Body.

Hemoglobin. This substance, which is a compound of a pigment with a protein (see Chap. I), is the functionally important part of the red corpuscle, the stroma serving merely as a framework upon which it is carried. Its importance lies in the fact that it combines readily with oxygen, forming a loose combination which can easily be broken up, thus it serves to transport oxygen from the lungs to the tissues of the Body (see Respiration). This property seems to be associated with the presence of iron in the pigment part of the hemoglobin molecule.

In the adult male about fourteen parts in the hundred by weight of the blood are hemoglobin. It has been estimated that a man weighing 68 kilograms (150 lbs.) has in his blood 750 grams (1.64 lbs.) of hemoglobin, which is distributed among' some $25,000,000,000,000$ red corpuscles, giving a total superficial area of about 3,200 sq. meters (3,800 sq. yds.) of hemoglobin. On account of the very rapid circulation of the blood (see Circulation) practically the whole of this great area of hemoglobin is poured through the capillaries of the lungs every thirty seconds, so it is apparent that we have here a remarkably efficient arrangement for supplying the Body with oxygen.

There is a pathological condition known as anemia in which there is a considerable reduction in the number of red corpuscles. This is usually accompanied by a diminution in the amount of hemoglobin contained in each corpuscle, so that as a result there is a serious shortage in the hemoglobin content of the Body. Per- 
sons suffering from this condition usually have little or no color, and because the oxygen-carrying mechanism of the Body is below normal there is a loss of bodily strength and endurance. The condition is more common between the ages of twelve and twenty years than at other periods, and in girls than in boys. It is not always easily overcome and should have the care of a physician. An outdoor life and plenty of nourishing food, in which iron containing substances are included, are beneficial in such cases.

Origin and Fate of the Red Corpuscles. Mammalian red corpuscles are cells which have lost their nuclei. In the red marrow of certain bones is the so-called hematopoietic (corpuscleforming) tissue where red corpuscles are constantly being formed. The cells of this corpuscle-forming tissue are continually multiplying by mitotic division (see Chap. II), and the daughter cells thus formed store up within themselves hemoglobin, lose their nuclei, either by disintegration or extrusion, and are cast off into the blood stream. It is not known how rapidly they are formed, nor how long any individual corpuscle remains actively at work in the blood stream; but it is known that sooner or later the red corpuscles become worn out, and disintegrate; the hemoglobin is decomposed in the liver in such fashion as to save the iron, and the residue is converted into the bile pigments and excreted (see Chap. XXXI).

After hemorrhage or as the result. of certain pathological conditions the rate of production of red corpuscles may be much increased. When, this occurs some corpuseles are liberated into the blood stream in an immature condition, and so the blood will be found at such times to contain nucleated as well as non-nucleated red corpuseles.

In the human embryo the labor of making red corpuscles is shared by many of the organs of the Body, notably the liver and spleen.

The Spleen. This large and conspicuous abdominal organ (L, Fig. 134) has presented to physiologists a problem of classification, in that its function has been and still is so obscure as to cause uncertainty under what general heading it should be discussed. The most satisfactory present view assigns it a function in connection with the blood, and it will, therefore, be described here. The spleen consists of an outer coating or sheath of con- 
nective tissue, part fibrous and part elastic, interspersed with smooth muscle-fibers. Projections of the sheath extend into the cavity of the organ, subdividing it into numerous spaces; these are filled with masses of cells which make up the spleen pulp. Numerous blood-corpuscles, both red and white, are found mingled with the cells of the spleen pulp. The spleen has a very rich blood supply which differs from that of all other organs of the Body in that the small arteries instead of communicating with capillaries, which lead in turn into veins, open directly into the spleen pulp. This tissue is bathed, therefore, with blood instead of with lymph as are all other tissues. The spleen pulp is drained by tiny veins which collect the blood into larger ones and so return it to the portal vein (p. 335) whence it passes through the liver and so on into the general circulation.

Function of the Spleen. The peculiarly intimate way in which the spleen cells are brought into relationship with the blood suggests that the organ is specially concerned somehow in maintaining the normal constitution of the blood. Moreover, this concern would appear to be with the formed elements rather than with the plasma, for the delicate membranes which form the capillary walls, and which, in all organs except this, stand between the blood and the tissue cells, oppose no difficulty to the passage of dissolved substances, but only to the passage of corpuscles. The spleen is the only region, then, aside from the red marrow, in which they were formed, that the red corpuscles have direct contact with tissue cells, other than those that form the lining membrane of the bloodvessels. The most satisfactory theory of spleen function that we have is that it picks out from the blood stream and disintegrates those red corpuscles that are "worn out" and no longer able to carry on efficiently their function as oxygen carriers. In support of this theory is the observation that the spleen always shows within its meshes numerous cells that have engulfed red corpuscles and are apparently in the process of disintegrating them. Moreover, in some cases of pernicious anemia, a blood disease in which there is excessive destruction of red corpuscles, virtual cures have been wrought by operative removal of the spleen. The hemoglobin that is set free by the disintegration of the corpuscles is carried to the liver and there decomposed as described above.

The spleen shows rhythmic contractions and relaxations which 
have been thought to aid the circulation of blood through it. It also becomes congested during the period of digestion. Whether this is important or merely incidental is not known.

The Colorless Blood-Corpuscles or Leucocytes (Fig. 97, F, $H, G)$. The colorless or white corpuscles of the blood are far less numerous than the red; in health there is on the average about one white to three hundred red, but the proportion may vary considerably. Each is finely granular and consists of a soft mass of protoplasm enveloped in no definite cell-wall, but containing a nucleus. The granules in the protoplasm commonly hide the nucleus in a fresh corpuscle, but dilute acetic acid dissolves most of them and brings the nucleus into view. These colorless corpuscles belong to the group of undifferentiated tissues, and differ in no important recognizable character from the cells which make up the whole very young Human Body, nor indeed from such a unicellular animal as an Amœba. They have the power of slowly changing their form spontaneously. At one moment a leucocyte will be seen as a spheroidal mass; a few seconds later (Fig. 98) processes will be seen radiating from this, and soon after these processes may be retracted and others thrust out; and so the corpuscle goes on changing its shape. These slow amoboid movements are greatly promoted by keeping the specimen of blood at the temperature of the Body. By thrusting out a process on one side, then drawing the rest of its body up to it, and then sending out a process again on the same side, the corpuscle can slowly change its place and creep across the field of the microscope. Inside the blood-vessels these corpuscles often execute similar movements; and they sometimes bore right through the capillary walls and, getting out into the lymph-spaces, creep about among the other tissues. This

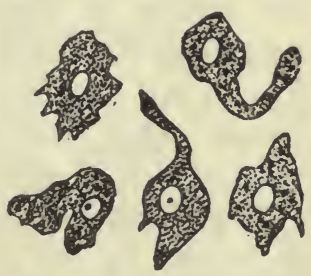

Fig. 98.-A white bloodcorpuscle sketched at successive intervals of a few seconds to illustrate the changes of form due to its amœboid movements. migration is especially frequent in inflamed parts, and the pus or "matter" which collects in abscesses is largely made up of white blood-corpuscles which have in this way got out of the blood-vessels. The average diameter of the white corpuscles is one-third greater than that of the red.

The colorless corpuscles, or some of them, are capable of tak- 
ing into themselves foreign particles present in the blood; this they do in a manner similar to that in which an amoba feeds: the process is known as phagocytosis and the cells exhibiting it as phagocytes. Among the substances observed to be taken up by white corpuscles are the minute organisms known as Bacteria, certain species of which have been proved to be the causes of some diseases. The white corpuscles doubtless in this way play an important part in the cure of such diseases, or in their prevention in persons exposed to infection. The accumulation of white corpuscles in inflamed or injured parts is probably primarily associated with the removal of dead and broken-down tissues, though it may be carried to excess as in the case of purulent accumulations.

The Blood-Plates. These are a third.kind of eorpuscle which remained undiscovered for a long time after the others were known because they break up and disappear.very soon after the blood is shed unless special precautions are taken to preserve them. They are smaller than the red corpuscles; in structure and composition they appear to resemble somewhat the colorless corpuscles, although they do not possess a well-marked nucleus. They are said to exhibit amœboid movements under certain conditions. The only function that is known for them is in connection with the process of blood-clotting (see page 317 ). They are fairly numerous, the blood containing perhaps one-tenth as many platelets as red corpuscles. The promptness with which they disintegrate when exposed to a foreign environment is their most marked characteristic.

The Blood-Plasma. This is a very complex liquid, containing as it does all the varied substances which are associated in the carrying out of the blood's many functions. The plasma is 90 per cent water; of the remaining 10 per cent the chief portion consists of organic compounds, mostly protein, serum albumin, paraglobulin, and fibrinogen; the remainder is sugar, about 0.15 per cent, and fat; the latter constituent varies greatly, being considerable after a meal rich in fats, and slight at other times. About 0.8 of 1 per cent of the plasma consists of sodium, potassium, and calcium salts, the sodium salts constituting by far the greatest part of the inorganic content. The small residue is made up mostly of the waste materials which have been received 
into the blood from the tissues, and which are to be gotten rid of in the excretory organs. The most important of these are urea, ereatinine, uric acid, and similar bodies. The plasma contains also the various hormones, mentioned in a previous paragraph, and a group of substances, known as biological reagents, which are part of the disease-resisting mechanism. These will be considered in detail in later paragraphs.

The plasma carries in solution a certain amount of oxygen, nitrogen, and carbon dioxid, but no more than a similar amount of pure water would dissolve at the same temperature. Most of the oxygen carried by the blood is in combination with the hemoglobin of the red corpuscles; most of the carbon dioxid is in combination with sodium, forming sodium carbonate or bicarbonate.

Summary. : Practically the composition of the blood may be thus stated: It consists of (1) plasma, consisting of watery solutions of serum albumin, paraglobulin, fibrinogen, grape-sugar, sodium and other salts, and extractives of which the most constant are urea, creatinine, and uric acid; (2) red corpuscles, containing rather more than half their weight of water, the remainder being mainly hemoglobin, other proteins, and potash salts; (3) white corpuscles, consisting of water, various proteins, glycogen, and potash salts; (4) the platelets; (5) gases, partly dissolved in the plasma or combined with its sodium salts, and partly combined (oxygen) with the hemoglobin of the red corpuseles.

Quantity of Blood. The total amount of blood in the Body is difficult of accurate determination. It is about $\frac{1}{20}$ of the whole weight of the Body, so the quantity in a man weighing 75 kilos (165 lbs.) is about 3.8 kilos $(8.5 \mathrm{lbs}$.$) . Of this at any given moment$ about one-fourth would be found in the heart, lungs, and larger blood-vessels; and equal quantities in the vessels of the liver, and in those of the muscles which move the skeleton; while the remaining fourth is distributed among the remaining parts of the Body.

Blood of Other Animals. In all animals with blood the white corpuscles are pretty much alike, but the red corpuseles, which with rare exceptions are found only in Vertebrates, vary considerably. In all the classes of the mammalia they are circular biconeave disks, with the exception of the eamel tribe, in which they are oval. They vary in diameter from $0.02 \mathrm{~mm}$. ( $\frac{1}{2} \frac{1}{20}$ inch) (musk deer) to $0.011 \mathrm{~mm}$. ( $\frac{1}{2} 70 \mathrm{inch}$ ) (elephant). In the dog they 
are nearly the same size as those of man. In no mammals do the fully-developed red corpuscles possess a nucleus. In all other vertebrate classes the red corpuscles possess a central nucleus, and are oval slightly biconvex disks, except in a few fishes in which they are circular. They are largest of all in the amphibia. Those of the frog are $0.022 \mathrm{~mm}$. ( ${ }_{1}^{1} 1_{00}^{1}$ inch) long and $0.015 \mathrm{~mm}$. ( ${ }_{16}^{1}{ }_{0}^{1} 0 \mathrm{inch}$ ) broad.

The blood of certain crustaceans contains instead of hemoglobin a substance of similar physiological action, hemocyanin, which is blue instead of red, and contains copper in place of iron.

Histology and Chemistry of Lymph. Pure lymph is a colorless watery-looking liquid; examined with a microscope it is seen to contain numerous white corpuscles closely resembling those of the blood, and no doubt many are leucocytes which have migrated. For the most part, however, these lymph-corpuscles or lymphocytes have another more important origin. In many parts of the Body there are collections of a peculiar lymphoid or adenoid tissue (p. 383), sometimes in nodular masses (lymphatic glands). This tissue consists essentially of a fine network, the meshes of which are occupied with lymphocytes which frequently shows signs of division. The meshes of the network communicate with lymphatic vessels and the lymph flowing through picks up and carries off the new-formed lymphocytes. The function of the lymphocytes is not clear. They are believed not to share in the phagocytic function of the leucocytes.

The lymph flowing from the intestines during digestion is, as already mentioned, not colorless, but white and milky. It will be considered with the process of digestion. During fasting the lymph from the intestines is colorless, like that from other parts of the Body.

Lymph is feebly alkaline, and has a specific gravity of about 1,045. The chief chemical difference between lymph and bloodplasma is that the former contains somewhat more waste materials and less food stuffs than the latter. This is because the consumption of food by the cells and their production of waste keep slightly ahead of the interchange of these substances between blood and lymph by the processes of filtration and dialysis. Lymph contains carbon dioxid in solution but no uncombined oxygen, the latter substance being taken up by the living cells as fast as it enters the lymph from the blood. 


\section{CHAPTER XVIII}

\section{THE HORMONE-CARRYING AND DISEASE-RESISTING FUNCTIONS OF THE BLOOD. BLOOD-CLOTTING}

Hormones. The chemical control of bodily processes by means of hormones has assumed great importance of recent years and is at present the subject of active investigation. For a long time it has been recognized that many processes are subject to hormone influence, but the number of such processes is being constantly added to as our knowledge advances. Although a few of the hormones have been isolated and their chemistry studied, by far the greater number are known only by their physiological effects. Most of the hormones are special substances, formed in organs whose sole function, so far as we can judge, is their production. A few of them exercise their hormone function only incidentally to their chief bodily destiny.

As stated previously the organs whose exclusive function is to secrete hormones are known as ductless glands. In spite of a great amount of investigation in recent years our knowledge of the chemical co-ordination of the Body is still very incomplete and there are some ductless glands concerning whose function we have virtually no information. Among these may be mentioned the parathyroids, small bodies, usually four in number, which are found on or near the thyroids, sometimes embedded within them. That these produce an essential hormone is proven by the fact that their complete removal is followed by acute toxic symptoms, with muscular convulsions, ending in death. Of the normal functioning of the hormone which they produce nothing significant is known.

There are some hormones, which, instead of being elaborated in specific ductless glands, are made by cells embedded in organs whose primary functions have no relation with those of the hormones made within their mass. The special hormone-producing cells in these cases, although anatomically parts of the organs within which they lie, are physiologically as distinct as though they 
were grouped into specific masses, recognizable as independent organs.

Since the hormones whose functions are at all understood are discussed in connection with the bodily processes with which they are associated no further account of them need be given here.

Infection. Bacteriology has taught us that we are continually surrounded by myriads of micro-organisms of various kinds. They are on the skin and mucous membranes; they are breathed in with the air and swallowed in the food and water; colonies of them flourish in the intestinal tracts. Not all of them are disease producing (pathogenic), but these are always present along with the harmless varieties.

Not only are these organisms always present, but small numbers of them frequently find their way into the lymph spaces of the Body, whence they get into the blood. The entry of pathogenic organisms into the Body does not constitute infection. It is only when they gain a foothold and begin to multiply that the infection is established and the disease under way.

It is recognized that the ill effects of an infection are not due to the presence of the organisms merely, but to poisonous substances, or toxins, which they produce as incidents in their vital processes. Some sorts give off this poison to the blood, themselves remaining out of the blood-stream; the diphtheria organism is of this sort. Others retain the toxin within themselves, and it is only when they die and decompose that the poison is liberated.

Resistance to Infection. In order for organisms to attack the Body they have first to get within it. So long as the skin and the lining membranes of the respiratory and digestive tracts are intact the entry of organisms is difficult, if not impossible. A prime feature in the resistance to infection, therefore, is the preservation of the membranes intact. The great danger from an ordinary cold, which in itself is usually a mild infection, is in the damage to the mucous membranes which accompanies it, and which may afford channels of entry to organisms which otherwise would not be able to gain admission. 'In uninjured membranes, then, we have the "first line of defense" against infection.

Even though organisms do succeed in penetrating the membranes infection does not always or even usually follow. If it did infection would be our fate much more frequently than it is. The 
tissues of the Body form, however, excellent culture media; organisms that do establish themselves flourish mightily, at least for a time. It follows, therefore, that ordinarily organisms are forcibly prevented from establishing themselves. This prevention of infection is in part a function of the tissue generally and in part a function of the blood. It must be sharply differentiated from an additional disease-resisting function possessed also by the blood, which is the overcoming of infection after it is once established. In the absence of this latter function every infection would result fatally.

The Infection-Resisting Mechanism. Although, as stated above, the bodily tissues form excellent culture media they do not all yield readily to the attacks of the invading micro-organisms. Some tissues are more susceptible than others, and some kinds of organisms attack certain tissues more readily than they do others. The curious fact has recently been demonstrated that the organism of "blood-poisoning" or septicemia, which often attacks nearly all the tissues of the Body, shows a decided preference for the particular tissue in which it formerly grew. Thus if from an animal killed by the infection some of the organisms found in the kidney be injected into the veins of a second animal, the kidneys of the latter will be first attacked. If the organisms came from the liver they will strike first at the liver.

The tissues of some people are in general more resistant than those of others. It is believed that this resistance is to a certain degree inherited. At any rate the experience of peoples exposed for the first time to particular infections suggests this. Whenever in the history of the world races have been brought into contact with new diseases they have suffered severely therefrom, although in many cases the diseases which wrought the havoc were lightly esteemed by races that had been accustomed to them for generations.

In addition to this general mode of resisting infection, which we may call tissue resistance, there are two sorts of structures in the blood specially devoted to the destruction of invading microorganisms. They work independently but in co-operation. The first of these are the phagocytes previously mentioned (p. 302) which engulf and thus dispose of the invading foreign bodies. The second sort is not made up of formed elements like the phagocytes, but is in solution in the plasma. It attacks and destroys bacteria 
by chemical action. To this substance is given the name alexin. It has been shown to be made up of two other substances. The first of these, the complements, are present in the blood in variable, but considerable, amounts and are actively destructive agents. Their destructive power is limited, however, by the circumstance that they are unable to attack foreign organisms directly, but must first be in combination with the second bodies, known as intermediary or immune bodies, through which they gain the necessary chemical grasp on the cells which are attacked. An important feature of the immune bodies is that each kind can combine with only one sort of foreign cell. Unless immune bodies of the right kind are present, the complements are helpless. The analogy of the yale lock which can be opened only by its own key suggests itself. Clearly the scope of this protective mechanism is limited to the varieties of immune bodies that happen to be present.

Why Does Infection Ever Occur? The establishment of an infection in the face of this elaborate protective mechanism can be explained in one of two ways. Either the mechanism falls off in efficiency, which is the condition present when we say "the resistance of the Body is lowered," or the invading organisms are so virulent that the Body is unable to overcome them. Lowered body resistance may result from a number of conditions; undernutrition, prolonged exposure to extremes of heat or cold, alcoholism, severe local inflammations, all of these may diminish the number of phagocytes or the quantity of alexins, or may lessen their activity. Bacteria may vary from time to time in virulence. It appears that the virulence of most sorts is much increased by a period of growth in a living Body. It is because of this increase of virulence that "exposure" to an infected individual is so often followed by infection. The fact of increased virulence explains also the occurrence of "epidemics."

Recovery from Infection involves two processes: 1 . Destroying and getting rid of the enormous numbers of bacteria which develop during the progress of the disease; 2 . Getting rid of or neutralizing the poison, or toxin, which the bacteria produce and which is the real cause of trouble. The course of every infection is a struggle between the Body on one hand and the micro-organisms on the other. The outcome is recovery or death according as one side or the other proves victorious. 
For destroying and getting rid of the bacteria the Body makes use of the same structures, the complements and phagocytes, that it uses in resisting infection in the first place; but the efficiency of these is enormously increased through the development of special aids to their activity.

Opsonins, Immune Bodies, and Agglutinins. The presence and growth of foreign organisms stimulate the cells of the Body to produce and set free in the blood large numbers of bodies of probably at least three sorts. The first of these, called opsonins, act upon the invading bacteria in such a way as to increase very greatly the "appetite" of the phagocytes for them. It is possible to obtain living phagocytes in salt solution, free from the other elements of blood. If to a slide containing some of these a number of bacteria be added and the whole kept at body temperature, the average number of bacteria ingested by each phagocyte can be determined by actual observation. It is found that if the bacteria, before being placed on the slide, are treated with a liquid containing the proper opsonin, the average ingestion per phagocyte is multiplied many fold. It is necessary, for this effect to be produced, that the opsonin be applied to the bacteria; treatment of the phagocytes with opsonin, with subsequent washing, does not increase at all their tendency to ingest bacteria.

Under the stimulus afforded by the presence of foreign organisms are produced, also, great quantities of the special immune bodies needed to give the complements access to those particular organisms. Thus a defensive agency which if present at all before the infection was only slightly effective becomes the chief reliance of the Body in its struggle to rid itself of the invaders.

In addition to opsonins and immune bodies, the cells under bacterial stimulation produce what is thought to be a third substance, agglutinin, which causes the bacteria to clump together, becoming thus more subject to the action of the phagocytes or complements. The development of these various bodies is the process of immunization.

Antitoxin. Beside the destruction of the invading bacteria it is necessary, before the Body is cured of an infection, that the toxins produced by the rapidly multiplying organisms be neutralized. This neutralization of poison is a simpler process than the destruction of formed elements as carried on by the phago- 
cytes and complements. It is brought about in the Body, however, in much the same way. The foreign toxin stimulates the cells of the Body to produce and pour into the blood an antitoxin which neutralizes the toxin. Antitoxins, like opsonins and immune bodies, are specific for the toxin which stimulated their development.

Immunity. An individual who has gone through an infection, and by the co-operation of the forces described above has overcome and destroyed the invaders with their harmful toxins, retains for a long time afterward in his blood the special opsonins, immune bodies, and antitoxins which were developed therein during the course of the infection. He is, during this time, immune toward that particular disease. The existence of this immunity has been known for centuries; its explanation is the result of the work of the last twenty years.

The duration of immunity varies greatly in different infections. There is every degree from the extremely brief immunity toward common colds, an immunity that apparently terminates with the period of convalescence; to an immunity that is life long as in the case of yellow fever.

Carriers. A fact of interest, as well as of great moment in the problem of caring for the public health, is that an occasional infected individual, instead of destroying the invading organisms, becomes so adapted to them that he continues in perfect health with his Body swarming with pathogenic organisms. Such a person is known as a carrier. He is a constant source of danger, since the organisms he carries, and by which he is unaffected, are liable to be transferred to some susceptible individual and cause severe illness or even a widespread epidemic.

The Use of Antitoxin in Disease. In some diseases, of which diphtheria is the best known example, the bacteria do not spread through the Body but take up their abode on a convenient surface where they develop and whence they discharge their toxin into the blood. Successful combating of such diseases requires only that the toxin be neutralized. In course of time the bacteria will reach the end of their development and die.

The antitoxin for any particular kind of toxin will neutralize it whether produced in the body which is infected or in some other body from which it is transferred to the infected one. This 
fact has made possible the development of the well-known antitoxin treatment. Animals, usually horses, receive doses of toxin obtained by growing the bacteria on culture media in proper vessels. These doses are small at first, but are gradually increased as the animal acquires immunity. In course of time the blood of an animal so treated contains large quantities of antitoxin. Considerable amounts of blood can be withdrawn from animals the size of horses without their suffering the slightest inconvenience. It is thus possible to obtain abundant supplies of antitoxin.

The methods of purifying antitoxin-containing solutions are so perfect at the present time that no one should feel the least hesitation at the prospect of its use. The percentage of deaths from diphtheria has fallen from more than fifty to about two since its introduction.

Protective Inoculation. It has been found practicable in some diseases, notably smallpox, to develop immunity by infecting the Body with an organism which is not virulent enough to endanger life but which produces immune substances that protect the Body against the more virulent infection. On account of the specific character of immunity this method can only be used where virtually the same organism occurs in virulent and non-virulent forms.

The most hopeful path of progress at present toward the mastery of disease is along the lines here indicated. We may look forward confidently to a time when most if not all the acute infections will be brought under medical control through application of the principles of immunity.

Anaphylaxis. Although in our discussion of immunity thus far emphasis has been laid on it as a means of destroying disease germs and their toxins, the fact is that the immunity reaction, considered as a reaction, may manifest itself toward foreign protein substances in general, whether they have any relation to disease or not. Thus it is possible by injection of egg-white into the blood to cause the Body to develop immunity toward that substance.

In the development of immunity toward toxins of disease the Body is under the influence of the toxin more or less continuously for a time, and this continuous influence seems essential to the normal progress of the immunity reaction. If, instead of such continuous influence the Body receives a single dose of foreign 
protein which is not repeated, there may appear a marked increase of sensitiveness toward the immunizing substance, so that although it may not have had any noteworthy effect on the Body formerly, after this sensitization has occurred injection of the protein may cause violent or even fatal disturbances. This reversal of the ordinary course of immunization is called anaphylaxis. The tissues which are most markedly affected are the involuntary muscles, and death, when it occurs, is the result of cardiac or bronchial spasms, or other smooth muscle involvements.

Anaphylaxis is of practical importance because the administration of antitoxin involves the introduction of foreign proteins into the system, and if sensitization should take place, a second dose would have serious, or even fatal consequences. The serum (see next paragraph) of horses forms the basis of diphtheria antitoxin. Sometimes persons who are much about horses develop the condition, apparently from inhaling the effluvium from the animals. Such persons cannot endure injections of antitoxin. They often suffer disagreeable bronchial disturbances from the mere presence of horses. Hay fever is a similar sensitization toward the proteins contained in the pollen grains of plants.

The Coagulation of Blood. When blood is first drawn from the living Body it is perfectly liquid, flowing in any direction as readily as water. This condition is, however, only temporary; in a few minutes the blood becomes viscid and sticky, and the viscidity becomes more and more marked until, after the lapse of five or six minutes, the whole mass sets into a jelly which adheres to the vessel containing it, so that this may be inverted without any blood whatever being spilled. This stage is known as that of gelatinization and is also not permanent. In a few minutes the top of the jelly-like mass will be seen to be hollowed or "cupped" and in the concavity will be seen a small quantity of nearly colorless liquid, the blood-serum. The jelly next shrinks so as to pull itself loose from the sides and bottom of the vessel containing it, and as it shrinks squeezes out more and more serum. Ultimately we get a solid clot, colored red and smaller in size than the vessel in which the blood coagulated though retaining its form, floating in a quantity of pale yellow serum. If, however, the blood be not allowed to coagulate in perfect rest, a certain number of red corpuscles will be rubbed out of the clot into the 
seirum and the latter will be more or less reddish. The longer the clot is kept the more serum will be obtained: if the first quantity exuded be decanted off and the clot put aside and protected from evaporation, it will in a short time be found to have shrunk to a smaller size and to have pressed out more serum; and this goes on until putrefactive changes commence.

Cause of Coagulation. If a drop of fresh-drawn blood be spread out very thin and watched for a few minutes with a microscope magnifying 600 or 700 diameters, it will be seen that the coagulation is due to the separation of very fine solid threads which run in every direction through the plasma and form a close network entangling all the corpuscles. These threads are composed of the protein substance fibrin. When they first form, the whole drop is much like a sponge soaked full of water (represented by the serum) and having solid bodies (the corpuscles) in its cavities. After the fibrin threads have been formed they tend to shorten; hence when blood clots in mass in a vessel, the fibrinous network tends to shrink in every direction just as a network formed of stretched india-rubber bands would, and this shrinkage is greater the longer the clotted blood is kept. At first the threads stick too firmly to the bottom and sides of the vessel to be pulled away, and thus the first sign of the contraction of the fibrin is seen in the cupping of the surface of the gelatinized blood where the threads have no solid attachment, and there the contracting mass presses out from its meshes the first drops of serum. Finally the contraction of the fibrin overcomes its adhesion to the vessel and the clot pulls itself loose on all sides, pressing out more and more serum, in which it ultimately floats. The great majority of the red corpuscles are held back in the meshes of the fibrin, but a good many leucocytes, by their amœboid movements, work their way out and get into the serum.

Whipped Blood. The essential point in coagulation being the formation of fibrin in the plasma, and blood only forming a certain amount of fibrin, if this be removed as fast as it forms the remaining blood will not clot. The fibrin may be separated by what is known as "whipping" the blood. For this purpose freshdrawn blood is stirred up vigorously with a bunch of twigs, and to these the sticky fibrin threads as they form, adhere. If the twigs be withdrawn after a few minutes a quantity of stringy 
material will be found attached to them. This is at first colored red by adhering blood-corpuscles: but by washing in water they may be removed, and the pure fibrin thus obtained is perfectly white and in the form of highly elastic threads. It is insoluble in water and in dilute acids, but swells up to a transparent jelly in the latter. The "whipped" or "defibrinated blood" from which the fibrin has been in this way removed, looks just like ordinary blood, but has lost the power of coagulating spontaneously.

The Buffy Coat. That the red corpuscles are not an essential part of the clot, but are merely mechanically caught up in it, seems clear from the microscopic observation of the process of coagulation; and from the fact that perfectly formed fibrin can be obtained free from corpuscles by whipping the blood and washing the threads which adhere to the twigs. Under certain conditions, moreover, one gets a naturally formed clot containing no red corpuscles in one part of it. The corpuscles of human blood are a little heavier, bulk for bulk, than the plasma in which they float; hence, when the blood is drawn and left at rest they sink slowly in it; and if for any reason clotting take place more slowly or the corpuscles sink more rapidly than usual, a colorlesis top stratum of plasma, with no red corpuscles in it, is left before gelatinization occurs and stops the further sinking of the corpuscles. The uppermost-part of the clot formed under such circumstances is colorless or pale yellow, and is known as the buffy coat; it is especially apt to be formed in the blood drawn from

7 febrile patients, and was therefore a point to which physicians paid much attention in the olden times when blood-letting was thought to be almost a panacea. In horse's blood the difference between the specific gravity of the corpuscles and that of the plasma is greater than in human blood, and horse's blood also coagulates more slowly, so that its clot has nearly always a buffy coat. The colorless buffy coat seen sometimes on the top of the clot must, however, not be confounded with another phenomenon. When a blood-clot is left floating exposed to the air its top becomes bright scarlet, while the part immersed in the serum has a dark purple-red color. The brightness of the top layer is due to the action of the oxygen of the air, which forms a scarlet compound with the coloring matter of the red corpuscles. If the clot be turned upside down and left for a short time, the pre- 
viously dark red bottom layer, now exposed to the air, becomes bright.

Uses of Coagulation. The clotting of the blood is so important a process that its cause has been frequently investigated; but it is not yet completely understood. The living circulating blood in the healthy blood-vessels does not clot; it contains no solid fibrin, but this forms in it, sooner or later, when the blood gets by any means out of the vessels or when the lining of these is injured. In this way the mouths of the small vessels opened in a cut are clogged up, and the bleeding, which would otherwise go on indefinitely, is stopped. So, too, when a surgeon ties up an artery before dividing it, the tight ligature crushes or tears its delicate inner surface, and the blood clots where that is injured, and from there a coagulum is formed reaching up to the next highest branch of the vessel. This becomes more and more solid, and by the time the ligature is removed has formed a firm plug in the cut end of the artery, which greatly diminishes the risk of bleeding.

The Source of Blood-Fibrin. Since fresh blood-plasma contains no fibrin but does contain considerable quantities of other proteins, we look first to these as a possible source of the fibrin formed during coagulation. If horse's blood be drawn directly from the living animal into a cold vessel and kept just above freezing temperature it does not clot and after a time the corpuscles settle to the bottom leaving a supernatant portion of clear plasma. This plasma retains the power of clotting, as is shown when it is warmed; but if before it clots it be saturated with sodium chlorid and filtered, the liquid that remains will no longer clot. The precipitate formed by the saturation with sodium chlorid must contain, therefore, some essential in the process of clotting. This precipitate if examined will be found to be a mixture containing all the fibrinogen of the plasma and part of the globulin. These two substances may be separated by proper treatment, and after this has been done it is found that a solution of the fibrinogen can be made to clot, while one containing only paraglobulin cannot. During the clotting of the fibrinogen solution the fibrinogen disappears, giving place to fibrin.

We are thus led to the conclusion that the natural clotting of fresh blood is due to the formation of fibrin from fibrinogen which existed in solution in the plasma of the circulating blood and has 
been altered in the clotted, giving origin to fibrin. But as normal blood circulating in healthy uninjured blood-vessels does not clot nor do pure solutions of fibrinogen, we have still to seek the exciting cause of the change.

If to a solution of fibrinogen there be added a few drops of blood or of blood-serum, or of the washings of a blood-clot, fibrin will be formed; therefore drawn blood and serum and natural clot each contain something which can effect the conversion of fibrinogen into fibrin. This substance is thrombin, frequently called also the fibrin ferment.

Thrombin. When blood-serum is treated with several times its volume of strong alcohol its various proteins and most of its salts are precipitated: if the precipitate be left standing in alcohol for some days the proteins become almost entirely insoluble in water, but a few drops of the watery extract cause clotting in a saline solution of fibrinogen, and clearly contain some of the thrombin. This substance was for a long time believed to be an enzym, hence its name of "fibrin ferment." Recent careful study shows, however, that it does not correspond to enzyms in either of their two cardinal characteristics, namely, the ability of a small amount of the substance to produce a very large amount of chemical activity, and the destruction of the substance by heating above $60^{\circ} \mathrm{C}$. It has been definitely proven that the amount of fibrinogen that is converted to fibrin bears a direct relationship to the amount of thrombin present, and that thrombin solutions free from protein impurities can be boiled without destroying the thrombin.

Source of Thrombin. If fresh blood is drawn directly from the veins of an animal into strong alcohol, and the resulting precipitate treated as described above for preparing thrombin from serum, it yields no thrombin; this substance, therefore, which is present in blood-serum, is absent from the blood within the Body and must be formed after the blood is shed and before the formation of the clot. When the process of clotting is watched under the microscope the fibrin threads will usually be seen to form about certain centers. These centers consist of disintegrating blood-plates, and the observation that fibrin formation proceeds from them in this fashion led to the idea that the blood-plates are in some way associated with the process. 
The natural conclusion drawn from this observation was that the blood-plates contain thrombin which is inactive so long as they are intact, and is liberated by their disintegration. This simple conclusion was upset by the further observation that fresh blood drawn into a solution of sodium oxalate will not clot. Sodium oxalate does not hinder the process of blood-plate disintegration. In fact its sole effect upon blood, so far as can be determined, is to precipitate out its calcium, as calcium oxalate. That the prevention of clotting is due to this precipitation of calcium is shown by the fact that addition of excess of a soluble calcium salt to "oxalate" blood causes it to clot with great promptness. The formation of active thrombin is dependent, then, upon the presence of calcium in the blood, and the substance contained in the blood-plates is not true thrombin, but a preparatory substance which we may call prothrombin.

Antithrombin. A feature of the coagulation process that presents some difficulty is that although the circulating blood contains all the essential factors of the process, clotting does not occur in it so long as it circulates normally, but only when it escapes from the vessels or when the lining of these is injured. To say that the prothrombin is stored in the platelets and so kept from combining with calcium to form thrombin seems an insufficient protection against the possible accident of a decomposition of platelets in the blood-stream.

Definite evidence has been obtained of the existence of a substance which will prevent clotting. This substance is present in the salivary glands of leeches, and serves to keep the blood which they ingest liquid in their stomachs. To it has been given the name antithrombin. Snake venom contains similar material. What is believed to be the same substance is produced within the bodies of some animals (dogs) by injecting unpurified peptone solutions into their veins. There is reason to believe that normal blood contains antithrombin in sufficient amounts to prevent clotting within the blood-vessels. If this substance is present in the blood it must be neutralized when the blood is shed to allow coagulation to proceed.

Thromboplastic substance. An observation that throws light on the manner in which antithrombin is neutralized when blood is shed is that if the blood is drawn from a vessel directly into a 
glass tube with care to avoid contamination from the wound clotting takes place very slowly, or in birds and reptiles may not occur at all. The deduction is that the tissues over which the blood flows in ordinary hemorrhage contain something that neutralizes the antithrombin. For this the name thromboplastic substance has been suggested. The leucocytes, and probably also the platelets, of mammalian blood contain enough of this substance so that their disintegration will neutralize the antithrombin and allow clotting to occur even though the blood may not have come at all into contact with the tissues. In ordinary bleeding, however, the escaping blood must flow directly over the raw tissue surfaces so that the antithrombin is promptly neutralized and clotting can proceed at once. Apparently all tissues contain thromboplastic substance except those that form the lining membranes of the blood-vessels. This rather cumbersome mechanism appears to be necessary to insure prompt clotting when the blood-vessels are ruptured and at the same time immunity from the disaster of clot-formation within the circulation.

The formation of blood-clots (thrombi) within the vessels is likely to be followed by serious effects, due to the plugging of important vessels by the clotted blood, but the occurrence of thrombi in the intact healthy circulation is unknown; their formation presupposes some injury to the walls of the blood-vessels, as by crushing them or tying ligatures about them.

Summary of the Process of Coagulation. We may picture the entire process of blood-clotting somewhat as follows:

1. As the result of rupture of the blood-vessels and contact of the blood with raw tissue surfaces the antithrombin is neutralized by thromboplastic substance and the blood-plates disintegrate, yielding prothrombin.

2. The prothrombin thus set free reacts with the calcium of the blood and forms thrombin.

3. By a reaction between thrombin and fibrinogen insoluble fibrin is precipitated in the form of a sticky network.

4. The fibrin network entangles corpuscles within it, forming a typical clot.

Methods of Hastening or Retarding Coagulation. Since the process of clotting is in several steps there are a corresponding number of points at which its normal course may be broken into, 
either with the effect of hastening the result or of retarding it or even preventing it altogether. Anything which quickens the disintegration of the blood-plates, as the application of a handkerchief to a wound, which acts by increasing the foreign surface in contact with the blood, makes the blood clot more quickly. The application of heat has this same effect; probably it acts both by increasing the rate of destruction of blood-plates and by hastening the chemical reactions involved in the process as a whole. Cold, as would be expected, has the converse effect. An increase in the calcium content of the blood shortens the coagulation time. Coagulation may be retarded, as we have seen, by cold or by depriving the blood of its calcium content. Blood drawn into a strong solution of sodium or magnesium sulphate and well mixed will not clot, these salts appearing to interfere in some way with the formation of the thrombin; such "salted" blood will clot if thrombin is added or if diluted sufficiently with water.

An interesting fact, recently established, is that an increase in the amount of adrenin (p. 199) in the blood hastens its coagulation. This result cannot be secured by adding the hormone to the blood as it is drawn, but only by introducing it into the circulation; showing that the quickening of the clotting process is not a direct result of the chemical action of adrenin on the blood, but is brought about indirectly through the influence of the adrenin on some of the tissues through which the blood circulates. This property of adrenin is looked upon as a phase of its general function as an emergency hormone, for in time of stress and possible bodily injury prompt coagulation of the blood would tend to stop a hemorrhage quickly and so conserve the precious liquid.

"Bleeders." There is a pathological condition, fortunately not very common, known as hemophilia, in which the blood will not clot. Persons suffering from this disease are called bleeders. Such persons are in danger of bleeding to death from slight wounds; a nosebleed, or the bleeding which follows the extraction of a tooth, becomes in such persons an affair of the utmost gravity. Various explanations have been offered to account for this disease; at present it is believed to be due to a deficiency of prothrombin.

This condition is usually hereditary. An interesting fact in connection with it is that whereas the disease itself appears only in males, its transmission seems to be confined wholly to females; 
a father who was a "bleeder" would have no children suffering from the condition nor would his sons, but if his daughters had sons they would probably be bleeders.

Blood Transfusion. The restoration of blood lost in severe hemorrhage, or the replacement of diseased blood by healthy blood through transfusion from the veins of one individual to those of another has long been a dream of physicians and physiologists. The early attempts to treat disease by this method were more often fatal than not because the blood to be introduced into the circulation had to be defibrinated. This process, as we have seen, preserves the blood in a liquid condition, but it leaves in it large quantities of the exciting agent to coagulation, thrombin. When such blood was introduced into the circulation it usually induced prompt clotting of the blood already there, with immediately fatal results. The fuller knowledge of the mechanism of blood-clotting gained of late years has made it clear that blood transfusion need not be followed by clotting if the transfer of blood be made without exposing it at any time to a foreign surface, such as favors the disintegration of the blood-plates. In accordance with this idea an ingenious method has recently been developed whereby an artery of one individual can be brought into communication with a vein of another and the blood allowed to flow naturally across the living channel thus formed. Many lives have been saved by this method during the few years since its first application, and it promises to fulfil in some degree, at least, the early hopes of the medical world. It should be noted that successful blood transfusion requires that the blood to be introduced be taken from an individual of the same species as the one who is to receive it; hence human beings who require blood must receive it from other human beings, and not from animals. One of the most curious facts brought out in connection with the study of the disease-resisting mechanism of the Body is that to this mechanism the red corpuscles of animals of a different species are as much foreign bodies to be attacked and destroyed as are the most malignant bacteria. The introduction of foreign blood, even if not attended by coagulation, is therefore more apt than not to be fatal, through the destruction of each kind of corpuscles by the liquid portion of the other sort of blood. Moreover, the operation is much more likely to prove successful if the donor is a 
near relative of the recipient; since different human strains may behave toward each other as do different species. Fortunately the operation of transfusing blood is neither excessively painful nor accompanied by untoward after effects to the donor, and persons can always be found who are willing to undergo the discomfort involved for the sake of restoring a fellow-being to health. 


\section{CHAPTER XIX}

\section{THE ANATOMY OF THE HEART AND BLOOD-VESSELS}

General Statement. During life the blood is kept flowing with great rapidity through all parts of the Body (except the few nonvascular tissues already mentioned) in definite paths prescribed for it by the heart and blood-vessels. These paths, which under normal circumstances it never leaves, constitute a continuous set of closed tubes (Fig. 99) beginning at and ending again in the heart, and simple only close to that organ. Elsewhere it is greatly branched, the most numerous and finest branches being the capillaries. The heart is essentially a bag with muscular walls, internally divided into four chambers (see figure). Those at one end receive blood from vessels opening into them and known as the veins. From there the blood passes on to the remaining chambers which have very powerful walls and, forcibly contracting, drive the blood out into vessels which communicate with them and are known as the arteries. The big arteries divide into smaller; these into smaller again (Fig. 100) until the branches become too small to be traced by the unaided eye, and these smallest branches end in the capillaries, through which the blood flows and enters the commencements of the veins; and these convey it again to the heart. At certain points in the course of the blood-paths valves are placed, which prevent a back-flow. This alternating reception of blood at one end by the heart and its ejection from the other go on during life steadily about seventy times in a minute, and so keep the liquid constantly in motion.

The vascular system is completely closed except at two points in the neck where lymph-vessels open into the veins; there some lymph is poured in and mixed directly with the blood. Accordingly everything which leaves the blood must do so by passing through the walls of the blood-vessels, and everything which enters it must do the same, except matters conveyed in by the lymph at the points above mentioned. This interchange through the 
walls of the vessels takes place only in the capillaries, which form a sort of irrigation system all through the Body. The heart, arteries, and veins are all merely arrangements for keeping the capillaries full and renewing the blood within them. It is in the capillaries alone that the blood does its physiological work.

The Position of the Heart. The heart ( $h$, Fig. 1$)$ lies in the chest immediately above the diaphragm and opposite the lower two-thirds of the breastbone. It is conical in form with its base or broader end turned upwards and projecting a little on the right of the sternum, while its narrow end or apex, turned downwards, projects to the left of that bone, where it may be felt beating between the cartilages of the fifth and sixth ribs. The position of the organ in the Body is therefore oblique with reference to its long axis. It does not, however, lie on the left side as is so commonly supposed but very nearly in the middle line, with the upper part inclined to the right, and the lower (which may be more easily felt beating -hence the common belief) to the left.

The Membranes of the Heart. The heart does not lie bare in the chest but is surrounded by a loose bag composed of connective tissue and called the pericardium. This

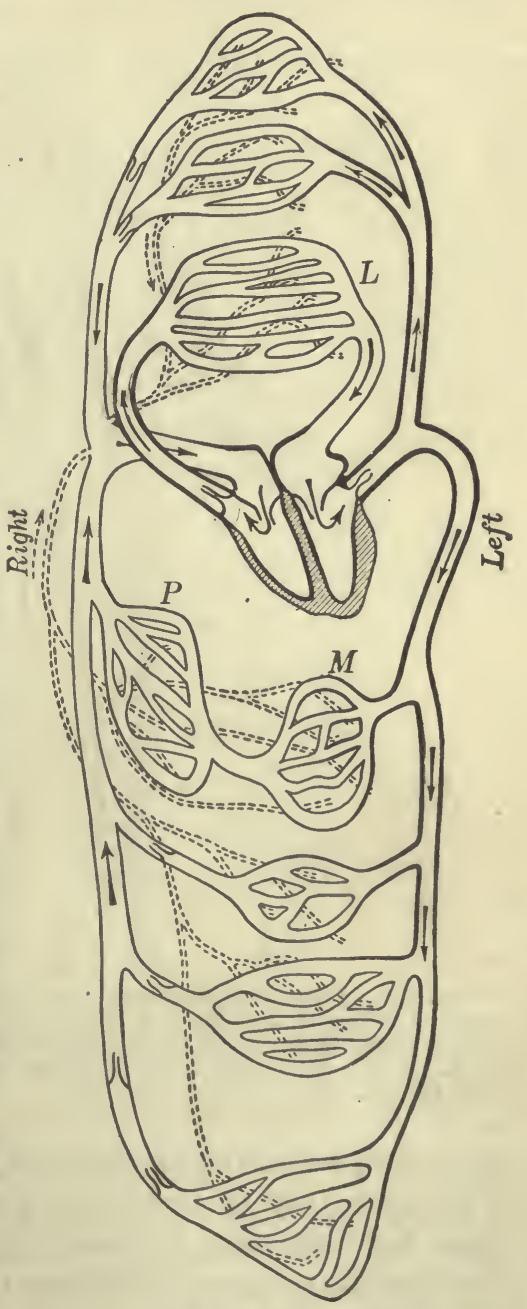

Fig. 99.-The heart and blood-vessels diagrammatieally represented. $L$, lung; $M$, intestine; $P$, liver; dotted lines repre$M$, intestine; $P$, liver;
sent lymphatie vessels. 
but turned the other way, its broad part being lowest and attached to the upper surface of the diaphragm. Internally it is lined by a smooth serous membrane like that lining the abdominal cavity, and a similar layer (the visceral layer of the pericardium)

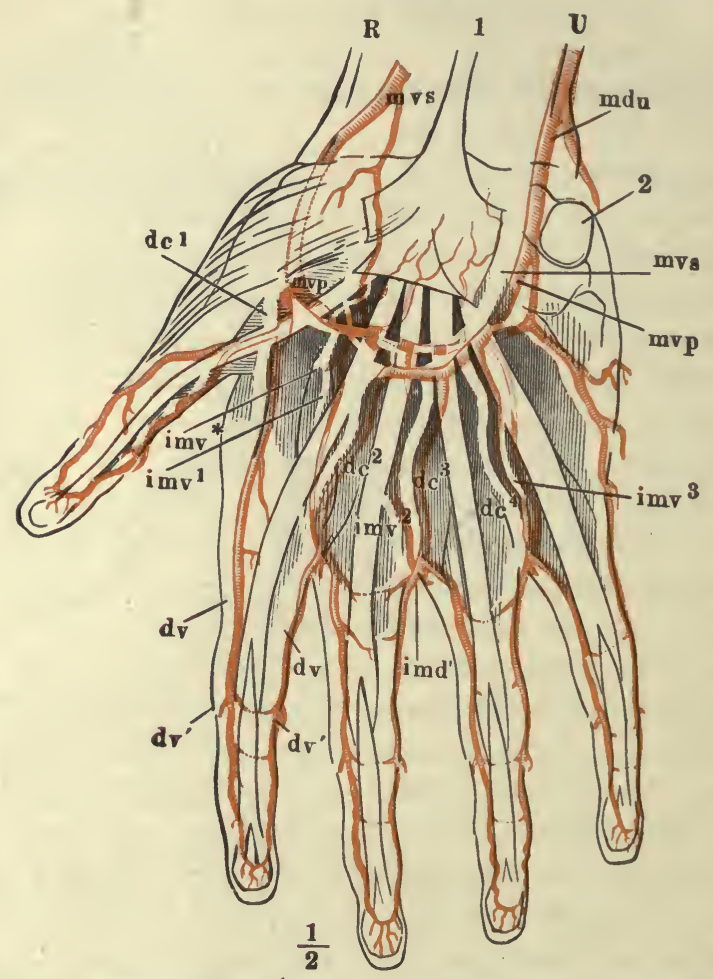

Fig. 100.-The arteries of the hand, showing the communications or anastomoses of different arteries and the fine terminal twigs given off from the larger trunks; these twigs end in the capillaries which would only become visible if magnified. $R$, the radial artery on which the pulse is usually felt at the wrist; $U$, the ulnar artery.

covers the outside of the heart itself, adhering closely to it. Each of the serous layers is covered by a stratum of flat cells, and in the space between them is found a small quantity of liquid which moistens the contiguous surfaces, and diminishes the friction which would otherwise occur during the movements of the heart.

Internally the heart is also lined by a fibrous membrane, covered with a single layer of flattened cells, and called the endocardium. 
Between the endocardium and the visceral layer of the pericardium the bulk of the wall of the heart lies and is made up mainly of the special cardiac muscular tissue previously described (p. 86); but connective tissues, blood-vessels, nerve-cells, and nerve-fibers are also abundant in it.

Note. Sometimes the pericardium becomes inflamed, this affection being known as pericarditis. It is extremely apt to occur in acute rheumatism, and great care should be taken never, even for a moment, except under medical advice, to expose a patient to cold during that disease, since any chill is then especially apt to set up pericarditis. In the earlier stages of pericardiac inflammation the rubbing surfaces on the outside of the heart and the inside of the pericardium become roughened, and their friction produces a sound which can be recognized through the stethoscope. In later stages great quantities of liquid may accumulate in the pericardium so as seriously to impede the heart's beat.

The Cavities of the Heart. On opening the heart (see diagram, Fig. 101) it is found to be subdivided by a longitudinal partition or septum into completely separated right and left halves, the partition running from about the middle of the base to a point a little on the right of the apex. Each of the chambers on the sides of the septum is again incompletely divided transversely, into a thinner basal portion into which veins open, known as the auricle, and a thicker apical portion from which arteries arise, called the ventricle. The heart thus consists of a right auricle and ventricle and a left

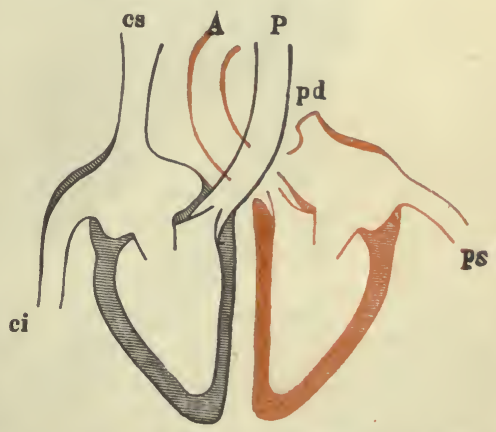

FIG. 101.-Diagram representing a section through the heart from base to apex. auricle and ventricle, each auricle communicating by an auriculoventricular orifice with the ventricle on its own side, and there is no direct communication whatever through the septum between the opposite sides of the heart. To get from one side to the other the blood must leave the heart and pass through a set of capillaries, as may readily be seen by tracing the course of the vessels in Fig. 99. 
The Heart as seen from its Exterior. When the heart is viewed from the side turned towards the sternum (Fig. 102) the two auricles, $A t d$ and $A s$, are seen to be separated by a deep groove from the ventricles, $V d$ and $V s$. A more shallow furrow runs between the ventricles and indicates the position of the internal

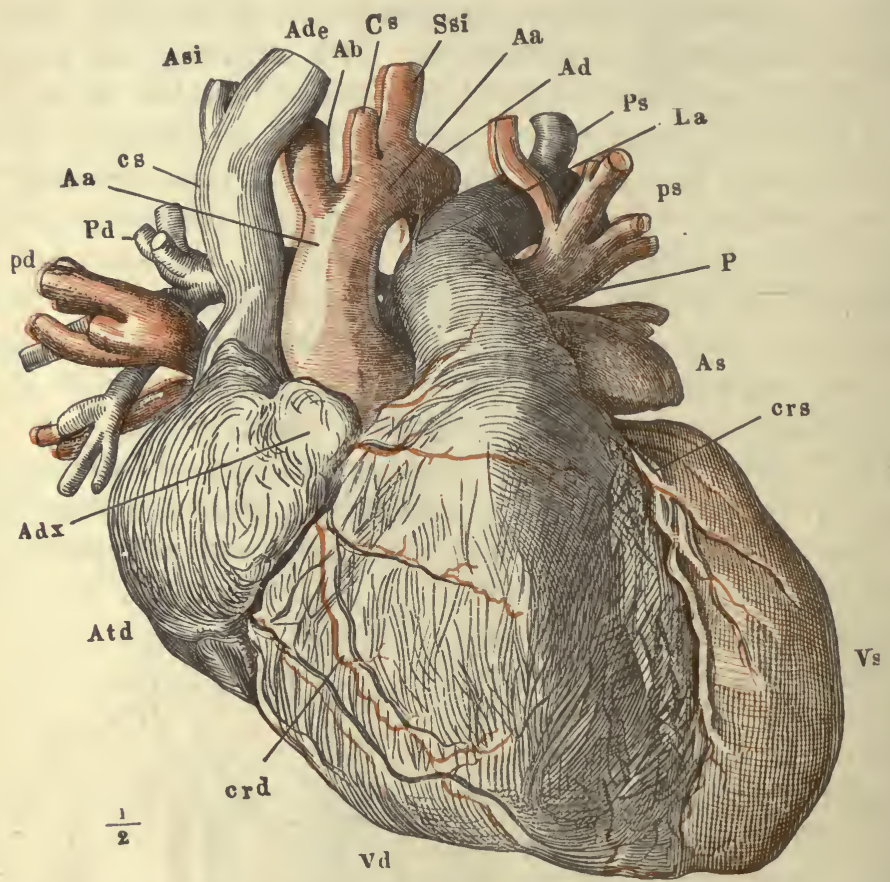

Fig. 102.-The heart and the great blood-vessel attached to it, seen from the side towards the sternum. The left cavities and the vessels connected with them are colored red; the right black. Atd, right auricle; $A d x$ and $A s$, the right and left auricular appendages; $V d$, right ventricle; $V s$, left ventricle; $A a$, aorta; $A b$, innominate artery; $C s$, left common carotid artery; $S$ si, left subclavian artery; $P$, main trunk of the pulmonary artcry, and $P d$ and $P s$, its branches to the right and left lungs; $c s$, superior vena cava; $A d e$ and $A s i$, the right and left innominate veins; $p d$ and $p s$, the right and left pulmonary veins; $c r d$ and $c r s$, the right and left coronary arteries.

longitudinal septum. On the dorsal aspect of the heart (Fig. 103) similar furrows may be noted, and on one or other of the two figures the great vessels opening into the cavities of the heart may be seen. The pulmonary artery, $P$, arises from the right ventricle, and very soon divides into the right and left pulmonary arteries, $P d$ and $P s$, which break up into smaller branches and enter the 
corresponding lungs. Opening into the right auricle are two great veins (see also Fig. 101), cs and ci, known respectively as the upper and lower vence cava, or "hollow" veins; so called by the older anatomists because they are frequently found empty after

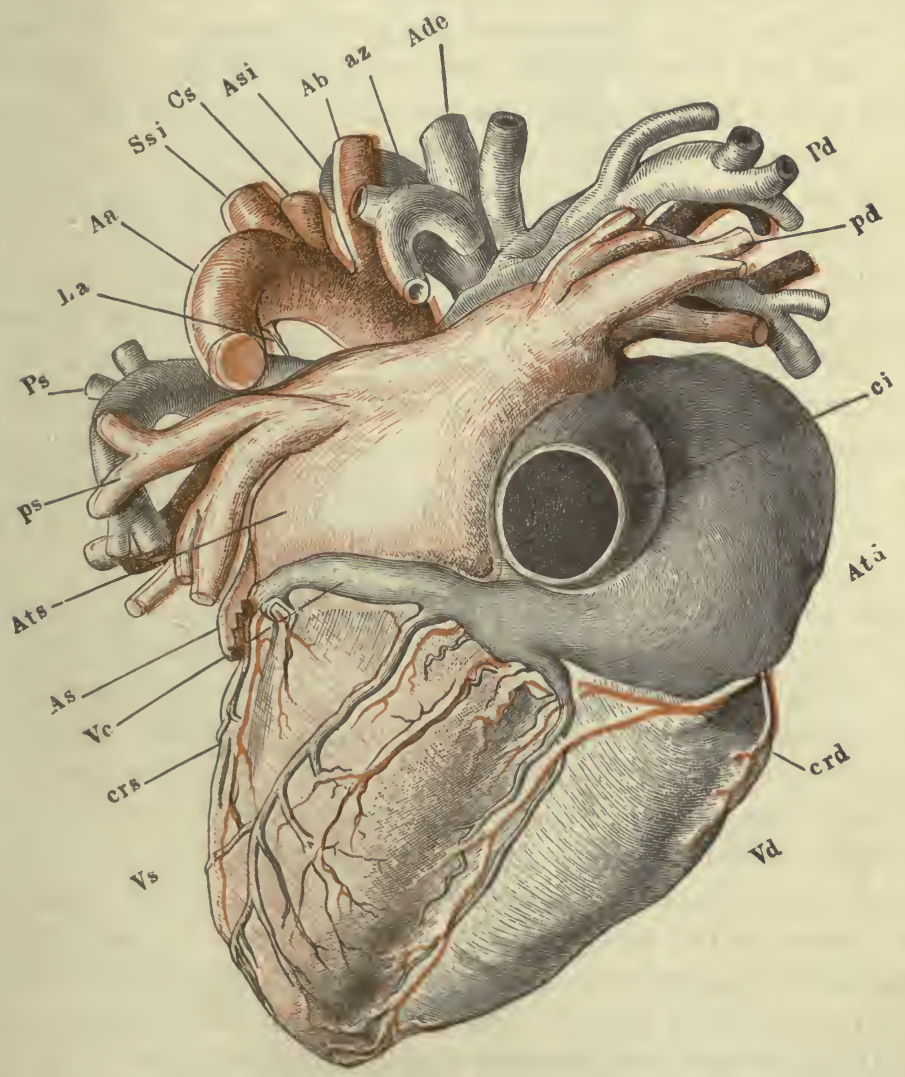

Fig. 103.- The heart viewed from its dorsal aspect. Atd, right auricle; $c$, inferior yena cava; $V c$, coronary vein. The remaining letters of reference have the same signification as in Fig. 102.

death. Into the back of the right auricle opens also another vein, $V c$, ealled the coronary vein or sinus, which brings back blood that has circulated in the walls of the heart itself. Springing from the left ventricle, and appearing from beneath the pulmonary artery when the heart is looked at from the ventral side, is a great 
artery, the aorta, $A a$. It forms an arch over the base of the heart and then runs down behind it at the back of the chest. From the convexity of the arch of the aorta several great branches are given off, $S s i, C s, A b$; but before that, close to the heart, the aorta gives off two coronary arteries, branches of which are seen at crd and crs lying in the groove over the partition between the ventricles, and which carry to the substance of the organ that blood which comes back through the coronary sinus. Into the left auricle open two right and two left pulmonary veins, $p s$ and $p d$, which are formed by the union of smaller veins proceeding from the lungs.

In the diagram Fig. 101 from which the branches of the great vessels near the heart have been omitted for the sake of clearness, the connection of the various vessels with the chambers of the heart can be better seen. Opening into the right auricle are the superior and inferior venæ cavæ (cs and $c i$ ) and proceeding from the right ventricle the pulmonary artery, $P$. Opening into the left auricle are the right and left pulmonary veins ( $p d$ and $p s)$ and springing from the left ventricle the aorta, $A$.

The Interior of the Heart. The communication of each auricle with its ventricle is also represented in the diagram Fig. 101, and the valves which are present at those points and at the origin of the pulmonary artery and that of the aorta. Internally the auricles are for the most part smooth, but from each a hollow pouch, the auricular appendage, projects over the base of the corresponding ventricle as seen at $A d x$ and $A s$ in Figs. 102 and 103. These pouches have somewhat the shape of a dog's ear and have given their name to the whole auricle. Their interior is roughened by muscular elevations, covered by endocardium, known as the fleshy columns (columnoe carnoe). On the inside of the ventricles (Fig. 104) similar fleshy columns are very prominent.

The Auriculoventricular Valves. These are known as right and left, or as the tricuspid and mitral valves respectively. The mitral valve (Fig. 104) consists of two flaps of the endocardium fixed by their bases to the margins of the auriculoventricular aperture and with their edges hanging down into the ventricle when the heart is empty. These unattached edges are not, however, free, but have fixed to them a number of stout connectivetissue cords, the cordo tendinex, which are fixed below to muscular 
elevations, the papillary muscles, $M p m$ and $M p l$, on the interior of the ventricle. The cords are long enough to let the valve flaps rise into a horizontal position and so close the opening between auricle and ventricle which lies between them, and passes up be-

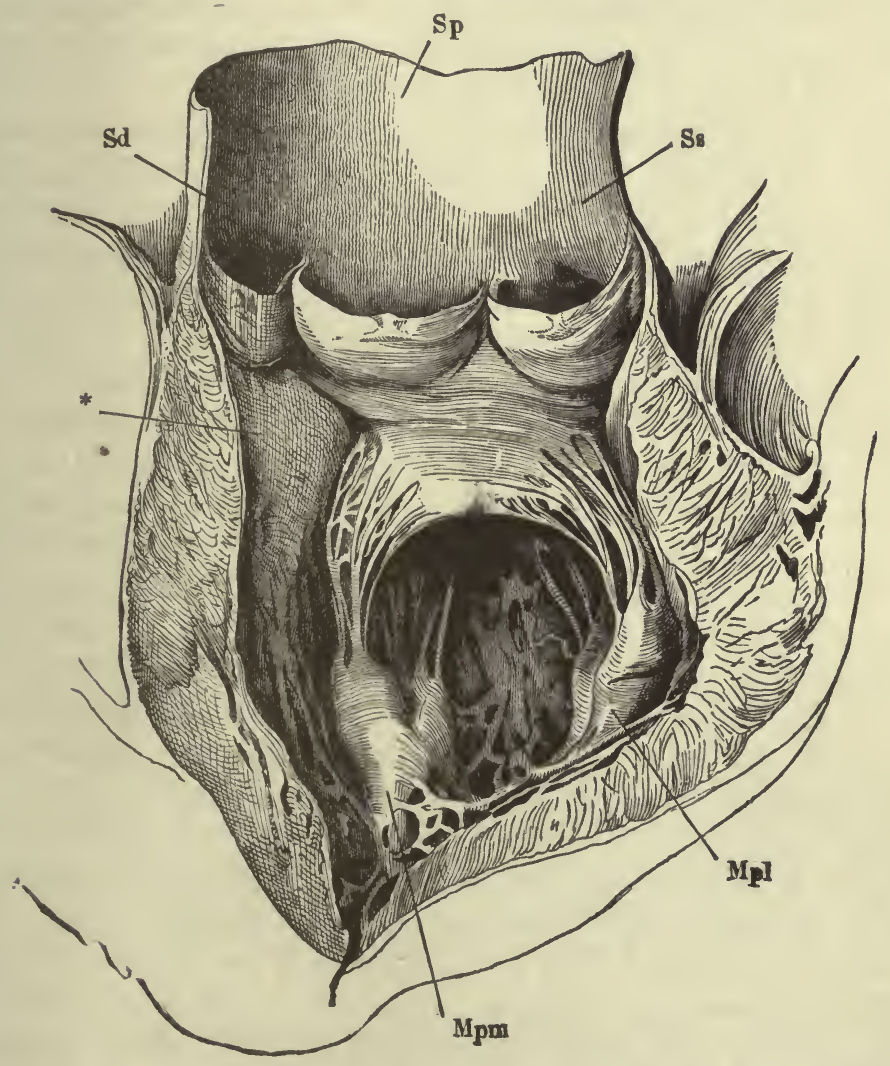

FIG. 104. - The left ventricle and the commencement of the aorta laid open. $M p m, M p l$, the papillary muscles. From their upper ends are seen the cordoe tendinee proceeding to the edges of the flaps of the mitral valve. The opening into the auricle lics between these flaps. At the beginning of the aorta are seen its three pouch-like semilunar valves.

hind the opened aorta, $S p$, represented in the figure. The tricuspid valve is like the mitral, but with three flaps instead of two.

Semilunar Valves. These are six in number: three at the mouth of the aorta, Fig. 104, and three, quite like them, at the mouth of the pulmonary artery. Each is a strong crescentic pouch fixed 
by its more curved border, and with its free edge turned away from the heart. When the valves are in action these free edges meet across the vessel and prevent blood from flowing back into the ventricle. In the middle of the free border of each valve is a little cartilaginous nodule, the corpus Arantii, and on each side of this the edge of the valve is very thin and when it meets its neighbor turns up against it and so secures the closure.

The Arterial System. All the arteries of the Body arise either directly or indirectly from the aorta or pulmonary artery, and the great majority of them from the former vessel. The pulmonary artery carries blood only to the lungs, to undergo exchanges with the air in them after it has circulated through the Body generally.

After making its arch the aorta continues back through the chest, giving off many branches on its way. Piercing the diaphragm it enters the abdomen and after supplying the parts in and around that cavity with branches, it ends opposite the last lumbar vertebra by dividing into the right and left common iliac arteries, which carry blood to the lower limbs. We have then to consider the branches of the arch of the aorta, and those of the descending aorta, which latter is for convenience described by anatomists as consisting of the thoracic aorta, extending from the end of the arch to the diaphragm, and the abdominal aorta, extending from the diaphragm to the final subdivision of the vessel.

Branches of the Arch of the Aorta. From this arise first the coronary arteries (crd and crs, Figs. 102 and 103) which spring close to the heart, just above two of the pouches of the semilunar valve, and carry blood into the substance of that organ. The remaining branches of the arch are three in number, and all arise from its convexity. The first is the innominate artery $(A b, \mathrm{Fig}$. 102), which is very short, immediately breaking up into the right subclavian artery, and the right common carotid. Then comes the left common carotid, Cs, and finally the left subclavian, Ssi.

Each subclavian artery runs out to the arm on its own side and after giving off a vertebral artery (which runs up the neck to the head in the vertebral canal of the transverse processes of the cervical vertebrx), crosses the armpit and takes there the name of the axillary artery. This continues down the arm as the brachial artery, which, giving off branches on its way, runs to the front of the arm, and just below the elbow-joint divides into the radial 
and ulnar arteries, the lower ends of which are seen at $R$ and $U$ in Fig. 100. These supply the forearm and end in the hand by uniting to form an arch, from which branches are given off to the fingers.

The common carotid arteries pass out of the chest into the neck, along which they ascend on the sides of the windpipe. Opposite the angle of the lower jaw each divides into an internal and external carotid artery, right or left as the case may be. The external ends mainly in branches for the face, scalp, and salivary glands, one great subdivision of it with a tortuous course, the temporal artery, being often seen in thin persons beating on the side of the brow. The internal carotid artery enters the skull through an aperture in its base and supplies the brain, which it will be remembered gets blood also through the vertebral arteries.

Branches of the Thoracic Aorta. These are numerous but small. Some, the intercostal arteries, run out between the ribs and supply the chest-walls; others, the bronchial arteries, carry blood to the lungs for their nourishment, that carried to them by the pulmonary arteries being brought there for another purpose; and a few other small branches are given to other neighboring parts.

Branches of the Abdominal Aorta. These are both large and numerous, supplying not only the wall of the posterior part of the trunk, but the important organs in the abdominal cavity. The larger are: the celiac axis which supplies stomach, spleen, liver, and pancreas; the su uperior mesenteric artery, which supplies a great part of the intestine; the 3 renal arteries, one for each kidney; and finally the inferior mesenteric artery, which supplies the rest of the intestine. Besides these the abdominal aorta gives off very many smaller branches.

Arteries of the Lower Limbs. Each common iliac divides into an internal and external iliac artery. The former ends mainly in branches to parts lying in the pelvis, but the latter passes into the thighs and there takes the name of the femoral artery. At first this lies on the ventral aspect of the limb, but lower down passes to the hask of the femur, and above the knee-joint (where it is called the popliteal artery) divides into the anterior and posterior tibial arteries, which supply the leg and foot.

The Capillaries. As the arteries are followed from the heart, their branches become smaller and smaller, and finally cannot be 
traced without the aid of a microscope. The smallest arteries are called arterioles. These pass into the capillaries, the walls of which are simpler than those of the arterioles, and which form very close networks in nearly all parts of the Body; their immense number compensating for their small size. The average diameter of a capillary vessel is $.016 \mathrm{~mm}$. ( $\frac{1}{1500}$ inch) so that only two or three

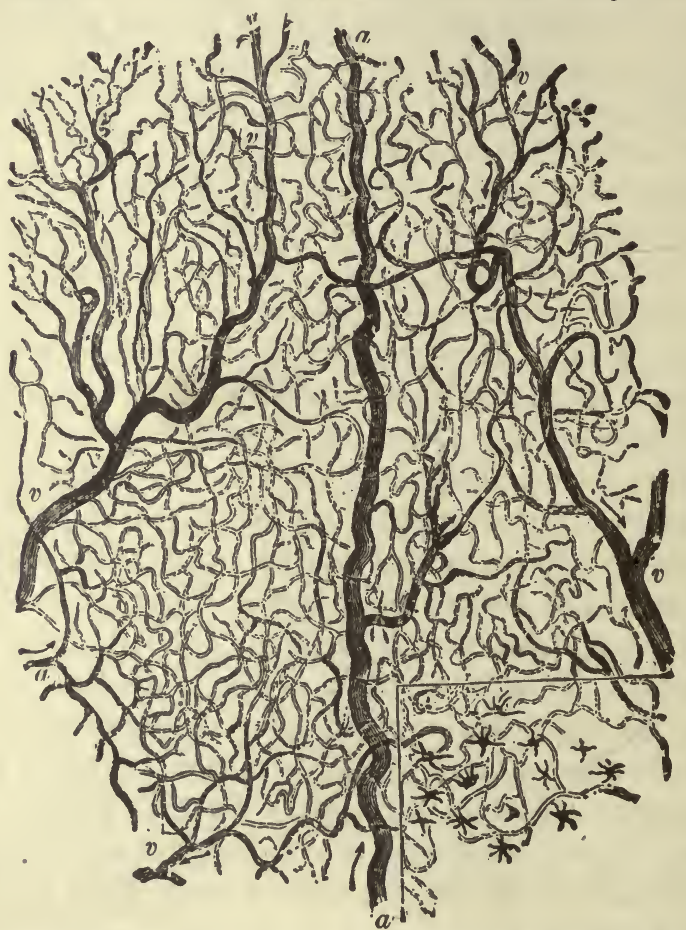

FIg. 105.-A small portion of the capillary network as seen in the frog's web when magnified about 25 diameters. $a$, a small artery feeding the capillaries; $v, v$, small veins carrying blood back from the latter.

blood-corpuscles can pass through it abreast, and in many parts they are so close that a pin's point could not be inserted between two of them (Fig. 105). It is while flowing in these delicate tubes that the blood does its nutritive work, the arteries being merely supply-tubes for the capillaries.

The Veins. The first veins arise from the capillary networks and like the last arteries are very small. They soon increase in size by union, and so form larger and larger trunks. These in 
many places lie near or alongside the main artery of the part, but there are many more large veins just beneath the skin than there are large arteries. This is especially the case in the limbs, the main veins of which are superficial, and can in many persons be seen as faint blue marks through the skin. Fig. 106 represents the arm at the front of the elbow-joint after the skin and subcutaneous areolar tissue and fat have been removed. The brachial artery, $B$, colored red, is seen lying tolerably deep, and accompanied by two small veins (venæ comites) which communicate by cross-branches. The great median nerve, 1 , a branch of the brachial plexus which supplies several muscles of the forearm and hand, the skin over a great part of the palm and the three inner fingers, is seen alongside the artery. The larger veins of the part are seen to form a more superficial network, joined here and there, as for instance at *, by branches from deeper parts. Several small nerve-branches which supply the skin $(2,3,4)$ are seen among these veins. It is from the vessel, cep, called the cephalic vein, just above the point where it crosses the median nerve, that surgeons usually bleed a patient.

A great part of the blood of the lower limb is brought back by the long saphenous vein, which can be seen in thin persons running from the inner side of the ankle to the top of the thigh. All the blood which leaves the heart by the aorta, except that flowing through the coronary arteries, is finally collected into the superior and inferior vence cave (cs and ci, Figs. 102 and 103), and poured into the right auricle. The jugular veins which run down the neck, carrying back the blood which went out along the carotid arteries, unite below with the arm-vein (subclavian) to form on each side an innominate vein (Asi and Ade, Fig. 102) and the innominates unite to form the superior cava. The coronary-artery blood after flowing through the capillaries of the heart itself also returns to this auricle by the coronary veins and sinus.

The Pulmonary Circulation ( $L$, Fig. 99). Through this the blood gets back to the left side of the heart and so into the aorta again. The pulmonary artery, dividing into branches for each lung, ends in the capillaries of those organs. From these the blood is collected by the pulmonary veins, which carry it back to the left auricle, whence it passes to the left ventricle to recommence its flow through the Body generally. 
The Course of the Blood. From what has been said it is clear that the movement of the blood is a circulation. Starting from any one chamber of the heart it will in time return to it; but to do this

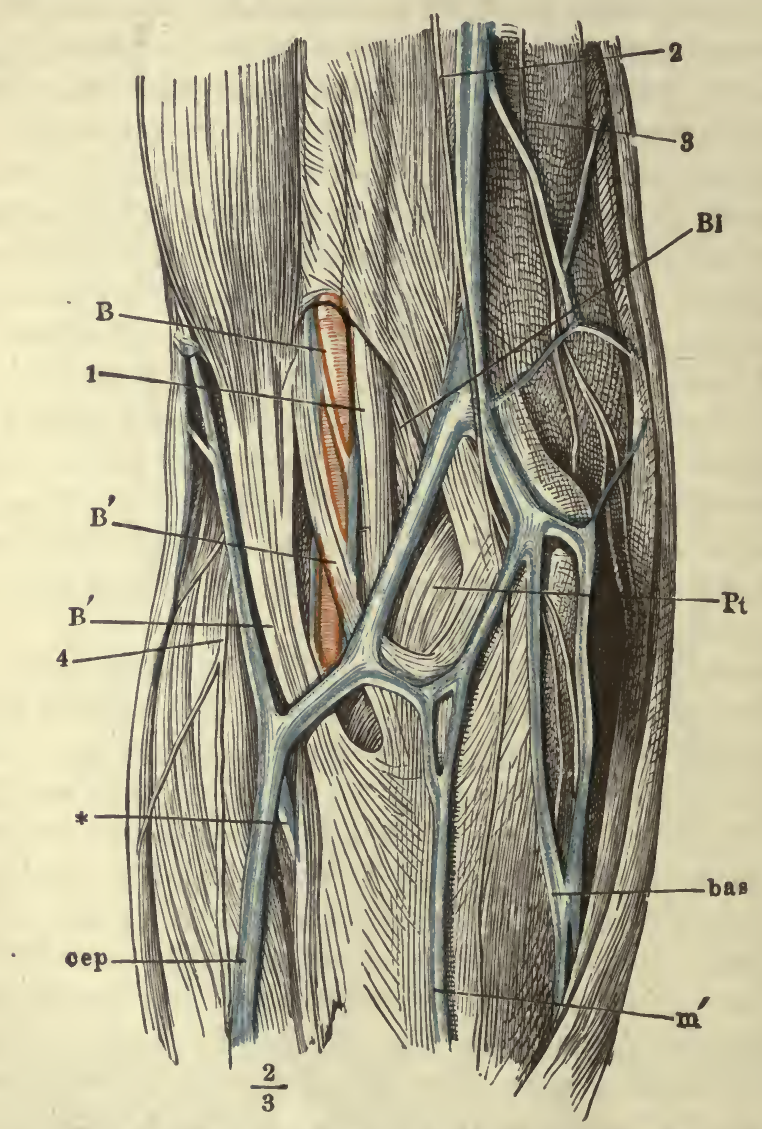

Fig. 106. - The superficial veins in front of the elbow-joint. $B^{\prime}$, tendon of biceps muscle; $B i$, brachialis internus muscle; $P t$, pronator teres muscle; 1 , median nerve; $2,3,4$, nerve-branches to the skin; $B$, brachial artery, with its small accompanying veins; $c e p$, cephalic vein; bas, basilic vein; $m^{\prime}$, median vein; $*$, junction of a deep-lying vein with the cephalic.

it must pass through at least two sets of capillaries; one of these is connected with the aorta and the other with the pulmonary artery, and in its circuit the blood returns to the heart twice. Leaving the left side it returns to the right, and leaving the right it returns to the left; and there is no road for it from one side of the 
heart to the other except through a capillary network. Moreover, it always leaves from a ventricle through an artery, and returns to an auricle through a vein.

There is then really only one circulation; but it is not uncommon to speak of two, the flow from the left side of the heart to the right, through the Body generally, being called the systemic circulation, and from the right to the left, through the lungs, the pulmonary circulation. But since after completing either of these alone the blood is not back at the point from which it started, but is separated from it by the septum of the heart, neither is a "circulation" in the proper sense of the word.

The Portal Circulation. A certain portion of the blood which leaves the left ventricle of the heart through the aorta has to pass through three sets of capillaries before it can again return there. This is the portion which goes through the stomach, spleen, pancreas, and intestines ( $M$, Fig. 99). After traversing the capillaries of those organs it is collected into the portal vein which enters the liver, and breaking up in it into finer and finer branches like an artery, ends in the capillaries of that organ, forming the second set which this blood passes through on its course ( $P$, Fig. 99). From these it is collected by the hepatic veins, which pour it into the inferior vena cava, which carries it to the right auricle, so that it has still to pass through the pulmonary capillaries to get back to the left side of the heart. The flow from the stomach and intestines through the liver to the vena cava is often spoken of as the portal circulation.

Diagram of the Circulation. Since the two halves of the heart are actually completely separated from one another by an impervious partition, although placed in proximity in the Body, we may conveniently represent the course of the blood as in the accompanying diagram (Fig. 107), in which the right and left halves of the heart are represented at different points in the vascular system. Such an arrangement makes it clear that the heart is really two pumps working side by side, each engaged in forcing the blood to the other. Starting from the left auricle, $l a$, and following the flow, we trace it through the left ventricle and along the branches of the aorta into the systemic capillaries, $s c$; from thence it passes back through the systemic veins, $v c$. Reaching the right auricle, $r a$, it is sent into the right 
ventricle, $r v$, and thence through the pulmonary artery, $p a$, to the lung capillaries, $p c$, from which the pulmonary veins, $p v$, carry it to the left auricle, which drives it into the left ventricle, $l v$, and this again into the aorta.

Arterial and Venous Blood. The blood when flowing in the pulmonary capillaries gives up carbon dioxid to the air and receives

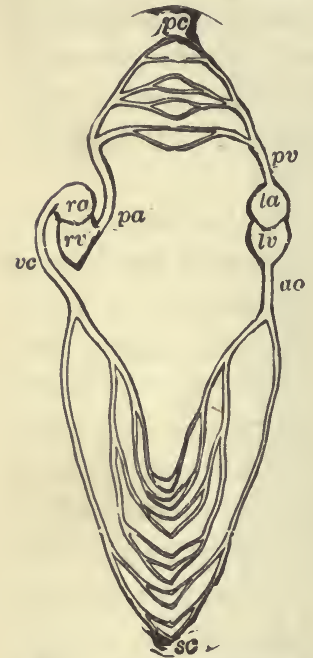

Fig. 107.-Diagram of the blood vascular system, showing that it forms a single closed circuit with two pumps in it, consisting of the right and left halves of the heart, which are represented separate in the diagram. ra and ro, right auricle and ventricle; $l a$ and $l v$, left auricle and ventricle; $a o$, aorta; $s c$, systemic capillaries; $v c$, venæ cavæ; $p a$, pulmonary artery; $p c$, pulmonary capillaries; $p v$, pulmonary veins. oxygen from it; and since its coloring matter (hemoglobin) forms a scarlet compound with oxygen, it flows to the left auricle through the pulmonary veins of a bright red color. This color it maintains until it reaches the systemic capillaries, but in these it loses much oxygen to the surrounding tissues and gains much carbon dioxid from them. But the blood coloringmatter which has lost its oxygen has a dark purple color, and since this unoxidized or "reduced" hemoglobin is now in excess, the blood returns to the heart by the venæ cavæ of a dark purple-red color. This hue it keeps until it reaches the lungs, when the reduced hemoglobin becomes again oxidized. The bright red blood, rich in oxygen and poor in carbon dioxid, is known as "arterial blood" and the dark red as "venous blood": and it must be borne in mind that the terms have this peculiar technical meaning, and that the pulmonary veins contain arterial blood, and the pulmonary arteries, venous blood; the change from arterial to venous taking place in the systemic capillaries, and from venous to arterial in the pulmonary capillaries. The chambers of the heart and the great vessels containing arterial blood are shaded red in Figs. 102 and 103.

The Structure of the Arteries. A large artery can by careful dissection be separated into three coats: an internal, a middle, and an outer. The internal coat tears readily across the long axis of the artery and consists of an inner lining of flattened nucleated cells, 
known as the intima, enveloped by a variable number of layers composed of membranes or networks of elastic tissue. The middle coat is made up of alternating layers of elastic fibers and plain muscular tissue; the former running for the most part longitudinally and the latter across the long axis of the vessel. The outer coat is the toughest and strongest because it is mainly made up of white fibrous connective tissue; it contains a considerable amount of elastic tissue also, and gradually shades off into a loose areolar tissue which forms the sheath of the artery, or the tunica adventitia, and packs it between surrounding parts. The smaller arteries have all the elastic elements less developed. The internal coat is consequently thinner, and the middle coat is made up mainly of smooth muscular fibers. As a result the large arteries are highly elastic, the aorta being physically much like a piece of indiarubber tubing, while the smaller arteries are highly contractile, in the physiological sense of the word.

Structure of the Capillaries. In the smaller arteries the outer and middle coats gradually disappear, and the elastic layers of the inner coat also go. Finally in the capillaries the intima alone is left, with a more or less developed layer of connective-tissue corpuscles around it, representing the remnant of the tunica adventitia. These vessels are thus extremely well adapted to allow of filtration or diffusion taking place through their thin walls.

Structure of the Veins. In these the same three primary coats as in the arteries are found; the inner and middle coats are less developed, while the outer one remains thick, and is made up almost entirely of white fibrous tissue. Hence the venous walls are much thinner than those of the corresponding arteries, and the veins collapse when empty while the stouter arteries remain open. But the toughness of their outer coats gives the veins great strength.

Except the pulmonary artery and the aorta, which possess the semilunar valves at their cardiac orifices, the arteries possess no valves. Many veins, on the contrary, have such, formed by semilunar pouches of the inner coat, attached by one margin and having the edge turned towards the heart free. These valves, sometimes single, oftener in pairs, and rarely three at one level, permit blood to flow only towards the heart, for a current in that 
direction (as in the upper diagram, Fig. 108) presses the valve close against the side of the vessel and meets with no obstruction from it. Should any back-flow be attempted, how-

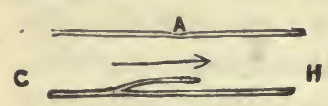
ever, the current closes up the valve and H bars its own passage as indicated in the lower figure. These valves are most

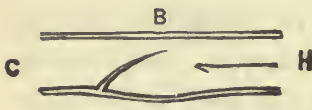

Fig. 108.-Diagram to illustrate the mode of action of the valves of the veins. $C$, the capillary end, and $H$, the heart end of the vessel. numerous in superficial veins and those of muscular parts. They are absent in the venæ cavæ and the portal and pulmonary veins. Usually the vein is a little dilated opposite a valve, and hence in parts where the valves are numerous gets a knotted look. On compressing the forearm so as to stop the flow in its subcutaneous veins and cause their dilatation, the points at which valves are placed can be recognized by their swollen appearance. They are most frequently situated where two veins communicate. 


\section{CHAPTER XX}

\section{THE ACTION OF THE HEART. THE REGULATION OF THE HEART-BEAT}

The Beat of the Heart. It is possible with some little skill and care to open the chest of a living narcotized animal, such as a rabbit, and see its heart at work, alternately contracting and relaxing. As observed under ordinary conditions these phases follow one another so rapidly as seemingly to defy analysis. When Harvey, the discoverer of the circulation, first looked upon the beating heart of a mammal he was so impressed by the complexity and rapidity of its action as to believe for the moment that the human mind could never fathom it.

By proper treatment the beat of the heart can be much slowed. When this has been done it is observed that each beat commences at the mouths of the great veins; from there runs over the rest of the auricles, and then over the ventricles; the auricles dilating the moment the ventricles commence to contract. Having finished their contraction the ventricles also dilate, and so for some time neither they nor the auricles are contracting, but the whole heart is at rest. The contraction of any part of the heart is known as its systole and the relaxation as its diastole.

The average heart-rate in man is 72 beats per minute, giving for each beat 0.8 second. The two sides of the heart work synchronously, the auricles together and the ventricles together. In describing the "cardiac cycle," therefore, the auricles are treated as one organ and the ventricles as one. The auricular systole occupies about 0.1 second, its diastole lasts 0.7 second. The ventricular systole begins at the end of the auricular contraction; it occupies about 0.3 second; the diastole of the ventricle lasts about 0.5 second. During fully half of each cardiac cycle, then, there is no muscular activity going on in any part of the heart. During diastole the heart if taken between the finger and thumb feels soft and flabby, but during systole it (especially its ventricular portion) becomes hard and rigid. 
Change of Form of the Heart. During its systole the heart becomes shorter and rounder, mainly from a change in the shape of the ventricles, which from having an elliptical cross-section take on a circular one. At the same time the length of the ventricles is lessened, the apex of the heart approaching the base and becoming blunter and rounder.

The Cardiac Impulse. The human heart lies with its apex touching the chest-wall between the fifth and sixth ribs on the left side of the breast-bone. At every beat a sort of tap, known as the "cardiac impulse" or "apex beat," may be felt by the finger at that point. There is, however, no actual "tapping, since the heart's apex never leaves the chest-wall. During the diastole the soft ventricles yield to the chest-wall where they touch it, but during the systole they become hard and tense and push it out a little between the ribs, and so cause the apex beat. Since the heart becomes shorter during the ventricular systole, it might be supposed that at that time the apex would move up a little in the chest. This, however, is not the case, the ascent of the apex towards the base of the ventricles being compensated for by a movement of the whole heart in the opposite direction. If water be pumped into an elastic tube, already moderately full, the tube will be distended not only transversely but longitudinally. This is what happens in the aorta: when the left ventricle contracts and pumps blood forcibly into it, the elastic artery is elongated as well as widened, and the lengthening of that limb of its arch attached to the heart pushes the latter down towards the diaphragm, and compensates for the upward movement of the apex due to the shortening of the ventricles. Hence if the exposed living heart be watched it appears as if during the systole the base of the heart moved towards the tip, rather than the reverse.

Events occurring within the Heart during a Cardiac Cycle. Let us commence at the end of the ventricular systole. At this moment the semilunar valves at the orifices of the aorta and the pulmonary artery are closed, so that no blood can flow back from those vessels. The whole heart, however, is soft and distensible and yields readily to blood flowing into it from the pulmonary veins and the venæ cavæ; this passes on through the open mitral and tricuspid valves and fills up the dilating ventricles, as well as 
the auricles. As the ventricles fill, back currents are set up along their walls and these carry up the flaps of the valves so that by the end of the pause they are nearly closed. At this moment the auricles contract, and since this contraction commences at and narrows the mouths of the veins opening into them, and at the same time the blood in those vessels opposes some resistance to a back-flow into them, while the still flabby and dilating ventricles oppose much less resistance, the general result is that the contracting auricles send blood into the ventricles, and not back into the veins. At the same time the increased direct current into the ventricles produces a greater back current on the sides, which, when the auricles cease their contraction and the filled ventricles become tense and press on the blood inside them, completely closes the auriculoventricular valves. That this increased filling of the ventricles, due to auricular contractions will close the valves may be seen easily in a sheep's heart. If the auricles be carefully cut away from this so as to expose the mitral and tricuspid valves, and water be then poured from a little height into the ventricles, it will be seen that as these cavities are filled the valve-flaps are floated up and close the orifices.

The auricular contraction now ceases and the ventricular commences. The blood in each ventricle is imprisoned between the auriculoventricular valves behind and the semilunar valves in front. The former cannot yield on account of the cordæ tendineæ fixed to their edges: the semilunar valves, on the other hand, can open outwards from the ventricle and let the blood pass on, but they are kept tightly shut by the pressure of the blood on their other sides, just as the lock-gates of a canal are by the pressure of the water on them. In order to open the canal-gates water is let in or out of the lock until it stands at the same level on each side of them; but of course they might be forced open without this by applying sufficient power to overcome the higher water pressure on one side. It is in this latter way that the semilunar valves are opened. The contracting ventricle tightens its grip on the blood inside it and becomes rigid to the touch. As it squeezes harder and harder, at last the pressure on the blood within it becomes greater than the pressure exerted on the other side of the valves by the blood in the arteries, the flaps are forced open and the blood begins to pass out: the ventricle continues its contrac- 
tion until it has obliterated its cavity and completely emptied itself; this total emptying appears, at least, to occur in the normally beating heart, but in some pathological conditions and under the influence of certain drugs the emptying of the ventricles is incomplete. After the systole the ventricle commences to relax and blood immediately to flow back towards it from the highly stretched arteries. This return current, however, catches the pockets of the semilunar valves, drives them back and closes the valve so as to form an impassable barrier; and so the blood which has been forced out of either ventricle cannot flow directly back into it.

Use of the Papillary Muscles. In order that the con'tracting ventricles may not force blood back into the auricles it is essential that the flaps of the mitral and tricuspid valves be maintained in position across the openings which they close, and be not pushed back into the auricles. At the commencement of the ventricular systole this is provided for by the cordæ tendineæ, which are of such a length as to keep the edges of the flaps in apposition, a position which is further secured by the fact that each set of cordæ tendineæ (Fig. 104) radiating from a point in the ventricle, is not attached around the edges of one flap but on the contiguous edges of two flaps, and so tends to pull them together. But as the contracting ventricles shorten, the cordæ tendineæ, if directly fixed to their interior, would be slackened and the valve-flaps pushed up into the auricle. The little papillary muscles prevent this. Shortening as the ventricular systole proceeds, they keep the cordæ taut and the valves closed.

Sounds of the Heart. If the ear be placed on the chest over the region of the heart during life, two distinguishable sounds will be heard during each cardiac cycle. They are known respectively as the first and second sounds of the heart. The first is of lower pitch and lasts longer than the second and sharper sound: vocally their character may be tolerably imitated by the words $l u b b, d \check{u} p$. The cause of the second sound is the closure, or, as one might say, the "clicking up," of the semilunar valves, since it occurs at the moment of their closure and ceases if they be hooked back in a living animal. The origin of the first sound is still uncertain: it takes place during the ventricular systole and is prob- 
ably due to vibrations of the tense ventricular wall at that time. It is not due, at least not entirely, to the auriculoventricular valves, since it may still be heard in a beating heart empty of blood, and in which there could be no closure or tension of those valves. In various forms of heart disease these sounds are modified or cloaked by additional "murmurs" which arise when the cardiac orifices are roughened or narrowed or dilated, or the valves inefficient. By paying attention to the character of the new sound then heard, the exact period in the cardiac cycle at which it occurs, and the region of the chest-wall at which it is heard most distinctly, the physician can often get important information as to its cause.

Action of the Heart Valves. The valves of the heart are entirely without rigidity. They consist of tough, but perfectly flaccid membranes, so that they respond perfectly to the forces which act upon them. This structure makes it inevitable that the valves will open whenever the pressure behind them is greater than that in front, and will close whenever the pressure in front is greater than that behind. During the whole diastole of the heart the pressure behind the auriculoventricular valves is greater than that in front of them; for in front is only the gradually filling cavity of the ventricle, while behind is the onward flow of blood from the great veins. During this time, therefore, these valves stand open. The systole of the auricle, by increasing the pressure behind, keeps them open until its end. During this same time the aortic valves are shut, because in front of them are arteries whose walls are stretched with their load of blood and which, therefore, exert high pressure upon the valves, while behind are only the ventricular cavities, filling with blood. At the instant the ventricles begin to contract the situation with regard to the auriculoventricular valves changes. The relaxing auricles make room for the blood coming in from the great veins and so release the pressure behind these valves; the contracting ventricle exerts pressure in front of them; they therefore close instantly. Since the semilunar valves remain closed until the rising pressure in the ventricle becomes greater than that in the aorta there is an instant at the beginning of ventricular systole when all the valves are shut. Again, at the beginning of ventricular diastole there is an instant when the ventricular pressure has fallen below that in the aorta but is still 
above the pressure in the auricles; during this time, again, the valves of the heart are all shut.

Effects of Valvular Insufficiency. The commonest heart troubles are due to failure of one or other of the valves to close perfectly. The mechanical effect of such inefficiency is, of course, a back rush of blood through the leaky valve. The effects on the Body at large will depend on which valve is inefficient. Leakage of the semilunars means a return into the ventricle of part of the blood just pumped out. The circulation is to that extent less effectively maintained. The heart usually compensates for this defect by muscular growth (hypertrophy) by which it becomes enough more powerful than normally to make up for the lessened efficiency. Leakage of an auriculoventricular valve is much more serious because it permits a jet of blood to be driven backward into the veins at each heart-beat under the driving force of the powerful ventricular contraction. The small veins and capillaries are not adapted to receive such a hammering and are injured thereby. If the leaky valve is the mitral the lung capillaries are the ones affected. If the tricuspid is inefficient the backward surge makes itself felt in distant organs. Kidney impairment is a common sequel to this type of valvular disease. Inflammatory rheumatism frequently brings on valve trouble. In fact the danger of this outcome is so great that every pains should be taken to avoid it, by giving the patient the best of care and treatment.

Diagram of the Events of a Cardiac Cycle. In the following table the phenomena of the heart's beat are represented with reference to the changes of form which are seen on an exposed working heart. Events in the same vertical column occur simultaneously; on the same horizontal line, from left to right, successively.

\begin{tabular}{|c|c|c|c|c|c|}
\hline & $\begin{array}{c}\text { Auricular } \\
\text { Systole }\end{array}$ & $\begin{array}{l}\text { Commence- } \\
\text { ment of } \\
\text { Ventricular } \\
\text { Systole }\end{array}$ & $\begin{array}{c}\text { Ventricular } \\
\text { Systole }\end{array}$ & $\begin{array}{l}\text { Cessation } \\
\text { of Ven- } \\
\text { tricular } \\
\text { Systole }\end{array}$ & Pause \\
\hline Auricles. . & $\begin{array}{c}\text { Contracting } \\
\text { and } \\
\text { emptying. }\end{array}$ & $\begin{array}{l}\text { Dilating and } \\
\text { filling. }\end{array}$ & $\begin{array}{l}\text { Dilating and } \\
\text { filling. }\end{array}$ & $\begin{array}{l}\text { Dilating and } \\
\text { filling. }\end{array}$ & $\begin{array}{l}\text { Dilating and } \\
\text { filling. }\end{array}$ \\
\hline Ventricles. & $\begin{array}{l}\text { Dilating and } \\
\text { filling. }\end{array}$ & Contracting. & $\begin{array}{c}\text { Contracting } \\
\text { and } \\
\text { emptying. }\end{array}$ & Dilating. & $\begin{array}{l}\text { Dilating and } \\
\text { filling. }\end{array}$ \\
\hline $\begin{array}{l}\text { Impulse.................. } \\
\text { Auriculoventricular valves } \\
\text { Semilunar valves......... } \\
\text { Sounds.............. }\end{array}$ & 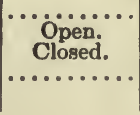 & $\begin{array}{l}\text { Apex beat. } \\
\text { Closed. } \\
\text { Closed. } \\
\text { First sound. }\end{array}$ & $\begin{array}{c}\text { Closed. } \\
\text { Open. } \\
\ldots \ldots \ldots\end{array}$ & $\begin{array}{l}\text { Closed. } \\
\text { Closed. } \\
\text { Second } \\
\text { sound. }\end{array}$ & $\begin{array}{c}\text { Open. } \\
\text { Closed. }\end{array}$ \\
\hline
\end{tabular}


Function of the Auricles. The ventricles have to do the work of pumping the blood through the blood-vessels. Accordingly their walls are far thicker and more muscular than those of the auricles; and the left ventricle, which has to force the blood over the Body generally, is stouter than the right, which has only to send blood around the comparatively short pulmonary circuit. The circulation of the blood is in fact maintained by the ventricles, and we have to inquire what is the use of the auricles. Not unfrequently the heart's action is described as if the auricles first filled with blood and then contracted and filled the ventricles; and then the latter contracted and drove the blood into the arteries. From the account given above, however, it will be seen that the events are not accurately so represented, but that during all the pause blood flows on through the auricles into the ventricles, which latter are already nearly full when the auricles contract; this contraction merely completing their filling. The real use of the auricles is to afford a reservoir into which the veins may empty while the comparatively long-lasting ventricular contraction is taking place.

If the heart consisted of the ventricles only, with valves at the points of entry and exit of the blood, the circulation could be maintained. During disatole the ventricle would fill from the veins, and during systole empty into the arteries. But in order to accomplish this, during the systole the valves at the point of entry must be closed, or the ventricle would empty itself into the veins as well as into the arteries; and this closure would necessitate a great loss of time which might be utilized for feeding the pump. This is avoided by the auricles, which are really reservoirs at the end of the venous system, collecting blood when the ventricular pump is at work. When the ventricles relax, the blood entering the auricles flows on into them; but previously, during the part of the cardiac cycle occupied by the ventricular systole, the auricles have accumulated blood, and when they at last contract they send on into the ventricles this accumulation. Even were the flow from the veins stopped during the auricular contraction this would be of comparatively little consequence, since that event occupies so brief a time. But, although no doubt somewhat lessened, the emptying of the veins into the heart does not seem to be, in health, stopped while the auricle is contracting. The heart in fact con- 
sists of a couple of "feed-pumps" - the auricles-and a couple of "force-pumps"- the ventricles; and so wonderfully perfect is the mechanism that the supply to the feed-pumps is never stopped. The auricles are never empty, being supplied all the time of their contraction, which is never so great as to obliterate their cavities; while the ventricles contain little or no blood at the end of their systole.

The Work Done by the Heart. According to the physical definition work is measured by the weight lifted times the height to which it is raised. In estimating the work of the heart we substitute for the height the resistance against which the heart works. This resistance is equivalent in the case of the left ventricle to that of a column of blood about 2 meters high, and for the right ventricle about 0.8 meter. The mass of blood ejected from each ventricle during systole probably averages about $100 \mathrm{gms}$. The work done by the left ventricle per beat equals, then, about $100 \times 2=200$ grammeters, and that by the right ventricle equals about $100 \times 0.8=80$ grammeters. Since the heart in addition to moving the weight of blood imparts to it a considerable velocity, it is necessary to add to the amounts of work calculated above an additional amount to represent that required to impart to the blood its velocity. This latter amount approximates 3 grammeters. The total work output of the heart per beat is, therefore, roughly 283 grammeters, equivalent in the English scale to about 2 foot-pounds. When the heart is beating at the rate of 70 per minute it does 140 foot-pounds per minute, making it a 240th horse-power engine. If it maintained this rate throughout the entire twenty-four hours of the day. it would do in that time 200,000 foot-pounds of work, an amount equivalent to that done by the leg muscles of a man weighing 150 pounds in climbing a mountain 1,300 feet high.

That the heart is able to do this amount of work daily without fatigue, and keep it up day in and day out for seventy or more years, is due to its ability to recover quickly from the effects of its activity, coupled with the fact that in a whole day its resting time considerably outweighs the time during which it is active. The heart-beat is ordinarily much slower during sleep than during bodily activity; as the result the heart enjoys an "eight hour day" if only its actual contraction time be counted. 
Relations of Nerve and Muscle Elements within the Heart. The heart-muscle consists, as previously stated, of muscle-cells of small size, intimately communicating with one another through their branches, and showing signs of cross-striation. At the junction of the great veins with the heart, a region, as we shall see, of great importance in the heart's activity, these muscular elements form thin sheets; in the auricles the heart-muscle is somewhat heavier and thicker; but it attains its greatest development in the ventricles, where the muscular walls are exceedingly heavy, and very stout. In mammals the only pulsating heart structures are the auricles and ventricles; in lower vertebrates, such as the frog, the great veins near the heart are differentiated into a pulsating structure, the sinus venosus, and the outlet from the ventricle, the bulbus arteriosus, also pulsates. Although in mammals these structures no longer pulsate, the region of the great veins which corresponds to the sinus venosus still seems to preserve to some degree the physiological properties it has in lower animals, and observations made upon frogs' hearts are interpreted for mammals' hearts upon that basis.

Embedded within the tissue of the heart are numerous nervecells. These are most numerous in the region of the sinus venosus and auricles; the base of the ventricles contains some of them, but the apex of the ventricles is said to be wholly free from them. Nerve-fibers, communicating with these cells, penetrate all parts of the cardiac musculature. It has not been possible by histologic means to show that these fibers are dendrites and axons such as occur in the general nervous system, and many histologists and physiologists believe that they form a continuous network or plexus involving all parts of the heart and so constituted that a stimulus applied at any point spreads over the whole organ. According to this view the nervous mechanism of the heart is not a "synaptic system" and so does not show the irreversibility of conduction which is a cardinal feature of the general nervous system. Some support for this idea is had in the fact that certain other viscera, notably the stomach and intestines, have within their walls nerve plexuses showing similar physiological properties.

Physiological Peculiarities of the Heart. The most striking of these is its automatic rhythmicity. The heart may be removed completely from the Body without its regular beating being at all in- 
terfered with. In cold-blooded animals such as frogs or turtles this activity outside the Body may continue for hours. While we refer to this activity as automatic we do not mean by the word anything more than the fact just stated, that the heart continues to beat independently of the rest of the Body. The rhythmic nature of the heart's activity is as characteristic as its automaticity. The regular succession of contractions and relaxations is its normal response to continuous or rapidly recurring stimulation. In this respect it differs strikingly from skeletal muscle, which remains strongly contracted throughout the period of such stimulation unless fatigue sets in to release it.

Another peculiarity of heart-muscle, and one that probably explains in part its rhythmic property, is that its contractions are always maximal. By this is meant that whenever heart-muscle contracts it always does so to the full extent of its ability at the time. In this respect we may compare its energy liberation with the discharge of a gun. When the trigger is pulled all the powder in the cartridge is exploded; similarly whenever the heart contracts it uses up all the energy available at the time. Because of this it is necessary that the contraction be followed by a relaxation during which an accumulation of energy may prepare for the next contraction.

The evidence that all the available energy of the heart-muscle is used up at each systole is furnished by the existence of the refractory period. During this period, which coincides with the systole, external stimulation of the heart-muscle is altogether ineffective, although during diastole the heart responds to adequate stimulation by contraction. It is observed, also, that the irritability of the heart increases steadily from the end of the refractory period to the beginning of the next systole. We may assume, then, that during diastole there is a gradual replacement of the energy supply used up during the preceding systole, and that the more energy has accumulated the more irritable is the tissue.

The Passage of the Beat over the Heart. In the first paragraph of the chapter it was stated that the beat of the heart takes a certain course, beginning at the mouths of the great veins, spreading thence over the auricles, and passing from them to the ventricles. In all vertebrates there is a distinct pause between the contraction of the auricles and of the ventricles. In animals, such 
as the frog and turtle that have a pulsating sinus, there is likewise a pause between the contraction of the sinus and of the auricles.

If in a beating heart a cut be made between the sinus and the auricles so that they are completely separated, the sinus continues to beat exactly as before; the other chambers of the heart may not beat for a moment, but after a short interval usually resume activity. The rate of beat of these chambers under such circumstances is slower than that of the sinus. Similarly the ventricles may be separated from the auricles without affecting the auricular beat, but with the result that the ventricles either fail to beat at all, or beat at a much slower rate than the auricles. Such experiments as these show that the rhythmic power increases the nearer we go toward the venous end of the heart, and also that in the normal heart the most rhythmic portion imposes its rate on the rest of the organ. In order for the heart-rate to be determined as a whole by the beat of the venous end it is evident that there must be a conduction of the impulse to activity from one chamber to the next throughout the heart. This conduction moves over the heart in the form of a wave.

There are in the frog's heart two places and in that of the mammal one place where there is a delay in the passage of the contraction wave. These are, as already noted, at the junction of the sinus with the auricles and of the auricles with the ventricles. Anatomical study shows that at these junctions most of the cardiac tissue proper is replaced by connective tissue, so that physiological communication between one chamber and another is restricted to small bundles of conducting heart tissue. The delay at the junctions is usually explained as resulting from the small size of these conducting paths, which offer on that account considerable resistance to the passage of the contraction wave.

Neurogenic and Myogenic Theories of the Heart Beat. There are two questions of fundamental importance to an understanding of the mechanism of the heart's action. These are: (1) Does the rhythmic property of the heart reside in its muscular elements or in its nervous elements? and (2) Is the contraction wave conducted over the heart by muscle or by nerve-tissue? By the early students of the heart both these properties were attributed to its nervous elements as being more like nerve activities in general than like those of muscle; and also because the venous end of 
the heart, where the beat originates, contains more nervous matter than do the other chambers. More recently, the view that both rhythmicity and conductivity are cardinal functions of the heart's musculature began to receive considerable attention, chiefly through such observations as that the apex of the ventricle, which is devoid of nerve-cells, may be made to show true rhythmicity, and that a series of zigzag cuts, sufficient to sever all direct nerve paths although leaving ample muscular connections, can be made in the ventricle without preventing the passage of the contraction wave over it. With recognition of the probability that the nervous elements of the heart form, not a synaptic system with irreversible conduction, but an intercommunicating plexus which may conduct in all directions, most of the evidence in favor of the myogenic theory seems less conclusive than it did at first, so that the problems of which is the rhythmic and conducting tissue, or whether both properties are possessed by both tissues, are still far from settled.

The Nature of Automatic Rhythmicity. It should be clearly understood that the question whether rhythmicity is a property of cardiac muscle or of cardiac nerve-tissue is quite distinct from the question of the underlying nature of rhythmicity itself. Much study has been given to this latter problem and here again two opposing views are held. One of these is that the heart is subject to the influence of a constant stimulus, its property of "maximal" contractions with their accompanying refractory periods sufficing to bring about rhythmic responses to such constant stimulation. The other view is that the heart is a truly automatic organ, the metabolic processes going on within the heart tissue being of such a nature as to produce rhythmic activity quite independently of "stimulation" as we ordinarily understand it.

Those who believe the heart to be under the influence of a constant stimulus look to the blood as its source, and especially to the inorganic blood-salts, it having been shown that the heartbeat can be maintained for an astonishing length of time when the heart is fed solutions containing only inorganic salts of sodium, potassium, and calcium in proper proportion. Those who look upon the heart as a truly automatic organ take the position that their view is more in accordance with general physiological principles than the other, and that no evidence yet brought forth 
disproves their claim. They put the burden of proof upon the supporters of the "constant stimulus" theory. It must be admitted that at present no conclusive evidence for either view is available, nor are the supporters of either able to picture a satisfactory mechanism of rhythmicity in terms of their particular theory.

The Extrinsic Nerves of the Heart. The heart, as stated previously, is under the control of the autonomic system. It receives nerve-fibers both from the cranial and thoracico-lumbar systems. The cranial autonomic fibers reach it by way of the tenth cranial nerves, the vagi, the thoracico-lumbar by way of sympathetic ganglia. The vagus nerves give off their cardiac branches in the neck; the cardiac nerves from the thoracico-lumbar system arise from the inferior cervical ganglion, a sympathetic ganglion lying in the lower neck region. Both anatomically and physiologically the two sets of nerve-fibers are distinct. Anatomically the vagus fibers are pre-ganglionic; they arise from cell-bodies in the nucleus of the tenth nerve in the medulla and are myelinated. They terminate about nerve-cells lying on or within the heart itself. The fibers from the sympathetic system are post-ganglionic; they arise from cell-bodies in sympathetic ganglia, the inferior cervical for the most part, and are non-myelinated. They terminate in the tissues of the heart directly. Since nicotine cuts the connection between pre- and post-ganglionic fibers, application of that drug to the nerve-cells of the heart abolishes the influence of the vagi, but does not affect the thoracico-lumbar control at all.

Physiologically the vagus fibers are inhibitory; their stimulation slows and weakens the heart-beat. When very strongly stimulated they may bring the heart to a complete standstill, although in mammals the standstill is maintained for a few seconds only, the heart soon "breaking through" the inhibition. The thoracico-lumbar fibers have precisely the opposite function, being augmentor; their stimulation accelerates and strengthens the beat of the heart.

In addition to the efferent autonomic innervation just described the heart is provided with a set of afferent nerve-fibers. These reach the central nervous system either by way of the vagus nerves, or in some species of animals, rabbits for example, as separate nerve-trunks known as the depressor nerves. The 
function of these afferent fibers will be discussed in Chap. XXII in connection with the nervous control of the blood-vessels.

The Inhibitory and Augmentor. Centers. The control of the heart-beat is reflex in its nature, and like most other "vital" processes which are subject to reflex control is vested in certain "centers" of the medulla. Two heart-regulating centers are recognized, the cardio-inhibitory center and the cardio-augmentor center. The inhibitory center is in the nuclei of the tenth nerve. It is bilateral, each side containing half of it. The exact position of the augmentor center has not been determined. It is probably not a compact mass of cells as is the inhibitory center, but is scattered diffusely through the medulla.

Both these centers are in the path of all incoming impulses, and there is evidence that both of them are kept in constant "tonic" activity through the incessant play of stimuli upon them. Of recent years the view has been gaining ground that the tonic activity of the "vital" centers is maintained, in part at least, by chemical influences exerted through the blood. This influence has long been known to exist in the case of the respiratory center. That it is a factor in the regulation of the heart is only now coming to be believed.

The heart is thus constantly receiving both inhibitory and augmentor impulses, the former tending to diminish its activity, the latter to increase it. The actual heart-beat is the expression, therefore, of the balance between two opposing tendencies, and its increase or decrease indicates that one or the other has gained the advantage.

In attempting to analyze the causes of changes in the heartrate it must be remembered that an increase in rate may mean either an increase in the activity of the augmentor center, or a depression of the inhibitory center. Conversely, a decrease in rate may mean either a depression of the augmentor center or an increase in the activity of the inhibitory center. An observation that helps us in deciding which of the centers may have been responsible for any observed change is that the inhibitory mechanism acts much more promptly than does the augmentor. Any change that follows quickly an exciting cause is, therefore, to be attributed to the inhibitory mechanism. Since the heart, as a matter of fact, responds almost instantly to most influences we are in 
the habit of looking upon the augmentor mechanism as affording a fairly steady background of augmentor excitation upon which the inhibitory mechanism may play in delicate adjustment to the needs of the circulation. There is a perceptible quickening of the beat with any muscular movement, at least with any as extensive as that required to press a telegraph key. The quickening shows itself in the next beat after the beginning of the movement. The suggestion has been made that during the discharge of the exciting nervous impulses from the brain to the muscles, there is irradiation in the brain stem unto the inhibitory center; that this irradiation depresses the center, and so allows a quickening of the beat.

There are certain conditions in which the augmentor center seems to show heightened activity. After muscular exercise there is a more or less persistent acceleration of the heart that appears to be due to stimulation of the augmentor center by the waste products of muscular activity which persist for a time in the circulating blood. The acceleration of the heart in time of emotional stress or of great pain is to be explained, as stated previously, through the connection of the augmentor mechanism with the thoracico-lumbar autonomic, the emergency, system.

The familiar changes of heart-rate with changes of posture, slowed when lying, quickened with sitting or standing, are apparently the results of the redistribution of the blood over the Body under the influence of gravity. The quickening of the beat when one stands erect is undoubtedly an adaptation designed to overcome the tendency of the blood to accumulate in the lower parts of the Body when in this position; but how the adaptation is brought about is not known. Successive swallowing, as in sipping water, increases the heart-rate by depressing the inhibitory center. A blow over the stomach (the solar plexus) gives rise to afferent impulses which stimulate the inhibitory center; the heartrate is therefore diminished.

These are all illustrations of the general rule that the heartbeat may be modified by sensory stimulations. It is a matter of ordinary observation that many experiences, particularly those involving sensory impressions of high intensity, are accompanied by marked changes in heart-rate.

In connection with this analysis of the control of the heart- 
beat the importance of obtaining the proper viewpoint for considering physiological processes may well be emphasized. If one who has not studied the subject particularly be asked why running makes the heart beat faster he will probably answer that exercising muscles require more blood than resting ones, and that the heart beats faster to furnish this extra amount. A moment's thought shows that this statement, though quite true, does not really answer the question. It implies that the heart has knowledge of the needs of the tissues, which, of course, it cannot have. The increased heart-rate which accompanies exercise is undoubtedly an adaptive response, as are most reflex responses, but its explanation resides, not in the adaptation, but in the reflex mechanism which brings it about. We should be continually on guard against the tendency to explain physiological processes by their results rather than by the means by which the results are accomplished. 


\section{CHAPTER XXI}

\section{THE CIRCULATION OF THE BLOOD. BLOOD PRESSURE AND BLOOD-VELOCITY. THE PULSE}

The Flow of the Blood Outside of the Heart. The blood leaves the heart intermittently and not in a regular stream, a quantity being forced out at each systole of the ventricles: before it reaches the capillaries, however, this rhythmic movement is transformed into a steady flow, as may readily be seen by examining under the microscope thin transparent parts of various animals, as the web of a frog's foot, a mouse's ear, or the tail of a small fish. In consequence of the steadiness with which the capillaries supply the veins the flow in these is also unaffected, directly, by each beat of the heart; if a vein be cut the blood wells out uniformly, while from a cut artery the blood spurts out not only with much greater force, but in jets which are much more powerful at regular intervals corresponding with the systoles of the ventricles.

The Circulation of the Blood as seen in the Frog's Web. There is no more fascinating or instructive phenomenon than the circulation of the blood as seen with the microscope in the thin membrane between the toes of a frog's hind limb. Upon focusing beneath the epidermis a network of minute arteries, veins, and eapillaries, with the blood flowing through them, comes into view (Fig. 105). The arteries, $a$, are readily recognized by the fact that the flow in them is fastest and from larger to smaller branches. The latter are seen ending in capillaries, which form networks, the channels of which are all nearly equal in size. While in the veins arising from the capillaries the flow is from smaller to larger trunks, and slower than in the arteries, but faster than in the capillaries.

The reason of the slower flow of the capillaries is that their united area is considerably greater than that of the arteries supplying them, so that the same quantity of blood flowing through them in a given time has a wider channel to flow in and moves slowly. The area of the veins is smaller than that of the 
capillaries but greater than that of the arteries, and hence the rate of movement in them is also intermediate. Almost always when an artery divides, the area of its branches is greater than that of the main trunk, and so the arterial current becomes slower and slower from the heart onwards. In the veins, on the other hand, the area of a trunk formed by the union of two or more branches is less than that of the branches together, and the flow becomes quicker and quicker towards the heart. But even at the heart the united cross-sections of the veins entering the auricles are greater than those of the arteries leaving the ventricles, so that, since as much blood returns to the heart in a given time as leaves it, the rate of the current in the pulmonary veins and the venæ cavæ is less than in the pulmonary artery and aorta. We may represent the vascular system as a double cone, widening from the ventricles to the capillaries and narrowing from the latter to the auricles. Just as water forced in at a narrow end of this would flow quickest there and slowest at the widest part, so the blood flows quickest in the aorta and slowest in the capillaries, which taken together form a much wider channel.

The Axial Current and the Inert Layer. If a small artery in the frog's web be closely examined it will be seen that the rate of flow is not the same in all parts of it. In the center is a very rapid current carrying along all the red corpuscles and known as the axial stream, while near the wall of the vessel the flow is much slower, as indicated by the rate at which the pale blood-corpuscles are carried along in it. This is a purely physical phenomenon. If any liquid be forcibly driven through a fine tube which it wets, water for instance through a glass tube, the outermost layers of the liquid will remain nearly motionless in contact with the tube; the next layers of molecules will move a little, the next faster still; and so on until a rapid current is found in the center. If solid bodies, as powdered sealing-wax, be suspended in the water, these will all be carried on in the central faster current or axial stream, just as the red corpuscles are in the artery. The white corpuscles, partly because of their less specific gravity, and partly because of their sometimes irregular form, due to amœboid movements, get frequently pushed out of the axial current, so that many of them are found in the inert layer.

The Resistance to the Blood-Flow. As liquid flows through a 
tube there is a certain amount of friction between the moving liquid and the walls of the tube. There is also friction between the different concentric layers of the liquid, since each of them is moving at a different rate from that in contact with it on each side. This form of friction is known in hydrodynamics as "internal friction," and it is of great importance in the circulation of the blood. The friction increases very fast as the caliber of the tube through which the liquid flows diminishes: so that with the same rate of flow it is disproportionately much greater in a small tube than in a larger one. Hence a given quantity of liquid forced in a minute through one large tube would experience much less resistance from friction than if sent in the same time through four or five smaller tubes, the united transverse sections of which were together equal to that of the single larger one. In the bloodvessels the increased total area, and consequently slower flow, in the smaller channels partly counteracts this increase of friction, but only to a comparatively slight extent; so that the friction, and consequently the resistance to the blood-flow, is far greater in the capillaries and arterioles than in the small arteries, and in the small arteries than in the large ones. Practically we may regard the arteries as tubes ending in a sponge: the united areas of all the channels in the latter might be considerably larger than that of the supplying tubes, but the friction to be overcome in the flow through them would be much greater.

\section{The Conversion of the Intermittent into a Continuous Flow.} Since the heart sends blood into the aorta intermittently, we have still to inquire how it is that the flow in the capillaries is continuous. In the larger arteries it is not, since we can feel them dilating as the "pulse," on applying the finger over the radial artery at the wrist, or over the temporal artery on the side of the brow.

The first explanation which suggests itself is that since the capacity of the blood-vessels increases from the heart to the capillaries, an acceleration of the flow during the ventricular contraction which might be very manifest in the vessels near the heart would become less and less obvious in the more distant vessels. But if this were so, then when the blood was collected again from the wide capillary sponge into the great veins near the heart, which together are but little bigger than the aorta, we 
ought to find a pulse, but we do not: the venous pulse which sometimes occurs having quite a different cause, being due to a backflow from the auricles, or a checking of the on-flow into them, during the cardiac systole. The rhythm of the flow caused by the heart is therefore not merely cloaked in the small arteries and capillaries, but abolished in them.

We can, however, readily contrive conditions outside the Body under which an intermittent supply is transformed into a continuous flow. Suppose we have two vessels, $A$ and $B$ (Fig. 109) containing water and connected below in two ways: through the tube $a$ on which there is a pump provided with valves so that it can only drive liquid from $A$ to $B$; and through $b$, which may be left wide open or narrowed by the clamp $c$, at will. If the apparatus be left at rest the water will lie at the same level, $d$, in each vessel.

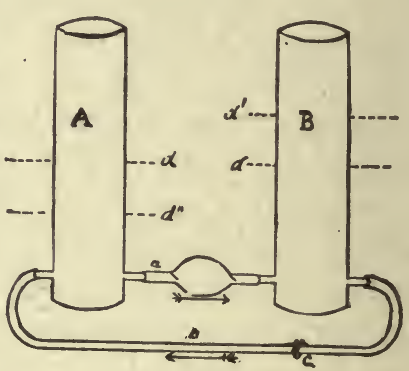

Fig. 109. If now we work the pump, at each stroke a certain amount of water will be conveyed from $A$ to $B$, and as result of the lowering of the level of liquid in $A$ and its rise in $B$, there will be immediately a return flow from $B$ to $A$ through the tube $b$. $A$, in these circumstances, would represent the venous system, from which the heart constantly takes blood to pump it into $B$, representing the arterial system; and $b$ would represent the capillary vessels through which the return flow takes place; but, so far, we should have as intermittent a flow through the capillaries, $b$, as through the heart-pump, $a$. Now imagine $b$ to be narrowed at one point so as to oppose resistance to the back-flow, while the pump goes on working steadily. The result will be an accumulation of water in $B$, and a fall of its level in $A$. But the more the difference of level in the two vessels increases, the greater is the force tending to drive water back through $b$ to $A$, and more will flow back, under the greater difference of pressure, in a given time, until at last, when the water in $B$ has reached a certain level, $d^{\prime}$, and that in $A$ has correspondingly fallen to $d^{\prime \prime}$, the current through $b$ will carry back in one minute just so much water as the pump sends the other way, and this back-flow will be nearly constant; 
it will not depend directly upon the strokes of the pump, but upon the head of water accumulated in $B$; which head of water will, it is true, be slightly increased at each stroke of the pump, but the increase will be very small compared with the whole driving force, and its influence will be inappreciable. We thus gain the idea that an incomplete impediment to the flow from the arteries to the veins (from $B$ to $A$ in the diagram), such as is afforded by friction in the capillaries, may bring about conditions which will lead to a steady flow along the latter vessels.

But in the arterial system there can be no accumulation of blood at a higher level than that in the veins, such as is supposed in the above apparatus; and we must next consider if the "head of water" can be replaced by some other form of driving force. It is in fact replaced by the elasticity of the large arteries. Suppose an elastic bag instead of the vessel $B$ connected with the pump " $a$." If there be no resistance to the back-flow the current through $b$ will be discontinuous. But if resistance be interposed, then the elastic bag will become distended, since the pump sends in a given time more liquid into it than it passes back through $b$. But the more it becomes distended the more will the bag squeeze the liquid inside and the faster will it send that back to $A$, until at last its squeeze is so powerful that each minute or two or five minutes it sends back into $A$ as much as it receives. Thenceforth the back-flow through $b$ will be practically constant, being immediately dependent upon the elastic reaction of the bag, and only indirectly upon the action of the pump which keeps it distended. Such a state of things represents very closely the phenomena occurring in the blood-vessels. The highly elastic large arteries are kept stretched with blood by the heart; and the reaction of their elastic walls, steadily squeezing on the blood in them, forces it continuously through the small arteries and capillaries. The steady flow in the latter depends thus on two factors: first, the elasticity of the large arteries; and secondly, the resistance to their emptying, dependent upon internal friction in the small arteries and the capillaries, which calls into play the elasticity of the large vessels. Were the capillary resistance or the arterial elasticity absent the blood-flow in the capillaries would be rhythmic.

Weber's Schema. It is clear from the statements made in the last paragraph that it is the pressure exerted by the elastic arteries 
upon the blood inside them which keeps up the flow through the capillaries, the heart serving to keep the big arteries tightly filled and so to call the elastic reaction of their walls into play. The whole circulation depends primarily, of course, upon the beat of the heart; but this only indirectly governs the capillary flow, and since the latter is the aim of the whole vascular apparatus, it is of great importance to know as much as possible about arterial pressure; not only how great it is on the average, but how it is altered in different vessels in various circumstances so as to make the flow through the capillaries of a given part greater or less according to circumstances; for, as blushing and pallor of the face (which frequently occur without any change in the skin elsewhere) prove, the quantity of blood flowing through a given part is not always the same, nor is it always increased or diminished in all parts of the Body at the same time. Most of what we know about arterial pressure has been ascertained by experiments made upon the lower animals, from which deductions are then made concerning what happens in man, since Anatomy shows that the circulatory organs are arranged upon the same plan in all the mammalia. A great deal can, however, be learnt by studying the flow of liquids through ordinary elastic tubes. Suppose we have a set of such (Fig. 110) supplied at one point with a pump, $c$, possessing valves of entry and exit which open only in the direction indicated by the arrows, and that the whole system is slightly overfilled with liquid so that its elastic walls are slightly stretched. These will in consequence press upon the liquid inside them and the amount of this pressure will be indicated by the gauges; so long as the pump is at rest it will be the same everywhere (and therefore equal in the gauges on $B$ and $A$ ), since liquid in a set of horizontal tubes communicating freely, as these do at $D$, always distributes itself so that the pressure upon it is everywhere the same. Let the pump $c$ now contract once, and then dilate: during the contraction it will empty itself into $B$ and during the dilatation fill itself from $A$. Consequently the pressure in $B$, indicated by the gauge $x$, will rise and that in $A$ will fall. But very rapidly the liquid will redistribute itself from $B$ to $A$ through $D$, until it again exists everywhere under the same pressure. Every time the pump works there will occur a similar series of phenomena, and there will be a disturbance of equilibrium causing a 
wave to flow round the tubing; but there will be no steady maintenance of a pressure on the side $B$ greater than that in $A$. Now let the upper tube $D$ be closed so that the liquid to get from $B$ to $A$ must flow through the narrow lower tubes $D^{\prime}$, which oppose considerable resistance to its passage on account of their frequent branchings and the great friction in them; then if the pump works frequently enough there will be produced and maintained in $B$ a pressure considerably higher than that in $A$. If, for example, the pump works 60 times a minute and at each stroke takes 180

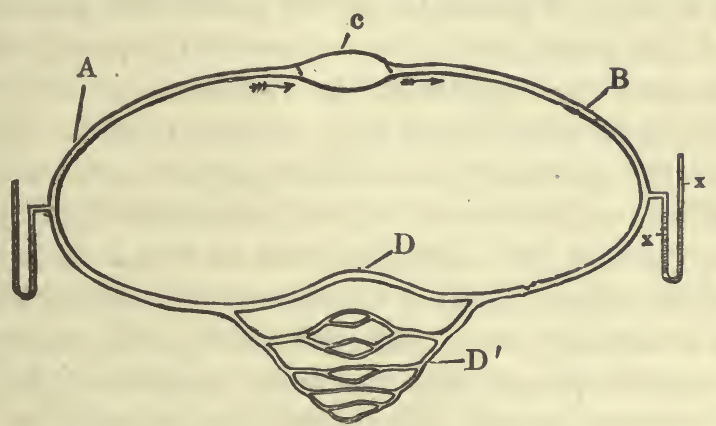

Fig. 110.-Diagram of Weber's Schema.

cubic centimeters of liquid (6 ounces) from $A$ and drives it into $B$, the quantity sent in at the first stroke will not (on account of the resistance to its flow offered by the small branched tubes), have all got back into $A$ before the next stroke takes place, sending 180 more cubic centimeters (6 ounces) into $B$. Consequently at each stroke $B$ will become more and more distended and $A$ more and more emptied, and the gauge $x$ will indicate a much higher pressure than that on $A$. As $B$ is more stretched, however, it squeezes harder upon its contents, until at last a time comes when this squeeze is powerful enough to force through the small tubes just 180 cubic centimeters ( 6 ounces) in a second. Then further accumulation in $B$ ceases. The pump sends into it 10,800 cubic centimeters (360 ounces) in a minute at one end and it squeezes out exactly that amount in the same time from its other end; and so long as the pump works steadily the pressure in $B$ will not rise, nor that in $A$ fall, any more. But under such circumstances the flow through the small tubes will be nearly constant since it depends upon the difference in pressure prevailing between $B$ and 
$A$, and only indirectly upon the pump which serves simply to keep the pressure high in $B$ and low in $A$. At each stroke of the pump it is true there will be a slight increase of pressure in $B$ due to the fresh 180 cubic centimeters (6 ounces) forced into it, but this increase will be but a small fraction of the total pressure and so have but an insignificant influence upon the rate of flow through the small connecting tubes.

Arterial Pressure. The condition of things just described represents very closely the phenomena presented in the blood-vascular system, in which the ventricles of the heart, with their auriculoventricular and semilunar valves, represent the pump, the smallest arteries and the capillaries the resistance at $D^{\prime}$, the large arteries the elastic tube $B$, and the veins the tube $A$. The ventricles constantly receiving blood through the auricles from the veins, send it into the arteries, which find a difficulty in emptying themselves through the capillaries, and so blood accumulates in them until the elastic reaction of the stretched arteries is able to squeeze in a minute through the capillaries just so much blood as the left ventricle pumps into the aorta, and the right into the pulmonary artery, in the same time. Accordingly in a living animal a pressure-gauge connected with an artery shows a much higher pressure than one connected with a vein, and this persisting difference of pressure, only increased by a small fraction of the whole at each heart-beat, brings about a steady flow from the arteries to the veins. The heart keeps the arteries stretched and the stretched arteries maintain the flow through the capillaries, and the constancy of the current in them depends on two factors: (1) the resistance experienced by the blood in its flow from the ventricles to the veins, and (2) the elasticity of the larger arteries which allows the blood to accumulate in them under a high pressure, in consequence of this resistance.

Since the blood flows from the aorta to its branches and from these to the capillaries and thence to the veins, and liquids in a set of continuous tubes flow from points of greater to those of less pressure, it is clear that the blood-pressure must constantly diminish from the aorta to the right auricle; and similarly from the pulmonary artery to the left auricle. At any point, in fact, the pressure is proportionate to the resistance in front, and since the farther the blood has gone the less of this, due to impediments 
at branchings and to internal friction, it has to overcome in finishing its round, the pressure on the blood diminishes as we follow it from the aorta to the venæ cavæ. In the larger arteries the fall of pressure is gradual and small, since the amount of resistance met with in the flow through them is but little. In the small arteries and capillaries the resistance overcome and left behind is (on account of the great internal friction due to their small caliber) very great, and consequently the fall of pressure.between the medium-sized arteries and the veins is rapid and considerable.

Modifications of Arterial Pressure by Changes in the Heart-beat. A little consideration will make it clear that the pressure prevailing at any time in a given artery depends on two things- the rate at which the vessel is filled, $i$. e., upon the amount of work done by the heart; and the ease or difficulty with which it is emptied, that is, upon the resistance in front. A third factor has to be taken into account in some cases; namely, that when the muscular coats of the small arteries contract the local capacity of the vascular system is diminished, and has to be compensated for by greater distention elsewhere, and vice versa. This would of itself of course bring about changes in the pressure exerted on the contained liquid, but for the present it may be left out of consideration. If we suppose a system such as represented in Fig. 110, to be in equilibrium, with the pump injecting into $B$ a certain volume of liquid per minute, and the elastic tension of the tube $B$ just sufficient to force that volume through the resistance $D^{\prime}$ in the same time, it is clear that the pressure indicated on the gauge $x$ will be very nearly constant. If, now, the volume of liquid forced into $B$ in a minute be increased, either by the pump working faster or by its pumping more at each stroke, there will evidently be an accumulation in $B$, since its tension is adjusted to force out the less volume per minute, but this accumulation, by stretching the tube still more, increases its elastic tension, so that this is presently great enough to force out the added volume as fast as it comes in. The pressure-gauge will now stand at a higher point, showing that the contents of the tube are under greater pressure than before. Similarly, a diminution in the influx of liquid into $B$ will be followed by a fall of pressure within it as the walls of the tube adjust themselves to the smaller volume to be forced out per minute. Precisely the same reasoning may 
be applied to the vascular system for determining the effects upon arterial pressure of changes in the heart-beat.

\section{Modifications of Arterial Pressure by Changes in the Peripheral} Resistance. If while the pump $c$ in Fig. 110 is steadily sending a given volume of liquid per minute into $B$ the resistance at $D^{\prime}$ increase, it is clear arterial pressure must rise. For $B$ is only stretched enough to squeeze out in a minute the given quantity of liquid against the original resistance, and cannot at first send out that quantity against the greater. Liquid will consequently accumulate in it until at last it becomes stretched enough to send out as much in a minute as before in spite of the greater resistance to be overcome. A new mean pressure at a higher level will then be established. If, on the contrary, the resistance diminishes while the pump's work remains the same, then $B$ will at first squeeze out in a minute more than it receives, until finally its elastic pressure is reduced to the point at which its receipts and losses balance, and a new and lower mean pressure will be established in $B$.

Similarly in the vascular system, increase of the peripheral resistance by narrowing of the small arteries will increase arterial pressure in all parts nearer the heart, while dilatation of the small arteries will have the contrary effect.

Summary. We find then that arterial pressure at any moment is dependent upon: (1) the quantity of blood forced into the arteries in a given time; (2) the caliber of the smaller vessels. Both of these and consequently the capillary circulation which depends upon arterial pressure, are under the control of the nervous system (see Chaps. XX and XXII).

The Pulse. When the left ventricle contracts it forces a certain amount of blood into the aorta, which is already distended and on account of the resistance in front cannot empty itself as fast as the contracting ventricle fills it. As a consequence its elastic walls yield still more-it enlarges both transversely and longitudinally and if exposed in a living animal can be seen and felt to pulsate, swelling out at each systole of the heart, and shrinking and getting rid of the excess during the pause. A similar phenomenon can be observed in all the other large arteries, for just as the contracting ventricle fills the aorta faster than the latter empties (the whole period of diastole and systole being 
required by the aorta to pass on the.blood sent in during systole), so the increased tension in the aorta immediately after the cardiac contraction drives on some of its contents into its branches, and fills these faster than they are emptying, and so causes a dilatation of them also, which only gradually disappears as the aortic tension falls before the next systole. Hence after each beat of the heart there is a sensible dilatation of all the larger arteries, known as the pulse, which becomes less and less marked at points on the smaller branches farther from the heart, but which in health can readily be recognized on any artery large enough to be felt by the finger through the skin. The radial artery near the wrist, for example, will always be felt tense by the finger, since it is kept overfilled by the heart in the way already described: But after each heart-beat it becomes more rigid and dilates a little, the increased distension and rigidity gradually disappearing as the artery passes on the excess of blood before the next heartbeat. The pulse is then a wave of increased pressure started by the ventricular systole, radiating from the semilunar valves over the arterial system, and gradually disappearing in the smaller branches. In the aorta the pulse is most marked, for the resistance there to the transmission onwards of the blood sent in by the heart is greatest, and the elastic tube in which it consequently accumulates is shortest, and so the increase of pressure and the dilatation caused are considerable. The aorta, however, gradually squeezes out the excess blood into its branches, and so this becomes distributed over a wider area, and these branches having less resistance in front find less and less difficulty in passing it on; consequently the pulse-wave becomes less and less conspicuous and finally altogether disappears before the capillaries are reached, the excess of liquid in the whole arterial system after a ventricular systole being too small to raise the mean pressure sensibly once it has been widely distributed over the elastic vessels, which is the ease by the time the wave has reached the small branches which supply the capillaries.

The pulse-wave travels over the arterial system at the rate of about 9 meters (29.5 feet) in a second, commencing at the wrist 0.159 second, and in the posterior tibial artery at the ankle 0.193 second, after the ventricular systole. The blood itself does not of course travel as fast as the pulse-wave, for that quantity sent 
into the aorta at each heart-beat does not immediately rush on over the whole arterial system, but by raising the local pressure causes the vessel to squeeze out faster than before some of the blood it already contains, and this entering its branches raises the pressure in them and causes them more quickly to fill their branches and raise the pressure in them; the pulse-wave or wave of increased pressure is transmitted in this way much faster than any given portion of the blood. How the wave of increased pressure and the liquid travel at different rates may be made clearer perhaps by picturing what would happen if liquid were pumped into one end of an already full elastic tube, closed at the other end. At the closed end of the tube a dilatation and increased tension would be felt immediately after each stroke of the pump, although the liquid pumped in at the other end would have remained about its point of entry; it would cause the pulsation not by flowing along the tube itself, but by giving a push to the liquid already in it. If instead of absolutely closing the distal end of the tube one brought about a state of things more nearly resembling that found in the arteries by allowing it to empty itself against a resistance, say through a narrow opening, the phenomena observed would not be essentially altered; the increase of pressure would travel along the distended tube far faster than the liquid itself.

The pulse being dependent on the heart's systole, "feeling the pulse" of course primarily gives a convenient means of counting the rate of beat of that organ. To the skilled touch, however, it may tell a great deal more, as for example whether it is a readily compressible or "soft pulse" showing a low arterial pressure, or tense and rigid ("a hard pulse") indicative of high arterial pressure, and so on. In adults the normal pulse-rate may vary from sixty-five to seventy-five, the most common number being seventytwo. In the same individual it is faster when standing than when sitting, and when sitting than when lying down. Any exercise increases its rate temporarily, and so does excitement; a sick person's pulse should not therefore be felt when he is nervous or excited (as the physician knows when he tries first to get his patient calm and confident), as it is then difficult to draw correct conclusions from it. In children the pulse is quicker than in adults, and in old age slower than in middle life. 
The Measurement of Blood-Pressure. Direct determinations of arterial and venous pressures are made in living, anesthetized animals by inserting into a large artery or vein a glass tube connected with a pressure-gauge. The usual form of gauge for such work is the mercury manometer represented in Fig. 111. This

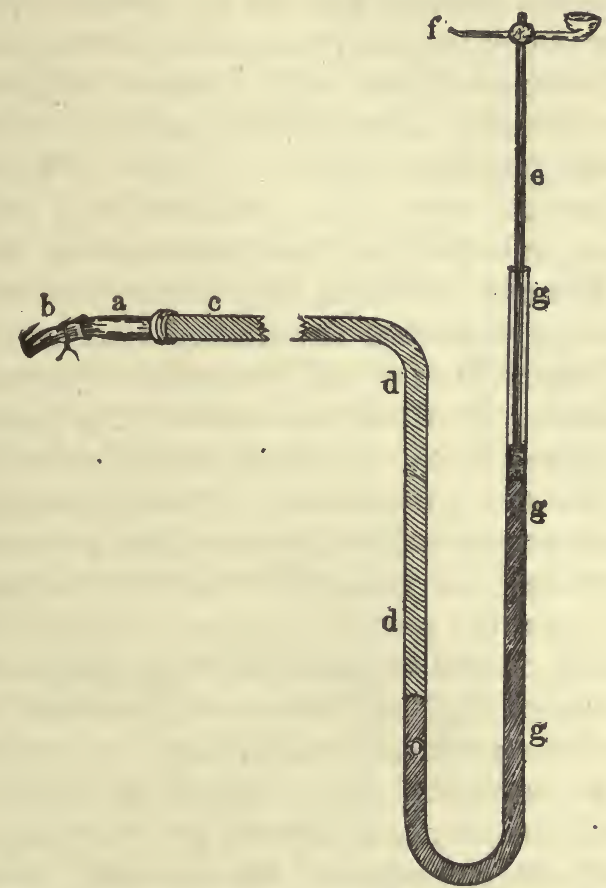

Fig. 111.-Mercury manometer for recording blood-pressure. $d g$, glass $U$-tube partly filled with mercury. In one limb is borne a float, $e$, bearing a recording device $f$; the other limb is filled with a suitable liquid and connected water-tight with the heart end of a divided artery $b$, by means of glass connection $a$. Changes in the mercury level indicate changes of arterial pressure.

instrument, on account of the great inertia of mercury, follows only slightly the rapid fluctuations of pressure due to the beats of the heart. It therefore gives mean or average pressures. Results obtained with mercury manometers are expressed in terms of the height of the mercury column sustained by the bloodpressure. To reduce them to columns of blood they must be multiplied by 13.6, the number of times mercury is heavier than blood. The mean aortic pressure in average-sized dogs is ordi- 
narily not far from 170 millimeters of mercury. 'The pressure in the veins diminishes from 3 or 4 millimeters of mercury in the large veins of the front leg to zero at the entrance to the auricle (see p. 362).

Blood-Pressure in Man. In man it is necessary to determine blood-pressures by methods that do not involve operative procedure. Various devices are in use for this purpose. Most of them depend on the fact that bodily tissues, being for the most part liquid, are virtually incompressible and so transmit throughout their extent pressures applied to them. For determining arterial pressures the upper arm is inclosed in a cuff of hollow rubber tubing so arranged that its inflation presses from all sides on the arm. The cuff is inflated until its pressure on the arm is just sufficient to squeeze shut the brachial artery. By means of a manometer attached to the cuff the amount of pressure applied can be determined. The differences between the various forms of instruments depend chiefly on their methods for determining exactly when the artery is occluded. These instruments do not give mean blood-pressures, as does the mercury manometer, but maximum (systolic) and minimum (diastolic) pressures. It is found that in man the systolic pressure averages from 110 to $120 \mathrm{mms}$. of mercury, and the diastolic about $65 \mathrm{mms}$. of mercury.

Determinations of capillary and venous pressures in man can be made more easily than determinations of arterial pressure because there are superficial capillaries and veins whose occlusion can be observed directly; in capillaries by whitening of the skin, in veins by the disappearance of the vein-ridge along it. The basis of the method is the same as for arterial pressure, namely, determination of the pressure necessary to occlude the vessel. Capillary pressures measured by this method average about $30 \mathrm{mms}$. of mercury; venous pressures $10 \mathrm{mms}$. or less.

The Rate of the Blood-Flow. As the vascular system becomes more capacious from the aorta to the capillaries the rate of flow in it becomes proportionately slower, and as the total area of the channels diminishes again from the capillaries to the venæ cavæ, so does the rate of flow quicken, just as a river current slackens when it spreads out, and flows faster when it is confined to a narrower channel; a fact taken advantage of in the construction of Eads' jetties at the mouth of the Mississippi, the object 
of which is to make the water flow in a narrower channel and so with a more rapid current in that part of the river. Actual measurements as to the rate of flow in the arteries cannot be made on man, but from experiments on lower animals it is calculated that in the human carotid the blood flows about 400 millimeters (16 inches) in a second. In the capillaries the current travels only from 0.5 to $0.75 \mathrm{~mm}$. $\left(\frac{1}{50}\right.$ to $\frac{1}{33}$ inch) in a second. The total time taken by a portion of blood in making a complete circulation has been measured by injecting some easily detected substance into an artery on one side of the body and noting the time which elapses before it can be found in a corresponding vein on the opposite side. In dogs this time is 15 seconds, and it is calculated for man at about 23 seconds. Of this total about a second is spent in the systemic and another second in the pulmonary capillaries, as each portion of blood on its course from the last artery to the first vein passes through a length of capillary which on the average is $0.5 \mathrm{~mm}$. ( $\frac{1}{50}$ inch). The rate of flow in the great veins is about $100 \mathrm{~mm}$. (4 inches) in a second, but is subject to considerable variations dependent on the respiratory and other movements of the Body; in the small veins it is much slower.

Secondary Factors Affecting the Circulation. While the heart's beat is the great driving force of the circulation, certain other things help more or less-viz., gravity, compression of the veins, and aspiration of the thorax. All of them are, however, quite subsidiary; experiment on the dead Body shows that the injection of defibrinated blood into the aorta under a less force than that exerted by the left ventricle during life is more than sufficient to drive it round and back by the venæ cavæ.

The Influence of Gravity. Under ordinary circumstances this may be neglected, since in parts of the Body below the level of the heart it will assist the flow in the arteries and impede it equally in the veins, while the reverse is the case in the upper parts of the Body. In certain cases, however, it is well to bear these points in mind. A part "congested" or gorged with blood should if possible be raised so as to make the back-flow in its veins easier; and sometimes when the heart is acting feebly it may be able to drive blood along arteries in which gravity helps, but not otherwise. Accordingly in a tendency to fainting it is best to lie down, and 
make it easier for the heart to send blood up to the brain, deficiency in its blood-supply being the cause of the loss of consciousness in a fainting-fit. 'In fact, so long as the breathing continues, the aspiration of the thorax will keep up the venous flow (see below), while, in the circumstances supposed, a slight diminution in the resistance opposed to the arterial flow may be of importance. The head of a person who has fainted should accordingly never be raised until he has undoubtedly recovered, a fact rarely borne in mind by spectators, who commonly rush at once to lift any one whom they see fall in the street or elsewhere.

The Influence of Transient Compression of the Veins. The valves of the veins being so disposed as to permit only a flow towards the heart, when external pressure empties a vein it assists the circulation. Continuous pressure, as by a tight garter, is of course bad, since it checks all subsequent flow through the vessel; but intermittent pressure, such as is exerted on many veins by muscles in the ordinary movements of the Body, acts as a pump to force on the blood in them.

The value of this pumping of the blood out of the veins by muscular movements is well illustrated by comparing two classes of workers whose occupations require that they be upon their feet continuously for hours. The condition of varicose veins, which is a stasis of blood in the superficial veins of the lower extremities, is very prevalent among motormen, and others who must stand still for long periods, but is virtually unknown among postmen, who are walking during the time spent on their feet.

The valves of the veins have another use in diminishing the pressure on the lower part of those vessels in many regions. If, for instance, there were no valves in the long saphenous vein of the leg the considerable weight of the column of blood in it, which in the erect position would be about a meter (39 inches) high, would press on the lower part of the vessel. But each set of valves in it carries the weight of the column of blood between it and the next set of valves above, and relieves parts below, and so the weight of the column of blood is distributed and does not all bear on any one point.

Aspiration of the Thorax (see also p. 399). Whenever a breath is drawn the pressure of the air on the vessels inside the chest is diminished, while that on the other vessels of the Body is unaf- 
fected. In consequence blood tends to flow into the chest. It cannot, however, flow back from the arteries on account of the semilunar valves of the aorta, but it can readily be pressed, or in common language "sucked," into the great veins close to the heart and into the right auricle of the latter. The details of this action must be omitted until the respiratory mechanism has been considered. All parts of the pulmonary circuit being within the thorax, the respiratory movements do not directly influence it, except in so far as the distention or collapse of the lungs alters the caliber of their vessels.

The considerable influence of the respiratory movements upon the venous circulation can be readily observed. In thin persons the jugular vein in the neck can often be seen to empty rapidly and collapse during inspiration, and fill up in a very noticeable way during expiration, exhibiting a sort of venous pulse. Every one, too, knows that by making a violent and prolonged expiration, as exhibited for example by a child with whooping-cough, the flow in all the veins of the head and neck may be checked, causing them to swell up and hinder the capillary circulation until the person becomes "black in the face," from the engorgement of the small vessels with dark-colored venous blood.

In diseases of the tricuspid valve another form of venous pulse is often seen in the superficial veins of the neck, since at each contraction of the right ventricle some blood is driven back through the right auricle into the veins.

Proofs of the Circulation of the Blood. The ancient physiologists believed that the movement of the blood was an ebb and flow, to and from each side of the heart, and out and in by both arteries and veins. They had no idea of a circulation, but thought pure blood was formed in the lungs and impure in the liver, and that these partially mixed in the heart through minute pores supposed to exist in the septum. Servetus, who was burnt alive by Calvin in 1553, first stated that there was a continuous passage through the lungs from the pulmonary artery to the pulmonary veins, but the great Englishman Harvey first, in lectures delivered in the College of Physicians of London about 1616, demonstrated that the movement of the blood was a continuous circulation as we now know it, and so laid the foundation of modern Physiology. In his time. however, the capillary vessel kad not been 
discovered, so that although he was quite certain that the blood got somehow from the final branches of the aorta to the radicles of the venous system, he did not exactly know how.

The proofs of the course of the circulation are at present quite conclusive, and may be summed up as follows: (1) Blood injected into an artery in the dead Body will return by a vein; but injected into a vein will not pass back by an artery. (2) The anatomical arrangement of the valves of the heart and of the veins shows that the blood can only flow from the heart, through the arteries and back to the heart by the veins. (3) A cut artery spurts from the end next the heart, a cut vein bleeds most from the end farthest from the heart. (4) A portion of a vein when emptied fills only from the end farthest from the heart. This observation can be made on the veins on the back of the hand of any thin person, especially if the vessels be first gorged by holding the hand in a dependent position for a few seconds. Select then a vein which runs for an inch or so without branching, place a finger on its distal end, and then empty it up to its next branch (where valves usually exist) by compressing it from below up. The vessel will then be found to remain empty as long as the finger is kept on its lower end, but to fill immediately when it is removed; which proves that the valves prevent any filling of the vein from its heart-end backwards. (5) If a bandage be placed around the arm, so as to close the superficial veins, but not tight enough to occlude the deeper-seated arteries, the veins on the distal side of the bandage will become gorged and those on its proximal side empty, showing again that the veins only receive blood from their ends turned towards the capillaries. (6) In the lower animals direct observation with the microscope shows the steady flow of blood from the arteries through the capillaries to the veins, but never in the opposite direction. 


\section{CHAPTER XXII}

\section{THE VASOMOTOR MECHANISM. SLEEP. THE LYMPHATIC SQYSTEM}

The Distribution of Blood Among Various Parts of the Body. In the nervous control of the heart-beat we have, as already noted, a mechanism whereby the blood-flow through the Body as a whole can be modified in accordance with the needs of the organism. In the vasomotor mechanism we have an arrangement, equally important, whereby individual organs or regions can be furnished with more or less blood as their activities require without the necessity of involving the whole circulation.

The Nerves of the Blood-Vessels. The arteries, as already pointed out, possess a muscular coat composed of fibers arranged around them, so that their contraction can narrow the vessels. This coat is most prominent in the smaller vessels, the arterioles. These vascular muscles are under the control of certain special nerves called vasomotor, and these latter can thus govern the amount of blood reaching any organ at a given time. The vasomotor nerves belong to the autonomic system. Their physiology is therefore the application to special structures of the general principles laid down in connection with that system (Chap. XII).

In the heart we had to consider a rhythmically contracting organ the force of whose contractions could be increased or diminished by the influence of extrinsic nerves; in the arteries, speaking broadly, we have to deal with muscle in a condition of tonic or constant contraction, which contraction can be increased by impulses coming through excitor or vasoconstrictor nerves, and diminished through the activity of inhibitory or vasodilator nerves. The general tonic contraction of the arterial muscle is, however, much more dependent on the vasoconstrictor nervefibers than is the beat of the heart on the cardio-excitor nerves. The inhibitory (dilator) set of vasomotor nerves have a much less extensive distribution over the arterial system than the constrictor. 
The Vasoconstrictor Nerves. If the ear of a white rabbit be held up against the light while the animal is kept quiet and not alarmed, the red central artery can be seen coursing along the translucent organ, giving off branches which by subdivision become too small to be separately visible, and the whole ear has a pink color and is warm from the abundant blood flowing through it. Attentive observation will show also that the caliber of the main artery is not constant; at somewhat irregular periods of a minute or more it dilates and coritracts a little.

If the sympathetic trunk have been previously divided on the other side of the neck of the animal, the ear on that side will present a very different appearance. Its arteries will be much dilated and the whole ear fuller of blood, redder, and distinctly warmer; the slow alternating variations in arterial diameter also have disappeared. We get thus evidence that the normal mean caliber of the artery is maintained by influences reaching its muscular coat through the cervical sympathetic. Stimulation of the upper end of the cut nerve confirms this opinion. It is then seen that the arteries of the corresponding ear gradually contract until even the main vessel can hardly be seen, and in consequence the whole ear becomes pale and cold. After the stimulation is stopped the arteries again slowly dilate until they have regained their full paralytic size.

Quite similar phenomena can be observed in transparent parts of other living animals, as in the web of a frog's foot, the arteries of which dilate after section of the sciatic nerve and constrict when the peripheral end of the nerve is stimulated. In the case of other parts changes in temperature may be used to detect alterations in the flow of blood. In a dog or cat, for example, a sensitive thermometer placed between the toes indicates a rise of temperature, owing to increased flow of warm blood through the skin, after section of the chief nerve of the limb, and a fall of temperature (usually) during stimulation of the peripheral end of the divided nerve.

When the vasoconstrictor nerves cut are those controlling a large number of arteries, the dilatation of the latter so much diminishes peripheral resistance to the blood-flow as to lead to a marked fall of general arterial pressure; and, due care being taken to avoid or to allow for concomitant variations in the rate or 
force of the heart's beat, this gives us another useful method of studying the distribution of the nerves concerned. For example, the splanchnic nerves are branches which spring from the thoracic portion of the sympathetic chain and pass through the diaphragm to end in the solar plexus from which nerves pass to the arteries of most of the abdominal viscera. The region whose blood-vessels are innervated by these nerves is often spoken of as the splanchnic region. When the splanchnic nerves are cut on both sides arterial pressure falls enormously, from say 120 millimeters of mercury in the carotid of a dog to 15 or 20 millimeters, most of the blood of the Body lying almost stagnant in the dilated blood-vessels of the abdomen. On the other hand, stimulation of the splanchnic nerves so diminishes the paths open for the circulation of the blood as to increase general blood-pressure enormously.

The skin and the abdominal organs seem to be the predominant localities of distribution of the vasoconstrictor nerves: other parts have them, but not in quantity sufficient to bring about any great general change in the blood-flow.

The Vasoconstrictor Center. This, one of the most important of the "vital" centers of the medulla, has not been identified anatomically with any particular group of nerve-cells, but its location is quite sharply defined physiologically. There is a small region of the medulla, known as the "vital knot," whose destruction is promptly fatal to the life of the organism. This region includes, in addition to at least one other "center," the vasoconstrictor center. From this center there is a constant outflow of impulses to all those arterioles of the Body whose muscles contain vasoconstrictor nerve-endings. This constant stream of constrictor impulses is the chief factor in the maintenance of socalled vasomotor tone, a condition of continuous moderate constriction of the arterioles by which general arterial pressure is kept at the proper level.

It is probable that the vasoconstrictor center consists physiologically of a number of associated centers which may act as a unit or separately. These "partial" centers are in connection with restricted vasomotor areas, and thus are enabled to bring about local vasomotor effects.

The Control of the Vasoconstrictor Center. This center, like the other "vital ' centers of the medulla, is kept in activity in 
part reflexly, and in part, probably, through chemical stimulation brought by way of the blood. The whole stream of afferent impulses passing through the medulla plays upon it. Like the centers for controlling the heart-beat its activity may be increased through the influx of stimuli into it, or it may suffer depression for the same cause. We divide afferent impulses affecting the vasoconstrictor center, therefore, into two groups: those increasing its activity, pressor impulses, and those diminishing it, depressor impulses. Certain sorts of stimuli are generally pressor in effect; pain, for example, usually brings about a reflex rise of bloodpressure through stimulating the vasoconstrictor center; cold on the skin acts similarly. It is possible that other stimuli may be pressor or depressor according to circumstances.

The Depressor Nerve. The best known nerve-tract which carries depressor impulses uniformly has already been mentioned. It is the afferent tract from the heart known, in animals where it is present as a separate trunk, as the depressor nerve. Stimulation of this nerve brings about, always, a reflex fall of blood-pressure, which is due mainly to vasodilation resulting from depression of the vasoconstrictor center. This nerve rises, not in heart tissue proper, but in the walls of the aorta near where that vessel springs from the heart. An undue increase in blood-pressure, such as might affect the heart injuriously, subjects the aortic wall to unusual tension. This seems to stimulate the depressor nerve mechanically. Thus the heart is protected against injury arising from working against too great resistance.

Taking Cold. This common condition is not unfrequently the indirect result of undue reflex excitement of the vasomotor center. Chilling of the skin beyond a certain point stimulates, through the afferent nerves, the portion of the vasomotor center governing the skin arteries, and the latter become contracted, as shown by the pallor of the surface. This has a twofold influence-in the first place, more blood is thrown into internal parts, and in the second, contraction of the arteries over so much of the Body considerably raises the general blood-pressure. Consequently the vessels of internal parts become overgorged or "congested," a condition which is especially favorable to invasion by the organisms which cause colds. The best preventive is to wear, when exposed to great changes of temperature, a woolen or at least a cotton gar- 
ment over the trunk of the Body; linen is so good a conductor of heat that it permits any change in the external temperature to act almost at once upon the surface of the Body. After an unavoidable exposure to cold or wet the thing to be done is of course to restore the cutaneous circulation; for this purpose movement should be persisted in, and a thick dry outer covering put on, until warm and dry underclothing can be obtained.

For healthy persons a temporary exposure to cold, as a plunge in a bath, is good, since in them the sudden contraction of the cutaneous arteries soon passes off and is succeeded by a dilatation causing a warm healthy glow on the surface. If the bather remain too long in cold water, however, this reaction passes off and is succeeded by a more persistent chilliness of the surface, which may even last all day. The bath should therefore be left before this occurs, but no absolute time can be stated, as the reaction is more marked and lasts longer in strong persons, and in those used to cold bathing, than in others.

Vasodilator Nerves. We have already noticed, in connection with the control of the vasoconstrictor center, one method by which dilation of arterioles may be secured, namely, by inhibition of the tonic activity of vasoconstrictor fibers. Frequently, however, in the Body this is managed in another way; by efferent vasodilator nerves which inhibit, not the vasoconstrictor center, but the muscles of the blood-vessels directly. The nerves of the skeletal muscles for example contain two sets of efferent fibers: one motor proper and the other vasodilator. When the muscle contracts in a reflex action or under the influence of the will both sets of fibers are excited; so that when the organ is set at work its arteries are simultaneously dilated and more blood flows through it. But if the animal have previously administered to it such a dose of curare as just to throw out of function the true motor-fibers, stimulation of the nerve produces dilation of the arteries without a corresponding muscular contraction. Quite a similar thing occurs in the salivary glands. Their cells, which form the saliva, are aroused to activity by special nerve-fibers; but the gland-nerve also contains a quite distinct set of vasodilator fibers which normally cause a simultaneous dilation of the gland-artery, though either can be artificially stimulated by itself and produce its effect alone. 
Since the effect of stimulating vasodilator nerves is the same as inhibiting the constrictor mechanism we might ask why there should be two distinct means thus provided for securing the same result. As a matter of fact the two mechanisms do not seem to overlap to any great extent; they rather supplement each other. The vasoconstrictor mechanism is confined, in the main, to the blood-vessels of the skin and viscera; the dilator mechanism is distributed chiefly to the muscles, the glands, and the genital organs.

Through such arrangements the distribution of the blood in the Body at any moment is governed: so that working parts shall have abundance and other parts less, while at the same time the general arterial pressure remains the same on the average; since the expansion of a few small local branches but little influences the total peripheral resistance in the vascular system. Moreover, commonly when one set of organs is at work with its vessels dilated, others are at rest with their arteries comparatively contracted, and so a general average blood-pressure is maintained. Few persons, for example, feel inclined to do brain-work after a heavy meal; for then a great part of the blood of the whole Body is led off into the dilated vessels of the digestive organs, and the brain gets a smaller supply.

The Vasodilator Center. There is reason to believe that the vasodilator nerves are under the control of a center in the medulla, which is in turn subject to the influence of afferent impulses of various sorts. The exact location of this center has not been determined. So far as can be judged from observation of vasodilator phenomena the vasodilator center is probably not in constant tonic activity, as is the constrictor center, but is aroused to activity only when afferent stimuli come to it from certain particular regions.

The Relation of Vasomotor Tone to Cerebral Activity. The circulation through the brain differs in some important respects from that of the rest of the Body. The differences arise from the fact that the brain, a fluid and therefore incompressible mass, is inclosed in an unyielding receptacle, the skull, which it fills completely. The result is that the cerebral blood-vessels occupy their allotted space, which cannot be either increased or diminished appreciably. The total volume of blood in the brain at any time is 
therefore practically constant, and the circulation through the brain can only be altered by changing the rate at which the blood flows through it. In such an arrangement as this, where.local vasodilation cannot occur, the only way in which the rate of blood-flow can be altered is by changes in the pressure at which the blood is forced into the region. The arteries feeding the brain spring directly from the aorta; it follows, therefore, that variations in aortic pressure, in other words, in general blood-pressure, are reflected exactly in the rate of cerebral blood-flow.

General blood-pressure, as we have seen, is maintained by vasomotor tone, the state of moderate constriction of arterioles generally. Variations in the tone of restricted areas, such as occur in connection with the functioning of individual organs, do not ordinarily affect general blood-pressure enough to alter the circulation through the brain to any extent.

There is good evidence that the degree of activity of the cells of the cerebral cortex is directly and immediately dependent upon the rate of blood-flow through the organ. A rapid circulation means alertness and efficiency of mental processes; as the flow becomes slower and slower the cells work less and less actively; when a certain point of sluggishness is reached consciousness disappears, the ceils, if not altogether quiescent, working too freely to arouse that state.

The phenomenon of fainting, which has already been mentioned, is the result usually of a sudden inhibition of the vasoconstrictor center whereby over a large area, the whole splanchnic region, for instance, there is general vasodilation and a resulting fall in blood-pressure. The rate of cerebral blood-flow falls to a point below that required for the maintenance of consciousness and the individual falls in a faint.

Sleep. This periodic loss of consciousness, so important for the proper restoration of the fatigued organs and tissues of the Body, has been the subject of considerable attention and investigation. Its explanation is not simple, involving as it does a number of questions, as, for instance, why fatigue, which ordinarily induces sleep, may, if extreme, prevent it; and what it is that causes one to awake after the proper number of hours of sleep.

Objectively sleep is marked by its well-known signs, which are not very instructive as to its cause, and also by certain vaso- 
motor changes which have been looked upon as very instructive; and as affording us, indeed, our only satisfactory method of studying sleep experimentally. Observations upon sleeping individuals have shown that normal sleep is frequently accompanied by a considerable fall in general blood-pressure, resulting from extensive vasodilation. This is itself sufficient to account for the diminished cerebral activity with its accompanying loss of consciousness which constitutes sleep, and many physiologists are inclined to believe, therefore, that the vasomotor changes may form the underlying basis for the phenomenon. A theory which expresses this view looks upon the vasoconstrictor center as the controlling mechanism of sleep. When this center is in good condition the constant stream of afferent impulses playing upon it maintains it in strong activity, and vasomotor tone is kept high. With the passage of hours of such ceaseless activity the center becomes fatigued and tends to respond less strongly to the afferent impulses coming to it. The result will be a falling off of vasomotor tone, unless by an effort of the will or an increase in the stream of afferent impulses, such as follows muscular exercise, for example, the center is whipped up to renewed activity. "Keeping awake" when one is sleepy is, according to this view, a matter of stimulating the tired vasoconstrictor center to continued effort. The effect may be produced by an artificial stimulant, such as coffee, or by an act of the will. The usual preparations for sleep are such as favor diminished activity of the vasoconstrictor center by lessening the afferent impulses coming to it. Lying in a comfortable position removes most of the impulses of muscle sense; by closing the eyes visual stimuli are gotten rid of. Thus unless the center is so irritable that the small stream of inevitable afferent impulses keeps it up to the mark the essential condition for sleep, loss of vasomotor tone, is fulfilled. The act of waking, according to this theory, results either from an undue stimulation of the vasoconstrictor center, as when one is waked by being violently shaken, or from a gradual restoration of the irritability of the center during its period of rest, to a point where the minimal stream of afferent impulses, inseparable from the living Body, is sufficient to stimulate it to the maintenance of waking vasomotor tone.

It must be admitted that not all experiments upon sleep have 
shown marked loss of vasomotor tone, but even if we consider vasomotor fatigue the primary factor we must grant, of course, that there are numerous additional factors modifying sleep. The condition of the cerebral cells and the nature of their activity doubtless have much to do with the phenomenon. These, however, are factors which physiology at present is unable to analyze completely, so that the vasomotor theory affords our most satisfactory explanation of sleep from the physiological standpoint.

Adrenin. The effect of this hormone upon the vascular system, as stated previously (Chap. XII), is to stimulate the vasoconstrictor fibers at their terminations in the muscles of the arterioles. The constant presence of this hormone in the blood is probably an important factor in maintaining that degree of vasomotor tone upon which the well-being of the Body depends. The great outpouring of adrenin into the blood under emotional stress so much increases the constriction of the blood vessels in the skin and the splanchnic area as to produce a pronounced rise in bloodpressure, with a correspondingly augmented cerebral circulation. The same influence acts to divert the blood largely from these regions to the skeletal muscles. The vessels of these latter being unprovided with vasoconstrictor fibers are not involved in the adrenin effect. Since the brain and the skeletal muscles are the regions specially in need of adequate nourishment in crises the adaptive character of this reaction is obvious. The substance adrenin as used experimentally shows several striking characteristics. In the first place a very small concentration of it (one part in ten thousand), introduced into a capillary region, brings about so strong a constriction in the immediate neighborhood as to stop the flow of blood completely through that region. It is possible thus to prevent troublesome bleeding in small operations. The effect of adrenin used in this way is, however, very transient; repeated injections are necessary to maintain the constricted state.

The Lymphatics. The living cells of the Body, as previously pointed out (Chap. XVII), are bathed in lymph, a liquid derived from the blood and serving as the intermediary by which interchanges of food materials, gases and waste substances between it and the cells are carried on. At the same place it was shown that there is a continuous movement of liquid from the blood into the lymph spaces, necessitating a system whereby the accumu- 
lation can be drained away from the tissues and carried back to the blood. This drainage is afforded by the lymphatic system. At its beginning this system is without definite structure, consisting simply of intercellular spaces. These communicate with one another, and at intervals with minute vessels having definite walls. These latter are the beginnings of definite lymph-channels.

The Structure of Lymph-Vessels. The smallest lymph-vessels proper are the lymph-capillaries; tubes rather wider than the blood-capillaries, but like them having a wall consisting of a single layer of flattened epithelium cells. The cells have, however, a wavy margin and are not as a.rule much longer in one diameter than another, in both of which respects they differ from the cells of the corresponding blood-vessels. In some regions, as in many glands, the lymph-capillaries are much dilated and form irregular lymph lacunce, everywhere bounded by their peculiar wavy cells, lying in the interstices of organs; and sometimes they form tubes around small blood-vessels, as in the brain (perivascular lymphchannel). In some places they commence by blind ends as in the lacteal vessels of the villi of the small intestine (Fig. 131) which are lymph-capillaries; but usually they branch and join to form networks. Lymph from the intercellular spaces enters them (probably by passing through their boundary cells) and is passed on to larger vessels which much resemble veins of corresponding size, having the same three coats, and being abundantly provided with valves.

The Thoracic Duct. The lymph-vessels proceeding from the capillaries in various organs become larger and fewer by joining together, and all end finally in two main trunks which open into the venous system on the sides of the neck, at the point of junction of the jugular and subclavian veins. The trunk on the right side is much smaller than the other and is known as the "right lymphatic duct." It collects lymph from the right side of the thorax, from the right side of the head and neck, and the right arm. The lymph from all the rest of the Body is collected into the thoracic duct. It commences at the upper part of the abdominal cavity in a dilated reservoir (the receptaculum chyli), into which the lacteals from the intestines, and the lymphatics of the rest of the lower part of the Body, open. From thence the thoracic duct, receiving tributaries on its course, runs up the thorax alongside of the aorta and, pass- 
ing on into the neck, ends on the left side at the point already indicated; receiving on its way the main stems from the left arm and the left side of the head and neck. The thoracic duct, thus, brings back to the blood much more lymph than the right lymphatic duct.

Lymph-Nodes. At intervals along the course of various lymphatic vessels are structures consisting of cells so arranged as to leave interspaces among them, through which interspaces the lymph is forced to flow. These structures are the lymph-nodes or lymph-glands and the peculiar tissue of which they are composed is lymphoid or adenoid tissue. Lymph-nodes occur in the neck, the groin, the axilla (arm pit) and in various other regions of the Body. Certain structures in the wall of the small intestine near its lower end, the so-called Peyer's Patches, are composed of lymphoid tissue as are also the structures in the throat making up the tonsillar ring.

Functions of Lymph-Nodes. Two quite different functions are attributed to the lymph-nodes. The first of these is that previously mentioned (p. 304) of serving as the seat of lymphocyte production.

The lymph-nodes have also the additional function of filtering the lymph that passes through them. This filtering action is probably of great importance in confining micro-organisms to the region which they first enter, since if they get into the lymph stream they are arrested at the first lymph-node. It is thought that the lymph-nodes are able also to arrest, for a time at least, the spread of cancer-cells over the Body. The lymph-nodes located on the channels draining the lungs become filled with dust that has worked its way through the pulmonary walls into the lymph, and that is prevented thus from spreading throughout the Body.

Tonsils and Adenoids. The irregular ring of lymphoid tissue surrounding the throat was referred to above. This at the front shows two enlargements, one on each side, known as the tonsils. At the back of the throat this same ring often in children becomes enlarged by overgrowth until it obstructs the nasal passage and interferes with the breathing. It may also obstruct the Eustachian tubes and cause partial deafness. This overgrowth is known as adenoids. The removal of adenoids is a simple matter surgically, and is advisable wherever there is evident obstruction of the breathing.

The tonsils, which function in the manner of lymphoid tissue 
generally, to filter out organisms from the lymph stream, are peculiarly liable to invasion by the organisms of common colds and also by those which form pus (streptococcus). When any of these become established in the tonsils and set up inflammation therein the very painful condition called tonsilitis results. In many cases the tonsils become permanently infected. In such there is a steady production of toxins which are discharged into the lymph stream and thence pervade the Body. Malnutrition in children and adults is often to be accounted for solely on the basis of chronic poisoning from infected tonsils. There is also reason to believe that acute rheumatism is caused similarly. In such cases the possible good that may come to the Body from the normal functioning of the tonsils is so far outweighed by the harm they do as seats of infection that they should obviously be removed.

The Movement of the Lymph. This is no doubt somewhat irregular in the commencing vessels, but, on the whole, sets on to the larger trunks and through them to the veins. In many animals (as the frog) at points where the lymphatics communicate with the veins, there are found regularly contractile "lymphhearts" which beat with a rhythm independent of that of the blood-heart, and pump the lymph into a vein. In the Human Body, however, there are no such hearts, and the flow of the lymph is dependent on less definite arrangements. It seems to be maintained mainly by three things: (1) The pressure on the blood-plasma in the capillaries is greater than that in the great veins of the neck; hence any plasma filtered through the capillarywalls will be under a pressure which will tend to make it flow to the venous termination of the thoracic or the right lymphatic duct. (2) On account of the numerous valves in the lymphatic vessels (which all only allow the lymph to flow past them to larger trunks) any movement compressing a lymph-vessel will cause an onward flow of its contents. The influence thus exerted is very important. If a tube be put in a large lymphatic, say at the top of the leg of an animal, it will be seen that the lymph only flows out very slowly while the animal is quiet; but as soon as it moves the leg the flow is greatly accelerated. (3) During each inspiration the pressure on the thoracic duct is less than that in the lymphatics in parts of the Body outside the thorax (see Chap. XXIII). Accordingly, at that time, lymph is pressed, or, 
in common phrase, is "sucked," into the thoracic duct. During the succeeding expiration the pressure on the thoracic duct becomes greater again, and some of its contents are pressed out; but on account of the valves of the vessels which unite to form the duct, they can only go towards the veins of the neck.

During digestion, moreover, contractions of the villi and of the intestinal walls press on the lymph or chyle within them and force it on; and in certain parts of the Body gravity, of course, aids the flow, though it will impede it in others.

The Action of Lymphagogues. Any substance that causes a pronounced increase in the rate of lymph formation is known as a lymphagogue. The source of lymph, we have already seen (p. 294), is in the main by filtration through the capillary walls. Evidently lymphagogues act by increasing this filtration. There are two ways in which this might be brought about, and lymphagogues are assigned to one of two clasises according to which of the ways they use. The first is by making the capillary walls more permeable, and so increasing the outpouring of lymph. Substances which have this effect are shell fish, strawberries, some meat extracts, egg-white and related organic compounds. Not all people are affected by these lymphagogues. Nor are those that are susceptible to one necessarily susceptible to all. Where the capillaries whose permeability is increased are superficial the outpouring lymph forms blotches on the skin. The condition is known as urticaria or hives. Mechanical injury to the capillaries may cause a similar outpouring, as seen in the swelling from a bruise.

The second method of increasing the flow of lymph is by producing an engorgement of the capillaries, a condition known as hydremic plethora. This can be brought about by raising the osmotic pressure of the blood, as by injecting into it a strong sugar solution. The effect is to cause a rush of lymph into the blood through the capillaries. The lymph thus withdrawn is made good by an outpouring of tissue fluids into the lymph spaces. It has been shown that in this situation the plethora is relieved chiefly by an increased filtration through the capillaries of the liver. The conclusion is drawn that these are the most permeable in the Body. The lymph thus formed passes to the thoracic duct and back to the blood, so that evidently no permanent advantage is gained. The excess of fluid is finally discharged through the kidneys. 


\section{CHAPTER XXIII}

\section{RESPIRATION. THE MECHANISM OF BREATHING. THE REGULATION OF BREATHING.}

Definitions. The blood as it flows from the right ventricle of the heart, through the lungs, to the left auricle, loses carbon dioxid and gains oxygen. In the systemic circulation exactly the reverse changes take place, oxygen leaving the blood to supply the living tissues; and carbon dioxid, generated in them, passing back into the blood capillaries. The oxygen loss and carbon dioxid gain are associated with a change in the color of the blood from bright scarlet to purple-red, or from arterial to venous; and the opposite changes in the lungs restore to the dark blood its bright tint. The whole set of processes through which blood becomes venous in the systemic circulation and arterial in the pulmonary -in other words, the processes concerned in the gaseous reception, distribution, and elimination of the Body-constitute the function of respiration; so much of this as is concerned in the interchanges between the blood and air being known as external respiration; while the interchanges occurring between the tissues and the systemic capillaries through the lymph, constitute internal respiration, and the processes in general by which oxygen is fixed and carbon dioxid formed by the living tissues, are known as tissue respiration. When the term respiration is used alone, without any limiting adjective, the external respiration only, is commonly meant.

Respiratory Organs. The blood being kept poor in oxygen and rich in carbon dioxid by the action of the living tissues, a certain amount of gaseous interchange will nearly always take place when it comes into close proximity to the surrounding medium; whether this be the atmosphere itself or water containing air in solution. When an animal is small there are often no special organs for its external respiration, its general surface being sufficient (especially in aquatic animals with a moist skin) to permit of all the gaseous exchange that is necessary. In the 
simplest creatures, indeed, there is even no blood, the cell or cells composing them taking up for themselves from their environment the oxygen which they need, and passing out into it their carbon dioxid waste; in other words, there is no differentiation of the external and internal respirations. When, however, an animal is larger many of its cells are so far from a free surface that they cannot transact this give-and-take with the surrounding medium directly, and the blood, or some liquid representing it in this respect, serves as a middleman between the living tissues and the external oxygen; and then one usually finds special respiratory organs developed, to which the blood is brought to make good its oxygen loss and get rid of its excess of carbon dioxid. In aquatic animals such organs take commonly the form of gills; these are protrusions of the body over which a constant current of water, containing oxygen in solution, is kept up; and in which blood capillaries form a close network immediately beneath the surface. In air-breathing animals a different arrangement is usually found. In some, as frogs, it is true, the skin is always moist and serves as an important respiratory organ, large quantities of venous blood being sent to it for aëration. But for the occurrence of the necessary gaseous diffusion, the skin must be kept very moist, and this, in a terrestrial animal, necessitates a great amount of secretion by the cutaneous glands to compensate for evaporation; accordingly in most land animals the air is carried into the body through tubes with narrow external orifices and so the drying up of the breathing surfaces is greatly diminished; just as water in a bottle with a narrow neck will evaporate much more slowly than the same amount exposed in an open dish. In insects (as bees, butterflies, and beetles) the air is carried by tubes which split up into extremely fine branches and ramify all through the body, even down to the individual tissue elements, which thus carry on their gaseous exchanges without the intervention of blood. But in the great majority of air-breathing animals the arrangement is different; the air-tubes leading from the exterior of the body do not subdivide into branches which ramify all through it, but open into one or more large sacs to which the venous blood is brought, and in whose walls it flows through a close capillary network. Such respiratory sacs are called lungs, and it 
is a highly developed form of them which is employed in the Human Body.

The Air-Passages and Lungs. In our own Bodies the es-

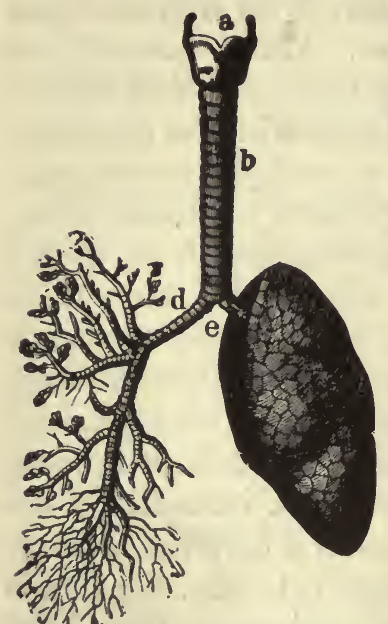

Fig. 112.-The lungs and airpassages seen from the front. On the left of the figure the pulmonary tissue has been dissected away to show the ramifications of the bronchial tubes. within the lung on its own side; and $a$, larynx; $b$, trachea; $d$, right the smallest bronchial tubes end in
bronchus. The left bronchus is seen entering the root of its lung. sacculated dilatations, the infundibula of the lungs, the sacculations (Fig. 114) being the alveoli. On the walls of the alveoli the pulmonary capillaries ramify, and it is in them that the interchanges of the external respiration take place.

Structure of the Trachea and Bronchi. The windpipe may readily be felt in the middle line of the neck, a little below Adam's apple, as a rigid cylindrical mass. It consists fundamentally of a fibrous tube in which cartilages are embedded, so as to keep it from collapsing; and is lined internally by mucous membrane covered by several

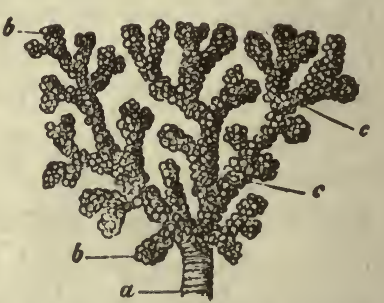

FIg. 113.-A small bronchial tube, $a$, dividing into its terminal branches, $c$; these have pouched branches, $c$; these have pouched
or sacculated walls and end in or sacculated walls and end
the sacculated infundibula, $b$. layers of epithelium cells, of which the superficial is ciliated. The elastic cartilages embedded in its walls are imperfect rings, 
each somewhat the shape of a horseshoe and the deficient part of each ring being turned backwards, it comes to pass that the deeper or dorsal side of the windpipe has no hard parts in it. Here the wall consists of smooth muscle. Against this the gullet lies, the absence of cartilage facilitating swallowing. The bronchi are similar in structure.

The Structure of the Lungs. These consist of the bronchial tubes and their terminal dilations; numerous blood-vessels, nerves, and lymphatics; and an abundance of connective tissue, rich in elastic fibers, binding all together. The bronchial tubes ramify in a tree-like manner (Fig. 112). The larger ones resemble the trachea, except that the cartilage rings do not have their open parts all turned one way, and the smooth muscle encircles the tube completely. As the tubes become smaller their constituents thin away; the cartilages become less frequent and finally disappear; the epithelium is reduced to a single layer of cells which, though still ciliated, are much shorter than the columnar superficial cell-layer of the larger tubes. The terminal alveoli ( $a, a$, Fig. 114) have walls composed mainly of elastic tissue and lined by a single layer of flat, non-ciliated epithelium, immediately beneath which is a very close network of capillary blood-vessels. The air entering by the bronchial tube is thus only separated from the blood by the thin capillary wall and the thin epithelium, both of which are moist, and well fitted for gaseous diffusion.

The Pleura. Each lung is covered, except at one point, by an elastic serous membrane which adheres tightly to it and is called the pleura; that point at which the pleura is wanting is called the root of the lung and is on

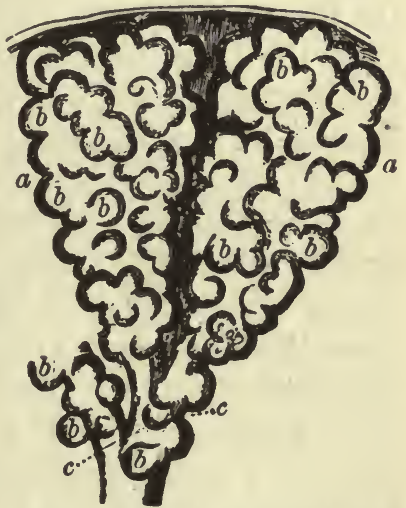

Frg. 114. - Two infundibula of the lung much magnified. $b, b$, hollow protrusions of the alveolus, opening into its central cavity; $c$, terminal branches of a bronchial tube. its median side; it is there that its bronchus, blood-vessels and nerves enter it. At the root of the lung the pleura turns back and lines the inside of the chest cavity, as represented by the heavy black line in the diagram Fig. 3. The part of the pleura at- 
tached to each lung is its visceral, and that attached to the chest-wall its parietal layer. Each pleura thus forms a closed sac surrounding a pleural cavity, in which, during health, there are found a few drops of lymph, keeping its surfaces moist. This lessens friction between the two layers during the movements of the chest-walls and the lungs; for although, to insure distinctness, the visceral and parietal layers of the pleura are represented in the diagram as not in contact, that is not the natural condition of things; the lungs are in life distended so that the visceral pleura rubs against the parietal, and the pleural cavity is practically obliterated. This is due to the pressure of the atmosphere exerted through the air-passages on the interior of the lungs. The lungs are extremely elastic and distensible, and when the chest cavity is perforated each shrivels up just as an india-rubber bladder does when its neck is opened; the reason being that then the air presses on the outside of each with as much force as it does on the inside. These two pressures neutralizing one another, there is nothing to overcome the tendency of the lungs to collapse. So long as the chest-walls are whole, however, the lungs remain distended. The pleural sac is air-tight and contains no air, and the pressure of the air around the Body is borne by the rigid walls of the chest and prevented from reaching the lungs; consequently no atmospheric pressure is exerted on their outside. On their interior, however, the atmosphere presses with its full weight, equal to about 90 centigrams on a square centimeter ( $14.5 \mathrm{lbs}$. on the square inch), and this is far more than sufficient to distend the lungs so as to make them completely fill all the parts of the thoracic cavity not occupied by other organs. S. Suppose $A$ (Fig. 115) to be a bottle closed airtight by a cork through which two tubes pass, one of which, $b$, leads into an elastic bag, $d$, and the other, $c$, provided with a stopcock, opens freely below into the bottle. When the stop-cock, $c$, is open the air will enter the bottle and press there on the outside of the bag, as well as on its inside through $b$. The bag will therefore collapse, as the lungs do when the chest cavity is opened. But if some air be sucked out through $c$ the pressure of that remaining in the bottle will diminish, and of that inside the bag will be unchanged, and the bag will thus be blown up, because the atmospheric pressure on its interior will not be balanced by 
that on its exterior. At last, when all the air is sucked out of the bottle and the stop-cock on $c$ closed, the bag, if sufficiently distensible, will be expanded so as to completely fill the bottle and press against its inside, and the state of things will then answer to that naturally found in the chest. If the bottle were now increased in size without letting air into it, the bag would expand still more, so as to fill it, and in so doing would receive air from outside through $b$; and if the bottle then returned to its original size, its walls would press on the bag and cause it to in shrink and expel some of its air through $b$. Ex- ships of the lungs in actly the same must of course happen, under

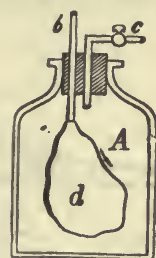

FIG. 115. - Diagram illustrating the the thorax. similar circumstances, in the chest, the windpipe answering to the tube $b$ through which air enters or leaves this elastic sac.

The Respiratory Movements. The air taken into the lungs soon becomes laden in them with carbon dioxid, and at the same time loses much of its oxygen; these interchanges take place mainly in the deep recesses of the alveoli, far from the exterior and only communicating with it through a long tract of narrow tubes. The alveolar air, thus become unfit any longer to convert venous blood into arterial, could only very slowly be renewed by gaseous diffusion with the atmosphere through the long airpassages-not nearly fast enough for the requirements of the Body, as one learns by the sensation of suffocation which follows holding the breath for a short time with mouth and larynx open. Consequently coöperating with the lungs is a respiratory mechanism, by which the air within them is periodically mixed with fresh air taken from the outside, and also the air in the alveoli is stirred up so as to bring fresh layers of it in contact with the walls of the air-cells. This mixing is brought about by the breathing movements, consisting of regularly alternating inspirations, during which the chest cavity is enlarged and fresh air enters the lungs, and expirations, in which the cavity is diminished and air expelled from the lungs. When the chest is enlarged the air the lungs contain immediately distends them so as to fill the larger space; in so doing it becomes rarefied and less dense than the external air; and since gases flow from points of greater to those of less pressure, some outside air at once flows in by the air-passages 
and enters the lungs. In expiration the reverse takes place. The chest cavity, diminishing, presses on the lungs and makes the air inside them denser than the external air, and so some passes out until an equilibrium of pressure is restored. The chest, in fact, acts very much like a bellows. When the bellows are opened air

Fig 116-Diagram to illustrate the entry of air to the lungs when the thoracic cavity enlarges. enters in consequence of the rarefaction of that in the interior, which is expanding to fill the larger space; and when the bellows are closed again it is expelled. To make the bellows quite like the lungs we must, however, as in Fig. 116, have only one opening in them, that of the nozzle, for both the entry and exit of the air; and this opening should lead, not directly into the bellows-cavity, but into an elastic bag lying in it, and tied to the inner end of the nozzle-pipe. This sac would represent the lungs and the space between its outside and the inside of the bellows, the pleural cavities.

We have next to see how the expansion and contraction of the chest cavity are brought about.

The Structure of the Thorax. The thoracic cavity has a conical form determined by the shape of its skeleton (Fig. 117), its narrower end being turned upwards. Dorsally, ventrally, and on the sides, it is supported by the rigid framework afforded by the thoracic vertebræ, the breast-bone, and the ribs. Between and over these lie muscles, and the whole is covered in, air-tight, by the skin externally, and the parietal layers of the pleuræ inside. Above, its aperture is closed by muscles and by various organs passing between the thorax and the neck; and below it is bounded by the diaphragm, which forms a movable bottom to the, otherwise, tolerably rigid box. In inspiration this box is increased in all its diameters-dorsiventrally, laterally, and from above down.

The Vertical Enlargement of the Thorax. This is brought about by the contraction of the diaphragm which (Figs. 1 and 118) is a thin muscular sheet, with a fibrous membrane, serving as a tendon, in its center. In rest, the diaphragm is dome-shaped, with its concavity towards the abdomen, being supported in that position by the pressure of the underlying abdominal organs. From the tendon on the crown of the dome striped muscular fibers 
radiate, downwards and outwards, to all sides; and are fixed by their inferior ends to the lower ribs, the breast-bone, and the vertebral column. In expiration the lower lateral portions of the diaphragm lie close against the chest-walls, no lung intervening between them. In inspiration the muscular fibers, shortening, flatten the dome and enlarge the thoracic cavity, room for the

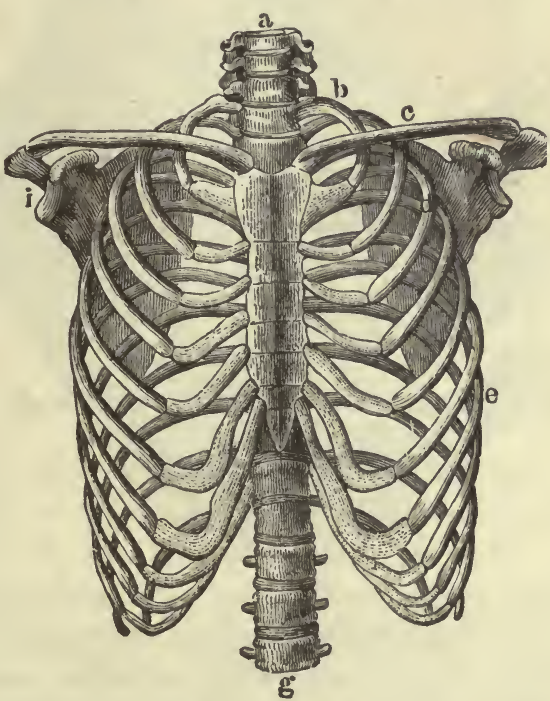

Fra. 117. - The skeleton of the thorax. $a, g$, vertebral column; $b$, first rib; $c$. clavicle; $e$, seventh rib; $i$, glenoid fossa.

viscera thus displaced being secured by stretching the abdominal walls; at the same time its lateral portions are pulled away from the chest-walls, leaving a space into which the lower ends of the lungs expand. The contraction of the diaphragm thus increases greatly the size of the thorax chamber by adding to its lowest and widest part.

The Dorsiventral Enlargement of the Thorax. The ribs on the whole slope downwards from the vertebral column to the breastbone, the slope being most marked in the lower ones. During inspiration the breast-bone and the sternal ends of the ribs attached to it are raised, and so the distance between the sternum and the vertebral column is increased. That this must be so will readily be seen on considering the diagram Fig. 119, where $a b$ represents the vertebral column, $c$ and $d$ two ribs, and st the ster- 
num. The continuous lines represent the natural position of the ribs at rest in expiration, and the dotted lines the position in inspiration. It is elear that when their lower ends are raised, so

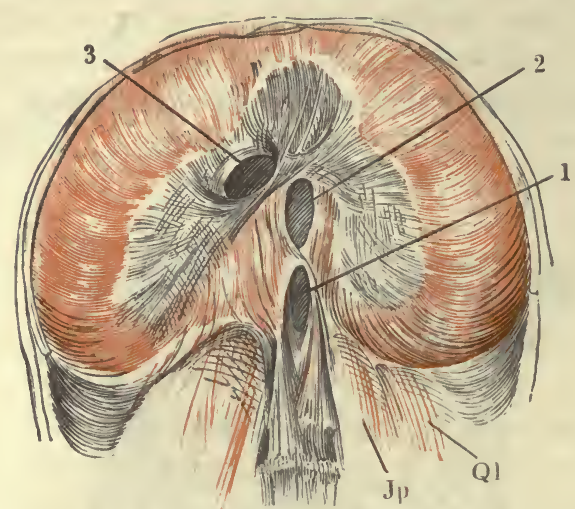

Fig. 118.-The diaphragm seen from below.

as to make the bars lie in a more horizontal plane, the sternum is pushed away from the spine, and so the chest cavity is increased dorsiventrally. The inspiratory elevation of the ribs is mainly due to the action of the scalene and external intercostal muscles.

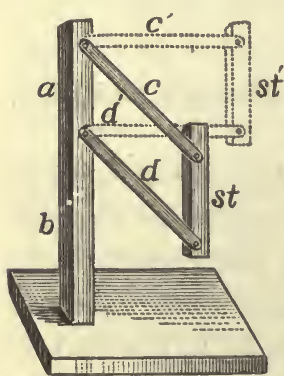

The scalene muscles, three on each side, arise from the cervical vertebræ, and are inserted into the upper ribs. The external intercostals (Fig. 120, A) lie between the ribs and extend from the vertebral column to the costal cartilages; their fibers slope downwards and forwards. During an inspiration the scalenes contract and fix the upper ribs firmly; then the external intercostals shorten and each raises the rib below it. The muscle, Fig. 119.- Diagramil- in fact, tends to pull together the pair of ribs ventral increase in the between which it lies, but as the upper one
diameter of the thorax but diameter of the thorax between which it lies, but as the upper one
when the ribs are raised. of these is held tight by the scalenes and other muscles above, the result is that the lower rib is pulled up, and not the upper down. In this way the lower ribs are raised much more than the upper, for the whole external intercostal muscles on each side may be regarded as one great muscle with many bellies, each belly. separated from the next by a tendon, 
represented by the rib. When the whole muscular sheet is fixed above and contracts, it is clear that its lower end will be raised more than any intermediate point, since there is a greater length of contracting muscle above it. The elevation of the ribs tends

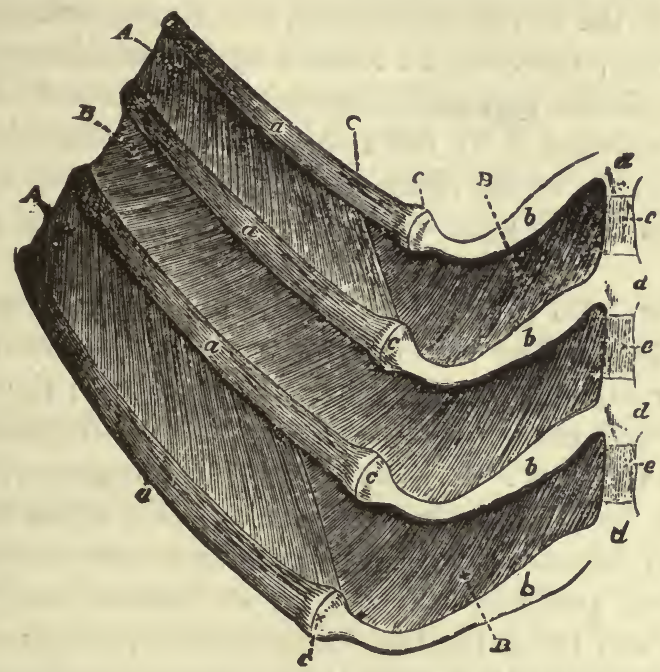

Fig. 120.-Portions of four ribs of a dog with the muscles between them. $a, a$, ventral ends of the ribs, joining at $c$ the rib cartilages, $b$, which are fixed to cartilaginous portions, $d$, of the sternum. $A$, external intercostal muscle, ceasing between the rib cartilages, where the internal intercostal, $B$, is seen. Between the middle two ribs the external intercostal muscle has been dissected away, so as to display the internal which was covered by it.

to diminish the vertical diameter of the chest; this is more than compensated for by the simultaneous descent of the diaphragm.

The Lateral Enlargement of the Chest is brought about by a rotation of the middle ribs which, as they are raised, roll round a little at their vertebral articulations and twist their cartilages. Each rib is curved and, if the bones be examined in their natural position in a skeleton, it will be seen that the most curved part lies below the level of a straight line drawn from the vertebral to the sternal attachment of the bone. By the rotation of the rib, during inspiration, this curved part is raised and turned out, and the chest widened. The mechanism can be understood by clasping the hands opposite the lower end of the sternum and a few inches in front of it, with the elbows bent and pointing downwards. Each arm will then answer, in an exaggerated way, to a 
curved rib, and the clasped hands to the breast-bone. If the hands be simply raised a few inches by movement at the shoulderjoints only, they will be separated farther from the front of the Body, and rib elevation and the consequent dorsiventral enlargement of the cavity surrounded will be represented. But if, simultaneously, the arms be rotated at the shoulder-joints so as to raise the elbows and turn them out a little, it will be seen that the space surrounded by the two arms is considerably increased from side to side, as the chest cavity is in inspiration by the similar elevation of the most curved part or "angle" of the middle ribs.

Expiration. To produce an inspiration requires considerable muscular effort. The ribs and sternum have to be raised; the elastic rib cartilages bent and somewhat twisted; the abdominal viscera pushed down; and the abdominal wall pushed out to make room for them. In expiration, on the contrary, no muscular effort is needed. As soon as the muscles. which have raised the ribs and sternum relax, these tend to return to their natural unconstrained position, and the rib cartilages, also, to untwist themselves and bring the ribs back to their position of rest; the elastic abdominal wall presses the contained viscera against the under side of the diaphragm, and pushes that up again as soon as its muscular fibers cease contracting. By these means the chest cavity is restored to its original capacity and the air sent out of the lungs, by the elasticity of the parts which were stretched or twisted in inspiration, and not by any special expiratory muscles.

Forced Respiration. When a very deep breath is drawn or expelled, or when there is some impediment to the entry or exit of the air, a great many muscles take part in producing the respiratory movements; and expiration then becomes, in part, an actively muscular act. The main expiratory muscles are the internal intercostals which lie beneath the external between each pair of ribs (Fig. 120, B), and have an opposite direction, their fibers running upwards and forwards. In forced expiration the lower ribs are fixed or pulled down by muscles running in the abdominal wall from the pelvis to them and to the breast-bone. The internal intercostals, contracting, pull down the upper ribs and the sternum, and so diminish the thoracic cavity dorsiventrally. At 
the same time, the contracted abdominal muscles press the walls of that cavity against the viscera within it, and pushing these up forcibly against the diaphragm make it very convex towards the chest, and so diminish the latter in its vertical diameter. In very violent expiration many other muscles may co-operate, tending to fix points on which those muscles which can directly diminish the thoracic cavity, pull. In violent inspiration, also, many extra muscles are called into play. The neck is held rigid to give the scalenes a firm attachment; the shoulder-joint is held fixed and muscles going from it to the chest-wall, and commonly serving to move the arm, are then used to elevate the ribs; the head is held firm on the vertebral column by the muscles going between the two, and then other muscles, which pass from the collar-bone and sternum to the skull, are used to pull up the former. The muscles which are thus called into play in labored but not in quiet breathing are called extraordinary muscles of respiration.

The Respiratory Sounds. The entry and exit of air are accompanied by respiratory sounds or murmurs, which can be heard on applying the ear to the chest-wall. The character of these sounds is different and characteristic over the trachea, the larger bronchial tubes, and portions of lung from which large bronchial tubes are absent. They are variously modified in pulmonary affections, and hence the value of auscultation of the lungs in assisting the physician to form a diagnosis.

The Capacity of the Lungs. Since the chest cavity never even approximately collapses, the lungs are never completely emptied of air: the space they have to occupy is larger in inspiration than during expiration, but is always considerable, so that after a forced expiration they still contain a large amount of air which can only be expelled from them by opening the pleural cavities; then they collapse almost completely, retaining within them only a small quantity of air imprisoned within the alveoli by the collapse of the small bronchi.

The capacity of the chest, and therefore of the lungs, varies much in different individuals, but in a man of medium height there remain in the lungs after the most violent possible expiration, about 1,000 cub. cent. of air, called the residual air. After an ordinary expiration there will be in addition to this about 1,600 cub. cent. of supplemental air; the residual and supplemental 
together forming the stationary air, which remains in the chest during quiet breathing. In an ordinary inspiration 500 cub. cent. (30 cub. inches) of tidal air are taken in, and about the same amount is expelled in natural expiration. By a forced inspiration about 1,600 cub. cent. (98 cub. inches) of complemental air can be added to the tidal air. After a forced inspiration, therefore, the chest will contain $1,000+1,600+500+1,600=4,700$ cub. cent. (300 cub. inches) of air. The amount which can be taken in by the most violent possible inspiration after the strongest possible expiration, that is, the supplemental, tidal, and complemental air together, is known as the vital capacity. For a healthy man 1.7 meters ( 5 feet 8 inches) high it is about 3,700 cub. cent. (225 cub. inches) and increases 60 cub. cent. for each additional centimeter of stature; or about $9 \mathrm{cub}$. inches for each inch of height. These figures are, of course, average figures. Individual variations from them are numerous.

The Quantity of Air Breathed Daily. Knowing the quantity of air taken in at each breath and expelled again (after more or less thorough admixture with the stationary air) we have only to know, in addition, the rate at which the breathing movements occur, to be able to calculate how much air passes through the lungs in twenty-four hours. The average number of respirations in a minute is found by counting on persons sitting quietly, and not knowing that their breathing rate is under observation, to be fifteen in a minute. In each respiration half a liter (30 cub. inches) of air is concerned; therefore $0.5 \times 15 \times 60 \times 24=10,800$ liters (375 cub. feet) is the quantity of air breathed under ordinary circumstances by each person in a day.

Hygienic Remarks. Since the diaphragm when it contracts pushes down the abdominal viscera beneath it, these have to make room for themselves by pushing out the soft front of the abdomen which, accordingly, protudes when the diaphragm descends. Hence breathing by the diaphragm, being indicated on the extcrior by movements of the abdomen, is often called "abdominal respiration," as distinguished from breathing by the ribs, called "costal" or "chest breathing." In both sexes the diaphragmatic breathing is the most important, but, as a rule, men and children use the ribs less than adult women. Since both abdomen and chest alternately expand and contract in healthy breathing, anything which impedes their free movement is to be avoided; and the tight lacing which used to be thought elegant a few years 
back, and is still indulged in by some who think a distorted form beautiful, seriously impedes one of the most important functions. of the Body, leading, if nothing worse, to shortness of breath and an incapacity for muscular exertion. In extreme cases of tight lacing some organs are often directly injured, weals of fibrous tissue being, for example, not unfrequently found developed on the liver, from the pressure of the lower ribs forced against it by a tight corset.

The Aspiration of the Thorax. As already pointed out, the external air cannot press directly upon the contents of the thoracic cavity, on account of the rigid framework which supports its walls; it still, however, presses on them indirectly through the lungs. Pushing on the interior of these with a pressure equal to that exerted on the same area by a column of mecury $760 \mathrm{~mm}$. (30 inches) high, it distends them and forces them against the inside of the chest-walls, the heart, the great thoracic blood-vessels; the thoracic duct, and the other contents of the chest cavity. The pressure against these organs is not equal to that of the external air, since some of the total air-pressure on the inside of the lungs is used up in overcoming their elasticity, and it is only the residue which pushes them against the things outside them. In expiration this residue is equal to that exerted by a column of mercury $754 \mathrm{~mm}$. (29.8 inches) high. On most parts of the Body the atmospheric pressure acts, however, with full force. Pressing on a limb it pushes the skin against the soft parts beneath, and these compress the blood-and lymph-vessels among them; and the yielding abdominal walls do not, like the rigid thoracic walls, carry the atmospheric pressure themselves, but transmit it to the contents of the cavity. It thus comes to pass that the blood and lymph in most parts of the Body are under a higher atmospheric pressure than they are exposed to in the chest, and consequently these liquids tend to flow into the thorax, until the extra distention of the vessels in which they there accumulate compensates for the less external pressure to which those vessels are exposed. An equilibrium would thus very soon be brought about were it not for the respiratory movements, in consequence of which the intrathoracic pressure is alternately increased and diminished, and the thorax comes to act as a sort of suction-pump on the contents of the vessels of the Borly outside it; thus the respira- 
tory movements influence the circulation of the blood and the flow of the lymph.

\section{Influence of the Respiratory Movements upon the Circulation.} Suppose the chest in a condition of normal expiration and the external pressure on the blood in the blood-vessels within it and in the heart, to have come, in the manner pointed out in the last paragraph, into equilibrium with the atmospheric pressure exerted on the blood-vessels of the neck and abdomen. If an inspiration now occurs, the chest cavity being enlarged the pressure on all of its contents will be diminished. In consequence, air enters the lungs from the windpipe, and blood enters the venæ cavæ and the right auricle of the heart from the outlying veins. When the next expiration occurs, and the pressure in the thorax again rises, air and blood both tend to be expelled from the cavity. Whatever extra blood has, to use the common phrase, been "sucked" into the intrathoracic venæ cavæ in inspiration and has not been sent already on into the right ventricle before expiration occurs, is, however, on account of the venous valves, prevented from flowing back whence it came, and is imprisoned in the cavæ under an increased pressure during expiration; and this tends to make it flow faster into the auricle during the diastole of the latter. How much the alternating respiratory movements assist the venous flow is shown by the dilatation of the veins of the head and neck which occurs when a person is holding his breath; and the blackness of the face, from distention of the veins and stagnation of the capillary flow, which occurs during a prolonged fit of coughing, which is a series of expiratory efforts without any inspirations.

The vencricles and arteries are not directly affected to any appreciable extent by the respiratory movements; their walls are too thick and the arterial pressure too great to respond to these small variations of intrathoracic pressure. The increase in venous flow which occurs during inspiration does, however, by supplying the heart with more blood at that time, bring about a small increase in arterial pressure during each inspiration. The increased blood-supply is handled by the heart through an augmentation of its beat. This has been shown to be brought about by an irradiation to the cardiac centers of the influences that govern the act of inspiration. To a marked extent the vigor of breathing and the heart-rate run parallel. 
Influence of Breathing Movements on the Lymph-Flow. During inspiration, when intrathoracic pressure is lowered, lymph is pressed into the thoracic duct from the abdominal lymphatics. In expiration, when thoracic pressure rises again, the extra lymph cannot flow back on account of the valves in the lymphatic vessels, and it is consequently driven on to the cervical ending of the thoracic duct. The breathing movements thus pump the lymph on.

The Respiratory Center. The respiratory movements are to a certain extent under the control of the will; we can breathe faster or slower, shallower or more deeply, as we wish, and can also "hold the breath" for some time-but the voluntary control thus exerted is limited in extent; no one can commit suicide by holding his breath. In ordinary quiet breathing the movements are quite involuntary; they go on perfectly without the least attention on our part, and, not only in sleep, but during the unconsciousness of fainting or of an apoplectic fit. The natural breathing movements are therefore either reflex or automatic.

The muscles concerned in producing the changes in the chest which lead to the entry or exit of air are of the ordinary striped kind; and these, as we have seen, only contract in the Body under the influence of the nerves going to them; the nerves of the diaphragm are the two phrenic nerves, one for each side of it; the external intercostal muscles are supplied by certain branches of the thoracic spinal nerves, called the intercostal nerves. If the phrenic nerves be cut the diaphragm ceases its contractions, and a similar paralysis of the external intercostals follows section of the intercostal nerves.

Since the inspiratory muscles only act when stimulated by nervous impulses reaching them, we have next to seek where these impulses originate; and experiment shows that it is in the medulla oblongata. All the brain of a cat or a rabbit in front of the medulla can be removed, and it will still go on breathing; and children are sometimes born with the medulla oblongata only, the rest of the brain being undeveloped, and yet they breathe for a time. If, on the other hand, the spinal cord be divided immediately below the medulla of an animal, all breathing movements of the chest cease at once. We conclude, therefore, that the nervous impulses calling forth contractions of the respiratory muscles arise in the medulla 
oblongata, and travel down the spinal cord and thence out along the phrenic and intercostal nerves. This is confirmed by the fact that if the spinal cord be cut across below the origin of the fourth pair of cervical spinal nerves (from which the phrenics mainly arise) but above the first thoracic spinal nerves, the respiratory movements of the diaphragm continue, but those of the intercostal muscles cease; this phenomenon has sometimes been observed on men so stabbed in the back as to divide the spinal cord in the region indicated. Finally, that the nervous impulses exciting the inspiratory muscles originate in the medulla, is proved by the fact that if a small portion of that organ, the so-called vital point, be destroyed, all the respiratory movements cease at once and forever, although all the rest of the brain and spinal cord may be left uninjured. This part of the medulla is known as the respiratory center.

Is the Respiratory Center Reflex? Since this center goes on working independently of the will, we have next to inquire, Is it a reflex center or not? Are the efferent discharges it sends along the respiratory nerves due to afferent impulses reaching it by centripetal nerve-fibers? Or does it originate efferent nervous impulses independently of excitation through afferent nerves?

We know, in the first place, that the respiratory center is largely under reflex control; a dash of cold water on the skin, the irritation of the nasal mucous membrane by snuff, or of the larynx by a foreign body, will each cause a modification in the respiratory movements - a long indrawn breath, a sneeze, or a cough. But, although thus very subject to influences reaching it by afferent nerves, the respiratory center seems essentially independent of such. In many animals, as rabbits (and in some men), marked breathing movements take place in the nostrils, which dilate during inspiration; and when the spinal cord of a rabbit is cut close to the medulla, thus cutting off all afferent nervous impulses to the respiratory center except such as may reach it through cranial nerves, the respiratory movements of the nostrils still continue until death. The movements of the ribs and diaphragm of course cease, and so the animal dies very soon unless artificial respiration be maintained. Moreover, if after cutting the spinal cord as above described, the chief sensory cranial nerves be divided, so as to cut off the respiratory center from almost all possible afferent nervous 
impulses, regular breathing movements of the nostrils continue. We conclude, therefore, that the activity of the respiratory center, however much it may be capable of modification through sensory nerves, is essentially independent of them.

What it is that Excites the Respiratory Center. It has long been recognized that the activity of the respiratory center is related to the condition of the blood flowing through it; arterial blood excites it feebly or not at all; venous blood excites it powerfully, and more and more strongly as its venosity increases. The difference between arterial and venous blood is wholly a difference in the relative amounts of oxygen and of carbon dioxid present therein. The question is: Does venous blood owe its ability to stimulate the respiratory center to its low oxygen content or to its high content of carbon dioxid? Experiment has shown that both factors enter somewhat, but that the center is more affected by small changes in the amount of carbon dioxid than by small changes in the amount of oxygen.

We might look upon carbon dioxid as the main regulator of the respiratory center, and for convenience of description shall do so. As a matter of exactness, however, not carbon dioxid as such but an acid condition of the blood, dependent chiefly on carbon dioxid, determines the activity of the center. Lack of oxygen may produce indirectly the same acid condition that is brought about by excess of carbon dioxid. So we see that we are not entitled to assign the control of the center exclusively to carbon dioxid, although that substance determines under ordinary circumstances, its stimulation.

Why are the Respiratory Discharges Rhythmic? If carbon dioxid is the stimulus for the respiratory center, why does that center act rhythmically? Does the carbon dioxid content of the circulating blood increase and decrease fifteen times or more a minute? The answer to this question is afforded by a simple experiment. If in an animal breathing naturally under anesthesia both vagus nerves are cut there is an immediate change in the character of the respirations. From being rapid and shallow they become very deep and take on a much slower rate. Under this condition we may properly assume that the respirations do follow the carbon dioxid content of the blood; the center begins to discharge when the blood contains enough carbon dioxid to stimulate 
it, and continues its discharge until the aëration of the blood, resulting from the inspiration, lowers the carbon dioxid below the point of stimulation. There follows a period of expiration and rest which continues until sufficient carbon dioxid has again accumulated to start the action anew.

Since with the vagus nerves cut the respirations follow the carbon dioxid concentration of the blood, but with the nerves intact do not, being much more shallow and rapid, we must determine the influence of the vagus nerves upon the center in order to understand ordinary breathing. It has been shown that the influence of the vagus nerves is a simple reflex one. These nerves contain sensory fibers arising in the lung tissue and so situated as to be stimulated mechanically every time the lung is inflated. 'The impulses conveyed over these fibers to the central nervous system are inhibitory to the respiratory center. Bearing this action of the vagus fibers in mind we may account for normal breathing thus; the blood contains enough carbon dioxid all the time, under ordinary circumstances, to stimulate the respiratory center; whenever the center discharges under this stimulus it brings about the movements of inspiration which result in expansion of the lungs; whenever the lungs expand the sensory fibers contained in their walls are stimulated and so inhibitory influences are sent to the respiratory center. Inspiration proceeds, then, until the inhibitory impulses from the lungs overcome the stimulus of carbon dioxid, when it comes to an end and the thorax falls back to the position of rest. This falling back, which constitutes normal expiration, collapses the lungs somewhat; the inhibitory impulses diminish or disappear; and the stimulating action of the carbon dioxid again becomes effective. Thus in normal breathing inspiration and expiration follow one another without any pause between, and the respirations are shallow because the inhibition cuts them off almost as soon as started.

The entire purpose of breathing is to ventilate the lungs. It is relatively a minor matter whether a system of rapid shallow breaths or of slow deep ones is used so long as the result is secured. The necessary amount of air would be taken into and discharged from the lungs every minute by either arrangement. There may possibly be some advantage to the Body in the rapid shallow type in avoiding such wide fluctuations in the concentrations of the respira- 
tory gases in the blood and the alveoli as would occur with slower and deeper breathing.

Forced Expiration. Although in ordinary quiet breathing, as we have seen, expiration is a passive collapse of the chest, active expiratory effort is of frequent occurrence. In talking, singing, whistling, as well as in coughing, sneezing, and straining, the expiratory muscles are functioning. The ease with which these are brought into play suggests that the part of the center which controls them, although not normally in action, is hung on a "hair trigger" so to speak, requiring very slight additional influence to arouse it to action.

Sensitiveness of the Respiratory Center. The respiratory center is responsive to very slight changes in the amount of carbon dioxid in the blood. A small increase quickens the breathing notably. The effect of the quickened breathing is to ventilate the lungs more thoroughly, and thus to bring about a more rapid movement of carbon dioxid from the blood to the alveolar air (see next chapter). In this manner the increased amount of carbon dioxid is gotten rid of. The respiratory center may be thought of as a delicate governor which serves to keep the carbon dioxid content of the blood at a uniform level. The chief source of carbon dioxid is in the active muscles. Muscular exercise is, therefore, the most common cause of quickened breathing. If so careful a regulation of the amount of carbon dioxid in the blood seems at first not very important we can better appreciate its significance by recalling that carbon dioxid is the end product of oxidation, so that any change in the amount of carbon dioxid in the blood means a corresponding change in the consumption of oxygen by the Body; and, furthermore, that the changes in lung ventilation which serve to keep the carbon dioxid level steady have the effect at the same time of increasing or diminishing the supply of available oxygen. Thus an increase in carbon dioxid brings about a more pronounced ventilation of the lungs, and thus, in turn provides more oxygen than is brought to the lungs during ordinary breathing. There will be no need for a change in the amount of oxygen unless there is a change in the bodily oxidations, so whenever more oxygen is needed the need is signalized by an increase in the carbon dioxid, and this acts to bring into play the mechanism by which the required oxygen is supplied. 
Eupnea, Hyperpnea, Dyspnea, Apnea. Ordinary quiet breathing is known as eupnea. Rapid breathing, such as follows moderate exercise, is designated as hyperpnea. When the breathing is forced, and especially when forced expiration enters, we have the condition called dyspnea. This results from abnormal excitement of the respiratory center either reflexly, as from stimulation of pain nerves, or by a greater increase in the carbon dioxid content of the blood than that which causes simple hyperpnea. The dyspnea of the early stages of suffocation arises from this latter cause. Apnea, or absence of breathing, may result from one of two conditions or from both acting together. The first of these is a deficiency of carbon dioxid in the blood, so that the respiratory center is not stimulated. The second is inhibition of the center through vigorous and repeated inflation of the lungs. Since inflation of the lungs with ordinary air brings about both conditions the apnea which results from this treatment is partly chemical and partly inhibitory. That inhibition enters in the production of apnea in this way is shown by the greater difficulty of producing the condition in animals with both vagi cut.

Holding the Breath. When one holds his breath he is sending impulses to the respiratory center which inhibit its discharge. Meanwhile the bodily oxidations go right on, so the longer this inhibition continues the greater becomes the amount of carbon dioxid in the blood, and the more powerfully does the normal excitation of the center act. In a very short time the carbon dioxid stimulation becomes more potent than the volitional inhibition, and when that time comes a breath must be taken in spite of the effort to hold it. Evidently any procedure that will diminish the amount of carbon dioxid in the blood to begin with will prolong the time the breath can be held. This can be done by forced breathing for several minutes. The over-ventilation of the lungs thus carried on sweeps out so much carbon dioxid from the blood that a much longer time elapses than ordinarily before the accumulation overcomes the volitional inhibition. Since, as we shall learn (p. 421), over-ventilation of the lungs does not materially increase the supply of available oxygen, this procedure may bring about severe oxygen deficiency, which shows itself by blueness of the skin and mucous membranes, a blueness caused by the venous condition of the blood in the arteries. 
Asphyxia. Asphyxia is death from suffocation, or want of oxygen by the tissues. . It may be brought about in various ways; as by strangulation, which prevents the entry of air into the lungs; or by exposure in an atmosphere containing no oxygen; or by putting an animal in a vacuum; or by making it breathe air containing a gas which has a stronger affinity for hemoglobin than oxygen has, and which, therefore, turns the oxygen out of the red corpuscles and takes its place. The gases which do the latter are very interesting since they serve to prove conclusively that the Body can live only by the oxygen carried around by the hemoglobin of the red corpuscles; the amount dissolved in the bloodplasma being insufficient for its needs. Of such gases carbon monoxid is the most important and best studied; in the frequent mode of committing suicide by stopping up all the ventilation holes of a room and turning on the gas, it is poisoning by carbon monoxid which causes death.

The Phenomena of Asphyxia. As soon as the carbon dioxid in the blood rises above the normal amount the breathing becomes hurried and deeper, and the extraordinary muscles of respiration are called into activity. The dyspnea becomes more.and more marked, and this is especially the case with the expirations which, almost or quite passively performed in natural breathing, become violently muscular. At last nearly all the muscles in the Body are set at work; the rhythmic character of the respiratory acts is lost, and general convulsions occur, but, on the whole, the contractions of the expiratory muscles are more violent than those of the in spiratory.

The violent excitation of the nerve-centers soon exhausts them, and all the more readily since their oxygen supply (which they like all other tissues need in order to continue their activity) is cut off. The convulsions therefore gradually cease, and the animal becomes calm again, save for an occasional act of breathing: these final movements are inspirations and, becoming less and less frequent, at last cease, and the animal appears dead. Its heart, however, though gorged with extremely dark venous blood still makes some slow feeble pulsations. So long as it beats artificial respiration can restore the animal, but once the heart has finally stopped restoration is impossible. There are thus three distinguishable stages in death from asphyxia. (1) The stage of dyspnea. 
The stage of convulsions. (3) The stage of exhaustion; the convulsions having ceased but there being from time to time an inspiration. The end of the third stage occurs in a mammal about five minute: after the oxygen supply has been totally cut off. If the asphyxia be due to deficiency, and not absolute want of oxygen, of course all the stages take longer.

Artificial Respiration. Asphyxia from drowning and other causes occurs with lamentable frequency these days, and there is no doubt that many lives are sacrificed through ignorance on the part of bystanders of the proper restorative procedures. There are several methods of applying artificial respiration to human beings. The method of Schaefer is as effective as any. The following description is quoted from his paper on the subject: "The method consists in laying the subject in the prone posture, preferably on the ground, with a thick folded garment underneath the chest and epigastrium. The operator puts himself athwart or at the side of the subject, facing his head and places his hands on each side over the lower part of the back (lowest ribs). He then slowly throws the weight of his Body forward to bear upon his own arms, and thus presses upon the thorax of the subject and forces air out of the lungs. This being effected, he gradually relaxes the pressure by bringing his own Body up again to a more erect position, but without moving the hands." These movements should be repeated about fifteen times a minute until normal breathing is resumed, and should not be given up for at least a half hour if recovery does not occur sooner. If there is water in the lungs it should be allowed to drain out before the artificial respiration is begun. Otherwise it may be churned into a foam by the movements, and defeat the desired ventilation of the lungs.

Modified Respiratory Movements. Sighing is a deep, longdrawn inspiration followed by a shorter but correspondingly large expiration. Yawning is similar, but the air is mainly taken in by the mouth instead of the nose, and the lower jaw is drawn down in a characteristic manner. Hiccough depends upon a sudden contraction of the diaphragm, while the aperture of the larynx closes; the entering air, drawn through the narrowing opening, causes the peculiar sound. Coughing consists of a full inspiration followed by a violent and rapid expiration, during the first part of which the laryngeal opening is kept closed; being afterwards suddenly opened, 
the air issues forth with a rush, tending to carry out with it anything lodged in the windpipe or larynx. Sneezing is much like coughing, except that, while in a cough the isthmus of the fauces is held open and the air mainly passes out through the mouth, in sneezing the fauces are closed and the blast is driven through the nostrils. It is commonly excited by irritation of the nasal mucous membrane, but in many persons a sudden bright light falling into the eye will produce a sneeze. Laughing consists of a series of short expirations following a single inspiration; the larynx is open all the time, and the vocal cords (Chap. XXXIII) are set in vibration. Crying is, physiologically, much like laughing and, as we all know, one often passes into the other. The accompanying contractions of the face muscles giving expression to the countenance are, however, different in the two.

All these modified respiratory acts are essentially reflex and they serve to show to what a great extent the discharges of the respiratory center can be modified by afferent nerve impulses; but, with the exception of hiccough, they are to a certain extent, like natural breathing, under the control of the will. Most of them, too, can be imitated more or less perfectly by voluntary muscular movements; though a good stage sneeze or cough is rare. 


\section{CHAPTER XXIV}

\section{RESPIRATION. THE GASEOUS INTERCHANGES}

Nature of the Problems. The study of the respiratory process from a chemical standpoint has for its object to discover what are, in kind and extent, the interchanges between the air in the lungs and the blood in the pulmonary capillaries; and the nature and amount of the corresponding gaseous changes between the living tissues, and the blood in the systemic capillaries. Neglecting some oxygen used up otherwise than in forming carbon dioxid, and some carbon dioxid eliminated by other organs than the lungs, these processes in the long run balance, the blood losing as much carbon dioxid gas in the lungs as it gains elsewhere, and gaining as much oxygen in the lungs as it loses in the systemic capillaries. To comprehend the matter it is necessary to know the physical and chemical conditions of these gases in the lungs, in the blood, and in the tissues generally; for only so can we understand how it is that in different localities of the Body such exactly contrary processes occur. So far as the problems connected with the external respiration are concerned our knowledge is tolerably complete; but as regards the internal respiration, taking place all through the Body, much has yet to be learnt; we know that a muscle at work gives more carbon dioxid to the blood than one at rest and takes more oxygen from it, but how much of the one it gives and of the other it takes is only known approximately; as are the conditions under which this greater interchange during the activity of the muscular tissue is effected: and concerning nearly all the other tissues we know even less than about muscle. In fact, as regards the Body as a whole, it is comparatively easy to find how great its gaseous interchanges with the air are during work and rest, waking and sleeping, while fasting or digesting, and so on, but when it comes to be decided what organs are concerned in each case in producing the greater or less exchange, and how much of the whole is due to each of them, the question is one far more difficult to settle and still very far from completely answered. 
The Changes Produced in Air by Being Once Breathed. These are fourfold-changes in its temperature, in its moisture, in its chemical composition, and its volume.

The air taken into the lungs is nearly always cooler than that expired, which has a temperature of about $36^{\circ} \mathrm{C}$. ( $97^{\circ} \mathrm{F}$.). The temperature of a room is usually less than $21^{\circ} \mathrm{C}$. $\left(70^{\circ} \mathrm{F}\right.$.). The warmer the inspired air the less, of course, the heat which is lost to the Body in the breathing process; its average amount is calculated as about equal to 50 Calories in twenty-four hours; a Calory being as much heat as will raise the temperature of one kilogram (2.2 lbs.) of water one degree centigrade $\left(1.8^{\circ} \mathrm{F}\right.$.).

The inspired air always contains more or less water vapor, but is rarely saturated; that is, rarely contains so much but it can take up more without showing it as mist; the warmer air is; the more water vapor is required to saturate it. The expired air is nearly saturated for the temperature at which it leaves the Body, as is readily shown by the water deposited when it is slightly cooled, as when a mirror is breathed upon; or by the clouds seen issuing from the nostrils on a frosty day, these being due to the fact that the air, as soon as it is cooled, cannot hold all the water vapor which it took up when warmed in the Body. Air, therefore, when breathed once, gains water vapor and carries it off from the lungs; the actual amount being subject to variation with the temperature and saturation of the inspired air: the cooler and drier that is, the more water will it gain when breathed. On an average the amount thus carried off in twenty-four hours is about 255 grams (9 ounces). To evaporate this water in the lungs an amount of heat is required, which disappears for this purpose in the Body, to reappear again outside it when the water vapor condenses. The amount of heat taken off in this way during the day is about 148 Calories. The total daily loss of heat from the Body through the lungs averages therefore 198 Calories, 50 in warming the inspired air and 148 in the evaporation of water.

The most important changes brought about in the breathed air are those in its chemical composition. Pure air when completely dried consists in each 100 parts of:

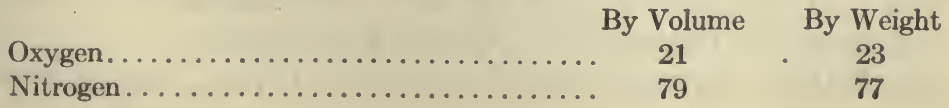


Ordinary atmospheric air contains in addition 4 volumes of carbon dioxid in 10,000 , or 0.04 in 100, a quantity which, for practical purposes, may be neglected. When breathed once, such air gains rather more than 4 volumes in 100 of carbon dioxid, and loses a little less than 5 of oxygen. More accurately, 100 volumes of expired air after drying contain:

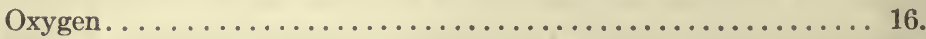

Nitrogen ................................. 79.

Carbon dioxid.............................. 4.4

Since 10,800 liters (375 cubic feet) of air are breathed in twentyfour hours and lose 5 per cent of oxygen, the total quantity of this gas taken up in the lungs daily is $10,800 \times 5 \div 100=540$ liters. One liter of oxygen measured at $0^{\circ} \mathrm{C}$. $\left(32^{\circ} \mathrm{F}\right.$.) and under a pressure equal to one atmosphere, weighs 1.43 grams, so the total weight of oxygen taken up by the lungs daily is $540 \times 1.43=772$ grams (27 ounces).

The amount of carbon dioxid excreted from the lungs being 4.4 per cent of the volume of the air breathed daily, is $10,800 \times$ $4.4 \div 100=475$ liters measured at the normal temperature and pressure. This volume weighs 930 grams, or 32.5 ounces. If all the oxygen taken in were breathed out again as carbon dioxid the volume of the latter should equal that of the oxygen breathed in. The discrepancy results from the fact that not all the oxygen combines with carbon; some of it unites with hydrogen to form water. The water thus formed simply adds itself to the general water content of the Body, and has no bearing on the amount discharged in the expired air; this latter depending, as already stated, on the rate of evaporation from the lung surface.

If the expired air be measured as it leaves the Body its bulk will be found greater than that of the inspired air, since it not only has water vapor added to it, but is expanded in consequence of its higher temperature. If, however, it be dried and reduced to the same temperature as the inspired air its volume will be found diminished, since it has lost 5 volumes per cent of oxygen and gained only 4.4 of carbon dioxid. In round numbers, 100 volumes of dry inspired air at zero, give 99 volumes of dry expired air measured at the same temperature and pressure.

Ventilation. Since at every breath some oxygen is taken from 
the air and some carbon dioxid given to it, were the atmosphere around a living man not renewed he would, at last, be unable to get from the air the oxygen he required; he would die of oxygen starvation or be suffocated, as such a mode of death is called, as surely, though not quite so fast, as if he were put under the receiver of an air-pump and all the air around him removed. Hence the necessity of ventilation to supply fresh air in place of that breathed, and clearly the amount of fresh air requisite must be determined by the number of persons collected in a room; the supply which would be ample for one person would be insufficient for two. Moreover, fires, gas, and oil lamps, all use up the oxygen of the air and give carbon dioxid to it, and hence calculation must be made for them in arranging for the ventilation of a building in which they are to be employed.

In order that air be unwholesome to breathe, it is by no means necessary that it have lost so much of its oxygen as to make it difficult for the Body to get what it wants of that gas. The evil results of insufficient air-supply are rarely, if ever, due to that cause even in the worst-ventilated room for, as we shall see hereafter, the blood is able to take what oxygen it wants from air containing comparatively little of that gas. The headache and drowsiness which come on from sitting in a badly ventilated room appear to be due chiefly to the high percentage of water vapor present under such circumstances, and the want of energy and general ill-health which result from permanently living in such surroundings are probably the result of a slow poisoning of the Body by absorption of gaseous substances given off to the air, not from the lungs, but from the skin in evaporating sweat and from the alimentary tract. The idea, formerly held very generally, that volatile poisons are given off by the lungs in quantities too small for chemical detection, has been largely abandoned partly because of the failure of the most careful experiments to demonstrate any such substances, but more because there are enough injurious materials given off from other channels of the Body to explain all the ill effects of insufficient ventilation.

That the air of rooms occupied by persons becomes injurious long before the amount of carbon dioxid in it is sufficient to do any harm has been abundantly demonstrated. Breathing air containing one or two per cent of that gas produced by ordinary 
chemical methods does no particular injury, but air containing one per cent of it produced by respiration is decidedly injurious, because of the other things present in it at the same time. Carbon dioxid itself, at least in any such percentage as is commonly found in a room, is not poisonous, as used to be believed, but, since it is tolerably easily estimated in air, while the actually: injurious substances also present are not, the purity or foulness of the air in a room is usually determined by finding the percentage of carbon dioxid in it: it must be borne in mind that to mean much this carbon dioxid must have been produced by. breathing; the amount of it found is in itself no guide to the quantity of really important injurious substances present. Of course when a great deal of carbon dioxid is present the air is irrespirable: as for example sometimes at the bottom of wells or brewing-vats.

In one minute $.5 \times 15=7.5$ liters $(0.254$ cubic feet) of air are breathed and this is vitiated with carbon dioxid to the extent of rather more than four per cent; mixed with three times its volume of external air, it would give thirty liters (a little over one cubic foot) vitiated to the extent of one per cent, and such air is not respirable for any length of time with safety. The result of breathing it for an evening is headache and general malaise; of breathing it weeks or months a lowered tone of the whole Body-less power of work, physical or mental, and less power of resisting disease; the ill effects may not show themselves at once, and may accordingly be overlooked, or considered scientific fancies, by the careless; but they are nevertheless there ready to manifest themselves. In order to have air to breathe in an even moderately pure state every man should get for his own allowance at least 23,000 liters of space to begin with (about 800 cubic feet) and the arrangements for ventilation should, at the very least, renew this at the rate of 30 liters (one cubic foot) per minute. In the more recently constructed hospitals, as a result of experience, twice the above minimum cubic space is allowed for each bed in a ward, and the replacement of the old air at a far more rapid rate, 100,000 liters per hour per person, is also provided for.

Ventilation does not necessarily imply draughts of cold air, as is often supposed. In warming by indirect radiation (the ordinary hot-air furnace) it may readily be secured by arranging, in addition to the registers from which the warmed air reaches the 
room, proper openings at the opposite side, by which the old air may pass off to make room for the fresh. An open fire in a room will always keep up a current of air through it, and is the healthiest, though not the most economical, method of warming an apartment.

In severe weather, when there is a tendency to keep rooms rather tightly closed, a good plan is to open widely all doors and windows for a few minutes each day, allowing fresh air to penetrate to every corner, sweeping out the old air before it. This daily renewing, helped out by such ventilation as is afforded by ill-fitting doors and windows, usually keeps the air of rooms in respirable condition when not occupied by too many persons. The modern habit of sleeping summer and winter in rooms with open windows is to be highly commended, and should be even more generally adopted. In fact the more outdoor air one can have, and at the same time keep warm, the better for the bodily well-being. The beneficial effects of fresh air and sunshine, especially in pulmonary tuberculosis, cannot be too strongly emphasized.

Reference was made above to the fact that discomfort in illy ventilated rooms is more a matter of the amount of water vapor present than of excess carbon dioxid or other poisons, or of deficient oxygen. Recent careful studies have emphasized this fact so clearly as to bring about marked changes in the practice of ventilation experts, particularly in their treatment of the problem of ventilating auditoriums, and other places where large numbers of people gather temporarily. To secure highest bodily comfort there should be a certain degree of humidity in association with a certain temperature. If the temperature changes the amount of water vapor in the air should change to correspond. Too low humidity is to be avoided as well as too high. An ordinary fault in ventilation is that the air is allowed to become too dry. This is particularly true during the winter months when artificial heat is used. To maintain the desired humidity in dwelling houses of ordinary size during cold weather from $1 \frac{1}{2}$ to 2 gallons of water should be evaporated in the house daily. Where large numbers of house plants are kept the evaporation from their leaves will contribute materially toward this amount.

While comfort depends on proper relationship of temperature and moisture, we must not lose sight of the fact that ultimate 
well-being requires that the air that we breathe be reasonably pure also. Provisions for renewing the air of occupied rooms must not be neglected, therefore, in working out ventilation plans.

Changes undergone by the Blood in the Lungs. These are the exact reverse of those undergone by the breathed air-what the air gains the blood loses, and vice versa. Consequently, the blood loses heat, and water, and carbon dioxid in the pulmonary capillaries; and gains oxygen. These gains and losses are accompanied by a change of color from the dark purple which the blood exhibits in the pulmonary artery, to the bright scarlet it possesses in the pulmonary veins.

The dependence of this color change upon the access of fresh air to the lungs while the blood is flowing through them, can be readily demonstrated. If a rabbit be rendered unconscious by chloroform, and its chest be opened, after a pair of bellows has been connected with its windpipe, it is seen that, so long as the bellows are worked to keep up artificial respiration, the blood in the right side of the heart (as seen through the thin auricle) and that in the pulmonary artery, is dark colored, while that in the pulmonary veins and the left auricle is bright red. Let, however, the artificial respiration be stopped for a few seconds and, consequently, the renewal of the air in the lungs (since an animal cannot breathe for itself when its chest is opened), and very soon the blood returns to the left auricle as dark as it left the right. In a very short time symptoms of suffocation show themselves and the animal dies, unless the bellows be again set at work.

In a former paragraph (p. 412) we saw that about 5 volumes in 100 of oxygen are absorbed from the alveolar air into the blood, and 4.4 in 100 of carbon dioxid given off to the alveolar air from the blood. If we put the amount of air inhaled and exhaled with each breath (tidal air) at 500 c.c. and the respiratory rate at 15 per minute, we have 7,500 c.c. of air involved each minute, 5 per cent of this, or 375 c.c. would give the oxygen consumption and 4.4 per cent, or 330 c.c. the carbon dioxid output in the same time. As a matter of fact direct determinations of the oxygen absorption and the carbon dioxid output of persons at rest ordinarily give somewhat smaller figures than these, 280-325 c.c. per minute for oxygen and 250-280 c.c. for carbon dioxid. This discrepancy can be explained by recalling that the figures for tidal air, for the 
breathing rate and for the percentages of oxygen and carbon dioxid, are round numbers and somewhat higher than the actual averages.

The Blood Gases. If fresh blood be rapidly exposed to as complete a vacuum as can be obtained, it gives off certain gases, known as the gases of the blood. These are the same in kind, but differ in proportion, in venous and arterial blood; there being more carbon dioxid and less oxygen obtainable from the venous blood going to the lungs by the pulmonary artery, than from the arterial blood coming back to the heart by the pulmonary veins. The gases given off by venous and arterial blood, measured under the normal pressure and at the normal temperature, amount to from 58 to 60 volumes for every 100 volumes of blood, and in the two cases are about as follows:

\begin{tabular}{|c|c|c|}
\hline & Venous Blood & Arterial Blood \\
\hline Oxygen. & 12 & 20 \\
\hline 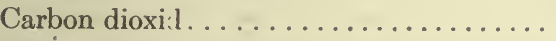 & 45 & 38 \\
\hline rogen $\ldots \ldots \ldots \ldots \ldots \ldots \ldots$ & 1.7 & 1.7 \\
\hline
\end{tabular}

It is important to bear in mind that while arterial blood contains some carbon dioxid that can be removed by the air-pump, venous blood also contains some oxygen removable in the same way; so that the difference between the two is only one of degree. When an animal is killed by suffocation, however, the last trace of oxygen which can be yielded up in a vacuum disappears from the blood before the heart ceases to beat. All the blood of such an animal is what might be called suffocation blood, and has a far darker color than ordinary venous blood.

The Cause of the Bright Color of Arterial Blood. The color of the blood depends on its red corpuscles, since pure blood-plasma or blood-serum is colorless, or at most a very faint straw yellow. Hence the color change which the blood experiences in circulating through the lungs must be due to some change in its red corpuscles. We have already seen (Chap. XVII) that the functional substance of the red corpuscles is hemoglobin, which has the property of combining with oxygen. Hemoglobin itself is of a dark purplish color, when combined with oxygen the resulting compound is a bright scarlet. Hemoglobin combined with oxygen is known as oxyhemoglobin, and it is on its predominance that the color of arterial blood depends. Hemoglobin uncombined with 
oxygen, sometimes named reduced hemoglobin, predominates in venous blood, and is the only kind found in the blood of a suffocated mammal.

The Laws Governing the Absorption of Gases by a Liquid. In order to understand the condition of the gases in the blood liquid it is necessary to recall the general laws in accordance with which liquids absorb gases. They are as follows:

1. A given volume of a liquid at a definite temperature if it absorbs any of a gas to which it is exposed, and yet does not combine chemically with it, takes up an amount of the gas which depends upon two things: (1) the solubility of the gas in the liquid; and (2) the pressure of the gas upon the surface of the liquid. As the pressure of the gas is increased the amount of it which goes in solution in the liquid is increased in exactly the same proportion. If a complete vacuum be formed above a liquid all the gas contained within it is given off. This law, that the quantity of a gas dissolved by a liquid varies directly as the pressure of that gas on the surface of the liquid is known as Henry's law.

2. The amount of a gas dissolved by a liquid depends, not on the total pressure exerted by all the gases pressing on its surface, but on the fraction of the total pressure which is exerted by the particular gas in question. For example, the average atmospheric pressure is equal to that of a column of mercury $760 \mathrm{~mm}$. (30 inches) high. But 100 volumes of air contain approximately 80 volumes of nitrogen and 20 of oxygen; therefore $\frac{1}{5}$ of the total pressure is due to oxygen and $\frac{4}{5}$ to nitrogen: and the amount of oxygen absorbed by water is just the same as if all the nitrogen were removed from the air and its total pressure therefore reduced to $\frac{1}{5}$ of $760 \mathrm{~mm}$. (30 inches) of mercury; that is, to $152 \mathrm{~mm}$. (6 inches) of mercury pressure. It is only the fraction of the total pressure exerted by the oxygen itself which affects the quantity of oxygen absorbed by water at any given temperature. So, too, of all the atmospheric pressure $\frac{4}{5}$ is due to nitrogen, and all the oxygen might be removed from the air without affecting the quantity of nitrogen which would be absorbed from it by a given volume of water. The atmospheric pressure would then be $\frac{4}{5}$ of $760 \mathrm{~mm}$. of mercury, or $608 \mathrm{~mm}$. ( 24 inches), but it would all be due to nitrogen gas - and be exactly equal to the fraction of the total pressure due to that gas before the oxygen was removed from the 
air. When several gases are mixed together the fraction of the total pressure exerted by each one is known as the partial pressure of that gas; and it is this partial pressure which determines the amount of each individual gas dissolved by a liquid. If a liquid exposed to the air for some time had taken up all the oxygen and nitrogen it could at the partial pressures of those gases in the air, and were then put in an atmosphere in which the oxygen had all been replaced by nitrogen, it would now give off all its oxygen, since, although the total gaseous pressure on it was the same, no part of it was any longer due to oxygen; and at the same time it would take up one-fifth more nitrogen, since the whole gaseous pressure on its surface was now due to that gas, while before only four-fifths of the total was exerted by it. If. on the contrary, the liquid were exposed to pure hydrogen under a pressure of one atmosphere it would give off all its previously dissolved oxygen and nitrogen, since none of the pressure on its surface would now be due to those gases; and would take up as much hydrogen as corresponded to a pressure of that gas equal to $760 \mathrm{~mm}$. of mercury (30 inches).

3. The amount of gas taken up by a liquid varies, other things being equal, inversely as the temperature.

4. A liquid may be such as to combine chemically with a gas. Then the amount of the gas absorbed is independent of the partial pressure of the gas on the surface of the liquid. The quantity absorbed will depend upon how much the liquid can combine with. Or, a liquid may be composed partly of things which simply dissolve a gas and partly of things which combine with it chemically. Then the amount of the gas taken up under a given partial pressure will depend on two things; a certain portion, that merely dissolved, will vary with the pressure of the gas in question; but another portion, that chemically combined, will remain the same under different pressures.

5. Bodies are known which combine chemically with certain gases when the partial pressure of these is considerable, forming compounds which break up, or dissociate, liberating the gas, when its partial pressure falls below a certain limit. Oxygen forms such a compound with hemoglobin.

6. A membrane, moistened by a liquid in which a gas is soluble, does not essentially alter the laws of absorption, by a liquid on one 
side of it of a gas present on its other side, whether the absorption be due to mere solution or to chemical combinations or to both.

The Absorption of Oxygen by the Blood. Applying the physical and chemical facts stated in the preceding paragraph to the blood, we find that the blood contains (1) plasma, which simply dissolves oxygen, and (2) hemoglobin, which combines with it under some partial pressures of that gas, but gives it up under lower.

Blood-plasma or, what comes to the same thing, fresh serum, exposed to the air, takes up no more oxygen than so much water: about 0.56 volumes of the gas for every 100 of the liquid, at a temperature of $20^{\circ} \mathrm{C}$. At the temperature of the Body the volume absorbed would be still less. This quantity obeys Henry's law.

If fresh defibrinated blood be employed, the quantity of oxygen taken up is much greater; this extra quantity must be taken up by the red corpuscles and it does not obey Henry's law. If the partial pressure of oxygen on the surface of the defibrinated blood be doubled, only as much more oxygen will be taken up as corresponds to that dissolved in the serum; and if the partial pressure of oxygen on its surface be reduced to one-half, only a very small amount of oxygen (onie-half of that dissolved by the serum) will be given off. All the much larger quantity taken up by the red corpuscles will be unaffected and must therefore be chemically combined with something in them. Since 90 per cent of their dry weight is hemoglobin, and this body when prepared pure is found capable of combining with oxygen, there is no doubt that it is the hemoglobin in the circulating blood which carries around most of its oxygen. The red corpuscles are so many little packages in which oxygen is stowed away.

The compound formed between oxygen and hemoglobin is, however, a very feeble one; the two easily separate, and always do so completely when the oxygen pressure in the liquid or gas to which the oxyhemoglobin is exposed falls below $25 \mathrm{~mm}$. of mercury. There is some slight dissociation at pressures of $70 \mathrm{~mm}$. of mercury. Hence, in an air-pump, the blood only gives off a little of its oxygen, until the pressure falls to about $\frac{1}{6}$ of an atmosphere, that is to $\frac{75}{6} 0=125 \mathrm{~mm}$. (5 inches) of mercury, of which total pressure one-fifth $(25 \mathrm{~mm}$. or $1 \mathrm{inch})$ is due to the oxygen present. As soon as this limit is passed the hemoglobin gives up its remaining oxygen with a rush. 
Consequences of the Peculiar Way in Which the Oxygen of the Blood is Held. The first, and most important, is that the blood can take up far more oxygen in the lungs than would otherwise be possible. Blood-serum exposed to the air would take up only onehalf volume of oxygen per hundred of liquid at ordinary temperatures, and still less at the temperature of the Body, were it not for its hemoglobin. In the lungs even less would be taken up, since the air in the air-cells of those organs is poorer in oxygen than the external air; and consequently the partial pressure of that gas in it is lower. The tidal air taken in at each breath serves merely to renew directly the air in the big bronchi; the deeper we examine the pulmonary air the less oxygen and more carbon dioxid will be found; in the layers farthest from the exterior and only renewed by diffusion with the air of the large bronchi, it is estimated that the oxygen only exists in such quantity that its partial pressure is equal to about $100 \mathrm{~mm}$. of mercury ( $\frac{1}{8}$ atmos.) instead of 152 ( $\frac{1}{5}$ atmos.) as in ordinary air. In the second place, on account of the way in which hemoglobin combines with oxygen, the quantity of that gas taken up by the blood is independent of such variations of its partial pressure in the atmosphere as we are subjected to in daily life. At the top of a high mountain, for example, the atmospheric pressure is greatly diminished, but still mountaineers can breathe freely and get all the oxygen they want; the distress felt for a time by persons unused to living in high altitudes is due in part to circulatory disturbances resulting from the low atmospheric pressure and in part to another condition to be described presently, but not at all to deficiency of oxygen. So long as the partial pressure of that gas in the lung air-cells is well above 25 $\mathrm{mm}$. of mercury, the amount of it taken up by the blood depends on how much hemoglobin there is in that liquid and not on how much oxygen there is in the air. So, too, breathing pure oxygen under a pressure of one atmosphere, or air compressed to one-half or a fourth its normal bulk, does not increase the quantity of oxygen absorbed by the blood, apart from the small extra quantity dissolved by the plasma.

The General Oxygen Interchanges in the Blood. Suppose we have a quantity of arterial blood in the aorta. This, fresh from the lungs, will have its hemoglobin practically saturated with oxygen and in the state of oxyhemoglobin. In the blood-plasma some 
more oxygen will be dissolved, viz., so much as answers to a pres. sure of that gas equal to $100 \mathrm{~mm}$. of mercury, which is the partial pressure of oxygen in the pulmonary air-cells. This tension of the gas in the plasma will be more than sufficient to keep the hemoglobin from giving off its oxygen. Suppose the blood now enters the capillaries of a muscle. In the liquid moistening this organ the oxygen tension is practically nil, since the tissue elements are steadily taking the gas up from the lymph around them. Consequently, through the capillary walls, the plasma will give off oxygen until the tension of that gas in it falls below $25 \mathrm{~mm}$. of mercury. Immediately some of the oxyhemoglobin is decomposed, and the oxygen liberated is dissolved in the plasma, and from there next passed on to the lymph outside; and so the tension in the plasma is once more lowered and more oxyhemoglobin decomposed. This goes on so long as the blood is in the capillaries of the muscle, but on account of the shortness of this interval, about one second, not all the oxyhemoglobin has time to decompose before the blood has passed on into the veins. Here further decomposition is quickly brought to an end by the rising tension of the oxygen dissolved in the plasma, the last oxygen given off from the corpuscles not being taken up by the lymph because of the passage of the blood on out of the capillaries. The blood will now go on as ordinary venous blood into the veins of the muscle and so back to the lungs. It will consist of (1) plasma with oxygen dissolved in it at a tension of about $25 \mathrm{~mm}$. (1 inch) of mercury. (2) A number of red corpuscles containing reduced hemoglobin. (3) A number of red corpuscles containing oxyhemoglobin. Or perhaps all of the red corpuscles will contain some reduced and some oxidized hemoglobin. This venous blood, returning to the heart, is sent on to the pulmonary capillaries. Here, the partial pressure of oxygen in the air-cells being $100 \mathrm{~mm}$. and that in the blood-plasma much less, oxygen will be taken up by the latter, and the tension of that gas in the plasma tend to be raised above the limit at which hemoglobin combines with it. Hence, as far as the plasma gets oxygen tho 3 red corpuscles which contain any reduced hemoglobin rob it, and so its oxygen tension is kept down below that in the air-cells until all the hemoglobin is saturated. Then the oxygen tension of the plasma rises to that of the gas in the air-cells; no more oxygen is absorbed, 
and the blood returns to the left auricle of the heart in the same condition, so far as oxygen is concerned, as when we commenced to follow it.

The Carbon Dioxid of the Blood. The same general laws apply to this as to the blood oxygen. The gas is partly merely dissolved and partly in a loose chemical combination with some one or more of the constituents of blood. Carbon dioxid is about twenty times as soluble in blood-plasma as is oxygen under equivalent conditions of temperature and pressure. We can therefore account for more of it than of oxygen in the state of simple solution. Not more than 6 per cent of the total amount present in venous blood can be accounted for, however, in this way. The remainder must be in some easily dissociable chemical combination. Two such combinations are known to exist in blood. The first is a combination of carbon dioxid with sodium, forming sodium carbonate; the second of carbon dioxid with the blood proteins, including hemoglobin, forming a compound somewhat analogous with oxyhemoglobin. This latter compound is more readily dissociable than sodium carbonate, and since, as we have seen, there is always, even in arterial blood, a considerable percentage of carbon dioxid, we may suppose that under ordinary circumstances the sodium carbonate circulates as such, and the protein compound serves as the carrier of carbon dioxid from tissues to lungs.

We may summarize the carbon dioxid interchanges as follows:

1. The tissues constantly produce and give off to the lymph carbon dioxid. It is present in lymph, therefore, at all times in considerable quantity, probably amounting to a carbon dioxid tension of $70 \mathrm{~mm}$. of mercury.

2. The blood entering the capillaries contains carbon dioxid under much less tension than this (about $35 \mathrm{~mm}$.), there is therefore a movement of carbon dioxid from lymph to blood. This movement, by raising the tension of carbon dioxid in the blood brings about conditions under which chemical combination may take place, chiefly with the blood proteins.

3. The venous blood as it enters the lungs contains carbon dioxid under a higher tension than that of alveolar air, $70 \mathrm{~mm}$. for venous blood, $35 \mathrm{~mm}$. for the alveoli; there is therefore a movement of carbon dioxid from the blood to the alveoli. This movement, by lowering the carbon dioxid tension of the blood, 
favors the dissociation of the chemical compounds formed during the passage of the blood through the tissue capillaries; thus the carbon dioxid taken up in the systemic capillaries is gotten rid of in the lung capillaries.

The Hormone Action of Carbon Dioxid. We have already learned (Chap. XXIII) that carbon dioxid has an important action in connection with maintaining the activity of the respiratory center. Recent work has shown that it has other functions as well. The carbon dioxid tension of alveolar air is ordinarily about $35 \mathrm{~mm}$. of mercury. The carbon dioxid tension of the blood does not, of course, fall below that of the alveoli, so that arterial blood under normal conditions contains a considerable amount of carbon dioxid. Under exceptional circumstances, as at high altitudes, where the atmospheric pressure as a whole is less than at the earth's surface, the tension of carbon dioxid in the alveoli may be considerably less than $35 \mathrm{~mm}$, and that of the blood correspondingly diminished. There is a condition known as mountain sickness, characterized by nausea and other distressing symptoms, which is due to this diminution of the carbon dioxid content of the blood. Any one, by taking a number of deep breaths in rapid succession, can lower the carbon dioxid tension of his alveolar air, and consequently of his blood, to a point where very disagreeable sensations are felt. Just how the carbon dioxid of the blood prevents these symptoms is not clear. That it has the power to do so is, however, well demonstrated.

The normal breathing mechanism is an adaptation by which the blood is continuously provided with all the oxygen it is able to carry, and by which also its carbon dioxid content, while never allowed to become excessive, is kept high enough for the proper performance of its hormone function. Deep breathing is therefore of no particular value from the standpoint of respiration. As an exercise for the chest muscles; as a means of insuring ventilation of the remotest alveoli; and most of all as an aid to the flow of venous blood and lymph, through the aspiration of the thorax, (p. 370) the practice has great value. We should remember, however, that shallow breathing is the normal mode, and that only while we are thinking about it can we breathe deeply. As soon as our attention is diverted to other matters we recur at once to the automatic shallow type. 
Tissue Respiration. Our knowledge of the use of oxygen and the production of carbon dioxid by the tissues is not very complete. The following general facts may be stated here:(1) The tissues take up oxygen from the lymph as fast as it is brought by the blood and use it in oxidative processes at the same rate; careful experiments fail to show that there is any storage of oxygen in the tissues for future use. (2) Tissue oxidations differ from ordinary oxidative processes, such as occur when fuel is burned in a furnace, for example, in that they are earried on through the agency of enzyms known as oxidases. The ehemical process of oxidation carried on thus is not direct as in ordinary burning; it occurs at a lower temperature, and requires a longer time; but it must be remembered that the amount of heat produced by the oxidation of a given weight of fuel is always the same whether the process be rapid or slow. Tissue oxidations, therefore, are not necessarily inefficient because they go on slowly.

(3) The amount of work that a man's organs do, is not dependent on the amount of oxygen supplied to them, but the amount of oxygen used by him depends on how much he uses his organs. It is necessary to emphasize this fact because of the notion, which seems to be rather widespread, that bodily processes are augmented by increasing the supply of oxygen to them. The man who goes from his ill-ventilated office to the open country, and feels the impulse to vigorous exercise as he breathes the pure country air, is apt to attribute his sensations of virility to an imagined augmentation of all his bodily processes through the increased amount of oxygen breathed in. The fact is that whatever augmentation of activity he may experience is the result of the agreeable sensory stimulations coming to him, which arouse his tissues to activity, either reflexly or voluntarily. Increased oxygen consumption is, therefore, never the cause, but always the result of augmented tissue activity.

Respiratory Changes in Muscular Exercise. With every increase in degree of muscular activity there is corresponding increase in oxygen consumption and carbon dioxid production up to a limit which is set by the ability of the blood to carry oxygen. Since, as already noted (p. 421), the blood as it leaves the lungs is virtually saturated with the gas under resting conditions, an increase in the amount transported by it can come about only by a more rapid flow of the blood or by a more complete use by the 
tissues of that brought to them. Both these methods enter as a matter of fact. The familiar increase in the rate of the heart, in combination with a slight increase in the amount of blood discharged with each beat, suffices to augment the blood-flow about $2 \frac{1}{2}-2 \frac{3}{4}$ times. We saw above (p. 417) that ordinarily venous blood contains about 60 per cent as much oxygen as arterial.- In exercise the amount of oxygen in venous blood is very much reduced; in extreme cases none at all may remain. By this more complete utilization, in connection with the more rapid flow, the total oxygen carrying power may be raised about $7-7 \frac{1}{2}$ times. The other factors, carbon dioxid transport, and lung ventilation, have much wider limits, so that the bound is established, as stated above, by the oxygen carrying power.

In connection with this an interesting point arises. Repeated reference has been made to the fact that ordinarily during the passage of the blood through the tissue capillaries it gives up only 40 per cent of its oxygen. The suggestion was made (p. 422) that this relatively small disbursement is due to the short stay of the blood in the capillaries. During muscular activity there is a much more rapid blood-flow, with a corresponding shortening of the time required for the blood to pass through the capillaries, yet in spite of this we find the blood giving up virtually all its oxygen, instead of only 40 per cent of it. A recent discovery may help us to explain this apparent paradox. It has been shown that the dissociation of oxyhemoglobin is much more rapid in an environment rich in carbon dioxid than in one containing only small amounts of this substance. One result of muscular exercise is a great outpouring of carbon dioxid from the active tissues. It may be supposed that in the presence of this outpouring the dissociation of oxyhemoglobin is so greatly accelerated that the increased rate of blood-flow is more than counter-balanced.

If, as may readily happen, the activity becomes so great that the oxygen supply cannot keep pace with the needs of the muscles we have the result already discussed in Chap. VII (p. 112), namely, an outpouring of sodium lactate into the blood. The effect of this is to make more pronounced that acid condition which, as stated previously (p.403), constitutes the real stimulus to the respiratory center. . The dyspnea is, therefore, markedly increased with the appearance of this substance in the blood. We would probably 
be safe in assuming the point of onset of marked respiratory distress as indicating the passage of the laboring muscles beyond the limit at which their immediate need for oxygen can be fully supplied.

Coal Gas Poisoning. In the paragraph on asphyxia (Chap. XXIII) the possibility of suffocation by carbon monoxid was mentioned. This substance, which is an important constituent of illuminating gas, has a greater affinity for hemoglobin than has oxygen, and forms with it a more stable compound, carbon monoxid hemoglobin. The result of breathing illuminating gas is, then, the conversion of hemoglobin of the blood into carbon monoxid hemoglobin, and the consequent abolishment of the oxygen-carrying function of the red corpuscles. If the breathing of carbon monoxid has gone on long enough for practically all the hemoglobin of the blood to be combined with it, death from lack of oxygen is inevitable unless by the prompt performance of blood transfusion a fresh supply of properly functioning red corpuscles be introduced into the circulation. Exposure to the gas for a shorter time, not enough to prove fatal, but to the point of unconsciousness, is often followed by a long period, weeks or months, of serious functional impairment of the tissues of the Body, due to the injury suffered by them during the period of oxygen deficiency. 


\section{CHAPTER XXV}

\section{FOODS: THEIR CLASSIFICATION}

What Constitutes Food. Material is taken into the Body in three physical states: solid, liquid, gaseous. We have considered the gaseous intake under the head of respiration, and turn now to the use by the Body of solid and liquid substances. From the standpoint of physiology we may include under the head of food everything, either solid or liquid, which is taken into the Body and used there for its normal functioning. This classification includes with the foods liquid substances, such as milk and water, which we ordinarily classify separately as drinks. It is clear, however, that from the standpoint of the Body a classification on this basis, the physical nature of the substance taken, is not very helpful, and we shall therefore disregard the distinction commonly made between liquid and solid foods.

The Function of Food. If we have gotten the viewpoint which the earlier chapters of this book have attempted to instil, and are able to look upon the Body as a piece of machinery, we appreciate that materials must be furnished it for at least two purposes: (1) to supply what it needs for the liberation of energy; and (2) to provide for its maintenance and repair. The first of these requirements is a simple fuel demand; anything that the Body is able to burn can be used if its burning or mere presence does not injure the delicate machinery. The second requirement is not so simple; the repair of the complex body mechanism calls for particular repair materials; in the carrying on of the Body's functions there is a continuous loss from it of substances, such as water, which must be continuously replaced; moreover, we often see fit to introduce substances which we think will aid the Body in carrying out its functions, as coffee, tea, spices, and condiments.

In the case of the child an additional factor enters, namely, growth, or the manufacture of new tissue. As we shall learn, this is not precisely equivalent to the repair of tissue already present, 
so that we shall have also to consider foods in their relationship to growth.

Classes of Foods. We are aware that the materials which compose our meals include indigestible substances as well as true foods. These indigestible materials serve, as we shall see, an important function through the bulk they impart to the food; it would be extremely difficult to maintain the Body in health upon a diet from which they were excluded; we may borrow for them an expressive term used by feeders of cattle for bulky stuffs of little nutritive value, and designate them as roughage.

The true foods fall into two classes, energy yielders and nonenergy yielders. The latter class includes all the inorganic constituents of the diet, such as water and the various salts; and a number of organic substances which serve definite purposes not involving the liberation of energy by them. These non-energy yielders are commonly classed as accessories of the diet, to signify their subordinate relation to the energy yielding food. We have to recognize, however, that some of the so-called accessories are necessary to health. These we may call the essential accessories. They include water, the various salts, and a group of organic substances to be described in detail in a later paragraph, known as the vitamines. The other accessories of the diet, chiefly organic, may be designated as occasional accessories. Among these are included the special substances which give flavor to the food, and by making it palatable aid in its digestion. All drugs, including the essential principles of tea, coffee, and cocoa, fall also into this class, as do the substances classed as condiments, pepper, mustard, etc.

The organic constituents of our food not included among the group of accessory articles of diet belong chemically to one or other of three great subdivisions. They are either carbohydrates, fats, or proteins. The entire supply of energy for the Body, and its repair and maintenance to great extent are derived from these three classes of food stuffs. Because of their prime importance they are usually set apart from the other foods as nutrients proper.

Occurrence of Nutrients in Food. The articles which in common language we call foods are, in most cases, mixtures of several nutrients with inorganic and organic accessory substances and with roughage. Bread, for example, contains water, salts, gluten 
(a protein), some fats, much starch, and a little sugar; all true food stuffs: but mixed with these is a quantity of cellulose (the chief chemical constituent of the walls which surround vegetable cells), and this is not a true food since it is incapable of digestion. Chemical examination of all the common articles of diet shows that the actual number of important food stuffs is but small: they are repeated in various proportions in the different things we eat, mixed with small quantities of different flavoring substances, and so give us a pleasing variety in our meals; but the essential substances are much the same in the fare of the workman and in the "delicacies of the season." These primary food stuffs, which are found repeated in so many different foods, belong to one or the other of the classes of nutrients mentioned above; and the food value of any article of diet depends on them far more than on the traces of flavoring matters which cause certain things to be especially sought after and so raise their market value. We cannot, however, conclude that the possession of flavor by foods is wholly unnecessary. We shall see it plays a very real and very important rôle in our use of foods in general.

The Inorganic Essential Accessories. Two inorganic substances, water and sodium chlorid (common salt); are taken separately and consciously as constituents of the diet. We require such large amounts of these substances that they have to be taken thus purposely to insure that enough be gotten. The other inorganic materials, the chlorids, phosphates, and sulphates of potassium, magnesium, and calcium, occur in most ordinary articles of diet, so that we do not swallow them in a separate form. Phosphates, for example, exist in nearly all animal and vegetable foods; moreover certain foods, as casein, contain phosphorus in combinations which in the Body yield it up to be oxidized to form phosphoric acid. The same is true of sulphates, which are partially swallowed as such in various articles of diet, and are partly formed in the Body by the oxidation of the sulphur of various proteins. Calcium salts are abundant in bread and milk, and are also found in many drinking-waters. That these salts are essential to life is proven by the results of feeding animals on diets which have been carefully made salt-free, but are otherwise fully adequate. Such diets invariably cause a steady decline, and, if not discontinued, death. The remarkable fact is that under these 
circumstances death comes much sooner than in complete starvation, if there is no lack of water. When starvation threatens the Body conserves all its substance carefully. This power is not shown when the only elements lacking are the salts. The Body then continues to eliminate them at the usual rate along with the waste products from the other food stuffs, and a fatal deficiency comes on quickly.

In general the craving for salt is associated with a vegetable diet. This is shown very strikingly in the case of grazing animals that in the wild state are known to travel long distances in quest of "salt licks." Carnivorous animals, on the other hand, not only have no craving for salt, but will reject food containing an excess of it. This is said to be true also of Eskimos, whose diet is exclusively of flesh.

The relationship of the salt craving to a vegetable diet is explained on the basis of the high potash content of vegetables. The potash salts react in the Body with the sodium chlorid, forming compounds which are rapidly eliminated by the kidneys. This constant drain on the sodium chlorid of the Body gives rise to a craving which insures its adequate replenishment. We must admit; nevertheless, that civilized man habitually consumes much inore salt than is absolutely necessary. The excess should be classed as a condiment, among the occasional accessories.

The Organic Essential Accessories. Vitamines. For a long time it has been known that rigid confinement to certain restricted diets leads to serious bodily disturbances, even though the amounts of food are ample and all the nutrients sufficiently represented. Outbreaks of scurvy among ships' companies on long voyages were early recognized as due to inadequacies of diet; specifically to lack of fresh meats and vegetables. The precise reason for the clisturbed metabolism of scurvy was not made clear until another dietary disease came under investigation in which the situation could be analyzed more exactly. This is the disease beri-beri, a disease in which the nerve trunks become inflamed, with consequent impairment of conductivity. Paralyses and various disturbances in the normal nutrition of the tissues follow. There is definite proof that this disease is the result of limiting the dict too strictly to polished rice. The inclusion of rice hulls, or of almost any other food substance prevents its occurrence or cures it if 
present. From rice hulls has been prepared a relatively simple extract which has the curative properties of the entire hulls. The explanation seems to be that certain organic substances are essential to normal metabolism. They are present in most foods, but are wanting from some. When the diet is restricted to these latter nutritional disturbances arise. A third dietary disease, pellagra, appears to be due to a diet composed too largely of the products of maize, grits, hominy, and cornmeal. For the substance or substances thus essential the name vitamines has been proposed. We have no definite knowledge as to their mode of action, although the suggestion has been made that they may either act directly as hormones, or may be essential constituents of some or all of the hormones manufactured in the Body.

Recently the interesting discovery has been made that growth of young animals is much favored by the presence in the diet of certain unpurified fats, notably the fat of milk (butter fat), of egg yolk, or of the liver (cod liver oil). Fats of exactly the same chemical composition as these but from other sources, or these same fats after careful purification, do not show this growth-favoring property. The conclusion is that a vitamine-like substance is present with these particular fats, and that the effect observed is due to it.

Occurrence of Occasional Accessories in Food. Variety in the diet depends practically altogether upon the occasional accessories, for the primary food stuffs are few in number and for the most part without very pronounced tastes or flavors, with the single exception of sugar, whose sweet taste makes it, to the eyes of most children at least, the most desirable of all foods. To civilized man variety of diet is a virtual necessity; the accessories, therefore, are to him of great importance. Both meats and vegetables owe their characteristic flavors, in the main, to organic substances present in them. We do not, however, depend wholly on these substances for securing the needed variety in our food. Condiments, pepper and mustard for example, and spices are used very largely in all civilized countries. Chocolate, coffee, and tea are taken by most people more for their agreeable flavor than for their stimulating properties.

The Nutrients. The actual nourishment of the Body depends, as stated above, primarily upon the taking of sufficient quantities 
of the nutrients proper. Of the three groups of nutrients two, carbohydrates and fats, are exclusively energy yielders. Their function is to be oxidized in the Body and thus to furnish the energy by which the machine does its work. The third nutrient group, the proteins, furnishes all the material by which waste of living tissues is made good, and provides likewise a very considerable proportion of the fuel supply of the Body. Because of the twofold function of proteins it is possible for a person or animal to live for a long time upon an exclusively protein diet. Since repair of tissue waste ean be made only by proteins, an animal or a man would starve to death upon a protein-free diet, no inatter how much of the other food stuffs he might have. For that matter not all proteins are tissue-formers; reference to the classification of proteins in Chap. I shows that only the first two classes, the albumins and globulins, are sufficiently complex to yield all the constituents needed for the formation or repair of living tissues. Albuminoids form a constant part of all flesh food, but they can be used by the Body, in the long run, only as it 'uses carbohydrates and fats, for fuel.

Carbohydrates. These are mainly of vegetable origin. The most important are starch, found in nearly all vegetable foods, and having the chemical formula $\left(\mathrm{C}_{6} \mathrm{H}_{10} \mathrm{O}_{5}\right)_{\mathrm{n}}$; the dextrins, or gums; and two classes of sugars; double sugars, having the formula $\mathrm{C}_{12} \mathrm{H}_{22} \mathrm{O}_{11}$, and represented by cane-sugar, sucrose, and milk-sugar, lactose; and single sugars, having the formula $\mathrm{C}_{6} \mathrm{H}_{12} \mathrm{O}_{6}$, and represented by grape-sugar, dextrose. Glycogen, animal starch, is a constituent of muscle tissue and is eaten as a part of flesh. It and milk-sugar are the only carbohydrates commonly eaten which are of animal origin. Cellulose, a very abundant vegetable carbohydrate, is to the human alimentary tract practically indigestible.

Fats. The most important are stearin, palmatin, and olein, which exist in various proportions in animal fats and vegetable oils; the more fluid containing more olein. Butter contains also a little of a fat named butyrin. Fats are compounds of glycerin and fatty acids, and any such substance which is fusible at the temperature of the Body will serve as a food. The stearin of beef and mutton fats is not by itself fusible at the body temperature, but is mixed in those foods with so much olein as to be melted in the alimentary canal. Beeswax, on the other hand, is a fatty body 
which will not melt in the intestines and so passes on unabsorbed; although from its composition it would be useful as a food could it be digested. A distinction is sometimes made between fats proper (the adipose tissue of animals consisting of fatty compounds inclosed in albuminous cell-walls) and oils, or fatty bodies which are not so organized.

Proteins occur as the chief constituent of animal foods, lean meat for example being 90 per cent protein after its large water content is removed. Eggs and milk contain considerable amounts of protein also. Proteins occur to a greater or lesser degree in most vegetable foods. The gluten of wheat is protein; beans and peas contain a larger percentage of protein than any other food except cheese.

The albuminoid of connective tissue, which is present in all meat, is by cooking converted into gelatin, a digestible protein.

Mixed Foods. These, as already pointed out, include nearly all common articles of diet; they contain more than one nutrient. Among them we find great differences; some being rich in proteins, others in starch, others in fats, and so on. The formation of a scientific dietary depends on a knowledge of these characteristics. The foods eaten by man are, however, so varied that we cannot do more than consider the most important.

Flesh. This, whether derived from bird, beast, or fish, consists essentially of the same things-muscular fibers, connective tissue and tendons, fats, blood-vessels, and nerves. It contains several proteins, especially myosin and myogen; gelatin-yielding matters in the white fibrous tissue; stearin, palmatin, and olein as representatives of the fats; and a small amount of carbohydrates in the form of glycogen and grape-sugar, or some chemically allied substances. Flesh also contains much water and a considerable number of salines, the most important and abundant being potassium phosphate. The nitrogenous extractives (Chap. I) give much of its taste to flesh; and small quantities of various of these substances exist in different kinds of meat. There is also more or less yellow elastic tissue in flesh; it is indigestible and useless as food.

When meat is cooked its white fibrous tissue is turned into gelatin, and the whole mass becomes thus softer and more easily disintegrated by the teeth. "When boiled some of the protein 
matters of the meat pass out into the broth, and there in part coagulate and form the scum; this loss may be prevented in great part by putting the raw meat at once into boiling water which coagulates the surface albumen before it dissolves out, and this keeps in the rest, while the subsequent cooking is continued slowly. In any case the myosin, being insoluble in water, remains behind in the boiled meat. In baking or roasting, all the solid parts of the flesh are preserved and certain agreeably flavored bodies are produced, as, to the nature of which little is known.

Eggs. These contain a large amount of egg albumen and, in the yolk, another protein, known as vitellin. Also fats, and a substance known as lecithin, which is important as containing a considerable quantity of phosphorus. Lecithin, or rather a substance yielding it, is an important constituent of the nervous tissues.

Milk contains at least two proteins, lactalbumin and casein; several fats in the butter; a carbohydrate; milk-sugar; much water; and salts, especially potassium and calcium phosphates. Butter consists mainly of the same fats as those in beef and mutton; but has in it about one per cent of a special fat, butyrin. In the milk it is disseminated in the form of minute globules which, for the most part, float up to the top when the milk is let stand and then form the cream. In this each fat-droplet is surrounded by a pellicle of albuminous matter; by churning, these pellicles are broken up and the fat-droplets then run together to form the butter. Casein is insoluble in water; in milk it is dissolved by the alkaline salts present. When milk is kept, its sugar ferments and gives rise to lactic acid, which neutralizes the alkali and precipitates the casein as curds. In cheese-making the casein is acted upon by a ferment present in the extract of stomach used, and converted into tyrein which is precipitated: this clotting does not take place unless a calcium salt be present. Tyrein, which forms the main bulk of a true cheese, is different from the curd precipitated from milk by acids; cheese made from the latter does not "ripen."

Vegetable Foods. Of these wheat affords the best; not that it contains more of any particular nutrient but because of a peculiar property of its protein. The protein of wheat is mainly gluten, 
which when moistened with water forms a tenacious mass, and this it is to which wheaten bread owes its superiority. When the dough is made, yeast is added to it, and produces a fermentation by which, among other things, carbon dioxid gas is produced. This gas, imprisoned in the tenacious dough, and expanded during baking, forms cavities in it and causes it to "rise" and make "light bread," which is not only more pleasant to eat but more digestible than heavy bread. Other cereals may contain a larger percentage of starch, but none have.so much gluten as wheat; when bread is made from them the carbon dioxid gas escapes so readily from the less tenacious dough that it does not expand the mass properly. Corn and rice are valuable chiefly for their high carbohydrate content; beans and peas, on the other hand, have a high per cent of protein. Potatoes contain less actual nutriment for their weight than do any of the other important foods. Their cheapness and digestibility have combined to give them a place in the average dietary out of all proportion to their real value. Other fresh vegetables, as carrots, turnips, and cabbages, are valuable mainly for the salts they contain; their weight is mainly due to water, and they contain but little starch, proteins, or fats. Fruits, like most fresh vegetables, are mainly valuable for their saline constituents, the other food stuffs in them being only. present in small proportion. The cellulose which they contain makes up the major portion of the roughage of the diet, and is valuable on that account.

The Cooking of Vegetables. This is of more importance even than the cooking of flesh, since in most the main alimentary principle is starch, and raw starch is difficult of digestion. In plants starch is stored up within the walls of the plant-cells, which are of cellulose and therefore indigestible. When vegetables are cooked the contents of the cells swell, the cellulose walls are ruptured and the starch is set free to be acted upon by the digestive mechanism of the Body.

Composition of Foods. The following table gives the percentage composition of some of the common foods. 


\begin{tabular}{|c|c|c|c|c|c|}
\hline In 100 Parts & Water & Protein & Fat & $\left|\begin{array}{c}\text { Digestible } \\
\text { Carbohydrate }\end{array}\right|$ & $\begin{array}{l}\text { Inorganic } \\
\text { Material }\end{array}$ \\
\hline 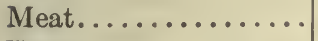 & 76.7 & 20.8 & 1.5 & 0.3 & 1.3 \\
\hline Eggs............... & 73.7 & 12.6 & 12.1 & .... & 1.1 \\
\hline Cheese............. & $36-60$ & 25-33 & $7-30$ & $3-7$ & $3-4$ \\
\hline Cow's Milk. . . . . . . . . & 87.7 & 3.4 & 3.2 & 4.8 & 0.7 \\
\hline Human Milk. ........ & 89.7 & 2.0 & 3.1 & 5.0 & 0.2 \\
\hline Wheat Flour. ........ & 13.3 & 10.2 & 0.9 & 74.8 & 0.5 \\
\hline Wheat Bread........ & 35.6 & 7.1 & 0.2 & 55.5 & 1.1 \\
\hline Rye Flour........... & 13.7 & 11.5 & 2.1 & 69.7 & 1.4 \\
\hline Rye Bread.......... & 42.3 & 6.1 & 0.4 & 49.2 & 1.5 \\
\hline Rice.............. & 13.1 & 7.0 & 0.9 & 77.4 & 1.0 \\
\hline Corn............... & 13.1 & 9.9 & 4.6 & 68.4 & 1.5 \\
\hline Macaroni........... & 10.1 & 9.0 & 0.3 & 79.0 & 0.5 \\
\hline Peas and Beans..... & $12-15$ & $23-26$ & $1 \frac{1}{2}-2$ & $49-54$ & $2-3$ \\
\hline Potatoes............. & 75.5 & 2.0 & 0.2 & 20.6 & 1.0 \\
\hline 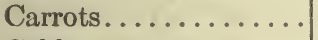 & 87.1 & 1.0 & 0.2 & 9.3 & 0.9 \\
\hline Cabbages............ & 90.0 & $2-3$ & 0.5 & $4-6$ & 1.3 \\
\hline Mushrooms.......... & $73-91$ & $4-8$ & 0.5 & $3-12$ & 1.2 \\
\hline Fruit.............. & 84.0 & 0.5 & $\ldots \ldots$ & 10.0 & 0.5 \\
\hline
\end{tabular}

In a bulletin of the U. S. Department of Agriculture more detailed analyses can be found. (Bull. 28.)

Alcohol. Perhaps no single question in physiology has aroused more discussion than that of the physiological position of alcohol. Its use from time immemorial as a beverage, and the long history of misery and crime which has followed its use to excess, make the problem of its true place one of very great practical importance.

We must recognize at the outset that alcohol has very diverse immediate effects according as it is taken in large or small amounts. The miserable spectacle presented by an intoxicated man emphasizes only too clearly the harm of excessive indulgence; on the other hand, the taking of a small quantity often leads to an appearance of heightened mental and physical ability. Both Mind and Body seem more alert than commonly. No one questions the injurious effects of large amounts of alcohol; the diversity of opinion is with reference to its use in small doses.

It has been demonstrated that alcohol in moderate amounts is oxidized in the Body with the liberation of energy, and is therefore a fuel in the true sense of the word. That it may serve as fuel is not in itself, however, justification for its use, even in small 
quantities. It must be shown that its direct physiological effects are not harmful to the Body, before it can be accepted as a food.

The action of alcohol in small doses appears to be chiefly upon the nervous system, and particularly upon the higher portions of the central nervous system. Its effect upon nerve-centers seems to be a depressing one; the generally accepted view that alcohol is a stimulant being based upon bodily effects which follow nervecenter depression rather than stimulation. For example, cutaneous vasodilation, with flushing of the skin, such as is commonly seen after taking alcohol, is the result of depression of the vasoconstrictor center. The rapid heart-beat, which is another usual phenomenon, results from depression of the cardio-inhibitory center. Even the sparkle of wit and repartee, which is reputed to be very marked after partaking of wine, is the result of removal of the brakes of judgment and caution through depression of those regions of the brain where these functions reside. It is intimated, in fact, that after-dinner wit is ordinarily appreciated at more than its due desert, because of the depression of judgment in the brains of the hearers.

The depressing effect of alcohol upon the brain appears to be progressively from higher to lower centers. The first traits to be dulled are those acquired through precept and moral training; therefore the individual is apt to reveal his "true self," stripped of the veneer of education. With increasing indulgence in alcohol lower and lower tendencies come to the fore, set free by the depression of the higher, and ordinarily controlling ones. Thus it comes to pass that man may sink to the level of the beast.

The question of the moderate use of alcohol resolves itself, then, from a physiological standpoint, into one of the desirability of setting free the lower mental traits and activities through depression of the higher inhibitory ones. It is sometimes argued that in America where the dominant mental obsession of a considerable proportion of the population is in affairs of business the setting free of the brain from business cares during leisure hours is a virtual necessity, and that the use of alcohol is the most direct method of bringing this about. Even though we grant the first part of the argument it does not necessarily follow that the second part is to be accepted also. For the real objection to the use of alcohol, even in small quantities, is that the desire for alcohol, 
unlike the desire for food, increases as more is taken instead of decreasing when satiety is reached. It thus requires a stronger effort of will to leave off as more is taken, and since the alcohol at the same time depresses the power of the will the danger of overindulgence is continually present. Only where the will is sufficiently strong to set a limit and adhere rigidly to it is the continuous moderate use of alcohol in any degree safe.

Returning to the question of the desirability of the practice of removing the brakes from the brain periodically, it may be said that the opinion seems to be becoming more and more prevalent among neurologists that the use of alcohol for such a purpose, particularly in early and middle life, is more of an injury than a benefit. The normal interactions among the different parts of the mental apparatus should be permitted, according to these observers, to proceed without artificial interference, at least during the period of the most active associative processes. There seems to be no vital objection to the moderate use of alcohol on the part of persons who have passed the age of fifty or thereabouts. The danger of acquiring the alcohol habit is practically nil at that age, and the predominant mental traits are by that time so completely in control that occasional release from them may operate as a distinct advantage. This is particularly true in the case of those alderly persons who find themselves disposed to a somewhat gloomy outlook upon life. The temperate use of alcohol may make life more enjoyable for themselves and also for those about them.

Tea, Coffee, and Cocoa: These beverages all owe their special physiological properties to certain alkaloids present in them. The active principle of tea and coffee is the same, caffein; that of cocoa, and its derivative, chocolate, is a closely related substance theobromin. Caffein and theobromin appear to be direct nervestimulants. They cause a rise of blood-pressure through stimulation of the vasoconstrictor center. Their use, like that of alcohol, constitutes an artificial interference with normal processes, and is subject, therefore, to the general objections which arise against such interference. Their effects are of varying intensity; cocoa is an exceedingly mild stimulant; tea, properly made, is somewhat stronger; and coffee, properly made, is stronger yet. Their use is borne much better by some persons than by others. 
They are not dangerous in the sense that alcohol is, through an increasing craving which readily leads to overindulgence and resulting disaster, although they, like alcohol, are often taken to excess. Temperance in the use of these beverages is as much the part of wisdom as in the use of alcohol. Again, like alcohol, they are best left alone during early life.

The improper preparation of tea and coffee, by boiling them in water, carries into solution, in addition to the stimulating principle, a substance, tannin, whose effect upon the system is apt to be distinctly harmful. These beverages should therefore always be prepared by methods which do not involve prolonged or even brief boiling while the tea leaves or coffee grounds are actually present in the liquid.

Food Poisoning. There are several conditions under which foods, instead of being of benefit to the Body, may become actually harmful. They need to be guarded against, both by individuals and by the public. The latter because, with the present organization of society, virtually all foods pass through many hands before they finally reach the consumer, and there are correspondingly many possibilities of contamination. The deliberate introduction into foods of injurious adulterants is probably much less common than some people have supposed. Unintentional contamination may occur, although it is much less likely under modern scientific conditions than formerly when rule-of-thumb methods obtained. A historical example of accidental contamination was the ergot poisoning that used occasionally to ravage certain parts of Europe. Ergot is a poisonous constituent of a parasitic growth sometimes found on rye. When the affected grain was made into rye flour and eaten regularly over a long period, whole populations underwent typical ergot poisoning. The symptoms were in many respects similar to those of leprosy; dry gangrene, with loss of fingers and toes, and ultimate death.

More dangerous, because more difficult to guard against, are chemical changes that may occur in food between the time of its preparation and its consumption. These are usually the result of bacterial growth within the food mass, this growth giving rise to toxins as waste products in much the same manner as does the growth of pathogenic organisms within the Body. There are at least two conditions of poisoning from such toxins. The first is 
ptomain poisoning; the toxins formed are known as ptomains. The chief symptom is the very pronounced gastro-intestinal upset. This is beneficial in that it acts to rid the Body promptly of the contaminated material, and so to reduce the amount of poison absorbed. A second form of poisoning from bacterial decomposition is botulism, so named from its occasional occurrence in sausage. The effect of this poison on the digestive tract is just opposite to that of ptomain. It paralyzes instead of exciting; so the poisoned mass is not expelled, but remains in the intestinal tract and allows absorption to continue. For this reason botulism has ordinarily much more serious effects than has ptomain.

An additional type of food poisoning is that seen in individual susceptibilities, or idiosyncracies. Some people are poisoned by veal, others by shell fish, occasionally a case of susceptibility to egg albumen is seen. Various other foods may act similarly. The poisonous elements in these cases appear to be identical with the lymphagogues described in a previous chapter (p. 385). The lymphagogue action was there stated to show itself only in susceptible individuals. In general acute poisoning is probably.only a more marked manifestation of the sensitiveness which takes the form of increased permeability of the capillaries in those in whom the action is purely that of a lymphagogue. 


\section{CHAPTER XXVI}

\section{THE ALIMENTARY CANAL AND ITS APPENDAGES}

General Arrangement. The alimentary canal is essentially a tube running through the Body (Fig. 2) and lined by a vascular membrane, most of which is specially adapted for absorption; it communicates with the exterior at three points (the nose, the mouth, and the anal aperture), at which the lining mucous membrane is continuous with the general outer integument. Supporting the absorbent membrane are layers which strengthen the tube, and are in part muscular and, by their contractions, serve to pass materials along it from one end to the other. In the walls of the canal are numerous blood and lymphatic vessels which carry off the matters absorbed from its cavity; and there also exist in connection with it numerous glands, whose function it is to pour into it various secretions by which the chemical act of digestion is carried on. Some of these glands are minute and embedded in the walls of the alimentary tube itself, but others (such as the salivary glands) are larger and lie away from the main channel, into which their products are carried by ducts of various lengths.

The alimentary tube is not uniform but presents several dilatations on its course; nor is it straight, since, being much longer than the Body, a large part of it is packed away by being coiled up in the abdominal cavity.

Subdivisions of the Alimentary Canal. The mouth-opening leads into a chamber containing the teeth and tongue, the mouthchamber or buccal cavity. This is succeeded by the pharynx or throat-cavity; which narrows at the top of the neck into the gullet or esophagus; this runs down through the thorax and, passing through the diaphragm, dilates in the upper part of the abdominal cavity into the stomach. Beyond the stomach the channel again narrows to form a long and greatly coiled tube, the small intestine, which terminates by opening into the large intestine, much shorter although wider than the small, and terminating by an opening on the exterior. 
The Mouth-Cavity (Fig. 121) is bounded in front and on the sides by the lips and cheeks, below by the tongue, $k$, and above

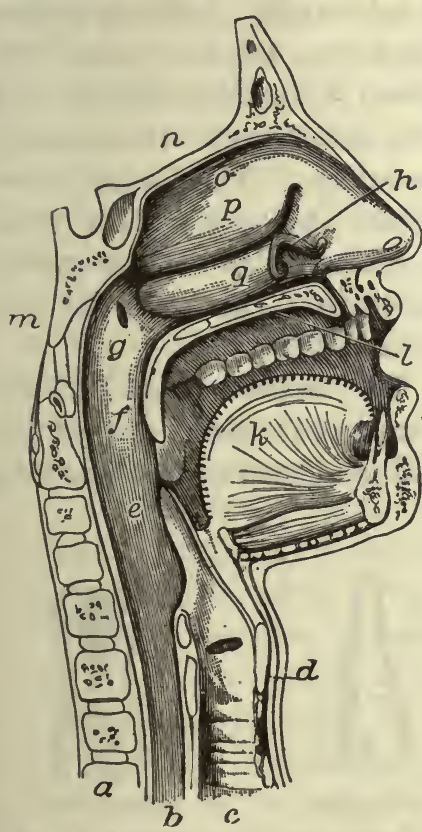

Fig. 121.-The mouth, nose and pharynx, with the commencement of the gullet and larynx, as exposed by a section, a little to the left of the median plane of the head. $a$, vertebral column; $b$, gullet; $c$, windpipe; $d$, larynx; $e$, epiglottis; $f$, soft palate; $g$, opening of Eustachian tube; $k$, tongue; $l$, hard palate; $m$, the sphenoid bone on the base of the skull; $n$, the fore part of the cranial cavity; $o, p, q$, the turbinate bones of the outer side of the left nostril-chamber.

the first or milk set appears soon after birth and is shed during childhood, when the second or permanent set appears.

The teeth differ in minor points from one another, but in each three parts are distinguishable; one, seen in the mouth and called the crown of the tooth; a second, embedded in the jaw-bone and called the root or fang; and between the two, embraced by the edge of the gum, is a narrowed portion, the neck or cervix. From differences in their forms and uses the teeth are divided into incisors, 
canines, bicuspids, and molars, arranged in a definite order in each jaw. Beginning at the middle line we meet in each half of each jaw with, successively, two incisors, one canine, and two molars in the milk set; making twenty altogether in the two jaws. The teeth of the permanent set are thirty-two in number, eight in each half of each jaw, viz.-beginning at the middle line-two incisors, one canine, two bicuspids, and three molars. The bicuspids, or premolars, of the permanent set replace the milk-molars, while the permanent molars are new teeth added on as the jaw grows, and not substituting any of the milk-teeth. The hindmost permanient molars are often called the wisdom-teeth.

Characters of Individual Teeth. The incisors (Fig. 122) are adapted for cutting the food. Their crowns are chisel-shaped and have sharp horizontal cutting edges, which become worn away by

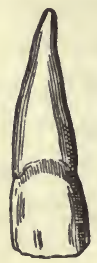

Fig. 122

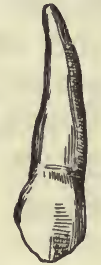

Fig. 123

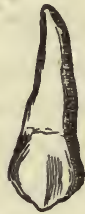

Fig. 124

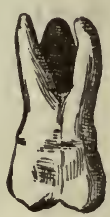

FIg. 125

Fra. 122.-An incisor tooth.

FIG. 123.-A canine or eye-tooth.

Fig. 124.-A bicuspid tooth seen from its outer side; the inner cusp is, accordingly, not visible.

Fig. 125. - A molar tooth.

use so that they are beveled off behind in the upper row, and in the opposite direction in the lower. Each has a long root. The canines (Fig. 123) are somewhat larger than the incisors. Their crowns are thick and somewhat conical, having a central point or cusp on the cutting edge. In dogs, cats, and other carnivora the canines are very large and adapted for seizing and holding prey. The bicuspids or premolars (Fig. 124) are rather shorter than the canines and their crowns are somewhat cuboidal. Each has two cusps, an outer towards the cheek, and an inner on the side turned towards the interior of the mouth. The root is compressed laterally, and has usually a groove partially subdividing it into two. At its tip the separation is often complete. The molar teeth or grinders (Fig. 125) have large crowns with broad surfaces, on which 
are four or five projecting tubercles, which roughen them and make them better adapted to crush the food. Each has usually several roots. The milk-teeth differ only in subsidiary points from those of the same names in the permanent set.

The Structure of a Tooth. If a tooth be broken open, a cavity extending through both crown and root will be found in it. This is filled during life with a soft vascular pulp, and hence is known as the "pulp-cavity" (c, Fig. 126). The hard parts of the tooth disposed around the pulp-cavity consist of three different tissues. Of these one immediately surrounds the cavity and makes up most of the bulk of the tooth; it is dentine (2, Fig. 126); covering the dentine on the crown is the enamel (1, Fig. 126) and on the root, the cement (3, Fig. 126).

The pulp-cavity opens below by a narrow aperture at the tip of the root, or at the tip of each if the tooth have more than one. The pulp consists mainly of connective tissue, but its surface next the dentine is covered by a layer of columnar cells. Through the opening on the root bloodvessels and nerves enter the pulp.

The dentine (ivory) yields on analysis the same materials as bone but is somewhat harder, earthy matters constituting 72 per cent of it as against 66 per cent in bone. Under the microscope it is recognized by the fine dentinal tubules which, radiating from the pulp-cavity, perforate it throughout, finally ending in minute branches which open into irregular cavities, the interglobular

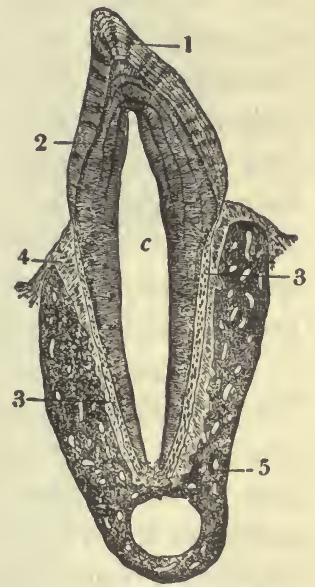

Fig. 126.-Section through a premolar tooth of the cat still embedded in its socket. 1, enamel; 2 , dentine; 3 , cement; 4 , the gum; 5 , the bone of the lower jaw; $c$, the pulpcavity. spaces, which lie just beneath the enamel or cement. At their widest ends, close to the pulp-cavity, the dentinal tubules are only about 0.005 millimeter $\left({ }_{45}^{1} 00\right.$ of an inch) in diameter. The cement is much like bone in structure and composition. It is thickest at the tip of the root and thins away towards the cervix. Enamel is the hardest tissue in the Body, yielding on analysis only from 2 per cent to 3 per cent of organic matter, the rest being mainly calcium phosphate and carbonate. Its histological elements are 
minute hexagonal prisms, closely packed, and set on vertically to the surface of the subjacent dentine. It is thickest over the free end of the crown, until worn away by use. Covering the enamel in unworn teeth is a thin structureless horny layer, the enamel cuticle.

The Tongue (Fig. 127) is a muscular organ covered by mucou:; membrane, extremely mobile, and endowed not only with a delicate tactile sensibility but with the terminal organs of the special sense of taste; it is attached by its root to the hyoid bone. On its upper surface are numerous small eminences or papillo, such as: are found more highly developed on the tongue of a cat, where they may be readily felt. On the human tongue there are three forms of papillæ, the circumvallate, the fungiform, and the filiform. The circumvallate papillæ, 1 and 2 (Fig. 127), the largest and leasi numerous, are from seven to twelve in number and lie near the root of the tongue arranged in the form of a $\mathrm{V}$ with its open angle turned forwards. Each is an elevation of the mucous membrane. covered by epithelium, and surrounded by a trench. On the sides of these papillæ, embedded in the epithelium, are many small oval bodies richly supplied with nerves and supposed to be concerned in the sense of taste, and hence called the taste-buds (Chap. XIV). The fungiform papillo, 3, are rounded elevations attached by somewhat narrowed stalks, and found all over the middle and fore part of the upper surface of the tongue. They are easily recognized on the living tongue by their bright red color. The filiform papilloe, 4, most numerous and smallest, are scattered all over the dorsum of the tongue except near its base. Each is a conical eminence covered by a thick horny layer of epithelium. It is these papillæ which are so highly developed on the tongues of Carnivora, and serve them to scrape bones clean of even such tough structures as ligaments.

In health the surface of the tongue is moist, covered by little, "fur," and in childhood of a red color. In adult life the natural color of the tongue is less red, except around the edges and tip; a bright-red glistening tongue being then, usually a symptom of disease. When the digestive organs are deranged the tongue is commonly covered with a thick yellowish coat, composed of a little mucus, some cells of epithelium shed from the surface, and numerous microscopic organisms known as bacteria; and there is frequently a "bad taste in the mouth." The whole alimen. 
tary mucous membrane is in close physiological relationship; and anything disordering the stomach is likely to produce a "furred tongue."

The Salivary Glands. The saliva, which is poured into the mouth and which, mixed with the secretion of minute glands em-

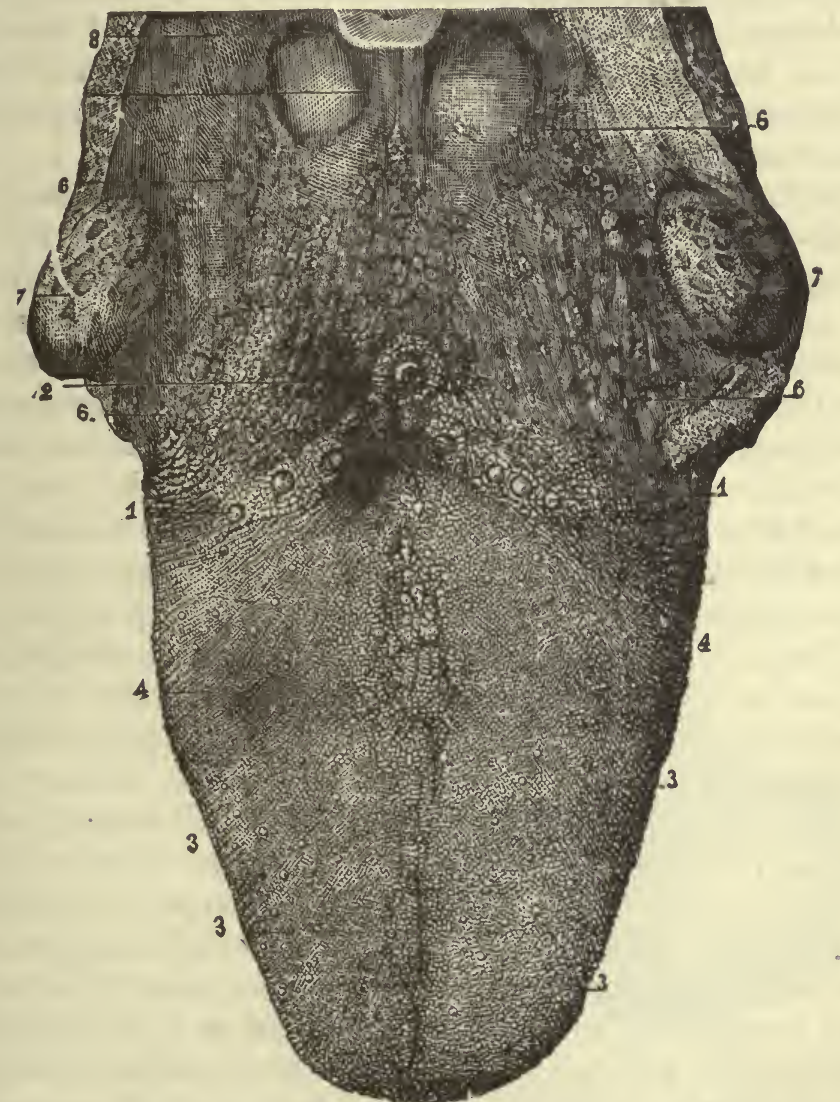

FIG. 127.-The upper surface of the tongue with part of the pillars of the fauces and the tonsils. 1,2 , circumvallate papillæ; 3 , fungiform papillæ; 4 , filiform papillæ; 6 , mucous glands; 7 , tonsils; 8 , tip of epiglottis.

bedded in its lining membrane, moistens it, is secreted by three pairs of glands, the parotid, the submaxillary, and the sublingual. The parotid glands lie in front of the ear behind the ramus of the lower jaw; each sends its secretion into the mouth by a tube known 
as Stenson's duct, which crosses the cheek and opens opposite the second upper molar tooth. In the disease known as mumps * the parotid glands are inflamed and enlarged. The submaxillary glands lie between the halves of the lower jaw-bone, near its angles, and their ducts open beneath the tongue near the middle line. The sublingual glands lie beneath the floor of the mouth, covered by its mucous membrane, between the back part of the tongue and the lower jaw-bone. Each has many ducts (8 to 20), some of which join the submaxillary duct, while the rest open separately in the floor of the mouth.

The Fauces is the name given to the aperture which can be seen at the back of the mouth below the soft palate (Fig. 121), and leading into the pharynx. It is bounded above by the soft palate and uvula, below by the root of the tongue, and on the sides by muscular elevations covered by mucous membrane, which reach from the soft palate to the tongue. These elevations are the pillars of the fauces. Each bifurcates below, and in the hollow between its divisions lies a tonsil (7, Fig. 127), a soft rounded body about the size of an almond, composed of lymphoid tissue (Chap. XXII).

The Pharynx or Throat-Cavity (Fig. 121). This portion of the alimentary canal may be described as a conical bag with its broad end turned upwards towards the base of the skull, and its narrow end downwards and passing into the gullet. Its front is imperfect, presenting openings which lead into the nose, the mouth, and (through the larynx and windpipe) the lungs. Except during swallowing or speech the soft palate hangs down between the mouth and pharynx; during deglutition it is raised into a horizontal position and separates an upper or respiratory portion of the pharynx from the rest. Through this upper part, therefore, air alone passes, entering it from the posterior ends of the two nostril-chambers; while through the lower portion both food and air pass, one on its way to the gullet, $b$, Fig. 121, the other through the larynx, $d$, to the windpipe, $c$; when a morsel of food "goes the wrong way" it takes the latter course. Opening into the upper portion of the pharynx on each side is an Eustachian tube, $g$ (p. 227); so that the apertures leading out of it are seven in number; the two posterior nares, the two Eustachian tubes, the fauces, the opening of the larynx, and that of the gullet. At the root of the tongue,

* Parotitis, in technical language. 
over the opening of the larynx, is a plate of cartilage, the epiglottis, $e$, which can be seen if the mouth is widely opened and the back of the tongue pressed down by some such thing as the handle of a spoon. During swallowing the epiglottis is pressed down like a lid over the air-tube and helps to keep food or saliva from entering it. In structure the pharynx consists essentially of a bag of connective tissue lined by mucous membrane, and having muscles in its walls which drive the food on.

The Esophagus or Gullet is a tube commencing at the lower termination of the pharynx and which, passing on through the neck and chest, ends below the diaphragm by joining the stomach. In the neck it lies close behind the windpipe. It consists of three coats - a mucous membrane within; next, a submucous coat of areolar connective tissue: and, outside, a muscular coat made up of two layers, an inner with transversely and an outer with longitudinally arranged fibers. In and beneath its mucous membrane are numerous small mucous glands whose ducts open into the tube.

The Stomach (Fig. 128) is a somewhat conical bag placed transversely in the upper part of the abdominal cavity. Its larger end is turned to the left and lies close beneath the diaphragm; opening into its upper border, through the cardiac orifice at $a$, is the gullet $d$. The narrower right end is continuous at $c$ with the small intestine; the aperture between the two is the pyloric orifice. The pyloric end of the stomach lies lower in the abdomen than the cardiac, and is separated from the diaphragm by the liver (see Fig. 1). The concave border between the two orifices is known as the small curvature, and the convex, as the great curvature of

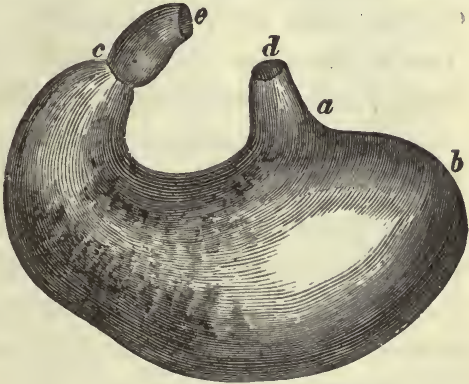

Fig. 128.- The stomach. $d$, lower end of the gullet; $a$, position of the cardiac aperture; $b$, the fundus; $c$, the pylorus; $e$, the commencement of the small intestine; along $a, b, c$, the great curvature; between the pylorus and $d$, the lesser curvature. the stomach. From the latter hangs down a fold of peritoneum known as the great omentum. It is spread over the rest of the abdominal contents like an apron. After middle life much fat frequently accumulates in the omentum, so that it is largely re- 
sponsible for the "fair round belly with good capon lin'd." The protrusion $b$ to the left side of the cardiac orifice, Fig. 128, is the fundus. The size of the stomach varies greatly with the amount of food in it; when empty it is little more than a tube; just after a moderate meal it is about ten inches long, by five wide at its broadest part.

Since the cardiac end of the stomach lies immediately beneath the diaphragm, which has the heart on its upper side, its overdistension, due to indigestion or flatulence, may impede the action of the thoracic organs, and cause feelings of oppression in the chest, or palpitation of the heart.

Structure of the Stomach. This organ has four coats, known successively from without in as the serous, the muscular, the submucous, and the mucous. The serous coat is formed by a reflection of the peritoneum, a double fold of which slings the stomach; after separating to envelop it the two layers again unite and, hanging down beyond it, form the great omentum. The muscular coat (Fig. 43) consists of unstriped muscular tissue arranged in three layers: an outer, longitudinal, most developed about the curvatures; a circular, evenly spread over the whole organ, except around the pyloric orifice where it forms a thick ring; and an inner, oblique and very incomplete, radiating from the cardiac orifice. The submucous coat is made up of lax areolar tissue and binds loosely the mucous coat to the muscular. The mucous coat is a moist pink membrane which is inelastic, and large enough to line the stomach evenly when it is fully distended. Accordingly, when the organ is empty and shrunken, this coat is thrown into folds, which disappear when the organ is distended. During digestion the arteries supplying the stomach become dilated and, its capillaries being gorged, its mucous membrane is then much redder than during hunger.

The blood-vessels of the stomach run to it between the folds of peritoneum which sling it. After giving off a few branches to the outer layers, most of the arteries break up into small branches in the submucous coat, from which twigs proceed to supply the close capillary network of the mucous membrane.

The nerves of the stomach belong to the autonomic system, and like most other structures supplied by this system, the stomach has double innervation (p. 19) ). The cranial autonomic fibers are 
derived from the vagi. In the lower part of the thorax these nerves consist mainly of non-medullated fibers, and lie on the sides of the gullet, across which they interchange fibers by means of several branches. On entering the abdomen the left vagus passes to the ventral side of the stomach, in which it ends: the right supplies the dorsal side of the stomach, but a considerable portion of it passes on to enter the solar plexus, which lies behind the stomach and contains several large ganglia. The thoracico-lumbar autonomic fibers pass to the stomach as branches from the great splanchnic nerves, which serve as the chief paths of distribution for these fibers in the abdomen.

Histology of the Gastric Mucous Membrane. Examination of the inner surface of the stomach with a hand lens shows it to be covered, except in the fundic region, with minute shallow pits. Into these open the mouths of minute tubes, the gastric glands, which are closely packed side by side in the mucous membrane; something like the cells of a honeycomb, except that each is open at one end. Between them lie a small amount of connective tissue, a close network of lymph-channels, and capillary bloodvessels. The whole surface of the mucous membrane is lined by a single layer of columnar mucus-making epithelium cells ( $m$, Fig. 129). These dip down and line the necks of the tubular glands. The deeper portions of the glands are lined by a layer of shorter and somewhat cuboidal cells, the central or chief cells. In specimens taken from a healthy animal killed during digestion these cells are large and do not stain deeply with carmine. Similar specimens taken from an animal an hour or two after a good meal has been swallowed show the chief cells shrunken and staining more deeply. They thus store up during rest a material which they get rid of when the gastric juice is being

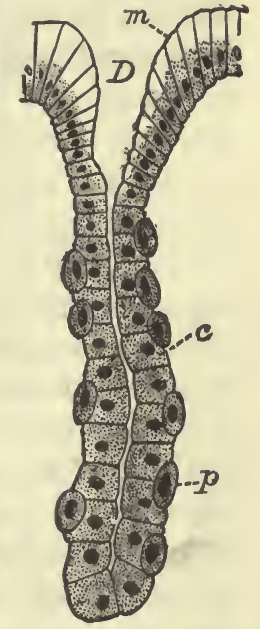

Fig. 129. - Diagram of a gastric gland. $D$, mouth of the gland; $m$, mucous cells lining the mouth of the gland and covering the inner surface of the mucous membrane; $c$, chief cells; $p$, oval cells. secreted.

In the pyloric end of the stomach only the chief cells line the glands, but elsewhere there is found outside of them, in most 
of the glands, an incomplete layer of larger oval cells ( $p$, Fig. 129). The glands frequently branch at their deeper ends.

The Pylorus. If the stomach be opened it is seen that the mucous membrane projects in a fold around the pyloric orifice and narrows it. This is due to a thick ring of the circular muscular layer there developed, and forming around the orifice a sphincter muscle, which, by its contraction, keeps the passage to the small intestine closed except when portions of food are to be passed on from the stomach to succeeding divisions of the alimentary canal.

The Small Intestine (Fig. 136), commencing at the pylorus, ends, after many windings, in the large intestine. It is about six meters (twenty feet) long, and about five centimeters (two inches) wide at its gastric end, narrowing to about two-thirds of that width at its lower portion. Externally there are no lines of subdivision on the small intestine, but anatomists arbitrarily describe it as consisting of three parts; the first twelve inches being the duodenum, $D$, the succeeding two-fifths of the remainder the jejunum, $J$, and the rest the ileum, $I$.

Like the stomach, the small intestine possesses four coats; a serous, a muscular, a submucous, and a mucous. The serous coat is formed by a duplicature of the peritoneum, but presents nothing answering to the great omentum; this double fold slinging the intestine is named the mesentery. The muscular coat is composed of plain muscular tissue arranged in two strata, an outer longitudinal, and an inner transverse or circular. The submucous coat is like that of the stomach; consisting of loose areolar tissue, binding together the mucous and muscular coats, and forming a bed in which the blood and lymphatic vessels (which reach the intestine in the fold of the mesentery) break up into minute branches before entering the mucous membrane.

The Mucous Coat of the Small Intestine. This is pink, soft and extremely vascular. It does not present temporary or effaceable folds like those of the stomach, but is, throughout a great portion of its length, raised up into permanent transverse folds in the form of crescentic ridges, each of which runs transversely for a greater or less way round the tube (Fig. 130). These folds are the valvulø conniventes. They are first found about two inches from the pylorus, and are most thickly set and largest in the upper half of the jejunum, in the lower half of which they become gradually 
less conspicuous; and they finally disappear altogether about the middle of the ileum. The folds serve greatly to increase the surface of the mucous membrane both for absorption and secretion, and they also delay the food somewhat in its passage, since it must collect in the hollows between them, and so be longer exposed to the action of the digestive liquids. Examined closely with the eye or, better, with aid of a lens, the mucous membrane of the small intestine is seen to be not smooth but shaggy, being covered everywhere (both over the valvulæ conniventes and between them) with closely packed minute processes, standing up somewhat like the "pile" on velvet, and known as the villi. Each villus is from 0.5 to 0.7 millimeter ( $\frac{1}{50}$ to $\frac{1}{35}$ inch) in length; some are conical

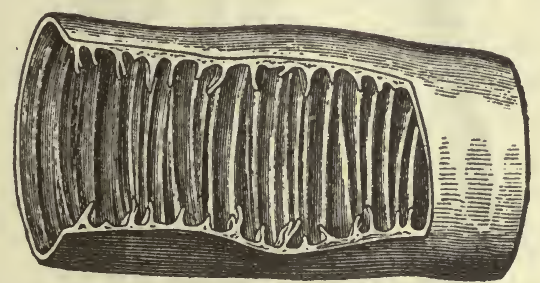

Fig. 130.-A portion of the small intestine opened to show the valvulce conniventes.

and rounded, but the majority are compressed at the base in one diameter (Fig. 131). In structure a villus is somewhat complex. Covering it is a single layer of columnar epithelial cells, the exposed ends of the majority having a peculiar bright striated border and being probably of great importance in absorption. Mixed with these cells are others in which most of the cell has become filled with a clear mass which does not stain readily with reagents; the deep narrow end of the cell stains easily and contains the nucleus. From time to time the clear substance (mucigen) is converted into mucus and discharged into the intestine, leaving behind only the nucleus and the protoplasm around it. These reconstruct the cell and form more mucigen. These mucus-forming cells are named goblet-cells, from their shape. Beneath the epithelium the villus may be regarded as made up of a framework of connective tissue, supporting the more essential constituents. Near the surface is an incomplete layer of plain muscular tissue, continuous below with a muscular stratum forming the deepest layer of the mucous membrane and named the muscularis mucose. In the center is an 
off-shoot of the lymphatic system; sometimes in the form of a single vessel with a closed dilated end, and sometimes as a network formed by two main vessels with cross-branches. During digestion these lymphatics are filled with a milky-white liquid absorbed from the intestines, and they are accordingly called the lacteals. They communicate with larger branches in the submucous coat, which end in trunks that pass out through the mesentery to join the main lymphatic system. Finally, in each villus,
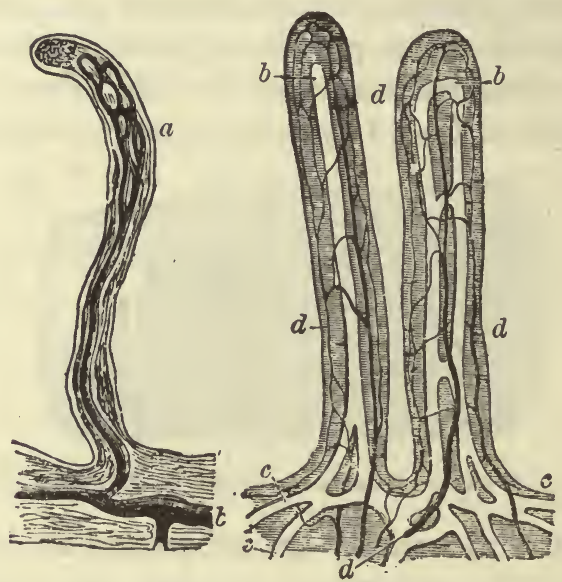

Fra. 131.-Villi of the small intestine; magnified about 80 diameters. In the right-hand figure the lacteals, $a, b, c$, are filled with white injection: $d$, bloodvessels. In the left-hand figure the lacteals alone are represented, filled with a dark injection. The epithelium covering the villi, and their muscular fibers, are omitted.

outside the lacteals and beneath the muscular layer of the villus, is a close network of blood-vessels.

Opening on the surface of the small intestine, between the bases of the villi, are small glands, the crypts of Lieberkühn. Each is a simple unbranched tube lined by a layer of columnar cells some of which have a striated free border, though less marked than that on the corresponding cells of the villi, and others are goblet-cells. The crypts of Lieberkühn are closely packed, side by side, like the glands of the stomach. In the duodenum are found other minute glands, the glands of Brunner. They lie in the submucous coat and send their ducts through the mucous membrane to open on its inner side. 
The Large Intestine (Fig. 136), forming the final portion of the alimentary canal, is about 1.5 meters ( 5 feet) long, and varies in diameter from about 6 to 4 centimeters (21/2 to $1 \frac{1}{2}$ inches). Anatomists describe it as consisting of the coecum with the vermiform appendix, the colon, and the rectum. The small intestine does not open into the commencement of the large but into its side, some distance from its closed upper end, and the cæcum, $C C$, is that part of the large intestine which extends beyond the communication. From it projects the vermiform appendix, a narrow tube not thicker than a lead pencil, and about 10 centimeters (4 inches) long. The colon commences on the right side of the abdominal cavity where the small intestine communicates with the large, runs up for some way on that side (ascending colon, $A C$ ), then crosses the middle line (transverse colon, $T C$ ) below the stomach, and turns down (descending colon, $D C$ ) on the left side and there makes an S-shaped bend known as the sigmoid flexure, SF; from this the rectum, $R$, the terminal straight portion of the intestine, proceeds to the anal opening, by which the alimentary canal communicates with the exterior. In structure the large intestine presents the same coats as the small. The external stratum of the muscular coat is not, however, developed uniformly around it, except on the rectum, but occurs in three bands separated by intervals in which it is wanting. These bands being shorter than the rest of the tube cause it to be puckered, or saccullated, between them. The mucous coat possesses no villi or valvulæ conniventes, but is usually thrown into effaceable folds, like those of the stomach but smaller. It contains numerous closely set glands much like the crypts of Lieberkühn of the small intestine.

The Ileocolic Valve. Where the small intestine joins the large there is a valve, formed by two flaps of the mucous membrane sloping down into the colon, and so disposed as to allow matters to pass readily from the ileum into the large intestine but not the other way.

The Nerves of the Intestines. The intestines, like the stomach, have the double autonomic innervation; the paths of approach are in general the same as for the stomach, by way of the vagus for the cranial autonomics, and the splanchnics for the thoracicolumbar. Both these sets of nerves ramify in the solar plexus; from here nerve strands pass to the intestine, as well as to the 
stomach, along the mesentery. This innervation extends throughout the small intestine and the ascending and transverse colons. The descending colon has its thoracico-lumbar innervation by way of the hypogastric nerve which extends to the hypogastric plexus in the lower portion of the abdominal cavity and ramifies thence over the descending colon and rectum. The opposing autonomic innervation for this region is derived from the sacral part of the system. The nervus erigens is the path from the sacral part of the

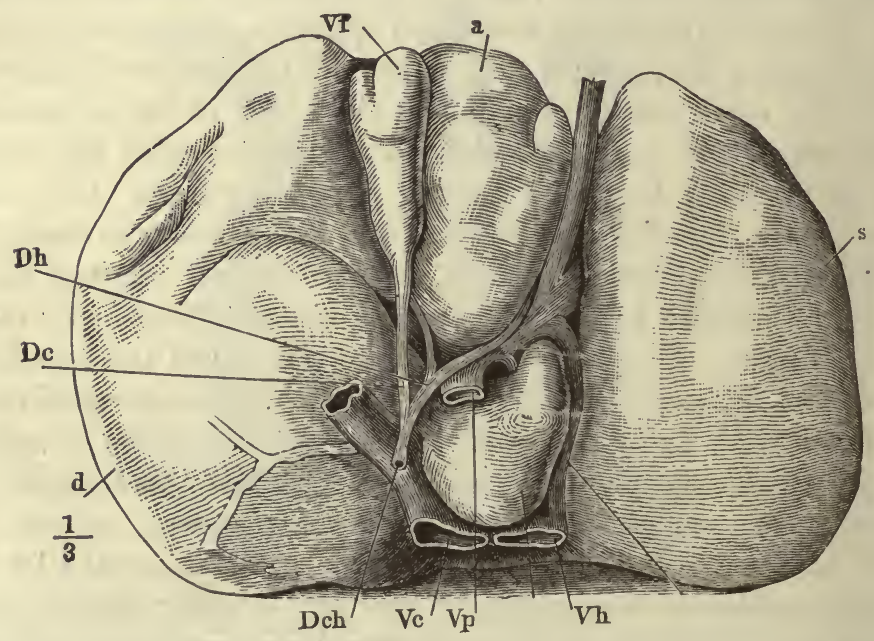

Fig. 132.-The under surface of the liver. $d$, right, and $s$, left lobe; $V h$, hepatic vein; $V p$, portal vein; $V c$, vena cava inferior; $D c h$, common bile-duct; $D c$, cystic duct; $D h$, hepatic duct; $V f$, gall-bladder.

spinal cord to the hypogastric plexus. Thence the distribution is the same as for the thoracico-lumber autonomics.

The intestines are provided, in addition, with an intrinsic innervation consisting of two nervous networks or plexuses lying, one between the mucosa and the muscular coat, the plexus of Meissner, and the other between the circular and longitudinal muscle layers, the plexus of Auerbach.

The Liver. Besides the secretions formed by the glands embedded in its walls, the small intestine receives those of two large glands, the liver and the pancreas, which lie in the abdominal cavity. The ducts of both open by a common aperture into the duodenum about 10 centimeters ( 4 inches) from the pylorus. 
The liver is the largest gland in the Body, weighing from 1,400 to 1,700 grams (50 to 64 ounces). It is situated in the upper part of the abdominal cavity (le, le', Fig. 1), rather more on the right than on the left side and immediately below the diaphragm, into the concavity of which its upper surface fits, and reaches across the middle line above the pyloric end of the stomach. It is of dark reddish-brown color, and of a soft friable texture. A deep fissure incompletely divides the organ into right and left lobes, of which

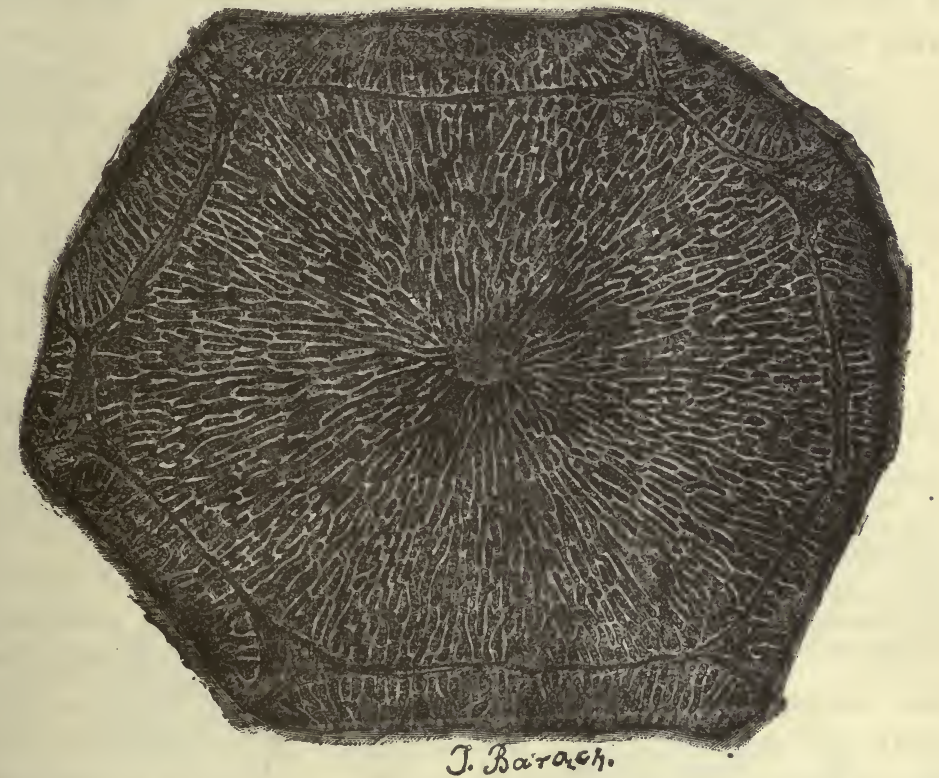

FIG. 133.-A lobule of the liver (pig), magnified, showing the hepatic cells radiately arranged around the central intralobular vein, and the connective tissue surrounding the lobule. (Scymonowicz.)

the right is much the larger; on its under surface (Fig. 132) shallower grooves mark off several minor lobes. Its upper surface is smooth and convex. The vessels carrying blood to the liver are the portal vein, $V p$, and the hepatic artery; both enter it at a fissure (the portal fissure) on its under side, and there also a duct passes out from each half of the organ. The ducts unite to form the hepatic duct, $D h$, which meets at an acute angle, the cystic duct, $D c$, proceeding from the gall-bladder, $V f$, a pear-shaped sac in which the bile, or gall, formed by the liver, accumulates when food is not 
being digested in the intestine. The common bile-duct, Dch, formed by the union of the hepatic and cystic ducts, opens into the duodenum. The blood which enters the liver by the portal vein and hepatic artery passes out by the hepatic veins, $V h$, which leave the posterior border of the organ close to the vertebral column, and there open into the inferior vena cava just before it passes up through the diaphragm.

The Structure of the Liver. On closely examining the surface of the liver, it will be seen to be marked out into small angular areas from one to two millimeters $\left(\frac{1}{25}\right.$ to $\frac{1}{12}$ inch) in diameter.

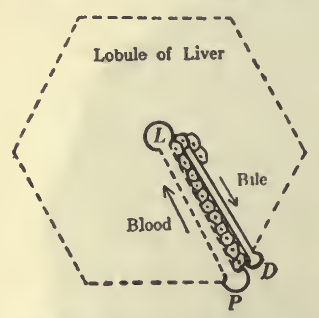

Fig. 134.-Diagram to illustrate the relationship of blood-capillaries, bilecapillaries, and liver-cells. $L$, intralobular vein at center of lobule; $P$, vessel of interlobular plexus from which a blood capillary extends to $L ; D$, a minute bile-duct with which a bilecapillary communicates.
These are the outer sides of the superficial layer of a vast number of minute polygonal masses, or lobules, of which the liver is built up; similar areas are seen on the surface of any section made through the organ. Each lobule (Fig. 133) consists of a number of hepatic cells supported by a close network of capillaries; and is separated from neighboring lobules by connective tissue, larger blood-vessels, and branches of the hepatic duct. The hepatic cells are the proper tissue elements of the liver, all the rest being subsidiary arrangements for their nutrition and protection. Each is polygonal, nucleated and very granular, and has a diameter of about 0.025 millimeter ( $\frac{1}{1000}$ of an inch). In each lobule they are arranged in rows or strings, which form a network, in the meshes of which the blood-capillaries and bile-capillaries run. The blood carried in by the portal vein (which has already circulated through the capillaries of the stomach, spleen, intestines and pancreas) is conveyed to a fine vascular interlobular plexus around the liver-lobules, from which it flows on through the capillaries of the lobules themselves. These (Fig. 134) unite in the center of the lobule to form a small intralobular vein, which carries the blood out and pours it into one of the branches of origin of the hepatic vein, called the sublobular vein. Each of the latter has many lobules emptying blood into it, and if dissected out with them would look something like a branch of a tree with apples attached to it by short stalks, 
represented by the intralobular veins. The blood is finally carried, as already pointed out, by the hepatic veins into the inferior vena cava. The hepatic artery, a direct off-shoot of the celiac axis

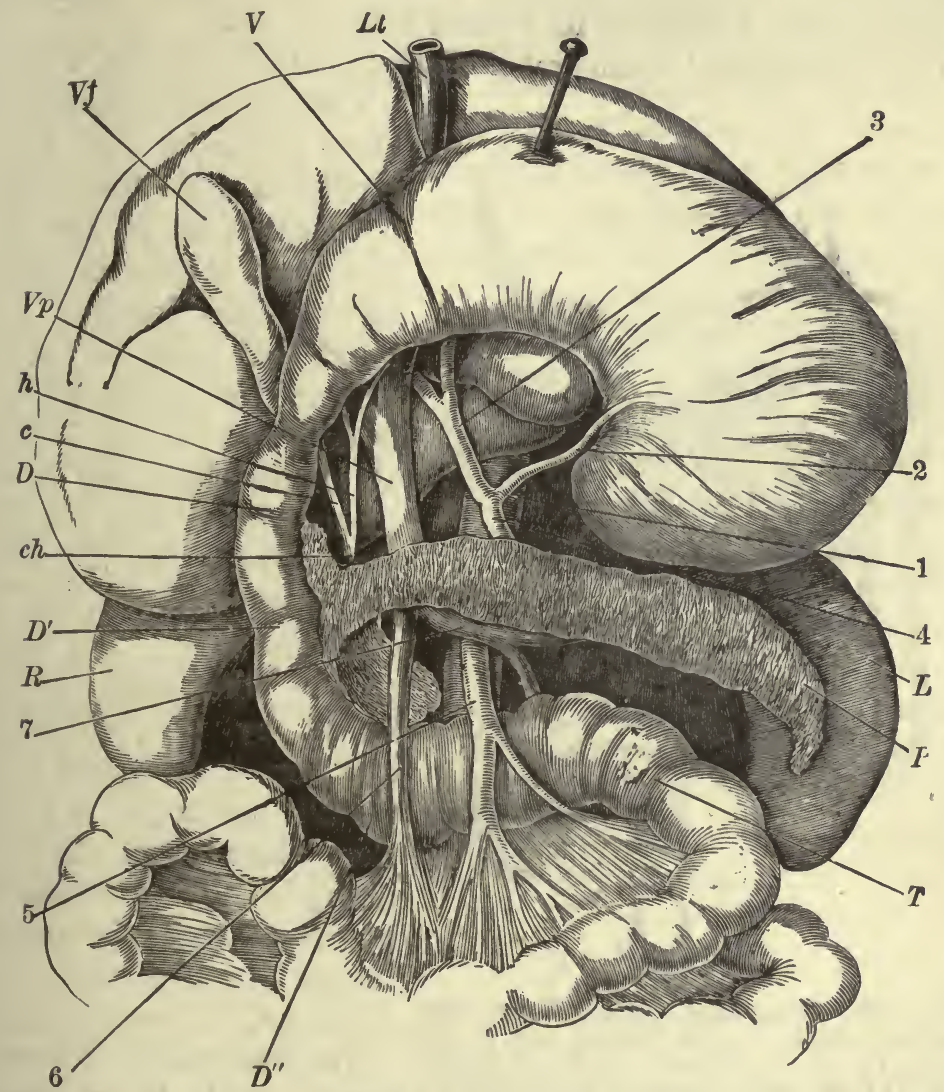

Fig. 135.-The stomach, pancreas, liver, and duodenum, with part of the rest of the small intestine and the mesentery; the stomach and liver have been turned up so as to expose the pancreas. $V$, stomach; $D, D^{\prime}, D^{\prime \prime}$, duodenum; $L$, spleen; $P$, pancreas; $R$, right kidney; $T$, jejunum; $V f$, gall-bladder; $h$, hepatic duct; $c$, cystic duct; ch, common bile-duct; 1, aorta, 2, an artery (left coronary) of the stomach; 3 , hepatic artery; 4 , splenic artery; 5 , superior mesenteric artery; 6 , superior mesenteric vein; 7 , splenic vein; $V p$, portal vein.

(p. 331) supplies some blood to the lobular plexuses, but by no means so much as the portal vein; it all finally leaves the liver by the hepatic veins.

The bile-ducts can be readily traced to the periphery of the 
lobules, and there communicate with a network of extremely minute commencing bile-capillaries, ramifying in the lobule between the hepatic cells composing it. The relation of the bilecapillaries to the blood-capillaries within the lobule is such that there is always a liver-cell interposed between them.

This arrangement is illustrated diagrammatically in Fig. 134 .

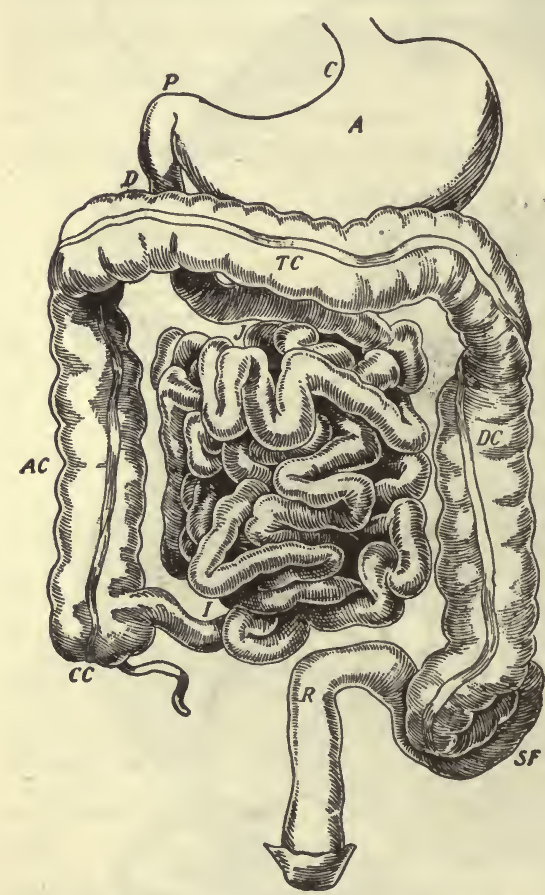

Frg. 136.-Diagram of abdominal part of alimentary canal. $C$, the cardiac, and $P$, the pyloric end of the stomach, $A ; D$, the duodenum; $J, I$, the convolutions of the small intestine; $C C$, the cæcum with the vermiform appendix; $A C$, ascending, $T C$, transverse, and $D C$, descending colon; $S F$, sigmoid flexure; $R$, the rectum.

the great curvature of the stomach. Its rewt end lying along the great curvature of the stomach. Its right end is the larger, and is embraced by the duodenum (Fig. 135), which there makes a curve to the left. A duct traverses the gland and joins the common bile-duct close to its intestinal opening. The pancreas produces a watery-looking secretion which is of great importance in digestion; the gland also secretes a hormone which exerts an 
important influence on the general nutritional processes of the Body (Chap. XXX).

The Blood-Vessels of Alimentary Canal, Liver, Spleen, and Pancreas. The portal vein ( $V p$, Fig. 135) has already been referred to as differing from all other veins in that it not only receives blood from a system of capillaries but ends in a second set of capillaries, which lie in the liver. The quantity of blood brought to supply the hepatic capillaries by the hepatic artery is in fact much less than that brought by the portal vein. The stomach, the intestines, the pancreas, and the spleen are supplied with arterial blood from three great branches of the aorta. The most anterior of these, the celiac axis, springs from the aorta close beneath the diaphragm and divides into the hepatic artery, splenic artery, and arteries for the stomach; some of these divisions may be seen in Fig. 135. The pancreas is supplied partly from the hepatic, partly from the splenic artery. The two other branches (superior and inferior mesenteric artery) are given off from the aorta lower down in the abdominal cavity; the former (5, Fig. 135) supplies the small intestine and half of the large, the latter the remainder of the large. The blood passing through all these arteries becomes venous in the capillaries of the organs they supply, and is gathered into corresponding veins (Fig. 135) which unite near the liver to form the portal vein. The further course of the blood carried to the liver (partly arterial from the hepatic artery, partly venous from the portal system) has been described already (p. 335). 


\section{CHAPTER XXVII}

\section{THE CHEMISTRY OF DIGESTION}

The Object of Digestion is twofold; to prepare the various foods for absorption by the lining of the digestive tract, which means that they must be made soluble if not already so; and to convert them into forms in which the Body can make use of them after they have been absorbed. Digestion is confined to the nutrients; the inorganic salts of the food are soluble, and are used by the Body in essentially the same form as eaten, they therefore need no digestion. The accessories either perform their function in connection with the process of digestion itself, or are absorbed and used by the Body in the form in which they are taken.

Nature of the Digestive Process. Although the foods requiring digestion are of very different sorts chemically, the method of digestion is at bottom the same for all of them. It consists of the process known in chemistry as hydrolysis. Hydrolysis is a chemical reaction in which one molecule of the substance involved combines with one molecule of water and the resulting compound splits into two or more simpler molecules. By repeated hydrolyses very complex substances may be split into comparatively simple ones.

Hydrolysis is a common reaction of organic chemistry. It is probable that it is the most frequently occurring reaction of the living Body. Not only the digestive processes, but many of the activities of living cells are of this nature. The digestive hydrolyses are all carried on through the agency of enzyms. There is a special and specific enzym for each particular reaction; the enzym that splits starch is without effect on protein or fat. These enzym reactions are all simple chemical reactions; they are carried on in the alimentary tract as in a chemical laboratory, and will go on just as well in test-tubes kept at Body temperature as in the Body itself. They are not therefore "vital" processes in the sense that they cannot occur except in the presence of living cells.

Digestion Products. Before beginning a detailed description 462 
of the digestive process as it affects the different food-stuffs it will perhaps be helpful to call attention to the comparatively few and simple substances which are finally produced as the result of the numerous reactions that go on in the alimentary tract. All carbohydrates (except the single sugars), the starches, gums, and double sugars, are hydrolyzed into single sugars during their digestion, so that absorption of carbohydrates is altogether in the form of single sugars. All fats are split into fatty acid and glycerin, in which state they are ready to be taken up by the intestinal walls. The proteins, as we saw in Chap. I, are complexes built up of a large number of amino acids. The digestive process splits them into simpler molecules each of which is composed either of a single amino acid, or of two or three of them together. We may say, in general, that proteins are split into their constituent amino acids.

Tabulating the digestion products we have:

from carbohydrates, single sugars;

from fats, fatty acid and glycerin;

from proteins, amino acids.

The Saliva. The first digestive fluid that the food meets with is the saliva, which, as found in the mouth, is a mixture of pure saliva, formed in parotid, submaxillary, and sublingual glands, with the mucus secreted by small glands of the buccal mucous membrane. This mixed saliva is a colorless, cloudy, feebly alkaline liquid, "ropy" from the mucin present in it, and usually containing air-bubbles. Pure saliva, as obtained by putting a fine tube in the duct of one of the salivary glands, is more fluid and contains no imprisoned air.

The uses of the saliva are in part physical and mechanical. It keeps the mouth moist and allows us to speak with comfort; it also dissolves such bodies as salt, and sugar, when they are taken into the mouth in solid form, and enables us to taste them; undissolved substances are not tasted, a fact which any one can verify for himself by wiping his tongue dry and placing a fragment of sugar upon it. No sweetness will be felt until a little moisture has exuded and dissolved part of the sugar.

In addition to such actions the saliva exerts a chemical one on an important food-stuff. It contains an enzym, ptyalin, which has the power of turning starch into a double sugar, maltose. This 
change, like all digestive reactions, is a hydrolysis. It does not occur in a single stage; that is, the starch molecule is not split directly into maltose, but first into a dextrin which is hydrolyzed into a simpler dextrin, and this in turn into maltose. In effecting the change the ptyalin is not altered; a very small amount of it can convert a vast amount of starch, and does not seem to have its activity impaired in the process.

In order that the ptyalin may act upon starch certain conditions are essential. Water must be present, and the liquid must be neutral or feebly alkaline; acids retard, or if stronger, entirely stop the process. The change takes place most quickly at about the temperature of the Human Body, and is greatly checked by cold. Boiling the saliva destroys its ptyalin and renders it quite incapable of converting starch. Cooked starch is changed more rapidly and completely than raw.

It will be noted that salivary digestion is only a stage in the preparation of starch for the use of the Body, since starch, in common with the other carbohydrates taken as food, is finally converted to single sugar before it is absorbed.

The Gastric Juice. The food having entered the stomach is subjected to the action of the gastric juice, which is a thin, colorless or pale yellow liquid, of a strongly acid reaction. It contains as specific elements free hydrochloric acid (about 0.2 per cent), and an enzym called pepsin which, in acid liquids, has the power of converting the ordinary proteins which we eat, by hydrolysis, into closely allied bodies, proteoses and peptones.

In neutral or alkaline media the pepsin is inactive; and cold checks its activity. Boiling destroys it. In addition to pepsin, gastric juice contains another enzym (rennin) which coagulates the casein of milk, as illustrated by the use of "rennet," prepared from the mucous membrane of the calf's digestive stomach, in cheese-making. The acid of the natural gastric juice would, it is true, precipitate the casein, but such precipitate is quite different from the true tyrein, and neutralized gastric juice still possesses this power; moreover, boiled gastric juice loses the milk-clotting property, and a very little normal juice can coagulate a great quantity of milk. The curdled condition of the milk regurgitated by infants is, therefore, not any sign of a disordered state of the stomach, as nurses commonly suppose. It is proper for milk to 
undergo this change, before the pepsin and acid of the gastric juice digest it.

Since muscle-fibers are enclosed within connective tissue (albuminoid) envelopes, it is necessary that the albuminoid coverings be digested off before the protein contents are exposed to the action of the digestive enzyms. There is reason to think that pepsin, which converts proteins, including albuminoids, into proteoses and peptones, soluble substances, but does not carry the digestion to completion, has as an important part of its function this removal from animal proteins of their albuminoid coverings.

The Pancreatic Juice. In the intestine the food is subjected to the action of the pancreatic juice. This is clear, watery, alkaline, and much like saliva in appearance. The Germans call the pancreas the "abdominal salivary gland." In digestive properties, however, the pancreatic secretion is far more important than the saliva, or even the gastric juice. It contains three digestive enzyms; amylopsin, a starch-splitting enzym whose action is identical with that of salivary ptyalin, and which is thought to be, perhaps, itself identical with ptyalin; lipase, a fat-splitting enzym, converting fats to fatty acid and glycerin; trypsin, a protein-splitting (proteolytic) enzym whose action is much more powerful than that of pepsin, as it is able to carry the process of protein hydrolysis clear to the amino acid stage. It acts upon such proteins as escape the influence of pepsin in the stomach.

The Bile. This fluid, which is poured into the intestine from the liver does not contain any digestive enzym, but it does have an important role in connection with fat digestion; it has been shown that pancreatic lipase splits fats several times as rapidly when bile is present as when it is absent.

The Succus Entericus (Intestinal juice). This fluid, which is secreted by the minute glands of the intestinal wall, is the last of the digestive fluids to come in contact with the food, and by its enzyms whatever foods are not completely digested must be finally prepared for absorption. By the enzyms thus far described none of the carbohydrate digestion is carried to completion, and only part of the proteins are made ready for use, for proteose and peptone are not end products, but only intermediate products of digestion. Fats are the only foods which do not require the aid of the succus entericus for their complete digestion. 
The digestive enzyms of the succus entericus are four; one proteolytic, erepsin, which acts particularly on proteoses and peptones, thus completing the work of the gastric pepsin; and three so-called inverting enzyms, which change double sugars to single sugars. These enzyms are specific in their action, each affecting only its particular sugar. Maltase inverts maltose, thus completing the starch digestion begun by ptyalin and amylopsin; sucrase splits cane-sugar or sucrose, and lactase converts milk-sugar, lactose, to single sugar. The result of the action of these three enzyms is to bring all the carbohydrates of the food, except cellulose, into the condition of single sugars, in which form they are ready for the use of the Body.

Summary of the Digestive Process. The chemical reactions by which the various food stuffs are made ready for absorption and use by the Body can be conveniently summarized in tabular form:

\begin{tabular}{|c|c|c|c|c|}
\hline Region & Secretion & Enzyms & $\begin{array}{c}\text { Substances } \\
\text { Affected }\end{array}$ & $\begin{array}{l}\text { Products } \\
\text { Formed }\end{array}$ \\
\hline \multirow{3}{*}{$\begin{array}{l}\text { Mouth } \\
\text { Stomach }\end{array}$} & Saliva & Ptyalin & Starch & Maltose ${ }^{1}$ \\
\hline & Gastric Juice & Pepsin & Albuminoid & Proteoses 1 \\
\hline & & & Protein & Peptones 1 \\
\hline \multirow{8}{*}{$\begin{array}{c}\text { Small } \\
\text { Intestine }\end{array}$} & Pancreatic & Amylopsin & Starch & Maltose ${ }^{1}$ \\
\hline & & Linese & Fots & Fotty acid 2 \\
\hline & & Lpase & Fats & $\begin{array}{l}\text { Fatty acid } \\
\text { Glycerin }{ }^{2}\end{array}$ \\
\hline & & Trypsin & Proteins & Amino Acids 2 \\
\hline & $\begin{array}{c}\text { Succus } \\
\text { Entericus }\end{array}$ & Erepsin & $\begin{array}{l}\text { Proteoses } \\
\text { Peptones }\end{array}$ & " \\
\hline & & Maltase & Maltose & Single Sugar ${ }^{2}$ \\
\hline & & Sucrase & Cane-Sugar & "6 \\
\hline & & Lactase & Milk-Sugar & " \\
\hline
\end{tabular}

${ }^{1}$ Intermediate products.

${ }^{2}$ Final products.

Bacterial Digestion. The human intestines normally contain enormous numbers of bacteria. In the small intestine the action of these is for the most part fermentation of carbohydrates with the production of carbon dioxid, alcohol, and acetic and lactic acids. There is no doubt that even in perfect health a considerable fermentation goes on in the intestine. So far as appears it is 
neither particularly harmful nor beneficial. The fermentation products are probably absorbed and used by the Body, but they would be used equally well if absorbed as sugar without fermentation. In the case of one particular carbohydrate, however, cellulose, bacterial fermentation affords the only means by which it can be made available in man for the use of the Body. It seems to be well established that tender cellulose, such as is eaten in lettuce, for example, may be digested by bacteria to a considerable extent; where it is less tender, as in most fruits and vegetables, it remains, as stated earlier, practically undigested.

Intestinal fermentation is not essential to health as is shown by the possibility of living normally in arctic regions, where, it is said, intestinal bacteria are sometimes wholly wanting. When the fermentation becomes excessive intestinal disturbances may readily result. The production of fermentation acids in too great concentration leads to irritation of the intestinal wall and causes diarrhea.

In the large intestine the bacterial action is chiefly putrefaction of proteins, rather than fermentation of carbohydrates. The difference is not due to the presence of different species of bacteria, but to the different nature of the available food. Where carbohydrate is present in excess, as in the small intestine, fermentation is the normal action. By the time the intestinal contents reach the large intestines the digestible carbohydrates are, as we shall learn (p. 500), all absorbed out into the blood. There remains, however, a portion of the protein, including all indigestible meat fragments. In this environment, largely protein, the normal bacterial action is of the nature of putrefaction. The characteristic features of the contents of the large intestine are the results of this putrefaction. In connection with it various toxic substances are formed which may be absorbed from the intestine into the blood. The symptoms of heaviness and general ill-feeling that frequently accompany sluggishness of the large intestine are to be referred to the presence of these toxins in the blood. The condition is known as autointoxication. The obvious method of avoiding this condition is by using care that material shall not stagnate in the colon.

The Prevention of Self-Digestion. A question of much interest to physiologists has been why the stomach and intestinal 
walls and the gastric and pancreatic glands are not themselves digested by the powerful proteolytic enzyms which they produce, in the case of the glands, or which are poured out unto them, in the case of the walls of the digestive organs. It has been shown that the prevention of self-digestion of stomach and intestine depends upon the continuance of life, for animals killed in the midst of digesting a meal often do digest great parts of their stomach and intestinal walls. Just how self-digestion of these structures is normally prevented is not clear, except in so far as the mechanism to be described presently (Chap. XXIX), which limits the outpouring of the secretions to periods when food is present, may be efficacious. The self-digestion of the pancreatic and gastric glands is, however, prevented by an interesting arrangement which has been recently analyzed. It appears that neither pepsin nor trypsin is formed in the gland as an active enzym but in an inactive pro-enzym or zymogen form, pepsinogen or trypsinogen, which becomes active only when converted into pepsin or trypsin by some activating agent. It has been shown that the conversion of trypsinogen to trypsin occurs only when the pancreatic juice is poured into the small intestine, and that it is brought about through a constituent of the succus entericus, enterokinase. This substance is believed to be an enzym having the sole function of activating trypsinogen to trypsin. The conversion of pepsinogen to pepsin is a similar activation, carried on by the hydrochloric acid of gastric juice. 


\section{CHAPTER XXVIII}

\section{MOVEMENTS OF THE ALIMENTARY CANAL}

Mastication serves to break the food into fine particles and by mixing it intimately with saliva to reduce it to a semi-liquid state. It consists primarily of cutting and grinding the food between the upper and lower teeth, a process which is performed by movements of the lower jaw. The articulation of the lower jaw with the skull and its equipment of muscles permit both up and down cutting movements and sidewise grinding movements. The actual chewing process involves, in addition, motions of the lips, cheeks, and tongue in holding the food in position for the teeth to act upon it. The whole process is carried on by skeletal muscles and is, therefore, under the control of the will.

It ought not to be necessary to emphasize the importance of thorough mastication of the food. Salivary digestion depends wholly, of course, upon the bringing of saliva into contact with the starch particles, and it can easily be shown experimentally that gastric digestion is several times more rapid when the material exposed to the action of gastric juice is finely divided than when it is in large masses.

The interesting fact has recently been brought out that the more the process of masticating each mouthful is prolonged the less food is required to satisfy the appetite. Since many people doubtless eat too much there is here a suggestion as to a way of reducing the amount taken without serious sacrifice of appetite.

Hygiene of the Mouth. The mouth cavity is almost never free from micro-organisms. The alkaline reaction of saliva is favorable to their growth, and they scarcely ever lack for food. The irregularly shaped teeth, packed closely along the jaw, have between them spaces where material that is being chewed readily lodges, and where it stays unless special care is taken to remove it. Such lodged food-masses shortly harbor flourishing colonies of bacteria. These in connection with their growth and multiplication produce substances which attack the protective enamel 
of the teeth and so gain foothold within the tooth substance itself, and we have under way the too-familiar process of tooth decay. Good teeth are so important for efficient mastication, as well as for the appearance of the face, that no pains should be spared to preserve them. Evidently the way to do this is to prevent the accumulation of bacteria in the spaces between them. Thorough cleaning, desirably after each meal, with the occasional use of an antiseptic mouth-wash is fairly but not completely satisfactory. Half yearly inspection and cleaning by a dentist are usually necessary to supplement one's own efforts, because of the practical impossibility of keeping every one of the small mouth spaces clear. Such inspection also insures the discovery of decay while the cavities are still small, and makes possible the preservation of the teeth in approximately normal condition for many years.

Recently evidence has been advanced showing that the saliva varies slightly in alkalinity in different people, and that the susceptibility of the teeth to decay depends largely on the degree of alkalinity. Three general groupings are suggested. Those who fall within the limits of the first group are likely to have perfect teeth even though no care is taken of them. The second group can have good teeth by the exercise of reasonable care. The third group have difficulty in preserving the teeth in good condition in spite of unremitting attention to them. This observation explains the frequent occurrence of perfect teeth in savages and others who never pay them the slightest attention, and the prevalence of decay among the most highly civilized. It is probable, although not proven, that the nature of the diet has much to do with the degree of alkalinity of the saliva.

- Of late years a great deal of attention has been paid to indirect harm that may follow neglect of the teeth. Allowing colonies of bacteria to flourish among them undisturbed means, of course, that any toxins these may produce will be absorbed into the system. The result of continuous absorption of such toxins is often manifested in lowering of the general health. Specifically, acute rheumatism is said frequently to follow.

Deglutition. A mouthful of solid food is broken up by the teeth, and rolled about the mouth by the tongue, until it is thoroughly mixed with saliva and made into a soft pasty mass. This mass is sent on from the mouth to the stomach by the process of 
deglutition, which is described as occurring in three stages. The first stage includes the passage from the mouth into the pharynx. The food being collected into a heap on the tongue, the tip of that organ is placed against the front of the hard palate, and then the rest of the tongue is raised from before back, so as to press the food-mass between it and the palate, and drive it back through the fauces. This portion of the act of swallowing is voluntary, or at least is under the control of the will, although it commonly takes place unconsciously. The second stage of deglutition is that in which the food passes through the pharynx; it is the most rapid part of its progress, since the pharynx has to be emptied quickly so as to clear the opening of the air-passages for breathing purposes. The food-mass, passing back over the root of the tongue, pushes down the epiglottis; at the same time the larynx (or voicebox at the top of the windpipe) is raised, so as to meet it, and thus the passage to the lungs is closed; muscles around the aperture probably also contract and narrow the opening. The raising of the larynx can be readily felt by placing the finger on the large cartilage forming "Adam's apple" in the neck, and then swallowing something. The soft palate is at the same time raised and stretched horizontally across the pharynx, thus cutting off communication with its upper, or respiratory portion, leading to the nostrils and Eustachian tubes. Finally, the isthmus of the fauces is closed as soon as the food has passed through, by the contraction of the muscles on its sides and the elevation of the root of the tongue. All passages out of the pharynx except the gullet are thus blocked, and by a sharp contraction of the mylohyoid muscles, in the floor of the mouth, such great pressure is put upon the food-mass as to shoot it clear through the pharynx into the opening of the esophagus. Liquids or very soft foods, under the impetus given by the contraction of these muscles, are propelled the whole length of the gullet to the sphincter which guards the entrance to the stomach; more solid masses are thrown only into the entrance of the gullet whence the third stage of swallowing conveys them to the stomach. The muscular movements concerned in this part of deglutition are all reflexly excited; food coming in contact with the mucous membrane of the pharynx stimulates afferent nerve-fibers in it; these excite efferent nervefibers proceeding to the muscles concerned and cause them 
to contract in proper sequence. The pharyngeal muscles, although of the striped variety, are but little under the control of the will; it is extremely difficult to go through the movements of swallowing without something (if only a little saliva) to swallow and thus excite the movements reflexly. Many persons, after having got the mouth completely empty cannot perform the movements of the second stage of deglutition at all. On account of the reflex nature of the contractions of the pharynx, any food which has once entered it must be swallowed: the isthmus of the fauces is a sort of Rubicon; food that has passed it must continue its course to the stomach, although the swallower learnt immediately that he was taking poison. The third stage of deglutition is that by which solid food is passed along the gullet, and is comparatively slow. The movements of the eosphagus are of the kind known as peristaltic. Its circular muscular fibers contract behind the morsel and narrow the passage there; and the constriction then travels along to the stomach, pushing the food in front of it. Simultaneously the longitudinal fibers, at the point where the food-mass is at any moment and immediately in front of that, relax, tending to widen the passage. This peristaltic wave requires about six seconds in man for its passage along the esophagus. It is part of the reflex act of swallowing and takes place whenever the act occurs, whether there be any food-mass to be conveyed to the stomach or not. The ring of smooth muscle of the circular coat at the entrance of the stomach acts as a sphincter (cardiac sphincter). This is ordinarily tightly contracted when there is food in the stomach, holding the esophagus shut, and only opens at the approach of the peristaltic wave to allow the food-mass to pass through into the stomach. Liquids, which pass very quickly down the esophagus (in 0.1 sec.), usually do not get into the stomach at once, but are held by the sphincter until the arrival of the peristaltic wave opens a passage for them. The relaxation of the cardiac sphincter under these circumstances does not open a free communication between the stomach and the throat, for there is always a descending peristaltic wave holding the esophagus closed. This is important because, as we shall see, the stomach contents are under pressure, and would be forced up into the esophagus were the sphincter to relax with no peristaltic wave present. As a matter of fact this sometimes happens, particularly in per- 
sons suffering from indigestion, or certain nervous disorders, or in users of tobacco. The upward rush of the acid stomach contents into the esophagus gives rise to a burning sensation which is generally known as "heart burn," although the heart has really nothing whatever to do with it.

Movements of the Stomach. When the stomach is empty of food its normal condition is as a flabby pouch. Its walls are neither much relaxed nor strongly distended. There are probably always a small amount of liquid and some bubbles of swallowed air in the stomach, even at the time when we speak of it as empty. Shortly before the usual time for taking a meal the circular muscle coat of the stomach goes into a state of tonus, probably as a result of a flow of impulses over the vagus nerve, which is the motor nerve of the organ. The effect of this tonus is to contract the stomach until it is little more than a tube. Usually about this same time the active contractions which give rise to hunger sensations (p. 209) begin. As food enters the contracted stomach it makes room for itself by stretching the walls, and the more food is taken, the more the stomach is distended. One result of this manner of filling the stomach is that the food is deposited in it in layers, the first food taken being next to the walls, subsequent amounts being toward the center, and further from the walls the more has entered before them.

The gastric glands are located in the middle and to some extent in the pyloric regions of the stomach. Such food as is in the fundus is not exposed directly, therefore, to the action of gastric juice, and so is not very rapidly acidified. The action of salivary ptyalin, which is brought to an end when the food becomes acid, may thus continue in the fundic region for a considerable time after the food is swallowed, especially in those portions of food which are swallowed late in the meal.

The movements of the stomach have been watched by means of the X-rays. Food which has been mixed with bismuth subnitrate is opaque to these rays and its movements in response to the movements of the stomach walls can be readily followed. By this means it has been learned that the walls of the stomach show peristaltic waves; these begin at about the middle, in a strong contraction of a ring of circular muscles at that point, and sweep to the pylorus. The fundic end is not involved at all in them. 
In man they recur regularly, so long as food is in the stomach, at intervals of about twenty seconds. For a considerable period after food enters the stomach the pyloric sphincter, which guards the exit into the small intestine, remains perfectly tight. During this time the peristaltic waves crowd the food caught by them up to the pylorus but cannot force any through. As the constriction approaches the pylorus the food-mass in front of it escapes back through the opening at its center, the waves not being deep enough to close this entirely, and so the food in the central and pyloric portions of the stomach is thoroughly churned.

During this churning the food, already semi-liquid from the mixture with saliva and with such liquid as was taken with the meal, is mixed with the gastric juice and made still more liquid, being called at this stage chyme. The effect of the gastric juice is to give the food an acid reaction, stopping the action of ptyalin and permitting that of the pepsin which it also pours out upon the food.

The Control of the Pyloric Sphincter. The way in which the sphincter of the pylorus is regulated so that after the food has been thoroughly mixed with gastric juice it opens and allows a small amount to pass, and then promptly closes to give opportunity for this to be influenced by the intestinal secretions before more is admitted, is one of the most interesting adaptations that we know of in the Body. The mechanism of this action is a special case of a peculiar reflex which apparently obtains throughout the alimentary canal, and is probably dependent on special properties of the nerve plexus which is embedded therein. This so-called myenteric reflex, is of such a sort that a stimulus applied to any point along the alimentary canal causes a contraction of the muscles immediately in front of (anterior to) the stimulated point, and a relaxation of those immediately behind (posterior to) it. The reflex was worked out first for the small intestine, and has since been shown to apply to the other parts of the canal. It is a so-called "local reflex," as the central nervous system has nothing whatever to do with it.

The adequate stimulus for arousing the reflex in the pyloric sphincter is the presence of free hydrochloric acid. When therefore the originally alkaline food in the pyloric part of the stomach has been completely neutralized by the acid of the gastric juice, 
and excess acid begins to accumulate, the pyloric sphincter is stimulated, but from the stomach side, and according to the working of the myenteric reflex a stimulus from that side produces relaxation of a region just posterior to it. As soon as the sphincter relaxes under this stimulation that part of the food lying in the pylorus is forced through into the intestine, but it carries with it the free acid with which the food is mixed and stimulates the sphincter from the intestinal side, namely, from behind, and therefore tends to cause it to close. A feature of the myenteric reflex is that where, as just described, a point is simultaneously stimulated from in front and from behind, the stimulus causing contraction, that from behind, is dominant. Therefore as soon as food enters the intestine the sphincter of the pylorus contracts and prevents more from passing. Before it will relax again the acid on its intestinal side must be neutralized; but this is rapidly done by the strongly alkaline bile and pancreatic juice, and so as fast as the food in the intestine is mixed with these juices more is admitted from the stomach.

The fundus of the stomach, which stores the bulk of the food while that in the pylorus is being thus treated and passed on to the intestine, is on the stretch all the time, so that as fast as food is passed out through the pyloric sphincter more is pushed to the pylorus from the fundus until at last the stomach is wholly emptied. The time required for emptying the stomach completely varies with different foods and under different bodily conditions. An average meal is probably all out of the stomach about four to six hours after eating.

An interesting incidental feature of this mechanism is that it operates automatically to pass quickly on into the small intestine carbohydrate food stuffs, which undergo no digestive action in the stomach, while proteins, upon which the pepsin of gastric juice acts, remain long enough to ensure their thorough mixture with the juice. This differentiation depends on the fact that the acid does not enter any chemical combination with carbohydrates, and therefore begins to appear in excess as soon as the alkali present has been neutralized. Proteins, on the other hand, do combine chemically with the acid, and there can be no excess, therefore, until this combination has occurred. Meanwhile thorough mixture with pepsin is taking place. This difference does not show, 
of course, in the case of a mixed meal, but a meal of pure carbohydrates will begin to leave the stomach much sooner after ingestion than a meal of pure protein (10-15 minutes as compared with $1 / 2$ hour), and will be discharged completely in half the time (2-21/2 hours as against 4-5); and a meal in which the carbohydrates are eaten before the proteins may show a definite interval between the discharge of the last carbohydrates and the first proteins. The admixture of fats with the other food stuffs delays considerably the rate of discharge.

The pyloric sphincter does not hold against pure water nor against substances of the consistency of raw egg-white or raw oysters. These, unless mixed with other materials, pass promptly, therefore, from the stomach into the small intestine.

Importance of the Stomach. Aside from its function of beginning the digestion of proteins, a function which, as we have seen (p. 465.) is subordinate to the more efficient digestive action of the small intestine, the chief significance of the stomach is that it enables us to take our daily supply of food in three meals, more or less, according to our habit. The small intestine is a narrow tube. The ducts of pancreas and liver open into its upper end. If our food when swallowed passed directly into the intestine each mouthful would crowd the preceding ones along at such a rate that no adequate admixture with the essential juices of the pancreas and liver could occur, and very little digestion would take place. To avoid this difficulty the food would have to be eaten little by little, and to get enough for the needs of the Body would require hours of steady nibbling. By affording storage to a considerable amount of food, which is automatically passed along to the intestine at just the rate at which that region can handle it, the stomach permits us to follow eating habits much less time consuming, and more convenient.

Movements of the Small Intestine. The food entering the small intestine is subjected to two sorts of movements whose combined effect is to churn it very thoroughly and to move it slowly along the gut so as to make room for more to come in from the stomach. The churning is effected mainly by movements of the intestine known as rhythmic segmentation. In these movements rings of the circular muscle coat about an inch apart constrict simultaneously, splitting the contained food into a series of 
segments; an instant later these constrictions disappear, and new ones, midway between the first, are formed, by which the food is again segmented, but in a shifted position. These rhythmic movements may recur as often as thirty times a minute. Their effect is to bring every particle of the contained food into intimate contact with the intestinal walls, insuring thorough mixing with the intestinal secretions, and also favoring absorption.

The onward movement of the food is secured by peristaltic waves which start at the pylorus and run rather slowly along the intestine. They are normally gentle movements, which do not carry the food bodily before them, but move it forward little by little. During digestion the two sorts of movements alternate more or less irregularly. After the segmentation has churned a food-mass thoroughly in one section it dies away and a peristaltic wave develops, which carries the food ahead of it into a fresh section; then the peristalsis, in turn, subsides, and segmentation is resumed.

The mechanism of these intestinal movements is not entirely clear, the peristaltic waves, and possibly also the segmentations, are special manifestations of the myenteric reflex described above, but the conditions that govern their appearance and disappearance, first in one part of the intestine and then in another, are not known.

Observations with the $\mathrm{X}$-rays have shown that the rate of progress of the food through the human small intestine is about $4 \frac{1}{2}$ feet in the hour, so that the first food from any meal may appear at the ileocolic valve about $4 \frac{1}{2}$ hours after it begins to leave the stomach.

Extrinsic Control of Stomach and Intestinal Movements. It has been shown that normal movements of both stomach and intestine may go on in animals in which the nerves leading to these organs from the central nervous system are cut. To a certain extent, therefore, they, like the heart, contain within themselves the essential requirements for normal activity. Like the heart, however, they are subject to reflex control through the central nervous system.

The vagus nerves carry cranial autonomic fibers which when stimulated arouse the stomach and intestine to activity. The opposing thoracico-lumbar autonomics, which, as we have already seen (p. 455), come by way of the splanchnics, are inhibitory. A 
part of the emergency reaction of the Body, therefore, consists in suspension of activity in these organs. This has been noted previously (p. 196).

Movements of the Large Intestine. During the passage of the food through the small intestine the greater part of its nutritive content is absorbed, but practically none of the water, so that it is delivered through the ileocolic valve to the large intestine in a very watery condition. The parts of the large intestine next to the small intestine, the ascending and transverse colon, show an interesting movement in the form of an antiperistalsis. This is a peristaltic wave which begins in the transverse colon and sweeps toward the ileocolic valve. It would tend to force the material within the colon back into the small intestine did not the ileocolic valve prevent. The result of this movement is a churning and mixing of the contents whereby the absorption of the last useful materials, including the water, is promoted. As the large intestine is filled more and more from the small, some of its contents are crowded, in spite of the antiperistalsis, into the descending colon, where regular peristaltic waves carry them on to the sigmoid flexure and the rectum, whence they are discharged from the Body. There is evidence that the stimulus for these intestinal waves is mechanical, depending on stretching of the walls by the intestinal contents.

Importance of Roughage. As the result of the absorption of water from the contents of the large intestine the material remaining, which consists of undigested substances, bacteria, the products of bacterial action, and some waste products excreted in the bile (p. 518), tends to become dry and closely packed. If the diet is poor in roughage (p. 429) so little room is occupied by this material that the necessary mechanical stimulation fails to be forthcoming for the movements by which it should be carried along to the region of discharge. There is, therefore, stagnation in the large intestine, and this, by permitting time for a more complete absorption of water, makes the condition of affairs still worse, and the evacuation of the colon still more difficult. The inclusion of considerable roughage in the diet (bran, the pulp of vegetables and fruits, particularly apples, popcorn) by increasing the bulk of the intestinal contents favors the onward movement of the material, and tends against stagnation. We need to remember 
in this connection that the colon is a smooth muscle structure, under the control of the autonomic system, and subject, therefore, to the disturbing influences characteristic of such structures. The inclusion of ample roughage in the diet does not always suffice to secure adequate evacuations, particularly where neglect or improper treatment has affected the colon so that it no longer responds normally to mechanical stimulation from its contents. The means commonly used to induce evacuations, the taking of purgative drugs, is objectionable, although sometimes necessary, because the drugs act by irritating the intestinal lining. Such irritation, if repeated regularly, brings on a chronic inflammation, which seriously impairs the ability of the colon to react normally. The habit of taking purgative drugs should be strenuously avoided. If persisted in it is sure to lead to much discomfort or even severe suffering. It is probable that much of the trouble from intestinal sluggishness could be avoided by proper supervision and care in childhood, when regular habits are easy to establish and enforce. Regularity, even more than ample roughage, is a prime requisite to the proper functioning of the colon. 


\section{CHAPTER XXIX}

\section{THE DIGESTIVE SECRETIONS AND THEIR CONTROL}

Organs of Secretion. The simplest form in which a secreting organ occurs ( $A$, Fig. 137) is that of a flat membrane provided with a layer of cells, $a$, on one side (that on which the secretion is poured out) and with a network of capillary blood-vessels, $c$, on the other. The dividing membrane, $b$, is known as the basement membrane and is usually made up of flat, closely fitting connective-tissue corpuscles; supporting it on its deep side is a layer of connective tissue, $d$, in which the blood-vessels and lymphatics are supported. Such simple forms of secreting surfaces are found on the serous membranes, but are not common; in most cases an extended area is required to form the necessary amount of secretion, and if this were attained simply by spreading out plane surfaces, these from their number and extent would be hard to pack conveniently in the Body. Accordingly in most cases, the greater area is attained by folding the secreting surface in various ways so that a large area can be packed in a small bulk, just as a Chinese lantern when shut up occupies much less space than when extended, although its actual surface remains of the same extent. In a few cases the folding takes the form of protrusions into the cavity of the secreting organ as indicated at $C$, Fig. 137, and found on some synovial membranes; but much more commonly the surface extension is attained in another way, the basement membrane, covered by its epithelium, being pitted in or involuted as at $B$. Such a secreting organ is known as a gland.

Forms of Glands. In some cases the surface involutions are uniform in diameter, or nearly so, throughout (B, Fig. 137). Such glands are known as tubular; examples are found in the lining coat of the stomach (Fig. 129); also in the skin (Fig. 142), where they form the sweat-glands. In other cases the involution swells out at its deeper end and becomes more or less sacculated; $(E)$ such glands are racemose or acinous. The small glands which form the oily matter poured out on the hairs are of this type. In both kinds 


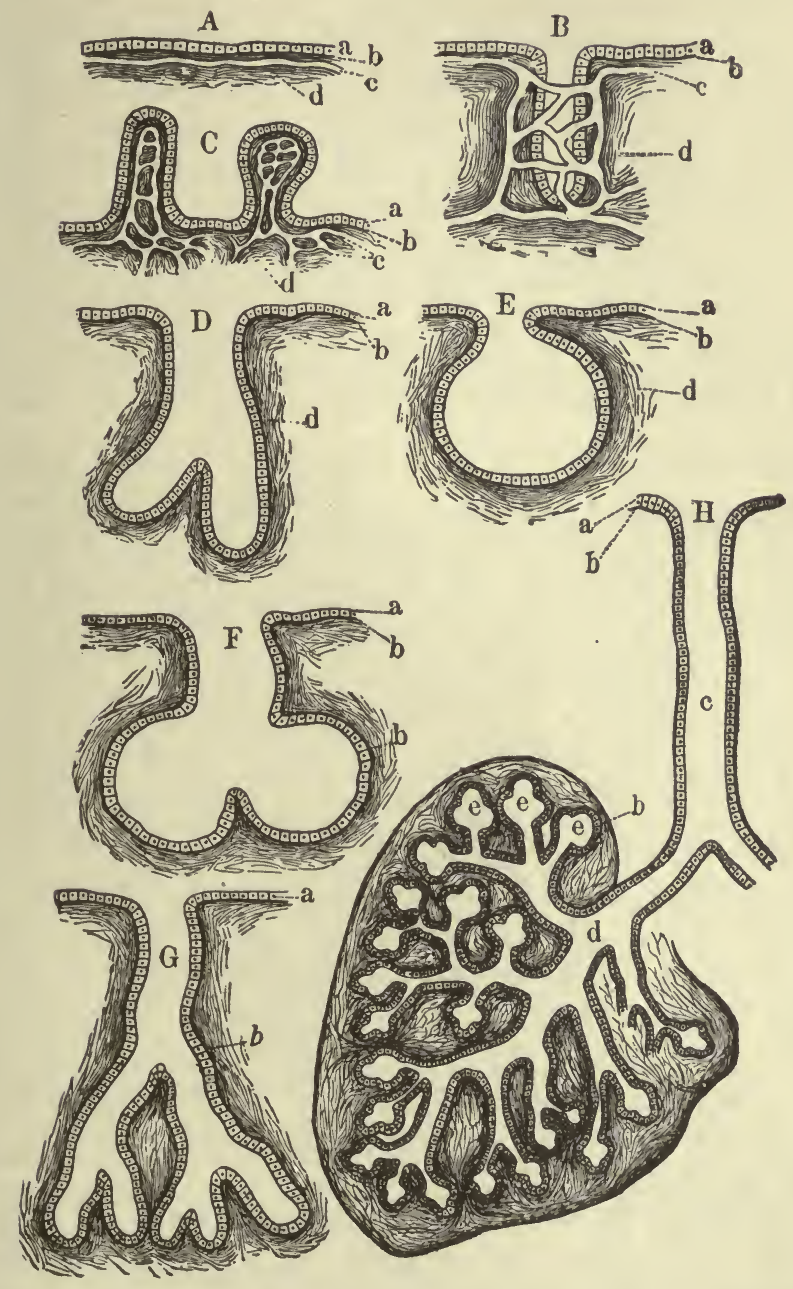

FIg. 137.-Forms of glands. $A$, a simple secreting surface; $a$, its epithelium; $b$, basement membrane; $c$, capillaries; $B$, a simple tubular gland; $C$, a secreting surface increased by protrusions; $E$, a simple racemose gland; $D$ and $G$, compound tubular glands; $F$, a compound racemose gland. In all but $A, B$, and $C$ the capillaries are omitted for the sake of clearness. $H$, half of a highly developed racemose gland; $c$, its main duct. 
the lining cells near the deeper end are commonly different in character from the rest; and around that part of the gland the blood-vessels form a closer network. These deeper cells form the true secreting elements of the gland, and the passage, lined with different cells, leading from them to the surface, and serving merely to carry off the secretion, is known as the gland-duct. When the duct is undivided the gland is simple; but when, as is more usual, it is branched and each branch has a true secreting part at its end, we get a compound gland, tubular $(G)$ or racemose $(F, H)$ as the case may be. In such cases the main duct, into which the rest open, is often of considerable length, so that the secretion is poured out at some distance from the main mass of the gland.

A fully formed gland, $H$, thus comes to be a complex structure, consisting primarily of a duct, $c$, ductules, $d d$, and secreting recesses, ee. The ducts and ductules are lined with epithelium which is merely protective and differs in character from the secreting epithelium which lines the deepest parts. Surrounding each subdivision and binding it to its neighbors is the gland stroma formed of connective tissue, a layer of which also commonly envelops the whole gland, as its capsule. Usually on looking at the surface of a large gland it is seen to be separated by partitions of its stroma, coarser than the rest, into lobes, each of which answers to a main division of the primary duct; and the lobes are often similarly divided into smaller parts or lobules. In the connective tissue between the lobes and lobules blood-vessels penetrate, to end in fine capillary vessels around the terminal recesses. They never penetrate the basement membrane. Lymphatics and nerves take a similar course; there is reason to believe that the nerve-fibers penetrate the basement membrane and become directly united with the secreting cells of some glands.

The Secretory Process. The function of glands is to elaborate and pour out a liquid, the secretion. It is obvious that the ultimate source of the secretion is the blood circulating through the gland. The digestive secretions, as we have already seen, contain, in addition to water, and inorganic salts, special chemical substances, the enzyms, which are different in different glands. It is easy to believe that the water and salts of the gland, since they are precisely the same as occur in blood, may be withdrawn from 
the blood through simple physical processes, filtration and dialysis (Chap. I). The special constituents of each secretion, being different from anything contained in the blood, must, on the other hand, be produced by chemical processes within the gland itself. It is easy to show microscopically that the cells of most glands during rest become filled with small granules, and that when the gland is active these granules for the most part disappear. We can picture the entire secretory process as occurring in two stages: the first, a chemical stage, during which the peculiar constituents of the secretion are elaborated and deposited within the cells of the gland; and a second physical stage consisting of a rapid flow of water with its dissolved salts from the blood through the gland into its duct, carrying with it the special materials previously prepared by the gland.

Nervous Control of the Secretory Process. Considerable evidence has accumulated indicating that gland tissue, like skeletal muscle tissue, carries on its function only when stimulated to do so, and that the stimulus is in many glands nervous. It has been shown for the salivary glands of dogs, for example, that proper stimulation of certain nerve-fibers leading to them causes them to produce and store within themselves granules, while stimulation of quite different nerve-fibers causes them to pour out their secretion. The chemical part of secretion is thus controlled by one set of nerves, often called trophic nerves, and the physical part by another. It is interesting to note that the nerves which cause the gland to pour out its secretion usually cause also vasodilation within it; an increased flow of blood through the gland therefore usually accompanies the physical part of secretion. That this increased blood-flow is not the sole cause of the outpouring of the secretion, as might easily be supposed, is proved by the possibility under proper conditions of stimulating a gland to pour out its fluid without any accompanying vasodilation. We must recognize, then, that the physical act of secretion is the result of the action of definite secretory nerves, as distinct from vasodilator nerves. Just how these function to bring about the more rapid passage of water and salts through the gland-cell is not clear. As we should expect, continued stimulation of the secretory fibers leading to a gland, without accompanying stimulation of the trophic fibers, results soon in the production of a secretion which is very watery, virtu- 
ally free from the special chemical substances that usually arc present in the secretion.

The efferent nerves to glands belong, without exception, to the autonomic system. Glands are, therefore, under reflex control, and not subject to the will.

Hormone Control of Gland Activity. Some of the digestive glands, notably the pancreas, appear to be wholly, or at least chiefly, independent of nervous influences. Their control is vested in hormones. The details of this method of control will be described in connection with the glands themselves. It may be noted here, however, that in general those glands whose secretions are needed early in the digestive process are under reflex control, and those whose secretions may not be required for some time after are under hormone control.

Control of the Salivary Secretion. The salivary glands are subject to reflex stimulation. We must inquire, therefore, what sensory stimuli may excite the reflex. At least three sorts of stimuli are effective to this end; mechanical, the presence of dry substances in the mouth, or merely the rubbing of the tongue against the palate and jaws; chemical, the presence of sapid substances upon the tongue; and psychic, the thought of savory food, as when the mouth "waters." It is an interesting fact that the character of the saliva varies somewhat with the nature of the exciting stimulus; mechanical stimulation causes the production of an abundant but very watery secretion; whereas the chemical stimulus of food in the mouth calls forth a secretion rich in ptyalin. By this mechanism the character of the secretion is adapted to the need which excites it. The mucous lining of the mouth and throat requires constant moistening. For this a watery saliva is adequate, and such a saliva is poured out whenever the dryness of the mouth becomes pronounced enough to act as a stimulus. When food is taken, on the other hand, the proper functioning of saliva requires that it be rich in ptyalin. Chemical stimulation, therefore, excites a secretion containing this substance.

The watering of the mouth at the thought of food is an example of an emotional reflex through the autonomic system such as was discussed earlier (Chap. XII). Inhibition of the salivary glands, leading to dryness of the mouth, as the result of excitation of the 
thoracico-lumbar autonomic system in time of stress, has also been described.

The Control of the Gastric Secretion. Our present knowledge of the mechanism for controlling the secretion of gastric juice is the result of some of the most interesting investigations of modern Physiology. Many workers have had a share in the solution of the problem but the name of one of them, the Russian physiologist Pawlow (Pâvloff), is more closely associated with it than that of any other one man. Pawlow's chief contribution was the demonstration that the secretion of gastric juice is in its early stages excited reflexly, and by only one particular sort of stimulus, namely, the psychical state accompanying the eating of food which is enjoyed. Pawlow gained this information through feeding experiments on dogs which had been prepared in a special way for the study. The preparation consisted of making a fistulous opening into the stomach, through which the secretion of gastric juice could be followed, and of cutting the esophagus in the neck and bringing the cut ends to the surface in such fashion that all the food swallowed reappeared at the upper esophageal opening, and none reached the stomach unless it was placed within the lower section of the esophagus through its opening. Dogs thus operated upon recovered promptly and completely and could be studied very satisfactorily. It was found that one of these dogs would eat with the greatest enjoyment, although none of the food reached the stomach, and that within a few minutes of the beginning of eating a secretion of gastric juice began to be poured into the stomach. That this secretion was excited reflexly was proved by cutting the vagus nerves, after which it never appeared. That it depends upon a certain psychical state, and not upon the mere eating of food was shown in various ways. Dogs which were not hungry would chew and swallow food, but without signs of much interest in it; no secretion was evoked. Meat which had been boiled till it was tasteless was eaten without the production of a secretion. These results make it clear that the stimulus is a psychical one, and that it depends upon active enjoyment of food. Equally important is the observation, made upon these same dogs, that unfavorable emotional states prevent the secretion of the juice. If the dog was angered while eating no juice appeared; even the presence of an attendant for whom he had an aversion 
sufficed to prevent the secretion. All these facts, established first upon dogs, have been proved true likewise for human beings.

Pawlow's studies showed, moreover, that the psychical secretion is not the only secretion of gastric juice which occurs during the digestion of a meal. This was proved by the simple observation that the amount of juice secreted during the eating of a "fictitious meal" is much less than that produced if the food eaten enters the stomach. We must look, then, for some other stimulating agency additional to the psychical one. In such a search the attention turns naturally to the foods swallowed. Do they serve as chemical stimuli for the production of the additional secretion? It has been shown that some foods, milk and water very slightly, the juices of meat more, do excite the secreting mechanism somewhat, but the really effective excitant appears to be something produced during the process of gastric digestion itself. Thus if the taking of food is attended with pleasure, so that a psychical secretion is produced, the digestive process is started and itself furnishes the stimulating agent for the additional secretion needed to complete the digestion. On the other hand, food eaten under conditions not favorable to the production of a psychical secretion may fail of digestion completely, through the absence of all factors which may lead to an outpouring of the juice.

Nature of the Chemical Stimulus to Gastric Secretion. It has been shown that the substances mentioned in the last paragraph as chemical excitants of gastric secretion do not stimulate the glands directly but indirectly through a hormone, gastric secretin. This hormone is apparently derived from some substance in the mucous membrane of the pyloric region, which reacts with the exciting substances derived from the food in such fashion as to produce the hormone, which is then taken up by the blood and carried to the gastric glands.

Control of the Pancreatic Secretion. Proper regulation of the outpouring of pancreatic juice requires that it begin about the time food begins to pass from the stomach into the small intestine. Since this may occur at a variable time after the eating of the meal, it would seem to call for a regulating mechanism quite independent of the act of eating. It has been shown that this requirement is fulfilled through the action of a hormone which is produced in active form during the time that food is passing 
from the stomach into the small intestine, and only then. The mucous membrane of the small intestine at its upper end contains a substance which has been named prosecretin. This substance reacts with hydrochloric acid to form pancreatic secretin, the hormone for exciting the pancreas to secrete. But this region of the small intestine comes in contact with hydrochloric acid only at the moment when a mass of food is entering it from the stomach; we have previously seen that this passage of food occurs only when the food is mixed with excess of hydrochloric acid. Thus the production of the hormone is confined to the time when its stimulating function is required.

The Control of the Bile Flow. It has been shown recently that the bile, which, although secreted continuously, is poured out only when food enters the small intestine, is controlled by the same hormone, secretin, which excites the flow of pancreatic juice. Under the stimulation of this hormone the gall bladder contracts, forcing its contents through the bile-duct into the intestine.

The Control of the Succus Entericus is at present wholly unknown. Whether it is constantly present in the intestine or whether its secretion is controlled by a hormone remains to be determined. It is worth noting, however, that there are probably not many periods, except during prolonged fasting, when intestinal digestion is not going on, so that a continuous secretion of intestinal juice would be less wasteful than of the other digestive juices.

Digestive History of a Meal. We can summarize the whole process of digestion as well, perhaps, by following the course of an ordinary meal through the digestive tract as in any other way. We shall disregard the accessories, and consider only the nutrients proper, since, as we have seen, the digestive process concerns itself with these alone. The meal, then, is a mixture of carbohydrates, proteins, albuminoids, and fats.

In the mouth the food is reduced to a semi-liquid alkaline mass, containing no large particles, by the combined action of chewing and mixing with the saliva. The salivary glands are reflexly excited to secrete their juice by the presence of the food in the mouth. The enzym of saliva, ptyalin, begins its digestive action on the starch, converting it to maltose. By the act of deglutition the food, when sufficiently mixed with saliva, is passed on to the stomach. If the chewing and swallowing of the food is attended 
with agreeable emotions, there is aroused a reflex secretion of gastric juice; the so-called "psychical" secretion.

The food enters the stomach in very much the same condition chemically as when taken into the mouth; a sinall amount of maltose added to it through the action of salivary ptyalin, and a correspondingly diminished amount of starch, being the only differences. That part of the food which is crowded down into the pyloric region begins at once to be churned by the peristaltic waves which sweep over that region; by the churning it is mixed with gastric juice. The food which remains in the fundic end of the stomach does not come into contact with the gastric juice; its reaction, therefore, continues alkaline, and the splitting of starch by pytalin goes on uninterruptedly. In the portion of food (chyme) which becomes impregnated with gastric juice there is an acid reaction and the changes which the gastric enzyms, pepsin, and rennin are capable of producing take place. Rennin clots any milk that may be present; pepsin attacks albuminoids and proteins, converting them into proteoses and peptones. Any fats present are liquefied, not by enzyms but by the stomach warmth. Some of the substances produced during this peptic digestion react with other substances in the mucosa of the pyloric region, forming a hormone, gastric secretin. This hormone is taken up by the blood, passes in the blood-stream to the gastric glands, and stimulates them to further outpouring of juice; thus enough for the whole meal is secured. Finally as the hydrochloric acid of the gastric juice accumulates in excess the pyloric sphincter is stimulated to relax; the mass of chyme next to it is pushed - through; and more material from the fundic end comes down to fill its place. Too much chyme is prevented from passing the sphincter at once by the powerful stimulus to contraction which is exerted on the sphincter by the acid chyme in contact with the upper intestine. The acid of this same chyme reacts with the prosecretin of the intestinal mucosa to form secretin, a hormone which is carried by the blood to the pancreas and excites it to activity.

The chyme which enters the intestine contains some, at least, of all the food stuffs originally making up the meal, and in addition maltose, proteose, and peptone. The strongly alkaline bile and pancreatic juice quickly neutralize its acid and the various en- 
zyms of the intestinal tract act upon it. The amylopsin of the pancreatic juice converts to maltose all starch not affected by ptyalin; the lipase of the same secretion splits the fats to fatty acid and glycerin; the trypsin of pancreatic juice, in co-operation with erepsin of the succus entericus reduces all proteins, including proteoses and peptones, to amino acids; the inverting enzyms, maltase, sucrase, and lactase, change all the double sugars, and therefore all the carbohydrates of the meal, to single sugars. The intestinal contents are churned and kept in onward progress by movements of segmentation and peristalsis performed by the muscular walls of the gut.

The Maintenance of Good Digestion. In the preceding paragraph the various activities essential to the proper performance of the digestive function have been outlined. If they are reviewed carefully it will be seen that most of them, after the food reaches the stomach, are affected, direetly or indirectly, by the conditions upon which depend the proper production of the psychical secretion of gastric juice. If, through anxiety or anger at meal-time, this secretion is inhibited, the whole sequence of the digestive process is upset. Without a psychic secretion little or no chemical secretion of gastric juice will appear; there is therefore not the necessary hydrochloric acid to stimulate the pyloric sphincter to relax, nor to react with prosecretin to form pancreatic secretin, should any food by any means get through into the intestine. Moreover, the same conditions which inhibit the psychical secretion inhibit also, as stated previously, the motions of the stomach and intestines. That indigestion usually follows the eating of meals under unfavorable emotional conditions is well known to all; the reason for it we have just seen. Of as great importance, though not so generally recognized, is that the psychical secretion, and hence good digestion, depends upon an active emotional state of enjoyment of the meal. Preoccupation, allowing the mind to dwell upon business or household cares, may interfere with the digestive processes only less seriously than worry or angry discussion.

The value of soups in aiding digestion is twofold. By exciting the appetite they help to arouse the psychical secretion; their content of meat juice is itself in some measure an excitant of the hormone to chemical gastric secretion, thus they are usually 
effective in starting the chain of events which make up the digestion of a meal. The practice of using them at the beginning rather than elsewhere in the meal, although long antedating our knowledge of their real value is thus seen to be physiologically sound. 


\section{CHAPTER XXX}

\section{THE ABSORPTION AND USE OF FOODS}

General Statement. The digestive process, as we have considered it in preceding chapters, is purely one of preparation. Its completion finds the food still within the alimentary tract, but ready for the use of the Body. It is conveyed to the tissues, as we have seen (Chap. XVII), by the blood. The passage of digested food from the alimentary tract, through its walls, into the blood or lymph, is known as absorption. The use of the food by the tissues, since it involves chemical activities on the part of the tissues themselves, is spoken of as metabolism. The discussion of these two processes is the purpose of the present chapter.

Absorption from the Stomach. Although the food remains in the stomach for several hours after each meal, in fact is often not wholly discharged before the taking of another one, it appears that absorption from the stomach into the blood normally occurs to a very limited degree, if at all. The fact that the digestive process is for no foods completed in the stomach affords sufficient reason why absorption should not take place there. We might suppose that the single great group of food-stuffs not requiring digestion, the single sugars, could advantageously be absorbed from the stomach, but experiment shows that even they are absorbed very slightly unless in rather high concentration, 5 per cent, in which case the walls of the stomach do take them up rather rapidly. The presence of alcohol in the stomach is said to increase markedly its absorptive power, but this is at best a doubtful benefit, since the single sugars form ordinarily a minor part of the meal, and the other food-stuffs are not ready for the use of the Body, and are wasted, therefore, if they are absorbed.

Absorption in the Small Intestine. The small intestine, being the chief and final digestive laboratory of the Body, is naturally the place from which absorption most largely goes on. It is, in fact, specially adapted structurally, as is no other region of the 
alimentary tract, for the absorptive processes. The innumerable projecting villi, each containing a capillary network and a lymphchannel, afford a total absorbing surface many times greater than would the same area if lined with ordinary mucous membrane; they also, by projecting into the intestinal cavity, are brought more readily into intimate contact with the intestinal contents.

Nature of the Absorptive Process. There is very good reason to believe that the process of absorption is not a simple physical one, involving only filtration, osmosis, and dialysis, but that it is carried on actively by the living cells which form the innermost intestinal lining, the columnar epithelium (Chap. XXVI). The support for this idea is chiefly experimental: the observation that blood-serum placed in the intestine is absorbed completely through its walls into the blood so long as the mucous lining is alive and functioning, but fails to be absorbed if the cells are injured, as by sodium fluorid, or some similar poison. Since the blood-serum placed in the intestine has presumably precisely the same osmotic pressure and percentage composition as the animal's own it is difficult to see how purely physical factors could bring about the absorption.

Channels of Absorption. We noted above that each villus contains a capillary network and a lymph-channel. The absorbed food stuffs might pass, therefore, either to the blood-stream directly or by the lymph-channels be conveyed to the receptaculum chyli (p. 382), and thence by way of the thoracic duct enter the bloodstream at the great vein of the shoulder. The essential difference between these two pathways is that the intestinal blood-stream drains into the portal vein, and must pass, therefore, through the capillaries of the liver before reaching the general circulation, while the lymph-stream reaches the general circulation without first traversing the liver. The significance of these two pathways will appear presently.

The entire phenomenon of absorption from the small intestine presents so many phases that it will be convenient to consider it in sections, one class of nutrients at a time.

The Absorption and Temporary Storage of Carbohydrates. Carbohydrate digestion reduces all foods of the class to single sugars. It is in this form, then, that they undergo absorption. However the process may be carried on it results in a flow of single 
sugars from the intestinal cavity into the blood-capillaries of the villi. These capillaries all drain, as previously stated (Chap. XIX), into the portal vein, which in turn passes to the liver and breaks up therein into the liver-capillaries (Chap. XXVI); so that all blood from the intestine, with whatever it may have taken up there, is forced to traverse the liver, and to come into intimate contact with the liver-cells, before it reaches any of the other living tissues of the Body.

The amount of sugar present in the blood of the portal vein is, of course, variable, there being a higher concentration at times when sugar is being actively absorbed from the intestine than at other times. Curiously, the blood flowing away from the liver, in the hepatic vein, is always found, normally, to contain a certain small percentage, about 0.15 per cent, of sugar, whether the sugar content of the portal vein is high or low.

It is evident that the liver must be able to store within itself the excess sugar that comes to it during active absorption from the intestine, and to give this out again between times. The sugar is retained in the liver, not as such, but in the form of glycogen or animal starch. The conversion of sugar into glycogen is a simple dehydration $\left(\mathrm{C}_{6} \mathrm{H}_{12} \mathrm{O}_{6}-\mathrm{H}_{2} \mathrm{O}=\mathrm{C}_{6} \mathrm{H}_{10} \mathrm{O}_{5}\right)$, and is doubtless easily effected by the liver-cells. The purpose of the change from sugar to starch seems to be to make the retention by the liver easier; sugar is too soluble to be held readily, whereas the liver can hold the glycogen without trouble. The liver is said to be able to hold 10 per cent of its weight of glycogen.

The use of the sugar is, as we have already seen, for fuel for the Body. Oxidations are constantly going on in the living tissues, therefore there is a steady withdrawal of sugar from the blood, and the liver must be continually making good the depletion by reconverting some of its glycogen into sugar. That the sugar content of the blood is kept up at the expense of liver-glycogen is proven by observations on fasting animals. A comparatively short period of starvation results in the complete disappearance of glycogen from the liver. That in fact is the first fuel supply to be drawn upon in the absence of food.

Just how the chemical process of converting glycogen to sugar is performed is not certain; although an enzym capable of effecting the transformation is said to be present in the liver. If the 
process is carried on by an enzym it is under closer control than the enzym reactions we have studied in connection with digestion, for it does not go on rapidly till all the glycogen is used up, but only so fast as is necessary to make good the loss of sugar from the blood.

Storage of Glycogen in the Muscles. These organs, as we learned when studying them (Chap. VII), perform their work through the oxidation of sugar, and since they are likely to be called upon for prolonged activity need to have immediately available a supply of their special fuel. Such a supply they have, in the form of glycogen, which makes up about 1 per cent of the weight of muscle tissue. This glycogen is, of course, derived from the sugar of the blood, so that the muscle-cells must have the same power that liver-cells have of changing sugar to glycogen and glycogen back to sugar.

The Relation of the Kidney to the Concentration of Sugar in the Blood. As we have seen, the sugar content of the blood remains practically constant all the time at a relatively low concentration, about 0.15 per cent. It is an interesting fact that the kidney, the great excretory organ of the Body, is so constructed that if for any reason the sugar content of the blood rises much above normal, to 0.2 per cent or more, the excess of sugar is withdrawn from the blood by the kidney and appears in the urine. The kidney stands to the sugar of the blood in the relation of a spillway; it allows the concentration to rise just so high, but no higher. This property of the kidney makes such a storage mechanism for sugar as we have described virtually, necessary to the Body, since without it the tissues could not be provided with fuel at once continuously and economically.

Just why the kidney should have this function is not very clear, but a suggestion is found in the observation that the continued presence of excess sugar in the tissue fluids, as in diabetes (p.496) is inimical to the highest welfare of the tissues.

The Assimilation Limit. Alimentary Glycosuria. The ability of the liver to convert into glycogen the sugar delivered to it by the portal vein is not without limit. If the absorption from the intestine is so rapid as to raise the sugar content of the portal blood to an abnormally high point, the liver is not able to handle all the sugar; and the excess escapes into the hepatic vein and so 
into the general circulation. Should this excess be sufficient to raise the sugar percentage of the blood above 0.2 per cent there is excretion of sugar from the kidney, a condition known as glycosuria. It is found that the rate of absorption of sugar depends chiefly on how much of it is present at one time in absorbable form in the intestine. Thus if large amounts of single sugar are eaten the essential condition for excessive absorption is likely to be fulfilled. Honey, a sweet containing considerable single sugar, is thus apt to cause glycosuria if too freely eaten. The greatest amount that can be eaten without causing glycosuria marks the assimilation limit. The other carbohydrates, since they require digestion before they are absorbed, are less apt to give rise to too rapid absorption. It is found, however, that there is a great difference in the amounts that can be taken without exceeding the assimilation limit. The inversion of milk-sugar gives rise to a special single sugar, galactose, which is converted into glycogen very slowly. The assimilation limit for milk-sugar is correspondingly low. Starch is digested so slowly that the assimilation limit for it is quite difficult to exceed. Glycosuria resulting, not from disease, but merely from overconsumption of carbohydrates, is called alimentary glycosuria.

Other Types of Glycosuria. An analysis of the carbohydratestorage mechanism just described reveals three points where an upset of the normal sequence might give rise to glycosuria; and three corresponding varieties are known. The three conditions which may cause glycosuria are: (1) A disturbance of the mechanism which controls the rate of conversion of liver-glycogen into sugar, so that more is poured into the blood than the tissues are able to use; (2) a diminution in the consumption of sugar by the tissues, so that more accumulates than the liver can store; (3) an alteration of the kidney such that it excretes all the sugar that comes to it, and thus drains sugar from the blood continuously. Much insight into the working of the carbohydrate-storing mechanism, as well as the use of carbohydrates by the Body, has been gained by study of these three forms of glycosuria.

Glycosuria from Disturbance of the Liver Function. Emotional Glycosuria. It has been shown that injury to a definite point in the medulla destroys the co-ordination between the output of sugar from the liver and the use of sugar by the tissues, with 
resulting glycosuria. This suggests, of course, that the liver carries on its function of storing and delivering sugar under the control of a reflex "center." Such a method of control seems reasonable inasmuch as increased activity of the tissues involves increased consumption of sugar, with a greater call upon the liver for supplies, and, as we know, the tissues most involved, the muscles, send into the medulla streams of afferent impulses whenever they are active, which would serve to excite the center. In corroboration of this idea it may be stated that certain diseases of the central nervous system in man result in an upset of the liver function of precisely this sort.

Recent observations have brought out the interesting fact that this "nervous control" of the conversion of glycogen to sugar by the liver, is not direct, but operates through the intervention of the hormone adrenin. Some time ago the discovery was made that during great emotional excitement sugar is apt to appear in the urine. A test was made recently on the members of the football squad of a great university immediately following the crucial game of the year. Of the men examined, players and substitutes, nearly all showed pronounced glycosuria. This fact, in conjunction with the known outpouring of adrenin in times of stress, suggested a causal relationship, and the demonstration was shortly afforded that the increased production of sugar from liver glycogen is the result of stimulation by the hormone. This we recognize at once as a part, and an important part, of the general emergency reaction of the Body. At a time when the utmost muscular exertion is likely to be demanded it is imperative that there be no failure from a shortage of fuel. The flooding of the blood with sugar as the result of the outpouring of adrenin assures that the fuel supply for the laboring muscles shall be ample. That there is an overproduction, so that much passes out by the kidneys and is wasted, merely emphasizes the general principle that in time of emergency the Body scorns economy, directing its resources lavishly toward successful meeting of the immediate situation.

Glycosuria from Inability of the Tissues to Use Sugar. Diabetes Mellitus. This condition, the usual pathological cause of glycosuria, and unfortunately not of rare occurrence, has been much studied, chiefly because it inṿolves the relation of the tissues to their chief fuel supply, sugar, and a complete understanding of 
the disease should throw much light on the mechanism of the consumption of fucl by them. The presence of sugar in the urine is only one of the symptoms of diabetes mellitus. A symptom of equal importance is the muscular weakness, and particularly the lack of endurance, which results from the failure of the tissues to make use of their fuel supply to advantage.

A very interesting feature of this condition is that it can be induced experimentally in a quite unexpected way, namely, by injuring or removing the pancreas. Complete destruction of this organ is followed by an apparent total loss of the power of the tissues to use sugar; there is excessive muscular weakness, and death occurs in a few days after the operation. The effects of partial destruction are less severe; in fact no symptoms appear unless fully three-fourths of the gland are destroyed. The function of the gland in connection with the prevention of diabetes is wholly independent of its function as a digestive gland. The duct of the pancreas may be tied without the production of diabetes, or the gland may be transplanted from its usual location to some other, quite abnormal one, where, if it lives and establishes connections with the circulation, it suffices to prevent diabetes perfectly.

The interpretation of this function of the pancreas is that it is a hormone action. The gland produces the hormone, and this, when carried by the blood to the tissues, in some way enables them to use sugar; perhaps by activating some tissue enzym or enzyms upon which the oxidation of sugar depends. It is not thought that the ordinary secreting cells of the pancreas produce the hormone, but that certain peculiar groups of cells embedded in the gland, the Islands of Langerhans, have this function. Although not all physiologists agree in assigning the production of the hormone to the Islands of Langerhans, the general trend of opinion seems to be that that is their function.

Diabetes mellitus in man not only shows symptoms agreeing precisely with those seen in animals with injuries to the pancreas, but many cases show on autopsy very well marked lesions of the Islands of Langerhans. We may thus conclude with fair certainty that the disease is one affecting these Islands, and that its symptoms are the result of more or less complete failure of the hormone formed by them. 
Glycosuria from Increased Permeability of the Kidney-Cells to Sugar. The injection of a certain drug, phlorhizin, into the circulation is followed by a glycosuria which is due chiefly, although probably not wholly, to alterations in the kidney. These are of such a sort that the kidney-cells, instead of removing only sugar in excess of 0.2 per cent, take all that comes to them. The result, of course, is a great waste of this valuable fuel, requiring greatly increased consumption of carbohydrates to make it good. This form of glycosuria has been produced experimentally in animals, for purposes of study, but occurs rarely, if at all, as a disease of man.

The Absorption of Proteins. We have learned that the digestive process splits proteins into their constituent amino acids (p. 466). The advantage of this is obvious, when we recall the fact that an important function of proteins is to repair tissue waste, and the further fact that to do this the food protein must be converted into the characteristic tissue protein of which it becomes a part. We saw in Chap. I (p. 11), that the difference between one protein and another is in the number, proportions, or arrangement of the amino acids which make up their molecules. While the food proteins, as such, would not serve for tissue repair, the amino acids of which they are composed are precisely what the Body needs for rebuilding its own substance. Furthermore, the different tissues must differ somewhat in the constitution of their characteristic proteins, and for the repair of all the different tissues a mixture of amino acids is evidently much more useful than a small number of undigested food proteins could possibly be.

There is abundant evidence that the digested amino acids are absorbed directly into the blood-stream, and not into the lacteals. This has been proven by inserting a tube into the thoracic duct of an animal and draining off all the lymph produced during the absorption of a protein-rich meal. No increase in the percentage of nitrogen (the characteristic element of proteins) could be detected in the lymph; conclusive proof that the amino acids do not follow that pathway. Moreover, chemical methods recently devised have proven the presence of amino acids in the blood-stream, and that during the absorption of a meal of meat, they are increased in amount. The use the Body makes of these amino acids 
will be considered in detail in a later paragraph, as will also the relation of the liver to them.

The Absorption of Fats. The result of fat digestion is to split the fats to fatty acid and glycerin. It is believed that they are taken up by the cells of the intestinal lining partly in this form; but not wholly so, since free fatty acid in the presence of free alkali, such as is furnished by the bile and pancreatic juice, reacts with the alkali to form soap. That there is in the small intestine a certain amount of soap formation cannot be doubted. The advantage of soap formation is one of increased solubility; fatty acids are insoluble in water, soap quite soluble. There is reason to believe, however, that only part of the fatty acid is combined into soap, and that the remainder is absorbed, as stated above, as fatty acid. This direct fatty acid absorption seems to be effected largely through the agency of the bile. It is known that fatty acids are soluble in bile, and can thus be brought in solution into contact with the absorbing cells; and a very common observation of physicians is that stoppage of the flow of bile into the intestine, as by occlusion of the bile-duct, is followed by an almost complete failure of fat absorption. The glycerin part of the decomposed fat is quite soluble in water and is doubtless absorbed readily.

After the absorbing cells of the intestinal wall have taken up the fatty acid and glycerin, these are recombined within the cells into fat. The presence of fat droplets in the absorbing cells can be demonstrated microscopically. We know that the fat droplets are not absorbed as such, but are formed after their constituents have been separately taken up, because these fat droplets are always observed in the part of the cells away from the intestinal cavity, and never in the part next to it; also because we know that the digestive splitting to acid and glycerin takes place, a meaningless process if not necessary to absorption.

The fat finds its way into the circulation by way of the lymphchannels of the villi, the lacteals, and the thoracic duct, entering the blood-stream at the point of emptying of the thoracic duct in the large vein of the shoulder. The fats alone, of all the food stuffs, take this course, and we may suppose the difference to mean that the liver has no special function to carry out in connection with the fats as it has for carbohydrates and proteins. 
Therefore the fats are shunted into another course which carries them into the blood stream without having first to traverse the liver.

Absorption from the Large Intestine. The mass that passes through the ileocolic valve into the large intestine contains conparatively little absorbable food material. The carbohydrates and fats are very completely removed during the passage of the small intestine, and fully ninety per cent of the proteins as well. There remains for the large intestine, then, only the absorption of the protein residue and the absorption of water. It is probable that this latter function, that of absorbing water, is in reality the chief one possessed by the large intestine. There is virtually no absorption of water in the small intestine; the intestinal contents pass the ileocolic valve as liquid as when leaving the stomach. This maintenance of a liquid consistency is, of course, essential to the absorptive processes, and it is only after all absorbable food has been removed that the water, which is also needed by the Body, is taken up.

The Food Requirement of the Body. If we know how much energy the Body liberates in a day, and how much tissue breakdown it suffers, we ought to be able to estimate how much energyyielding food, and how much tissue-repair food is required daily; assuming, of course, that we know the amount of energy yielded by definite weights of food stuffs. By the use of devices called calorimeters the total energy liberation of the Body per day has been determined under various conditions, and the energy content of the various foods has also been found. . We learned in a previous chapter (p. 107) that a unit of heat energy commonly used in physiology is the Calorie; the amount of heat required to raise 1,000 grams of water through $1^{\circ}$ centigrade. In terms of this unit the energy output of man in 24 hours averages from about 2,400 Calories for men of sedentary occupation to 5,000 Calories for those doing heavy manual labor. The energy yield of the various foods is as follows:

Carbohydrates...................... 4.1 C. per gram.

Proteins............................ 4.1 " "

Fats.............................. 9.3 C. " "

It is therefore a matter of simple calculation to determine how much of any one food stuff is needed to supply the required en- 
ergy, or to arrange suitable mixtures of the three. By reference to the table of food compositions (Chap. XXV), the amounts of actual food materials needed can be found.

The Protein Requirement of the Body. Before proceeding with a further discussion of the energy relationships of the Body it will be well to consider the tissue-maintenance requirement, which as we have seen, is wholly a protein need. In order to analyze this requirement intelligently we need to know, first of all, what use the Body makes of protein, and second, how much is required. We have already seen that protein can be oxidized in the Body with the liberation of energy, and constitutes, therefore, a good fuel. In this respect, however, it is in no degree superior to the other nutrients, fats and carbohydrates. Our special interest in it is for the function which it alone can exercise, that of making good tissue wear and tear.

Since protein is the only food stuff that contains nitrogen we can tell how much of it is used up in the Body by measuring the amount of nitrogen eliminated (p. 512). All except a very small portion (roughly 2 per cent) is discharged in the urine. Chemical tests of the urine will furnish us, then, with the data we seek. Evidently if a man abstains wholly from food for a while all the nitrogen in his urine must come from tissue break-down. We have a means, thus, of finding out how rapidly this break-down occurs. A moment's thought will show us, however, that the tissue breakdown in complete starvation is not necessarily the same as in ordinary life. When no food is eaten the energy requirements of the Body must be met at the expense of its own tissues; particularly in prolonged starvation, when the stored fuels, fat and glycogen have been used up; so that in addition to the usual loss of substance by wear and tear there is a further consumption of material as fuel. To get at the amount of tissue break-down under ordinary conditions by this method the starvation must not be complete. The subject must be given abundant supplies of fat, earbohydrates, and essential accessories, but no proteins. When this is done the nitrogen eliminated from the Body can be așsumed to represent the normal tissue break-down. Experiments conducted along this line have shown that in an adult man of ordinary size (70 kilos, 165 lbs.) the protein lost from the Body daily by tissue wear and tear amounts to about 20-25 grams 


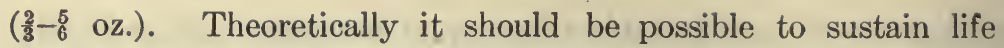
indefinitely on a diet containing this amount of protein, provided adequate fats and carbohydrates for the fuel requirements of the Body are also furnished. This theoretical minimum of protein does not agree at all well with the amounts of protein actually consumed. In fact dietary studies show that most people take four to five times this amount. The great discrepancy between the amount of protein theoretically required and that actually ingested has occasioned a great deal of discussion among dietitians as to whether the human race is habitually consuming proteins to excess. Since the proteins are the most expensive food stuffs the question is one of great economic importance.

Numerous experiments have been performed to see what is the effect of cutting the protein intake approximately to the theoretical minimum. In all the early experiments along this line when the protein content of the diet was reduced to about 35-40 grams daily, a figure well above the theoretical minimum, the tissue wear and tear was no longer made good completely. This was evidenced by the daily elimination from the Body of more nitrogen than was taken in. The only possible source of the excess was from break-down of the Body's own tissues. There was also a steady loss of body weight. When an explanation of this was sought it was found that the chief difficulty lay in the manner of administering the protein. This substance when taken with the diet in the usual rather large amounts functions in part to replace worn-out tissues and in part, as we have seen, as fuel for supplying energy, the latter use being made of all surpius after the tissue repair has been provided for. The wear and tear of tissues goes on throughout the twenty-four hours of the day. The ordinary method of taking the protein, on the other hand, is in three meals during the daytime portion of the day. When the consumption is cut down to a low level evidently if there is any use of protein as fuel a shortage for tissue repair is likely to occur. When the protein is ingested in connection with the usual meals there is absorbed into the Body after each an amount which is in excess of the immediate needs for tissue repair, and accordingly some is used as fuel. The result is that before the time for the next meal arrives all the absorbed protein is used up and there is none to carry on the work of tissue repair. To avoid this the 
experiment was tried of dividing the protein into six equal parts and administering one part every four hours. When this was done, so that there was a practically continuous though small absorption of protein into the Body, it was found possible to make good completely the tissue break-down with an amount of protein very little in excess of the theoretical requirement.

The Replacement Value of Different Proteins. Obviously to make good the wear and tear of the tissues with the least possible amount, the proteins ingested must correspond as closely as possible in composition with those of the Body itself. We have already noted ( $p .11$ ) that the differences between different proteins are in the number or relative amounts of amino acids present. These differences are in some cases very pronounced. In general meat proteins resemble those of man more closely than do proteins of vegetable origin. Any protein that contains only a small proportion of some amino acid that is present in human protein in large proportion must evidently be fed in sufficient amount to satisfy the requirement for that particular amino acid. The other constituents, meanwhile, are in excess and afford a surplus to be used as fuel. It appears that the Body possesses a limited ability to convert some kinds of amino acids into others, but this is applicable to so few of the many which make up the protein molecule as to have little practical bearing.

Maintenance Proteins and Growth Proteins. Evidently, from what has been said above, any protein that is completely lacking in some essential amino acid or acids cannot serve to replace wornout tissues. Such a protein is ordinary table gelatin. This is a protein derived from bone and connective tissue (p. 49). It is deficient in three of the amino acids which are essential to living protein (tryptophan, tyrosin, and cystein). No matter how much gelatin may be included in the diet, if there is not provided also some protein which contains these essential acids there will be a wasting of the tissues.

Related to this fact is the even more remarkable discovery that there are certain proteins which are fully adequate for the maintenance of the Body, but will not suffice for the formation of new tissues. Young animals fed upon diets whose protein components are of this character will maintain a constant weight, but will not grow. A good example of such a protein is gliadin, one of the 
proteins of wheat. This protein lacks the amino acid lysin. The conclusion drawn from this observation is that the lysin which is one of the constituents of living protein is not involved in the processes of tissue break-down. After the tissue is once formed, therefore, it does not require continual supplies of this substance. No new tissue can be made, however, unless lysin is provided. A curious incidental discovery in connection with the experiments by which this was established was that the rats which were used as subjects could be maintained in health with the weight and bodily dimensions of young animals for months after they would have become full grown on an ordinary diet. If then they were changed from maintenance proteins to proteins that were adequate for growth they promptly began growing and presently attained full size. The significance of this is that the ability to grow is not restricted to the early periods of life and does not come to an end with the attainment of a certain age.

Fuel Protein. We have learned that proteins are absorbed from the digestive tract into the blood as amino acids. Of these a portion are destined to provide for tissue repair and growth. The excess is used as fuel. The nitrogenous portion is virtually devoid of value as a source of energy. To fit the remainder to serve as fuel the nitrogen-containing radicals are dissociated from the molecules, leaving non-nitrogenous residues of high energy value. This process of setting aside the nitrogenous radicals is known as deaminization. It was formerly believed to occur during the passage of the amino acids through the intestinal walls in the process of absorption, but recent investigations have shown that the amino acids are absorbed as such and that deaminization is probably carried on by the tissues generally. The further history of the nitrogen-containing radicals will be considered in a later chapter (p. 517). The non-nitrogen residues join themselves with the other energy-yielding food stuffs and will be discussed together with them (p. 507).

Should the Diet Include Much or Little Protein? We have seen that it is possible to maintain the tissues adequately upon a diet containing only a fraction of the amount of protein ordinarily taken. Are we to conclude from this that the human race eats too much protein? To this question no final answer can be given at present. Eminent dietitians have argued on both sides of it. 
One consideration that has been suggested as probably significant is that the low protein diet, although adequate for immediate maintenance, does not afford the Body sufficient reserve vitality to place it in the best situation for resisting infections or other debilitating influences. Emphasis has also been placed on the fact that the poorer inhabitants of Bengal, who live of necessity on a low-protein diet, are deficient both in strength and endurance. Conservative students of the subject are inclined to the opinion that our present dietary habits, based as they are upon centuries of experience, are probably in the long run better suited to our needs than radically altered dietaries, which may be theoretically sound, but lack the confirmation of long experience.

The allowance of protein in standard diets varies from 80-90 grams daily, which is the average amount consumed by American College students, to the 115-120 grams considered by some European dietitians suitable for the European laborer. In contrast with these figures are the allowances of 40-60 grams proposed by the advocates of a low-protein diet. While we may properly adopt a conservative attitude with reference to the low-protein controversy, we are not thereby justified in going to the opposite extreme. Excessive consumption of meat, particularly by people who lead sedentary lives, undoubtedly is attended by various evils, although most of these are referable to other causes than overconsumption of proteins.

The Liberation of Energy in the Body. We have seen that all the energy liberated by the Body can be expressed in terms of heat-units, but it is not to be concluded, therefore, that heat energy is the only form manifested by the Body. As a matter of fact the Body undoubtedly converts the potential energy of the food into at least three forms of kinetic energy; chemical, the carrying on of the digestive and other chemical processes of the Body; mechanical, the working of the skeletal muscles, as well as of the heart, the muscles of respiration, and the muscles of the viscera; and thermal, the direct production of heat by oxidation processes. This latter form of energy, although far exceeding in amount both the others together, may be looked upon as in large degree a by-product of the mechanical work of the Body, and arising through the inefficiency of the body machinery. We know that most of the heat of the Body is produced in the muscles, and that though these are 
producing some heat even when at rest, they produce enormously more when they are active. A characteristic of all machines is that they work more or less wastefully; not all the energy imparted to them appears again as useful work; the part that is lost, moreover, appears always as hest. In the Body there is this same inability to convert food energy into mechanical energy without there being at the same time a large heat production.

Studies of the metabolism of the Body must necessarily take into account these two main forms in which the energy of the food is manifested. For physical reasons which need not be considered here all muscular and chemical activities occurring wholly within the Body manifest themselves ultimately to the exterior in the form of heat. The total energy turnover of the Body can be determined, therefore, if the external mechanical work and the entire heat output can be measured. Theoretically these should exactly balance the energy content of the ingested food. Metabolism studies are devoted in part to demonstrating that this balance actually exists, and in part to determinations of the individual factors concerned.

Basal Metabolism. A necessary starting point for any study of energy manifestation in the Body is the determination of the amount liberated when the Body is as inactive as possible. The metabolism which gives rise to this energy represents that which is essential to the life processes. It is known as the basal metabolism. Its energy all appears in the form of heat. As measured in an adult man of average size, who eats nothing during the day of observation, it amounts to about 1,700 Calories (p. 500). The necessary activities of eating and digesting enough food to maintain the Body involve an expenditure of about 10 per cent additional energy, bringing the total practical basal metabolism up to about 1,870 Calories per day. Any energy liberation in excess of this amount must represent either actual muscular work or the by-product of heat which always attends it on account of the inefficiency of the muscles.

We shall see in the chapter on Heat Regulation (Chap. XXXII) that the Body makes very good use of this by-product of heat in keeping itself at a proper temperature the year round, and so the extra amounts of food we have to eat on account of the inefficiency of our bodily machines are not wholly wasted after all. 
The Metabolism of Muscular Work. The total energy turnover per day of any individual is made up, as we have just seen, of his basal metabolism, together with the metabolism of his active muscles. The first factor is practically constant; the second is extremely variable. Some average figures may, however, be presented. If we reckon the muscular efficiency at 20 per cent every Calorie of energy manifested in the form of muscular work means a consumption of 5 Calories altogether, and a liberation of 4 Calories as the by-product of heat. A man who leads a decidedly sedentary life, making no more movements than necessary, is calculated to do an amount of work in a day equivalent to about 40 Calories $(120,000)$ foot-pounds. This work consists in large part of the labor involved in the maintenance of the sitting and standing positions. The performance of 40 Calories of muscular work requires, on account of the bodily inefficiency, previously noted, an energy liberation of 200 Calories. This, added to the practical basal metabolism of 1,900 Calories, brings the total to 2,100 Calories. When allowance is made for a moderate amount of excrcise; no more than must be taken if good health is to be maintained; the daily metabolism amounts to 2,500 Calories. This figure is believed to represent the average for adults of all classes other than manual laborers. An interesting fact is that calculations of the average daily metabolism per individual of the inhabitants of cities, based on estimates of the amounts of food brought in each day to the markets, indicate this same figure, 2,500 Calories, as the average metabolism for the city dweller. The energy liberation of the manual laborer varies greatly, of course, with the nature of the toil. The range is usually set at 3,500 to 5,000 Calories per day. The latter figure probably represents a high limit which is rarely exceeded by any worker day after day for long periods, although trained men may show a much greater metabolism for a day or two. An output at the rate of 10,000 Calories is believed not to be impossible for a brief spurt.

The Relative Food Values of Proteins, Carbohydrates and Fats. Disregarding the use of protein as a tissue-repairer, and considering all three varieties of food simply as furnishers of energy, we may inquire whether any one of them is superior to the others, or whether any particular proportion of the three food stuffs is 
specially desirable. From the purely mechanical standpoint there is evidently no choice among them; the Body requires 2,500 or more Calories of energy each day; each food stuff yields definite amounts of energy; therefore all we have to do to supply the Body's requirement is to eat enough grams of one or the other food stuff, or of a mixture of them. The answer to the question goes back, then, to other considerations than that of the energy content of the foods. The first of these is the matter of relative digestibility and absorbability; it is of little avail to eat a food if it fails to be properly digested and absorbed. Experiments have shown that carbohydrates, exclusive, of course, of cellulose, are the most completely absorbed of all foods, 97 per cent of the amount eaten finding its way into the Body; fats come next in order, 94.4 per cent being absorbed; proteins are taken up least completely of all, the Body getting only 92.6 per cent of the protein eaten. There are also differences of digestibility and absorbability of different foods within the same class; the protein of lean meat, for example, being more readily digested and absorbed than that of beans and peas. Cheese, which contains the highest per cent of protein of any common food, has a reputation, perhaps undeserved, for indigestibility. Graham bread is, by many, supposed to be more nutritious than white. It is true that graham flour contains a higher percentage of protein than does white flour, but the extra protein of the graham flour is in the bran, whence the human digestive process fails to extract it; so as a matter of fact white bread yields more actual nourishment to the Body than does graham. The special importance of graham flour or of whole wheat is in the roughage it contains. Some fats are much more digestible than others; olive oil and pork fat, for example, are more completely utilized by the Body than is mutton fat. Fat of any sort, taken in the meal with other foods, seems for some reason to delay the whole digestive process, and the delay is greater the more fat is present. For this reason it is desirable to limit somewhat the amount of fat used.

Another question which may affect the choice of foods is the degree to which they tax the excretory organs of the Body. We have seen that fuel proteins yield a nitrogenous residue which must be gotten rid of by the excretory organs. There seems to be a rather general belief that this task constitutes a somewhat serious 
strain upon these organs, and if it does tend to throw upon them excessive labor it is clear that the consumption of proteins ought on this account to be kept as low as possible. The idea that the excretory organs are endangered by ordinary amounts of protein in the diet is not sustained by any very convincing evidence. In fact there is at least one race of men, the Eskimos, in which huge consumption of flesh proteins is the rule and in which no tendency to gout and the other diseases ordinarily attributed to overuse of meat is discoverable.

In the matter of cost, which must also be taken into consideration, carbohydrates have a marked advantage over the other food stuffs. For example, bread, which is chiefly carbohydrate, yields, dollar for dollar, about ten times as many Calories as lean beef, a protein. The cheapest proteins are the vegetable ones; a given weight of protein costing about five times as much when bought as beef as when purchased in the form of beans.

Still another factor to be taken into account is the appetizing quality of the different foods. The dependence of the whole digestive process upon a proper initial psychic secretion of gastric juice emphasizes the importance of the use of appetizing foods. Boiled meat contains as much nourishment as the same weight of roasted meat, but the former is less desirable as a food because the process of boiling extracts from it the substances which impart to meat its flavor. Eggs are exceedingly nutritious, but to some people they are practically valueless as food, because they inspire aversion rather than appetite.

The Specific Dynamic Action of Proteins. A feature of protein metabolism that is both interesting and of great dietary importance is a stimulating power it exercises toward the whole metabolic process. Whenever in the Body active consumption of proteins is going on there occurs, in addition to the metabolism of the proteins themselves a further metabolism of some of the reserve fuel supply of the Body, with, of course, a corresponding increase in the total heat production. This stimulating property of protein has been called its specific dynamic action. Practically it is important in regulating the heat production at different seasons of the year. In winter, when we naturally eat protein freely, a large amount of heat is necessary to maintain the Bodily warmth. In summer, when we wish to produce no more heat within our 
Bodies than absolutely necessary, the amount of protein is cut down. The very large protein intake of Eskimos probably serves to insure for them a heat production adequate to the extreme climate in which they live.

The Nutritive Value of Albuminoids. These proteins, as stated above (p. 503), lack some of the essential constituents of cell proteins, and cannot, therefore, serve as tissue-restorers. We can imagine, however, that they ought to satisfy the Body's demand for protein fuel, and so be substituted for the major part of the protein of the diet. Various attempts have been made to substitute gelatin for proteins in this way, and it seems to be highly efficacious in satisfying the Body's protein-fuel demand. But curiously gelatin can be used thus for only a few meals; presently there is a revolt of the appetite against it and no more can be eaten. Experiments have shown that dogs will starve rather than take continuously a diet whose chief constituent is gelatin.

The Special Metabolism of Fats. Fats are very useful fuel foods. Their energy content is twice that of the other nutrients. As we saw in an early chapter (p. 106) there is no present reason to suppose that they have to be changed to sugar before they can be used as sources of muscular energy. There is, however, a feature of their metabolism which negatives their consumption in large excess. In the process of oxidation of fats there is a stage in which certain organic acids are formed. These, if produced in amounts so large that the alkalies of the Body cannot neutralize them successfully, bring about a condition known as acidosis, which is harmful and, when pronounced, fatal. The acid formation is kept in check if there is an accompanying metabolism of carbohydrates. Acidosis is not so likely to occur on a mixed diet, therefore, as on one in which fat is the chief item.

A practical difficulty that arises in prescribing a diet in diabetes (p. 496) is due to this feature of fat metabolism. The diabetic, as we have seen, cannot utilize carbohydrates. To feed him upon a carbohydrate diet is, therefore, not only wasteful but positively harmful, since it involves the constant presence in his body fluids of injurious quantities of sugar. The same difficulty inheres, although in less degree, in a diet of protein, since the fuel residue of this substance is, as we have noted (p. 504) essentially carbohydrate. The most feasible source of energy to the diabetic is, 
therefore, fat, and his diet usually consists largely of this substance. He is thus confronted with the ever-present possibility of developing acidosis. As a matter of fact sooner or later practically every pronounced diabetic has this experience. Fatal acidosis is the recognized cause of death in the disease.

Principles of Dietetics. From the various considerations presented above we may summarize the general rule that the choice of food should be such as to yield sufficient protein for the Body's protein requirement, without containing an amount so excessive as to throw an undue burden on the excretory organs; that the amount of fat should be somewhat limited; and that enough carbohydrate should be added to bring the sum total up to the Body's energy requirement; finally, that the most appetizing foods obtainable within a reasonable limit of cost should be selected. Fortunately for the well-being of the race, mankind has always selected just such a diet under no other guidance than his appetite and his means, and these, to a healthy person, make trustworthy guides, so long as they are accompanied by temperance as a third.

The importance of dietetics as a science is chiefly in connection with the fceding of the sick, or providing for the maintenance of large numbers of individuals, as in armies or public institutions, where a slight error in selecting food, in greater amounts, or at greater cost than needed, amounts in the aggregate to a very large waste.

The Maintenance of Constant Weight. It is the experience of most adults that during periods of unbroken health the body weight remains practically unchanged day in and day out. It is clear that this condition depends on the maintenance of an exact balance between the intake and outgo of the Body, since if more is taken in than is given out there must be a gain in wcight, and vice versa. It is customary to consider the question of weight maintenance under three heads: water equilibrium, nitrogen equilibrium, and carbon equilibrium.

Water Equilibrium. For a Body to be in water.equilibrium the amount of water lost per day must be exactly replaced by the amount drunk. In large measure the sudden and transient changes of weight which occur are due to upsets of water equilibrium. Any violent exercise in hot weather reduces the weight 
by inducing a profuse perspiration with resulting loss of water. The intense thirst which follows the exercise leads to abundant ingestion of water and a speedy restoration of the lost weight.

Nitrogen Equilibrium. Those metabolic activities of living tissues which result in tissue break-down are particularly associated with the use of protein foods, since, as we have seen, their repair can be accomplished only by proteins. The characteristic constituent of protein is nitrogen; and the simplest way to estimate the amount of protein contained in any food mass, or represented by any particular amount of excretion, is to determine the nitrogen and multiply the weight of it present by 6.25 , the fraction of protein which is nitrogen. We shall learn in the chapter on Excretion (Chap. XXXI), that in the healthy Body an accumulation of nitrogen-containing excretory products never occurs; as fast as wastes are formed they are gotten rid of. It follows, then, that if there is less nitrogen being given off than taken in, the living tissues of the Body must be increasing in amount, and if more is given off than is obtained in the food the living tissues must be wasting away. In the healthy adult Body, neither of these conditions is at all usual; the intake and outgo of nitrogen balance each other and the Body is in nitrogen equilibrium.

It has been chiefly through experimental studies of nitrogen equilibrium that our ideas of the twofold function of protein, as tissue-restorer and as fuel, have been gained. If an animal be fed large enough quantities of protein he requires no other food, and if healthy maintains nitrogen equilibrium upon this high level, the large nitrogen intake being exactly balanced by an equally large outgo. Now by substituting other foods, as carbohydrates or fats, for part of the protein, the nitrogen intake and outgo are each less in quantity, but they still balance; the animal is in nitrogen equilibrium upon a lower level. If the substitution of other foods for protein is increased a point is presently reached when the nitrogen outgo exceeds its intake; the animal is not getting enough protein for his needs, and so his own tissues are breaking down (p. 501). During the growth period, on the other hand, or after a wasting illness, when new tissue is being formed, the nitrogen balance is the other way; the amount of balance lost from the Body daily is less than that consumed. The hearty appetites of children and convalescents are associated with this necessity of 
taking sufficient nourishment to insure a supply of protein for tissue building.

Carbon Equilibrium. For an animal to be in carbon equilibrium only needs that all the fuel taken in be burned, and that no reserve store be called upon. Aside from the temporary storage of carbohydrate food as glycogen all the fuel taken into the Body must look forward to one of two fates, either to be oxidized promptly or to be stored in the form of fat for future use. Just as nitrogen equilibrium may be established on a high or a low level so carbon equilibrium can be maintained in the face of variations in the intake of fuel. It is easily seen, however, that the limits of carbon equilibrium must be narrower than of nitrogen equilibrium. The actual protein requirement of the Body is so much less than the usual protein intake that considerable variations in the protein consumed can be made without affecting the nitrogen equilibrium; but the energy requirement of the Body is quite definite, varying with the work done rather than with the food eaten. Thus it follows that the fuel intake and the energy requirement are harder to keep balanced than are the nitrogen intake and outgo. It may easily be a matter of astonishment how successfully the Body, under the guidance of the appetite, manages to make its fuel consumption balance its fuel need.

There is a difference of opinion among Physiologists as to whether every accidental excess consumption of fuel results in the normal individual in the deposition of the surplus in the form of fat, or whether the Body has the power to carry on oxidations in excess of the normal basal metabolism and of the amount of muscular exercise.

Such positive information as we have on this point (see next paragraph) is based on observations on abnormal individuals and cannot be taken as necessarily applying to persons in normal health.

The Influence of the Thyroid Hormone upon Metabolism. Whether excess fuel shall be stored as fat or be burned, has been shown to depend, to a large extent, at least, on the amount of the thyroid hormene that is produced. When the hormone is abundant the bodily oxidations are so vigorous that no surplus of fuel remains to be converted into fat. An inactive thyroid gland, on the other hand, signifies a likelihood 
to fat formation whenever the consumption of food happens to exceed the immediate energy requirement. In the disease known as exophthalmic goiter (Grave's Disease) the thyroid gland is abnormally active. The chief symptoms of the disease are those that are associated with a greatly augmented metabolism. Sufferers from the condition eat hugely and yet are emaciated. Measurements of the daily energy turn-over show a heat produc. tion that may be virtually double that of normal persons.

Recently the interesting fact has been brought out that the thyroid gland is subject to nervous stimulation by way of the thoracico-lumbar autonomic system. Artificial Grave's Disease has been produced in animals by causing persistent excitation of those branches of the system that innervate the thyroid. Similarly, the hormone adrenin, which stimulates tissues innervated by the thoracico-lumbar autonomics, has been shown to excite the thyroid to activity. The suggestion has been made that this reaction is a part of the general emergency function of the Body. Evidently a heightened metabolism, by increasing the outpouring of energy, might be beneficial in time of stress. At present, however, this emergency action of the thyroid must be looked upon as suggested rather than proved.

The Treatment for Obesity is obviously to make the energy requirement equal, or even exceed, the fuel intake. Vigorous muscular exercise accompanied by strict dietary limitation may produce the desired result, but the good effects continue only so long as the flesh-reducing measures are persisted in. Exercise and - dieting are both conducive to good appetite, therefore as soon as the treatment is relaxed a return to the former condition is virtually inevitable. Persistent semi-starvation, unaccompanied by active exercise, is an efficient weight reducer. It should be rcsorted to with intelligence, however, for undesirable impairment of strength may follow its injudicious employment. A good rule for those who wish to avoid gaining flesh is never to satisfy the appetite wholly. On account of its specific dynamic action (p. 509) protein is usually made the chief constituent of the diet in the treatment of obesity.

The administration of thyroid extract is a means of reducing flesh by stimulating the oxidation processes of the Body. Since the thyroid hormone has effects upon the nervous system (p. 202) 
as well as upon general metabolism this treatment should never be undertaken except under competent medical advice.

Source of the Body Fat. For a long time there was much discussion as to which of the three sorts of food stuffs, proteins, carbohydrates, or fats, is the source of the fat which is stored in the Body. The natural conclusion that body fat is derived from food fat is shown to be not universally true, at any rate, by the ability of cattle to produce milk, with its abundant fat content, upon a diet of hay and grain in which no trace of fat occurs. The question whether in these animals the protein or the carbohydrate of the food gives rise to the fat was formerly much studied; but with the rise of the modern view of normal protein metabolism, according to which all but a small percentage of the protein taken in the food is deaminized and used as carbohydrate, the question has lost much of its force. There can be little doubt that body fat represents stored fuel; and since the whole fuel supply of the bovine Body is often represented by carbohydrates, these must be the source of the fat which the Body elaborates.

It seems to be the general opinion that even in animals whose diet includes some fat the normal source of the body fat is for the most part carbohydrate. It is supposed, without very definite evidence to prove it, that the fat absorbed after a meal is retained in the blood till taken up by the tissues and burned, and that the somewhat leisurely process of fat deposition is carried on in connection with the carbohydrate, which is transferred from its temporary storehouse in the liver to a more permanent one in the adipose tissues. There is no reason to doubt that when large amounts of fat are included in the diet there may be direct storage of some of the fat absorbed. In fact it has been shown that under these circumstances foreign fats, such as linseed-oil, for example, can be deposited in the adipose tissues of animals. 


\section{CHAPTER XXXI}

\section{EXCRETION AND THE EXCRETORY ORGANS}

Exogenous and Endogenous Excreta. It is usual to include - under the general head of excreta all waste materials of any kind that are given out from the Body. We shall see, however, that under this general definition come two very distinct classes of materials. Many substances are taken into the Body with the food which have of themselves no food value, and escape absorption during the passage of the food through the alimentary tract; thes' appear, of course, among the excreta. Other substances have an accessory food value, in arousing appetite, or in stimulating some of the bodily processes; these may be absorbed from the alimentary tract into the blood, but they do not enter in any intimate fashion into the metabolic activities of the living tissues, and after a longer or shorter sojourn in the blood they appear among the excreta. The third substances to be grouped with those just described are the nitrogen containing compounds which are split off from the fuel-proteins in the process of deaminization. These, from the moment of their separation, are waste products, to be conveyed as rapidly as possible to the excretory organs and gotten rid of. All these excretory materials are grouped together as exogenous excreta, the term suggesting that they are derived from sources outside the actual life processes of the tissues.

The second group of excreta, the endogenous excreta, includes those substances that are produced by the living cells of the Body in the course of their metabolic activities. Most of our knowledge of cell metabolism has been gained through studies of the endogenous excreta.

The Channels of Excretion. Four channels are recognized through which the body discharges waste materials; these are: the lungs, the skin, the urinary system, the rectum. The lungs are the channel for the discharge of gaseous wastes, carbon dioxid, and water vapor; the skin eliminates a part of the water and traces of the nitrogenous excreta; the urinary system disposes of the major part of the endogenous excreta other than gaseous, and also of 
those exogenous excreta that are absorbed from the alimentary tract into the blood. From the rectum are discharged all exogenous excreta that fail of absorption, and likewise a number of endogenous excretory substances received into the intestine from the liver, by way of the bile duct. The chapter on Respiration contains the discussion of the excretory function of the lungs. It is not necessary, therefore, to consider it here.

The Liver as an Excretory Organ. To the functions previously described of aiding the digestive and absorptive processes, and of serving as a temporary storehouse for carbohydrates, the liver adds a very important excretory function. This is in part direct, the separation from the blood of waste materials contained in it, and in part the working over of harmful excretory substances into harmless ones which it does not excrete but returns to the blood to be discharged through the urinary system and skin. This latter function will be considered before the direct excretions of the liver are discussed. It will be recalled that by the process of deaminization the "fuel-protein" is split into a nitrogenous waste portion, and a non-nitrogenous oxidizable portion. The nitrogenous part takes the form largely of ammonia compounds, chief of which is ammonium carbonate $\left(\mathrm{NH}_{4}\right)_{2} \mathrm{CO}_{3}$. These ammonia compounds are discharged into the blood. In connection with the putrefactive processes that go on in the large intestine there is a considerable production of ammonia which is also absorbed into the blood. It is well known that ammonia compounds are very poisonous to animals into whose circulating blood they are introduced, and it has been proven that an animal would be seriously affected if all the ammonia produced in the Body were allowed to remain in the circulation in that form. It is through the action of the liver that the Body is protected from the harmful effects of ammonia. During the passage of the blood through the liver its ammonia is converted by dehydration into urea, a compound harmless to the Body if not present in the blood in too great concentration. The conversion of ammonium carbonate by dehydration to urea is made clear if we compare the chemical formulæ of the two substances:

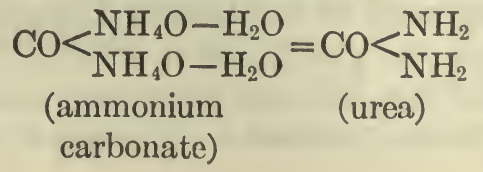


The urea formed thus from the ammonia compounds of the blood belongs to the group of exogenous excreta, since it does not represent a product of true cell metabolism in the Body. From the liver it is delivered to the blood of the general circulation where it remains till excreted by the kidneys.

The direct excretory function of the liver consists in the withdrawal from the blood and the delivery to the intestine through the bile of certain endogenous excretory substances. The most marked of these are the bile-pigments, which, as stated in Chap. XVII, are derived from the worn-out red corpuscles of the blood, and consist essentially of the pigment portion of hemoglobin minus its iron. Two bile-pigments occur, of very similar chemical constitution; bilirubin, golden-brown in color, is the predominating pigment of carnivorous bile, and of human bile on a mixed diet; biliverdin, a green pigment, predominates in the bile of herbiverous animals. Recent investigations have indicated that the bilepigments, although primarily waste products, serve some useful purpose during their stay in the alimentary tract. The nature of their use is not yet clear.

Beside the bile-pigments the liver excretes small amounts of various substances which are interesting chiefly on account of their insolubility in the ordinary fluids of the Body, and the fact that they are soluble in bile. These are found in the Body for the most part in nervous tissues, and they may be excretory products of nerve-cell metabolism. The most abundant of them is the nonnitrogenous substance cholesterin.

The chief constituents of bile not heretofore mentioned are the bile salts, sodium salts of peculiar acids found only. in bile, glycocholic acid and taurocholic acid. These do not appear to be excreta pure and simple, inasmuch as they are reabsorbed in part by the intestinal walls, and returned by the portal vein to the liver whence they again appear as constituents of the bile. They are thought to give to bile its special ability to promote fat absorption by dissolving the fatty acids, and it is also by virtue of their presence that the bile is able to dissolve cholesterin.

General Arrangement of the Urinary Organs. These consist of (1) the kidneys, the glands which secrete the urine; (2) the ureters or ducts of the kidneys, which carry their secretion to (3) the urinary bladder, a reservoir in which it accumulates and 
from which it is expelled from time to time through (4) an exit tube, the urethra. The general arrangement of these parts, as

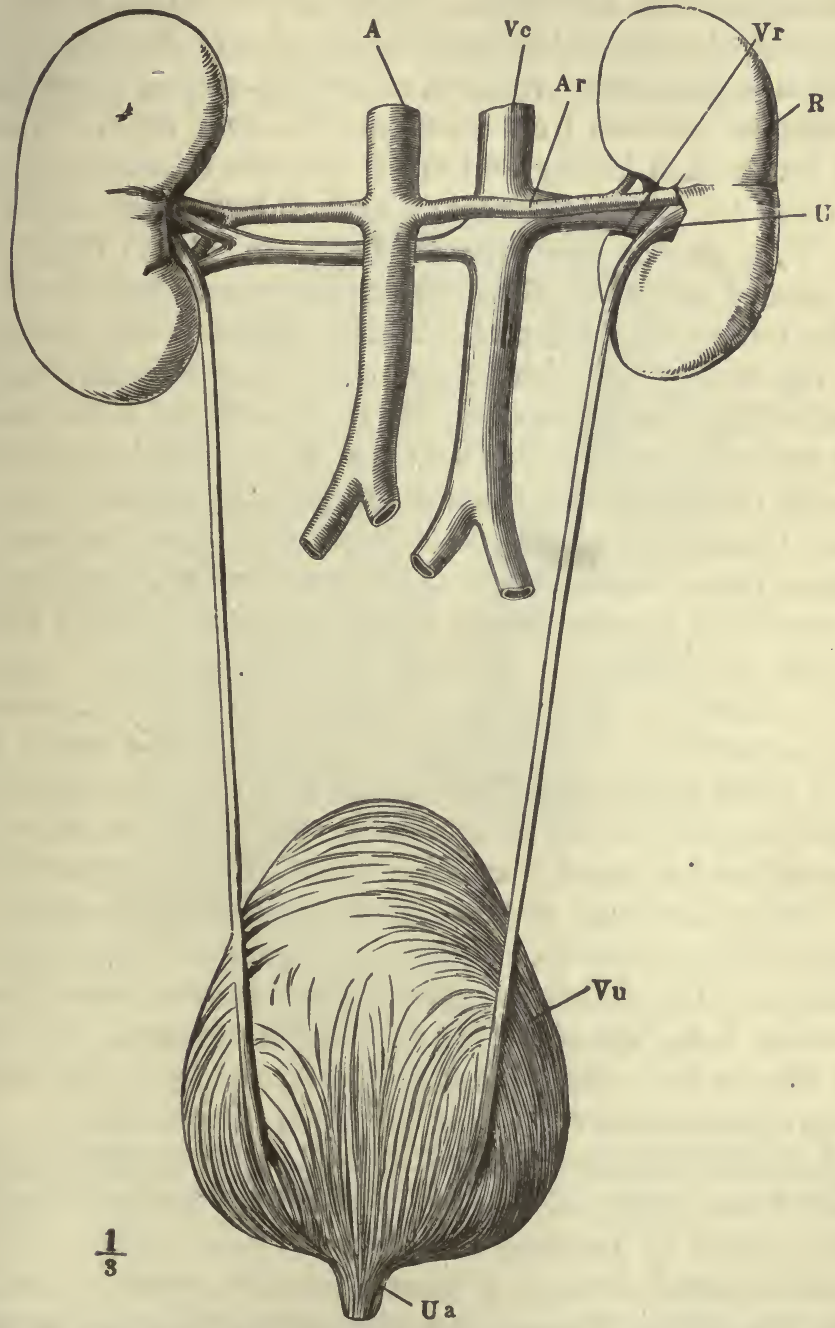

Fra. 138. - The renal organs, viewed from behind. $R$, right kidney; $A$, aorta; $A r$, right renal artery; $V c$, inferior vena cava; $V r$, right renal vein; $U$, right ureter; $V u$, bladder; $U a$, commencement of urethra.

seen from behind, is represented in Fig. 138. The two kidneys, $R$, lie in the dorsal part of the lumbar region of the abdominal 
cavity, one on each side of the middle line. Each is a solid mass, with a convex outer and a concave inner border, and its upper end a little larger than the lower. From the abdominal aorta, A, a renal artery, $A r$, enters the inner border of each kidney, to break up within it into finer branches, ultimately ending in capillaries. The blood is collected from these into the renal veins, $V r$, one of which leaves each kidney and opens into the inferior vena cava, $V c$. From the concave border of each kidney proceeds also the ureter, $U$, a slender tube from 28 to $34 \mathrm{~cm}$. (11 to 13.5 inches) long, opening below into the bladder, $V u$, on its dorsal aspect, and near its lower end. From the bladder proceeds the urethra, at $U a$. The channel of each ureter passes very obliquely through the wall of the bladder to open into it; accordingly if the pressure inside the latter organ rises above that of the liquid in the ureter, the walls of the oblique passage are pressed together and it is closed. Usually the bladder, which has a thick coat of unstriped muscular tissue lined by a mucous membrane, is relaxed, and the urine flows readily into it from the ureters. While urine is collecting, the beginning of the urethra is kept closed, in part at least, by bands of elastic tissue around it: some of the muscles which surround the commencement of the urethra assist, being kept in reflex contraction; it is found that in a dog the urinary bladder can retain liquid under considerably higher pressure when the spinal cord is intact than after destruction of its lumbar portion. The contraction of these urethra constricting muscles can be reinforced voluntarily. When some amount of urine has accumulated in the bladder, it contracts and presses on its content; the ureters being closed in the way above indicated, the elastic fibers closing the urethral exit are overcome, and the urethral muscles simultaneously relaxing, the liquid is forced out.

Naked Eye Structure of the Kidneys. These organs have externally a red-brown color, which can be seen through the transparent capsule of peritoneum which envelops them. When a section is carried through a kidney from its outer to its inner border (Fig. 139) it is seen that a deep fissure, the hilus, leads into the latter. In the hilus the ureter widens out to form the pelvis, $D$, which breaks up again into a number of smaller divisions, the cups or calices. The cut surface of the kidney proper is seen to consist of two distinct parts: an outer or cortical portion, and an 
inner or medullary. The medullary portion is less red and more glistening to the eye, is finely striated in a radial direction, and loes not consist of one continuous mass but of a number of conical portions, the pyramids of Malpighi, 2', each of which is separated from its neighbors by an inward prolongation, 4, of the

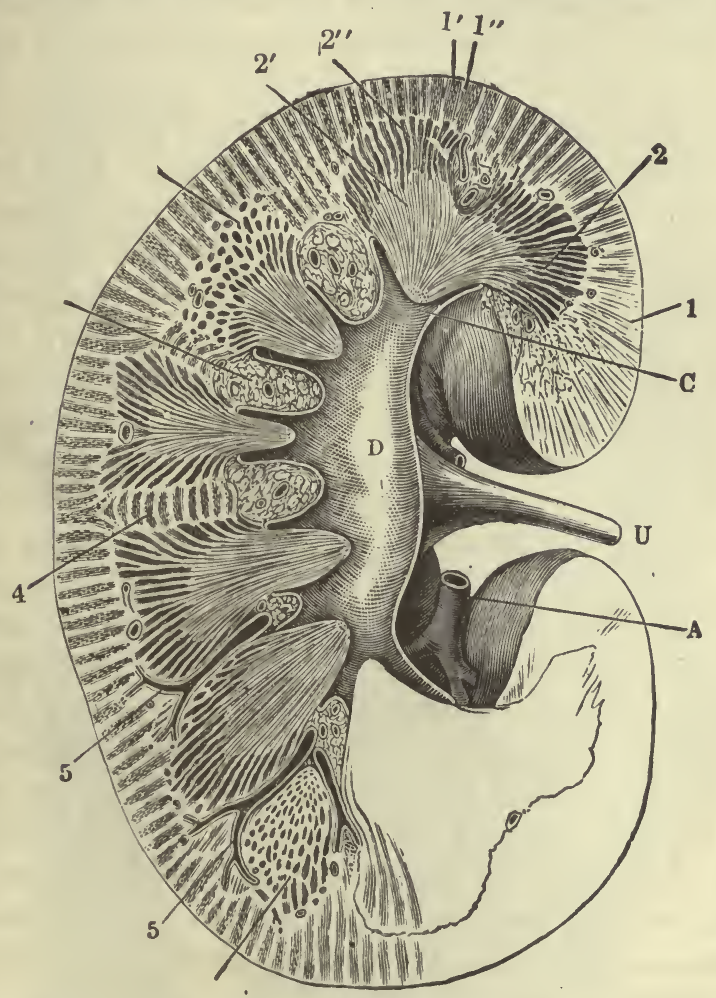

Fig. 139.-Seetion through the right kidney from its outer to its inner border, 1 , cortex; 2 , medulla; $2^{\prime}$, pyramid of Malpighi; $2^{\prime \prime}$, pyramid of Ferrein; 5 , small branches of the renal artery entering between the pyramids; $A$, a branch of the renal artery; $D$, the pelvis of the kidney; $U$, ureter; $C$, a calyx.

cortical substance: this, however, does not reach to the inner end of the pyramid, which projects, as the papilla, into a calyx of the ureter. At its outer end each pyramid separates into smaller portions, the pyramids of Ferrein, $2^{\prime \prime}$, separated by thin layers of cortex and gradually spreading everywhere into the latter. The cortical substance is redder and more granular looking and less 
shiny than the medullary, and forms everywhere the outer layer of the organ next its capsule, besides dipping in between the pyramids in the way described.

The renal artery divides in the hilus into branches (5) which run into the kidney between the pyramids, giving off a few twigs to the latter and ending finally in a much richer vascular network in the cortex. The branches of the renal vein have a similar course.

The Minute Structure of the Kidney. The kidneys are compound tubular glands, composed essentially of branched microscopic uriniferous tubules, lined by epithelium. Each tubule commences at a small opening on a papilla and from thence has a very complex course to its other extremity: usually about twenty open, side by side, on one papilla, where they have a diameter of about $0.125 \mathrm{~mm}$. ( $\frac{1}{200}$ inch). Running from this place into the pyramid each tubule divides repeatedly; the ultimate branches, which are the secreting tubules, pursue a tortuous course (Fig. 140) to terminations in the cortex of the kidney in peculiar spherical dilatations, the Malpighian capsules, each containing a tuft of capillaries, the glomerulus (Fig. 141). Throughout its course the tubule is lined by a single layer of epithelium cells differing in character in its different sections: they are flat and clear in the capsules, and very granular in the convoluted parts, where their appearance suggests that they are not mere lining cells but cells with active work to do; in the collecting and discharging tubules they are somewhat cuboidal in form and have no active secretory function. All the tubes are bound together by a sparse amount of connective tissue and by blood-vessels to form the gland. The lymph-spaces are large and numerous, especially about the convoluted portions of the tubules.

The Blood-Flow Through the Kidney. The amount of blood brought to the kidney is large relatively to the size of the organ and enters under a very high pressure almost direct from the aorta, and leaves under a very low, into the inferior cava (Fig. 138). The final twigs of the renal artery in the cortex, giving off a few branches which end in a capillary network around the convoluted tubules and in the pyramids, are continued as the afferent vessels of Malpighian capsules, the walls of which are doubled in before them (Fig. 141); there each breaks up into a little knot of 


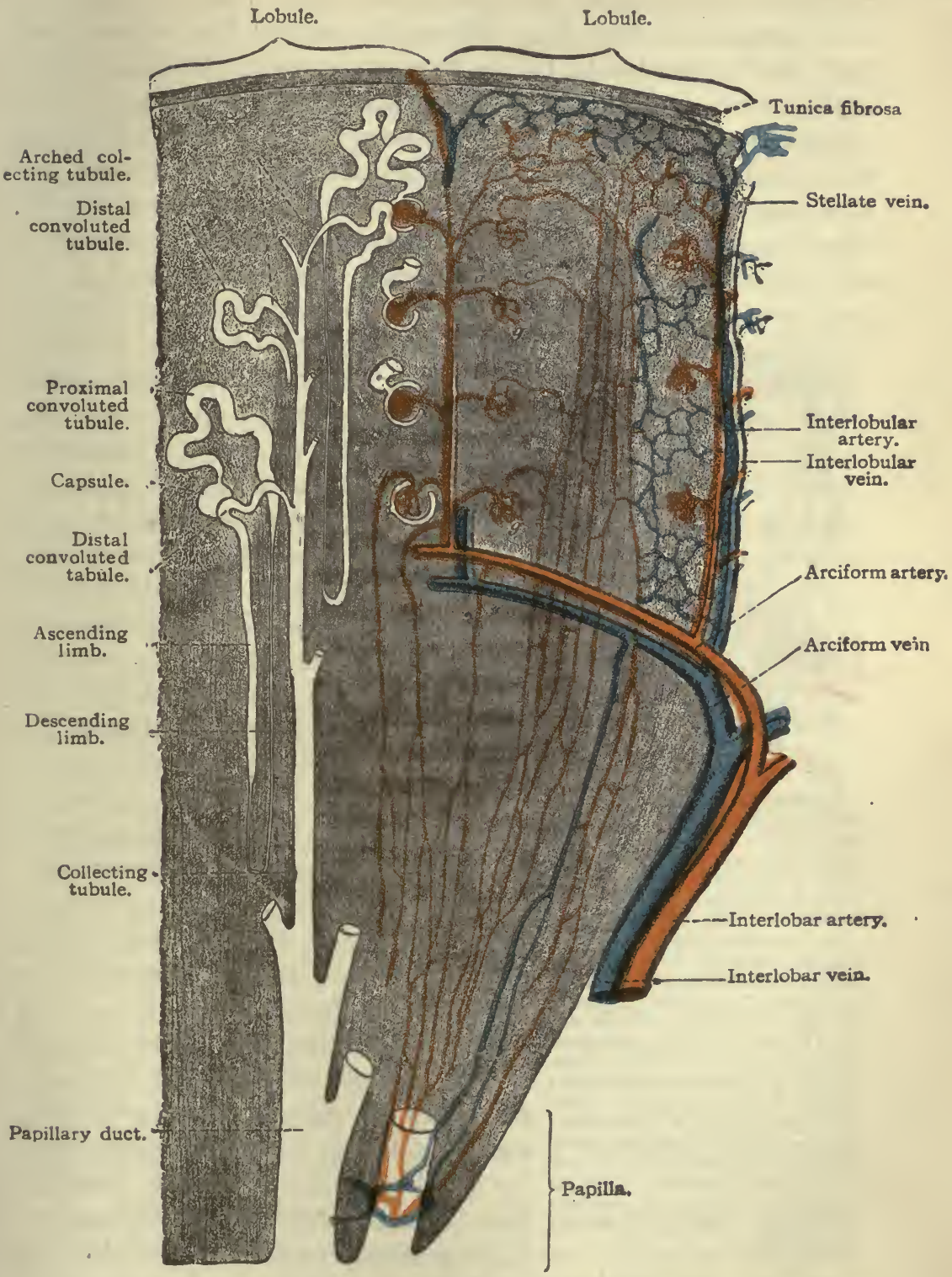

Fig. 140.-Diagram of kidney tubule and renal blood-vessels (Lewis and Stöhr). 
capillary vessels called the glomerulus, from which ultimately an efferent vessel proceeds. Where the wall of the capsule, $w$, Fig. 141, is doubled in before the blood-vessels, its lining cells continue as a covering, $c$, to the latter, closely adhering to the vascular walls. A space, $A$, is left between the epithelial cells of the outside of the capsulc and those involuted on the vessels, as there would be in the interior of a rubber ball one side of which was pushed in so as to nearly meet the other; this cleft, into which any liquid transuded from the vessels must enter, opens by a narrow neck, $d$, into the commencement of the first contorted

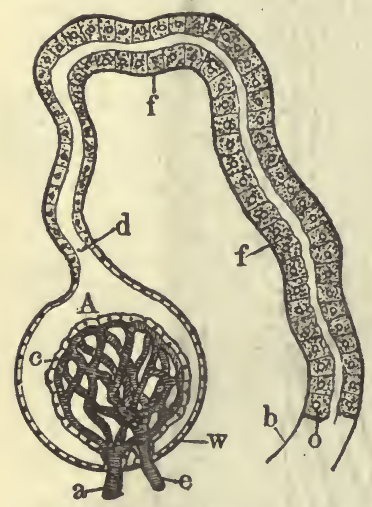

FIg. 141.-Diagram showing a kidney glomerulus and the commencement of an uriniferous tubule. $a$, afferent bloodvessel pushing in the wall, $w$, of a Malpighian capsule and ending in the capillary tuft from which the vein $e$ issues; $c$, involuted epithelium covering the vascular tuft; for the sake of distinctness it is represented as a general wräpping for the whole tuft, but in nature it forms a close investment around each vessel of the glomerulus; $A$, space in capsule into which liquid transuded from the vessels of the glomerulus passes; $d$, neck of capsule passing into commencement of first convoluted portion, $f f$, of an uriniferous tubule; $o$, granular epithelial cells; $b$, basement membrane.

skin excretion. Its average daily quantity varies from 1,200 to

1,750 cub. cent. (40 to 60 fluid ounces). The urine is a clear amber-

part of an uriniferous tubule. The efferent vein, carrying blood away from the glomerulus, breaks up into a close capillary network around the neighboring tubules of the cortex (Fig. 140). From these capillaries the blood is collected into the renal vein. Most of the blood flowing through the kidney thus goes through two sets of capillaries; one found in the capsules, and the second formed by the breaking up of their efferent veins. The capillary network in the pyramids is much less close than that in the cortex, which gives reason to suspect that most of the secretory work of the kidneys is done in the capsules and convoluted tubules. The pyramidal blood flows only through one set of capillaries, there being no glomeruli in the kidney medulla.

The Renal Excretion. The amount of this carried off from the Body in 24 hours is subject to considerable variation, being especially diminished by anything which promotes perspiration, and increased by conditions, as cold to the surface, which diminish the 
colored liquid, of a slightly acid reaction; its specific gravity is about 1,022, being higher when the total quantity excreted is small than when it is greater, since the amount of solids dissolved in it remains nearly the same in health; the changes in its bulk being dependent mainly on changes in the amount of water separated from the blood by the kidneys.

Normal Urine consists of about 96 per cent water and 4 per cent dissolved solids. Chemically it is a very complex liquid, the 4 per cent of dissolved materials including a large variety of different substances. This is to be expected when, we recall that the kidney is the excretory channel, not only for the chief part of the endogenous excreta, but also for virtually all the exogenous waste materials that are absorbed into the blood-stream. Among these latter are found the substances that lend flavor to our food; likewise most drugs that are taken find their way ultimately into the urine. One group of exogenous urinary substances, the ethereal sulphates, are interesting since they are derived from compounds formed in the large intestine in the course of the putrefactive processes which normally go on there; these compounds are absorbed into the blood-stream and are excreted by the kidney. The extent of their occurrence in the urine measures the amount of putrefaction in the large intestine. These substances are toxic if present in quantity and it may be that the ill feeling which often accompanies constipation is the result of their presence in considerable concentration in the blood.

Urea is the constituent of urine most abundant next to the water. About two per cent of urine, half of all the dissolved materials, is urea. The greater part of this is of exogenous origin, being formed in the liver from the ammonia residues of fuelprotein. The amount of exogenous urea varies from time to time according as the amount of protcin undergoing absorption varies. It is thought that a certain amount of endogenous urea is produced during the course of cell metabolism. How much of the total urea of the excretion is of this origin cannot be told.

Creatinin. In some respects the most interesting of the endogenous excreta found in the urine is the compound creatinin. This substance, as stated in Chap. I, is excreted during health at a rate which is practically constant for a given individual, and 
which appears to be determined chiefly by the amount of muscle. tissue present in the Body. The conclusion with regard to creatinin which has been drawn from these facts is that it is a product of the life of muscles as distinct from their special function. In other words, the muscle in doing its work uses up sugar and produces carbon dioxid and water, but in living it uses up protein and produces, among other things, creatinin. Since the amount of creatinin is constant, regardless of the extent to which the muscles are used, unless they are used to excess, it is believed that muscle-cells, and perhaps other cells as well, live at a rate which varies scarcely at all from day to day, and is independent of their functional activity. The interesting observation that the amount of creatinin excreted is roughly proportional to the bulk of the muscle tissues may be taken to indicate that all muscle-cells live at about the same rate, the temperamental differences noted in different individuals not involving differences in the metabolic activities of their muscle-tissues.

The Purin Bodies, of which uric acid is the best known, are other endogenous excreta found in urine. They show chemical characteristics which indicate that they represent probably the end products of the metabolism of cell nuclei. Caffein, the active principle of coffee and tea, and theobromin, the active principle of cocoa, are very closely related chemically to the purin bodies excreted from the kidney.

Since all the endogenous excreta are produced in the living tissues they occur in the flesh of animals eaten for food. In fact the flavor of meat is largely the result of their presence. When eaten with meat they are, of course, absorbed into the blood from the intestine and become part of the exogenous excreta. For this reason it is often necessary, when studying metabolism experimentally, to exclude meat from the diet, so that the endogenous excreta may be obtained pure.

The Urinary Salts are chiefly sodium chlorid, and the sulphates and acid phosphates of sodium, potassium, calcium, and magnesium. Whatever salt is taken with the food, unless stored permanently in the Body, as in bone formation, finally is excreted.by the kidneys. The acid phosphates of sodium and potassium are in part responsible for the acid reaction of urine.

In various diseases abnormal substances are found in the urine: 
the more important are albumens in albuminuria or nephritis; grape sugar or glucose in diabetes; bile salts; bile pigments.

The Secretory Actions of Different Parts of a Uriniferous Tubule. The microscopic structure of the kidneys is such as to suggest that in those organs we have to do with two essentially distinct secretory apparatuses: one represented by the glomeruli, with their capillaries separated only by a single laver of flat epithelial cells from the cavity of the capsule and especially adapted for filtration and dialysis; the other represented by the contorted portions of the tubules, with their large granular cells, which clearly have some more active part to play than that of a mere passive transudation membrane. And we find in the urine substances which like the water and mineral salts may easily be accounted for by mere physical processes, and others, urea especially, which are present in such proportion as must be due to some active physiological work of the kidney. More direct evidence does, in fact, justify us in saying that in general the glomeruli are transudation organs, the contorted portions of the tubuli secretory organs, while the collecting and discharging tubules are merely passive channels for the gathering and transmission of liquid. In calling the capsules transudation organs we do not intend to assert that the passage of water and salts through them is necessarily a physical process pure and simple. Although many physiologists have supposed it to be nothing more, there is abundant evidence that the cells of the capsule exercise a controlling function over the passage of the salts through them if not of the water.

Several lines of evidence indicate that the organic constituents of urine are excreted through the secretory portions of the tubules. One of the best of these has come from work on frogs. Urea, the most important and most abundant of the characteristic ingredients of urine, has a very marked influence on kidney activity, the injection of some of it into blood causing a greatly increased secretion of urine, in which the injected urea is quickly passed out. In amphibia the blood carried to the kidney, like that supplying the mammalian liver, has two sources, one venous and one arterial; the arterial supply comes from the renal arteries, the venous from the veins of the leg by the reniportal vein. Both bloods leave the organ by the renal veins, but their distribution in it is in great part distinct; the arteries supply the glomeruli, 
the reniportal vein the tubules of the cortex, though mixed there with blood from the efferent vessels of the glomeruli. On tying the renal arteries of one of these animals urinary secretion ceases, there being then no blood-pressure in the glomeruli to cause the transudation of liquid; but if some urea be now injected into the blood the epithelial cells of the tubules are stimulated to secrete, and urine rich in urea is formed; but in these circumstances it cannot come from the Malpighian bodies. It would seem then that urea is a special stimulant to some cells of the tubules, and that an excess of it in the blood can stir them up to its elimination along with some water, quite independently of any formation of transudation urine.

The Relation of Renal Blood-Flow to the Secretion of Urine. The kidneys have probably a richer blood supply than any other organs of the Body. It has been estimated that under proper circumstances their own weight of blood may flow through them each minute. This rich blood supply is, of course, an adaptation to secure the withdrawal of waste substances from the blood at a rapid rate. From the structure of the glomeruli and the fact that most of the water of the urine is derived from them it is a priori probable that anything tending to increase the pressure of blood in them will increase the bulk of urine secreted, and anything diminishing that pressure will decrease the urine. The structure of the glomeruli themselves is such that the pressure of blood with them tends to be higher than in the capillaries in general. Reference to Figure 141 shows that the vessel which drains the glomerulus, the efferent vessel $e$, is smaller than the afferent vessel $a$. This means that there is a resistance to the outflow from the capsule greater than that at the point of entrance. According to the relation between pressure and resistance (p. 364) there must be a correspondingly greater pressure in the glomeruli than in the other capillaries whose outlet is not similarly restricted. This high glomerular pressure favors filtration. Experiment shows, moreover, that the vigor of urine formation depends on the pressure of blood within the capsule. The kidney is supplied with both vasoconstrictor and vasodilator nerves which reach it mainly through the solar plexus. When the spinal cord is cut in the neck region of a dog the kidney vessels as well as those of the rest of its Body dilate and blood-pressure everywhere is very low. Under these 
circumstances the secretion of urine is suppressed. If the lower end of the cut cord be stimulated the vessels all over the Body of the animal contract, and blood-pressure everywhere becomes very high. But the kidney vessels being constricted with the rest allow very little blood to enter the glomeruli in spite of the high aortic pressure, and little or no urine is secreted. If, however, the vasoconstrictor nerves of the kidney be cut before the stimulation of the cord, we get a dilation of the kidney vessels. with a constriction of vessels elsewhere, and abundant blood flows through the glomeruli under high pressure: the whole kidney swells and abundant urine is formed. When the skin vessels contract on exposure to cold, more blood flows through internal organs, the kidneys included, and the blood-pressure in these is if anything increased, the expansion of internal arteries not at the most more than counterbalancing the constriction of the cutaneous. Hence the greater secretion of urine in cold weather.

Diuretics. Various substances, caffein, digitalis, urea, salts, and even water, stimulate the kidney to increased activity. Substances which have this effect are known as diuretics. It appears that these act for the most part by stimulating the secreting cells of the tubules to greater activity.

The Skin, which covers the whole exterior of the Body, consists everywhere of two distinct layers; an outer, the cuticle or epidermis, and a deeper, the dermis, cutis vera, or corium. A blister is due to the accumulation of liquid between these two layers. The hairs and nails are excessively developed parts of the epidermis.

The Epidermis, Fig. 142, consists of cells, arranged in many layers, and united by a small amount of cementing substance. The deepest layer, $d$, is composed of elongated or columnar cells, set on with their long axes perpendicular to the corium beneath. To it succeed several layers of roundish cells, $b$, the deepest of which, prickle-cells, are covered by minute processes (not indicated in the figure) which do not interlock but join end to end so as to leave narrow spaces between the cells; in more external layers the cells become more and more flattened in a plane parallel to the surface. The outermost epidermic stratum is composed of many layers of extremely flattened cells from which the nuclei (conspicuous in the deeper layers) have disappeared. These superficial cells are dead and are constantly being shed from the surface of 
the Body, while their place is taken by new cells, formed in the deeper layers, and pushed up to the surface and flattened in their progress. The change in the form of the cells as they travel outwards is accompanied by chemical changes, and they finally con-

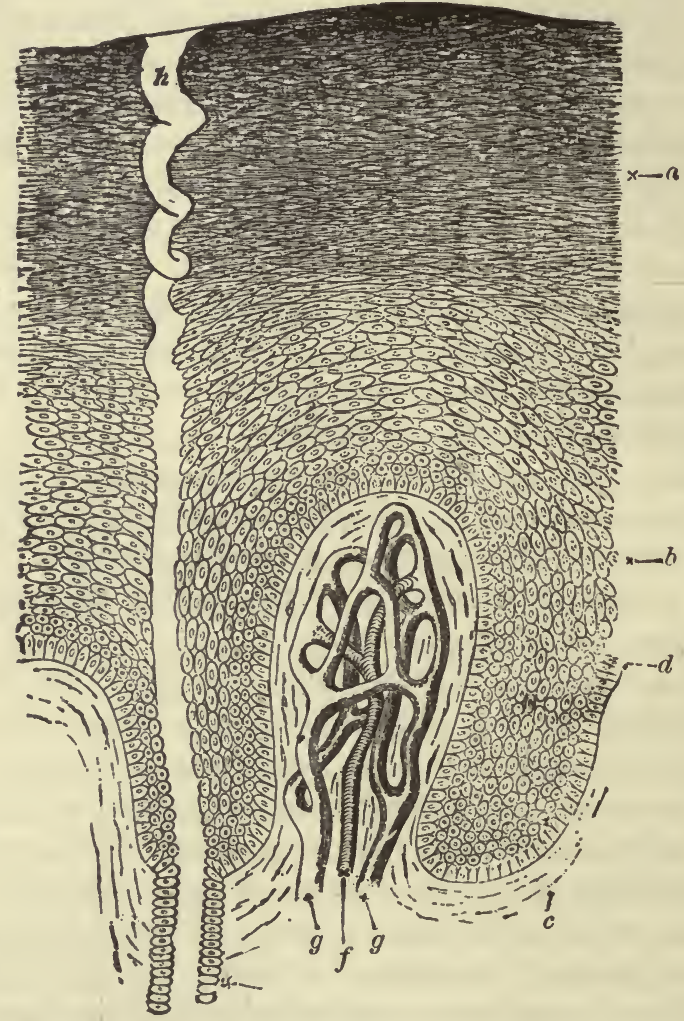

FIg. 142.-A section through the epidermis, somewhat diagrammatic, highly magnified. Below is seen a papilla of the dermis, with its artery, $f$, and veins, $g g$; $a$, the horny layer of the epidermis; $b$, the rete mucosum or Malpighian layer; $d$, the layer of columnar epidermic cells in immediate contact with the dermis; $h$, the duct of a sweat-gland.

stitute a semitransparent dry horny stratum, $a$, distinct from the deeper, more opaque and softer Malpighian or mucous layer, $b$ and $d$, of the epidermis.

The rolls of material which are peeled off the skin in the "shampooing" of the Turkish bath, or by rubbing with a rough towel 
after an ordinary warm bath, are the dead outer scales of the horny stratum of the epidermis.

In dark races the color of the skin depends mainly on minute pigment-granules lying in the cells of the deeper part of the Malpighian layer.

No blood or lymphatic vessels enter the epidermis, which is entirely nourished by matters derived from the subjacent corium. Fine nerve-fibers run into it and end there among the cells.

The Corium, Dermis, or True Skin, Fig. 143, consists fundamentally of a close feltwork of elastic and white fibrous tissue, which, becoming wider meshed below, passes gradually into the subcutaneous areolar tissue (Chap. IV) which attaches the skin loosely to parts beneath. In tanning it is the dermis which is turned into leather, its white fibrous tissue forming an insoluble and tough compound with the tannin of the oak-bark employed.

Wherever there are hairs, bundles of smooth muscular tissue are found in the corium; it contains also a close capillary network and numerous lymphatics and nerves. In shaving, so long as the razor keeps in the epidermis there is no bleeding; but a deeper cut shows at once the vascularity of the true skin.

The outer surface of the corium is almost everywhere raised into minute elevations, called the papillo, on which the epidermis is molded, so that its deep side presents pits corresponding to the projections of the dermis. In Fig. 142 is shown a papilla of the corium containing a knot of blood-vessels, supplied by the small artery, $f$, and having the blood carried off from them by the two little veins, $g g$. Other papillæ contain no capillary loops but special organs connected with nerve-fibers, and supposed to be concerned in the cutaneous senses (Chap. XIII). On the palmar surface of the hand the dermic papillæ are especially well developed (as they are in most parts where the sense of touch is scute) and are frequently compound, or branched at the tip. On the front of the hand, they are arranged in rows; the epidermis fills up the hollows between the papillæ of the same row, but dips down between adjacent rows, and thus are produced the finer ridges seen on the palms. In many places the corium is also furrowed, as opposite the finger-joints and on the palm. Elsewhere such furrows are less marked, but they exist over the whole skin. The epidermis closely follows all the hollows, and thus they are made visible 
from the surface. The wrinkles of old persons are due to the absorption of subcutaneous fat and of other soft parts beneath the skin, which, not shrinking itself at the same rate, is thrown into folds.

Hairs. Each hair is a long filament of epidermis developed on the top of a special dermic papilla, seated at the bottom of a depression reaching down from the skin into the tissue beneath, and

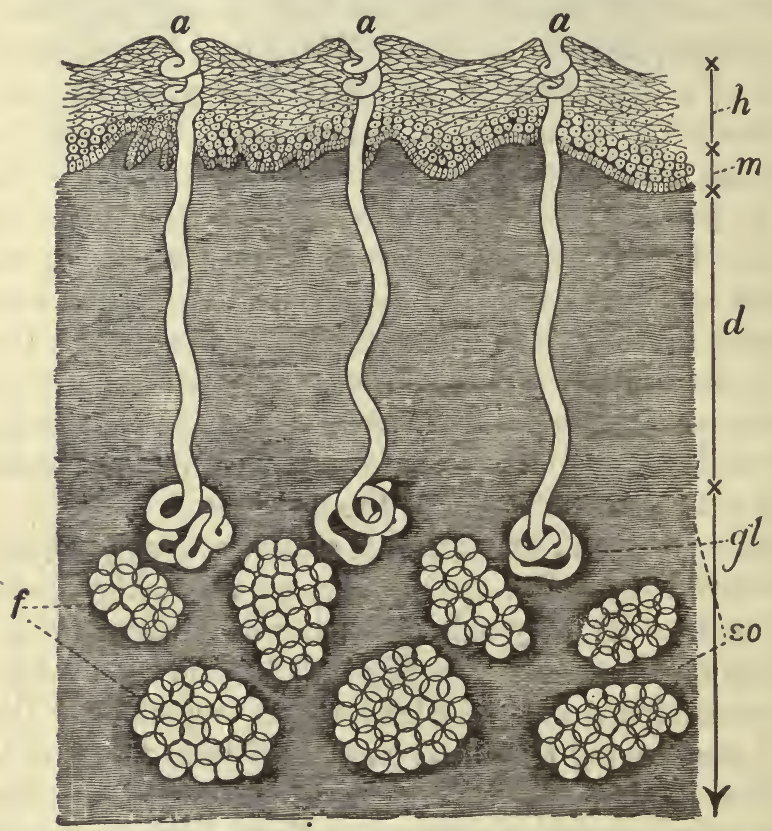

Frg. 143.-A section through the skin and subcutancous areolar tissue. $h$, horny stratum, and $m$, deeper more opaque layer of the epidermis; $d$, dermis passing below into $s c$, loose areolar tissue, with fat, $f$, in its meshes; above, dermic papillæe are seen, projecting into the epidermis which is molded on them. $a$, opening of a sweat-gland; $g l$, the gland itself.

called the hair-follicle. The portion of a hair buried in the skin is called its root; this is succeeded by a stem which, in an uncut hair, tapers off to a point. The stem is covered by a single layer of overlapping scales forming the hair-cuticle; the projecting edges of these scales are directed towards the top of the hair. Beneath the hair-cuticle comes the cortex, made up of greatly elongated cells united to form fibers; and in the center of the shaft there is found, in many hairs; a medulla, made up of more or less rounded cells. 
The color of hair is mainly dependent upon pigment-granules lying between the fibers of the cortex. All hairs contain some air cavities, especially in the medulla. They are very abundant in white hairs and cause the whiteness by reflecting all the incident light, just as a liquid beaten into fine foam looks white because of the light reflected from the walls of all the little air cavities in it. In dark hairs the air cavities are few.

The hair-follicle (Fig. 144) is a narrow pit of the dermis, projecting down into the subcutaneous areolar tissue, and lined by an involution of the epidermis. At the bottom of the follicle is a papilla, and the epidermis, turning up over this, becomes continuous with the hair. On the papilla epidermic cells multiply rapidly so long as the hair is growing, and the whole hair is there made up of roundish cells. As these are pushed up by fresh ones formed beneath them, the outermost layer become flattened and form the hair-cuticle; several succeeding layers elongate and form the cortex; while, in hairs with a medulla, the middle cells retain pretty much their original form and size. Pulled apart by the elongating cortical cells, these central ones then form the medulla with its air-cavities. The innermost layer of the epidermis lining the follicle, has its cells projecting, with overlapping edges turned downwards. Accordingly these interlock with the upward directed edges of the cells of the haircuticle; consequently when a hair is pulled out the epidermic lining of the follicle is usually brought with it. So long as the dermic papilla is left intact a new hair will be formed, but not otherwise. Slender bundles of smooth muscle

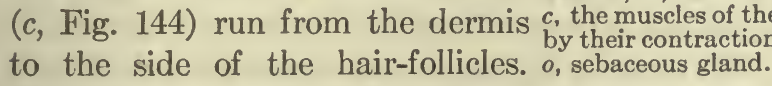

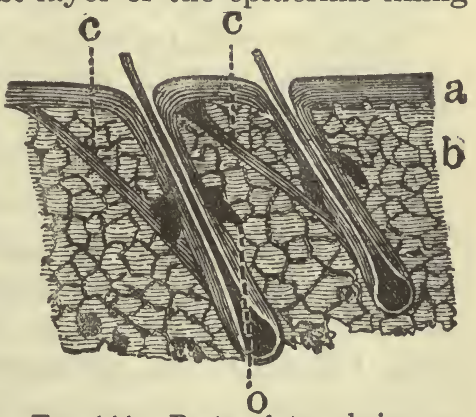

Frg. 144.-Parts of two hairs embedded in their follicles. $a$, the skin, which is seen to dip down and line the follicle; $b$, the subcutaneous tissue; $c$, the muscles of the hair-follicle, which
by their contraction can erect the hair; The latter are in most regions obliquely implanted in the skin so that the hairs lie down on the surface of the Body, and the muscles are so fixed that when they shorten, they erect the hair and cause it to bristle, as may be seen in an angry cat, or sometimes in a greatly terrified man. Opening into each hair-follicle are usually a 
couple of sebaceous or oil-glands. Hairs are found all over the skin except on the palms of the hands and the soles of the feet; the back of the last phalanx of the fingers and toes, the upper eyelids, and one or two other regions.

Nails. Each nail is a part of the epidermis, with its horny stratum greatly developed. The back part of the nail fits behind into a furrow of the dermis and is called its root. The visible part consists of a body, fixed to the dermis beneath (which forms the bed of the nail), and of a free edge. Near the root is a little area whiter than the rest of the nail and called the lunula. The whiteness is due in part to the nail being really more opaque there and partly to the fact that its bed, which seen through the nail causes its pink color, is in this region less vascular.

The portion of the corium on which the nail is formed is called its matrix. Posteriorly this forms a furrow lodging the root, and it is by new cells added on there that the nail grows in length. The part of the matrix lying beneath the body of the nail, and called its bed, is highly vascular and raised up into papillæ which, except in the region of the lunula, are, arranged in longitudinal rows, slightly diverging as they run towards the tip of the finger or toe. It is by new cells formed on its bed and added to its under surface that the nail grows in thickness, as it is pushed forward by the new growth in length at its root. The free end of a nail is therefore its thickest part. If a nail is "cast" in consequence of an injury, or torn off, a new one is produced, provided the matrix is left.

The Glands of the Skin are of two kinds, the sudoriparous or sweat-glands, and the sebaceous or oil-glands. The former belong to the tubular, the latter to the racemose type. The sweat-glands, Fig. 145, lie in the subcutaneous tissue, where they form little globular masses composed of a coiled tube. From the coil a duct (sometimes double) leads to the surface, being usually spirally twisted as it passes through the epidermis. The secreting part of the gland consists of a connective-tissue tube, continuous along the duct with the dermis; within this is a basement membrane; and the final secretory lining consists of several layers of glandcells. A close capillary network intertwines with the coils of the gland. Sweat-glands are found on all regions of the skin, but more closely set in some places, as the palms of the hands and on the brow, than elsewhere: there are altogether about 
two and a half millions of them opening on the surface of the Body.

The sebaceous glands nearly always open into hair-follicles, and are found wherever there are hairs. Each consists of a duct opening near the mouth of a hair-follicle and branching at its other end: the final branches lead into globular secreting saccules, which, like the ducts, are lined with epithelium. In the saccules the substance of the cells becomes charged with oil-drops, the protoplasm disappearing; and finally the whole cell falls to pieces, its detritus constituting the secretion. New cells are, meanwhile, formed to take the place of those destroyed. Usually two glands are connected with each hair-follicle, but there may be three or only one. A pair of sebaceous glands are represented on the sides of each of the hair-follicles in Fig. 142.

The Skin Secretions. The skin besides forming a protective covering and serving as a sense organ (Chap. XIII) also plays an important part in regulating the temperature of the Body, and a less important function as an excretory organ, in carrying off water and traces of other waste products.

The sweat poured out by the sudoriparous glands is a transparent colorless liquid, with a peculiar odor, varying in different races and, in the same individual, in different regions of the Body. Its quantity in twenty-four hours is subject to great variations, but usually lies between 700 and 2,000 grams (10,850 and 31,000 grains). The amount is influenced mainly by the sur-

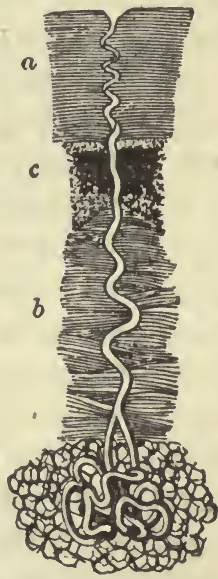

Fig. 145.-A sweatgland. $a$, horny layer of cuticle; $c$, Malpighian layer; $b$, dermis. The coils of the gland proper, embedded in the subcutaneous fat, are seen below the derrounding temperature, being greater when this mis.

is high; but it is also increased by other things tending to raise the temperature of the Body, as muscular exercise. The sweat may or may not evaporate as fast as it is secreted; in the former case it is known as insensible, in the latter as sensible perspiration. By far the most passes off in the insensible form, drops of sweat only accumulating when the secretion is very profuse, or the surrounding atmosphere so humid that it does not readily take up more moisture. The perspiration 
is acid, and in 1,000 parts contains 990 of water to 10 of solids. Among the latter are found urea (1.5 in 1,000), fatty acids, sodium chlorid, and other salts. In diseased conditions of the kidneys the urea may be greatly increased, the skin supplementing to a certain extent deficiencies of those organs.

The Nervous and Circulatory Factors in the Sweat Secretion. It used to be believed that an increased flow of blood through the skin would suffice of itself to cause increased perspiration; but against this view are the facts that, in terror for example, there may be profuse sweating with a cold pallid skin; and that in many febrile states the skin may be hot and its vessels full of blood, and yet there may be no sweating.

Direct experiment shows that the secretory activity of the sweat-glands is under immediate control of nerve-fibers, and is only indirectly dependent on the blood-supply in their neighborhood. Stimuating the sciatic nerve of the freshly amputated leg of a cat will cause the balls of its feet to sweat, although there is no blood flowing through the limb. On the other hand, if the sciatic nerve be cut so as to paralyze it, in a living animal, the skin arteries dilate and the food gets more blood and becomes warmer; but it does not sweat. The sweat-fibers doubtless communicate with sweat-centers in the medulla, which may either be directly excited by blood of a higher temperature than usual flowing through them or, reflexly, by warmth acting on the exterior of the Body and stimulating the sensory nerves there. Both of these agencies commonly also excite the vasodilator nerves of the sweating part, and so the increased blood-supply goes along with the secretion; but the two phenomena are fundamentally independent. Since the sweat-glands are innervated through the autonomic system they share in the emotional reactions which are characteristic of this system. The effect of embarrassment to cause profuse sweating is too well known to require comment.

The Sebaceous Secretion. This is oily, semifluid, and of a special odor. It contains about 50 per cent of fats (olein and palmatin). It lubricates the hairs and usually renders them glossy. No doubt, too, it gets spread more or less over the skin and makes the cuticle less permeable by water. Water poured on a healthy skin does not wet it readily but runs off it, as "off a duck's back" though to a less marked degree. 
Hygiene of the Skin. The sebaceous secretion, and the solid residue left by evaporating sweat, constantly form a solid film over the skin, which must tend to choke the mouths of the sweatglands (the so-called "pores" of the skin) and impede their activity. Hence the value to health of keeping the skin clean: a daily bath should be taken by every one.

Bathing. The general subject of bathing may be considered here. One object of it is that above mentioned-to cleanse the skin; but it is also useful to strengthen and invigorate the whole frame. For strong healthy persons a cold bath is the best, except in extremely severe weather, when the temperature of the water should be raised to $15^{\circ} \mathrm{C}$. (about $60^{\circ} \mathrm{F}$.), at which it still feels quite cold to the surface. The first effect of a cold bath is to contract all the skin-vessels and make the surface pallid. This is soon followed by a reaction, in which the skin becomes red and congested, and a glow of warmth is felt in it. The proper time to come out is while this reaction lasts, and after emersion it should be promoted by a good rub. If the stay in the cold water be too prolonged the state of reaction passes off, the skin becomes cold and pale and the person feels chilly, uncomfortable, and depressed all day. Then bathing is injurious instead of beneficial; it lowers instead of stimulating the activities of the Body. How long a stay in the cold water may be made with benefit depends greatly on the individual: a vigorous man can bear and set up a healthy reaction after much longer immersion than a feeble one; moreover, being used to cold bathing renders a longer stay safe, and, of course, the temperature of the water has a great influence: water called "cold" may vary within very wide limits of temperature, as indicated by the thermometer; and the colder it is the shorter is the time which it is wise to remain in it. Persons who in the comparatively warm water of Narragansett during the summer months stay with benefit and pleasure in the sea, have to content themselves with a single plunge on parts of the coast where the water is colder. The nature of the water has some influence; the salts contained in sea-water stimulate the skin-nerves and promote the afterglow. Many persons who cannot stand a simple cold fresh-water bath take one with benefit when some salines are previously dissolved in the water. The best for this purpose are probably those sold in the shops under the name of "sea-salts." 
It is perfectly safe to bathe when warm, provided the skin is not perspiring profusely, the notion commonly prevalent to the contrary notwithstanding. On the other hand, no one should enter a cold bath when feeling chilly, or in a depressed vital condition. It is not wise to take a bath immediately after a meal, since the afterglow tends to draw away too much blood from the digestive organs, which are then actively at work. The best time for a long bath is about three hours after breakfast; but for an ordinary daily dip, lasting but a short time, there is no better period than on rising and while still warm from bed.

The shower-bath abstracts less heat from the skin than an ordinary cold bath and, at the same time, gives it a greater stimulus: hence it has certain advantages.

Persons in feeble health may diminish the shock to the system by raising the temperature of the water they bathe in up to any point at which it still feels cool to the skin. The very hot bath is occasionally useful as the most efficient means for cleansing the skin. There is no doubt, however, that its effect tends to be enervating, and it should not be indulged in too frequently. 


\section{CHAPTER XXXII}

\section{THE PRODUCTION AND REGULATION OF THE HEAT OF THE BODY}

Cold- and Warm-Blooded Animals. All animals, so long as they are alive, are the seat of chemical changes by which heat is liberated; hence all tend to be somewhat warmer than their ordinary surroundings, though the difference may not be noticeable unless the heat production is considerable. A frog or a fish is a little hotter than the air or water in which it lives, but not much; the little heat that it produces is lost, by radiation or conduction, almost at once. Hence such animals have no proper temperature of their own; on a warm day they are warm, on a cold day cold, and are accordingly known as changeable-temperatured (poikilothermous) or, in ordinary language, "cold-blooded" animals. Man and other mammals, as well as birds, on the contrary, are the seat of very active chemical changes by which much heat is produced, and so maintain a tolerably uniform temperature of their own, much as a fire does whether it be burning in a warm or a cold room; the heat production during any given time balancing the loss, a normal body temperature is maintained, and usually one considerably higher than that of the medium in which they live; such animals are commonly named "warm-blooded." This name, "however, does not properly express the facts; a lizard basking in the sun on a warm summer's day may be quite as hot as a man usually is; but on the cold day the lizard becomes cold, while the average temperature of the healthy Human Body is, within a degree, the same in winter or summer; within the arctic circle or on the equator. Hence it is better to call such animals "homothermous" or of uniform temperature.

Moderate warmth accelerates protoplasmic activity; compare a frog dormant in the winter with the same animal active in the warm months: what is true of the whole frog is true of each of its living cells. Its muscles contract more rapidly when warmed, and the white corpuscles of its blood when heated up to the tem- 
perature of the Human Body are seen (with the microscope) to exhibit much more active amœboid movements than they do at the temperature of frog's blood. In summer a frog or other coldblooded animal uses much more oxygen and evolves much more carbon dioxid than in winter, as shown not only by direct measurements of its gaseous exchanges, but by the fact that in winter a frog can live a long time after its lungs have been removed (being able to breathe sufficiently through its moist skin), while in warm weather it dies of asphyxia very soon after the same loss. The warmer weather puts its tissues in a more active state; and so the amount of work the animal does, and therefore the amount of oxygen it needs, depend to a great extent upon the temperature of the medium in which it is living. With the warm-blooded animal the reverse is the case. Within very wide limits of exposure to heat or cold it maintains its temperature at that at which its tissues live best; accordingly in cold weather it uses more oxygen and sets free more carbon dioxid because it needs a more active internal combustion to compensate for its greater loss of heat to the exterior. And it does not become warmer in warm weather, partly because its oxidations are less than in cold (other things being equal), and partly because of physiological arrangements by which it loses heat faster from its body. In fact the living tissues of a man may be compared to hothouse plants, living in an artificially maintained temperature; but they differ from the plants in the fact that they themselves are the seats of the combustions by which the temperature is kept up. Since, within wide limits, the Human Body retains the same temperature no matter whether it be in cold or warm surroundings, it is clear that it must possess an accurate arrangement for heat regulation; either by controlling the production of heat in it, or the loss of heat from it, or both.

The Temperature of the Body. The parts of the Body are all either in contact with one another directly or, if not, at least indirectly through the blood, which, flowing from part to part, carries heat from warmer to colder regions. Thus, although at one time one group of muscles may especially work, liberating heat, and at other times another, or the muscles may be at rest and the glands the seat of active oxidation, the temperature of the whole Body is kept pretty much the same. The skin, however, 
which is in direct contact with external bodies, usually colder that itself, is cooler than the internal organs; its temperature in health is from $36^{\circ}$ to $37^{\circ} \mathrm{C}$. $\left(96.8-98.5^{\circ} \mathrm{F}\right.$.), being warmer in more protected parts, as the hollow of the armpit. In internal organs, as the liver and brain, the temperature is somewhat higher. In the lungs there is loss of the heat carried out by the expired air and that used up in evaporating the water carried out in the breath, so the blood returned to the heart by the pulmonary veins is slightly colder than that carried from the right side of the heart to the lungs.

The Sources of Animal Heat. Apart from heat received from its surroundings and in hot food and drink, the source of heat in the Body is the oxidation of fuel. The 1,750 Calories which represent the basal metabolism (p. 506) all appear as heat; whenever muscular work is done there is an additional by-product of heat. Moreover, except in those who store surplus fuel as fat, any excess of food consumed is burned, with the production of still more heat.

The Maintenance of a Uniform Temperature. Obviously if the Body is to preserve the same temperature during any period of time the production of heat within it must exactly balance the loss of heat from it during that time. In ourselves this balance is actually maintained within narrow limits of fluctuation throughout healthy life. Only in fevers, or as the result of prolonged exposure to cold, is the balance upset. In fact its preservation is necessary for the continuance of the life of a warm-blooded animal; should the temperature rise above certain limits chemical changes, incompatible with life, occur in the tissues; for example, at about $49^{\circ} \mathrm{C}$. $\left(120^{\circ} \mathrm{F}\right.$.) the muscles begin to become rigid. On the other hand, death ensues if the Body be cooled down to about $19^{\circ} \mathrm{C} .\left(66^{\circ} \mathrm{F}\right.$ 。)

Since we live in an environment of constantly varying temperature a rather delicate adjustment between heat production and heat loss is required.

This adjustment is attained through the interaction of two sorts of regulatory devices, one for controlling the loss of heat from the Body, the other its production in the Body. Both of these are partly voluntary and partly involuntary. As regards heatloss, by far the most important regulating organ is the skin: under ordinary circumstances nearly 90 per cent of the total heat given 
off from the Body in 24 hours goes by the skin ( 73 by radiation and conduction, 14.5 by evaporation). This loss may be controlled:

1. By clothing; we naturally wear more in cold and less in warm weather; the effect of clothes being, of course, not to warm the Body but to diminish the rate at which the heat produced in it is lost.

2. Warmth through reflex vasomotor actions leads to dilation of the skin vessels and cold to contraction. In a warm room the vessels on the surface dilate as shown by its redness, while in a cold atmosphere they contract and the skin becomes pale. But the more blood that flows through the skin the greater will be the heat lost from the surface-and vice versa.

3. Heat induces sweating and cold checks it; the heat appears to act, for the most part, reflexly through afferent cutaneous nervefibers exciting the sweat-centers from which the secretory nerves for the sudoriparous glands arise; it may also act to some extent directly on those centers, as they are thrown into activity, at least in health, as soon as the temperature of the blood flowing through the spinal cord is raised. In fever of course we may have a high temperature with a dry non-sweating skin. The more sweat is poured out, the more heat is used up in evaporating it and the more the Body is cooled.

Of less importance in man, but of great importance in furbearing animals, is the loss of heat through the lungs. In warm weather there is quickened respiration, brought about reflexly through the play of cutaneous sensory impulses of warmth upon the respiratory center. This quickened respiration carries off heat more rapidly both by increasing the amount of air warmed to body temperature in a given time, and by increasing the evaporation of water from the lungs.

Our sensations induce us to add to or diminish the heat in the Body according to circumstances; as by cold or warm baths, and iced or hot drinks.

As regards temperature regulation by modifying. the rate of heat production in the Body, the following points may be noted; on the whole, such regulation is far less important than that brought about by changes in the rate of loss, since the necessary vital work of the Body always necessitates the continuance of 
oxidative processes which liberate a tolerably large quantity of heat. The Body cannot therefore be cooled by diminishing such oxidations; nor, on the other hand, can it be safely warmed by largely increasing them. Still, within certain limits, the heat production may be controlled in several ways:

1. In cold weather there is an increased appetite for protein foods. The increased consumption of proteins leads, through their specific dynamic action (p. 509) to greater oxidative activity, and so to increased heat production.

2. Cold inclines us to voluntary exercise; warmth to muscular idleness; and the more the muscles are worked the more heat is produced in the Body.

3. Cold tends to produce reflex muscular movements, and so increased heat production; as chattering of the teeth and shivering.

4. Certain drugs, as salicylic acid, and perhaps quinine, diminish the heat production of the Body. Their mode of action is still obscure.

On the whole, however, the direct heat-regulating mechanisms of the Human Body itself are not very efficient, especially as protections against excessive cooling. Man needs to supplement them in cold climates by the use of clothing, fuel, and exercise.

Local Temperatures. Although, by the means above described, a wonderfully uniform bodily temperature is maintained, and by the circulating blood all parts are kept at nearly the same warmth, variations in both respects do occur. The arrangements for equalization are not by any means fully efficient. External parts, as the skin, the lungs (which are really external in the sense of being in contact with the air), the mouth, and the nose chambers, are always cooler than internal, and even all parts of the skin have not the same temperature, such hollows as the armpit being warmer than more exposed regions. On the other hand, a secreting gland or a working muscle becomes warmer, for the time, than the rest of the Body, because more heat is liberated in it than is carried off by the blood flowing through. In such organs the venous blood leaving is warmer than the arterial coming to them; while the reverse is the case with parts, like the skin, in which the blood is cooled. An organ colder than the blood is of course warmed by an increase in its circulation, as seen in the local rise of temperature in the skin of the face in blushing. 
Fever. The condition of fever or pyrexia, as an abnormally high temperature is named, could conceivably be brought about by increased heat production, decreased heat loss, or both; or by a greater increase of production than of loss. Direct experiments on animals prove that there is always increased production of heat, in febrile diseases. This is shown by the fact that the animal uses more oxygen and gives off more carbon dioxid in a given time than when in health. It also usually gives off more heat, but not enough to compensate for the increase of oxidative processes going on in its body, and so its temperature rises. The regulating mechanism which in health keeps heat production and heat dissipation proportionate is out of gear. The increased heat production during fever is usually attributed to stimulation of the oxidative processes of the Body by toxins in the blood, but the mechanism of their action is not known. It has been suggested that fever is a protective reaction in that it raises the body temperature above that which is most favorable to the growth of the invading organisms, while at the same time favoring the development of the resisting mechanism of the Body itself.

Clothing. While the majority of other warm-blooded animals have coats of their own, formed of hairs or feathers, over most of man's Body his hairy coating is merely rudimentary and has lost nearly all physiological importance as a protection from cold; except in tropical regions he has to protect himself by artificial garments, which his esthetic sense has led him to utilize also for purposes of adornment. Here, however, we must confine ourselves to clothes from a physiological point of view. In civilized societies every one is required to cover most of his Body with something, and the question is what is the best covering; the answer will vary, of course, with the climatic conditions of the country dwelt in. In warm countries, clothing, in general terms, should allow free radiation or conduction of heat from the surface; in cold it should do the reverse; and in temperate climates, with varying temperatures, it should vary with the season. If the surface of the Body be exposed so that currents of air can freely traverse it much more heat will be carried off (under those usual conditions in which the air is cooler than the skin) than if a stationary layer of air be maintained in contact with the surface. As every one knows, a "draught" cools much faster than air of the same tem- 
perature not in motion. All clothing, therefore, tends to keep up the temperature of the Body by checking the renewal of the layer of air in contact with it. Apart from this, however, clothes fall into two great groups: those which are good, and those which are bad, conductors of heat. The former allow changes in the external temperature to cool or heat rapidly the air stratum in actual contact with the Body, while the latter only permit thesie changes to act more slowly. Of the materials used for clothes, linen is a good conductor; calico not quite so good; and silk, wool, and fur are bad conductors.

Whenever the surface of the Body is suddenly chilled the skinvessels are contracted and those of internal parts reflexly dilated; hence internal organs tend to become congested; this within limits is a protective physiological process, but if excessive it is dangerous since the congested membranes of the nose, throat, and lungs are especially liable to fall victims to the agencies which produce colds, influenza or even pneumonia. When hot, therefore, the most unadvisable thing to do is to sit in a draught, throw off the clothing, or in other ways to strive to get suddenly cooled. Moreover, while in the American summer it is tolerably safe to wear good-conducting garments, and few people take cold then, this is by no means safe in the spring or autumn, when the temperature of the air is apt to vary considerably within the course of a day. A person going out, clad only for a warm morning, may have to return in a very much colder evening; and if his clothes be not such as to prevent a sudden surface chill, will get off lightly if he only "take" one of the colds so prevalent at those seasons. In the great majority of cases, no doubt, he suffers nothing worse, but persons, especially of the female sex; often thus acquire far more serious diseases. When sudden changes of temperature are at all probable, even if the prevailing weather be warm, the trunk of the Body should be always protected by some tolerably closefitting garment of non-conducting material. Those whose skins are irritated by anything but linen should wear immediately outside the under-garments a jacket of silken or woolen material. 


\section{CHAPTER XXXIII}

\section{VOICE AND SPEECH}

Voice consists of sounds produced by the vibrations of two elastic bands, the true vocal cords, placed in the larynx, an upper modified portion of the passage which leads from the pharynx to the lungs. When the vocal cords are put in a certain position, air driven past them sets them in vibration, and they emit a musical note; the lungs and respiratory muscles are, therefore, accessory parts of the vocal apparatus: the strength of the blast produced by them determines the loudness of the voice. The larynx itself is the essential voice-organ: its size primarily determines the pitch of the voice, which is lower the longer the vocal cords; and, hence, shrill in children, and usually higher pitched in women than in men; the male larynx grows rapidly at commencing manhood, causing the change commonly known as the "breaking of the voice." Every voice, while its general pitch is dependent on the length of the vocal cords, has, however, a certain range, within limits which determine whether it shall be soprano, mezzo-soprano, alto, tenor, baritone, or bass. This variety is produced by muscles within the larynx which alter the tension of the vocal cords. Those characters of voice which we express by such phrases as harsh, sweet, or sympathetic, depend on the structure of the vocal cords of the individual; cords which in vibrating emit only harmonic partial tones (Chap. XIV) are pleasant; while those in which inharmonic partials are conspicuous are disagreeable.

The vocal cords alone would produce but feeble sounds; those that they emit are strengthened by sympathetic resonance of the air in the pharynx and mouth, the action of which may be compared to that of the sounding-board of a violin. By movements of throat, soft palate, tongue, cheeks, and lips the sounds emitted from the larynx are altered or supplemented in various ways, and converted into articulate language or speech.

The Larynx lies in front of the neck, beneath the hyoid bone and above the windpipe; in many persons it is prominent, caus- 
ing the projection known as "Adam's apple." It consists of a framework of cartilages, partly joined by true synovial joints and partly bound together by membranes; muscles are added

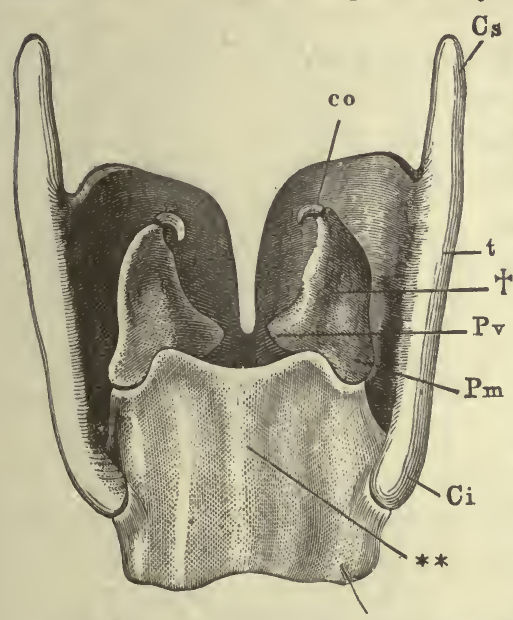

FIG. 146.-The more important cartilages of the larynx from behind. $t$, thyroid; $C s$, its superior, and $C i$, its inferior, horn of the right side; $* *$, cricoid cartilage; $\dagger$, ary tenoid cartilage; $P v$, the corner to which the posterior end of a vocal cord is attached; $\mathrm{Pm}$, corner on which the muscles which approximate or separate of Santorini. the vocal cords are inserted; $c o$, cartilage

which move the cartilages with reference to one another; and the whole is lined by a mucous membrane.

The cartilages of the larynx (Fig. 146) are nine in number; three single and median, and three pairs. The largest $(t)$ is called the thyroid, and consists of two halves which meet at an angle in front, but separate behind so as to inclose a V-shaped space, in which most of the remaining cartilages lie. The epiglottis (not represented in the figure) is fixed to the top of the thyroid cartilage and overhangs the entry from the pharynx to the larynx; it may be seen, covered by mucous membrane, projecting at the base of the tongue, if the latter be pushed down while the mouth is held open in front of a mirror; and is, similarly covered, represented, as seen from behind, at $a$ in Fig. 147. The cricoid, the last of the unpaired cartilages, has the shape of a signet-ring; its broad part (**, Fig. 146) is on the posterior side and lies at the lower part of the opening between the halves of the thyroid; in front and on the sides it is narrow, and a space, occupied by the cricothyroid membrane, intervenes between its upper border and the lower edge of the thyroid cartilage. The angles of the latter are produced above and below into projecting horns ( $\mathrm{Cs}$ and $\mathrm{Ci}$, Fig. 146), and the lower horn on each side forms a joint with the cricoid. The thyroid can be rotated on an axis, passing through the joints on each side, and rolled down so that its lower front edge shall come nearer the cricoid cartilage, the membrane there intervening being folded. The arytenoids ( $\dagger$, Fig. 146) are the 
largest of the paired cartilages; they are seated on the upper edge of the posterior wide portion of the cricoid, and form true joints with it. Each is pyramidal with a triangular base, and has on its tip a small nodule (co, Fig. 146), the cartilage of Santorini. From the tip of each arytenoid cartilage the aryteno-epiglottic fold of mucous membrane (10, Fig. 147) extends to the epiglottis; the cartilage of Santorini causes a projection (8, Fig. 147) in this, and a little farther on (9) is a similar eminence on each side, caused by the remaining pair of cartilages, known as the cuneiform, or cartilages of Wrisberg.

The Vocal Cords are bands of elastic tissue which reach from the inner angle ( $P v$, Fig. 146) of the base of each arytenoid cartilage to the angle on the inside of the thyroid where the sides of the $V$ unite; they thus meet in front but are separated at their other ends. 'The cords are not, however, bare strings, like those of a harp, but covered over with the lining mucous membrane of the larynx, a slit, called the glottis (c, Fig. 147), being left between them. It is the projecting cushions formed by them on each side of this slit which are set in vibration during phonation. Above each

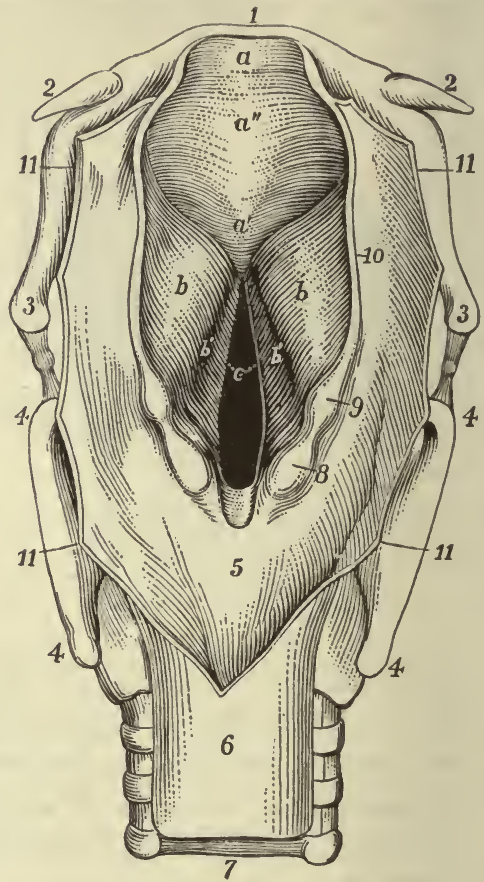

Fig. 147.-The larynx viewed from its pharyngeal opening. The back wall of the pharynx has been divided and its edges (11) turned aside. 1, body of hyoid; 2 , its small, and 3 , its great, horns; 4 , upper and lower horns of thyroid cartilage; 5 , mucous membrane of front of pharynx, covering the back of the cricoid cartilage; 6, upper end of gullet; 7 , windpipe, lying in front of the gullet; 8 , eminence caused by cartilage of Santorini; 9 , eminence caused by cartilage of Wrisberg; both lie in, 10, the arytenoepiglottic fold of mucous membrane, surrounding the opening (aditus laryngis) from pharynx to larynx. $a$, projecting tip of epiglottis; $c$, the glottis, the lines leading from the latter point to the free vibratory edges of the vocal cords. $b^{\prime}$, the ventricles of the larynx; their upper edges, marking them off from the eminences $b$, are the false vocal cords. vocal cord is a depression, the ventricle of the larynx ( $b^{\prime}$, Fig. 147); this is bounded above by a somewhat prominent edge, the false 
vocal cord. Over most of the interior of the larynx its mucous membrane is thick and covered by ciliated epithelium, and has many mucous glands embedded in it. Over the vocal cords, however, it is represented only by a thin layer of flat nonciliated cells, and contains no glands. In quiet breathing, and after death, the free inner edges of the vocal cords are thick and rounded, and seem very unsuitable for being readily set in vibration. They are also tolerably widely separated behind, the arytenoid cartilages, to which their posterior ends are attached, being separated. Air under these conditions passes through without producing voice. If they are watched with the laryngoscope during phonation, it is seen that the cords approximate behind so as to narrow the glottis; at the same time they become more tense, and their inner edges project more sharply and form a better-defined margin to the glottis, and their vibrations can be seen. These changes are brought about by the delicately coördinated activity of a number of small muscles, which move the cartilages to which the cords are fixed.

The Muscles of the Larynx. In describing the direction and action of these it is convenient to use the words front or anterior and back or posterior with reference to the larynx itself (that is, as equivalent to ventral and dorsal) and not with reference to the head, as usual. The base of each arytenoid cartilage is triangular and fits on a surface of the cricoid, on which it can slip to and fro to some extent, the ligaments of the joint being lax. One corner of the triangular base is directed inwards and forwards (i. e., towards the thyroid) and is called the vocal process ( $P v$, Fig. 146), as to it the vocal cords are fixed. The outer posterior angle $(P m$, Fig. 146) has several muscles inserted on it and is called the muscular process. If it be pulled back and towards the middle line the arytenoid cartilage will rotate on its vertical axis, and roll its vocal processes forwards and outwards, and so widen the glottis; the reverse will happen if the muscular process be drawn forwards. The muscle producing the former movement is the posterior crico-arytenoid (Cap, Fig. 148); it arises from the back of the cricoid cartilage, and narrows to its insertion into the muscular process of the arytenoid on the same side. The opponent of this muscle is the lateral crico-arytenoid, which arises from the side of the cricoid cartilage, on its inner surface, and passes up- 
wards and backwards to the muscular process. The posterior crico-arytenoids, working alone, pull inwards and downwards the muscular processes, turn upwards and outwards the vocal processes, and separate the posterior ends of the vocal cords. The lateral cricothyroid, working alone, pulls downwards and forwards the muscular process, and rotates inwards and upwards the vocal process, and narrows the glottis; it is the chief agent in producing the approximation of the cords necessary for the production of voice. When both pairs of muscles act together, however, each neutralizes the tendency of the other to rotate the

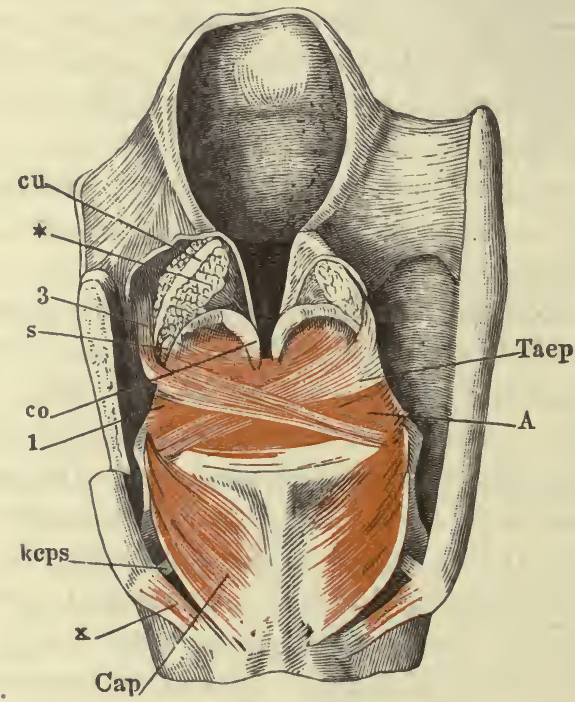

FIG. 148.-The larynx seen from behind and dissected so as to display some of its muscles. The mucous membrane of the front of the pharynx (5, Fig. 146) has been dissected away, so as to display the laryngeal muscles beneath it. Part of the left half of the thyroid cartilage has been cut away. co, cartilage of Santorini; cu, cartilage of Wrisberg.

arytenoid cartilage; the downward part of the pull of each is, thus, alone left, and this causes the arytenoid to slip downwards and outwards, off the eminence on the cricoid with which it articulates, as far as the loose capsular ligament of the joint will allow. The arytenoid cartilages are thus moved apart and the glottis greatly widened and brought into its state in deep quiet breathing. 
Other muscles approximate the arytenoid cartilages after the cartilages have been separated. The most important is the transverse arytenoid ( $A$, Fig. 148), which runs across from one arytenoid cartilage to the other. Another is the oblique arytenoid (Taep), which runs across the middle line from the base of one arytenoid to the tip of the other; thence certain fibers continue in the arytenoepiglottic fold (10, Fig. 147) to the base of the epiglottis; this, with its fellow, embraces the whole entry to the larynx; when they contract they bend inwards the tips of the arytenoid cartilages, approximate the edges of the aryteno-epiglottic fold, and draw down the epiglottis, and so close the passage from the pharynx to the larynx. When the epiglottis has been removed, food and drink rarely enter the larynx in swallowing, the folds of mucous membrane being so brought together as to effectually close the aperture between them.

Increased tension of the vocal cords is produced by the cricothyroid muscles, one of which lies on each side of the larynx, over the cricothyroid membrane. Their action may be understood by help of the diagram, Fig. 149, in which $t$ represents the thyroid

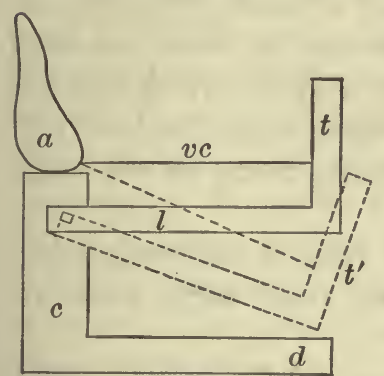

FIG. 149. cartilage, $c$ the cricoid, $a$ an arytenoid, and $v c$ a vocal cord. The muscle passes obliquely backwards and upwards from about $d$ near the front end of $c$, to $t$, about $l$, near the pivot (which represents the joint between the cricoid cartilage and the inferior horn of the thyroid). When the muscle contracts it pulls together the anterior ends of $t$ and $c$; either by depressing the thyroid (as represented by the dotted lines) or by raising the front end of the cricoid; and thus stretches the vocal cord, if the arytenoid cartilages be held from slipping forwards. The antagonist of the cricothyroid is the thyro-arytenoid muscle; it lies, on each side, embedded in the fold of elastic tissue forming the vocal cord, and passes from the inside of the angle of the thyroid cartilage in front, to the anterior angle and front surface of the arytenoid behind. If the latter be held firm, the muscle raises the thyroid cartilage from the position into which the cricothyroid pulls it down, and so slackens the vocal cords. If the thyroid be held fixed by the 
cricothyroid muscle, the thyro-arytenoid will help to approximate the vocal cords, rotating inwards the vocal processes of the arytenoids.

The lengthening of the vocal cords when the thyroid cartilage is depressed tends to lower their pitch; the increased tension, however, more than compensates for this and raises it. There seems, however, still another method by which high notes are produced. Beginning at the bottom of his register, a singer can go on up the scale some distance without a break; but, then, to reach his higher notes, must pause, rearrange his larynx, and begin again. What happens is that, at first, the vocal processes are turned in, so as to approximate but not to meet; the whole length of each edge of the glottis then vibrates, and its tension is increased, and the pitch of the note raised, by increasing contraction of the cricothyroid. At last this attains its limit and a new method has to be adopted. The vocal processes are more rolled in, until they touch. This produces a node (see Physics) at that point and shortens the length of vocal cord which vibrates. The shorter string emits a higher note; so the cricothyroid is relaxed, and then again gradually tightened as the notes sung are raised in pitch from the new starting-point. To pass easily and imperceptibly from one such arrangement of the larynx to another is a great art in singing. There is some reason to believe that a second node may, for still higher notes, be produced at a more anterior point on the vocal cords.

The method of production of falsetto notes is uncertain; during their emission the free border of the vocal cords alone vibrates.

The range of the human voice is about three octaves, from $\boldsymbol{e}\left(80\right.$ vib. per $\left.1^{\prime \prime}\right)$ on the unaccented octave, in male voices, to $c$ on the thrice-accented octave $\left(1,024\right.$ vib. per $\left.1^{\prime \prime}\right)$, in female. Great singers of course go beyond this range; basses have been known to take $a$ on the great octave (55 vib. per $1^{\prime \prime}$ ); and Nilsson in "Il Flauto Magico" used to take $f$ on the fourth accented octave $\left(1,408\right.$ vib. per $\left.1^{\prime \prime}\right)$. Mozart heard at Parma, in 1770, an Italian songstress whose voice had the extraordinary range from $g$ in the first accented octave (198 vib. per $\left.1^{\prime \prime}\right)$ to $c$ on the fifth accented octave $\left(2,112\right.$ vib. per $\left.1^{\prime \prime}\right)$. An ordinary good bass voice has a compass from $f$ (88 vib. 
per $\left.1^{\prime \prime}\right)$ to $d^{\prime \prime}$ (297 vib. per $\left.1^{\prime \prime}\right)$; and a soprano from $b^{\prime}$ (248 vib. per $\left.1^{\prime \prime}\right)$ to $g^{\prime \prime \prime}(792)$.

Vowels are, primarily, compound musical tones produced in the larynx. Accompanying the primary partial of each, which determines its pitch when said or sung, are a number of upper partials, the first five or six being recognizable in good full voices. Certain of these upper partials are reinforced in the mouth to produce one vowel, and others for other vowels; so that the various vowel sounds are really musical notes differing from one another in timbre. The mouth and throat cavities form an airchamber above the larnyx, and this has a note of its own which varies with its size and form, as may be observed by opening the mouth widely, with the lips retracted and the cheeks tense; then gradually closing it and protruding the lips, meanwhile tapping the cheek. As the mouth changes its form the note produced changes, tending in general to pass from a higher to a lower pitch and suggesting to the ear at the same time a change from the sound of $\dot{a}$ (father) through $\overline{0}$ (more) to $\overline{00}$ (moor). When the mouth and throat chambers are so arranged that the air in them has a vibratory rate in unison with any partial in the laryngeal tone, it will be set in sympathetic vibration, that partial will be strengthened, and the vowel characterized by it uttered. As the mouth alters its form, although the same note be still sung, the vowel changes. In the above series $(\dot{a}, \overline{0}, \overline{00})$ the tongue is depressed and the cavity forms one chamber; for $\dot{a}$ this has a wide mouth opening; for $\bar{o}$ it is narrowed; for $\overline{o 0}$ still more narrowed, and the lips protruded so as to increase the length of the resonance chamber. The partial tones reinforced in each case are, according to Helmholtz-

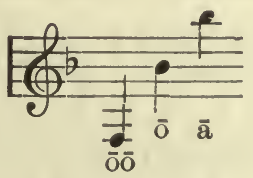

In other cases the mouth and throat cavity is partially subdivided, by elevating the tongue, into a wide posterior and a narrow anterior part, each of which has its own note; and the vowels thus produced owe their character to two reinforced partials. 
This is the case with the series $\breve{a}$ (man), e (there), and $\mathrm{i}$ (machine), the tones reinforced by resonance in the mouth being-

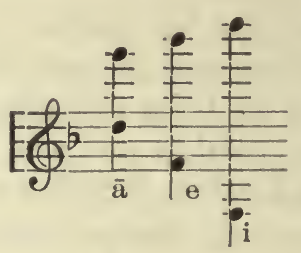

The usual $\bar{i}$ of English, as in spire, is not a true simple vowel but a diphthong, consisting of $\breve{a}$ (pad) followed by $\bar{e}$ (feet), as may be observed by trying to sing a sustained note to the sound $\mathbf{i}$; it will then be seen that it begins as $\breve{a}$ and ends as $\overline{\mathrm{ee}}$., A simple vowel can be maintained pure as long as the breath holds out.

In uttering true vowel sounds the soft palate is raised so as to cut off the air in the nose, which, thus, does not take part in the sympathetic resonance. For some other sounds (the semi-vowels or resonants) the initial step is, as in the case of the true vowels, the production of a laryngeal tone; but the soft palate is not raised, and the mouth exit is more or less closed by the lips or the tongue; hence the blast partly issues through the nose, and the air there takes part in the vibrations and gives them a special character; this is the case with $m, n$, and $n g$.

Consonants are sounds produced not mainly by the vocal cords, but by modifications of the expiratory blast on its way through the mouth. The current may be interrupted and the sound changed by the lips (labials); or, at or near the teeth, by the tip of the tongue (dentals); or, in the throat, by the root of the tongue and the soft palate (gutturals). Consonants are also characterized by the kind of movement which gives rise to them. In explosives an interruption to the passage of the air-current is suddenly interposed or removed (P, T, B, D, K, G). Other consonants are continuous (as F, S, R), and may be subdivided into: (1) Aspirates, characterized by the sound produced by a rush of air through a narrow passage, as when the lips are approximated $(\mathrm{F})$, or the teeth (S), or the tongue is brought near the palate (Sh), or its tip against the two rows of teeth, they not being quite in contact (Th). For $\mathrm{L}$ the tongue is put against the hard palate and the 
air escapes on its sides. For Ch (as in the proper Scotch pronunciation of loch) the passage between the back of the tongue and the soft palate is narrowed. To many of the above pure consonants answer others, in whose production true vocalization (i.e., a laryngeal tone) takes a part. $\mathrm{F}$ with some voice becomes $\mathrm{V}$; $\mathrm{S}$ becomes Z, Th soft (teeth) becomes Th hard; and Ch becomes Gh. (2) Resonants; these have been referred to above. (3) Vibratories (the different forms of $\mathrm{R}$ ), which are due to vibrations of parts bounding a constriction put in the course of the air-current. Ordinary $\mathrm{R}$ is due to vibrations of the tip of the tongue held near the hard palate; and guttural $\mathrm{R}$ to vibrations of the uvula and parts of the pharynx.

The consonants may physiologically be classified as in the following table (Foster):

Explosives. Labials, without voice ....... with voice......... B.

Dentals, without voice ...... T.

" with voice ........ D.

Gutturals, without voice.....K.

" with voice.......G (hard).

Aspirates. Labials, without voice .......F.

" with voice ......... V.

Dentals, without voice ......S, L, Sh, Th (hard).

" with voice ....... Z, Zh (azure), Th (soft),

Gutturals, without voice..... Ch (loch).

" with voice........ Ch.

Resonants. Labial ..................

Dental. .................

Gutteral. . ................

Vibratories. Labial-not used in European languages.

Dental................ (common).

Guttural...............R (guttural).

$\mathrm{H}$ is a laryngeal sound: the vocal cords are separated for its production, yet not so far as in quiet breathing. The air-current then produces a friction sound but not a true note, as it passes the glottis; and this is again modified when the current strikes the wall of the pharynx. Simple sudden closure of the glottis, attended with no sound, is also a speech element, though we do not indicate it with a special letter, since it is always understood when a word begins with a vowel, and only rarely is used at other times. The Greeks had a special sign for it, ', the soft breathing; 
and another, ', the hard breathing, answering somewhat to our $h$ and indicating that the larynx was to be held open, so as to give a friction sound, but not voice.

In whispering there is no true voice; the latter implies true tones, and these are only produced by periodic vibrations; whispering is a noise. To produce it the glottis is considerably narrowed but the cords are not so stretched as to produce a sharply defined edge on them, and the air driven past is then thrown into irregular vibrations. Such vibrations as coincide in period with the air in the mouth and throat are always present in sufficient number to characterize the vowels; and the consonants are produced in the ordinary way, though the distinction between such letters as $\mathrm{P}$ and $\mathrm{B}, \mathrm{F}$ and $\mathrm{V}$, remains imperfect. 


\section{CHAPTER XXXIV}

\section{REPRODUCTION}

Reproduction in General. In all cases reproduction consists, essentially, in the separation of a portion of living matter from a parent; the separated part bearing with it, or inheriting, certain tendencies to repeat, with more or less variation, the life history of its progenitor. In the more simple cases a parent merely divides into two or more pieces, each resembling itself except in size; these then grow and repeat the process; as, for instance, in the case of Amœba and our own white blood corpuscles (p. 19). Such a process may be summed up in two words as discontinuous growth; the mass, instead of increasing in size without segmentation, divides as it grows, and so forms independent living beings. In some tolerably complex multicellular animals we find essentially the same thing; at times certain cells of the freshwater Polyp multiply by simple division in the manner above described, but there is a certain concert between them: they build up a tube projecting from the side of the parent, a mouth-opening forms at the distal end of this, tentacles sprout out around it, and. only when thus completely built up and equipped is the young Hydra set loose on its own career. How closely such a mode of multiplication is allied to mere growth is shown by other polyps in which the young, thus formed, remain permanently attached to the parent stem, so that a compound animal results. This mode of reproduction (known as gemmation or budding) may be compared to the method in which many of the ancient Greek colonies were founded; carefully organized and prepared at home, they were sent out with a due proportion of artificers of various kinds; so that the new commonwealth had from its first separation a considerable division of employments in it, and was, on a small scale, a repetition of the parent community. - In the great majority of animals, however (even those which at times multiply by budding), a different mode of reproduction occurs, one more like that by which our western lands were settled and grad- 
ually built up into Territories and States. The new individual in the political world began with little differentiation; it consisted of units, separated from older and highly organized societies, and these units at first did pretty much everything, each man for himself, with more or less efficiency. As growth took place development also occurred; persons assumed different duties and performed different work until, finally, a fully organized State was formed. Similarly, the body of one of the higher animals is, at an early stage of life, merely a collection of undifferentiated cells, each capable of multiplication by division, and more or less retaining all its original protoplasmic properties; and with no specific individual endowment or function. The mass (Chap. III) then slowly differentiates into the various tissues, each with a predominant character and duty; at the same time the majority of the cells lose their primitive powers of reproduction, though exactly how completely is a problem not yet sufficiently studied. In adult Vertebrates it seems certain that the white blood corpuscles multiply by division: and in some cases (in the newts or tritons, for example) a limb is reproduced after amputation. But exactly what cells take part in such restorative processes is uncertain; we do not know whether or not the old bone corpuscles left form new bones, old muscle-fibers new muscles, and so on. In Mammals no such restoration occurs; an amputated leg may heal at the stump but does not form again. In the healing processes the connective tissues play the main part, as we might expect; their cellular elements being but little modified from their primitive state can still multiply and develop. New bloodcapillaries, however, sprout out from the sides of old, and new epidermis seems only to be formed by the multiplication of epidermic cells; hence the practice, frequently adopted by surgeons, of transplanting little bits of skin to points on the surface of an extensive burn or ulcer. In blood-capillaries and epidermis the departure from the primary undifferentiated cell is but slight; and, as regards the cuticle, one of the permanent physiological characters of the cells of the rete mucosum is their multiplication throughout the whole of life; that is a main physiological characteristic of the tissue: the same is very probably true of the protoplasmic cells forming the walls of the capillaries. When a highly differentiated tissue is replaced in the body of mammals after 
breaking down or removal, it is usually by the activity of special cells set apart for that purpose, or by repair or outgrowth of the cells affected and not by their division. The red blood-corpuscles are constantly being broken down and replaced, but the new ones are not formed by the division of already fully formed corpuscles but by certain special hematoblastic cells retained throughout life in the red marrow of bone. The nervous tissues are highly differentiated and a nerve is often regenerated after division, but this is by outgrowth of the ends of axons still attached to their cells and by secondary formation of a myelin sheath around these, and not by division or multiplication of already existing fibers. A striped muscle when cut across is healed by the formation of a band of connective tissue; after a very long time it is said that true muscular fibers may be found in the cicatrix, but their origin is not known; it is probably not from previously developed musclefibers. On the other hand, the less differentiated unstriated muscle has been observed to be repaired in some cases after injury by true karyokinetic division of previously formed musclecells. Although many gland-cells in the performance of their physiological work are partially broken down and lost in their secretion, and then repaired by the residue of the cell, multiplication by division of fully differentiated gland-cells does not appear to occur, if we except such organs as the testes, the secretion of which consists essentially of cells. An excised portion of a salivary or parotid gland is never regenerated: the wound is repaired by connective tissues.

We find, then, as we ascend in the animal scale a diminishing reproductive power in the tissues generally: with the increasing division of physiological labor, with the changes that fit preeminently for one work, there is a loss of other faculties, and this one among them. The more specialized a tissue the less the reproductive power of its elements, and the most differentiated tissues are either not reproduced at all after injury, or only by the specialization of amœboid cells, and not by a progenitive activity of survivors of the same kind as those destroyed. In none of the higher animals, therefore, do we find multiplication by simple division, or by budding: no one cell, and no group of cells used for the physiological maintenance of the individual, can build up a new complete living being; but 
the continuance of the race is specially provided for by setting apart certain cells which shall have this one property - cells whose duty is to the species and not to any one representative of it-an essentially altruistic element in the otherwise egoistic whole.

Germ-Cells Compared with Tissue-Cells. Those cells which are set apart for the maintenance of the race are called germ-cells to distinguish them from the cells which make up the Body generally and which are designated as somatic cells. Each individual is derived from a single germ-cell, as noted in an earlier chapter (p. 29), by a process of cell multiplication and cell differentiation. The controlling factor in these processes was shown to be the chromatin network of the nucleus, made up of a definite number of chromosomes. An important feature of the difference between germ-cells and somatic cells is that the former contain a much larger amount of chromatin material than do the latter. At the beginning of cell multiplication in the ovum (p. 25) the daughter cells are alike in chromatin content, but a stage is soon reached, very early in some forms, in which many of the daughter cells discharge a part of their chromatin. The part eliminated passes out of the nucleus into the mass of the cell and is dissolved. Those cells in which this occurs are destined to develop into the somatic or general tissues. Those that retain their full complement of chromatin become the germ-cells of the adult organism.

Sexual Reproduction. In some cases, especially among insects, the specialized reproductive cells can develop, each for itself, under suitable conditions, and give rise to new individuals; such a mode of reproduction is called parthenogenesis: but in the majority of cases, and always in the higher animals, this is not so; the fusion of two cells, or of products of two cells, is a necessary preliminary to development. Commonly the coalescing cells differ considerably in size and form, and one takes a more direct share in the developmental processes; this is the egg-cell or ovum; the other is the sperm-cell or spermatozoön. The fusion of the two is known as fertilization. Animals producing both ova and spermatozoa are hermaphrodite; those bearing ova only, female; and those spermatozoa only, male: hermaphroditism is not found in Vertebrates, except in rare and doubtful cases of monstrosity.

Maturation of the Germ-Cells. In the germinal tissues of the sexually mature individual cell multiplication goes on actively 
by the process of mitosis previously described (p. 24). At a certain stage, however, every germ-cell passes through a modified mitosis to fit it for taking part in the reproductive cycle. An essential feature of reproduction in higher forms, as noted in the last paragraph, is fertilization, or the fusion of two germ-cells, male and female. This fusion, by adding the chromosomes of the male cell to those of the female, would double the number in the fertilized egg were not some arrangement provided to avoid it. This arrangement is found in the modified mitosis mentioned above, to which is given the name maturation or ripening of the germ-cell. We will recall from the earlier description of mitosis that the chromatin forms itself into a definite number of chromosomes, and that each chromosome splits lengthwise in such fashion that half of it goes to each daughter cell. Thus the daughter cells are exactly alike so far as chromosome content goes. In the process of maturation the chromosomes do not split lengthwise. Instead half of them pass to one daughter cell and half to the other. Thus we have a reduction of the number of chromosomes to half the original. Moreover the daughter cells of this division are not alike in chromosome content, a fact that is supposed to be highly significant in heredity, since the chromosomes are looked upon as determiners of hereditary traits.

In the formation of sperm the daughter cells of the maturation or reduction division (so called because it reduces the number of chromosomes), are of equal value. Each undergoes an additional mitosis of the ordinary type, so that from each primary spermforming cell four functional spermatozoa are derived. The ovum is the active agent in the reproductive process. Its maturation proceeds in such a fashion that the cell mass as a whole is undisturbed by the changes taking place in the chromatin. The reduction division occurs with the chromosomes of the ovum, precisely as in the primary sperm-cell; but instead of this chromosome division being followed by ordinary cell division, the division is unequal. Most of the cell substance continues as before, retaining half the chromosomes. A very small amount is set apart with the other half of the chromosomes, and serves no useful purpose. This is known as the first polar body. At this stage the ovum and the polar body are comparable to the daughter cells of the reduction division of the primary sperm-forming cell. There is an addi- 
tional mitosis in ovum and polar body just as in the sperm formers. The polar body divides into two of equal size. The division of the ovum is again unequal, and an additional polar body is formed. At the end of maturation of the egg, therefore, we have four cells corresponding to the four sperm, but one of them has retained virtually all the cell substance, and is the functional ovum. Figure 150 is a diagram illustrating the stages of maturation. The ovum and the sperm each contain half the original number of chromosomes. When they fuse in the process of fertilization the full number is restored.

Accessory Reproductive Organs. The organ in which ova are produced is known as the ovary, that forming spermatozoa, as the testis or testicle; but in different groups of animals many addi-

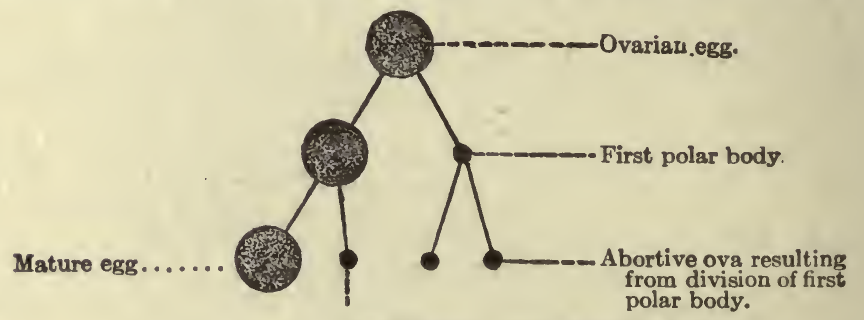

Second polar body (abortive ovum).

Frg. 150.-Diagram showing the genesis of the egg (after Boveri). A similar diagram in which all the daughter cells were of equal size would serve to illustrate the genesis of spermatozoa.

tional accessory parts may be developed. Thus, in all but the very lowest Mammalia, the offspring is nourished for a considerable portion of its early life within the body of its mother, a special cavity, the uterus or womb, being provided for this purpose: the womb communicates with the exterior by a passage, the vagina; and two tubes, the oviducts or Fallopian tubes, convey the eggs to it from the ovaries. In addition, mammary glands provide milk for the nourishment of the young in the first months after birth. In the male mammal we find as accessory reproductive organs, vasa deferentia which convey from the testes the seminal fluid containing spermatozoa; vesicula seminales (not present in all Mammalia), glands whose secretion is mixed with that of the testes or is expelled after it in the sexual act; a prostate gland, whose secretion is added to the semen; and an erectile organ, the penis, by which the fertilizing liquid is conveyed into the vagina of the female. 
The Male Reproductive Organs. The testes in man are paired tubular glands, which lie in a pouch of skin called the scrotum. This pouch is subdivided internally by a partition into right and left chambers, in each of which a testicle lies. The chambers are lined inside by a serous membrane, the tunica vaginalis, and this doubles back (like the pleura round the lung) and covers the exterior of the gland. Between the external and reflected layers of the tunica vaginalis is a space containing a small quantity of lymph.

The testicles develop in the abdominal cavity, and only later (though commonly before birth) descend into the scrotum, passing through apertures in the muscles, etc., of the abdominal wall, and then sliding down over the front of the pubes, beneath the skin. The cavity of the tunica vaginalis at first is a mere offshoot of the peritoneal cavity, and its scrous membrane is originally a part of the peritoneum. In the early years of life the passage along which the testis passes usually becomes nearly closed up, and the communication between the peritoneal cavity and that of the tunica vaginalis is also obliterated. Traces of this passage can, however, readily be observed in male infants; if the skin inside the thigh be tickled a muscle lying beneath the skin of the scrotum is made to contract reflexly, and the testis is jerked up some way towards the abdomen and quite out of the scrotum. Somctimes the passage remains permanently open and a coil of intestine may descend along it and enter the scrotum, constituting an inguinal hernia or rupture. A hydrocele is

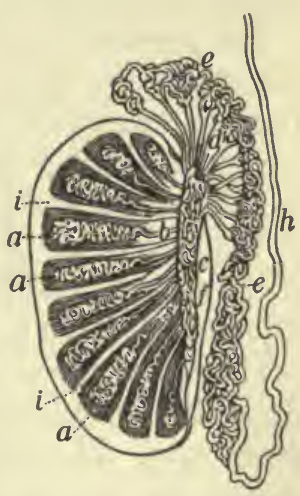

Frg. 151.-Diagram of a vertical section through the testis. $a, a$, tubuli seminiferi; $b$, vasa recta; $d$, vasa efferentia ending in the coni vasculosi; $e, e$, epididymis. $h$, vas deferens.

an excessive accumulation of liquid in the serous cavity of the tunica vaginalis.

Beneath its covering of serous membrane each testis has a proper fibrous tunic of its own. This forms a thick mass on the posterior side of the gland, from which partitions or septa (i, Fig. 151) radiate, subdividing the gland into many chambers. In each chamber lie several greatly coiled seminiferous tubules, $a$, $a$, averaging in length 0.68 meter ( 27 inches) and in diameter only 
$0.14 \mathrm{~mm}$. ( $1 \frac{1}{80}$ inch). Their total number in each gland is about 800. Near the posterior side of the testis the tubules unite to form about 20 vasa recta (b), and these pass out of the gland at its upper end, as the vasa efferentia $(d)$, which become coiled up into conical masses, the coni vasculosi; these, when unrolled, are tubes from 15 to $20 \mathrm{~cm}$. (6-8 inches) in length; they taper somewhat from their commencements at the vasa efferentia, where they are $0.5 \mathrm{~mm}$. ( $\left.{ }_{5}^{1} \mathrm{inch}\right)$ in diameter, to the other end where they terminate in the epididymis ( $e, e$, Fig. 151). The latter is a narrow mass, slightly longer than the testicle, which lies along the posterior side of that organ, near the lower end of which it passes $(g)$ into the vas deferens, $h$. If the epididymis be carefully unravelled it is found to consist of a tube about 6 meters (20 feet) in length, and varying in diameter from 0.35 to $0.25 \mathrm{~mm}$. ( $\frac{1}{80}$ to $\frac{1}{90}$ inch).

The vas deferens ( $h$, Fig. 151) commences at the lower part of the epididymis as a coiled tube, but it soon ceases to be convoluted and passes up beneath the skin covering the inner part of the groin, till it gets above the pelvis and then, passing through the abdominal walls, turns inwards, backwards, and downwards, to the under side of the urinary bladder, where it joins the duct of the seminal vesicle; it is about 0.6 meter ( 2 feet) in length and $2.5 \mathrm{~mm}$. ( $\left.{ }_{10}^{1} \mathrm{inch}\right)$ in diameter. Its lining epithelium is ciliated.

The vesicula seminales, two in number, are membranous receptacles which lie, one on each side, beneath the bladder, between it and the rectum. They are commonly about $5 \mathrm{~cm}$. ( 2 inches) long and a little more than a centimeter wide (or about 0.5 inch) at their broadest part. 'The narrowed end of each enters the vas deferens on its own side, the tube formed by the union being the ejaculatory duct, which, after a course of about an inch, enters the urethra near the neck of the bladder. In some animals the vesicula seminales form a liquid which is added to the secretion of the testis. In man they appear to be merely reservoirs in which the semen collects.

The prostate gland is a dense body, about the size of a large chestnut, which surrounds the commencement of the urethra; the ejaculatory ducts pass through it. It is largely made up of fibrous and unstriped muscular tissues, but contains also a number of small secreting saccules whose ducts open into the urethra. The prostatic secretion though small in amount would appear to be of importance: at least the gland remains undeveloped in per- 
sons who have been castrated in childhood; and atrophies after removal of the testicles later in life.

The male urethra leads from the bladder to the end of the penis, where it terminates in an opening, the meatus urinarius. It is described by anatomists as made up of three portions, the prostatic, the membranous, and the spongy. The first is surrounded by the prostate gland and receives the ejaculatory ducts. On its posterior wall, close to the bladder, is an elevation containing erectile tissues (see below) and supposed to be dilated during sexual congress, so as to cut off the passage to the urinary receptacle. On this crest is an opening leading into a small recess, the utricle, which is of interest, since the study of its embryology shows it to be an undeveloped male uterus. The succeeding membranous portion of the urethra is about $1.8 \mathrm{~cm}$. ( $\frac{3}{4} \mathrm{inch}$ ) long; the spongy portion lies in the penis.

The penis is composed mainly of erectile tissue, $i . e$. , tissues so arranged as to inclose cavities which can be distended by blood. Covered outside by the skin, internally it is made up of three elongated cylindrical masses, two of which, the corpora cavernosa, lie on its anterior side; the third, the corpus spongiosum, surrounds the urethra and lies on the posterior side of the organ for most of its length; it, however, alone forms the terminal dilatation, or glans, of the penis. Each corpus cavernosum is closely united to its fellow in the middle line and extends from the pubic bones, to which it is attached behind, to the glans penis in front. It is enveloped in a dense connective-tissue capsule from which numerous bars, containing white fibrous, elastic, and unstriped muscular tissues, radiate and intersect in all directions, dividing its interior into many irregular chambers called venous sinuses. Into these blood is conveyed partly through open capillaries, partly directly by the open ends of small arteries; this blood is carried off by veins proceeding from the sinuses.

The arteries of the penis are supplied with vasodilator nerves, the nervi erigentes, derived from the sacral plexus. Under certain conditions these are stimulated and, the arteries expanding, blood is poured into the venous sinuses faster than the veins drain it off; the latter are probably also at the same time compressed where they leave the penis by the contraction of certain muscles passing over them. Simultaneously the involuntary muscular 
tissue of the bars ramifying through the erectile masses relaxes. As a result the whole organ becomes distended and finally rigid and erect. The co-ordinating center of erection lies in the lumbar region of the spinal cord, and may be excited reflexly by mechanical stimulation of the penis, or under the influence of nervous impulses originating in the brain and associated with sexual emotions. The corpus spongiosum resembles the corpora cavernosa in essential structure and function.

The skin of the penis is thin and forms a simple layer for some distance; towards the end of the organ it separates and forms a fold, the foreskin or prepuce, which doubles back, and, becoming soft, moist, red, and very vascular, covers the glans to the meatus urinarius, where it becomes continuous with the mucous membrane of the urethra; in it, near the projecting posterior rim of the glans, are embedded many sebaceous glands. It possesses nerve end organs (genital corpuscles) which must resemble end bulbs in structure.

The Seminal Fluid. The essential elements of the testicular secretion are much modified cells, the spermatozoa, which are passed out with some albuminous liquid. The spermatozoa (Fig. 152) are motile bodies about $0.04 \mathrm{~mm} .\left(\frac{1}{00}\right.$ inch) in length.

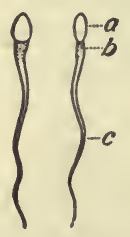

Frg. 152.- Spermatozoa, seen from the front and inside view. $a$, head; $b$, neck; $c$, tail.
They have a flattened clear body or head and a long vibratile tail or cilium; the portion of the tail nearest to the head is thicker than the rest, and is known as the neck. The mode of development of a spermatozoön shows that the head is a cell-nucleus and the neck and tail a modified cellbody.

On cross-section a seminiferous tubule pre-sents externally a well-marked basement membrane, upon which are borne several layers of cells; the lumen or bore of the tubule is in great part occupied by the tails of spermatozoa projecting from some of the lining cells. The outer cells, those next the basement membrane, are arranged in a single layer, and are usually found in one or other stage of active mitosis (p. 24). The result of the division is an outer cell, which remains next the basement membrane to repeat the process, and an inner, which is the mother-cell of spermatozoa. The latter by the process of maturation described in a former 
paragraph (p. 561) gives rise to four cells each of which develops into a functional spermatozoön.

The Reproductive Organs of the Female. Each ovary ( $o$, Fig. 153) is a dense oval mass about $3.25 \mathrm{~cm}$. (1.5 inches) in length, $2 \mathrm{~cm}$. (0.75 inch) in width, and $1.27 \mathrm{~cm}$. $(0.5$ inch) in thickness; it weighs from 4 to 7 grams (60-100 grains). The organs lie in the pelvic cavity enveloped in a fold of peritoneum (the broad ligament), and receive blood-vessels and nerves along one border. From time to time ova reach the surface, burst through the enveloping peritoneum, and are received by the wide fringed aperture, $f i$, of the oviduct or Fallopian tube, od. This tube narrows towards its inner end, where it communicates with the uterus, and is lined by a mucous membrane, covered by ciliated epithelium; plain muscular tissue is also developed in its wall. The uterus ( $u, c$, Fig. 153) is a hollow organ, with relatively thick muscular walls (left unshaded in the figure); it contains the fetus during pregnancy and expels it at birth; it lies in the pelvis between the urinary bladder and the rectum (Fig. 154); the Falopian tubes open into its anterior corners. It is free above, but its lower end is attached to and projects into the vagina. In the fully developed virgin state the organ is somewhat pear-shaped, but flattened from before back; about $7.5 \mathrm{~cm}$. (3 inches) in length, $5 \mathrm{~cm}$. (2 inches) in breadth at its upper widest part, and $2.5 \mathrm{~cm}$. ( 1 inch) in thickness; it weighs from 25 to 42 grams $\left(\frac{7}{8}\right.$ to $1 \frac{1}{2} \mathrm{oz}$.). The upper wider portion of the womb is known as its body; the cavity of this is produced at each side to meet the openings of the Fallopian tubes, and narrows below to the neck, or cervix uteri, opposite $c$ (Fig. 153), the communication between neck and body cavities being known as the os internum. Below this the neck dilates somewhat: it forms no part of the cavity in which the embryo is retained and nourished. The lowest part of the cervix reaches into the vagina and communicates with it by a transverse aperture, the os uteri. During gestation or pregnancy the fetus develops in the body of the womb, which becomes greatly enlarged and rises high into the abdomen: the virgin womb lies mainly below the level of the bones of the pelvis.

The chief bulk of the non-gravid uterus consists of a coat of plain muscular tissue, arranged in a thin outcr longitudinal layer, and an inner, thicker, consisting of oblique and circular fibers. 
Between the layers is an extensive vascular network, with many dilated veins or venous sinuses. The muscular coat is lined internally by a ciliated mucous membrane, and is covered externally by the peritoneum, bands of which project from each side of it as the broad ligaments ( $l l$, Fig. 153). The outer layer of the mucous membrane presents a very well developed muscularis mucosce, much thicker than the corresponding layer in the gastric or intestinal mucous membranes and much less sharply marked off from the true muscular coat outside it. The main thickness of the mucous membrane consists of closely set, simple or slightly branched, tubular glands; between these is a close blood-vascular

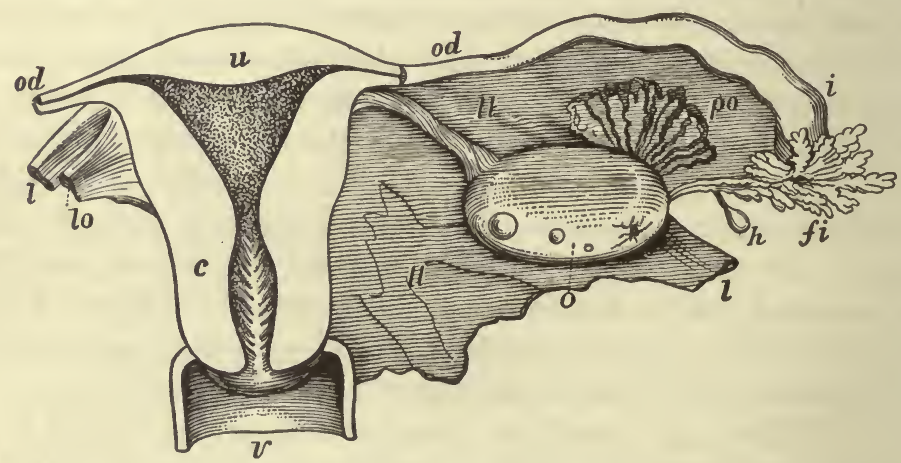

FIG. 153.- The uterus, in section, with the right Fallopian tube and ovary, as seen from behind, about $\frac{2}{3}$ the natural size. $u$, upper part of uterus; $c$, cervix; $v$, upper part of vagina; od, Fallopian tube; $f$, its fimbriated extremity; $o$, ovary; po, parovarium.

and lymphatic network. The glands open on the interior of the womb; they and the mucous membrane between their mouths are lined by a single layer of columnar ciliated cells, with some goblet cells between them. In the cervix the glands are shorter, and many of the epithelial cells not ciliated. The viscid mucus secreted by the uterine glands is alkaline or neutral.

The vagina is a distensible passage, extending from the uterus to the exterior; dorsally it rests on the rectum, and ventrally is in contact with the blaidder and urethra. It is lined by mucous membrane, the epithelium of which is much like the epidermis but thinner; outside the mucous membrane the vagina is made up of areolar, erectile, and unstriped muscular tissues. Around 
its lower end is a ring of striated muscular tissue, the sphincter vaginoe.

The vulva is a general term for all the portions of the female generative organs visible from the exterior. Over the front of the pelvis the skin is elevated by adipose tissue beneath it, and forms the mons Veneris. From this two folds of skin (l, Fig. 154), the labia

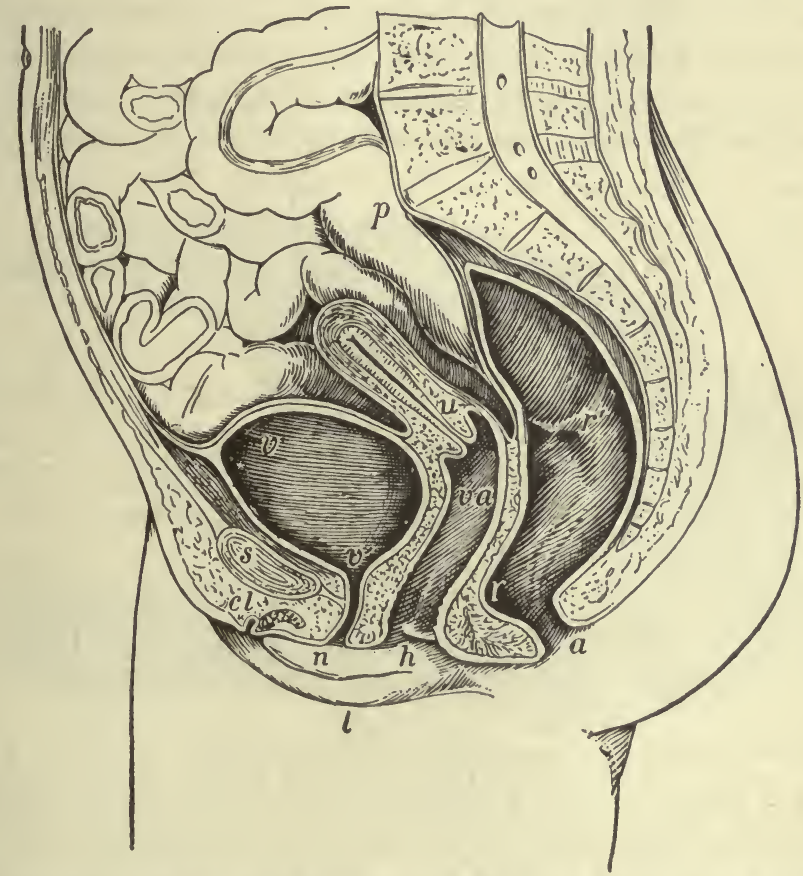

Fig. 154.-The viscera of the female pelvis as exposed by a dorsiventral median section. $s$, symphysis pubis; $v, v^{\prime}$, urinary bladder; $n$, urethra; $u$, uterus; $v a$, vagina; $r, r^{\prime}$, rectum; $a$, anal opening; $l$, right labium major; $n$, right nympha; $h$, hymen; $c l$, divided cilitoris.

majora, extend downwards and backwards on each side of a median cleft, beyond which they again unite. On separating the labia majora a shallow genito-urinary sinus, into which the urethra and vagina open, is exposed. At the upper portion of this sinus lies the clitoris, a small and very sensitive erectile organ, resembling a miniature penis in structure, except that it has no corpus spongiosum and is not traversed by the uretha. From the clitoris descend two folds of mucous membrane, the nymphe or labia interna, 
between which is the vestibule, a recess containing above, the opening of the short female urethra, and, below, the aperture of the vagina, which is in the virgin more or less closed by a thin duplicature of mucous membrane, the hymen.

Microscopic Structure of the Ovary. The main mass of the ovary consists of a dense connective-tissue stroma, containing unstriped muscle, blood-vessels, and nerves: it is covered externally hy a peculiar germinal epithelium, and contains embedded in it many minute cavities, the Graafian follicles, in which ova lie. If a thin section of an ovary be examined with the microscope many

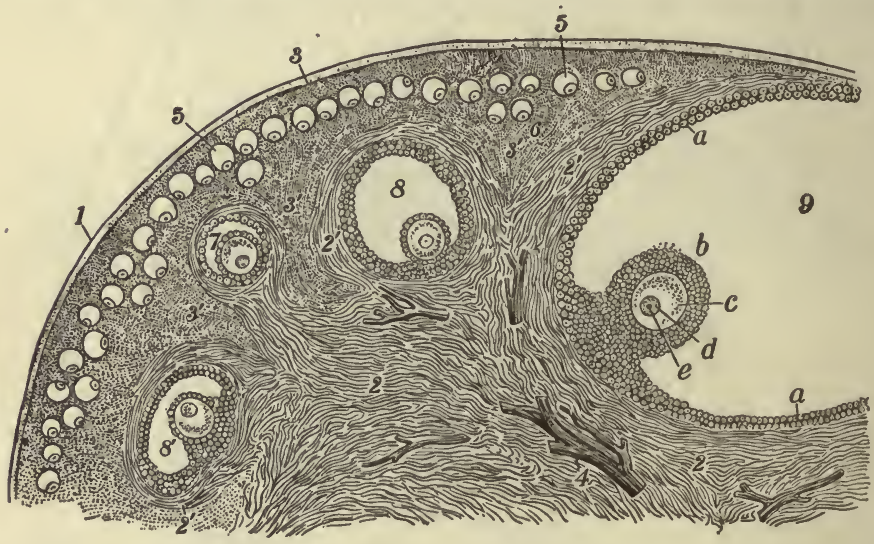

Frg. 155.-A section of a Mammalian ovary, considerably magnified. 1, outer capsule of ovary; $2,3,3^{\prime}$, stroma; 4 , blood-vessels; 5 , rudimentary Graafian follicles; $6,7,8$, follicles beginning to enlarge and mature, and receding from the surface; 9 , a nearly ripe follicle which is extending towards the surface preparatory to discharging the ovum; $a$, membrana granulosa; $b$, discus proligerus; $c$, ovum, with $d$, germinal vesicle, and $e$, germinal spot. The general cavity of the follicle (in which 9 is printed) is filled with lymph-like transudation liquid during life.

hundreds of small Graafian follicles, each about $0.25 \mathrm{~mm}$. $\left(\frac{1}{100}\right.$ inch) in diameter, will be found embedded in it near the surface. These are lined by cells, and each contains a single ovum. In a woman of child-bearing age there will be found also, deeper in, larger follicles (7, 8, 9, Fig. 155), their cavities being distended, during life, by liquid; in these the essential structure may be more readily made out. Each has an external fibrous coat constituted by a dense and vascular layer of the ovarian stroma; within this come several layers of lining cells (9, $a$, Fig. 155) constituting the membrana granulosa. At one point, $b$, the cells of this layer are 
heaped up, forming the discus proligerus, which projects into the liquid filling the cavity of the follicle. Buried among the cells of the discus proligerus the ovum, $c$, lies.

The Mammalian Ovum. As the Graafian follicles enlarge the ova grow but not proportionately, so that they occupy relatively less of the cavities of the larger follicles: the cells of the discus proligerus probably elaborate food for the egg-cell from material derived from the blood-vessels which form a close network around most of each enlarging Graafian follicle and transude crude nutritive matter into the liquid filling most of the follicle. The fully formed ovum (Fig. 156) is about $0.2 \mathrm{~mm}$. ( $\frac{1}{120}$ inch) in diameter: it has a well-marked outer coat or sac, a, the zona radiata, zona pellucida or vitelline membrane, surrounding a very granular cellbody or vitellus, $b$, in which is a conspicuous nucleus, $c$, with its characteristic network of chromatin. The main bulk of the vitellus or yolk consists of highly refracting spheroidal particles of nutritive matter (deutoplasm) embedded in and concealing a true protoplasmic reticulum. In the eggs of birds and reptiles the deutoplasm is in very large amount and forms nearly all the yolk, the protoplasm being for the most part aggregated around the nucleus at a small area on one

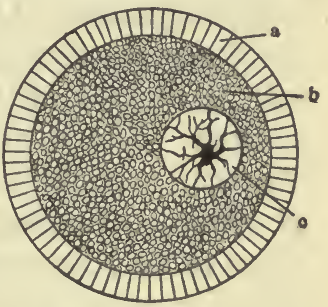

Fig. 156. - A human ovum; somewhat diagrammatic. $a$, zona radiata; $b$, vitellus or yolk; $c$, nucleus. side of the yolk. It is in this area that new cell-formation occurs and the embryo is built up, the rest of the yolk being gradually absorbed by it; such eggs are known as mesoblastic or partly dividing eggs. In all the higher mammalia the deutoplasm is relatively sparse and tolerably evenly mingled with the protoplasm, and the whole fertilized ovum divides to form the first cells of the embryo: such eggs are named holoblastic.

Ovulation. From puberty, during the whole child-bearing period of life, certain comparatively very large Graafian follicles may nearly always be found either close to the surface of the ovary or projecting on its exterior. These, by accumulation of liquid within them, have become distended to a diameter of about $4 \mathrm{~mm}$. ( $\frac{1}{6}$ inch); finally, the thinned projecting portion of the wall of the follicle, which differs from the rest in containing few blood-vessels, gives way and the ovum is discharged, surrounded by some cells of 
the discus proligerus. The emptied follicle becomes filled up with a reddish-yellow mass of cells, and constitutes the corpus luteum, which recedes again to the interior of the ovary and disappears in three or four weeks, unless pregnancy occur; in that case the corpus luteum increases for a time, and persists during the greater part of the gestation period.

The discharged ovum enters the Fallopian tube and passes down it to the uterus. Just how the passage from the ovary to the tube occurs is not clear, although it is suggested that the cilia which line the tube set up by their motion a current sufficient to convey the ovum across the intervening space and into its mouth. Having entered the Fallopian tube the egg slowly passes on to the uterus, moved by the cilia lining the oviduct; its descent probably takes about four or five days; if not fertilized, it dies and is passed out.

Menstruation. Ovulation occurs during the sexual life of a healthy. woman at intervals of about four weeks, and is attended with important changes in other portions of the generative apparatus. The ovaries and Fallopian tubes become congested. The mucous membrane of the uterus at or just before the periods of ovulation becomes swollen and soft, and minute hemorrhages occur in its substance. The superficial layers are broken down, and discharged along with more or less blood, constituting the menses, or monthly sickness, which commonly lasts from three to five days. During this time the vaginal secretion is also increased, and, mixed with the blood discharged, more or less alters its color and usually destroys its coagulating power. Except during pregnancy and while suckling, menstruation occurs at the above intervals, from puberty up to about the forty-fifth year; the periods then become irregular, and finally the discharges cease; this is an indication that ovulation has come to an end, and that the sexual life of the woman is completed. This time, the climacteric or "turn of life," is a critical one; various local disorders are apt to supervene, and even mental derangement.

Hygiene of Menstruation. During menstruation there is apt to be more or less general discomfort and nervous irritability; the woman is not quite herself, and those responsible for her happiness ought to watch and tend her with special solicitude, forbearance, and tenderness, and protect her from anxiety and agitation. Any strong emotion, especially of a disagreeable character, is apt to 
check the flow, and this is always liable to be followed by serious consequences. A sudden chill of ten has the same effect; hence a menstruating woman ought always to be warmly clad, and take more than usual care to avoid draughts or getting wet. At these periods, also, the uterus is enlarged and heavy, and being (as may be seen in Fig. 152) but slightly supported, and that near its lower end, it is especially apt to be displaced or distorted; it may tilt forwards or sideways (versions of the uterus), or be bent where the neck and body of the organ meet (flexion). Hence violent exercise at this time should be avoided, though there is no reason why a properly clad woman should not take her usual daily walk.

The absence of the menstrual flow (amenorrhea) is normal during pregnancy and while suckling; and in some rare cases it never occurs throughout life, even in healthy women capable of childbearing. Usually, however, the non-appearance of the menses at the proper periods is a serious symptom, and one which calls for prompt measures. In all such cases it cannot be too strongly impressed upon women that the most dangerous thing to do is to take drugs tending to induce the discharge, except under skilled advice; to excite the flow, in many cases, as for example occlusion of the os uteri, or in general debility (when its absence is a conservative effort of the system), may have the most disastrous results.

Fertilization. As the ovum descends the Fallopian tube the changes of menstruation are taking place in the uterus. Fertilization usually takes place in a Fallopian tube. The spermatozoa are carried along partly, perhaps, by the contractions of the muscular walls of the female cavities, but mainly by their own activity. Occasionally the ovum is fertilized before reaching the Fallopian tube and fails to enter it, giving rise to an extra-uterine pregnancy.

The actual process of the fertilization of the ovum has only been observed in the lower animals, but there is no doubt that the phenomena are the same in all essentials in all cases. Some of the spermatozoa penetrate the zona radiata and one of them enters the ovum. After the entrance of a single spermatozoan a membrane forms inside the zona radiata (Fig. 156) which prevents others from entering. The head of the spermatozoan, which is chiefly chromatin, becomes separated from the tail; the latter disappears, probably by absorption into the substance of the ovum. The head, now known as the male pronucleus, approaches the chromatin mass 
of the ovum, at this stage called the female pronucleus, and the two fuse into a single fertilization or segmentation nucleus. This process restores to the ovum the typical number of chromosomes.

In addition to the chromatin material the spermatozoan also brings with it the stimulus to cell division, so that immediately after the formation of the fertilization nucleus segmentation begins. In the first and subsequent divisions, which, as stated earlier, are by the process of mitosis, the chromatin is so distributed that each daughter cell receives equal amounts from ovum and sperm.

Heredity. The relative influence of the two parents upon the characteristics of the offspring has been studied and speculated upon for ages. With the discovery of the chromosomes it has become evident that to them we must look largely, if not wholly, for the agency of hereditary transmission. So far as paternal characters impress themselves they must do so through the chromosomes since the sperm contributes virtually nothing else. To the Austrian monk Mendel and the Dutch botanist DeVries we owe the conception of the machinery of heredity which has clarified our ideas on the subject more than all the previous work has done.

According to this conception the chromosomes are to be looked upon as made up of groups of determiners of hereditary traits. If in the union of maternal and paternal chromosomes all the factors are harmonious the offspring will be a perfect blend of the parents. This condition is not realized, however, unless the parents are alike in practically all respects. Thus if one is light haired and the other dark, or if one has blue eyes and the other brown the chromosomes which bear these traits are in conflict. Mendel found that under these circumstances usually one of the conflicting traits appears in the offspring and the other is suppressed. The one which appears is called dominant, the other recessive. More rarely there is a blending of the characters, as seen in the intermediate skin coloration in mulattos. Even though the recessive traits are not apparent in the presence of dominant conflicting characters the chromosomes which determine them persist unchanged, and will be found in the germ-cells. An individual whose germ plasm contains such conflicting chromosomes is known as a hybrid. Experiment has shown that during the development of the germ plasm of hybrids there is a separation of conflicting char- 
acters so that any given germ-cell may contain either the determiners for the dominant character or those for the recessive, but not both. This principle of "the purity of the germ-cell" is the corner stone of Mendelian inheritance. When such hybrids mate it is evident that there are four possible combinations of germ-cells that may occur. If we designate the dominant by $D$ and the recessive by $R$, the maternal germ-cell by $m$ and the paternal by $p$, we may represent the four possible combinations thus $D m+$ $D p ; D m+R p ; R m+D p ; R m+R p$. Of these four the first and last are pure; the second and third are hybrid. Since the dominant character is present in the hybrids they will have the same appearance as number 1 , which is pure dominant. Number 4, however, which is pure recessive, will have the appearance characteristic of the recessive trait. A simple illustration is furnished by eye color. Brown eyes are dominant and blue eyes recessive. According to the principles just stated brown-eyed persons may be pure dominant or hybrid, but all blue-eyed persons are pure recessive. If both parents are blue-eyed all the offspring must therefore be blue-eyed also. If both parents are brown-eyed the eye color of the offspring will depend on whether the parents are pure dominant or hybrid. If one or both are pure dominant all offspring will have brown eyes. If both are hybrid one in four of the offspring may have blue eyes.

The actual situation is complicated by the numerous factors that may be in conflict, but extension of the principle stated above is believed to cover all forms of hereditary transmission that are susceptible of modification by breeding. Whether the fundamental features of inheritance; those that make the offspring of dogs dogs and of roses roses, are also Mendelian; is at present a subject of discussion.

Sex Determination. An interesting application of the principles of Mendel is in the determination of sex. It appears that in general in the germ-cells of males there is one less chromosome than in the cells of females of the same species. Human females, for example, have 48 chromosomes and human males 47 . In the reduction division that occurs in connection with maturation (p. 561) one of the daughter cells that is formed from the division of the primary sperm cell has only 23 chromosomes, while the other has 24. The subsequent division of the daughter cells to 
form sperm preserves the same relation of numbers. Half of the spermatozoa, therefore, will have 23 chromosomes and the other half 24 . Since the female germ-cells contain 48 chromosomes each ovum will have 24 . In the fertilization of the ovum, if the penetrating sperm contains 24 chromosomes the offspring will be female; if only 23 the offspring will be male. Obviously this has little practical bearing on the problem of artificial sex determination, except in so far as it shows the futility of attempting to bring it about. It serves, however, to explain a number of facts of inheritance. For example, in certain species of insects all the fertilized eggs give rise to females; the males being derived from eggs that develop without fertilization. This is explained by the fact that only those spermatozoa that have the full number of chromosomes develop to functional maturity.

Impregnation. The fertilized ovum, which, as we have seen (p. 573), receives the sperm in the Fallopian tube, continues its descent to the uterine cavity, but, instead of lying dormant like the unfertilized, segments (p. 29), and forms a morula. This becomes embedded in the soft, vascular uterine mucous membrane from which it imbibes nourishment, and which, instead of being cast off in subsequent menstrual discharges, is retained and grows during the whole of pregnancy, having important duties to discharge in connection with the nutrition of the embryo.

Sexual congress is most apt to be followed by pregnancy if it occur immediately after a menstrual period; at those times a ripe ovum is usually in the Fallopian tube, near the upper end of which it is probably fertilized in the majority of cases. There is some difference of opinion as to whether the rupture of the Graafian follicle occurs most frequently immediately before the appearance of the menstrual flow, or towards its close; but the preponderance of evidence favors the latter view. The menstrual process probably is a special preparation of the womb for the reception of an embryo and its nourishment. There is, however, evidence that ova are occasionally discharged at other than the regular monthly periods of ovulation and may be fertilized and cause a pregnancy.

Pregnancy. When the mulberry mass reaches the uterine cavity the mucous membrane lining the latter grows rapidly and forms a new, thick, very vascular lining to the womb, known as the decidua. At one point on this the morula becomes attached, the 
decidua growing up around it. As pregnancy advances and the embryo grows, it bulges out into the uterine cavity and pushes before it that part of the decidua which has grown over it (the decidua reflexa); at about the end of the third month this coalesces with the decidua lining the opposite sides of the uterine cavity so that the two can no longer be separated. That part of the decidua (decidua serotina) against which the morula is first attached subsequently undergoes a great development in connection with the formation of the placenta (see below). Meanwhile the whole uterus enlarges; its muscular coat especially thickens. At first the organ still lies within the pelvis, where there is but little room for it; it accordingly presses on the bladder and rectum (see Fig. 152) and the nerves in the neighborhood, frequently causing considerable discomfort or pain; and, reflexly, often exciting nausea or vomiting (the morning sickness of pregnancy). Later on, the pregnant womb escapes higher into the abdominal cavity, and although then larger, the soft abdominal walls more readily make room for it, and less discomfort is usually felt, though there may be shortness of breath and palpitation of the heart from interference with the diaphragmatic movements. All tight garments should at this time be especially avoided; the woman's breathing is already sufficiently impeded, and the pressure may also injure the developing child. Meanwhile, changes occur elsewhere in the Body. The breasts enlarge and hard masses of developing glandular tissue can be felt in them; and there may be mental symptoms: depression, anxiety, and an emotional nervous state.

During the whole period of gestation the woman is not merely supplying from her blood nutriment for the fetus, but also, through her lungs and kidneys, getting rid of its wastes; the result is a strain on her whole system which, it is true, she is constructed to bear and will carry well if in good health, but which is severely felt if she be feeble or suffering from disease. The healthy married woman who endeavors to evade motherhood because she thinks she will thus preserve her personal appearance, or because she dislikes the trouble of a family, deserves but little sympathy; she is trying to escape a duty voluntarily undertaken, and owed to her husband, her country, and her race; but she whose strength is undermined and whose life is made one long discomfort for the sexual gratification of her husband deserves every consideration, and the 
family physician ought perhaps to warn the husband more frequently than he does of the risk to a delicate wife's health, or even life, of frequent pregnancies: and the husband should control himself accordingly.

The Intra-Uterine Nutrition of the Embryo. At first the embryo is nourished by absorption of materials from the soft vascular lining of the womb; as it increases in size this is not sufficient, and a new organ, the placenta, is formed for the purpose. A fetal outgrowth, the allantois, plants itself firmly against the decidua serotina, and villi developed on it burrow from its surface into the uterine mucous membrane. In the deeper layer of this latter are large sinuses through which the maternal blood flows, and into which the allantoic villi project. Blood is brought from the fetus to the allantois by arteries and carried back by veins after traversing the capillaries of the villi, and while flowing through these receives, by dialysis, oxygen and food materials from the maternal blood, and gives up to it carbon dioxid, urea, and other wastes. There is thus no direct intermixture of the two bloods; the embryo is from the first an essentially separate and independent organism. The allantois and decidua serotina becoming inseparably united together form the placenta, which in the human species is, when fully developed, a round thick mass about the size of a large saucer, connected to the embryo by a narrow stalk, the umbilical cord, in which blood-vessels run to and from the placenta.

Parturition. At the end of from 275 to 280 days from fertilization of the ovum (conception) pregnancy terminates, and the child is expelled by powerful contractions of the uterus, assisted by those of the muscles in the abdominal walls. When the child is born, it has attached to its navel the umbilical cord, which is then usually ligatured and cut across: some good authorities, however, maintain that this should not be done until after the contractions which expel the placenta, as.otherwise a quantity of the infant's blood remains in that organ; the loss of which might be serious to a feeble infant. Shortly after the birth of the child renewed uterine contractions detach and expel the placenta, both its fetal or allantoic and maternal or decidual part, as the afterbirth. Where it is torn loose from the uterine wall large blood sinuses are left open; hence a certain amount of bleeding occurs, 
but in normal labor this is speedily checked by firm contraction of the uterus. Should this fail to take place profuse hemorrhage occurs (flooding) and the mother may bleed to death in a few minutes unless prompt measures are adopted.

For a few days after delivery there is some discharge (the lochia) from the uterine cavity: the whole decidua being broken down and carried off, to be subsequently replaced by new mucous membrane. The muscular fibers developed in the uterine wall in such large quantities during pregnancy undergo rapid fatty degeneration and are absorbed, and in a few weeks the organ returns almost to its original size. The parturient woman is especially apt to take infectious diseases; and these, should they attack her, are fatal in a very large percentage of cases. Very special care should therefore be taken to keep all contagion from her.

There is a current impression that a pregnancy, once commenced, can be brought to a premature end, especially in its early stages, without any serious risk to the woman. That belief is erroneous. Premature delivery, early or late in pregnancy, is always more dangerous than natural labor at the proper term; the physician has sometimes to induce it, as when a malformed pelvis makes normal parturition impossible, or the general derangement of health accompanying the pregnancy is such as to threaten the mother's life; but the occasional necessity of deciding whether it is his duty to procure an abortion is one of the most serious responsibilities he meets with in the course of his professional work.

The production of abortion, even in the first stages of pregnancy, by the taking of drugs, the so-called abortifacients, a practice which seems to have gained considerable headway through the widespread advertisement of their wares by unscrupulous vendors of "patent medicines," is so dangerous to the health, and even the life, of the woman who practices it that no consideration sanctions it.

Lactation. The mammary glands for several years after birth remain small, and alike in both sexes. Towards puberty they begin to enlarge in the female, and when fully developed form in that sex two rounded eminences, the breasts, placed on the thorax. A little below the center of each projects a small eminence, the 
nipple, and the skin around this forms a colored circle, the areola. In virgins the areolæ are pink; they darken in tint and enlarge during the first pregnancy and never quite regain their original hue. The mammary glands are constructed on the compound racemose type. Each consists of from fifteen to twenty distinct lobes, made up of smaller divisions; from each main lobe a separate galactophorous duct, made by the union of smaller branches from the lobules, runs towards the nipple, all converging beneath the areola. There each dilates and forms a small elongated reservoir in which the milk may temporarily collect. Beyond this the ducts narrow again, and each continues to a separate opening on the nipple. Embedding and enveloping the lobes of the gland is a quantity of firm adipose tisslie which gives the whole breast its rounded form.

During maidenhood the glandular tissue remains imperfectly developed and dormant. Early in pregnancy it begins to increase in bulk, and the gland-lobes can be felt as hard masses through the superjacent skin and fat. Even at parturition, however, their functional activity is not fully established. The oil-globules of the milk are formed.by a sort of fatty degeneration of the glandcells, which finally fall to pieces; the cream is thus set free in the watery and albuminous secretion formed simultaneously, while newly developed gland-cells take the place of those destroyed. In the milk first secreted after accouchement (the colostrum) the cell destruction is incomplete, and many cells still float in the liquid, which has a yellowish color; this first milk acts as a purgative on the infant, and probably thus serves a useful purpose, as a certain amount of substances (biliary and other), excreted by its organs during development, are found in the intestines at birth.

Human milk is undoubtedly the best food for an infant in the early months of life; and to suckle her child is useful to the mother if she be a healthy woman. Many women refuse to suckle their children from a belief that so doing will injure their personal appearance, but skilled medical opinion is to the contrary effect; the natural course of events is the best for this purpose, unless lactation be too prolonged. Of course in many cases there are justifiable grounds for a mother's not undertaking this part of her duties; a physician is the proper person to decide. 
In a healthy woman, not suckling her child, ovulation and menstruation recommence about six weeks after childbirth; a nursing mother usually does not menstruate for ten or twelve months; the infant should then be weaned.

When an infant cannot be suckled by its mother or a wet-nurse an important matter is to decide what is the best food to substitute. Good cow's milk contains rather more fats than that of a woman, and much more casein; the following table gives averages in 1,000 parts of milk:

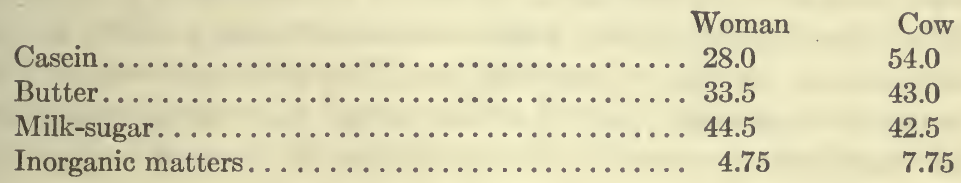

The inorganic matters of human milk yield, on analysis, in 100 parts-calcium carbonate, 6.9; calcium phosphate, 70.6; sodium chlorid, 9.8; sodium sulphate, 7.4; other salts, 5.3. The lime salts are of especial importance to the child, which has still to build up nearly all its bony skeleton.

When undiluted cow's milk is given to infants they rarely bear it well; the too abundant casein is vomited in loose coagula. The milk should therefore be diluted with half or, for very young children, even two-thirds its bulk of water. This, however, brings down the percentage of sugar and fat below the proper amount. The sugar is commonly replaced by adding cane-sugar; but sugar of milk is readily obtainable and is better for the purpose. If used at all it should, however, be employed from the first; it sweetens much less than cane-sugar, and infants used to the latter often refuse milk in which milk-sugar is substituted. In order to bring the percentage of fat up to normal it is usual to dilute, not "whole milk" but "top milk." The latter, after the milk has stood for a few hours, contains enough of the rising cream to supply the needed fat. As the infant grows older less diluted cow's milk may gradually be given; after the seventh or eighth month no water need be added.

It should not be necessary to emphasize the vital importance of giving to infants only the purest milk obtainable. It is unfortunately true that the milk produced in the average dairy is not 
only dirty but swarming with micro-organisms. In cities it has become the practice for medical societies to inspect various dairies and set their seal of approval upon those that fulfil the sanitary conditions essential to the production of pure, clean milk. The slightly higher cost of such "certified" milk should not be allowed to bar it from homes where children are to be fed except where extreme poverty makes its procurement impossible. In small towns and in the country personal inspection of the source of the milk supply on the part of parent or physician should give assurance of its cleanliness. Where it is impossible to procure milk free from suspicion, pasteurization (heating to $120^{\circ} \mathrm{F}$. for 20 minutes) should be resorted to. This destroys most of the dangerous organisms, but of course is not a complete substitute for cleanliness and care in the production of the milk in the beginning.

In the first weeks after birth it is no use to give an infant starchy foods, as arrowroot. The greater part of the starch passes through the bowels unchanged; apparently because the pancreas has not yet fully developed, and has not commenced to make its starchconverting enzym. Later on, starchy substances may be added to the diet with advantage, but it should be borne in mind that they cannot form the chief part of the child's food; it needs proteins for the formation of its tissues, and amyloid foods contain none of these. Many infants are, ignorantly, half starved by being fed almost entirely on such things as corn-flour or arrowroot.

Puberty. The condition of the reproductive organs of each sex described in preceding pages is that found in adults; although mapped out, and, to a certain extent, developed before birth and during childhood, these parts grow but slowly and remain functionally incapable during the early years of life; then they comparatively rapidly increase in size and become physiologically active; the boy or girl becomes man or woman.

This period of attaining sexual maturity, known as puberty, takes place from the eleventh to the sixteenth year, and is accompanied by changes in many parts of the Body. Hair grows more abundantly on the pubes and genital organs, and in the armpits, in the male also on various parts of the face. The lad's shoulders broaden; his larynx enlarges, and lengthening of the vocal cords causes a fall in the pitch of his voice; all the reproductive organs 
increase in size; fully formed seminal fluid is secreted, and erections of the penis occur. As these changes are completed spontaneous nocturnal seminal emissions take place from time to time during sleep, being usually associated with voluptuous dreams. Many a young man is alarmed by these; he has been kept in ignorance of the whole matter, is too bashful to speak of it, and getting. some quack advertisement thrust into his hand in the street is alarmed to learn that his strength is being drained off, and that he is on the highroad to idiocy and impotence unless he place himself in the hands of the advertiser. Lads at this period of life should have been taught that such emissions, when not too frequent and not excited by any voluntary act of their own, are natural and healthy. They may, however, occur too often; if there is any reason to suspect this, the family physician should be consulted, as the healthy activity of the sexual organs varies so much in individuals as to make it impossible to lay down numerical rules on the subject. The best preventives in any case are, however, not drugs, but an avoidance of too warm and soft a bed, plenty of muscular exercise, and keeping out of the way of anything likely to excite the sexual instincts.

In the woman the pelvis enlarges considerably at puberty, and, commonly, more subcutaneous adipose tissue develops over the Body generally, but especially on the breasts and hips; consequently the contours become more rounded. The external generative organs increase in size, and the clitoris and nymphæ become erectile. The uterus grows considerably, the ovaries enlarge, some Graafian follicles ripen, and menstruation commences.

Hormones of the Reproductive System. The interrelations of various processes in the functioning of the reproductive mechanism are many of them very striking and they have long been the subject of investigation. The development of the so-called secondary sexual characters at puberty, where in a few weeks the vocal cords change and hair develops over various parts of the body, is a good example of the sort of interrelations that occur in this system. The fact, known for centuries, that castration in early life prevents the appearance of the secondary sexual characters, shows that they are directly dependent on the reproductive organs. Before the idea of hormone action had crystallized to its present form some such mechanism had been postulated for the reproductive 
system. For it is difficult to explain such effects as those of castration on any other basis than that the generative organs elaborate some control-exercising substance of which the body is deprived by castration. Perhaps the best known examples of hormones concerned with reproduction are those that have to do with lactation. It has been proven that the development of the mammary glands during pregnancy is caused by a hormone produced in the body of the embryo. This hormone is attended apparently by another one, which, while permitting the development of the glands inhibits their active functioning. At the birth of the child this second hormone is withdrawn, and the glands are thus left free to pour forth their secretion.

The Stages of Life. Starting from the ovum each human being, apart from accident or disease, runs through a life-cycle which terminates on the average after a course of from 75 to 80 years. The earliest years are marked not only by rapid growth but by differentiating growth or development; then comes a more stationary period, and finally one of degeneration. The life of various tissues and of many organs is not, however, coextensive with that of the individual. At birth numerous bones are represented mainly by cartilage. The pancreas has not attained its full development; and some of the sense-organs seem to be in the same case; at least new-born infants appear to hear very imperfectly. The reproductive organs only attain full development at puberty, and degenerate and lose all or much of their functional importance as years accumulate. Certain organs have even a still shorter range of physiological life; the thymus, for example, attains its fullest development at the end of the second year and then gradually dwindles away, so that in the adult scarcely a trace of it is to be found. The milk-teeth are shed in childhood, and their so-called permanent successors rarely last to ripe old age.

During early life the Body increases in mass, at first very rapidly, and then more slowly, till the full size is attained, except that girls make a sudden advance in this respect at puberty. Henceforth the woman's weight (excluding exceptional cases of accumulation of non-working adipose tissue) remains about the same until the climacteric. After that there is often an increase of weight for several years due mainly to increased formation of fat; a man's weight usually slowly increases until forty. 
As old age comes on a general decline sets in, the rib cartilages become calcified, and lime salts are laid down in the arterial walls, which thus lose their elasticity; the refracting media of the eye become more or less opaque; the physiological irritability of the sense-organs in general diminishes; and fatty degeneration, diminishing their working power, occurs in many tissues. In the brain we find signs of less plasticity; the youth in whom few lines of least resistance have been firmly established is ready to accept novelties and form new associations; but the longer he lives, the more difficult does this become to him. A man past middle life may do good, or even his best work, but almost invariably in some line of thought which he has already accepted; it is extremely rare for an old man to take up a new study or change his views, philosophical, scientific, or other. Hence, as we live, we all tend to lag behind the rising generation.

Death. After the prime of life the tissues dwindle (or at least the most important ones) as they increased in childhood.

Before any great diminution takes place, however, a breakdown occurs somewhere, the enfeebled community of organs and tissues forming the man is unable to meet the contingencies of life, and death supervenes. "It is as natural to die as to be born," Bacon wrote long since; but though we all know it, few realize the fact until the summons comes. To the popular imagination the prospect of dying is often associated with thoughts of extreme suffering; personifying life, men picture a forcible and agonizing rending of it, as an entity, from the bodily frame with which it is associated. As a matter of fact, death is probably rarely associated with any immediate suffering. The sensibilities are gradually dulled as the end approaches; the nervous tissues, with the rest, lose their functional capacity, and, before the heart ceases to beat, the individual has commonly lost consciousness.

The actual moment of death is hard to define: that of the Body generally, of the mass as a whole, may be taken to be the moment when the heart makes its last beat; arterial pressure then falls irretrievably, the capillary circulation ceases, and the tissues, no longer nourished from the blood, gradually die, not all at one instant, but one after another, according as their individual respiratory or other needs are great or little.

While death is the natural end of life, it is not its aim-we should 
not live to die, but live prepared to die. Life has its duties and its legitimate pleasures, and we better play our part by attending to the fulfilment of the one and the enjoyment of the other, than by concentrating a morbid and paralyzing attention on the inevitable, with the too frequent result of producing indifference to the work which lies at hand for each. Our organs and faculties are not talents which we may justifiably leave unemployed; each is bound to do his best with them, and so to live that he may most utilize them. An active, vigorous, dutiful, unselfish life is a good preparation for death; when that time, at which we must pass from the realm controlled by physiological laws, approaches, when the hands tremble and the eyes grow dim, when "the grasshopper shall be a burden and desire shall fail," then, surely, the consciousness of having quitted us like men in the employment of our faculties while they were ours to use, will be no mean consolation. 


\section{APPENDIX \\ SUGGESTIONS FOR LABORATORY WORK}

To a greater and greater extent colleges and normal schools are supplementing text-book instruction in Physiology with practical work in the laboratory. For such work laboratory instructions must be provided. There are upon the market numerous excellent laboratory manuals of Physiology, but most of them have been prepared for use in Medical Schools, and with the needs of Medical Students primarily in mind. The aim of this appendix is to furnish a basis for the preparation of a manual suited to the needs of the students who are pursuing the subject non-professionally. The complete equipment of a Physiological laboratory is rather extensive, and in many cases not available in entirety. The time which can be devoted to laboratory work in Physiology varies greatly in different institutions. For these two reasons it has seemed best not to offer in this connection a manual of selected experiments, but rather to suggest simple exercises that can be adapted by the teacher to his particular requirements.

The special character of much of the work of the physiological laboratory demands a certain amount of special preparation on the part of the teacher. In describing the following experiments this preparation is assumed. The majority of teachers of Biology have it. For those who have not, and wish to introduce in their classes laboratory work in Physiology, resort may be had to one of the summer courses in Physiology now offered by the larger Universities in various parts of the country.

Fortunately for the successful development of laboratory Physiology in America the special apparatus required can be obtained of American manufacture and at a moderate price. Information as to where such apparatus may be sought can be had upon application to the department of Physiology in any of the larger Universities. 


\section{INTRODUCTION}

General Histology of Body. Microscopic study of typical cells and tissues. Desirable for students who have no previous histological training.

General Chemistry of Body. Inorganic Constituents. Water. The percentage of water in flesh may be determined roughly by placing a weighed piece of meat in a dessicator in a warm place and weighing it at intervals until thoroughly dry.

Inorganic Salts. A weighed piece of meat may be incinerated under a hood and the ash weighed. The solubility of the ash in water may be ascertained. Tests for chlorids with silver nitrate, and for calcium with sodium oxalate solution may be applied.

Organic Constituents. Carbohydrates. Dextrose is present i: honey and corn syrup. The test for its presence is by means cf Fehling's Solution (see any laboratory manual of Organic Chemistry). The presence of a reddish precipitate in the test tube in which mixed Fehling's Solution (alkaline copper tartrate) and dextrose are combined and heated to boiling is the test.

Glycogen is a constituent of oysters. Grind a raw oyster with sand in a mortar. Extract with water. Add to a few drops of the juice in a test tube a few drops of iodine solution (tincture may be used). A mahogany-brown color is the test for glycogen.

Lactose. If sweet milk is clotted with rennet and filtered the whey contains milk sugar. Test with Fehling's solution as for dextrose.

Fats. Solubilily of Fats. Shake a small piece of lard with some ether in a test tube. Pour some of the solution on filter paper and allow it to evaporate. If the ether dissolved the fat, a grease spot will be left on the filter paper after the evaporation of the ether.

Moisten a second filter paper with ether, allow it to evaporate. Compare the two filter papers. The test may be repeated with olive oil instead of lard.

Test for Fats. Melt a small piece of lard in a test tube and to it add a drop of dilute osmic acid. This acid turns fat black.

Composition of Fats. Fats are compounds of fatty acids with glycerin. To separate the constituents heat on a water bath a small piece of lard with an alcoholic solution of caustic potash. 
After forty minutes pour the mixture into a dish containing $100 \mathrm{c} . \mathrm{c}$. water. Drive off the alcohol by heating over water. While still hot acidify with sulphuric acid. On cooling the fatty acid forms a solid crust on the surface of the liquid. Remove it. The glycerin is dissolved in the liquid.

The fatty acid is insoluble in water as may be shown by test.

Saponification. Soap is a compound of fatty acid with alkali. Shake some of the fatty acid produced above with warm dilute caustic soda. Filter. The filtrate shows the characteristic features of a soap solution. Soaps form an insoluble compound with calcium chlorid. This can be shown by adding a few drops of calcium chlorid to some of the soap solution in a test tube. Hard water contains calcium salts. This explains the difficulty of using soap with hard water.

Proteins. Tests for Proteins. Raw egg white is a satisfactory protein for these tests.

Heat Test. To a small amount of a solution of protein add a drop of a two-tenths per cent solution of acetic acid. Heat the upper portion of the solution. The protein is rendered insoluble and is now a coagulated protein.

Biuret Test. To a solution of protein add a drop or two of a one per cent solution of copper sulphate $\left(\mathrm{CuSO}_{4}\right)$ and then strong sodic hydrate $(\mathrm{NaOH})$. A characteristic violet color is the test.

Xanthoproteic Reaction. To a solution of protein add a few drops of strong nitric acid $\left(\mathrm{HNO}_{3}\right)$, and boil; after cooling, add ammonic hydrate $\left(\mathrm{NH}_{4} \mathrm{OH}\right)$. A yellow color which deepens to orange when the ammonia is added is the test.

Physico-Chemical Principles. Osmosis. Tie an osmotic membrane (gold beater's skin, bladder) across the mouth of a funnel or thistle tube. Fill with a sugar solution of the consistency of syrup. Attach a glass tube to the nozzle of the funnel. Fasten in an upright position and surround the funnel with distilled water in a beaker.

Drops of blood on a microscope slide may be used to demonstrate osmosis indirectly. Three such drops should be examined; one diluted with distilled water; a second with 0.9 per cent sodium chlorid; the third with 10 per cent sodium chlorid. Avoid excessive evaporation. The destruction of the corpuscles in the dilute solution by over-distension (plasmolysis); their preservation in the 0.9 
per cent saline; and their shrinkage (crenation) in the concentrated solution illustrate osmosis.

Dialysis.-Sausage casings make good dialyzing tubes, or special tubes may be purchased from chemical supply houses. To illustrate the separation of crystalloids from colloids by dialysis make a mixture of raw egg white with moderately concentrated sodium chlorid solution. Place in the dialyzing tube; suspend the tube in a beaker of distilled water. Stir the water frequently. After a sufficient interval the presence of sodium chlorid in the water can be demonstrated with silver nitrate, but the biuret test for protein (a very delicate test) continues negative.

\section{THE SUPPORTING TISSUES}

Gross and microscopic studies of bones and cartilage, and microscopic studies of various forms of connective tissue may be made as the time and available material permit, and as the previous training of the students requires.

That bone consists of inorganic salts deposited in an organic matrix may be shown by dissolving out the inorganic salts with dilute hydrochloric acid. That calcium is an important constituent of the inorganic portion may be shown by testing the hydrochloric acid solution with sodium oxalate.

Gelatin is a product obtained from bones by cooking them in water heated above the normal boiling point by inclosing in a sealed vessel. The ordinary protein tests applied to gelatin show that it is a member of the group of proteins.

\section{The Skeleton}

The general arrangement of the bones of the skeleton, and details of selected regions, as the skull, may be studied.

Joints. In connection with work on the skeleton the various types of joints should be studied in detail.

Typical joints are those at hip, knee, and ankle.

Hip Joint. Observe on the outer surface of the innominate bone a deep depression, the acetabulum, into which fits the smooth. nearly spherical head of the femur, making a ball and socket joint. Study the possible movements of the joint. Note (1) flexion, simple bending of the joint as in walking; (2) extension, the opposite of 
flexion; (3) abduction, drawing the leg outward from the body; (4) adduction, the opposite of abduction; (5) rotation, twisting at the joint as in placing ankle on knee.

In addition to these elementary joint movements, the hip joint permits various combination movements, as flexion with abduction. Note these.

Knee Joint. Observe the surfaces on femur and tibia which come together at the knee. Analyze the possible movements of this joint.

Ankle Joint. Observe the possible movements of this joint.

\section{MUSCLES}

For dissection studies the cat is the most available mammal.* Detailed descriptions are given in "The Anatomy of the Cat" by Reighard and Jennings. For purposes of illustration directions for a single region are included here.

Dissection of Hind Leg of Cat. Directions for dissection. Muscles are inclosed in and bound together by sheets of connective tissue, which make up the fascia. To dissect out a muscle cut or tear through the fascia which joins it to its neighboring muscles until the desired muscle can be lifted clear except for the attachments at its ends. Do not cut attachments until you have finished the study of the muscle.

The attachment of the muscle nearer the trunk is called the origin; the attachment further from the trunk is the insertion.

In dissecting the hind leg of the cat, consider it as made up of four regions: pelvis, thigh, shank, and foot. The muscles are to be dissected in order as directed below. As each is cleared from its surrounding muscles the region in which it has its origin is to be determined, also the region of its insertion.

Muscles are grouped functionally, according to the joint-motions they produce, as flexors, extensors, abductors, adductors, and rotators. $\dagger$ Determine for each muscle the region it moves and the motion it produces.

On the outside of the thigh is the biceps femoris. Abductor of

* If there is a pork-packing establishment in the vicinity embryo pigs can usually be obtained in ample numbers. These make exceedingly satisfactory dissection material, especially for elementary classes.

$\dagger$ Elevators, depressors, and sphincters occur in the Body but not in the leg. 
leg and flexor of shank. Directly beneath the biceps femoris observe the large nerve, the sciatic.

Along the front edge of the thigh and on its inner surface is the sartorius or tailor's muscle. Extensor of shank, adductor of leg, and rotator of leg.

The entire mass of muscle in front of the femur after the sartorius has been removed is the quadriceps femoris. Extensor of shank.

On the inner surface of the thigh toward the back is the gracilis. Adductor of leg.

On the inner surface of the thigh after the removal of the gracilis appear the following muscles from front to back. Small adductor longus. Adductor of leg. The larger triangular adductor femoris. Extensor of thigh. Large flat semi-membranosus. Extensor of thigh.

After dissection of above muscles there remains on the thigh only the semi-tendinosus. Flexor of shank.

The great mass of muscle forming the calf consists of three muscles, the plantaris, gastrocnemius, and soleus. Do not try to separate these. The tendo achilles is their common tendon. Extensor of foot.

After removing above muscles there will be found against the shank bones at the back the flexor longus digitorum. Flexor of toes.

Along the front of the shank, a superficial muscle with its tendon toward the inner side, is the tibialis anterior. Flexor of foot.

Partly underneath the above, with its tendon toward the outer side, is the extensor digitorum longus. Extensor of toes.

The Contraction of Muscles. The tissues of cold-blooded animals are well suited for studies of function since they survive, sometimes for hours, the general death of the animal.

From the hind leg of a recently killed frog make a femur-gastrocnemius preparation. This preparation is used for the study of muscular contraction.

To study muscular contraction adequately the motions of the muscle must be magnified and must be recorded. For obtaining a magnified record of its movements the muscle is fastened in a clamp and its tendon attached to the short arm of a lever, whose long arm presses lightly at its tip against a smoked paper on which every movement of the muscle is recorded as a line (p. 94). To avoid superposing separate tracings the smoked paper is mounted 
on a drum which can be moved by hand, or driven at various speeds by a clockwork. This apparatus is called a kymograph. Tracings made with it can be preserved by passing the paper bearing them through a solution of shellac in alcohol.

Skeletal muscles contract only when stimulated. A suitable artificial stimulus is the shock from an induction coil. Induced currents are generated in the secondary coil of an inductorium when, and only when, a current is made or broken in the primary coil. An ordinary dry cell is a good source of current. The strength of the induced current varies with the position of the secondary coil relative to the primary, being greatest when the secondary is directly over the primary, and least when the secondary is withdrawn as far as possible from the primary and turned at right angles to it.

Mount the frog's gastrocnemius muscle in readiness for obtaining tracings of its contractions. By means of fine copper wires establish a circuit from the secondary coil of the inductorium through the tissue. Be careful to avoid short circuits.

With the secondary coil in the position of least effectiveness make and break the primary circuit. If the muscle does not respond shift the secondary, little by little, toward the position of greatest effectiveness, making and breaking the primary circuit with each shift. As soon as the muscle responds, recording its contraction on the smoked paper, move the drum forward by hand so that new contractions will not be superposed on the first one.

Continue increasing the strength of the shock, obtaining records of each contraction.

Thus the relation of contraction height to stimulation strength is shown.

A current is induced in the secondary at make and at break of the primary current. Determine which is a more powerful stimulus, a "make" shock or a "break" shock.

The inductorium as used in the physiological laboratory is provided with an automatic circuit breaker which can be included in the primary circuit. Make the proper connections for doing this. Now when the circuit is closed shocks are sent into the muscle in rapid succession.

Set the clockwork of the kymograph to drive the drum at a slow speed. Obtain a tracing showing the response of the muscle to 
rapidly repeated stimuli. This sort of contraction is known as a physiological tetanus or a tetanic contraction (p. 102).

The Staircase Effect, Contracture, Fatigue. Prepare and mount a gastrocnemius muscle as in the previous exercise. Set the secondary of the inductorium at a position that gives a sharp but not excessive break stimulus. With the drum moving at its slowest speed make and break the primary circuit twice per second, allowing the contractions to record themselves on the slowly moving drum. Continue the series of stimuli until the muscle ceases to respond. Make the record permanent.

The increase in the height of the contractions during the first part of the record, due to "warming up" is called the "staircase effect."

A rise in the base line during the later part of the curve is the phenomenon of contracture (p. 100).

The final failure of the muscle to contract is the result of fatigue. This may be explained either as due to the exhaustion of the fuel supply or to the accumulation of harmful "fatigue products."

To distinguish between these alternatives replace the fatigued muscle with a fresh one. Arrange the primary circuit for rapidly repeated shocks of moderate intensity. With the muscle recording on a slowly moving drum close the key, throwing the muscle into tetanus. Continue the stimulation until the muscle is well fatigued. Let the muscle rest for five minutes. Repeat the stimulation. Recovery under this condition proves that the previous fatigue could not have been due to exhaustion of the fuel supply.

The production of acid in an active muscle may be demonstrated with sensitive litmus paper. A resting isolated muscle is neutral or slightly alkaline to litmus. The same muscle, exercised to fatigue, is acid to litmus.

The Influence of Temperature on Contraction. For this study arrangement must be made for immersing the muscle in a liquid and at the same time recording its contractions. A method of doing this is to mount an L-shaped glass rod in a clamp. Fasten the femur end of the frog's gastrocnemius to the horizontal end of the L. A small pulley must be mounted above the rod, over which a thread can be led from the tendon of the muscle to the recording lever. Connect the ends of the muscle by fine copper wire to the terminals of the secondary coil. Set this so that the 
muscle gives a vigorous contraction upon stimulation. Bring around the muscle a beaker of ice-cold Ringer's solution $(\mathrm{NaCl}$ $0.7 \% ; \mathrm{CaCl}_{2} 0.026 \% ; \mathrm{KCl} 0.03 \%$ ), which has the same osmotic pressure as frog's blood.

With the drum moving at the fastest clockwork speed obtain a record of a single contraction.

Withdraw the cold solution and allow the muscle tissue to return to room temperature. With the drum moving at the same rate as before obtain a record of a single contraction. Now surround the muscle with Ringer's solution warmed to $30^{\circ} \mathrm{C}$., and obtain a record of a single contraction, again with the drum at the same speed as before.

Let the drum make a complete revolution at the speed used in making those records. Determine as accurately as possible the time consumed. After the smoked paper has been removed measure its length, and calculate the speed of the drum in centimeters per second.

Varnish the tracings and mount side by side the records of contraction at the three temperatures used.

Compute the time required at each temperature for a complete contraction.

Set the drum moving at its slowest speed. Surround the muscle with Ringer's solution, and without stimulating the muscle allow it to record on the drum while the solution is gradually warmed to $65^{\circ} \mathrm{C}$. The contraction brought about by warming above $40^{\circ}$ is called heat rigor.

\section{THE NERVOUS SYSTEM}

Dissections of PERIPHERAL NERVES may be made according to the description in Reighard and Jennings. By way of suggestion directions for dissecting a typical spinal nerve and a typical cranial nerve are given.

A representative spinal nerve is the great sciatic with its branches. (To be dissected in the cat.)

Cut through the skin on the outer side of the hind leg from the heel to the middle of the back. Remove the biceps femoris muscle. The nerve trunk thus exposed is the sciatic. . Follow the nerve trunk upward, cutting away overlying tissues where necessary. 
The nerve can be traced to where it passes through a hole in the pelvic bone. Thrust a seeker through this hole and then cut away the tissues on the front surface of the pelvis until the seeker is exposed. The nerve will thus be brought into view, and can be traced for a short distance to the point where it emerges from the spinal canal.

Returning to the nerve on the outer surface of the thigh follow it downward, noting that it gives off occasional branches to contiguous muscles. A short distance behind the knee the nerve divides into two branches. One of these, the peroneus, passes across the gastrocnemius muscle on its outer surface, then plunges beneath it and passes down the shank close to the fibula. It can be followed by cutting away the overlying tissue. The second branch of the sciatic, the tibialis, passes directly into the muscular mass of the calf, then turns downward toward the foot. Follow it as far as possible, cutting away overlying tissues carefully.

A Typical Cranial Nerve: The Vagus. Make an incision along the mid-line of the under surface of the body its entire length. Separate the muscles of the neck until the trachea (wind-pipe) is exposed in the mid-line. Follow around the left side of the trachea, separating the muscles, but not cutting them, until a sheath of connective tissue inclosing blood-vessels and nerves is found. This sheath is usually in close contact with the side of the trachea. With a blunt instrument open the sheath and separate the nerves from the adjacent artery for the space of an inch or more. Careful observation discloses two nerves in close contact with each other. The larger is the vagus. Follow it toward the head. At the level of the larynx (vocal apparatus) it gives off a branch, the superior laryngeal. Continue the dissection forward to the point where the nerve enters the skull. An enlargement, the ganglion nodosum, is seen just here.

Return to the point where the vagus was first separated, and carry the dissection backward. Separate overlying tissues, but do not cut through them except when absolutely necessary for progress. At the junction of neck with thorax there are some large veins which may bleed. If any such are cut accidentally the blood should be carefully wiped away with cotton to keep the field of dissection clear. Follow the nerve into the thorax to the level of the root of the lung. A short distance above this point a branch of 
the vagus, the inferior laryngeal, passes behind a large artery, the aorta, and turns back toward the head.

Branches of the vagus can be traced into the root of the lung. At about the level of the root of the lung the nerve divides into two branches, each of which can be traced to the surface of the esophagus, where each unites with a corresponding branch from the right vagus. The nerve trunks thus formed continue backward along the esophagus to the stomach where they break up into fine branches which supply the stomach and upper portion of the small intestine.

For the CENTRAL NERvous SYSTEM the sheep's brain is a satisfactory object of dissection. The brain should be carefully removed from the skull and hardened in formalin before use.

Sheep's Brain. With the aid of the figures on pages 144, 145, 146, and 151 make out the grand divisions of the brain: cerebrum, or fore brain; midbrain, overlain in front by the pons, and behind by the cerebellum; medulla oblongata, forming the connecting link between brain and spinal cord.

Observe on the surface of the cerebrum the irregular convolutions which serve to increase its surface relative to its bulk.

On the base of the brain make out with the aid of the figure on page 151 the optic tracts and optic chiasma; also as many other roots of cranial nerves as possible. Note that all cranial nerves behind the optic nerve spring from the brain stem (midbrain and medulla). In front of the optic tracts and springing from the cerebrum, the olfactory lobes may be seen.

Cut the brain through the vertical median plane. Note the corpus callosum.

Conduction in the Nerve Trunk. The sciatic nerve in the frog can be exposed by removing the skin from the leg and separating carefully the two large muscles on the dorsal surface of the thigh. Dissect the nerve out carefully from the upper end of the leg toward the knee. Use great care to avoid injuring the nerve by stretching or squeezing. Cut the nerve away at its upper end. Leave it in connection below with the muscles of the shank. The ability of the nerve to conduct impulses may be demonstrated by stimulating it as far as possible from the muscles and observing their response. The susceptibility of the nerve to different forms of energy may be shown by stimulating it with forceps (mechanical), a hot rod 
(thermal), or shocks from a pair of electrodes leading from the terminals of the induction coil (electrical).

The impairment of conductivity by cold may be shown by bringing against the nerve, between the point of stimulation and the muscle, a small test tube filled with cold brine.

Motor Points on the Body. To one terminal of the secondary coil of an inductorium attach a flat electrode which has been wrapped with gauze well moistened with saline solution. Bare the forearm and lay it, palm up, in contact with the electrode. To the other terminal of the inductorium attach a rod electrode. With the use of tetanizing stimuli of moderate strength explore the surface of the forearm by means of the rod electrode. At certain points individual muscles will be thrown into contraction. These points are "motor points."

\section{Spinal Reflexes}

Suspend a frog, whose brain has been recently destroyed, by means of a hook through the jaw. Pinch the toes of the right foot. The foot is retracted. Repeat the experiment on the other toes. In each case note the relation of the muscles that respond to the region stimulated.

Tie two fine copper wires $1 / 4$ inch $(6 \mathrm{~mm}$.) apart, about the right hind toes. Carry these to the terminals of the secondary coil. Send in tetanizing shocks of increasing strength. As more and more widespread movements are elicited note the order in which various parts of the body become involved.

Moisten a bit of porous paper with acid. Place the acid on the frog's back near the legs. Note the adaptive response.

Wash the skin thoroughly with water. Repeat the experiment, this time placing the acid paper on the belly.

Destroying the frog's brain has destroyed his intelligence. These responses, though adaptive, are purely automatic.

By. means of a fine-pointed pipette introduce a few drops of strychnine solution under the skin of the frog's back. After allowing a few minutes for the drug to take effect pinch one of the toes.

The widespread convulsive responses signify the breaking down of synaptic resistances to a uniform level (p. 162). 


\section{Summation and Inhibition of Reflexes}

Summation. Suspend a frog, whose brain has recently been destroyed, by a hook through the lower jaw. Tie fine copper wires $1 / 4$ inch $(6 \mathrm{~mm}$.) apart, about the right toes. Connect the wires with the secondary coil of an inductorium, taking care to avoid short-circuits. The primary circuit should be arranged to give single shocks.

Set the secondary coil so that a small twitch follows each stimulus. Make and break the primary circuit repeatedly and rapidly.

Note that a series of stimuli produce an effect that a single stimulus could not. This is summation.

Inhibition. Bring dilute acid in a beaker in contact with the left toes of the frog used in the preceding experiment.

Determine in seconds the time required for the foot to be withdrawn.

Immediately wash thoroughly with water the acidified foot.

Now bring the beaker of acid again in contact with the left toes, at the same time stimulating the right toes with an interrupted current of moderate strength.

Determine the time for withdrawal of the foot from the acid. A delay is due to inhibition. To prove that the acid has not injured the foot repeat the immersion without simultaneous stimulation. Prompt withdrawal should occur.

\section{Neuro-muscular Fatigue}

Dissect out a gastrocnemius-sciatic preparation (p. 597). Arrange to secure a record of the contraction of the muscle. Moisten the nerve frequently with salt solution to prevent drying and consequent loss of irritability. With the drum moving at a slow speed stimulate the nerve of the preparation with fairly strong stimuli until the muscle no longer responds. Now quickly bring the electrodes in contact with the muscle itself. A contraction shows that the muscle is not fatigued.

Since nerve trunks are indefatigable the fatigue must have occurred in the neuro-muscular junctions (p. 198). 


\section{Time Relations in Nervous Processes}

Determine accurately by repeated trials the time in seconds required for a single revolution of the kymograph drum at its highest speed. Be careful to use this determined speed in the observations below. Each experiment requires a subject and an operator.

Simple Reaction Time. Arrange the inductorium for single shocks, and select a strength of stimulus distinctly felt on the tongue at the break of the primary circuit. The apparatus is so arranged that the operator can make and break the circuit at one place and the subject at another (see diagram, Fig. 157). A signal records on the drum the instant of making and breaking

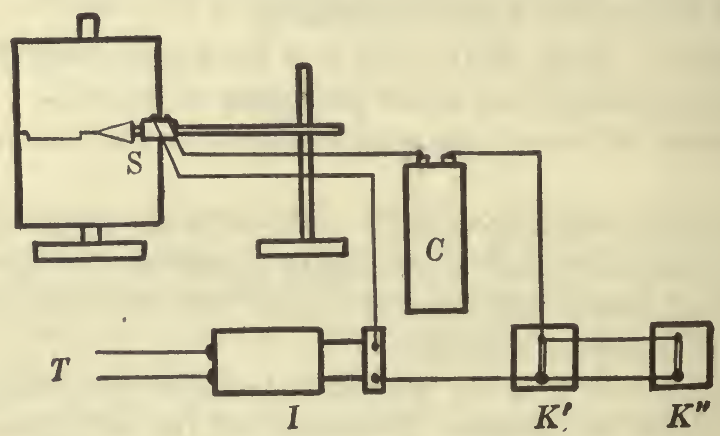

Fig. 157.-Diagram of reaction time apparatus. $K^{\prime}$ and $K^{\prime \prime}$, keys for making or breaking primary circuit; $C$. dry cell; $I$, inductorium; $T$, wires to tongue electrodes; $S$, signal magnet, writing on drum.

the circuit. Let the subject press the electrodes on his tongue, place his hand on his key, and close his eyes. The operator should now start the drum at known speed and close the circuit at his contact. While the drum is in motion the operator should break the circuit at his contact. This break shock stimulates the subject. The instant the stimulus is felt the subject should close his key. The points of stimulus and of response are shown in the record traced by the signal on the drum. Repeat the experiment several times with each member of the pair acting as subject. After the tracing has been varnished measure with care the length of each reaction record. By comparing these lengths with the drum circumference compute the reaction times in hundredths of a second. Average the results from each individual. 
Thought Time Compared with Speech Time. With the apparatus arranged as before let the subject, when stimulated, think the first ten letters of the alphabet before pressing his key. Repeat the experiment; this time having the subject say the ten letters aloud. Make a number of trials and determine the average results.

\section{THE SPECIAL SENSES}

In the following experiments one member of a pair is to act as subject, the other as experimenter. Members of the pair should alternate in these functions.

\section{Touch}

Localizing delicacy. Let the subject sit with his hand on the table, and with eyes closed. Apply carefully to the back of the hand the points of small dividers separated about $1 / 2 \mathrm{~mm}$. The subject reports whether he feels one point or two points, or is in doubt. Record the result. Change the distance between the points gradually, in successive tests applied to the same region, until the subject reports a change in sensation. The minimal distance at which the two points can be felt as two points is the threshold.

Record the results of testing for the threshold on the finger-tips, palm, flexor and extensor surfaces of the forearm, cheek, and lips.

\section{Temperature}

Cold and Warmth Spots. Outline an area on the back of the wrist about $2 \mathrm{~cm}$. square. Let a blunt pointed metal rod stand in cold water until it has become cooled. Dry it and examine point by point the selected area. Mark the spots at which the cool rod causes sensations of cold.

Let the rod stand in hot water until it can be felt as hot when dried and touched to the skin lightly, but not so hot as to cause burning or pain. Explore point by point with very light contact an equal area contiguous to that examined for cold spots. Mark with ink the spots at which the rod causes sensations of warmth. 


\section{Equilibrium Sense}

Compensating Movements. Let the subject with head erect rotate his body for 15 seconds about the vertical axis. When rotation is stopped, note the movements of the eyeballs and the arms and legs. Describe the after-sensation.

Influence of Vision on the Maintenance of Equilibrium. Try to stand on one foot for a minute with the eyes closed. Repeat the trial with the eyes open. Record the experiences.

\section{HEARING}

Threshold. In a quiet room determine the greatest distance at which the subject, who sits with eyes closed and a hand pressed tightly over the right ear can hear the ticking of a watch held opposite the left ear. Repeat the experiment with the right ear, holding the left ear tightly closed.

Bone Transmission. Hold a ticking watch between the teeth. Close both ears with finger-tips. Note the effect on loudness of closing both ears.

Unstop one ear. Compare the loudness in the two ears.

Space Perception. Let a student click together two coins in various positions with reference to the ears of another student, who acts as subject and keeps his eyes closed. The subject should point in the direction to which he refers the sound. Compare the accuracy of judgment at the sides with that in the median plane.

With a finger-tip stop the ear on one side, and observe whether the power of localizing sound is diminished.

\section{TASTE}

Localization. Apply to different parts of the tongue samples of the following solutions: a solution of quinine sulphate (bitter), a 5 per cent solution of cane sugar (sweet), a 10 per cent solution of $\mathrm{NaCl}$ (saline), and a 1 per cent solution of acetic acid (sour). Note the region on the tongue on which each substance is tasted most acutely.

- Let the student wipe the surface of his tongue as dry as possible, and then let another student apply crystals of salt and of sugar to the dry surface.

Undissolved substances are not tasted. 


\section{SMELL}

Fatigue. With one nostril stopped, smell tincture of iodine through the other. Hold the bottle near the nose, inhale evenly and somewhat rapidly, and exhale through the mouth.

Note the time required to produce exhaustion.

Allow a minute for recuperation and repeat the above test. Repeat until a minute does not suffice for recuperation.

Note the successive exhaustion times.

This experiment explains failure to perceive closeness in a room through fatigue of the sense of smell.

\section{VISION}

A good introduction to the study of vision is the dissection of the eye.

Sheep's eyes hardened in formalin are satisfactory. Directions for dissection are given below.

Conjunctiva. This is the smooth membrane which is loosely attached to the eye in front. It lines the lids, and is reflected from the lid upon the surface of the ball.

Muscles. Remove the fat which is adherent to the ball, so that the external smooth coat will be exposed. The cut ends of several muscles will be seen.

The Cornea and Sclera. On the free surface of the ball the elliptical area includes the cornea, transparent during life but rendered opaque during preservation; the rest of the surface of the ball is constituted by the naturally white and opaque sclera, commonly called the sclerotic coat.

Optic Nerve. With the finger and forceps tear apart the muscular masses surrounding the optic nerve, and remove with the scissors. Notice the fibrous constitution of the nerve and the firmness of the sheath; also, that the nerve does not enter the center of the eye.

Aqueous Humor. Press the eye so as to make the cornea tense. Cut through the cornea with the point of the scalpel; a clear fluid will ooze out.

Iris and Pupil. Raise the cut edge of the cornea with the forceps and remove it with the scissors; a dark lamina, the iris, with a central orifice, the pupil, will be seen. 
Anterior Chamber. This is the space between the iris and cornea and is filled with the aqueous humor. Through the pupil will be seen the crystalline lens. The space between the iris and the lens is called the posterior chamber and also contains aqueous humor.

The Crystalline Lens and the Coats of the Eye. Make a median section through the remaining part of the eye. The lens in cross section, the cut edges of three coats, and a transparent jelly-like mass, the vitreous humor, will be seen.

The coats from within outward are:

1. The retina, a very thin, white membrane, covering the inside of the eye, except the anterior part. The retina is a continuation of the optic nerve. At its posterior part where the nerve enters may be seen a small area, the blind spot, from which several minute blood-vessels radiate.

2. The choroid coat, which is the middle tunic of the eye, is pigmented, and firmer than the retina. This coat appears black or blue in the specimen, and is continued into the ciliary body and the iris, the former supporting and controlling the shape of the lens in accommodation.

3. The outer coat is the sclera, the anterior part of which is transparent and called the cornea. It is thick and fibrous, giving strength and form to the eye. It is white in appearance, is pierced by the optic nerve at the back, and gives attachment to the muscles on its outer surface.

Lens. Separate the halves of the lens from the vitreous humor in which they are embedded. Note that the lens is composed of concentric layers, like an onion. It is surrounded by a capsule. Note that the anterior surface is flatter than the posterior.

\section{Refraction in the Eye}

The eye is an instrument for producing upon a sensitive surface, the retina, images of objects in space. The production of an image requires a device for focussing. In the eye the cornea and lens together make up the focussing apparatus. The eye is so constructed that rays of light coming from points more than 18 feet away are focussed naturally upon the retina.

The fundamental fact of vision, the formation of images by lenses can be demonstrated with the aid of a double convex lens, 
a candle flame, and a screen in a darkened room. The visual defects of myopia and hypermetropia (p. 262) may be illustrated by shifting the screen in such a manner as to throw the image on it out of focus. When the screen is too far away from the lens for the image to be clear the situation is as in myopia. A double concave lens placed in the path of the rays illustrates the correction for this defect. Throwing the image out of focus by bringing the screen too near the lens gives the situation seen in hypermetropia. This may be corrected with a double convex lens.

\section{Some Phenomena of Vision}

Visual Reference. The eye learns by experience to refer visual stimuli outward through a point called the "nodal point" (p. 267). This point is within the crystalline lens, three-quarters of the distance from retina to cornea.

Scheiner's Experiment. The fact that images on the right side of the retina are interpreted as coming from objects to the left of the visual axis, and vice versa, was demonstrated by Scheiner in 1619 .

Pierce a card with two pin holes about one-tenth inch apart. Look through the pin holes at a distant object. Place in the line of vision about a foot from the eye a pin mounted in a block. Do not accommodate for the pin. Two images of the pin are seen. Slide a card over the right hand pin hole. The left hand image disappears.

Place the pin four feet from the eye. Look through the pin holes at a second pin in line with the first one, but only four to six inches distant from the eye. Two images of the far pin are seen. Slide a card over the right hand pin hole. Now the right hand image disappears. For the explanation see diagram, Fig. 158.

The Blind Spot. Make a small black spot near the left margin of a sheet of note paper. Place the paper on the desk. Let one student of a pair look fixedly at the spot with the right eye, holding the head stationary, about twelve inches over the spot. The other member of the pair should move a black-headed hatpin from a point in the right margin of the paper directly opposite the black spot toward the spot itself. The subject should report the instant the head of the pin disappears, and the place should be marked 
with a pencil. Continue moving the pin head toward the black spot and fix in the same manner the place of reappearance.of the head of the pin. These two spots indicate the lateral extremities of the blind spot. Its outline is to be determined by moving the pin head toward it from various directions and fixing the point of disappearance in each of them.

The Field of Color Vision. Let one student of a pair look fixedly with the right eye at a spot in an upright screen, supporting the chin firmly. The other student moves a small square of colored paper toward the "spot" from the margin of the screen. The squares of paper should be fixed to straws or stiff wires so they
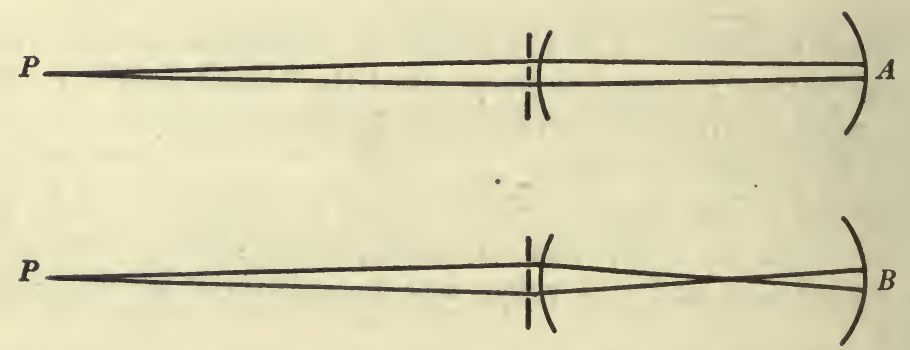

FrG. 158.-Diagrams illustrating Scheiner's experiment. In $A$ the eye is accommodated for distant vision and rays from the pin $P$ strike the retina before they meet. The image through the right hand pin hole falls upon the right hand side of the retina, just opposite to the usual manner. In $B$ the rays cross before striking the retina. The relation of images to pin holes is therefore the same as in ordinary vision.

can be handled readily. Red, green, blue, yellow, and white should be the colors provided. The subject should not know which color is being used. As soon as he recognizes the color he should report it, and the color should be marked on the screen. If the color is incorrectly named, continue moving the square inward until it is correctly perceived. Repeat the test along different meridians and with different colors until the field of each color has been roughly outlined.

\section{BLOOD}

Histological Structure. Blood is composed of a liquid, the plasma, in which are several kinds of minute structures, the corpuscles, red and colorless, and the platelets.

A sample of blood for observation is prepared as follows: Provide 
two clean microscopic slides. Congest the blood in the middle finger of the left hand by wrapping a handkerchief tightly about it, beginning at the base. Prick the congested region sharply with a clean needle and squeeze out a drop of blood. Bring the surface of one of the slides near one end in contact with the drop of blood. The blood will adhere to the slide. Quickly place an edge of the second slide against the surface of the first one and move it along till it comes in contact with the blood-drop. The latter should spread out along the edge of the second slide. Now draw this slide along the first one. The blood will follow, and thus be spread out in a thin layer. Let the slide dry for a few minutes before beginning observations. No cover glass is needed, but the slide should be kept free from dust.

Place the prepared slide on the stage of the microscope and focus on it with the low power. Numerous pale yellow specks will be seen. These are red corpuscles. Some idea of their great numbers in the blood can be gained by comparing the area of the field of the microscope with that over which the original blood-drop was spread, and that drop, in turn, with the whole volume of blood in the body.

Change from the low-power objective to the high power. Note that by this change the field is much reduced. After obtaining a sharp image, study individual red corpuscles carefully. Compare the margin of a corpuscle with its center. The different appearance of the two regions signifies that the corpuscle is a disk thicker at the edges than in the center.

Look for groups of corpuscles arranged in rows, edge to edge. These are rouleaux. Corpuscles in shed blood tend to cling together thus.

By exploring the slide carefully, colorless corpuscles can be found and studied. They are transparent, colorless bodies about twice the diameter of red corpuscles. They are much less numerous than red corpuscles. The ratio is about 1 to 300 .

The various kinds of colorless corpuscles can be distinguished by their appearance after treatment with suitable stains. On prepared slides different types of colorless corpuscles can be studied.

Chemical Structure. Blood plasma* is an exceedingly complex

* The liquid part of clotted blood is called serum. That of unclotted blood is called plasma. 
liquid. It carries in solution all substances absorbed from the digestive tract; all waste products of cell activity; all hormones; and all of the great group of unidentified materials that are concerned with the control of infection. The most prominent constituents of blood belong in the chemical group of proteins.

Tests for Blood Proteins. The Xanthoproteic Reaction. Pour in a test tube concentrated blood serum to the depth of $1 / 4$ inch. Add 3 or 4 drops of concentrated nitric acid $\left(\mathrm{HNO}_{3}\right)$. (Handle with care.) Boil and cool. A yellow precipitate is formed and the solution becomes yellow. Add strong sodium hydrate $(\mathrm{NaOH})$. (Handle with care.) When sufficient has been added the color becomes much deeper.

The Biuret Reaction. Pour a little blood serum into a test tube. Add a few drops of very dilute copper sulphate $\left(\mathrm{CuSO}_{4}\right)$. Make alkaline with sodium hydrate $(\mathrm{NaOH})$. A rose color is produced. These two tests are characteristic for proteins.

Iron in Hemoglobin. The essential substance of red corpuscles is an iron-containing pigment compound, hemoglobin. The property of hemoglobin as an oxygen-carrier depends on its iron content.

A Chemical Test for Iron. Place a few iron filings in a test tube. Pour into the test tube, under the hood, a few drops of aqua regia (nitric and hydrochloric acids). The fumes of aqua regia are very irritating and the fluid is very corrosive. Handle with great care, always under the hood, and avoid inhaling the fumes. After allowing a few minutes for some iron to be dissolved, dilute with an inch of water and add a few drops of potassium ferrocyanide solution. A characteristic deep blue precipitate of iron ferrocyanide (prussian blue) is the test for iron.

To demonstrate the presence of iron in blood, place a few lumps of dried blood in a porcelain crucible over a bunsen flame under a hood. Continue heating the mass, stirring occasionally with a glass rod, until only a reddish ash is left. Allow the crucible to cool. Add a few drops of aqua regia. Warm gently. After the solution has cooled again dilute with water, pour into a test tube and add potassium ferrocyanide solution. The appearance of abundant prussian blue shows the presence of iron in the ash of blood. 


\section{Coagulation of Blood}

Draw a drop of blood, as described above, but do not remove it from the finger. Rest the hand in a comfortable position. Test the consistency of the blood-drop by drawing a hair through it Repeat the test at intervals of one minute till no further change occurs. Observe the changes that take place in the drop of blood during the process of coagulation.

Coagulation Time. When blood clots it sets first into a soft jelly which is firm enough, however, to support a small weight. Advantage is taken of this fact in the Dale Coagulometer. A fine glass tube, about $1 / 2$ inch long, is to be filled, by suction, with freshly drawn blood. A small shot placed in the tube will run along it when the tube is inclined so long as the blood is uncoagulated, but will remain stationary as soon as clotting occurs.

Congest the finger as above, and after two sharp prickings with a needle, near together, squeeze out a good sized drop of blood. Suck the fine tube nearly full. Insert the small shot as quickly as possible. The ends of the tube need not be plugged, since surface tension will retain the shot in the blood. Note the minute and second at which the blood was drawn. Thirty seconds thereafter hold the tube upright with the shot at the top. If the shot runs down repeat at thirty second intervals until it fails to move. The elapsed time is coagulation time. To check the result the experiment may be repeated.

The Importance of Calcium in the Coagulation Process. Prepare to repeat the above described experiment on coagulation time. After the blood is drawn and before sucking it into the tube sprinkle into it three or four grains of powdered sodium oxalate. This substance removes the calcium from the blood by precipitation. Continue the experiment for twice the coagulation time previously determined. If the blood has not then clotted discontinue the experiment. Interpret the result.

Coagulation Time in a Lower Animal. The experiment on coagulation time may be varied by using, instead of human blood, blood drawn directly from the vessels of a turtle, with brain destroyed and plastron removed, into the tube of the coagulometer. Determine carefully the coagulation time for the turtle. Compare with the coagulation time of human blood. 


\section{THE CIRCULATORY SYSTEM}

A good introduction to the study of the circulation is the dissection of the chief arteries and veins of the cat.

Preparation. Inject the arterial system with a starch mass colored red. To do this expose the heart and tie the nozzle of the injecting syringe directly into the tip of the left ventricle. The injection drives the blood into the veins so that they retain their natural blue color. Trace the vessels by tearing cautiously with the handle of a scalpel or some blunt instrument. Do not cut unless directions are given. Lay the thorax and abdomen wide open by a median incision.

Heart. The process of injection mutilates the heart. A separate exercise on the sheep's heart is described below.

\section{ARTERIES OF the Thorax}

The Aorta. This is a single great artery arising from the left chamber of the heart. It curves sharply to the left, thus making the arch of the aorta.

Coronary Artery. Two in number arising within the heart; they are small and the first branches seen. They supply the heart.

Innominate. This arises from the convexity of the arch very near its origin; it gives rise to the right and left carotid arteries.

Subclavians. The right subclavian is a continuation of the innominate; the left subclavian, the next large branch of the aorta, is given off close to the innominate.

Intercostal Arteries. These are seen, one below each pair of ribs.

\section{Arteries of the Abdomen}

Abdominal Aorta. This is a continuation of the thoracic aorta. Turn the stomach and intestines to the right, press upon the median line against the spinal column, and the injected aorta will be felt. Tear away the peritoneum and follow the vessel from the diaphragm and note branches.

Celiac Axis. As the aorta enters the abdomen there is given off a large branch, the celiac axis. This divides into three branches, the first being the gastric, which goes to the stomach. The second goes to the liver and is called the hepatic artery. Turn 
the liver upward, and near the lesser curvature of the stomach this vessel will be seen. The third and largest is the splenic.

Superior Mesenteric. Turn the stomach and intestines to the right. The artery rises from the aorta just below the celiac axis. It has an extensive distribution to the coils of the small intestine.

Renal Arteries. Rise from the sides of the aorta and enter the hilum of the kidneys.

Inferior Mesenteric. It arises from the abdominal aorta about opposite the iliac crest, and has two large branches which supply the large intestine.

\section{Arteries of the Lower Extremity}

Just before leaving the abdomen the aorta sends off four branches, two external iliacs and two internal iliacs, and then it becomes the caudal.

External Iliac. This passes downward a short distance and becomes the femoral artery, it runs down the leg as the femoral, and behind the knee it becomes the popliteal, which divides into the anterior and posterior tibial. The anterior tibial becomes the dorsalis pedis on the upper surface of the foot.

Internal Iliac. This arises from the aorta just below the origin of the preceding and passes obliquely downward into the pelvis, and supplies the organs of the pelvis with the following branches: vesical to bladder, internal pubic to internal genital organs, external pubic to external genital organs, sciatic, with gluteal branch, to muscles of back of pelvis, hip, and thigh.

Caudal Artery. A continuation of the aorta to the tail.

\section{Arteries of the Head and Upper Extremities}

Carotid Arteries. These arise from the innominate artery, a short distance above the arch of the aorta, and pass upward on either side of the trachea, supplying the neck and head. Follow one of these arteries forward, noting its branches.

Subclavian Arteries. The right subclavian is a continuation of the innominate. The left arises from the arch of the aorta. They supply the upper extremities. Follow the artery down one arm, note the vertebral, a branch running up the foramina in the transverse processes of the cervical vertebræ to the brain. The sub- 
clavian changes its name to axillary in axilla, brachial in upper arm, and divides at elbow into radial and ulnar.

These arteries give off branches to muscles and surrounding tissues in their course.

\section{Veins of the Extremities}

These veins follow the general course of the arteries and usually have similar names in their corresponding positions.

Common Iliac Veins. These form the inferior vena cava at a point opposite the junction of the sixth and seventh lumbar vertebræ, where the internal and external iliac veins which bring the blood from the leg and pelvis unite to form the common iliac vein.

Inferior Vena Cava. This is formed by the union of the common iliacs. Turn stomach and intestines to the left. It will be seen accompanying the aorta, and running to the right auricle of the heart.

Renal Veins. These extend laterally from the kidneys and empty into the inferior vena cava.

Portal Vein. Formed near the outlet from the stomach by union of veins from the stomach and intestines, and goes to the liver.

Veins of the Thorax. The superior vena cava is a prominent vessel extending from a point opposite the first rib to the upper part of the right auricle. It conducts the blood from the head and upper extremities back to the heart. It is formed by the union of the two innominate veins.

Innominate. Formed by union of the subclavian and jugular veins.

Subclavian. This returns the blood from the arm.

External Jugular. Returns the blood from head and brain.

\section{ANATOMY OF THE SHEEP'S HEART}

Removal of the Pericardium. At about the middle of the length of the heart, slit the pericardium and with the scissors girdle it completely. Remove the lower portion and note the smoothness of its internal surface. It and the apposed external surface of the heart are covered by a serous membrane which secretes a fluid during life. Turn the upper portion of the pericardium inside out, 
like the finger of a glove. At varying distances from the base it is attached to the heart and vessels. Trim the pericardium along or near the line of attachment.

General Topography of the Heart. The apex is conical, smooth, firm, and fleshy, and is formed by the muscular ventricles. Notice that the left ventricle is larger and has thicker walls than the right ventricle. The base is irregular and wider. It presents not only the thin-walled auricles but also vessels and fat.

The Vessels. The aorta and pulmonary artery maintain a cylindrical form and their cut ends are naturally circular. The great veins have thinner walls in proportion to their size and collapse more or less completely. The inferior vena cava forms nearly a right angle with the long axis of the heart. The superior vena cava is at the base on the ventral surface. The pulmonary artery is the prominent vessel on the ventral aspect between the two auricles, extending from the base of the right ventricle. The aorta, with its principal branch, will be seen more distinctly at a later stage.

Dissection of the Heart. The order of dissection follows the course of the blood through the organ. Bear in mind that, although anatomically united and acting together as muscles, as to their cavities, the right and left sides of the heart are entirely separated by complete partitions between the two auricles and between the two ventricles. When the cavities are open wash their interiors with running water. The flowing water will show how the valves close the openings between the cavities and also the action of both sets of semilunar valves.

Opening the Right Auricle. Hold the heart with its ventral side toward you. Push the point of the scissors into the upper and outer angle of the appendix and cut toward the median line of the heart. Lift the edge of the flap and note the wide mouths of the great veins. The right, the superior; the left, the inferior vena cava. In the back wall of the auricle at the base of the inferior vena cava will be found an oval scar, the fossa ovalis. Near the orifice of the inferior vena cava is a ridge, the eustachian valve.

Opening the Right Ventricle. This must be done very carefully. At a point on the ventricle at a short distance from the pulmonary artery, insert the point of a scalpel and cut parallel with the furrow, extending to the apex of the right ventricle. Keep the cut edges of ventricular walls apart while studying the cavity. 
Musculi Papillares. These are muscular columns attached at one end to the walls of the ventricle, and at the other to the chordoe tendinece. These are delicate tendinous cords which pass from the musculi papillares to the edge of the valve segments. The muscles and cords prevent the segments of the valve being forced into the auricle by the weight of the blood behind them.

The Tricuspid Valve. Pass the finger from the auricle into the ventricle and distend the auriculo-ventricular orifice. Note that it is surrounded by three fibrous sheets which hang down into the ventricle and are connected at the sides to the musculi papillares by the chordoe tendinea. This is the tricuspid valve.

The Pulmonary Artery. With the scissors, extend the incision upward through the pulmonary artery and note that the mouth of the artery is surrounded by three membranous cups, the semilunar valve. Each constitutes a sort of pocket, and all three together when distended close the opening of the artery completely.

Opening the Left Auricle. Make an incision in the appendix so as to see the interior of the auricle. Note the two portions, the appendix and the atrium. It resembles the right auricle.

The Pulmonary Veins. Hold the heart so as to see the depth of the atrium and note that it presents a ridge at right angles to it. Hold the organ to the light and note the openings of the pulmonary veins near the ridge.

Opening the Left Ventricle. With the scalpel transect the left ventricle, carrying the incision to the apex. The left ventricle forms the apex of the heart. The auriculo-ventricular opening of this side is surrounded by the mitral or bicuspid valves. Note the chordoe tendinece and musculi papillares.

The Aorta. Pass a probe into the aorta and follow the course of the probe with the scissors, thus opening the aorta. Note the semilunar valves guarding its orifice. Note the openings of other blood vessels from the aorta, the coronary arteries, just above the valve. Observe the smooth lining of the aorta, as well as of the auricles and ventricles.

\section{THE CIRCULATION OF BLOOD IN THE FROG'S FOOT}

A live frog is wrapped carefully in moist cloth except one leg. The projecting foot is secured on the microscope stage in such 
fashion that a portion of the web is in the field. Study the field with the low power.

Note the rate and character of the blood flow in different vessels. The larger vessels are either arteries or veins. In arteries the blood flow is intermittent, and the rate of pulsation agrees with that of the heart-beat. In veins the flow is more or less steady. The smallest vessels are capillaries. These communicate, in general, between arteries and veins. In the frog's web, however, the circulation is anastomosing. In such a circulation some capillaries can be seen connecting one vein with another. The direction of flow in anastomosing capillaries is sometimes forward, and sometimes backward.

Study a region showing arteries, veins, and typical, non-anastomosing capillaries.

\section{THE CIRCULATION OF BLOOD IN MAN}

Pulse Tracings. To obtain a graphic record of the pulse the pressure changes in the beating artery are transmitted to a delicate recording device known as a tambour. For the transmitter a thistle tube, with sheet rubber tied tightly, without stretching, over the mouth, may be used. Secure a bone or wood button to the center of the rubber. Connect the transmitter with the recorder by a rubber tube. Insert in the course of this tube a glass $\mathrm{T}$ with a short rubber tube on the side neck.

Gently press the button on the transmitter on the front surface of the wrist directly over the point where the pulse can be felt in the radial artery. The carotid artery in the neck may be used if preferred. When the system is made air tight by closing the side tube the tambour lever should pulsate in synchronism with the artery. Obtain a record of these pulsations on a drum moving at moderate speed. The notch in the descending limb of the pulse curve marks the closing of the semilunar valves, and therefore the end of systole.

Determinations of Pulse Rate. In these determinations two students should alternate as subject and observer. Trustworthy readings cannot be made upon oneself. The pulse should be taken by pressing the tips of three fingers against the radial artery at the wrist. Do not use the thumb. 
With the subject sitting quietly in a comfortable position count the pulse during the first twenty seconds of three consecutive minutes. Compute the rate per minute for each minute. If there is much variation wait three minutes and try again for three minutes. When the pulse is reasonably steady for three consecutive minutes the average rate may be taken as the pulse rate for that subject in that particular condition.

The Effect of Posture. Using the method described above determine the pulse rate with the subject lying down, sitting, and standing.

The Effect of Exercise. Determine the pulse rate with the subject in the sitting position. When the rate is uniform let the subject raise and lower his legs six times without rising from his seat. Count the pulse for twenty seconds as quickly as possible after the movement ceases.

This shows the effect of slight exercise.

After the pulse rate has returned to normal and become steady let the subject run up and down stairs for two or three minutes. Count the pulse for twenty seconds as soon as possible after he returns to his seat and at two-minute intervals for ten minutes thereafter.

This shows the effect on pulse rate of vigorous exercise.

The Direction of Blood Flow in Arteries and Veins. Expose the arm to the shoulder. Find in the upper arm a place where the pulse can be felt. Learn by practice to occlude the artery by pressing it against the arm bone. Now with one hand on the artery in the upper arm and the other on the artery at the wrist determine the direction of arterial flow by showing which pulse disappears when the other artery is occluded.

Occlude a prominent vein on the hand or forearm by pressing upon it with a finger.

Observe on which side of the occluded point the vein becomes congested.

Attempt to empty the vein above and below the occluded point by pressing a finger along it.

Note on which side of the point this can be done.

Thus is determined the direction of venous flow.

The Control of Hemorrhage. Bleeding can be checked by com- 
pressing the ruptured blood vessel on the side of the injury from which blood comes.

Arterial bleeding can be distinguished from venous by the brighter color of the blood and by the fact that the blood escapes in jets. When bleeding is from veins the flow is steady and the blood is dark.

Veins which are likely to be injured are on the surface. A bandage tied tightly around the arm or leg in proper position relative to the injury usually suffices to check venous bleeding. Select a point on the arm as a possible seat of injury. Adjust.a bandage in such a position as to cut off venous flow to the chosen point. Show on a diagram the position of injury point and of bandage. Indicate direction of venous flow by arrows.

Arteries are deep seated through most of their course, and blood pressure within them is high. To check arterial bleeding strong pressure on properly selected points is necessary. Expose the entire arm. Find a point near the upper and inner margin of the biceps muscle where the pulse can be felt. Strong pressure on this point if properly applied will check arterial bleeding below. For a more permanent check make a hard knot the size of an egg in the middle of a handkerchief. Tie firmly around the arm just above the elbow with the knot in front. Bend the forearm so that it presses hard against the knot. If the procedure is successful the pulse at the wrist disappears. Another method is to make a hard knot of cloth the size of a fist. A round stone or other hard substance may be used. Push it hard into the arm pit. Bring the elbow straight down and hold it firmly against the side. The success of the procedure should be tested by observing whether the pulse at the wrist disappears. (For the control of bleeding in other regions than the arm see Dulles: Accidents and Emergencies, Philadelphia, 1899.)

The Effect of Muscular Movement on Venous Flow. Tie a cloth tightly about the arm at the elbow. Note the rate at which the superficial veins become congested. Loosen the cloth until the veins return to normal. Replace the cloth about the arm and close and open the hand several times.

Compare the rate of venous congestion with that seen in the quiet arm. 


\section{HEART BEAT AND BLOOD PRESSURE IN THE TURTLE}

A turtle whose brain has been destroyed and plastron (lower shell) removed, is fastened to a board back down, and with neck extended. The heart can be seen beating through its enclosing membrane, the pericardium. With a fine scissors cut away the pericardium, taking care not to injure the heart. The turtle has two auricles and a single ventricle. Identify these. Determine by careful observation the sequence of beat of the different chambers.

Compare the periods of systole and diastole.

In the turtle the great veins pulsate as well as do auricles and ventricle. To observe these veins the ventricle must be lifted out of the way. Find the connective tissue frenum which attaches the tip of the ventricle to the pericardium. Cut this as far from the heart as possible. Seize the frenum with a pair of forceps and by means of it lift the ventricle till the underlying veins can be seen. The beat is seen to originate in these veins whence it sweeps over the heart in the form of a wave.

Vagus Inhibition. Find the carotid artery where it passes up the neck. Associated with it is a nerve, the vagus. Expose carefully a short portion of this nerve and stimulate it with repeated induction shocks. In a normal preparation one or the other vagus nerve contains inhibitory fibers whose stimulation will stop the heart.

Note whether the standstill occurs in systole or in diastole.

Graphic Record. Catch a pin-hook either through the frenum or through the very tip of the ventricle. Connect this hook by thread or fine wire to the short arm of the heart lever, which should be directly above the heart. Obtain on a slowly moving drum a record of the heart beat. While the record is being traced stimulate the vagus nerve. Indicate on the record the period of stimulation.

Determine carefully the interval between the beginning of stimulation and the cessation of beat. Note also how long it takes the heart to recover after cessation of vagus stimulation.

The Effect of Nicotine. By means of a camel's hair brush apply a few drops of nicotine solution to the surface of the heart. In two minutes repeat the vagus stimulation. The inhibition is no 
longer effective. The junctions of preganglionic and postganglionic neurons of the vagus path are in the heart tissue itself (p. 194).

Arterial Pressure. Disconnect the heart from the recording lever. If the heart is beating feebly or very slowly bathe with warm water. If no response take a freshly prepared turtle. Trace one of the large arteries to a point at least one inch from the heart. At this point separate the artery carefully from surrounding tissues for another inch. Squeeze the artery shut at the cardiac end of the prepared portion by means of a spring clip. Pass a stout thread around the prepared artery. Fill with salt solution a small L-shaped glass tube, 15 inches high. Hold the solution in the tube by closing the long end. Make a transverse cut halfway through the prepared artery, slip the short end of the glass tube through this out into the artery and tie it in place by means of the thread previously prepared. The tube may now be opened and the clip removed from the artery. The height at which the column of salt solution is maintained measures arterial pressure.

\section{THE RESPIRATORY SYSTEM}

Dissection of Air Passages and Lungs of Sheep. Note the large trachea (windpipe) with open rings of cartilage in its wall. Observe carefully the surface of the lung. Note the cellular appearance and the delicate texture. Note the subdivision of each lung into lobes.

Inflate the specimen.

Dissection. In one lung, follow the trachea down the bronchus to its smallest branches.

Starting at the heart follow the pulmonary artery and vein to their finest branches. Use a probe and scissors.

On the other lung tear away the tissues so as to show the interlacing of these vessels. Use the forceps to pick with.

Miscroscopic Study. The final subdivisions of the bronchi, and their terminations in infundibula and alveoli, are microscopic. Observe in prepared and stained sections the relatively thick walled bronchioles, and the extremely delicate walls of the air cells (infundibula and alveoli). The capillaries which run in the alveolar walls cannot be seen in ordinary microscopic sections.

Breathing Movements in Man. The following observations may 
be made by a group of students on a single subject. Let the latter strip the upper part of the body to the undershirt. With the subject sitting erect on a stool observe the chest and abdomen closely during quiet breathing.

Note the general direction of movement of the body walls during inspiration, and during expiration.

Observe the movements of chest and abdomen during forced inspiration and expiration.

Abdominal movements are caused by contraction and relaxation of the diaphragm. Explain the relationship.

Costal and Diaphragmatic Breathing. Whereas in normal involuntary breathing the diaphragm and the chest muscles are in action simultaneously, it is possible in voluntary breathing movements to use one or the other at will. Compare abdominal movements in normal breathing and in breathing with the chest held stationary (diaphragmatic breathing). Compare chest movements in normal breathing and in breathing with abdomen held stationary (costal breathing).

Volumes of Respired Air. These are:

1. Volume passing in and out in quiet breathing - tidal air.

2. Volume that can be breathed in after a normal inspiration-complemental air.

3. Volume that can be breathed out after a normal expiration - supplemental air.

4. Sum of above: volume that can be breathed out after forced inspiration-vital capacity.

Determine the supplemental air by blowing into a spirometer after normal expiration. Determine tidal air by blowing into the spirometer after normal inspiration and subtracting previously determined supplemental air. Make several trials. Determine vital capacity by blowing into spirometer after forced inspiration.

Determine complemental air by subtracting combined supplemental and tidal air from above.

Graphic Record of Breathing. For recording breathing an apparatus known as a pneumograph is fastened around the chest so that its volume will be changed by breathing movements. It is connected with a recording tambour which indicates on a kymograph the movements of breathing. 
With the pneumograph in place and the back to the apparatus get a record of normal breathing.

Note the rate per minute.

Sip the contents of a glass of water. Observe the effect of swallowing on breathing.

Obtain records of reading aloud, of coughing. Compare these with normal, quiet breathing.

The Control of Breathing. The students of a pair should act alternately as subject and observer in the following tests. Obtain, by counting the breathing movements, the normal rate per minute. Let the subject run down and up stairs. Determine the rate per minute every three minutes till the normal rate is restored.

Determine how many seconds the subject can hold his breath. Note the difference according to whether he begins to hold it at the end of inspiration or the end of expiration.

Let the subject breathe rapidly and deeply for two or three minutes and then hold the breath.

Note the time of holding it as compared with the former trials.

Repeat the experiment, this time breathing into and from a paper bag. Compare the time the breath can be held with former trials.

The automatic stimulus for breathing depends on the amount of carbon dioxide in the blood, and indirectly on the amount in the alveoli of the lungs. The experiments on breath-holding can be explained on this basis.

Artificial Respiration. The Schafer Method. In this experiment the subject should cease breathing so far as possible. The treatment may be considered successful when no active breathing movements are necessary.

Lay the subject prone, with a thick roll of clothing under the chest and the epigastrium. Take a position over or beside the subject's legs, and facing his head. Place the hands on either side over his lowest ribs. Slowly throw the weight of the body onto the hands, and thus compress the subject's thorax and force air from the lungs. Without removing the hands, release the pressure. The chest by its own elasticity will perform the function of inspiration. Repeat this procedure at the normal rate of respiration until the issue is determined. In case of drowning it may be a half hour before respiration is restored. Schafer's 
method is specially applicable to cases of drowning because the face is downwards and water in the air passages readily runs out.

Carbon Dioxide $\left(\mathrm{CO}_{2}\right)$ in Expired Air. Dip a tube below the surface of a bottle containing lime water $\left(\mathrm{CaO}_{2} \mathrm{H}_{2}\right)$. Exhale through the tube. The $\mathrm{CO}_{2}$ in the expired air combines with the lime water in the bottle and forms an insoluble carbonate of lime $\left(\mathrm{CaCO}_{3}\right)$ which makes the solution cloudy, later this will settle as a precipitate (powdered chalk).

When present in excess the $\mathrm{CO}_{2}$ makes the solution acid and the $\mathrm{CaCO}_{3}$ redissolves.

\section{THE DIGESTIVE SYSTEM}

\section{Dissection of the Digestive System in the Cat}

Exposure of the Viscera. Make an incision the length of the abdomen in the mid line. Separate the edges of the opening so as to get a good view of the abdominal contents.

Peritoneum. This is a membrane lining the abdomen. It gives the abdominal wall a smooth glistening appearance and may be easily separated from the muscles forming the walk. The mesenteries and the ligaments of the liver, the bladder and uterus are formed by duplicatures of the peritoneum.

The Great Omentum. This is a double fold of peritoneum forming a sac which is called the lesser peritoneal cavity. It is attached to the posterior abdominal wall and the greater curvature of the stomach. Demonstrate the sac-like character of the omentum by tearing it open. Each wall of the sac is also composed of two layers. Notice the distribution of fat through the omentum.

Spleen. This is a deep red, usually single-lobed organ, situated on the left of the stomach in the great omentum.

Drawing. Make a drawing without disturbing anything, showing position of liver, stomach, spleen, and great omentum covering the coils of small intestine.

Stomach. Turn the left lobe of the liver toward the head and the abdominal œsophagus will be seen emerging from the diaphragm and entering the cardiac end of the stomach. The stomach, as a whole, is pear-shaped and curved upon itself. The great curvature is at the lower border of the stomach, and has the great omentum attached to it, while the lesser curvature is the upper border. The 
larger or cardiac end is next to the diaphragm. The pyloric or smaller end is curved sharply upon itself. It is firm to the touch and appears as an annular constriction.

Small Intestine. Very carefully turn the omentum over toward the thorax. The greatly coiled cylindrical small intestine will be exposed. It is divided into three regions, the duodenum, the jejunum, and the ileum.

Duodenum. This is the first portion of the small intestine along which the pancreas extends. It is held rather firmly in position. Into the duodenum empty the common bile duct and the pancreatic duct.

Jejunum. This is an ill-defined portion of the small intestine immediately following the duodenum. It is so called because in man it is often found empty after death.

The Ileum. This is the last part of the small intestine. It terminates in the large intestine, entering it obliquely. At its termination is the ileo-coecal valve which allows the alimentary contents to pass from the small to the large intestine, but not easily in the opposite direction.

Large Intestine. Turn the coil of small intestine toward the left leg. The large intestine extends from the cæcum to the anus. It is divided into five parts,-crecum, ascending, transverse and descending colon, and rectum.

Coecum. This is a somewhat conical blind sac at the beginning of the large intestine. It lies on the right side and in about the middle of the abdominal cavity.

Ascending Colon. This is the part of the large intestine which extends upward from the cæcum.

Transverse Colon. This is a continuation of the preceding. It extends transversely across the abdomen in front of the duodenum and below the stomach.

Descending Colon and Rectum. After extending nearly across the abdomen from right to left, the large intestine passes obliquely downward. The last and straighter part is called the rectum.

Pancreas. The pancreas will appear as a pinkish, finely lobulated and elongated body after the great omentum has been turned toward the thorax. It extends from the spleen under the stomach to the pylorus, in the great omentum, and then downward for a short distance along the duodenum in the mesentery. 
Mesentery. This is a duplicature of peritoneum supporting the different portions of the small intestine. It is a double-walled membrane and carries blood-vessels and lymphatics.

Mesenteric Glands. The so-called mesenteric glands belong to the lymphatic system. They are between the layers of the mesentery and are especially large near the cæcum.

Internal Structure of the Stomach. Open the stomach and wash out its contents. It is composed of a muscular coat covered by the peritoneum, and an internal, mucous coat, which is thrown into folds or rugee.

Interior of the Small Intestine. Open and wash in water. It is composed of three coats like the stomach. It has a velvety feel due to villi, which are microscopic finger-like processes found only in the small intestine and most abundantly in the upper part.

Interior of the Cacum. Open the cæcum and observe the ileocæcal valve.

Interior of the Large Intestine. Wash the contents out. The structure of the large intestine is like the small, excepting that it has no villi.

The Liver. The liver is a deep red and multi-lobular organ occupying nearly all the upper part of the abdomen but especially the right side. It is supported in various parts by folds of peritoneum. It is composed of right and left lobes, each of which is subdivided into smaller lobes by fissures. The cystic lobe is one of the divisions of the right lobe near the front, and contains the gall-bladder.

If good sections are available the gross study outlined above may be followed by microscopic studies of the structure of the stomach wall, the small intestine, salivary gland, pancreas, and liver.

\section{STUDY OF FOODS}

Carbohydrates. The food carbohydrates are starch, glycogen, dextrin, double sugars, single sugars.

The following experiments illustrate tests for the different carbohydrates, and the application of these tests to different foods to determine which of the carbohydrates is present.

Test for Starch. To a solution of starch add a drop or two of a solution of iodine. A deep blue color shows the presence of starch. Various foods such as potato, bread, egg white, may be tested 
for starch by adding hot water to a small amount of each (solids), and then, after cooling, applying the iodine test.

Dextrin. This is an intermediate product in the conversion of starch into sugar, and during the change different forms of dextrin are produced.

Test for Dextrin. To a solution of commercial dextrin add a few drops of a solution of iodine. A reddish color is the dextrin test.

Tests for glycogen, single sugars, and lactose (milk-sugar) are described above (p. 588). Cane-sugar (sucrose) does not give the Fehling test. It can be split into single sugars (dextrose and levulose) by boiling with a mineral acid, and the sugars thus produced will respond to the Fehling test. Tests for proteins and fats are described above (p. 589).

\section{STUDY OF DIGESTION}

Salivary Digestion. Add to an inch of dilute starch solution in a test tube a quarter of an inch of saliva. At four minute intervals test a few drops of this mixture with dilute iodine solution. Note the time it takes for the appearance of dextrin, and then for the disappearance of the dextrin.

The nature of the final product of the salivary digestion of starch may be shown by keeping a test tube of starch solution and saliva, as prepared above, at a temperature of $40^{\circ} \mathrm{C}$. for 30 minutes or more. Fehling's test will show the presence of sugar.

Gastric Digestion of Protein. Gastric digestion proceeds slowly. Allow ample time for the following observation; a good plan is to begin the experiment one day and complete it on the next.

Fill each of four test tubes about one-third full of water. Add to the first a few drops of commercial pepsin solution; to the second ten drops of a $0.5 \%$ solution of hydrochloric acid; to the third and fourth both pepsin and acid. Place in each tube a cube of boiled egg white. The cubes should be approximately the same size. Mark the tubes and place numbers one, two, and three in a thermostat at body temperature, number four in a cool place. Shake each tube at intervals. Several hours later examine all the tubes for evidences of digestion. It will be found that acid pepsin is essential, and warmth desirable for gastric digestion.

Movements of the Stomach. Expose the stomach of a recently 
killed frog. Tie a ligature about the pyloric end. Make an opening into the stomach at the cardiac end and by means of a pipette fill the stomach with $0.7 \%$ salt solution. Tie a second ligature so as to close the opening into the stomach, and cut the stomach from the body.

By means of a thread attached to the pylorus hang the stomach so that the cardiac end just dips into a solution of $0.7 \%$ sodium chloride and $0.1 \%$ sodium carbonate. Look for the peristaltic waves passing over the stomach. Note the time required for a single wave to pass, and the number of waves per minute. This observation is more striking in early fall or late spring than in mid winter when the frogs are in the midst of hibernation.

Absorption from Stomach and from Small Intestine. Lay bare the stomach and intestines of a large turtle whose brain has been destroyed and plastron removed without loss of blood.

Using great care to avoid tearing the mesentery (supporting membrane of stomach and intestines) find the points of union of stomach and intestine and of intestine and rectum. Tie stout threads tightly about these points.

Find the junction of esophagus with stomach. Place a thread about the junction ready for tying. Make an opening into the esophagus above the thread. Introduce into the stomach through this opening by means of a graduated pipette, water to moderate distension. Note the exact amount of water introduced. Withdraw the pipette and tie off the junction with care that no water escapes.

Place a thread ready for tying about the intestine one-half inch below the one previously tied about the point of union of stomach with intestine. Introduce a known amount of water into the intestine through a hole made just above the thread last placed. Tie this thread. Allow absorption to proceed for one hour. At the expiration of the period of absorption cut between the threads tied about the upper end of the intestine and dissect out the stomach and intestine. Empty each into a separate vessel. Compare the amount of water introduced with the amount recovered. If the mesentery is intact and the circulation good the intestine will be virtually empty at the end of an hour. The stomach will contain practically the entire amount introduced. Similar tests may be made with solutions of -dextrose. 


\section{METABOLISM}

The Influence of Muscular Exercise on Carbon Dioxide Production. Make the first observation described below after at least one hour of relative inactivity.

Fill a large wide-mouthed bottle with water and invert over a reservoir of water. Insert a good sized rubber or bent glass tube into the neck of the bottle. Place the other end in the mouth. Hold the nose during each expiration and blow all the expired air into the bottle. Note carefully the number of expirations and the time required for filling the bottle with expired air. Cork the bottle tightly and turn right side up.

Fill a narrow test tube with strong sodium hydroxide solution. Cork. Tie a thread about the test tube and by means of this thread lower the tube carefully into the bottle of expired air. Restopper this bottle tightly and then by jarring it break the test tube, liberating the alkali. Shake thoroughly. The alkali takes up the carbon dioxide. Invert the bottle again over the reservoir of water. With the neck under water remove the stopper. Water rushes in to replace the absorbed carbon dioxide. Lower the bottle till the water is at the same level inside and outside. With the bottle in this position replace the stopper. The volume of water enclosed equals that of the carbon dioxide in the entire bottle of expired air. Determine this volume with the aid of a graduated vessel. Calculate the volume of carbon dioxide exhaled with each breath and also the volume exhaled per minute.

Rinse the bottle out thoroughly to remove all traces of sodium hydroxide. Take several minutes of very brisk exercise. As quickly as possible after the cessation of the exercise repeat the experiment above. Care must be taken that all the air expired during the period of collection enters the bottle. The augmented breathing makes this a matter of some difficulty. There are numerous sources of error in this experiment, but if carefully performed it demonstrates a marked increase in the carbon dioxide production per minute with exercise, and a suggestion as to the amount produced.

A Study of Urine. Urine is the chief excretion of the body. It contains the greater portion of the end-products of protein metab- 
olism and is also the medium in which the accessories of the diet are discharged from the body.

The chief end-products of protein metabolism are urea, creatinin, uric acid, and ammonia. Among the chief excreted accessories are (a) inorganic: water, and sodium, potassium, calcium, and magnesium chlorides, sulphates, and phosphates; (b) organic: nonnutrient constituents of food which serve to give it flavor; drugs. Examples of this class of excreta are the purin bodies, which represent the excreted alkaloids of tea, coffee, and cocoa; and the substance which gives urine its peculiar odor after the eating of asparagus.

Test for Urea. To 15 c.c. of urine add $1 / 2$ its volume of baryta mixture to remove inorganic constituents which would interfere with the test. Filter. To a portion of the filtrate add a solution of mercuric nitrate, a precipitate forms which is a compound of urea and mercury.

Test for Creatinin. To 8 c.c. of urine add 2 c.c. of a solution of sodium nitro-prussiate, then 1 c.c. $\mathrm{NaOH}$; a red color appears. Boil the solution. The color fades; while boiling add about 1 c.c. of acetic acid; the color changes to blue.

Test for Ammonia. To 4 c.c. of fresh urine in a test tube add a little dry sodium carbonate. Heat. Hold in the neck of the test tube, without touching the sides, a strip of moistened, neutral litmus paper. The blue coloration shows the presence of ammonia gas.

Test for Chlorides. To 4 c.c. of urine add an excess of nitric acid $\left(\mathrm{HNO}_{3}\right)$, then a drop or two of silver nitrate solution $\left(\mathrm{AgNO}_{3}\right)$.

Test for Sulphates. Add to 4 c.c. of urine a few drops of barium chloride solution and then an excess of $\mathrm{HCl}$. The latter redissolves the phosphates, leaving the sulphate of barium alone in the precipitate.

Test for Phosphates. Make 10 c.c. of urine alkaline with ammonium hydroxide $\left(\mathrm{NH}_{4} \mathrm{OH}\right)$. Heat. The precipitate that forms is a mixture of calcium and magnesium phosphates. Filter. To the filtrate add a small amount of magnesia mixture $\left(\mathrm{MgSO}_{4}\right.$, $\mathrm{NH}_{4} \mathrm{Cl}$, and $\mathrm{NH}_{4} \mathrm{OH}$ in water). Heat. The precipitate is due to the presence of sodium, potassium, and ammonium phosphates.

Test for Purin Bodies. Add to 10 c.c. of urine an excess of magnesia mixture. Filter off the precipitate of phosphates. Add 
to the filtrate ammoniacal silver nitrate solution. The precipitate consists of the silver salts of the purin bodies, and will be more abundant after tea, coffee, or cocoa have been taken.

\section{A STUDY OF MILK}

This is a secretion of the mammary gland and contains Protein, Fat, Carbohydrate (Sugar), and salts in solution.

The opaque white appearance of milk is due to the presence in it of a protein, caseinogen. This may be precipitated out with acid or by the enzyme rennin. In the latter case the caseinogen combines with some of the calcium of the milk forming casein.

Fill each of two test tubes $1 / 3$ full of milk and add an equal volume of water.

To one of the tubes add five drops of hydrochloric acid $(\mathrm{HCl})$; to the other ten drops rennin solution. Allow both tubes to stand in a water bath at body temperature.

After twenty minutes filter the contents of both tubes. Make the following tests on each.

Demonstrate the presence of protein in the precipitate (curd) with the xanthoproteic test.

Test for fat with osmic acid.

Apply the biuret test to a few drops of the filtrate (whey).

Test 2 c.c. of filtrate for sugar with Fehling's test.

Add to 5 c.c. of the filtrate three drops ammonia $\left(\mathrm{NH}_{4} \mathrm{OH}\right)$, and 5 drops sodium oxalate solution. A white precipitate proves the presence of calcium in the filtrate. A careful comparison of acid whey with rennin whey will show that the former contains more calcium. 



\section{INDEX}

Abdominal cavity, 4; contents of, 5 . Abdominal respiration, 398.

Abducens nerve, 152.

Abduction, 72, 74 .

Aberration, chromatic, 262; spherical, 263.

Abortion, danger of, 579 .

Absorption, 491; of carbohydrates, 492 ; channels of, 492; nature of, 492; of proteins, 498; of fats, 499; from small intestine, 491; from stomach, 491; from large intestine, 500.

Accessories of diet, 429; inorganic, 430; organic, 431.

Accessory reproductive organs, 562 .

Accommodation, 260.

Acetabulum, 65, 73, 74.

Acetic acid, 16.

Achromatic lenses, 263.

Achromatic spindle, 25.

Acid, acetic, 16; animo, 11, 463; butyric, 16 ; fatty, 463; formic, 16 ; glychocolic, 518; hydrochloric, 19, 464 ; lactic, 16, 90, 92, 435; taurocholic, 518; uric, 14, 526.

Acidosis, 510.

Acromegaly, 50.

Action currents, 103.

Activity, maintenance of, 38 .

Adam's apple, 547.

Adaptation, 25, 42.

Adaptive systems, 37 .

Addison's disease, 199.

Adduction, 72, 74 .

Adenoids, 228, 383.

Adenoid tissue, 304.

Adipose tissue, 44.

Adrenals, 199.

Adrenin, 199, 319; effect of, on vascular system, 381 .

Afferent nerve paths, 170.

After birth (placenta), 578 .

After images, 281.

Agglutinins, 309.

Air, composition of, 411; changes in when breathed, 411; quantity breathed daily, 398 .

Albumin, 12; serum, 302.

Albuminoid, 12; nutritive value of, 510.
Albuminuria, 471.

Alcohol, 437.

Alexin, 308.

Alimentary canal, 4, 39; general arrangement of, 442 ; blood-vessels of, 461 ; subdivisions of, 442 .

Alimentary glycosuria, 494 .

Allantois, 578.

Alveoli of lungs, 388, 389.

Ameba, 21.

Ameboid movements, 301 .

Amenorrhea, 573.

Animo acid, 11, 463.

Ammonia compounds, 517.

Ampulla, 232.

Amylopsin, 465.

Anal opening, 455.

Anaphylaxis, 311.

Anatomy, definition of, 1.

Anatomy, of alimentary canal, 442; of brain, 145; of ear, 226; of eye, 248 ; of joints, 72 ; of lymphatic system, 382; of nervous system, 138; of respiratory organs, 388; of skeleton, 53 ; of skin, 529 ; of urinary organs, 518; of vascular system, 322.

Anemia, 298.

Animal heat, sources of, 541 .

Animal, normal compared with "reflex," 169 .

Animals compared with plants, 40 .

Ankle bones, 66 .

Antiperistalsis, 478.

Antithrombin, 317.

Antitoxin, 309; uses of, in disease, 310.

Anus, 455.

Anvil bone, 229.

Aorta, 328, 331; abdominal, 330; thoracic, 330; branches of, 331 .

Apex beat of heart, 340 .

Aphasia, 188.

Apnea, 406.

Apparatus, lachrymal, 246.

Appendages of eye, 244.

Appendicular skeleton, 64.

Appendix, vermiform, 455 .

Appetite, 209.

Aqueous humor, 254.

Arachnoid, 5, 141; space, 142.

Arborization, terminal, 137. 
Arc, reflex, 158; variability in, 159.

Areas of cerebrum, association, 181; motor, 177; sensory, 177.

Areola, 580.

Areolar tissue, 41.

Arm, skeleton of, 66 .

Arterial blood, 336; color of, 417.

Arterial pressure, 362 ; influence of capillary resistance on, 364 ; influence of heart-rate on, 363; measurement of, 367 .

Arterial system, 330 .

Arterioles, 332.

Artery, axillary, 330; brachial, 330; bronchial, 331; carotid, 330, 331; celiac, 331, 461 ; coronary, 328, 330; femoral, 331; hepatic, 457, 461; iliac, 330, 331; innominate, 330; intercostal, 331; mesenteric, 331, 461; popliteal, 331; pulmonary, 326, 333; radial, 330; renal, 331; splenic, 461 ; subclavian, 330 ; temporal, 331 ; tibial, 331 ; ulnar, 330 ; vertebral, 330 .

Artery, 332; structure of, 336.

Articular cartilage, 73.

Articulations, 71 ; of skull, 61.

Artificial respiration, 408.

Aryteno-epiglottic fold, 548.

Arytenoid cartilages, 547.

Arytenoid muscles, 551 .

Asphyxia, 407.

Aspirates, 554.

Aspiration of thorax, 370, 399 .

Assimilation, 21.

Assimilation limit, 494.

Association areas of cerebrum, 181; fibers of cerebrum, 176 .

Association, nature of, 181.

Association neurons, 137.

Associative memory, 182; functions of, 183; interactions of, 184 .

Astigmatism, 264.

Astragalus, 66.

Atlas, 58, 75.

Attraction sphere, 24.

Auditory apparatus, 62.

Auditory area of cerebrum, 177.

Auditory nerve, 152.

Auditory ossicles, 229; functions of, 230.

Auditory perceptions, 235.

Auerbach's plexus, 456.

Augmenter center, 352; nerves, 351.

Auricle, 325; function of, 345.

Auriculo-ventricular valves, 328 .

Auscultation of lungs, 397.

Automatic rhythmicity of heart, 347; nature of, 350.
Autonomic nervous system, 139, 154, 193; divisions of, 195; reflex control of, 194; cranial, 195; sacral, 195; thoracico-lumbar, 195; in relation to emotions, 197.

Axial current, 356 .

Axial ligament, 230.

Axillary artery, 330.

Axis, 58, 75.

Axis, visual, 275.

Axon, 136; collaterals of, 160.

Bacterial digestion, 466.

Ball-and-socket joints, 74 .

Basal metabolism, 506.

Basement membran๑, 480, 566.

Basilar membrane, 233.

Bathing, 537.

Beat, "apex," 340.

Beat of heart, 339 .

Beef tea, 90 .

Beri-beri, 431.

Biceps, 79, 82 .

Bicuspid teeth, 444.

Bile, 457, 465; capillaries, 460; control of, 487; duct, 458; pigments, 14, 518; acids, 518; salts, 518.

Bilirubin, 14, 518.

Biliverdin, 14, 518 .

Binocular vision, 287.

Biological chemistry, definition of, 9.

Biuret reaction, 11.

Blackness, sensation of, 276.

Bladder, urinary, 39, 518.

Bleeders, 319.

Blind spot, 269.

Blood, 39, 292; of animals other than man, 303; arterial, 336; carbon dioxid of, 423; changes in, in lungs, 416; chemical composition of, 295; coagulation of, 312 ; course of, 334 ; distribution of, in body, 373; fibrin, source of, 315 ; functions of, 292; gases, 417; microscopic characters of, 295; oxygen interchanges in, 420; plates, 302; plasma, 295, 302 ; quantity of, 303 ; reaction of, 295; serum, 312; specific gravity of, 295; structure of, 295; transfusion, 320 ; venous, 336 ; whipped, 313.

Blood-clot, 312.

Blood-corpuscles, 295; colorless, 301; red, 295.

Blood-fibrin, 313.

Blood-flow, rate of, 368 .

Blood-flow, see Circulation.

Blood-plasma, 295, 302.

Blood-plates, 302.

Blood-pressure, 362 ; in man, determination of, 368 ; measurement of, 367 . 
Blood-vessels, 35, 39, 322.

Blood-vessels of alimentary tract, 461.

Blood-vessels, nerves of, 373 .

Body, composition of, 8; compounds in, 9 ; elements in, 9 ; microscopic structure of, 7 ; physico-chemical constitution of, 16; physiological properties of, 21; liquid environment of, 16; water in, 10; levers in, 119 ; food requirements of, 500; liberation of energy in, 505; protein requirement of, 500 ; temperature of, 540 .

Body fat, source of, 515 .

Body senses, 163, 172; tracts of, 172 .

Body sense area, 177.

Body temperature, 540.

Bone, $31,46,49$; chemistry of, 49 ; formation of, 46,51 ; structure of, 47 ; repair of, 48,71 .

Bones, of cranium, 60 ; of face, 60 ; of limbs, 66; of pectoral arch, 64 ; of pelvic girdle, 64 ; of skull, 60 ; of vertebral column, 55 .

Bony labyrinth, 231.

Botulism, 441.

Bow legs, cause of, 51 .

Brachial artery, 330 .

Brachial plexus, 148.

Brain, 5, 36, 138, 145, 174; membranes of, 138; nourishment of, 190 ; ventricles of, 142 ; convolutions of, 176; white and gray matter in, $174,175$.

Brain stem, 192.

Bread, 436.

Breast, 579.

Breast-bone, 64.

Breath, holding, 406.

Breathing, 391; forced, 396; hygiene of, 398.

Broad ligament, 567, 568.

Bronchial artery, 331.

Bronchial tubes, 39,388 ; structure of, 388.

Brunner's glands, 454.

Buccal cavity, 442.

Buffy coat, 314 .

Bulbus arteriosus, 347.

Burdach, column of, 172.

Butter, 433, 435.

Butyric acid, 16.

Caffein, 439.

Calcium phosphate, 10, 49 .

Calcium salts, relation of to bloodclotting, 317 .

Calorie, 107, 500.

Calorimeter, 500 .
Camera, photographic, 258.

Canals, lachrymal, 246; semicircular, 232.

Canal, neural, 56 ; central of spinal cord, 141, 144.

Canine teeth, 444.

Canthi of eyelids, 245.

Capacity of lungs, 397.

Capillaries, bile, 460; blood, 293, 322, 331 ; structure of, 337.

Capillary blood-flow, 357.

Capsule, internal, 176.

Carbohydrates, $15,429,433$; absorption of, 492; food value of, 507 ; storage of, 492.

Carbohydrate foods, 433.

Carbon dioxid, 16; of blood, 423; influence of on respiratory center, 403; hormone action of, 424.

Carbon equilibrium, 513.

Carbon monoxid hemoglobin, 427; poisoning by, 427 .

Carbonate of sodium, 90.

Cardiac cycle, diagram of, 344 ; events of, 340 ; time relations of, 339.

Cardiac impulse, 340 .

Cardiac murmurs, 343.

Cardiac muscle, 86; physiology of, 117.

Cardiac orifice of stomach, 449 .

Cardiac plexus, 154.

Cardio-augmentor center, 352 ; nerves, 351.

Cardio-inhibitory center, 352 ; nerves, 351.

Care of teeth, 470.

Carotid artery, 330, 331.

Carpals, 66.

Carriers of infection, 310.

Cartilage, $31,44,49$; articular, 73; arytenoid, 547; costal, 64; cricoid, 547; cuneiform, 548; elastic, 45; ensiform, 55; fibro-, 46; hyaline, 45 ; structure of, 45 ; temporary and permanent, 44; thyroid, 547; of Wrisberg, 548.

Caruncula lachrymalis, 245.

Casein, 13, 435.

Castration, 583.

Cataract, 264.

Cauda equina, 148.

Caudate nucleus, 153.

Celiac axis, 331,461 .

Cells, 7 ; structure of, 23 ; ciliated, 86

Cell-body, of neuron, 136.

Cell division, 23.

Cell growth, 22.

Cell membranes, 17. 
Cell-nucleus, 23.

Cellulose, 16, 430, 433, 467.

Cement of tooth, 445.

Center of gravity of Body, 125.

Centers, cardio-augmentor, 352; cardio-inhibitory, 352 ; respiratory, 401; sweat, 536; vasoconstrictor, 375 ; vasodilator, 378 .

Central nervous system, 138; membranes of, 139 .

Centrosome, 24.

Cephalic vein, 333.

Cerebellar reflexes, cerebral control of, 186.

Cerebellum, 165; functions of, 166.

Cerebral activity, relation of to vasomotor tone, 378 .

Cerebral circulation, relation of to consciousness, 191.

Cerebral control of spinal and cerebellar reflexes, 186 .

Cerebral functions compared in man and animals, 189.

Cerebrospinal liquid, 141, 142.

Cerebrum, 145; afferent paths of, 170 ; cortex of, 174 ; development of, 182; projection fibers of, 175 ; commissural fibers of, 176; lobes of, 176; relation of, to muscular activity, 169 ; relation of, to receptor system, 170; motor areas of, 177; reflex paths of, 179; white matter of, 175 .

Cervical plexus, 148.

Cervical vertebrae, 57 .

Channels, of absorption, 492; of excretion, 516.

Characters, hereditary, 574.

Characteristics of human skeleton, 68.

Cheeks, 443; bones of, 60, 62 .

Cheese, 435.

Chemical changes in respired air, 411.

Chemical composition of body, 8 .

Chemical co-ordination, 28, 39 .

Chemistry, biological, definition of, 9.

Chemistry, of bile, 518 ; of blood, 302 ; of bone, 49 ; of fats, 15; of gastric juice, 464 ; of lymph, 304 ; of muscle, 89 ; of pancreatic juice, 465 ; of saliva, 418; of teeth, 445 ; of urine, 525.

Chemistry of muscular contraction, 106.

Chest, 4

Childbirth, 578.

Chloroform rigor, 109.

Cholesterin, 518.
Chorea, 94.

Choroid, 249.

Chromatic aberration, 262.

Chromatin, 23, 560.

Chromosomes, 25, 560.

Chyme, 488.

Cilia, 33,87 .

Ciliary muscle, 195, 255; action of, in accommodation, 261.

Ciliary processes, 249.

Ciliated cells, 86 .

Circle of dispersion, 260.

Circulation, 334; appearance under microscope, 355; diagram of, 335 ; influence of gravity on, 369; of vein compression on, 370 ; of respiratory movements on, 370,400 ; portal, 335; proofs of, 371 ; pulmonary, 333; resistance to, 356; rate of, 368 ; renal, 522 ; systemic, 335 ; outside heart, 355 .

Circulation scheme, 359 .

Circulatory system, 39 .

Circumvallate papillae, 446.

Classification of tissues, 30 .

Clavicle, 64.

Climacteric, 572 .

Clitoris, 569.

Clot, of blood, 312 .

Clothing, 542, 544 .

Coagulation of blood, 312 ; cause of, 313 ; summary of, 318 ; use of, 315 ; methods of hastening and retarding, 318; within blood-vessels, 318 ; influence of adrenin on, 319 .

Coal-gas poisoning, 427.

Coccyx, 60.

Cochlea, bony, 231; membraneous, 233; functions of, 234.

Cocoa, 439.

Coecum, 455.

Coffee, 439.

Cold-blooded animals, 539.

Cold receptors, 218.

Colds, common, 376, 545.

Collagen, 41.

Collar-bone, 64.

Collaterals, of axon, 160.

Colliculi, 146, 153.

Colloids, 17.

Colon, 455.

Color blindness, 280; tests for, 281.

Color, sensations, 277; sense, distribution of, in retina, 279; vision, 276 ; peculiarities of, 278 ; theories of, 281.

Colors, complementary, 278.

Colostrum, 580.

Columnae carnae, 328. 
Columns, of spinal cord, 144, 172; of Burdach, 172; of Goll, 172.

Commissures, of cerebrum, 176; of spinal cord, 144.

Common bile duct, 458 .

Common sensations, 205.

Complemental air, 398.

Complemental colors, 278.

Complements, of blood, 308 .

Conception, 576, 578.

Concepts, 182.

Concha, 226.

Condiments, 429.

Conduction, nervous, irreversibility of, 161 .

Conductive system, 38.

Conductive tissues, 32 .

Condyle, occipital, 62, 69.

Cones, 252; functions of, 279 ; excitation of, 268.

Coni vasculosi, 564.

Conjugate focus, 258.

Conjunctiva, 245.

Connective tissue, 31, 41, 49.

Consciousness, 189; dependence of on blood supply, 190.

Consonants, 554.

Constant weight, maintenance of, 511 .

Contact senses, 206.

Contractile tissues, see Muscles.

Contractility, 21.

Contraction of muscle, 94 ; effect of temperature on, 97 ; effect of increasing stimuli on, 96 ; graphic record of, 95 .

Contraction, maximal, 97; tetanic, 102; voluntary, 102; summary of, 113.

Contracture, 100.

Contrasts, 281.

Convolutions of brain, 146, 176.

Cooking of meats, 434 ; of vegetables, 436.

Co-ordination, 27, 28; chemical, 28, 39 ; nervous, 28.

Cordæ tendinæ, 328.

Cord, spinal, 5, 138, 142.

Cords, vocal, 546.

Corium, 5, 531 .

Cornea, 249.

Corona radiata, 176.

Coronary artery, 328, 330; vein, 327.

Corpora quadrigemina, 146, 153, 251.

Corpora striata, 146.

Corpus, arantii, 330; callosum, 176; cavernosum, 565; luteum, 572; spongiosum, 565.

Corpuscles of blood, 295; colorless, 301; red, 295.
Corpuscles, Pacinian, 213.

Corresponding points of retina, 287.

Cortex, of cerebrum, 174; development of, 182; of cerebellum, 165 .

Corti, organ of, 233; rods of, 234.

Cortical localization, $176^{\circ}$.

Cortical reflex paths, 179.

Cortical reflexes, 180.

Costal breathing, 398.

Costal cartilages, 64.

Coughing, 408.

Course of blood, 322.

Cranial autonomics, 195.

Cranial nerves, 139, 150.

Cranium, 55.

Cream, 435.

Creatine, 14, 90.

Creatinine, 14, 525.

Cretinism, 202.

Crico-ary tenoid muscles, 549.

Cricoid cartilage, 547.

Cricothyroid membrane, 547; muscle, 551.

Crossed pyramidal tracts, 177.

Crura cerebri, 146.

Crying, 409.

Crypts of Lieberkühn, 454.

Crystalline lens, 254.

Crystalloids, 17.

Cuneate nucleus, 173.

Cuneiform cartilage, 548 .

Currents of action, 103; of injury, 103.

Curve of muscular contraction, 95.

Cutaneous senses, 211.

Cuticle, 529.

Cystic duct, 457.

Cytoplasm, 8.

Deaminization of protein, 504 .

Death, 585; rigor, 92.

Decidua, 576.

Decussation, of pyramids, 177; sensory, 173.

Defects of eye, optical, 262

Degeneration of nerves, 171.

Deglutition, 470.

Dendrites, 136.

Dentals, 554.

Dentate nucleus, 153.

Dentine, 445.

Depressor nerve, 351, 376; impulses, 376.

Depth, perception of, 287.

Dermis, 5, 531.

Desperation, strength of, 201.

Determination of sex, 575 .

Development, 29.

De Vries, 574.

Dextrin, 433, 164. 
Dextrose, 15, 90, 433.

Diabetes, 114, 496, 527.

Dialysis, 19.

Diaphragm, 4, 5, 392.

Diastole of heart, 339 .

Dietary accessories, 429.

Dietetics, 511.

Differentiation of tissues, 25, 30.

Diffusion, 19.

Digastric muscles, 82.

Digestion, 462; auto, 467; bacterial, 466 ; of cellulose, 467 ; good, maintenance of, 489 ; in intestine, 488 ; in mouth, 487; object of, 462 ; products, 463; in stomach, 488; summary of, 466 .

Digestive system, 39.

Dioptrics of eye, 244 .

Direct cerebellar tract, 173.

Discus proligerus, 571.

Disks, intervertebral, 56, 72.

Dislocations, 73.

Dispersion circles, 260.

Dispersion of light, 256.

Dissimulation, 23.

Distance, perception of, 286.

Distribution of blood over body, 373 .

Diuretics, 529.

Diversion, importance of, 199.

Division of labor, physiological, 30 , 87.

Divisions of autonomic system, 195.

Doctrine of specific nerve energies, 204.

Dominant characters, 574 .

Dorsal (neural) cavity, 5 .

Dorsal (thoracic) vertebrae, 58 .

Drum of ear, 226.

Duct, bile, 458; cystic; 457 ; hepatic, 457 ; of pancreas, 460 ; of salivary glands, 448; of Stenson, 448; thoracic, 382 .

Ductless glands, 39, 305 .

Duodenum, 452.

Dura mater, 139.

Duration of luminous sensations, 273.

Dwarfishness, 50 .

Dynamic action of protein, 509, 543 .

Dyspnea, 406.

Ear, 226; drum, 226; external, 226; functions of, 223; internal, 231; middle, 227.

Ear-ache, 228.

Efferent nerve paths, 171.

Efficiency, of muscle, 106.

Eggs, 435.

Elastic cartilage, 45.

Elastic connective tissue, 44.

Elastin, 44.
Electrical phenomena of muscle, 103.

Elements found in body, 9 .

Embryo, nutrition of, 578.

Emergency mechanism of body, 196; reaction, 478 .

Emmetropia, 262.

Emotion, 189; in relation to autonomic system, 197.

Emotional glycosuria, 495.

Enamel, 33, 445.

End arborization, 137.

End plate, 85, 198.

Endocardium, 324.

Endogenous excreta, 516.

Endolymph, 231, 233.

Endoskeleton, 53.

Energy, manifestation in body, 22, 38 ; in contracting muscle, 106, 108; units, 107.

Energy-yielding foods, 429.

Ensiform cartilage, 55.

Enterokinase, 468.

Entoptic phenomena, 265.

Environment, relation of man to, 36 .

Enzyms, 14; digestive, 462.

Epidermis, 5, 33, 529.

Epididymis, 564.

Epiglottis, 449, 547.

Epithelium, 5, 33.

Equilibrium, maintenance of, 125.

Equilibrium, of carbon, 513; of nitrogen, 512; of water, 511.

Equilibrium, of opposing muscles, 122.

Equilibrium organs, 164, 236.

Equilibrium sense, 205, 206, 237.

Erect posture, 124.

Erectile tissue, 565.

Erepsin, 466.

Ergot, 440.

Esophagus, 442, 449.

Etherial sulphates, 525 .

Ethmoid bone, 60.

Eupnea, 406.

Eustachian tube, 227, 448.

Excitability, a physiological property, 21.

Excitation of visual apparatus, 267.

Excreta, endogenous and exogenous, 516.

Excretion, channels of, 516; from lungs, 516; renal, 524 .

Excretory function of liver, 517.

Excretory system, 39; tissue, 31.

Exercise, beneficial effects of, 100; proper kinds for various ages, 132, 133; respiratory changes in, 425; varieties of, 131.

Exogenous excreta, 516. 
Exophthalmic goiter, 202, 514.

Exoskeleton, 53.

Expiration, 396.

Expired air, composition of, 412.

Extension, of joint, 72, 74 .

Extensor muscles, 118; relation of to posture, 126.

External auditory meatus, 226.

External ear, 226.

External medium, 290.

External rectus muscle, 246.

External respiration, 386 .

External senses, 206.

Extract of meat, food value of, 91 .

Extractives, 13.

Extrinsic reference of sensations, 220.

Eye, 248; appendages of, 244; defects of, 262; hygiene of, 265; motions of, 247 ; muscles of, 246 ; nodal points of, 267; physiology of, 267; refracting media of, 244, 254, 259 ; structure of, 243, 248; wide range of clear vision in, 259.

Eyelashes, 245.

Eyelids, 245; muscles of, 245.

Eyestrain, 265.

Face, bones of, 60 .

Facial nerve, 152, 241.

Fallopian tube, 567.

False vocal cords, 549 .

Falsetto, 552.

Far-sightedness, 262.

Fasciculus cuneatus, 172; gracilis, 172.

Fat, absorption of, 499; food value of, 507 ; food 429,433 ; of body, source of, 515; chemistry of, 15; special metabolism of, 510 .

Fatigue, of muscle, 100; of nerves, 156 ; nature of, 101 ; neuro-muscular, 198; sense of, 207.

Fatty tissue, 44.

Fauces, 448.

Fechner's law, 205, 214.

Feeding of infants, 581 .

Female reproductive organs, 567 .

Femoral artery, 331.

Femur, 86; dislocation of, 73.

Fermentation, 466.

Ferments, see Enzyms.

Ferrein, pyramids of, 521 .

Fertilization, 573.

Fetus, nutrition of, 578 .

Fever, 544.

Fiber, of muscle, 83.

Fibrin, 313; source of, 315 .

Fibrin ferment, 316.

Fibrinogen, 302.

Fibrocartilage, 46.
Fibula, 66.

Filiform papillae, 446.

Fillet, 173.

Filtration, 18.

Filum terminale, 143, 148.

First order levers in body, 119.

Fissures, of cerebrum, 176; of spinal cord, 143.

Flavor, 241; importance of, in food, 432.

Flesh food, 434.

Flexion, 72, 74.

Flexors, 118.

Flexure, sigmoid, 455.

Flooding, 579 .

Fluid, cerebrospinal, 141, 142; synovial, 74 ; seminal, 566 .

Focal plane of lens, 258.

Focus, of lens, 207; conjugate, 258.

Follicle, Graafian, 570; Meibomian, 245.

Follicle of hair, 532 .

Fontanelles, 72 .

Food, carbohydrate, 433; composition of, 436; classes of, 429; definition of, 428; energy yielding, 429; fat, 389; flesh, 434; functions of, 428; inorganic, 430; maintenance, 429; protein, 434; requirement of body, 500 ; the source of energy, 38 ; values, 507; vegetable, 435 .

Food poisoning, 440.

Foot-pound, 108.

Foot, skeleton of, 68.

Foramen magnum, 61, 139; oval, 227, 229 ; round, 227.

Fore brain, 145.

Fore limb, 66.

Fore skin, 566.

Formation of bone, 46 .

Formic acid, 16.

Forms of muscles, 82.

Fossa, glenoid, 64, 74 .

Fovea centralis, 250, 251, 254, 271.

Fractured bone, repair of, 48 .

Franklin theory of color vision, 285.

Frontal bone, 60 .

Frontal lobe, 176.

Fuel of body, 428; of muscles, 104.

Fundamental vibrations, 224.

Fundus of stomach, 450 .

Fungiform papillae, 446 .

Fur on tongue, 446.

Gall (bile), 457, 465 .

Gall bladder, 457.

Ganglia, spinal, 147; sympathetic, 154.

Ganglion, definition of, 153; Gasserian, 150; semilunar, 150. 
Gas, absorption of, by liquid, 418; partial pressure of, 419.

Gases of blood, 417.

Gasserian ganglion, 150.

Gastric, digestion, 488; glands, 451; juice, 464; secretin, 486; secretion, control of, 485 .

Gastric mucous membrane, histology of, 451.

Gastrocnemius muscle, 93.

Gelatin, 49.

Gemmation, 557.

Geniculate bodies, 153, 251.

Germ cells, compared with tissue cells, 560; maturation of, 560 .

Gestation, 567.

Gigantism, 50.

Girdle, pelvic, 64, 69.

Gland-duct, 482 .

Glands, 480; of Brunner, 454; ductless, 39; gastric, 451; mammary, 579 ; prostate, 564; pancreatic, 39; salivary, 39, 447; sebaceous, 245 , 535 ; of skin, 534; sweat, 480, 534; tear, 246; thyroid, 201.

Glenoid fossa, 64, 74 .

Gliadin, 503.

Gliding joints, 76 .

Globe of eye, 248 .

Globin, 12.

Globulin, 12 .

Glomerulus, 522, 524.

Glossopharyngeal nerve, 152, 240.

Glottis, 548 .

Glucose, see Dextrose.

Gluten, 435.

Glycerin, 433, 463.

Glycocholic acid, 518 .

Glycogen, $16,90,433$; storage of in liver, 493; in muscles, 494.

Glycoprotein, 13.

Glycosuria, alimentary, 494; emotional, 495; pancreatic, 496; phlorhizin, 498.

Goblet cells, 453 .

Goiter, 201; exophthalmic, 202.

Golgi, tendon organs of, 85 .

Goll, column of, 172 .

Gower's tract, 173.

Graafian follicle, 570 .

Gracile nucleus, 173.

Graded synaptic resistance, 161.

Graham flour, 508.

Gram-centimeter, 108.

Grape sugar (dextrose), 15, 433.

Graphic record, 94.

Grave's Disease, 202, 514.

Gravity, influence of, on circulation, 369.
Gray matter, 153; definition of, 153; distribution of, 153; of spinal cord, 144.

Growth of cells, 22.

Growth proteins, 503 .

Gullet, $442,449$.

Gums, 443.

Gustatory area of cerebrum, 177.

Gutterals, 554 .

Gyri, 146, 176.

Habit formation, 187.

Hairs, 33, 532.

Hair cells of cochlea, 234; of semicircular canals, 237.

Hammer bone, 229.

Hand, see Fore limb.

Harmonic partials, 224.

Haversian system, 47.

Hay fever, 312.

Head senses, 163,172 ; relation of to control of reflexes, 163; tracts of, 173.

Hearing, 163, 172, 205, 206; nerve paths of, 174; range of, 224 .

Heart, $5,35,39,293,322$; anatomy of, 328; augmentor center of, 352 ; augmentor nerves of, 351 ; automaticity of, 347; beat of, 339 ; cavities of, 325 ; change in form of, 340 ; contractions maximal, 348 ; extrinsic nerves of, 351; hypertrophy of, 344; influence of salts on, 350 ; inhibitory center of, 352 ; inhibitory nerves of, 351 ; interior of, 328 ; membranes of, 323; passage of beat over, 348 ; physiological peculiarities of, 347; position of, 323; rate, 339; refractory period of, 348 ; relation of nerve and muscle elements within, 347 ; rhythmic action of, 347 ; septum of, 325 ; sounds of, 342 ; valves of, 328 ; work of, 346 .

Heart-beat, theories of, 349 .

Heart-valves, action of, 343 .

"Heart burn," 472 .

Heat, animal, sources of, 541; loss, regulation of, 542; production, control of, 542 .

Heat rigor, 98; in smooth muscle, 116.

Hematin, 14.

Hematopoietic tissue, 298.

Hemianopia, 251.

Hemispheres, cerebral, 145.

Hemoblastic cells, 559 .

Hemochromogen, 14.

Hemocyanin, 304.

Hemoglobin, 13, 298, 336, 417, 420; absorption of oxygen by, 420; amount of in body, 298; carbon monoxid, 427; reduced, 418. 
Hemophilia, 319.

Henry's law, 418.

Hepatic artery, 457, 461; cells, 458; duct, 457 ; vein, 335,458 .

Heredity, 574.

Hering's theory of color vision, 284.

Hermaphrodite, 560 .

Hernia, inguinal, 563.

Hiccough, 408.

Hilus of kidney, 520 .

Hind-brain, 146.

Hind limb, structure of, 66 .

Hinge joints, 75 .

Hip joint, 72 .

Histological methods, 7.

Histology, definition, 2; of adipose tissue, 44; of adenoid tissue, 304; of areolar tissue, 43; of blood, 295; of bone, 47 ; of cardiac muscle, 86 ; of cartilage, 45; of connective tissue, 43; of ear, 232, 237; of elastic tissue, 44; of hairs, 532; of heart, 86; of kidney, 522; of liver, 458 ; of lungs, 389 ; of lymph, 304 ; of lymph glands, 304; of nails, 534 ; of nervous tissue, 136; of retina, 252 ; of skeletal muscle, 83 ; of small intestine, 452; of smooth muscle, 85; of stomach, 451; of tongue, 446.

Histon, 12.

Hives, 385 .

Holding the breath, 406:

Holmgren test for color blindness, 281.

Holoblastic ova, 571.

Homothermous animals, 539 .

Hormones, definition, 39, 292, 305; action of on glands, 484; affecting metabolism, 496, 513; emergency, 200,319 ; production of, 305 ; of reproductive system, 583; of skeletal muscle, 114; of supporting system, 49 .

Hormone action of carbon dioxid, 424.

Horopter, 288.

Humerus, 66, 79.

Humor, aqueous and vitreous, 254.

Hunger, 205, 206, 209.

Hyaline cartilage, 45 .

Hyaloid membrane, 254.

Hybrid, 574.

Hydremic plethora, 385 .

Hydrocarbons, 15.

Hydrocele, 563.

Hydrocephalus, 142.

Hydrochloric acid, 10, 464.

Hydrogen, 9.
Hydrolysis, 462.

Hygiene, definition, 1; of bones, 51; of clothing, 544; of digestion, 489 ; of exercise; 131; of eyes, 265; of joints, 76; of menstruation, 572; of muscles, 130; of mouth, 469; of respiration, 398; of skeleton, 70 ; of skin, 537 .

Hymen, 570.

Hyoid bone, 55, 62.

Hypermetropia, 262.

Hyperpnea, 406.

Hypertrophy of heart, 344 .

Hypogastric nerve, 456; plexus, 456.

Hypoglossal nerve, 152.

Hypophysis, 50.

Idiosyncracy, 441.

Ileocolic valve, 455 .

Ileum, 452.

Iliac artery, 330, 331.

Ilium, 52, 65 .

Illusions, sensory, 221.

Images, after, 281.

Immune bodies, 308, 309.

Immunity, 310.

Immunization, 309.

Impregnation, 576.

Impulse, cardiac, 340; nervous, 135 ; passage of along neuron, 155 ; nature of, 157; how aroused, 156; speed of, 156; spread of in both directions, 156.

Incisor teeth, 444.

Incus, 229.

Index of refraction, 256.

Inert layer, 356.

Infant feeding, 581 .

Infection, 306; carriers of, 310; recovery from, 308; resistance to, 306.

Infection-resisting mechanism, 307.

Inferior maxilla, 60 .

Inferior maxillary nerve, 151.

Inferior mesenteric artery, 331.

Inferior oblique muscle, 247.

Inferior rectus muscle, 246.

Inferior turbinate bone, 60 .

Inflammatory rheumatism, 344 .

Infundibulum, 388.

Inhibition, 184.

Inhibitory center, 352 ; nerves, 351 .

Injury currents, 103.

Innervation of iris, 250.

Innominate artery, 330; vein, 333.

Inoculation, protective, 311 .

Inorganic food, 430.

Insertion of muscles, 82 .

Inspiration, 391.

Insufficiency, valvular, effects of, 344 . 
Instinctive reactions, 190 .

Intensity of sensations. 205; visual, 271.

Intercellular spaces, 18.

Intercostal arteries, 331; muscles, 394.

Interior of heart, 328 .

Intermediary bodies, 307.

Internal capsule, 176 .

Internal ear, 231.

Internal medium, 291.

Internal rectus muscle, 246.

Internal senses, 206; effect of, in consciousness, 207.

Intervertebral pads, 56, 72.

Intestinal digestion, 489; juice, 465.

Intestine, 5, 442; large, 455; absorption from, 500; movements of, 478; small, 452; absorption from, 491; digestion in, 488; movements of, 476; nervous control of, 477; mucous coat of, 452 .

Intestines, nerves of, 455 .

Intermittent flow converted to continuous, 357.

Intima, 337.

Iodothyrin, 201.

Iris, 249, 250; innervation of, 250; muscles of, 250; pigment of, 214 .

Irradiation, nervous, 353.

Irritable tissues, 32 .

Irritability, 21.

Ischium, 52, 65.

Islands of Langerhans, 497.

Jaw, 60.

Jejunum, 452.

Joint motions, 72 .

Joints, 72; ball-and-socket, 74; gliding, 76; hip, 72; hinge, 75; hygiene of, $76 ;$ knee, 75 ; pivot, 75 .

Judgments, 221.

Jugular vein, 333.

Kidney, 5, 39, 518; blood-flow through, 522; blood supply of, 520 ; relation of to sugar in blood, 494; structure of, 520,522 .

Kidney secretion, mechanism of, 527; relation of to blood-flow, 528 .

Kilocalorie, 107.

Knee-cap, 66.

Knce joint, 75 .

Labia majora, 569; interna, 569.

Labials, 554.

Labyrinth, 226; bony, 231; membraneous, 232 .

Lachrymal apparatus, 246; bone, 60; canals, 246; papilla, 245; sac, 246.

Lactase, 466.

Lactation, 579.
Lacteals, 294, 454.

Lactic acid, 90, 92, 435; significance of in contraction, 112; precursor, 111.

Lactose, 15, 466.

Lamina spiralis, 232.

Langerhans, Islands of, 497.

Language, 187.

Large intestine, 455; absorption from, 500 ; movements of, 478 .

Larynx, 546; cartilages of, 547; muscles of, 549 .

Latent period, 96.

Laughing, 409.

Leaping, 128.

Lecethin, 16.

Leg bones, 66 .

Lens, crystalline, 254

Lens, refraction by, 257.

Lenticular nucleus, 153.

Leucocytes, 301; movements of, 301.

Levator palpebrae superioris, 245.

Levers in body, 119.

Lieberkühn, crypts of, 454 .

Life, stages of, 584 .

Ligament, 53, 73; axial, 230; broad, 567 ; capsular, 73 ; round, 73 ; suspensory, of eye, $254,261$.

Light, 255; dispersion of, 256 ; monochromatic, 255; refraction of, 255; wave-length of, 255.

Limbs, 6; skeleton of, 66 .

Lime, in diet, 50, 581.

Linin, 23.

Lipase, 465.

Lips, 443.

Liquid environment of body cells, 16 .

Liver, $5,39,456$; excretory function of, 517; glycogenetic function of, 493; histology of, 458 .

Lobes, of cerebrum, 176; olfactory, 145.

Lobules of liver, 458.

Local sign in sensation, 205, 215.

Localization of function in cerebrum, 176.

Localizing power of retina, 274; of skin, 215.

Local temperatures, 543 .

Lochia, 579 .

Locomotion, 118, 123, 126; .sensory basis of, 164 .

Locomotor reflexes, 166.

Long-sight, 262.

Lumbar plexus, 149; vertebrae, 59.

Lungs, $5,36,39$, 388; capacity of, 397 ; changes of blood in, 416; excretory functions of, 517 ; structure of, 389 . 
Lymph, 18, 142, 293; chemistry of, 304 ; histology of, 304 ; movements of, 384 ; nodes, 383 ; relation of to blood, 294; renewal of, 293; vessels, $322,382$.

Lymphagogue, 385, 441.

Lymphatics, 294, 381.

Lymph-flow, influence of respiratory movements on, 401.

Lymph-nodes, 383; functions of, 383; -vessels, 322,382

Lymphocytes, 304.

Lymphoid tissue, 304.

Lysin, 504.

Maintenance food, 429; proteins, 503 ; systems, 38, 40.

Malaise, 207.

Malar bone, 60 .

Male reproductive organs, 563.

Malleus, 229.

Malphigian capsule, 522

Malphigian layer of epidermis, 530; pyramids of kidney, 521 .

Maltase, 466.

Maltose, 463, 487.

Mammal, characteristics of, 4 .

Mammary gland, 579.

Man, zoölogical position, 2; relation to environment, 36 .

Manometer, 367.

Mastication, 123, 469.

Mastoiditis, 229.

Maturation of germ cells, 560 .

Maxilla, 60.

Maximal contraction, 97; of heart, 348.

Meal, digestive history of, 487.

Measurement of blood-pressure, 367 .

Meatus, external auditory, 226.

Meatus urinarius, 565, 566.

Media, refracting, of eye, 254; refractive indices of, 259.

Median nerve, 333.

Medium, external, 290; internal, 291.

Medulla oblongata, 146, 192; centers of, 192, 352, 401; nuclei in, 154 .

Medullated (myelinated) nerve fibers, 138.

Meibomian follicles, 245.

Meissner's plexus, 456.

Membrane, aryteno-epiglottic, 548; basilar, 233; cell, 17; cricothyroid, 547; hyaloid, 254; mucous, 5; nictitating, 245; permeable, 19; of Reissner, 233; semipermeable, 19; serous, 5; synovial, 74; tectorial, 234; tympanic, 226, 228; vitelline, 571 .

Membraneous labyrinth, 232.
Membranes of central nervous sys. tem, 139; of heart, 323

Mendel, 574.

Memory, 180; associative, 182.

Menstruation, 572; hygiene of, 572 .

Mesenteric artery, 331. 461.

Mesentery, 452.

Mesoblastic ova, 571.

Metabolism, 40; basal, 506; of fats, 510 ; of muscular work, 507 ; relation of thyroid to, 202, 513.

Metacarpals, 66.

Metatarsals, 66.

Microscopic anatomy, see Histology.

Mid brain. 192.

Middle ear, 227.

Milk, composition of, 435,580 ; for infants, 581 ; in diet, 51 ; pasteurization of, 582 ; pure, importance of, 581 .

Millon's test for proteins, 12 .

Mitosis, 24.

Mitral valve, 328 .

Modality of sensations, 205

Modified respiratory movements, 408 .

Modiolus, 232.

Molar teeth, 444.

Mons Veneris, 569

Monochromatic light, 255.

Morula, 29.

Motion, 27; in animals, 78.

Motions of joints, 72 .

Motor area of cortex, 177; neurons, 136; system, 37; tissues, 32 .

Motores oculi, 150.

Mountain sickness, 424 .

Mouth, 443; digestion in, 487; hygiene of, 469.

Movements, intestinal, 476, 478; respiratory, 391 ; influence of on circulation, 400; on lymph flow, 401.

Mucin, 13.

Mucous layer of epidermis. 529; of intestine, 452 ; of stomach, 451 .

Mucous membrane, 5 .

Mulberry mass, 29.

Mumps, 448.

Murmurs, cardiac, 343.

Muscæ volitantes, 264.

Muscle, anatomy of, 82 ; chemistry of, 89 ; cardiac, 86,347 ; electrical phenomena of, 103; end plates of, 85; energy output of, 108; fatigue of, 100 ; forms of, 82 ; fuel of, 104 , 105; glycogen in, 494; heat rigor of, 98 ; histology of, 83; hormones of, 114; oxidation in, 114; plasma, 84; relation of form to working power, 99 ; relaxation of, 111 ; skeletal, 79 ; smooth, 79; spindles, 85 ; stroma, 89. 
Muscles, classification of, 79 ; biceps, 79 ; ciliary, 195, 255, 261 ; digastric, 82 ; extensor, 126; of eyeball, 246 ; flexor, 118; hygiene of, 130; of iris, 249 ; of larynx, 549; opposing, equilibrium of, 122; origin and insertion, 82 ; papillary, 329,342 ; paralysis of, 130 ; relation of to bones, 79; respiratory, 394; special physiology of, 118.

Muscle-fiber, 83.

Muscle groups, functional, 123.

Muscle sense, 164, 172, 205, 206, 207.

Muscle, smooth, 79; heat rigor in, 116; mechanism of contraction of, 116; physiology of, 114 .

Muscle spindle, 85.

Muscle stroma, 89; tissue, 33.

Muscular contraction, 94 ; chemistry of, 106; energy relations in, 106; extent of, 96 .

Muscular efficiency, 106.

Muscular energy, source of, 104; exercise, respiratory changes in, 425.

Muscular tissue, 33.

Muscular work, 98; metabolism of, 507.

Muscularis mucosæ, 453.

Myelin sheath, 138.

Myelination, successive, 172.

Myenteric reflex, 474

Myogen, 89.

Myogenic theory of heart-beat, 349 .

Myopia, 262.

Myosin, 89.

Myxedema, 202.

Nails, 33, 534.

Nares, 62.

Nasal bone, 60; duct, 246.

Nausea, 207.

Nearsightedness, 262.

Nerve, 135; abducens, 150; auditory, 152; depressor, 351, 376; facial, 152 ; glossopharyngeal, 152; hypoglossal, 152; inferior maxillary, 151; median, 333; oculomotor, 150; olfactory, 150 ; ophthalmic, 150 ; optic, 35, 150; patheticus, 150; phrenic, 149; pneumogastric, 152; sciatic, 150 ; spinal accessory, 152 ; splanchnic, 375,451 ; superior maxillary, 151; trigeminal, 150; vagus, 152.

Nerve-cells, 136; sensory, cell-bodies of, 136.

Nerve end plate, 85, 198.

Nerve elements within heart, 347.

Nerve energies, specific, 204.
Nerve-fibers, indefatigability of, 156 .

Nerve impulse, definition, 135; how aroused, 156 ; nature of, 157 ; speed of, 156; spread of in both directions, 156; methods of studying, 155.

Nerve paths, afferent, 170; efferent, 170 ; method of tracing, 171 .

Nerves, autonomic, 139, 193; cardiac, 351 ; cranial, 139, 150; of intestines, 455 ; of respiration, 402 ; secretory. 483; spinal, 139, 147; sympathetic, 139, 193; trophic, 483; vasoconstrictor, 374 ; vasodilator, 377 ; vasomotor, 373 .

Nervus erigens, 456,565 .

Nervous co-ordination, 28, 38.

Nervous fatigue, 156, 198.

Nervous irradiation, 353.

Nervous system, 135; autonomic. $139,154,193$; central and peripheral, 138; membranes of, 139; sympathetic, 139, 154, 193.

Neural canal, 56; tube, 141.

Neurilemma, 138.

Neurogenic theory of heart-beat, 349.

Neuroglia, 139.

Neuromuscular fatigue, 198.

Neurons, 136; association, 137; bipolar, 137; conduction in, 155; motor, 136; post ganglionic, 194; preganglionic, 194; sensory, 136.

Nicotine, 194, 351.

Nictitating membrane, 245.

Nipple, 580.

Nitrogen, 9.

Nitrogen equilibrium, 512 .

Nitrogenous extractives, 13.

Nodal points of eye, 267.

Nodes of Ranvier, 138.

Noise, 223.

Nose, bones of, 60 .

Notes, musical, 223.

Nourishment, of brain, 190.

Nuclear spindle, 25.

Nuclei, nervous, 153; in medulla, 154; auditory, 174 ; caudate, 153 ; cuneate, 173; dentate, 153; gracile, 173; lenticular, 153; red, 153; of cerebellum, 165.

Nucleoprotein, 13.

Nucleus of cell, 8; structure of, 23.

Nutrients, 429, 432 ; functions of, 433 ; occurrence of in foods, 429.

Nutrition, see Metabolism.

Nutrition of embryo, 578 .

Nutritive tissues, 31 .

Nutritive value, of albuminoids, 510; of carbohydrates, fats, and proteins, 507. 
Nymphæ, 569.

Obesity, 514 .

Oblique muscles of eye, 247.

Occipital bone, 60; condyle, 62, 69; lobe of cerebrum, 176.

Oculomotor nerve, 150.

Odontoid process, 58, 75 .

Odors, nature of, 240.

Old-sightedness, 266.

Olecranon, 66.

Olein, 15.

Olfactory areas of cerebrum, 157.

Olfactory lobes, 145; nerves, 150; organ, 239.

Omentum, 449.

Ophthalmic nerve, 150.

Opsonins, 309.

Optical defects of eye, 262.

Optical system, 258; of eye, 259.

Optic chiasma, 150, 251; disk, 250; nerve, $35,150,251$; thalami, 146, 153 ; tracts, 150, 251.

Optogram, 273.

Orbicularis oris, 119; palpebrarum, $119,245$.

Orbit, 62.

Organ of Corti, 233.

Organs, 1, 34; of circulation, 322 ; of digestion, 442; of excretion, 516 ; of movement, 78; of nervous system, 135; of reproduction, 562 ; of respiration, 386 ; of secretion, 480 ; of sensation, 204, 226, 243.

Origin of muscles, 82 .

Os innominatum, 52, 65, 67.

Osmosis, 19.

Osmotic pressure, 19, 93.

Os orbiculare, 229.

Ossein, 49.

Ossicles, auditory, 229; functions of, 230.

Osteoblast, 46.

Osteoclast, 48.

Otoliths, 237.

Oval foramen, 227.

Ovary, 567; structure of, 570 .

Overtones, 224.

Oviduct, 567.

Ovulation, 571.

Ovum, 29, 571; fertilization of, 573 ; maturation of, 560 .

Oxidase, 425.

Oxidation, 38; in muscle, 104, 114; as source of animal heat, 541; "recovery," 110.

Oxygen, 9 ; absorption of by blood, 420 ; interchanges in blood, 421 .

Oxyhemoglobin, 417.

Pacinian corpuscles, 213.
Pain, 172, 205, 206, 211; internal, 213; localization of, 213.

Palate, 443.

Palatine bones, 60 .

Palmatin, 15.

Pancreas, 5, 39, 114, 460.

Pancreatic juice, 465; secretin, 487; secretion, control of, 486 .

Papillæ, lachrymal, 245; of skin, 531; of tongue, 241, 446.

Papillary muscles, 329; use of, 342.

Paraglobulin, 302.

Parathyroids, 305.

Parietal bones, 60; lobe, 176.

Parieto-occipital fissure, 176.

Parotid gland, 447.

Parthenogenesis, 560.

Partial tones, 224.

Parturition, 578.

Pasteurization of milk, 582 .

Patella, 66.

Patheticus nerve, 150.

Pathogenic organisms, 306.

Pathology; definition, 1.

Paths, nervous, of various senses, 172.

Pawlow (Pavloff), 485.

Peas, 508.

Pectoral arch, 64.

Peduncles of cerebellum, 165.

Pelvic girdle, 64, 67.

Pelvis of kidney, 520.

Penis, 565.

Pepsin, 15, 464.

Pepsinogen, 468.

Peptone, 13, 464.

Perceptions, 219; auditory, 235; visual, 285, 286, 287, 288.

Pericarditis, 325.

Pericardium, 323.

Perichondrium, 45.

Perilymph, 231, 233.

Perimeter, 279.

Perimysium, 83.

Periosteum, 46.

- Peripheral nervous system, 139; reference of sensations, 218 .

Peristalsis, 472.

Peritoneum, 5, 450.

Permanent cartilage, 44.

Permeable membrane, 19.

Perspiration, 535.

Peyer's patches, 383.

Phagocytes, 302, 307; action of in resisting infection, 307.

Phagacytosis, 302.

Phalanges, 66.

Pharynx, 442, 448.

Phlorhizin, 498.

Phosphoproteins, 13. 
Photochemical substances, 282.

Phrenic nerve, 149.

Physico-chemistry of Body, 16; of skeletal muscle, 88 .

Physiological division of labor, $30,87$.

Physiological systems, 35.

Physiology, 1; of brain, 166, 179; of digestion, 462; of ear, 226, 237; of eye, 267; of heart, 347; of kidney, 527; of metabolism, 500; of muscle, 93; of nerve, 155; of respiration, 386 ; of sensation, 204; of skin, 535 ; of smell, 239 ; of spinal cord, 157; of taste, 240; of touch, 214.

Pia mater, 141.

Pigment, 14; of iris, 250.

Pitch, audible limits of, 224; definition of, 223; range of, in human voice, 552.

Pituitary body, 50 .

Pivot joints, 75.

Placenta, 578.

Plain muscular tissue, see Smooth Muscle.

Plantigrade animals, 70 .

Plants compared with animals, 40.

Plasma, 295, 302.

Platelets, blood, 302.

Pleura, 5, 6, 389.

Plexus, 148; of Auerbach, 456; brachial, 148; cardiac, 154; cervical, 148; lumbar, 149; of Meissner, 456; sacral, 150; solar, 154, 411.

Pneumogastric nerves, 152.

Poikilothermous animals, 539.

Poisoning, by coal gas, 427; by food, 440.

Polar bodies, 561 .

Pons varolii, 146.

Popliteal artery, 331.

Portal circulation, 335 ; vein, 335,457 , 461.

Post ganglionic neurons, 194.

Postural reflexes, 167.

Posture, 123, 124; the task of extensor muscles, 126.

Potassium chlorid, 10.

Precursor, lactic acid, 111.

Preganglionic neurons, 194.

Pregnancy, 567, 576; extra-uterine, 573.

Prehension, 123, 129.

Prepuce, 566.

Presbyopia, 266.

Pressor impulses, 376.

Pressure, of blood, 362,367 ; intrar thoracic, 399; osmotic, 19; partial, of gases, 419.

Pressure sense, 214.
Primates, 2.

Principles of dietetics, 511.

Process, olecranon, 66; odontoid, 58, 75; ciliary, 249.

Production of heat in Body, 40, 541 .

Projecting senses, 207:

Projection fibers of cerebrum, 175.

Pronation, 76.

Proofs of circulation, 371.

-Properties of Body, 21.

Prosecretin, 487

Prostate, 564.

Protamin, 12.

Protective inoculation, 311.

Protective tissues, 33.

Protein, 10, 429, 434; absorption of, 498 ; amount of in diet, 504; conjugated, 12 ; deaminization of, 504 ; derived, 13 ; digestion of, 464 ; foods, 434 ; food value of, 507 ; fuel, 504 ; growth, 503; maintenance, 503; of muscle, 89 ; requirements of Body for, 500; specific dynamic action of, 509, 543; subdivisions of, 10 ; tests for, 11; use of in Body, 501.

Proteose, 13, 464.

Prothrombin, 317.

Protoplasm, 8.

Psychic secretion, 485.

Psycho-physical law, 205, 214, 271.

Ptomain, 441.

Ptosis, 248.

Ptyalin, 15, 463, 487.

Pulerty, 582.

Pubis, 52, 65.

Pulmonary artery, 326, 333, 334; circulation, 333, 335; veins, 323, $333,336$.

Pulse, 364; use of in diagnosis, 360 ; -wave, rate of movement, 365 .

Pupil, 244, 249.

Purin bodies, 14, 526.

Purkinje's experiment, 269.

Pus, 301.

Putrefaction, 467.

Pyloric sphincter, 474 ; control of, 474 .

Pylorus, 449, 452.

Pyramidal nerve cells, 175; tracts, 177.

Pyramids, decussation of, 177.

Pyramids of Ferrein, 521; of Malphigi, 521.

Pyrexia, 544.

Quadriceps femoris, 120.

Qualities of sensation, 205.

Quantity, of air breathed daily, 398; of blood, 303; of food needed daily, 500,511 .

Racemose glands, 480 . 
Radial artery, 330.

Radio-ulnar articulation, 76.

Radius, 66.

Range of human voice, 552 .

Ranvier, nodes of, 138.

Rate of blood flow, 368; of nerve impulse, 156; of pulse-wave, 365 .

Reaction of blood, 295.

Reactions, emotional, 197.

Reason, faculty of, 190.

Receptaculum chyli, 382.

Receptive tissues, see Irritable tissues.

Receptor system, 204.

Receptors, classification of, 206.

Recessive characters, 574.

Record, graphic, 94 .

"Recovery" oxidation in muscle, 110.

Rectum, 455.

Rectus abdominis, 83.

Rectus muscles of eye, 246 .

Red blood corpuscles, 295; color, 296; composition, 297; consistency, 297; form and size, 295; number, 297; origin and fate, 299; structure, 296.

Red nucleus, 153.

Reduced hemoglobin, 418.

Reduction division, 561 .

Reflex, definition of, 157; myenteric, 474.

Reflex animal, compared with normal, 169.

Reflex control of autonomic system, 194.

Reflex arcs, 158; of cortex, 179; variability in, 159 .

Reflex time, 180.

Reflexes, control of by head senses, 163; cortical compared with spinal, 180 ; locomotor, 166; mediated by spinal cord, 162; postural, 167; spreading of, 162 .

Refracting media of eye, 254.

Refraction, 255; in the eye, 259; index of, 256; law of, 256; of lenses, 257; of light, 255.

Refractory period of heart, 348 .

Regeneration, 558.

Regio olfactoria, 239.

Regulation of temperature, 40, 539 .

Reissner, membrane of, 233.

Relaxation of muscle, 111.

Renal artery, 331, 520; excretion, 524 ; organs, 518 ; vein, 520.

Rennin, 464.

Repair of fractured bone, 48.

Reproduction, 21, 557; sexual, 560.

Reproductive organs, accessory, 562; female, 567 ; male, 563 .
Reproductive system, 40; hormones of, 583 .

Reproductive tissues, 34 .

Residual air, 397.

Resistance, capillary, 356.

Resistance, synaptic, 161; to infection, 306.

Resonance, 225.

Resonants, 554, 555.

Respiration, 386; abdominal, 398; artificial, 408; chemistry of, 410 ; costal, 398; external, 386; forced, 396 ; hygiene of, 398 ; influence of on circulation, 400; on lymph flow, 401 ; internal, 386 ; nerves of, 402 ; rhythmic character of, 403; tissue, 386,425 .

Respiratory center, 401; action of vagi on, 404; excitation of, 403; reflex influence on, 402 ; sensitiveness of, 405; changes in muscular exercise, 425; movements, 391; movements, modified, 408; organs, 386 ; sounds, 397 ; tissue, 31 .

Response of muscle to rapidly repeated stimuli, 102.

Reticular membrane, 234.

Retina, 35, 243, 250; blood vessels of, 251 ; distribution of color sense over, 279 ; localizing power of, 274 ; microscopic structure of, 252 ; nervous elements of, 253.

Rheumatism, 344 .

Rhythmic segmentation, 476.

Rhythmicity of heart, 347; nature of, 350.

Rib cartilage, 64 .

Ribs, 55, 63.

Rigor, chloroform, 109; mortis. 92.

Rods of Corti, 234.

Rods of retina, 252; excitation of, 268; function of, 272 .

Rolando, fissure of, 176 .

Roots of spinal nerves, 147.

Rotation, movements of, 72, 74; of radius over ulna, 66, 75, 129.

Roughage, 429; importance of, 478.

Round foramen, 227.

Running, 128.

Rupture, 563.

Sac, lachrymal, 246.

Sacculus, 232.

Sacral autonomics, 195; plexus, 150; vertebræ, 60.

Sacrum, 52, 55, 59, 67.

Saint Vitus' dance, 94.

Saliva, 463.

Salivary glands, $39,447$.

Salivary secretion, control of, 484 . 
Salt, common, 10; importance of in diet, 431.

Salts, of Body, 10; of urine, 526.

Santorini, cartilages of, 548 .

Saphenous vein, 333.

Sarcolemma, 84, 88.

Sarcoplasm, 84, 88.

Sarcostyle, $84,85,88$; behavior of in contraction, 113.

Scalæ of cochlea, 233.

Scalene muscles, 394.

Scapula, 64, 129.

Sciatic nerve, 150.

Sclerotic, 248.

Scrotum, 563.

Scurvy, 431.

Sebaceous glands, 245,535 ; secretion, 536.

Secondary sexual characters, 583 .

Secretin, gastric, 486; pancreatic, 487.

Secretion, 482; cutaneous, 535; gastric, 464; control of, 485 ; intestinal, 465 ; control of, 487; organs of, 480 ; pancreatic, 465; control of, 486; psychic, 485; renal, 527; salivary, 463 ; control of, 484; sebaceous, 536 ; sweat, 535 .

Secretory nerves, 483.

Secretory process, 482 ; hormone control of, 484; nervous control of, 483 .

Secretory tissues, 31 .

Sections of Body, 5,6 .

Segmentation, of ovum, 29, 576; rhythmic, of intestine, 476 .

Self-digestion, prevention of, 467 .

Semicircular canals, 164, 231; bony, 232; epithelium of, 237 ; function of, 237; membraneous, 232; nerve endings in, 236.

Semilunar ganglion, 150; valves of heart, 329 .

Seminal fluid, 566 ; vesicle, 564 .

Seminiferous tubule, 563 .

Semipermeable membrane, 19.

Sensations, 27; of color, 277 ; common, 205; differences between, 204; extrinsic reference of, 220 ; intensity of, 205; local sign of, 205, 215; modality of, 205 ; peripheral reference of, 218; quality of, 205; visual, duration of, 273; intensity of, 271.

Sense, of equilibrium, 237; of hearing, 226,234 ; of hunger, 209; muscular, 207 ; of pain, 211; of sight, 267; of smell, 239; of taste, 240; of thirst, 210 ; of touch, 214 ; of temperature, 206, 217.
Senses, 205; classification of, 206; contact, 206; cutaneous, 211; external, 206; internal, 206, 207; nerve paths of, 172; projecting, 207.

Sensory areas of cortex, 177.

Sensory decussation, 173; illusions, 221 ; neurons, 136.

Septicemia, 307.

Septum of heart, 325.

Serous membranes, 5,450 .

Serum, 312; albumin, 302.

Sex determination, 575 .

Sexual characters, secondary, 583; reproduction, 560 .

Sheath, myelin, 138.

Shivering, 543 .

Short sight, 262.

Shoulder-blade, 64; -girdle, 64; attachment to axial skeleton, 66 .

Sighing, 408.

Sight, 163, 164, 172, 205, 206; nerve paths of, 173; hygiene of, 265.

Sigmoid flexure, 455 .

Simple contraction, 94 .

Sinus venosus, 347 .

Size, perception of, 287

Skeletal muscles, 79; chemistry of, 89 ; histology of, 83 ; hormone of, 114; physico-chemistry of, 88 ; special physiology of, 118.

Skeleton, 53; appendicular, 60; axial, 55 ; of face, 60 ; hygiene of, 70 ; peculiarities of, 68; of skull, 60; of thorax, 63, 392.

Skin, 5, 39, 529; glands, 534; hygiene of, 537 ; localizing power of, 215 ; papillæ of, 531 ; secretions, 535 .

Skull, 55; details of, 60; articulations of, 61 .

Sleep, 379.

Small intestine, 452; absorption from, 491; digestion in, 488; movements of, 476 ; control of, 477 .

Smell, 163, 172, 205, 206, 239; fatigue of, 240 ; keenness of, 240 .

Smooth muscle, 85 ; heat rigor in, 116 ; mechanism of contraction of, 116 ; physiology of, 114 .

Sneezing, 408.

Soap, production of from fat, 15 ; in small intestine, 499 .

Sodium carbonate, 90, 423.

Sodium chlorid, 10, 431.

Sodium lactate, formation of in muscle, $112,426$.

Solar plexus, $154,375,451$; effect of blow on, 353 .

Solidity, perception of, 288 .

Somatic cells, 560 . 
Sound, 223; intensity of, 223; pitch of, 223; quality of, 223 .

Sounds, of heart, 342 ; respiratory, 397.

Soup, value of, 498 .

Source, of animal heat, 541 ; of body fat, 515; of glycogen, 493; of muscular energy, 104; of urea, 517.

Spaces, arachnoid, 142; intercellular, 18.

Special senses, 205.

Specific dynamic action of proteins, $509,543$.

Specific gravity of blood, 295.

Specific nerve energies, 204.

Spectacles, 265.

Speech, 546.

Speed of nerve impulse, 156.

Sperm, 561.

Spermatozoa, 560, 566.

Sphenoid bone, 60 .

Sphere, attraction, 24.

Spherical aberration, 263.

Sphincter, 115; pyloric, 474.

Spinal accessory nerve, 152.

Spinal column, see Vertebral column.

Spinal cord, $5,138,142$; central canal of, 141,144 ; columns of, 144 ; commissures of, 144; fissures of, 143; functions of, 160,172; gray matter of, 144; membranes of, 139; white matter of, 144.

Spinal cord reflexes, 162; cerebral control of, 186; compared with cortical, 180.

Spinal ganglia, 147; nerve roots, 147; nerves, 139, 147; distribution of, 148.

Spindle, nuclear, 25.

Spindles, muscle, 85.

Spine, curvature of, 70.

Splanchnic nerves, 375, 451; region, 375.

Spleen, 5, 299; function of, 300.

Splenic artery, 461.

Sprains, 77.

Spread of nerve impulse in both directions, 156.

Squinting, 248.

Staining, differential, 7.

Stages of life, 584 .

Staircase phenomenon, 100.

Stapedius muscle, 230.

Stapes, 229.

Starch, animal, 16; digestion of, 463 , 465,487 ; as food, 433 .

Stearin, 15.

Stenson's duct, 448.

Stereoscopic vision, 289.

Sternum, 55, 64.
Stimulation, necessity of, 93.

Stimuli, 94, 204; increasing, influence on contraction, 96 ; rapidly repeated, effect of, 102.

Stirrup bone, 229.

Stomach, 5, 442, 449; absorption from, 491; digestion in, 488; histology of, 451 ; importance of, 476 ; movements of, 473 ; nervous control of, 477; salivary digestion in, 473.

Storage, of carbohydrates, 493; of glycogen in muscles, 494 .

Storage tissues, 32.

Strabismus, 248.

Strength of desperation, 201.

Structure, vertebrate, 3.

Strychnine poisoning, 162.

Subclavian artery, 330; vein, 333.

Subcutaneous areolar tissue, 531 .

Sublingual gland, 448.

Submaxillary gland, 448.

Successive myelination, 172.

Succus entericus, 465 ; control of, 487.

Sucrase, 466.

Sucrose, 433.

Sudoriparous glands, 534 .

Sugar, 433; as food, 493; as fuel for muscles, 105; elimination of through kidney, 494.

Sulcus spiralis, 233.

Superior maxillary nerve, 151 ; mesenteric artery, 461; oblique muscle, 247 ; rectus muscle, 246.

Supination, 76.

Supplemental air, 397.

Supporting tissue, 31 ; system, 37.

Suprarenal capsules, 199.

Suspensory ligament of eye, 254, 261.

Sutures, 71.

Swallowing, 470.

Sweat, 535; center, 536; glands, 480 , 534 ; nervous and circulatory factors in, 536 .

Sweating, relation of to heat loss, 542 .

Sweet bread, 460.

Sylvius, fissure of, 176 .

Sympathetic ganglion, 154.

Sympathetic resonance, 225, 227.

Sympathetic system, see Autonomic system.

Synapse, 137; relation of to gray matter, 153.

Synaptic fatigue, 198; resistance, 161.

Synovial fluid, 74; membrane, 74:

System, alimentary, 39, 442; autonomic, 139, 154, 193; circulatory, 39,322 ; conductive, 38,135 ; digestive, 39,442 ; excretory, 39,516 ; 
motor, 37,78 ; nervous, 38,135 ; receptor, 37, 204; respiratory, 39, 386 ; supporting, 37,43 .

Systemic circulation, 335.

Systems, Haversian, 47.

Systems, physiological, 35; adaptive, 37 ; maintenance, 38 .

Systole of heart, 339 .

Taking cold, 376,545 .

Tannin, 440.

Tarsus, 66, 70.

Taste, 163, 172, 205, 206, 240; -buds, $241,446$.

Taurocholic acid, 518.

Tea, 439.

Tear-glands, 246.

Tears, 246.

Tectorial membrane, 234.

Teeth, 443; structure of, 445 ; care of, 470.

Temperature, of Body, 540; effect of on muscular contraction, 97 ; sensation zero, 217; sense, 206, 217; bodily, regulation of, 541 ; local, 543 .

Temporal artery, 331; bone, 60; lobe, 176.

Temporary cartilage, 44.

Tendon-organs of Golgi, 85.

Tendons, 79.

Tension of blood-gases, 422 .

Tensor tympani muscle, 230.

Test for color blindness, 281.

Testis, 563.

Tests for proteins, 12.

Tetanus, 102.

Thalami, optic, 153.

Theobromin, 439.

Theories, of color vision, 281; of heart-beat, 349 ; of sleep, 380 .

Thirst, 205, 206, 210.

Thoracic cavity, 4 ; duct, 382 ; vertebræ, 58.

Thoracico-lumbar autonomics, 195.

Thorax, aspiration of, 370, 399; contents of, 5 ; movements of, 392 ; skeleton of, 392 .

Throat, 448.

Thrombin, 316; source of, 316.

Thromboplastic substance, 317.

Thumb, articulation of, 66 .

Thyro-arytenoid muscle, 551.

Thyroid cartilage, 547.

Thyroid gland, 50, 201.

Thyroid hormone, emergency function of, 202; influence of on metabolism, 513.

Tibia, 66.

Tibial artery, 331.

Tidal air, 398.
Timbre, 223, 224.

Tissue cells compared with germ cells, 560 .

Tissue differentiation, 25; resistance, 307 ; respiration, 425.

Tissues, 1, 8; adenoid, 304, 383; adipose, 44; areolar, 43; assimilative, 31 ; bony, 46 ; cartilaginous, 45 ; classification of, 30 ; conductive, 32 ; connective, 31 , 41 ; contractile, 32 , 78; elastic, 44; erectile, 565; excretory, 31, 518; irritable, 32; lymphoid, 304, 383; motor, 32, 78; nervous, 135 ; nutritive, 31 ; protective, 33 ; reproductive, 34 ; respiratory, 31, 389; secretory, 31 , 480 ; storage, 32 ; supporting, 31 , 41; undifferentiated, 30.

Tone, vasomotor, 375 ; relation of to cerebral activity, 378 .

Tones; number distinguishable, 235.

Tongue, 123, 240, 443, 446.

Tonsil, 383, 448.

Touch, 164, 172, 205, 206, 214.

Toxins, 308.

Trachea, 39, 388.

Tracing nerve paths, 171.

Tracts, of body sense, 172; of Gower, 173 ; of head senses, 173 ; pyramidal, 177.

Training, 124 .

Transfusion of blood, 320 .

Triceps, 82.

Tricuspid valve, 328,329 .

Trigeminal nerve, 150, 240.

Trochlear muscle, 247.

Trophic nerves, 483.

Trypsin, 465.

Trypsinogen, 468.

Tube, Eustachian, 227, 228; Fallopian, 567; neural, 141.

Tubular glands, 480 .

Tubules, uriniferous, 522 ; seminiferous, 563.

Tunica, adventitia, 337; vaginalis, 563 .

Turbinate bones, 60 .

Twitch of muscle, 94.

Tympanic membrane, 226, 228; functions of, 226.

Tyrein, 39.

Ulna, 66.

Ulnar artery, 331.

Umbilical cord, 578 .

Undifferentiated tissues, 30.

Uniform temperature, maintenance of, 541 .

Units of energy, 107.

Unstriped muscle, see Smooth muscle. Urea, 13, 90, 517, 525. 
Ureter, 518.

Urethra, 519; male, 565.

Uric acid, 14, 526.

Urinary organs, 518 ; salts, 526 .

Urine, 525.

Uriniferous tubules, 522; secretory action of, 527 .

Urobilin, 14.

Urticaria, 385.

Uterus, 567.

Utriculus, 232.

Uvula, 443.

Vaccination, 311.

Vagina, 568.

Vagus nerve, 152, 477; relation of to heart, 351.

Valve, ileocolic, 455.

Valves of heart, 328; action of, 343.

Valves of veins, 337.

Valvulæ conniventes, 452.

Valvular insufficiency, effects of, 344.

Varicose veins, 370.

Vasa efferentia, 564.

Vasa recta, 564.

Vas deferens, 564 .

Vaso constrictor center, 375; control of, 375 ; nerves, 374 .

Vasodilator center, 378; nerves, 377.

Vasomotor tone, 375 ; relation of to cerebral activity, 378 .

Vegetable foods, 435; cooking of, 436.

Veins, 322, 332; cephalic, 333; coronary, 327; hepatic, 335,458 ; innominate, 333 ; jugular, 333 ; portal, 335, 457; pulmonary, 328, 333; saphenous, 333; structure of, 337; subclavian, 333; valves of, 337 ; varicose, 370 .

Vena cava, 327, 333.

Venous blood, 336.

Venous sinus, 565 .

Ventilation, 412.

Ventricle of heart, 325; functions of, $341,346$.

Ventricles of brain, 142.

Vermiform appendix, 455.

Vertebræ, 56; cervical, 57; lumbar, 59 ; sacral, 59 ; structure of, 56 ; thoracic, 58.

Vertebral artery, 330; column, 3, 55.

Vertebrata, 3 .

Vesicle, seminal, 564.

Vessels, blood, 322; lymphatic, 294.

Vestibule of ear, 164, 231, 232; function of, 237; nerve endings in, 236.

Vibrations, analysis of, 224; the basis of sound, 223; sympathetic, 225, 227.

Vibratories, 555.

Villi of intestines, 453.
Vision, 123; binocular, 287; color, 276 ; stereoscopic, 289; wide range of, 259 .

Visual angle, 274 ; apparatus, excitation of, 267; area of cerebrum, 177; axis, 275; contrasts, 281; defects, 262 ; perceptions, 285 ; purple, 250 , 252, 273; sensations, 271.

Vital capacity, 398; centers, 193; point, 402; processes, 192.

Vitamines, 429, 431.

Vitelline membrane, 571 .

Vitreous humor, 254.

Vocal cords, 546, 548; false, 548; relation of, to pitch, 552 .

Voice, 546; production, 123; range of, 552.

Volition, 166, 169, 184.

Voluntary acts, reflex at bottom, 184.

Voluntary muscular contraction, 102.

Vomer, 60.

Vowels, 553.

Vulva, 569.

Walking, 126.

Wallerian degeneration, 171.

Warm-blooded animals, 539.

"Warming up," 100.

Warmth receptors, 218.

Water, equilibrium, 511; proportion of in Body, 10.

Wave-length of light, 255.

Waves, peristaltic, 472 .

Wax of ear, 226.

Weber's law, 205, 214; circulation scheme, 359.

Weeping, 246.

Weight, maintenance of, 511.

Whipped blood, 313.

Whispering, 556.

White, sensations, 276; blood-corpuscles, 301 ; of eye, 249; fibrous connective tissue, 31 , 43; matter, 138, 153; matter of cerebrum, 175; of spinal cord, 144.

Will power, 185.

Wind pipe, 36,388 .

Work, of heart, 346; muscular, measure of, 98.

Worry, significance of, 197.

Wrisberg, cartilage of, 548.

Wrist bones, 66.

Xanthoproteic test, 12.

Yawning, 408.

Yellow elastic tissue, 31, 44.

Yolk, 571.

Young-Helmholtz theory of color vision, 282.

Zona pellucida, 571 ; radiata, 571 .

Zoölogical position of man, 2.

Z.ymogen, 468. 




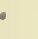





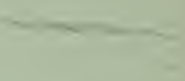

- 


\section{DATE DUE SLIP}

UNIVERSITY OF CALIFORNIA MEDICAL SCHOOL LIBRARY

THIS BOOK IS DUE ON THE IAST DATE STAMPED BELOW

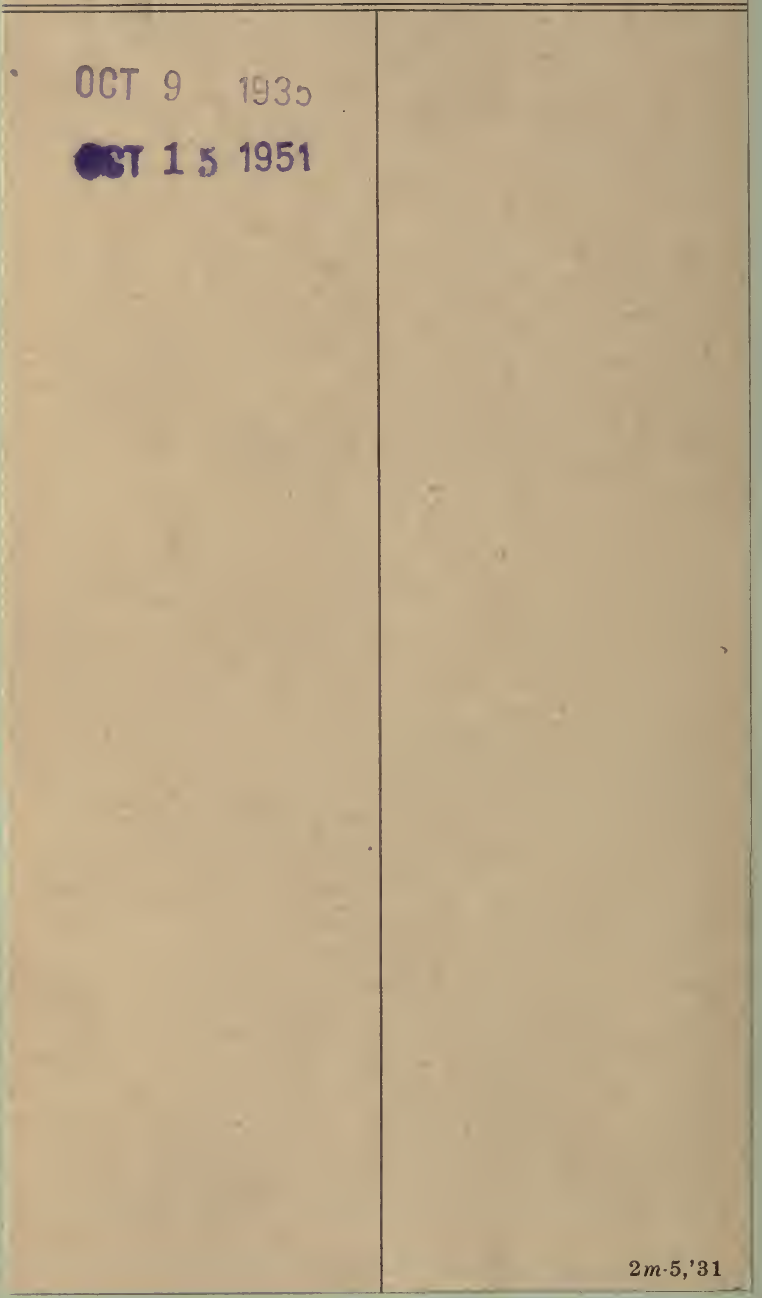



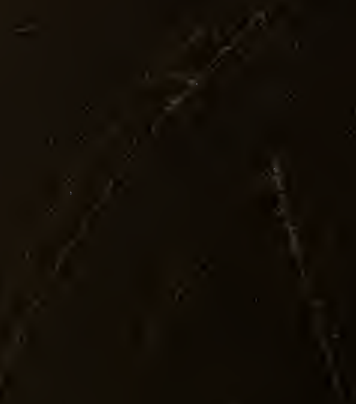